Systematic ID SPBTRNATHR.07 SPCTRNATHR.08 SPATRNATRP.01 SPBTRNATRP.03 SPATRNAILE.03 SPATRNATHR.01 SPATRNATHR.03 SPCTRNASER.09 SPCTRNASER.13 SPCTRNASER.07

SPCTRNASER.08 SPATRNAPRO. 01 SPBTRNASER.06 SPATRNASER.04 SPBTRNAPRO.08 SPRRNA.32

SPATRNAILE.04

SPBTRNAARG 07 SPATRNAARG.01 SPATRNASER.02 SPAPJ760.03C

SPATRNATHR.02 SPATRNATHR.04 SPBTRNATRP.02 SPBTRNAMET.06 SPCTRNATHR.09 SPBTRNAASN.03 SPATRNAMET 03 SPCTRNASER.12 SPATRNAALA.03 SPATRNATHR.05 SPATRNAILE.01 SPAC821.09

SPATRNAGLY.0 SPATRNASER 03 SPBTRNAASN.02 SPCTRNAMET.07 SPBTRNASER.05 SPCTRNAASN.06 SPATRNAVAL.02 SPCTRNAVAL.11 SPCTRNALYS.11 SPRRNA.36

SPCTRNASER.11 SPCTRNAGLY.10 SPBTRNAARG.05
Gene

SPBTRNATHR.07 SPCTRNATHR.08 SPATRNATRP.01 SPBTRNATRP.03 SPATRNAILE.03 SPATRNATHR.01 SPATRNATHR.03 SPCTRNASER.09 SPCTRNASER.13 SPCTRNASER.07 SPCTRNASER.08 SPATRNAPRO. 01 SPBTRNASER.06 SPATRNASER.04 SPBTRNAPRO.08 SPRRNA.32 PATRNAILE.04 SPBTRNAARG.07 SPATRNAARG.01 SPATRNASER.02 adg1

SPATRNATHR.02 SPATRNATHR.04 SPBTRNATRP.02 SPBTRNAMET.06 SPCTRNATHR.09 SPBTRNAASN.03 SPATRNAMET.03 SPCTRNASER.12 scn1

SPATRNATHR.05 SPATRNAILE.01 eng1

SPATRNAGLY.01 sup3

SPBTRNAASN.02 SPCTRNAMET.07 SPBTRNASER. 05 SPCTRNAASN.06 SPATRNAVAL.02 SPCTRNAVAL.11 SPCTRNALYS.11 SPRRNA.36

up9

SPCTRNAGLY.10 SPBTRNAARG.05
Description

RNA Threonine

tRNA Threonine

tRNA Tryptophan

tRNA Tryptophan

tRNA Isoleucine

tRNA Threonine

RNA Threonine

tRNA Serine

tRNA Serine

tRNA Serine

tRNA Serine

tRNA Proline

tRNA Serine

RNA Serine

tRNA Proline

5S rRNA

RNA Isoleucine

tRNA Arginine

tRNA Arginine

tRNA Serine

Schizosaccharomyces specific protein Adg1

tRNA Threonine

tRNA Threonine

tRNA Tryptophan

tRNA Methionine

tRNA Threonine

tRNA Asparagine

tRNA Methionine

tRNA Serine

tRNA Alanine

RNA Thre

tRNA Isoleucine

cell septum surface endo-1,3-beta-glucanase Eng1

RNA Glycine

tRNA Serine

tRNA Asparagine

RNA Methionine

tRNA Serine

tRNA Asparagine

tRNA Valine

tRNA Valine

tRNA Lysine

5S rRNA

tRNA Serine

tRNA Glycine

tRNA Arginine
logFC logCPM PValue FDR

$7.302136892 .98254886 \quad 2.60 \mathrm{E}-14$

$\begin{array}{llll}7.05184184 & 2.77382839 & 1.79 \mathrm{E}-12 & 1.74 \mathrm{E}-11\end{array}$

$\begin{array}{llll}6.96016403 & 2.70399118 & 1.83 \mathrm{E}-12 & 1.78 \mathrm{E}-11\end{array}$

$\begin{array}{llll}6.96016403 & 2.70399118 & 1.83 \mathrm{E}-12 & 1.78 \mathrm{E}-11\end{array}$

$\begin{array}{llll}6.95817202 & 2.70380494 & 3.52 \mathrm{E}-12 & 3.28 \mathrm{E}-11\end{array}$

$\begin{array}{llll}6.67787747 & 2.47501117 & 9.88 \mathrm{E}-10 & 6.90 \mathrm{E}-09\end{array}$

$6.30808157 \quad 2.20086998 \quad 5.47 \mathrm{E}-08$

$\begin{array}{llll}5.81975273 & 1.86249656 & 3.19 \mathrm{E}-06 & 1.38 \mathrm{E}-05\end{array}$

$\begin{array}{llll}5.74541083 & 1.81912221 & 1.45 \mathrm{E}-05 & 5.56 \mathrm{E}-05\end{array}$

$\begin{array}{llll}5.61432361 & 1.72959961 & 1.74 \mathrm{E}-05 & 6.60 \mathrm{E}-05\end{array}$

$\begin{array}{llll}5.61432361 & 1.72959961 & 1.74 \mathrm{E}-05 & 6.60 \mathrm{E}-05\end{array}$

$\begin{array}{llll}5.45583127 & 1.63338275 & 6.24 \mathrm{E}-05 & 0.000212934\end{array}$

$\begin{array}{llll}5.45250991 & 1.63320147 & 8.06 E-05 & 0.000269441\end{array}$

$\begin{array}{llll}5.36924958 & 1.58274486 & 0.00012393 & 0.000402739\end{array}$

$\begin{array}{lllll}5.28434898 & 1.53078682 & 0.00022719 & 0.000704673\end{array}$

$\begin{array}{llllll}5.28337577 & 1.53070785 & 0.00023208 & 0.000718841\end{array}$

$\begin{array}{llll}5.08486191 & 1.41993685 & 0.00044565\end{array}$

$\begin{array}{llll}5.07539911 & 1.41935756 & 0.00089813 & 0.002439833\end{array}$

$\begin{array}{lllll}4.84850619 & 1.299574 & 0.0014035 & 0.003635647\end{array}$

$\begin{array}{lllll}4.84504892 & 1.29932341 & 0.00268904 & 0.006528333\end{array}$

$\begin{array}{llll}4.52458565 & 6.28501313 & 5.17 \mathrm{E}-112 & 2.07 \mathrm{E}-109\end{array}$

$\begin{array}{llrr}4.49673202 & 2.96301526 & 4.24 \mathrm{E}-12 & 3.92 \mathrm{E}-11\end{array}$

$\begin{array}{llll}4.40678554 & 1.09805371 & 0.00955197 & 0.020211806\end{array}$

$4.36585837 \quad 2.8604366 \quad 5.99 \mathrm{E}-11$

$\begin{array}{llll}4.35063804 & 2.83979542 & 3.19 \mathrm{E}-10 & 2.36 \mathrm{E}-09\end{array}$

$\begin{array}{llll}4.20349169 & 2.72825058 & 1.40 \mathrm{E}-09 & 9.57 \mathrm{E}-09\end{array}$

$4.1935804 \quad 3.48568797 \quad 2.02 \mathrm{E}-15$

$\begin{array}{llll}4.0670198 & 3.37174036 & 1.56 \mathrm{E}-13 & 1.65 \mathrm{E}-12\end{array}$

$\begin{array}{llll}4.01425098 & 0.94617604 & 0.03500389 & 0.063707261\end{array}$

$4.010894261 .98441841 \quad 1.26 \mathrm{E}-05 \quad 4.90 \mathrm{E}-05$

$3.962748792 .96325849 \quad 1.45 \mathrm{E}-10 \quad 1.11 \mathrm{E}-09$

$\begin{array}{llll}3.94494236 & 1.94506089 & 1.93 \mathrm{E}-05 & 7.23 \mathrm{E}-05\end{array}$

$\begin{array}{llll}3.89530086 & 8.7113161 & 7.65 \mathrm{E}-168 & 1.04 \mathrm{E}-164\end{array}$

$3.85825593 \quad 3.19903723 \quad 3.91 \mathrm{E}-13 \quad 4.02 \mathrm{E}-12$

$3.80704815 \quad 3.16434916 \quad 4.08 \mathrm{E}-11 \quad 3.37 \mathrm{E}-10$

$\begin{array}{llll}3.75433108 & 3.95897437 & 6.92 \mathrm{E}-19 & 1.17 \mathrm{E}-17\end{array}$

$3.6897811 \quad 3.53944971 \quad 5.08 \mathrm{E}-14$

$\begin{array}{llll}3.67945637 & 1.7754652 & 0.00015844 & 0.000505232\end{array}$

$\begin{array}{lllr}3.60025581 & 3.82558172 & 5.69 \mathrm{E}-18 & 8.94 \mathrm{E}-17\end{array}$

$\begin{array}{llll}3.53731629 & 2.94316086 & 1.27 \mathrm{E}-09 & 8.78 \mathrm{E}-09\end{array}$

$\begin{array}{llll}3.51127976 & 2.92303542 & 1.50 \mathrm{E}-09 & 1.02 \mathrm{E}-08\end{array}$

$\begin{array}{llll}3.48549565 & 2.20135254 & 4.26 \mathrm{E}-06 & 1.80 \mathrm{E}-05\end{array}$

$\begin{array}{llll}3.43611949 & 1.63394842 & 0.00113608 & 0.003008188\end{array}$

$\begin{array}{llll}3.40567037 & 3.31065528 & 3.79 \mathrm{E}-10 & 2.77 \mathrm{E}-09\end{array}$

$\begin{array}{llll}3.37227696 & 3.6292627 & 1.16 \mathrm{E}-14 & 1.34 \mathrm{E}-13\end{array}$

$\begin{array}{llll}3.36973287 & 2.81813354 & 3.51 \mathrm{E}-08 & 2.01 \mathrm{E}-07\end{array}$ 
SPATRNALEU.02 SPATRNAALA.02 SPCTRNAASN.05 SPBTRNAGLY.03

SPBTRNAILE.06 SPATRNAPRO.02 SPBTRNAILE.05

SPBTRNAILE.08

SPNCRNA.1415

SPBTRNAILE.07

SPCTRNASER.10

SPBTRNAGLY.07

SPBTRNAGLY.08

SPNCRNA.1307

SPBTRNALYS.07

SPBTRNALYS.08

SPBTRNAGLY.06

SPBTRNALYS.06

SPAC5H10.03

SPNCRNA.452

SPNCRNA.941

SPBPB10D8.02C

SPATRNAARG.02

SPNCRNA.236

SPNCRNA.385

SPATRNAMET.01

SPNCRNA.489

SPNCRNA.1190

ScpofMt22

SPCC74.04

SPCTRNAGLN.06

SPCTRNAARG.08

SPAC26H5.09C

SPRRNA.12

SPCTRNAARG.09

SPAPB1E7.04C

SPCC18.01C

SPCTRNAGLN.05

SPBTRNAVAL.05

SPBTRNAVAL.08

SPATRNAPRO.03

SPNCRNA.482

SPNCRNA.485

SPAPJ695.02

SPATRNALYS.03

SPCTRNAILE.09

SPRRNA.33
SPATRNALEU.02

$\operatorname{scn} 2$

SPCTRNAASN.05

SPBTRNAGLY.03

SPBTRNAILE.06

SPATRNAPRO.02

SPBTRNAILE.05

SPBTRNAILE.08

\#N/A

SPBTRNAILE.07

SPCTRNASER.10

SPBTRNAGLY.07

SPBTRNAGLY.08

\#N/A

SPBTRNALYS.07

SPBTRNALYS.08

SPBTRNAGLY.06

SPBTRNALYS.06

SPAC5H10.03

\#N/A

$$
\# N / A
$$

SPBPB10D8.02C

SPATRNAARG.02

$\# \mathrm{~N} / \mathrm{A}$

$\# N / A$

SPATRNAMET.01

$\# \mathrm{~N} / \mathrm{A}$

$\# \mathrm{~N} / \mathrm{A}$

SPCC74.04

SPCTRNAGLN.06

SPCTRNAARG.08

SPAC26H5.09c

SPRRNA.12

PCTRNAARG.09

SPAPB1E7.04C

adg 3

SPCTRNAGLN.05

SPBTRNAVAL.05

SPBTRNAVAL.08

SPATRNAPRO.03

$\# \mathrm{~N} / \mathrm{A}$

$\# \mathrm{~N} / \mathrm{A}$

SPAPJ695.02

SPATRNALYS.03

SPCTRNAILE.09

SPRRNA.33
tRNA Leucine

tRNA Alanine

tRNA Asparagine

tRNA Glycine

tRNA Isoleucine

tRNA Proline

tRNA Isoleucine

tRNA Isoleucine

tRNA Isoleucine

\#N/A

tRNA Serine

tRNA Glycine

tRNA Glycine

tRNA Lysine

tRNA Lysine

tRNA Glycine

tRNA Lysine

phosphoglycerate mutase/6-phosphofructo-2-kinase family

$$
\# N / A
$$

$\# N / A$

arylsulfatase (predicted)

tRNA Arginine

$$
\# N / A
$$

$\# N / A$

tRNA Methionine

\section{$\#$ N/A}

\#N/A

amino acid transmembrane transporter (predicted)

tRNA Glutamine

tRNA Arginine

oxidoreductase involved in NADPH regeneration (predicted) $5 S$ rRNA

tRNA Arginine

chitinase (predicted)

beta-glucosidase Adg3 (predicted)

tRNA Glutamine

tRNA Valine

tRNA Valine

tRNA Proline

$\# N / A$

$\# \mathrm{~N} / \mathrm{A}$

Schizosaccharomyces pombe specific protein

tRNA Lysine

tRNA Isoleucine

5S rRNA $\begin{array}{llll}3.34194609 & 1.5825827 & 0.00200955\end{array}$

$\begin{array}{llll}3.33075531 & 2.09692618 & 2.65 \mathrm{E}-05\end{array}$

$\begin{array}{lll}3.28911938 & 3.97013639 & 1.81 \mathrm{E}-17\end{array}$

$\begin{array}{lll}3.26962883 & 3.82578426 & 1.58 \mathrm{E}-16\end{array}$

$\begin{array}{lll}3.21645401 & 2.70435042 & 3.96 \mathrm{E}-07\end{array}$

$\begin{array}{lll}3.21274245 & 2.02266588 & 8.25 \mathrm{E}-05\end{array}$

$3.20588742 .94297672 \quad 3.53 \mathrm{E}-08$

$3.2058874 \quad 2.94297672 \quad 3.53 \mathrm{E}-08$

$\begin{array}{llll}3.14534871 & 1.4760402 & 0.00492191\end{array}$

$\begin{array}{lll}3.07231509 & 2.83971851 \quad 6.67 \mathrm{E}-08\end{array}$

$3.02275367 \quad 1.90463094 \quad 0.00027666$

$\begin{array}{lll}3.01465693 & 3.84749835 & 2.63 \mathrm{E}-15\end{array}$

$\begin{array}{lll}3.01465693 & 3.84749835 & 2.63 \mathrm{E}-15\end{array}$

$2.957379695 .10641622 \quad 2.60 \mathrm{E}-23$

$\begin{array}{llll}2.92192974 & 2.2018837 & 0.00016818\end{array}$

$\begin{array}{llll}2.92192974 & 2.2018837 & 0.00016818\end{array}$

$\begin{array}{lll}2.90058298 & 3.24837399 & 2.92 \mathrm{E}-09\end{array}$

$2.86848241 \quad 2.44652796 \quad 1.79 \mathrm{E}-05$

$\begin{array}{lll}2.8558236 & 7.1561947 & 2.13 \mathrm{E}-115\end{array}$

$\begin{array}{llll}2.77728638 & 1.77477048 & 0.00887338\end{array}$

$2.77205913 \quad 3.64217874 \quad 6.60 \mathrm{E}-09$

$2.73205452 \quad 5.18883517 \quad 7.00 \mathrm{E}-21$

$\begin{array}{llll}2.72554163 & 1.72991398 & 0.00228812\end{array}$

$\begin{array}{lll}2.72539128 & 3.60486276 & 1.86 \mathrm{E}-08\end{array}$

$2.64310537 \quad 1.68245606 \quad 0.00382054$

$2.61474735 \quad 3.96990283 \quad 8.30 \mathrm{E}-12$

$\begin{array}{llll}2.53446695 & 1.633754 & 0.00820438\end{array}$

$2.5336471 \quad 2.65822113 \quad 4.25 \mathrm{E}-05$

$\begin{array}{llll}2.52909881 & 4.30977699 & 2.82 \mathrm{E}-14\end{array}$

$\begin{array}{lll}2.52817846 & 7.37006481 & 1.76 \mathrm{E}-30\end{array}$

$\begin{array}{lll}2.50810062 & 4.28557988 & 3.28 \mathrm{E}-16\end{array}$

$2.50759377 \quad 3.64301096 \quad 1.58 \mathrm{E}-10$

\begin{tabular}{lll}
$2.50193593 \quad 8.49755573 \quad 4.01 \mathrm{E}-172$ \\
\hline
\end{tabular}

$\begin{array}{lll}2.4923572 & 4.1543153 & 7.48 \mathrm{E}-15\end{array}$

$2.4739155 \quad 1.16863057 \quad 0.04602063$

$\begin{array}{llll}2.44077933 \quad 8.52647827 \quad 4.30 \mathrm{E}-114 & 0\end{array}$

$\begin{array}{lll}2.42988641 & 8.49375266 & 2.60 \mathrm{E}-58\end{array}$

$2.41651096 \quad 4.21287927 \quad 1.17 \mathrm{E}-14$

$\begin{array}{llll}2.37415287 & 2.1336531 & 0.00194502\end{array}$

$\begin{array}{llll}2.37290055 & 2.1337201 & 0.00247737\end{array}$

$\begin{array}{llr}2.36689445 & 2.70518789 & 1.90 \mathrm{E}-05\end{array}$

$2.34711249 \quad 1.53075175 \quad 0.02346546$

$\begin{array}{llll}2.34711249 & 1.53075175 & 0.02346546\end{array}$

$\begin{array}{lll}2.3459032 & 6.22671448 & 3.32 \mathrm{E}-28\end{array}$

$2.33098285 \quad 2.68124005 \quad 8.21 \mathrm{E}-05$

$\begin{array}{lll}2.32665277 & 2.09690279 & 0.00076\end{array}$

$\begin{array}{llll}2.30346332 & 1.81983277 & 0.00594325\end{array}$
0.005038688

$9.74 \mathrm{E}-05$

2.69E-16

2.16E-15

$1.93 \mathrm{E}-06$

0.000275111

2.01E-07

$2.01 \mathrm{E}-07$

0.0112065

3.63E-07

0.0008431

3.23E-14

3.23E-14

$6.20 \mathrm{E}-22$

0.000533056

0.000533056

$1.91 \mathrm{E}-08$

6.77E-05

1.03E-112

0.018911167

4.13E-08

1.47E-19

0.005676515

1.09E-07

0.008911136

$7.42 \mathrm{E}-11$

0.017606692

0.000149359

3.16E-13

6.53E-29

4.39E-15

$1.21 \mathrm{E}-09$

$6.82 \mathrm{E}-169$

$8.85 \mathrm{E}-14$

0.080589395

1.83E-111

3.93E-56

1.35E-13

0.004896735

0.00607952

7.14E-05

0.045082566

0.045082566

$1.05 \mathrm{E}-26$

0.000273842

0.002108157

0.013286398 
SPBPB10D8.01 SPAC19D5.07 SPBTRNALEU.0 SPAC869.01

SPBTRNAVAL.07 SPBTRNALEU.07 SPNCRNA.1666

SPCTRNALYS.10

SPBTRNAASN.04 SPBTRNATHR.06 SPCC757.06

SPCTRNALEU.13

SPNCRNA.1672

SPCTRNALEU.12

SPCTRNATHR.10

SPATRNALYS.05

SPATRNAARG.03

SPBC1861.02

SPNCRNA.163

SPATRNASER.01

SPCC191.05C

SPAC2E1P3.05C

SPBC36.01C

SPBTRNAASN.01

SPATRNALYS.02

SPRRNA.24

SPNCRNA.421

SPATRNATYR.01

SPATRNALYS.01

SPNCRNA.1609

SPAC19G12.05

SPBTRNAALA.08

SPBTRNAALA.10

SPRRNA.15

SPNCRNA.694

SPAC6G10.12C

SPBPB2B2.05

SPAC22A12.04C

SPAC19G12.16C

SPBTRNAHIS.02

SPNCRNA.113

SPBPB2B2.01

SPNCRNA.1674

SPAC8E11.10

SPRRNA. 27

SPAC23H4.06

ScpofMt15
SPBPB10D8.01

uga1

SPBTRNALEU.06

SPAC869.01

SPBTRNAVAL.07

SPBTRNALEU.07

\#N/A

PCTRNALYS.10

SPBTRNAASN.04

SPBTRNATHR.06

SPCC757.06

SPCTRNALEU.13

$\# \mathrm{~N} / \mathrm{A}$

SPCTRNALEU.12

PCTRNATHR.10

SPATRNALYS.05

SPATRNAARG.03

abp2

$\# N / A$

SPATRNASER.01

SPCC191.05C

SPAC2E1P3.05C

SPBC36.01C

SPBTRNAASN.01

SPATRNALYS.02

SPRRNA.24

\#N/A

SPATRNATYR.01

SPATRNALYS.01

$\# \mathrm{~N} / \mathrm{A}$

mce1

SPBTRNAALA.08

SPBTRNAALA.10 SPRRNA.15

$\# \mathrm{~N} / \mathrm{A}$

ace2

SPBPB2B2.05

rps2201

adg2

SPBTRNAHIS.02

\#N/A

SPBPB2B2.01

$\# \mathrm{~N} / \mathrm{A}$

SPAC8E11.10

SPRRNA.27

gln1

\#N/A cysteine transmembrane transporter (predicted)

$2.29077014 \quad 4.92185253 \quad 1.84 \mathrm{E}-20$

$\begin{array}{lllll} & 2.29 \\ \text {-aminobutyrate aminotransferase (GABA transaminase) } & 2.28647426 & 8.98402213 & 4.20 \mathrm{E}-150\end{array}$

tRNA Leucine

$\begin{array}{lll}2.25784046 & 4.13555807 & 5.14 \mathrm{E}-11\end{array}$

hydrolase activity, implicated in cellular detoxification (prec $2.257731396 .80189575 \quad 4.82 \mathrm{E}-68$

tRNA Valine

tRNA Leucine

tRNA Lysine

$\# \mathrm{~N} / \mathrm{A}$

$2.25589057 \quad 2.06148133 \quad 0.00360085$

$\begin{array}{lll}2.24689908 & 4.12689331 & 6.70 \mathrm{E}-11\end{array}$

$\begin{array}{lll}2.23624795 & 3.34335141 & 2.60 \mathrm{E}-07\end{array}$

$2.21874423 \quad 1.77586156 \quad 0.0072329$

$2.19605356 \quad 4.60161117 \quad 7.30 \mathrm{E}-16$

$\begin{array}{lll}2.19548071 & 3.31288689 & 1.98 \mathrm{E}-07\end{array}$

tRNA Threonine

Schizosaccharomyces pombe specific protein, similar to a $r_{1} \quad 2.19208962 .02294011 \quad 0.00754829$

tRNA Leucine

$\#$ N/A

tRNA Leucine

$2.16665168 \quad 4.22023653 \quad 1.16 \mathrm{E}-10$

$\begin{array}{lll}2.16303625 & 4.12808496 & 7.13 \mathrm{E}-11\end{array}$

$2.15630571 \quad 4.21207338 \quad 1.51 \mathrm{E}-10$

$2.1445789 \quad 3.94118877 \quad 2.41 \mathrm{E}-10$

$\begin{array}{lll}2.14228147 & 2.20212783 & 0.00239305\end{array}$

$\begin{array}{llll}2.13928578 & 1.72998456 & 0.01265165\end{array}$

$2.13463707 \quad 6.84966478 \quad 6.66 \mathrm{E}-66$

$2.13358526 \quad 1.73017816 \quad 0.01742508$

$\begin{array}{lll}2.13049263 & 4.33851714 & 6.86 \mathrm{E}-11\end{array}$

$2.12380216 \quad 5.64780032 \quad 6.06 \mathrm{E}-17$

$\begin{array}{llll}\text { nucleoside 2-deoxyribosyltransferase (predicted) } & 2.12380216 & 5.64780032 & 6.06 \mathrm{E}-17 \\ \text { fungal cellulose binding domain protein } & 2.11946234 & 7.21236989 & 1.46 \mathrm{E}-73 \\ \text { spermidine family transmembrane transporter (predicted) } & 2.11926041 & 6.94753489 & 1.75 \mathrm{E}-55\end{array}$

$\begin{array}{llll}\text { spermidine family transmembrane transporter (predicted) } & 2.11926041 & 6.94753489 & 1.75 \mathrm{E}-55 \\ \text { tRNA Asparagine } & 2.11379527 & 4.13634986 & 1.30 \mathrm{E}-10\end{array}$

RNA Lysine

5S rRNA

\#N/A

$\begin{array}{lll}2.11189239 & 2.68135494 & 0.0002228\end{array}$

$\begin{array}{llll}2.08493751 & 2.16762658 & 0.00128393\end{array}$

$\begin{array}{lll}2.06460354 & 3.61847941 & 8.39 \mathrm{E}-08\end{array}$

$\begin{array}{llll}2.04658951 & 2.32971356 & 0.00158103\end{array}$

$\begin{array}{llll}2.03767603 & 1.68260221 & 0.01844237\end{array}$

$\begin{array}{lll}1.99093375 & 3.63079376 \quad 2.42 \mathrm{E}-07\end{array}$

$1.9857223 \quad 5.52449849 \quad 1.90 \mathrm{E}-15$

$\begin{array}{llll}1.97777577 & 1.36085874 & 0.08438818\end{array}$

$\begin{array}{llll}1.97777577 & 1.36085874 & 0.08438818\end{array}$

$\begin{array}{llll}1.97465569 & 4.22186002 & 4.92 \mathrm{E}-11\end{array}$

$1.973062342 .44899455 \quad 0.00493986$

$\begin{array}{lll}1.96595386 & 6.64027552 & 2.10 \mathrm{E}-40\end{array}$

$1.955078043 .91241305 \quad 2.70 \mathrm{E}-08$

$1.95408934 \quad 6.86628746 \quad 4.91 \mathrm{E}-54$

$\begin{array}{lll}1.92383236 & 8.55341927 \quad 4.48 \mathrm{E}-52\end{array}$

$\begin{array}{lll}1.92115689 & 1.86297231 & 0.01116042\end{array}$

$1.9112858 \quad 4.12886886 \quad 6.03 \mathrm{E}-10$

$1.9064528 \quad 8.39727059 \quad 5.32 \mathrm{E}-92$

$\begin{array}{lll}1.8991248 & 4.51807225 & 5.27 \mathrm{E}-12\end{array}$

$\begin{array}{lll}1.89263433 & 6.39663749 & 3.12 \mathrm{E}-28\end{array}$

$\begin{array}{lll}1.87789551 & 3.09646132 & 0.0001238\end{array}$

$\begin{array}{lll}1.86159094 & 7.92982414 & 5.95 \mathrm{E}-75\end{array}$

$\begin{array}{llll}1.84929904 & 3.24873812 & 0.00013141\end{array}$

3.69E-19

$3.57 \mathrm{E}-147$

4.15E-10

9.94E-66

0.008464167

$5.31 \mathrm{E}-10$

1.30E-06

0.015760454

9.46E-15

$1.00 \mathrm{E}-06$

0.016374288

8.99E-10

5.63E-10

1.16E-09

1.81E-09

0.005900262

0.026018283

$1.22 \mathrm{E}-63$

0.03469212

$5.43 \mathrm{E}-10$

8.63E-16

3.31E-71

2.39E-53

$1.00 \mathrm{E}-09$

0.000693248

0.003364327

4.49E-07

0.004052325

0.036570433

$1.21 \mathrm{E}-06$

2.38E-14

0.135598948

0.135598948

$3.98 \mathrm{E}-10$

0.011231454

$1.42 \mathrm{E}-38$

$1.56 \mathrm{E}-07$

$6.08 \mathrm{E}-52$

5.17E-50

0.023253714

4.31E-09

$1.72 \mathrm{E}-89$

4.80E-11

9.91E-27

$5 S$ rRNA

glutamate-ammonia ligase $\mathrm{G} \ln 1$

$\# N / A$

$1.40 \mathrm{E}-72$

0.000425209 
SPAC27D7.09c SPCC $31 \mathrm{H} 12.02 \mathrm{C}$ SPNCRNA.231 SPCTRNAPHE.04

SPNCRNA.1375 SPCTRNAVAL.09 SPNCRNA.51

SPAC869.04

SPRRNA.14

SPBC24C6.04

SPBTRNAGLN.04

SPBC1734.11

SPCC11E10.01

SPBTRNAGLN.01

SPBTRNALEU.10

SPBP8B7.26

SPBTRNALEU.05

SPATRNAVAL.01

SPAC821.08C

SPATRNALYS.04

SPBTRNALEU.08

SPAC56F 8.12

SPAC31G5.03

SPAC23C11.05

SPATRNAVAL.03

SPBTRNAGLN.02

SPCTRNALYS.12

SPRRNA.39

SPBC530.02

SPAC750.01

SPAC56E4.03

SPAC1B3.03C

SPBC3E7.02C

SPBTRNAARG.04

SPBC839.13C

SPBC409.08

SPAC14C4.01C

SPNCRNA.1036

SPNCRNA.1388

SPBP22H7.08

SPBTRNAGLY.05

SPBC660.14

SPBP4H10.13

SPATRNAVAL.04

SPCC24B10.09

ScpofMt21

SPAC23A1.11
SPAC27D7.09c

mug73

$\# N / A$

SPCTRNAPHE.04

\#N/A

SPCTRNAVAL.09

$\#$ N/A

PAC869.04

SPRRNA.14

put2

SPBTRNAGLN.04

mas5

SPCC11E10.01

SPBTRNAGLN.01

SPBTRNALEU.10

SPBP8B7.26

SPBTRNALEU.05

SPATRNAVAL.01

slp1

SPATRNALYS.04

SPBTRNALEU.08

SPAC56F8.12

rps1101

ipp1

SPATRNAVAL.03

SPBTRNAGLN.02

SPCTRNALYS.12

SPRRNA.39

SPBC530.02

SPAC750.01

SPAC56E4.03

wis2

hsp16

SPBTRNAARG.04

rpl1601

SPBC409.08

SPAC14C4.01C

$\# \mathrm{~N} / \mathrm{A}$

$\# \mathrm{~N} / \mathrm{A}$

rps1002

SPBTRNAGLY.05

mik1

rps2302

SPATRNAVAL.04

rps1702

\#N/A

rpl1602
But2 family protein, similar to cell surface molecules

1.848372116 .87606285

$\begin{array}{lll}1.846634 & 3.66742878 \quad 7.68 \mathrm{E}-06\end{array}$

tRNA Phenylalanine

$\# \mathrm{~N} / \mathrm{A}$

\#N/A

tRNA Valine

$\# \mathrm{~N} / \mathrm{A}$

$183068878 \quad 1.29944668 \quad 0.12922588$

$\begin{array}{lll}1.82886387 & 1.82108159 & 0.04402998\end{array}$

$\begin{array}{lll}1.79312354 & 2.47535905 & 0.00351961\end{array}$

formamidase-like protein, implicated in cellular detoxificati $1.78216793 \quad 4.24789093 \quad 7.87 \mathrm{E}-09$

$5 S$ rRNA

$1.74142905 \quad 2.7966946 \quad 0.00056912$

delta-1-pyrroline-5-carboxylate dehydrogenase Put2 (predic $1.73903542 \quad 8.543841 \quad 4.44 \mathrm{E}-83$

$\begin{array}{lll}1.73166206 & 4.35609295 & 4.95 \mathrm{E}-10\end{array}$

DNAJ domain protein Mas5 (predicted) $\quad 1.72438767 \quad 7.88094077 \quad 5.01 \mathrm{E}-53$

$\begin{array}{llll}\text { cystathionine beta-lyase (predicted) } & 1.72028413 & 8.10084356 & 2.45 \mathrm{E}-80\end{array}$

$\begin{array}{llll}\text { tRNA Glutamine } & 1.7082444 & 4.370776 & 9.25 \mathrm{E}-10\end{array}$

$1.69378973 \quad 2.6575265 \quad 0.00146435$

$\begin{array}{lllll}\text { Schizosaccharomyces specific protein } & 1.69058815 & 6.29587135 & 2.94 \mathrm{E}-24\end{array}$

tRNA Leucine

tRNA Valine

$\begin{array}{rrr}1.69058815 & 6.29587135 & 2.94 \mathrm{E}-24 \\ 1.68947408 & 1.94520751 & 0.02145409\end{array}$

$1.68366944 \quad 3.28189457 \quad 5.49 \mathrm{E}-05$

substrate-specific mitotic metaphase/anaphase APC coacti $1.68099315 \quad 6.89283603 \quad 1.87 \mathrm{E}-42$

$\begin{array}{lllll}\text { tRNA Lysine } & 1.67982583 & 2.26712689 & 0.00910418\end{array}$

$\begin{array}{llll}\text { tRNA Leucine } & 1.6782949 & 1.2354933 & 0.1237315\end{array}$

DUF2434 family conserved fungal multispanning membran $1.678206894 .7182597 \quad 2.41 \mathrm{E}-11$

$\begin{array}{lllll}40 \mathrm{~S} \text { ribosomal protein S11 (predicted) } & 1.67803239 & 6.52261936 & 1.13 \mathrm{E}-26\end{array}$

inorganic pyrophosphatase Ipp1 (predicted) $\quad \begin{array}{llll}1.67787857 & 8.18427382 & 1.65 \mathrm{E}-76\end{array}$

tRNA Valine

tRNA Glutamine

tRNA Lysine

5S rRNA

$1.67595443 \quad 1.23558162 \quad 0.12205669$

$\begin{array}{llr}1.67432285 & 4.34876718 \quad 1.41 \mathrm{E}-09\end{array}$

$\begin{array}{lll}1.65562744 & 2.09784029 & 0.02101027\end{array}$

$\begin{array}{lll}1.65473451 & 2.09772787 \quad 0.01961153\end{array}$

$\begin{array}{llll}\text { transmembrane transporter (predicted) } & 1.64874148 & 5.07001892 & 2.61 \mathrm{E}-10 \\ \text { NADP-dependent aldo/keto reductase, unknown biological I } 1.64365061 & 5.76685808 & 5.22 \mathrm{E}-17\end{array}$

aromatic aminotransferase (predicted) $\quad$\begin{tabular}{lllll}
\hline & 1.63079883 & 7.93273337 & $4.43 \mathrm{E}-31$
\end{tabular}

cyclophilin family peptidyl-prolyl cis-trans isomerase Wis2 $1.62289204 \quad 7.96177826 \quad 1.91 \mathrm{E}-42$

heat shock protein Hsp16

tRNA Arginine

60S ribosomal protein L13/L16 (predicted)

spermine family transmembrane transporter (predicted)

DUF1770 family protein

\section{$\# N / A$}

$\#$ N/A

$40 \mathrm{~S}$ ribosomal protein $\mathrm{S} 10$ (predicted)

tRNA Glycine

mitotic inhibitor kinase Mik1

40S ribosomal protein S23 (predicted)

tRNA Valine

40S ribosomal protein S17 (predicted)

\#N/A

$60 S$ ribosomal protein L13/L16 (predicted) $\begin{array}{lll}1.61798652 & 7.61580616 \quad 5.30 \mathrm{E}-38\end{array}$

$\begin{array}{llll}1.61370617 & 3.07835755 & 0.00019687\end{array}$

$\begin{array}{lll}1.6113427 & 7.2532594 & 7.04 \mathrm{E}-46\end{array}$

$\begin{array}{lll}1.610446 & 7.91898683 & 2.22 \mathrm{E}-52\end{array}$

$\begin{array}{lll}1.60867957 & 9.30030649 \quad 2.81 \mathrm{E}-94\end{array}$

$\begin{array}{lll}1.60633838 & 7.97338142 \quad 1.58 \mathrm{E}-40\end{array}$

$\begin{array}{lll}1.60530373 & 3.55531641 \quad 2.99 \mathrm{E}-05\end{array}$

$\begin{array}{lll}1.60250789 & 6.92525947 \quad 5.39 \mathrm{E}-42\end{array}$

$\begin{array}{llll}1.58105046 & 2.79790608 & 0.00145857\end{array}$

$1.57311543 \quad 5.39965034 \quad 3.11 \mathrm{E}-16$

$\begin{array}{lll}1.56040813 & 6.98832069 & 2.90 \mathrm{E}-41\end{array}$

$\begin{array}{llll}1.55377853 & 1.68297886 & 0.12982573\end{array}$

$1.54777236 \quad 7.04606713 \quad 8.67 \mathrm{E}-43$

$\begin{array}{lll}1.54626244 & 4.88286811 & 2.64 \mathrm{E}-11\end{array}$

$1.540541497 .60977955 \quad 6.02 \mathrm{E}-55$
$1.49 \mathrm{E}-42$

$5.30 \mathrm{E}-06$

$3.11 \mathrm{E}-05$

0.077747957

0.195114734

0.077622802

0.008307689

$4.88 \mathrm{E}-08$

0.001621807

$1.26 \mathrm{E}-80$

$3.58 \mathrm{E}-09$

$5.98 \mathrm{E}-51$

$6.42 \mathrm{E}-78$

$6.48 \mathrm{E}-09$

0.003775785

7.29E-23

0.04165337

0.000189319

$1.50 \mathrm{E}-40$

0.019348506

0.188185168

2.05E-10

3.26E-25

4.01E-74

0.185982031

9.60E-09

0.040873321

0.038526692

1.96E-09

$7.48 \mathrm{E}-16$

$1.69 \mathrm{E}-29$

$1.51 \mathrm{E}-40$

3.00E-36

0.000617663

$6.39 \mathrm{E}-44$

2.61E-50

1.01E-91

$1.08 \mathrm{E}-38$

0.000108289

4.03E-40

0.00376397

4.17E-15

2.05E-39

0.195933488

7.11E-41

2.23E-10

8.03E-53 
SPNCRNA.383 SPBTRNATYR.02 SPCP20C8.02C SPBC29A3.12

SPATRNAILE.02 SPBTRNAGLN.03 SPNCRNA.539

SPNCRNA.1673

ScpofMt23

SPBC21B10.08C

SPAC25G10.05C

SPNCRNA.06

SPAC4G9.03

SPCTRNAPHE.05

SPATRNALEU.04

SPCTRNALEU.11

SPNCRNA.264

SPBC1271.09

SPBTRNAALA.09

SPBTRNAALA.11

SPRRNA.10

SPCC1827.03C

SPNCRNA.897

SPCTRNAGLY.12

SPAC1071.07c

SPNCRNA.122

SPBTRNAHIS.01

SPCTRNAHIS.04

SPAC513.07

SPAC1F8.07C

SPBTRNAMET.05

SPAC23H4.05C

SPNCRNA.1260

SPNCRNA.215

SPNCRNA.1134

SPAC1687.06C

SPBP4G3.02

SPBP26C9.02C

SPNCRNA.609

SPNCRNA.1166

SPAC11D3.14C

SPBC354.15

SPBC1773.03C

SPATRNAASP.02

SPBTRNATYR.03

SPNCRNA.716

SPBC776.11
SPBTRNATYR.02 SPCP20C8.02C

rps902

SPATRNAILE.02

SPBTRNAGLN.03

$\# N / A$
$\# N / A$

$\# \mathrm{~N} / \mathrm{A}$

SPBC21B10.08C his1

\#N/A

adk1

SPCTRNAPHE.05

SPATRNALEU.04

SPCTRNALEU.11

\#N/A

gp1

SPBTRNAALA.09

SPBTRNAALA.11

SPRRNA.10

SPCC1827.03C

$\# N / A$

SPCTRNAGLY.12

rps 1502

$\# \mathrm{~N} / \mathrm{A}$

SPBTRNAHIS.01

SPCTRNAHIS.04

SPAC513.07

pdc101

SPBTRNAMET.05

SPAC23H4.05C

$\# \mathrm{~N} / \mathrm{A}$

rpl44

pho1

car1

$\#$ N/A

$\# \mathrm{~N} / \mathrm{A}$

SPAC11D3.14c

fap1

SPBC1773.03C

SPATRNAASP.02

SPBTRNATYR.03

$\# \mathrm{~N} / \mathrm{A}$

rpl2801
\#N/A

RNA Tyrosine

S. pombe specific UPF0321 family protein 1

$40 \mathrm{~S}$ ribosomal protein $\mathrm{S} 9$ (predicted)

tRNA Isoleucine

tRNA Glutamine

\section{$\# N / A$}

$\# N / A$

\#N/A

antibiotic biosynthesis monooxygenase-like domain (predic

ATP phosphoribosyltransferase

$\# \mathrm{~N} / \mathrm{A}$

adenylate kinase Adk1

tRNA Phenylalanine

RNA Leucine

tRNA Leucine

\#N/A

$\begin{array}{llll}1.53793337 & 2.65853358 & 0.00920174\end{array}$

$\begin{array}{llll}1.53691937 & 2.44738253 & 0.01112197\end{array}$

$\begin{array}{lll}1.53651748 & 3.84809577 \quad 2.36 \mathrm{E}-05\end{array}$

$\begin{array}{lll}1.53160696 & 7.35137211 & 1.43 \mathrm{E}-40\end{array}$

$\begin{array}{llll}1.52772452 & 3.02304691 & 0.00070414\end{array}$

$\begin{array}{lll}1.5241242 & 4.27120881 & 3.97 \mathrm{E}-08\end{array}$

$\begin{array}{llll}1.52202612 & 3.43274297 & 0.00023373\end{array}$

$1.512394575 .05020935 \quad 1.56 \mathrm{E}-11$

$1.510833383 .65614327 \quad 8.03 \mathrm{E}-05$

$\begin{array}{lll}1.51031029 & 6.96681209 & 1.38 \mathrm{E}-35 \\ 1.50544027 & 7.34418697 & 2.92 \mathrm{E}-39\end{array}$

$\begin{array}{llll}1.50335192 & 2.92529245 & 0.00150608\end{array}$

$\begin{array}{lll}1.49580735 & 7.29373427 & 8.77 \mathrm{E}-46\end{array}$

$\begin{array}{llll}1.49462185 & 1.16832164 & 0.20273754\end{array}$

$\begin{array}{llll}1.49277897 & 1.1682533 & 0.19968721\end{array}$ $\begin{array}{llll}1.491439 & 1.16818496 & 0.19947276\end{array}$ $\begin{array}{llll}1.491439 & 1.16818496 & 0.19947276\end{array}$

plasma membrane glycerophosphodiester transmembrane $1.49013271 \quad 6.13177703 \quad 1.09 \mathrm{E}-16$

$\begin{array}{lllll}\text { tRNA Alanine } & 1.48915252 & 1.16852069 & 0.20298359\end{array}$

SS rRNA

acetyl-CoA ligase (predicted)

$\# \mathrm{~N} / \mathrm{A}$

tRNA Glycine

40S ribosomal protein S15 (predicted)

\#N/A

tRNA Histidine

tRNA Histidine

flavonol reductase/cinnamoyl-CoA reductase family

pyruvate decarboxylase (predicted)

tRNA Methionine

Schizosaccharomyces pombe specific protein

$$
\# N / A
$$

$\# N / A$

$\# \mathrm{~N} / \mathrm{A}$

60S ribosomal protein L28/L44 (predicted)

acid phosphatase Pho1

arginase Car1

$\# N / A$

$\# N / A$

5-oxoprolinase (ATP-hydrolizing) (predicted)

L-pipecolate oxidase

$\begin{array}{lll}1.48915252 & 1.16852069 & 0.20298359 \\ 1.48915252 & 1.16852069 & 0.20298359\end{array}$

$\begin{array}{llll}1.48606437 & 1.16901075 & 0.19770939\end{array}$

$1.483774497 .88444369 \quad 1.93 \mathrm{E}-57$

$\begin{array}{lll}1.47413855 & 6.97952029 & 1.28 \mathrm{E}-29\end{array}$

$\begin{array}{llll}1.47100498 & 2.72972277 & 0.00389388\end{array}$

$\begin{array}{lll}1.46564215 & 7.49992511 & 4.39 \mathrm{E}-45\end{array}$

$\begin{array}{llll}1.46278411 & 1.63407034 & 0.09125165\end{array}$

$\begin{array}{llll}1.46248659 & 1.63365747 & 0.09638616\end{array}$

$\begin{array}{llll}1.46248659 & 1.63365747 & 0.09638616\end{array}$

$1.461902397 .18508581 \quad 7.95 \mathrm{E}-26$

$\begin{array}{lll}1.45896226 & 9.91088554 \quad 1.99 E-92\end{array}$

$\begin{array}{llll}1.45851744 & 3.26570297 & 0.00024355\end{array}$

$\begin{array}{llll}1.45763978 & 1.98534455 & 0.03650469\end{array}$

$\begin{array}{lll}1.45715621 & 2.13382219 & 0.02965261\end{array}$

$\begin{array}{llll}1.45415355 & 1.98515369 & 0.03391372\end{array}$

$1.45250695 \quad 4.46376186 \quad 8.00 \mathrm{E}-08$

$\begin{array}{lll}1.42929874 & 7.1412101 & 4.82 \mathrm{E}-37\end{array}$

$\begin{array}{lll}1.42253581 & 8.97342804 & 5.24 \mathrm{E}-46\end{array}$

$1.4205018 \quad 7.20239787$

$1.41106274 \quad 7.94472912 \quad 3.70 \mathrm{E}-48$

$\begin{array}{lll}1.40736713 & 2.94543453 & 0.00253867\end{array}$

$1.404613197 .94725094 \quad 1.74 \mathrm{E}-48$

$\begin{array}{llll}1.40229189 & 6.34473697 & 2.89 \mathrm{E}-20\end{array}$

aminotransferase class-III, possible transaminase, unknowr $\begin{array}{lll}1.39789059 & 8.0360416 & 2.90 \mathrm{E}-54\end{array}$

tRNA Aspartic acid

$\begin{array}{lll}1.39582263 & 4.21513695 & 1.20 \mathrm{E}-06\end{array}$

$\begin{array}{lll}1.39045447 & 2.47545803 & 0.01937021\end{array}$

$\begin{array}{llll}1.38949673 & 4.88391658 \quad 1.22 \mathrm{E}-08\end{array}$

$1.388802257 .17918175 \quad 5.19 \mathrm{E}-32$

0.01953146

0.023180704

8.74E-05

9.83E-39

0.001971867

2.25E-07

0.000723614

$1.35 \mathrm{E}-10$

0.000268595

$6.80 \mathrm{E}-34$

$1.84 \mathrm{E}-37$

0.003877736

$7.86 \mathrm{E}-44$

0.283774982

0.280295268

0.280109812

0.280109812

$1.51 \mathrm{E}-15$

0.283926691

0.283926691

0.27809268

$2.79 \mathrm{E}-55$

4.50E-28

0.009062193

$3.83 \mathrm{E}-43$

0.145323542

0.152005062

0.152005062

2.14E-24

6.79E-90

0.000752325

0.066050091

0.055162925

0.062105175

4.30E-07

2.56E-35

4.82E-44

$3.40 \mathrm{E}-25$

$3.65 \mathrm{E}-46$

0.006212016

1.77E-46

$5.72 \mathrm{E}-19$

3.73E-52

5.50E-06

0.038151735

7.31E-08

$60 S$ ribosomal protein L27/L28

$2.12 \mathrm{E}-30$ 
SPAPJ760.02C SPATRNALEU.03 SPBTRNAMET.04 SPCTRNAASP.07

SPNCRNA.1181

SPCC1795.05C

SPBC839.14c

SPNCRNA.1400

SPAC922.07c

SPCTRNAGLU.10

SPNCRNA.34

SPAC2E1P3.04

SPBTRNAPRO.04

SPNCRNA.1096

SPAC1F7.13C

SPCC4G3.06C

SPBC1289.03C

SPNCRNA. 219

SPAC977.14c

SPNCRNA.1090

SPCC1795.06

SPAC1786.02

SPAC1486.07C

SPCC1682.08C

SPBTRNAPRO.07

SPNCRNA.232

SPAC23G3.04

SPCC970.05

SPBCPT2R1.08C

SPAP7G5.05

SPBC3E7.07C

SPNCRNA.373

SPAC9.02C

SPBC887.07

SPATRNAPHE.01

SPAC27F1.05C

SPNCRNA.977

SPNCRNA.1659

SPNCRNA.199

SPNCRNA.1022

SPAC13G7.06

SPCC306.11

SPNCRNA.1139

SPCC70.09C

SPBTRNAGLY.04

SPBC4F6.12

SPCC569.07 abp1

PATRNALEU.03

SPBTRNAMET.04

SPCTRNAASP.07

\#N/A

ura6

efm4

\#N/A

atd2

SPCTRNAGLU.10

$\# \mathrm{~N} / \mathrm{A}$

cao1

SPBTRNAPRO.04

\#N/A

rpl801

mrpl4

spi1

\#N/A

SPAC977.14C

\#N/A

map2

SPAC1786.02

mrpl19

$\mathrm{mpf} 2$

SPBTRNAPRO.07

$\# \mathrm{~N} / \mathrm{A}$

ies4

rpl3601

tlh2

rpl1002

SPBC3E7.07C

$\# \mathrm{~N} / \mathrm{A}$

SPAC9.02

mrpl38

SPATRNAPHE.01

SPAC27F1.05C

$\# N / A$

$\# N / A$

$\# N / A$

$\# N / A$

met16

SPCC306.11

$\# N / A$

mug9

PBTRNAGLY.04

pxl1

SPCC569.07 cofilin/tropomyosin family, drebrin ortholog Abp1

tRNA Leucine

tRNA Methionine

tRNA Asparagine

\#N/A

uridylate kinase Ura6

elongation factor EF-1 alpha (eEF1A) I methyltransferase E. $\begin{array}{rrr}1.36491533 & 4.6667547 & 9.12 \mathrm{E}-08\end{array}$

$$
\# N / A
$$

aldehyde dehydrogenase (predicted)

tRNA Glutamic acid

$\#$ N/A

copper amine oxidase Cao1

tRNA Proline

$\#$ N/A

60S ribosomal protein L8 (predicted)

mitochondrial ribosomal protein subunit L4 (predicted)

Ran GTPase Spi1

\#N/A

aldo/keto reductase, predicted calcium channel regulato $\# \mathrm{~N} / \mathrm{A}$

P-factor pheromone Map2

phospholipase (predicted)

mitochondrial ribosomal protein subunit L19 (predicted) meiotic pumilio family RNA-binding protein Mpf2

tRNA Proline

$$
\# N / A
$$

Ino80 complex subunit les 4

$60 \mathrm{~S}$ ribosomal protein $\mathrm{L} 36$

RecQ type DNA helicase TIh1

$60 \mathrm{~S}$ ribosomal protein $\mathrm{L} 10$

DUF757 family protein, human PBDC1 ortholog

$$
\text { \#N/A }
$$

polyamine $\mathrm{N}$-acetyltransferase (predicted)

mitochondrial ribosomal protein subunit L38 (predicted)

tRNA Phenylalanine

aminotransferase class-III, unknown specificty

$\# \mathrm{~N} / \mathrm{A}$

$\# N / A$

$\#$ \#N/A

phosphoadenosine phosphosulfate reductase

Schizosaccharomyces specific protein

$$
\# N / A
$$

DUF2406 family conserved fungal protein

RNA Glycine

paxillin-like protein Pxl1

aromatic aminotransferase (predicted) $\begin{array}{lll}1.38787263 & 7.38295647 \quad 6.62 \mathrm{E}-36\end{array}$

$\begin{array}{llll}1.38118102 & 2.097782 & 0.03694349\end{array}$

$\begin{array}{llll}1.37649835 & 2.09783147 & 0.05109806\end{array}$

$\begin{array}{lll}1.3724373 & 4.23177238 & 1.28 \mathrm{E}-06\end{array}$

$1.36815825 \quad 6.5481767 \quad 5.45 \mathrm{E}-26$

$\begin{array}{lll}1.3620331 & 2.55593134 & 0.01089474\end{array}$

$\begin{array}{lll}1.36086404 & 8.29717591 & 1.89 \mathrm{E}-56\end{array}$

$\begin{array}{lll}1.36001631 & 3.643985 & 9.81 \mathrm{E}-05\end{array}$

$\begin{array}{llll}1.35674629 & 3.32923408 & 0.00102133\end{array}$

$1.34709436 \quad 9.23348583 \quad 3.13 \mathrm{E}-78$

$\begin{array}{llll}1.34236192 & 3.37386731 & 0.00073962\end{array}$

$1.34002275 \quad 5.51696728 \quad 1.22 \mathrm{E}-09$

$1.33750735 \quad 8.24906126 \quad 7.69 \mathrm{E}-52$

$\begin{array}{lll}1.33459209 & 4.99297735 & 5.09 \mathrm{E}-09\end{array}$

$\begin{array}{lll}1.3296759 & 7.13382793 \quad 3.29 E-22\end{array}$

$\begin{array}{llll}1.32836303 & 2.33064104 \quad 0.04919468\end{array}$

$\begin{array}{llll}1.32711962 & 8.26251207 \quad 2.10 \mathrm{E}-50\end{array}$

$\begin{array}{lll}1.32704441 & 7.78810013 & 2.87 \mathrm{E}-46\end{array}$

$\begin{array}{lll}1.32638033 & 6.19192309 & 3.31 \mathrm{E}-20\end{array}$

$1.32564893 \quad 8.12396075 \quad 1.51 \mathrm{E}-37$

$\begin{array}{llll}1.3213439 & 3.96331338 & 0.00094686\end{array}$

$\begin{array}{lll}1.31999572 & 7.35675766 & 1.98 \mathrm{E}-31\end{array}$

$\begin{array}{llll}1.31772521 & 3.56828252 & 0.00024621\end{array}$

$\begin{array}{llll}1.30986083 & 3.91154876 & 0.00073045\end{array}$

$\begin{array}{llll}1.3097177 & 3.26912552 & 0.05537971\end{array}$

$1.30912266 \quad 6.44658164 \quad 2.01 \mathrm{E}-18$

$\begin{array}{lll}1.30112753 & 4.21450449 \quad 1.05 \mathrm{E}-05\end{array}$

$\begin{array}{lll}1.29503704 & 7.67210255 & 9.02 \mathrm{E}-44\end{array}$

$1.29480135 \quad 4.81703155 \quad 1.75 \mathrm{E}-07$

$\begin{array}{llll}1.28816338 & 2.79755088 & 0.03637374\end{array}$

$\begin{array}{lll}1.2823111 & 5.80770409 & 2.34 \mathrm{E}-13\end{array}$

$\begin{array}{lll}1.27645664 & 4.83690833 \quad 4.30 \mathrm{E}-08\end{array}$

$1.27176585 \quad 1.09849593 \quad 0.30990765$

$1.27048205 \quad 8.11326772 \quad 2.19 \mathrm{E}-31$

$\begin{array}{lll}1.27048205 & 8.11326772 & 2.19 E-31\end{array}$

$1.269557148 .63546024 \quad 1.69 \mathrm{E}-34$

$\begin{array}{llll}1.26706118 & 1.73049978 & 0.11489794\end{array}$

$\begin{array}{lll}1.26548891 & 4.53684238 & 5.66 \mathrm{E}-05\end{array}$

$1.26503325 .88189553 \quad 1.81 \mathrm{E}-11$

$1.2582847 \quad 7.33645311 \quad 1.11 \mathrm{E}-29$

$\begin{array}{lll}1.25765304 & 5.04502197 & 3.60 \mathrm{E}-08\end{array}$

$\begin{array}{llll}1.25753687 & 3.00433158 & 0.00876657\end{array}$

$\begin{array}{llll}1.25566774 & 2.77594073 & 0.01209782\end{array}$

$\begin{array}{lll}1.2551951 & 6.86247663 \quad 7.02 \mathrm{E}-28\end{array}$

$\begin{array}{lll}1.2549068 & 7.07164449 \quad 8.57 \mathrm{E}-27\end{array}$
3.31E-34

0.066702163

0.088261248

5.83E-06

$1.50 \mathrm{E}-24$

4.60E-19

4.85E-07

0.02276287

2.63E- 54

0.000324414

0.002735204

$7.89 \mathrm{E}-76$

0.002060227

8.46E-09

$8.73 \mathrm{E}-50$

3.24E-08

$7.45 \mathrm{E}-21$

0.08544406

$2.20 \mathrm{E}-48$

$2.68 \mathrm{E}-44$

$6.49 \mathrm{E}-19$

$8.15 \mathrm{E}-36$

0.002557917

7.70E-30

0.000759157

0.002038632

0.094640621

3.27E-17

4.14E-05

$7.58 \mathrm{E}-42$

$8.92 \mathrm{E}-07$

0.065848178

$2.45 \mathrm{E}-12$

2.42E-07

0.403466917

$8.40 \mathrm{E}-30$

$8.40 \mathrm{E}-30$

$8.04 \mathrm{E}-33$

0.176456892

0.000194872

1.56E-10

3.95E-28

2.05E-07

0.018712836

0.02497745

2.19E-26

2.49E-25 
SPAC6G9.09C

SPNCRNA.1551

SPAC1071.08

SPBPJ4664.02

SPNCRNA.933

SPCTRNAPRO.09

SPAC977.08

SPBC1348.09

SPNCRNA.1693

SPAC25B8.12C

SPBC29A10.11C

SPAC4H3.07C

SPBC685.06

SPBC2F12.04

SPNCRNA.1532

SPACUNK4.09

SPNCRNA.39

SPAC212.11

SPNCRNA.843

SPCP31B10.08C

SPBC21C3.10C

SPAC1F12.07

SPAPYUG7.06

SPBC18H10.13

SPBTRNAPRO.05

SPAC343.14C

SPBC19C2.07

SPBC19G7.04

SPRRNA.30

SPCC576.09

SPAC57A10.12C

SPCC576.11

SPAPYUG7.03C

SPNCRNA.687

SPBC8E4.03

SPBC215.09c

SPNCRNA.559

SPNCRNA.1182

SPBC2F12.10

SPAC26A3.07C

SPNCRNA.870

SPAC694.05C

SPCC1884.01

SPNCRNA.629

SPBC1921.01C

SPNCRNA.31

SPAC806.07 rpl2401

$\#$ N/A

rpp203

SPBPJ4664.02

\#N/A

SPCTRNAPRO.09

ayr2

SPBC1348.09

$\# \mathrm{~N} / \mathrm{A}$

SPAC25B8.12C

vps902

rdl2

rps001

rpl1701

$\# \mathrm{~N} / \mathrm{A}$

SPACUNK4.09

$\# \mathrm{~N} / \mathrm{A}$

tlh1

\#N/A

rpl35a

rib7

ser1

sdu1

rps1402

SPBTRNAPRO. 05

tif222

fba 1

SPRRNA.30

rps20

ura3

mid2

\#N/A

SPBC8E4.03

erg10

$\# \mathrm{~N} / \mathrm{A}$

$\# N / A$

SPBC2F12.10

rpl1101

$\# N / A$

rps 2502

SPCC1884.01

\#N/A

rpl35b

$\# \mathrm{~N} / \mathrm{A}$

ndk1
60 S ribosomal protein L24 (predicted)

\#N/A

605 acidic ribosomal protein $A 2$

crazy cell surface glycoprotein

\#N/A

RNA Proline

1-acyl DHAP reductase Ayr2 (predicted)

short chain dehydrogenase, implicated in cellular detoxifica

$$
\text { \#N/A }
$$

HAD superfamily hydrolase, unknown role

guanyl-nucleotide exchange factor, CUE domain absent Vps

mitochondrial outer membrane, thiosulfate sulfurtransfera

$40 \mathrm{~S}$ ribosomal protein SOA (p40)

$60 \mathrm{~S}$ ribosomal protein $\mathrm{L} 17$

\#N/A

methyltransferase with rhodanese domain, unknown specif $\#$ N/A

RecQ type DNA helicase

$\# \mathrm{~N} / \mathrm{A}$

$60 S$ ribosomal protein L35a$$
\text { N/A }
$$

5-amino-6-(5-phosphoribosylamino) uracil reductase Rib7 ( $1.21770822 \quad 5.10713579$

phosphoserine aminotransferase (predicted)

$1.25054753 \quad 7.02240996 \quad 1.55 E-27$

$1.24876715 \quad 2.504487190 .02605587$

$\begin{array}{lll}1.24780162 & 5.59608273 \quad 7.75 \mathrm{E}-11\end{array}$

$\begin{array}{lll}1.24745721 & 8.95692744 \quad 3.49 E-32\end{array}$

$1.24502395 \quad 7.73887468 \quad 6.01 \mathrm{E}-42$

1.24194343 .417950590 .00180743

$\begin{array}{lll}1.24162291 & 2.3902447 & 0.03463569\end{array}$

$2.3902447 \quad 0.03463569$

$1.240297316 .28373033 \quad 9.93 \mathrm{E}-18$

$\begin{array}{lll}.23560873 & 7.77235291 & 2.88 \mathrm{E}-39\end{array}$

$1.30 \mathrm{E}-11$

$1.23307 \quad 6.13493376 \quad 1.52 \mathrm{E}-13$

$\begin{array}{lll}1.23202952 & 8.1184343 \quad 3.44 \mathrm{E}-42\end{array}$

$1.228464877 .69658197 \quad 1.02 \mathrm{E}-38$

$225233145.01646725 \quad 2.45 \mathrm{E}-06$

$1.15 \mathrm{E}-11$

$\begin{array}{lll}1.22433374 & 2.26772692 & 0.06276837\end{array}$

$\begin{array}{lll}1.22304995 & 4.16451702 \quad 5.45 \mathrm{E}-05\end{array}$

$1.22280278 \quad 7.60319927 \quad 7.02 \mathrm{E}-35$

$\begin{array}{lll}1.2222347 & 6.75613418 & 4.02 \mathrm{E}-20\end{array}$

40S ribosomal protein S14 (predicted)

1.217323727 .73908069

$8.94 \mathrm{E}-05$

$1.213393657 .74292304 \quad 3.66 \mathrm{E}-39$

$1.21283545 \quad 3.23375227 \quad 0.00255496$

$1.21252252 \quad 6.17247584 \quad 1.43 \mathrm{E}-15$

$\begin{array}{lll}1.21161148 & 9.24636751 \quad 5.68 \mathrm{E}-40\end{array}$

$1.21100153 \quad 6.48867873 \quad 7.58 \mathrm{E}-18$

$\begin{array}{llll}1.20245324 & 3.63298968 & 0.00081741\end{array}$

$\begin{array}{lll}1.19968817 & 7.36562374 & 5.45 \mathrm{E}-33\end{array}$

$1.19865707 \quad 7.42129882 \quad 2.67 \mathrm{E}-27$

$1.19761897 \quad 7.94928223 \quad 2.16 \mathrm{E}-26$

$\begin{array}{lll}1.19743548 & 8.79044517 & 6.01 \mathrm{E}-48\end{array}$

$1.19651314 \quad 5.46663898 \quad 3.61 \mathrm{E}-11$

$1.19382013 \quad 8.47362628 \quad 7.16 \mathrm{E}-49$

$1.19249814 \quad 8.63343143 \quad 7.02 \mathrm{E}-51$

$\begin{array}{lll}1.19049816 & 5.69751526 & 9.03 \mathrm{E}-12\end{array}$

$1.18656223 \quad 3.32852564 \quad 0.00346164$

$\begin{array}{lll}1.18231503 & 5.96483248 & 3.76 \mathrm{E}-10\end{array}$

$\begin{array}{lll}1.17962756 & 7.4091751 & 3.11 E-32\end{array}$

$1.17679405 \quad 8.27864894 \quad 1.07 \mathrm{E}-42$

$1.16964349 \quad 6.54260343 \quad 1.16 \mathrm{E}-19$

$\begin{array}{lll}1.16852385 & 4.75530479 \quad 9.74 \mathrm{E}-06\end{array}$

$\begin{array}{lll}1.16839654 & 6.87928239 & 1.01 \mathrm{E}-17\end{array}$

$1.16526375 \quad 6.54523566$

$1.16358921 \quad 5.09830689 \quad 3.90 \mathrm{E}-08$

$1.16277957 \quad 6.902476 \quad 8.26 \mathrm{E}-18$

\#ncleoside diphosphate kinase Ndk1
4.78E-26

0.049293917

6.10E-10

1.44E-30

4.45E-40

0.004570631

0.063189239

0.063189239

$1.52 \mathrm{E}-16$

$1.83 \mathrm{E}-37$

1.14E-10

1.61E-12

$2.66 \mathrm{E}-40$

$6.14 \mathrm{E}-37$

$1.08 \mathrm{E}-05$

$1.02 \mathrm{E}-10$

0.105235624

0.000188101

3.39E-33

$7.83 \mathrm{E}-19$

5.30E-06

4.07E-36

0.000296814

$2.24 \mathrm{E}-37$

0.006240661

$1.81 \mathrm{E}-14$

$3.72 \mathrm{E}-38$

1.17E-16

0.002242929

$2.41 \mathrm{E}-31$

8.03E-26

$6.10 \mathrm{E}-25$

5.84E-46

2.99E-10

7.39E-47

7.47E-49

$8.05 \mathrm{E}-11$

0.008196668

2.75E-09

1.30E-30

8.64E-41

2.17E-18

3.86E-05

1.54E-16

4.33E-14

2.21E-07

1.27E-16 
SPCTRNAALA.12 SPBTRNALYS.09 SPRRNA.06

SPAC23G3.03

SPCC1223.05C

SPBC2F12.07c

SPCC965.14C

SPAC4F8.14C

SPBP16F5.08C

SPAC4G9.07

SPCC830.06

SPRRNA. 53

SPNCRNA.1261

SPBC1711.06

SPBC428.11

SPBC428.03C

SPNCRNA.1410

SPAC3G6.08

SPAC15E1.08

SPBTRNAPRO.06

SPNCRNA.1021

SPAC222.09

SPBC27.04

SPAC806.03C

SPBC3E7.12C

SPCC330.14C

SPNCRNA.718

SPBC25H2.05

SPBC1A4.02C

SPBC12C2.07C

SPAC227.18

SPCC4G3.14

SPBC365.03C

SPCC18.14C

SPNCRNA.1107

SPBC1711.08

SPAC25H1.08C

SPBC839.04

SPBC215.08c

SPCC191.06

SPBTRNATYR.04

SPBC800.04c

SPAC110.04c

SPAC24H6.08

SPBC1271.04c

SPNCRNA.1517

SPCC330.05c
SPCTRNAALA.12

SPBTRNALYS.09

SPRRNA.06

sib2

rpl3702

rpl802

SPCC965.14c

hcs 1

mo1

mug133

cnb1

SPRRNA. 53 \#N/A

rpl401

met17

pho4

$\# \mathrm{~N} / \mathrm{A}$

erv1

nato

SPBTRNAPRO.06

$\#$ N/A

seb1

uds 1

rps2601

ch4

rpl2402

\#N/A

egd2

leu1

srm1

lys3

mdj1

rpl2101

rpp0

\#N/A

aha1

sqt1

rpl803

arg 4

SPCC191.06

SPBTRNATYR.04

rpl4301

pss1

SPAC24H6.08

dys 1

$\# \mathrm{~N} / \mathrm{A}$

ura4
tRNA Alanine

tRNA Lysine

$5 S$ rRNA

ornithine N5 monooxygenase (predicted)

$60 S$ ribosomal protein L37 (predicted)

$60 S$ ribosomal protein L8 (predicted)

cytosine deaminase (predicted)

3-hydroxy-3-methylglutaryl-CoA synthase

ER flavin-containing $\mathrm{N}, \mathrm{N}-\mathrm{dime}$ -

UPF0300 family protein 2

calcineurin regulatory subunit (calcineurin $B$ )

$5 \mathrm{~S}$ rRNA (predicted)

\section{\#N/A}

60S ribosomal protein L4 (predicted)

homocysteine synthase Met17

thiamine-repressible acid phosphatase Pho4

$\# N / A$

$16159458 \quad 1.29952317 \quad 0.24911386$

$\begin{array}{llll}1.1611739 & 1.98579394 & 0.13228101\end{array}$

$\begin{array}{llll}1.16102463 & 1.29980186 & 0.24452958\end{array}$

$1.15389423 \quad 6.81197625 \quad 8.30 \mathrm{E}-20$

$\begin{array}{lll}1.15238107 & 6.58917747 & 2.33 \mathrm{E}-18\end{array}$

$\begin{array}{lll}1.1514201 & 7.50476207 & 7.21 \mathrm{E}-26\end{array}$

$1.150067325 .81460421 \quad 1.89 \mathrm{E}-08$

$1.14966704 \quad 8.47147594$

$2.28 \mathrm{E}-29$

$\begin{array}{lll}1.14737802 & 5.39240694 & 4.42 \mathrm{E}-07\end{array}$

$1.146932835 .48262877 \quad 3.56 \mathrm{E}-09$

$1.145434875 .10201892 \quad 7.08 \mathrm{E}-08$

$\begin{array}{lll}1.14515432 & 7.51833359 & 1.62 \mathrm{E}-32\end{array}$

$1.135360548 .15137971 \quad 3.95 \mathrm{E}-33$

$1.134042685 .99312266 \quad 9.61 \mathrm{E}-11$

$\begin{array}{lll}1.12933847 & 6.10572598 & 3.20 \mathrm{E}-11\end{array}$

$1.128464375 .38102133 \quad 1.64 \mathrm{E}-09$

mitochondrial Mia40-Erv1 disulfide relay system sulfhydryl $1.12807726 \quad 5.24118218 \quad 3.65 \mathrm{E}-07$

Naa10 1.127763846 .20291665

tRNA Proline

\#N/A

$\begin{array}{lll}1.12706157 & 3.44676318 & 0.00246165\end{array}$

$1.12525604 \quad 8.95533862 \quad 5.98 \mathrm{E}-43$

RNA-binding and 3'-end processing protein Seb1 $\quad 1.1240453 \quad 7.81655474 \quad 4.10 \mathrm{E}-33$

septation protein Uds 1

40 S ribosomal protein S26 (predicted)

chitin synthase regulatory factor Cfh4 (predicted)

$60 S$ ribosomal protein L24 (predicted)

$\# \mathrm{~N} / \mathrm{A}$

$1.12228344 \quad 8.22301019$

1.120060416 .37372161

$4.10 \mathrm{E}-33$
$1.44 \mathrm{E}-20$

$1.75 \mathrm{E}-15$

$3.05 \mathrm{E}-23$

$\begin{array}{lll}1.1195082 & 7.66737314 & 3.05 \mathrm{E}-23 \\ 1.11853673 & 6.97376126 & 6.15 \mathrm{E}-20\end{array}$

$\begin{array}{lll}1.11853673 & 6.97376126 & 6.15 \mathrm{E}-20 \\ 1.11799125 & 7.32816228 & 4.83 \mathrm{E}-25\end{array}$

$\begin{array}{lll}1.11799125 & 7.32816228 & 4.83 \mathrm{E}-25\end{array}$

nascent polypeptide-associated complex alpha subunit Egd: $1.11625784 \quad 7.27923772$

3-isopropylmalate dehydrogenase Leu1

spermidine synthase Srm1 (predicted)

saccharopine dehydrogenase Lys3

mitochondrial DNAJ domain protein Mdj1 (predicted)

$60 S$ ribosomal protein L21 (predicted)

$60 \mathrm{~S}$ acidic ribosomal protein $\mathrm{Rpp0}$ (predicted)

$$
\# N / A
$$

chaperone activator Aha1

ribosome assembly protein Sqt1 (predicted)

$60 S$ ribosomal protein $L 8$ (predicted)

arginine specific carbamoyl-phosphate synthase Arg4

Schizosaccharomyces pombe specific protein

tRNA Tyrosine

60 S ribosomal protein L37a (predicted)

heat shock protein Pss1

Schizosaccharomyces specific protein

elF-5A-deoxyhypusine synthase Dys1 (predicted)

$$
\text { \#N/A }
$$

orotidine 5'-phosphate decarboxylase Ura4
$1.11456077 \quad 7.56924019 \quad 5.17 \mathrm{E}-30$

$\begin{array}{lll}1.11394719 & 8.16589943 \quad 3.41 \mathrm{E}-41\end{array}$

$1.11211176 \quad 7.98854725 \quad 1.29 \mathrm{E}-38$

$1.11175284 \quad 6.94949094 \quad 8.35 \mathrm{E}-18$

$\begin{array}{lll}1.10863657 & 7.45217473 & 3.48 \mathrm{E}-27\end{array}$

$1.107093828 .63446138 \quad 1.39 E-36$

$1.10641379 \quad 1.47629843 \quad 0.32549278$

$1.103536597 .27441541 \quad 3.55 \mathrm{E}-16$

$\begin{array}{lll}1.10241029 & 7.1152539 & 6.49 \mathrm{E}-18\end{array}$

$1.10000083 \quad 7.98175838 \quad 6.91 \mathrm{E}-37$

$1.09444015 \quad 9.42417736 \quad 4.63 \mathrm{E}-32$

$\begin{array}{lll}1.09359938 & 6.31486599 & 3.56 \mathrm{E}-10\end{array}$

$\begin{array}{llll}1.09118837 & 2.50327269 & 0.06045325\end{array}$

$\begin{array}{lll}1.08961925 & 6.20542699 \quad 3.71 \mathrm{E}-12\end{array}$

$1.089177319 .17345046 \quad 1.42 \mathrm{E}-41$

$\begin{array}{lll}1.08833959 & 5.3808777 & 2.20 \mathrm{E}-06\end{array}$

$1.08797526 \quad 7.3685894$

$\begin{array}{lll}1.08622782 & 8.17223438 & 3.11 \mathrm{E}-39\end{array}$

$\begin{array}{lll}1.07802697 & 6.87812027 \quad 1.02 \mathrm{E}-16\end{array}$
0.338908406

0.199241323

0.33379522

1.56E-18

$3.76 \mathrm{E}-17$

1.96E-24

$1.11 \mathrm{E}-07$

$2.22 \mathrm{E}-42$

$7.77 \mathrm{E}-28$

2.14E-06

2.30E-08

$3.83 \mathrm{E}-07$

$6.86 \mathrm{E}-31$

1.77E-31

7.50E-10

2.66E-10

$1.11 \mathrm{E}-08$

$1.78 \mathrm{E}-06$

2.29E-10

0.006045307

4.96E-41

$1.82 \mathrm{E}-31$

$2.91 \mathrm{E}-19$

2.20E-14

7.22E-22

1.17E-18

$1.26 \mathrm{E}-23$

$3.82 \mathrm{E}-25$

$1.88 \mathrm{E}-28$

$2.39 \mathrm{E}-39$

7.55E-37

$1.28 \mathrm{E}-16$

1.04E- 25

7.13E-35

0.420010016

4.72E-15

$1.01 \mathrm{E}-16$

3.62E-35

$1.90 \mathrm{E}-30$

2.62E-09

0.101786933

$3.45 \mathrm{E}-11$

$1.03 \mathrm{E}-39$

9.74E-06

4.44E-24

1.92E-37

1.43E-15 
SPRRNA.31

SPNCRNA.1447

SPNCRNA.1201

SPAC13G6.15C

SPAC56F8.04C

SPBC2G2.17C

SPAC22H12.03

SPCC1223.07C

SPBP8B7.03C

SPCC794.03

SPAC21E11.04

SPBC1709.01

SPCC576.08C

SPAC1F12.04C

SPCC1753.04

SPAC31G5.17C

SPBC428.05C

SPNCRNA.1534

SPAC3G9.17

SPAPJ698.02C

SPBC646.16

SPBC11C11.07

SPNCRNA.1594

SPBC1198.02

SPAC926.05C

SPCC16A11.09C

SPBC17G9.09

SPCC1259.05C

SPBC1105.09

SPCC11E10.06C

SPAC16C9.05

SPBC409.06

SPAC15F9.03C

SPBC1778.01C

SPBP16F5.07

SPBC1773.10C

SPAC3H1.07

SPAPB1E7.12

SPRRNA.03

SPAC11E3.04C

SPNCRNA.1507

SPAC922.03

SPCP1E11.03

SPNCRNA.616

SPNCRNA.1340

SPBC25H2.16C

SPCC962.06c
SPRRNA.31

$\# \mathrm{~N} / \mathrm{A}$

$\# N / A$

SPAC13G6.15C

ppt1

SPBC2G2.17C

SPAC22H12.03

drs1

rpl402

SPCC794.03

aca 1

chs 2

rps2

SPAC1F12.04C

tol1

rps1001

$\arg 12$

$\#$ N/A

new8

rps002

$\mathrm{scl} 1$

rpl1801

$\# \mathrm{~N} / \mathrm{A}$

dea2

dph4

$\operatorname{tim} 23$

tif213

cox 9

ubc15

elp4

cph1

uch2

nxt2

zuo1

apm1

nrs1

aru1

rps602

SPRRNA.03

ubc13

$\#$ N/A

SPAC922.03

$\operatorname{mug} 170$

$\# N / A$

gga22

bpb1
$5 S$ rRNA

\#N/A

calcipressin (predicted)

$\# N / A$

para-hydroxybenzoate--polyprenyltransferase Ppt1

beta-glucosidase Psu2 (predicted)

mitochondrial hydrolase (predicted)

cytoplasmic aspartate-tRNA ligase Drs1 (predicted)

$60 S$ ribosomal protein L4 (predicted)

amino acid transmembrane transporter (predicted)

L-azetidine-2-carboxylic acid acetyltransferase Aca

chitin synthase homolog Chs2

40S ribosomal protein S2 (predicted)

peroxisomal protein Pex11 paralog (predicted)

$\begin{array}{lll}07489613 & 2.29944982 & 0.07800655\end{array}$

$\begin{array}{lll}1.07264988 & 8.24986488 & 1.10 \mathrm{E}-19\end{array}$

$\begin{array}{lll}1.06975674 & 4.89339984 & 2.63 \mathrm{E}-05\end{array}$

$\begin{array}{lll}1.06756382 & 4.852735 & 9.26 \mathrm{E}-05\end{array}$

$1.06381454 \quad 6.48424087 \quad 1.53 \mathrm{E}-13$

$\begin{array}{lll}1.06077128 & 4.48168102 & 9.85 \mathrm{E}-05\end{array}$

$\begin{array}{lll}1.05905288 & 5.09898822 & 2.26 \mathrm{E}-05\end{array}$

$1.05730879 \quad 8.09477229-1.27 \mathrm{E}-33$

$\begin{array}{lll}1.05707103 & 8.8929918 & 3.82 \mathrm{E}-47\end{array}$

$\begin{array}{llll}1.05579886 & 4.49974226 & 0.00017609\end{array}$

$\begin{array}{lll}1.05272612 & 6.37100868 & 2.86 \mathrm{E}-12\end{array}$

$1.05176433 \quad 6.4586595 \quad 1.94 \mathrm{E}-14$

$\begin{array}{lll}1.05129039 & 8.4827045 \quad 1.22 \mathrm{E}-37\end{array}$

$1.05064599 \quad 5.31664021 \quad 7.06 \mathrm{E}-08$

'

$40 \mathrm{~S}$ ribosomal protein $\mathrm{S} 10$ (predicted)

argininosuccinate synthase $\operatorname{Arg} 12$

$$
\# \mathrm{~N} / \mathrm{A}
$$

$2.35 \mathrm{E}-24$
$1.82 \mathrm{E}-18$

$6.64 \mathrm{E}-37$

$1.04863281 \quad 3.7867999 \quad 0.00465368$

$\begin{array}{llll}1.04845613 & 3.58106306 & 0.0049223\end{array}$

$1.04178844 \quad 8.47348284 \quad 1.28 \mathrm{E}-32$

$1.03691988 \quad 6.90893983-4.53 \mathrm{E}-20$

$1.03589885 \quad 7.8928411 \quad 1.56 \mathrm{E}-31$

$\begin{array}{lll}1.03463226 & 7.89421222 & 1.68 \mathrm{E}-31\end{array}$

$\begin{array}{lll}1.03173142 & 6.51184547 & 6.77 \mathrm{E}-14\end{array}$

$1.02531057 \quad 4.98782567 \quad 5.57 \mathrm{E}-05$

$1.02433834 \quad 6.37686365 \quad 1.34 \mathrm{E}-14$

$\begin{array}{lll}1.02341646 & 8.29141366 & 1.02 \mathrm{E}-36\end{array}$

$1.022906835 .04030794 \quad 2.78 \mathrm{E}-05$

$1.01860535 \quad 5.49740956 \quad 1.03 \mathrm{E}-08$

$\begin{array}{lll}1.01797042 & 5.75158394 & 1.73 \mathrm{E}-09\end{array}$

$1.015408 \quad 6.5290478 \quad 4.75 \mathrm{E}-12$

$1.014532796 .69845434 \quad 1.70 \mathrm{E}-15$

$\begin{array}{lll}1.01446676 & 6.06128354 & 7.05 \mathrm{E}-12\end{array}$

$1.012190197 .56976775 \quad 3.52 \mathrm{E}-27$

$1.012148326 .55424527 \quad 1.14 \mathrm{E}-15$

$\begin{array}{lll}1.0111216 & 8.38769106 & 1.39 \mathrm{E}-34\end{array}$

$1.01055058 \quad 8.09707606 \quad 2.65 \mathrm{E}-33$

$1.010509727 .26788026 \quad 6.02 \mathrm{E}-23$

$1.00813068 \quad 4.16539554 \quad 0.00040252$

$\begin{array}{lll}1.00779826 & 6.27320537 \quad 1.84 \mathrm{E}-12\end{array}$

$\begin{array}{crrr}\text { \#N/A } & 1.0076137 & 5.41125114 & 4.09 \mathrm{E}-08 \\ \text { 1-aminocyclopropane-1-carboxylate deaminase (predicted) } & 1.00667497 & 7.1855607 & 1.97 \mathrm{E}-19\end{array}$

arrestin family Schizosaccharomyces specific protein Mug1 $1.00645545 \quad 5.29216463 \quad 1.51 \mathrm{E}-06$

$\# N / A$

$1.006173995 .15474043 \quad 1.66 \mathrm{E}-06$

\#N/A $\quad 1.005906882 .68091978 \quad 0.13445506$

Golgi localized Arf binding gamma-adaptin ortholog Gga22 $1.0043333 \quad 7.0118915 \quad 2.42 \mathrm{E}-16$

$\mathrm{KH}$ and $\mathrm{CC} / \mathrm{hC}$ domain splicing factor $\mathrm{Bpb} 1$

1.002637617 .0709583

$1.15 \mathrm{E}-20$
0.126481322

$2.06 \mathrm{E}-18$

9.67E-05

0.000306629

$1.62 \mathrm{E}-12$

0.000325396

$8.38 \mathrm{E}-05$

$5.80 \mathrm{E}-32$

3.61E-45

0.000555806

2.69E-11

2.19E-13

6.71E-36

3.82E-07

$5.85 \mathrm{E}-23$

2.97E-17

3.50E-35

0.010673946

0.0112065

5.50E-31

8.79E-19

6.13E-30

$6.56 \mathrm{E}-30$

7.31E-13

0.000191829

1.54E-13

5.26E-35

0.000101283

6.26E-08

1.17E-08

4.34E-11

2.14E-14

6.37E-11

1.05E-25

1.46E-14

6.67E-33

1.20E-31

$1.41 \mathrm{E}-21$

0.001187306

$1.78 \mathrm{E}-11$

2.31E-07

$3.60 \mathrm{E}-18$

6.82E-06

7.44E-06

0.201845725

3.28E-15

2.34E-19 
SPAC631.01C

SPAC22G7.09C

SPBC16E9.05

SPBC14C8.01C

SPAP11E10.01

SPBP4H10.14C

SPBP8B7.17C

SPAC521.05

SPBC1348.08C

SPAC644.16

SPBC428.04

SPCC74.05

SPCC13B11.02C

SPBC3B9.05

SPCC13B11.01

SPAC23A1.12C

SPAC23C4.02

SPCC736.13

SPBC839.05c

SPNCRNA.1245

SPCC5E4.05C

SPAC4H3.06

SPNCRNA.171

SPBC31F10.05

SPBC23E6.06C

SPBC31F10.06C

SPCC965.09

SPNCRNA.1561

SPAC5H10.05C

SPCC330.03C

SPCC1223.14

SPBC106.11C

SPNCRNA.300

SPAC17A2.14

SPBC17G9.10

SPCC584.01C

SPCC285.05

SPAC6C3.02C

SPNCRNA.1533 tam11

acp2

nup45

erg6

cut2

SPAP11E10.01

SPBP4H10.14C

SPBP8B7.17C

rps802

SPBC1348.08c

rna15

apq12

rpl2702

SPCC13B11.02C

hot15

adh1

frs1

crn1

SPCC736.13

rps1701

$\mathrm{mgl1}$

SPAC4H3.06

$$
\# \mathrm{~N} / \mathrm{A}
$$

mug37

rib3

sar1

SPCC965.09

$\#$ N/A

SPAC5H10.05C

SPCC330.03C

aro2

plg7

$\# N / A$

$\mathrm{mnr} 2$

rpl1102

met10

SPCC285.05

$\operatorname{mix} 17$

\#N/A
$\# \mathrm{~N} / \mathrm{A}$

no80 complex subunit les 6

cell surface glycoprotein, flocculin PfI6

transcription corepressor Ncb2 (predicted)

6-phosphofructokinase pfk1

tRNA Asparagine

\section{$\# N / A$}

Schizosaccharomyces specific protein Tam11

F-actin capping protein beta subunit Acp2

nucleoporin Nup45

sterol 24-C-methyltransferase Erg6

securin, sister chromatid separation inhibitor

ornithine cyclodeaminase-like protein (predicted)

Schizosaccharomyces specific protein

phosphomethylpyrimidine kinase (predicted)

$40 \mathrm{~S}$ ribosomal protein $\mathrm{S} 8$ (predicted)

cell surface glycoprotein, adhesion molecule (predicted)

RNA-binding protein Rna15

nuclear membrane organization protein Apq12 (predicted)

60 S ribosomal protein L27 (predicted)

Schizosaccharomyces pombe specific protein

helper of TIM Hot15 (predicted)

alcohol dehydrogenase Adh1

cytoplasmic phenylalanine-tRNA ligase beta subunit Frs1 ( $F$

actin binding protein, coronin $\mathrm{Crn} 1$

short chain dehydrogenase (predicted)

$40 \mathrm{~S}$ ribosomal protein S17 (predicted)

$$
\text { \#N/A }
$$

mitochondrial acylglycerol lipase Mgl1 (predicted)

human REX1BD ortholog, implicated in DNA repair

$$
\text { \#N/A }
$$

conserved fungal protein

3,4-dihydroxy-2-butanone 4-phosphate synthase Rib3 (pred

ADP-ribosylation factor Sar1

mitochondrial omega-amidase (predicted)

\#N/A

FAD binding oxidoreductase, implicated in cellular detoxific $0.97267126 \quad 7.0239829 \quad 4.34 \mathrm{E}-19$

$\begin{array}{llll}\text { NADPH-hemoprotein reductase (predicted) } & 0.97201622 & 7.31113788 & 1.33 \mathrm{E}-17\end{array}$

chorismate synthase Aro2 (predicted)

phospholipase A2, PAF family homolog

$$
\text { \#N/A }
$$

vacuolar CorA family magnesium ion transmembrane trans $0.97107785 \quad 7.28111136 \quad 6.27 \mathrm{E}-21$

$60 S$ ribosomal protein $L 11$ (predicted)

sulfite reductase NADPH flavoprotein subunit (predicted) $\quad \begin{array}{lllll}0.96875739 & 8.64227808 & 3.07 \mathrm{E}-38\end{array}$

purine nucleoside transmembrane transporter (predicted) $0.96730568 \quad 5.89297178$

mitochondrial $\mathrm{CHCH}$ domain protein Mix17 (predicted)

\#N/A
$1.00257527 \quad 4.59432116 \quad 0.00020584$

$1.00041828 \quad 5.82676985 \quad 2.45 \mathrm{E}-06$

$0.99926815 \quad 3.31307416 \quad 0.01273224$

$0.99809751 \quad 5.57985426 \quad 9.57 \mathrm{E}-08$

$0.9979864 \quad 9.82445822 \quad 2.60 \mathrm{E}-40$

$0.9977953 \quad 4.24085095 \quad 0.00029209$

$0.99626118 \quad 4.39192053 \quad 0.01569485$

$0.99619065 \quad 5.99590957 \quad 2.36 \mathrm{E}-11$

$0.99511345 \quad 6.63269473 \quad 1.74 \mathrm{E}-14$

$0.994547996 .76676347 \quad 7.37 \mathrm{E}-14$

$0.994500568 .29134292 \quad 1.16 \mathrm{E}-32$

$0.99430355 \quad 5.26106372 \quad 197 \mathrm{E}-06$

$0.99360902 \quad 6.38885851 \quad 4.36 \mathrm{E}-12$

$0.99348707 \quad 5.51831767 \quad 6.07 \mathrm{E}-05$

$0.9930838 \quad 6.15437314 \quad 4.00 \mathrm{E}-12$

$0.98972329 \quad 7.73886956 \quad 2.10 \mathrm{E}-24$

$\begin{array}{lll}0.98782854 & 3.4030565 & 0.01028985\end{array}$

$98723769 \quad 7.06597283 \quad 3.13 \mathrm{E}-18$

$\begin{array}{lll}0.98627285 & 3.97134133 & 0.00761263\end{array}$

$\begin{array}{lll}0.98588038 & 7.28830414 & 2.26 \mathrm{E}-19\end{array}$

$0.98484965 \quad 9.65980678 \quad 1.25 \mathrm{E}-37$

$\begin{array}{llll}0.98376741 & 4.55334999 & 0.00041588\end{array}$

$0.98376552 \quad 9.65775092 \quad 5.91 \mathrm{E}-38$

$\begin{array}{lll}0.98345782 & 8.28457668 & 6.40 \mathrm{E}-32\end{array}$

$\begin{array}{llll}0.98103422 & 7.70421247 \quad 2.49 \mathrm{E}-23\end{array}$

$\begin{array}{lll}0.98026262 & 7.22978125 & 2.52 \mathrm{E}-15\end{array}$

$0.980202647 .05715112 \quad 4.04 \mathrm{E}-15$

$0.97924981 \quad 7.29596741 \quad 1.45 \mathrm{E}-19$

$0.97912412 \quad 6.88463557 \quad 5.39 \mathrm{E}-14$

$\begin{array}{lll}0.97906666 & 6.58431254 & 8.92 \mathrm{E}-11\end{array}$

$\begin{array}{llll}0.97854697 & 2.13265545 & 0.13396433\end{array}$

$0.97810155 \quad 6.92023723 \quad 4.17 E-17$

$\begin{array}{lll}0.9778729 & 6.70359932 & 7.11 \mathrm{E}-16\end{array}$

$0.97773837 \quad 7.58118316 \quad 1.09 \mathrm{E}-26$

$\begin{array}{lll}0.97477673 & 6.30807652 & 2.22 \mathrm{E}-11\end{array}$

$\begin{array}{llll}0.97322175 & 4.40237821 & 0.00041246\end{array}$

$0.97195307 \quad 7.85261939 \quad 182 \mathrm{E}-27$

$\begin{array}{llll}0.97160946 & 6.4144461 & 5.50 \mathrm{E}-10\end{array}$

$\begin{array}{llll}0.97112871 & 3.72773988 & 0.00426352\end{array}$

$0.96991575 \quad 7.73309479 \quad 6.42 \mathrm{E}-24$

0.000644031

$1.08 \mathrm{E}-05$

0.026160288

5.07E-07

$1.74 \mathrm{E}-38$

0.000884195

0.03154267

2.01E-10

1.97E-13

$7.93 \mathrm{E}-13$

5.00E-31

8.72E-06

4.01E-11

0.000207581

$3.71 \mathrm{E}-11$

5.26E-23

0.021625215

5.03E-17

0.016492827

$4.10 \mathrm{E}-18$

$6.80 \mathrm{E}-36$

0.001222485

3.30E-36

2.56E-30

$5.97 \mathrm{E}-22$

3.10E-14

4.89E-14

2.67E-18

5.87E-13

$6.98 \mathrm{E}-10$

0.201331107

6.02E-16

9.24E-15

3.15E-25

1.90E-10

0.001214014

$7.58 \mathrm{E}-18$

2.00E-16

5.54E-26

3.95E-09

0.00984836

1.33E-19

$1.57 \mathrm{E}-22$

1.77E-36

7.27E-07

$\begin{array}{lll}0.96686831 & 6.8253505 & 9.33 \mathrm{E}-15\end{array}$

$\begin{array}{lll}0.96553204 & 5.0069293 & 0.00025986\end{array}$ 


\begin{tabular}{|c|c|c|c|c|c|c|}
\hline SPNCRNA.1614 & \#N/A & \#N/A & 0.96407424 & 4.96035345 & $2.01 \mathrm{E}-05$ & 7.53E-05 \\
\hline SPAC23H3.09c & gly1 & threonine aldolase Gly1 (predicted) & 0.96395799 & 8.16854949 & $7.71 E-26$ & $2.08 \mathrm{E}-24$ \\
\hline SPNCRNA.947 & \#N/A & $\# N / A$ & 0.9638959 & 5.02651406 & 0.00012428 & 0.000403485 \\
\hline SPAC1805.02c & etf2 & electron transfer flavoprotein beta subunit EtfB (predicted) & 0.96365946 & 7.06420618 & $4.01 \mathrm{E}-16$ & $5.31 \mathrm{E}-15$ \\
\hline SPCC14G10.03c & ump1 & proteasome maturation factor Ump1 (predicted) & 0.9624085 & 5.97752258 & $1.96 \mathrm{E}-09$ & 1.30E-08 \\
\hline SPAC9E9.12c & ybt1 & $\mathrm{ABC}$ transmembrane transporter Ybt1 & 0.96135194 & 8.04639496 & $3.04 \mathrm{E}-26$ & $8.48 \mathrm{E}-25$ \\
\hline SPBC365.01 & csr103 & sec14 cytosolic factor family, phospholipid-intermembrane & 0.96080235 & 6.75560581 & $5.31 \mathrm{E}-16$ & $6.95 \mathrm{E}-15$ \\
\hline SPBC16C6.11 & rpl3201 & $60 S$ ribosomal protein $\mathrm{L} 32$ & 0.96001843 & 7.15321117 & 8.54E-19 & 1.45E-17 \\
\hline SPCC613.08 & SPCC613.08 & CDK regulator, involved in ribosome export (predicted) & 0.95963316 & 5.64679243 & 7.47E-07 & $3.50 \mathrm{E}-06$ \\
\hline SPCTRNAASP.05 & SPCTRNAASP.05 & tRNA Asparagine & 0.95900862 & 4.21623472 & 0.0005357 & 0.001538756 \\
\hline SPAC869.10c & put4 & plasma membrane proline transmembrane transporter Put. & 0.95825216 & 7.78665052 & $1.15 \mathrm{E}-22$ & $2.67 \mathrm{E}-21$ \\
\hline SPAC57A10.06 & mug15 & Schizosaccharomyces specific protein Mug15 & 0.95306741 & 2.75255497 & 0.05069522 & 0.087781412 \\
\hline SPCC191.09c & gst1 & glutathione S-transferase Gst1 & 0.95212299 & 2.96610737 & 0.05809285 & 0.098485767 \\
\hline SPAC5H10.04 & SPAC5H10.04 & NADPH dehydrogenase, (Old yellow enzyme) involved in sm & 0.95193191 & 7.03245142 & $2.68 \mathrm{E}-18$ & 4.31E-17 \\
\hline SPNCRNA.90 & $\# N / A$ & \#N/A & 0.95136528 & 1.42019484 & 0.28779001 & 0.380513323 \\
\hline SPNCRNA.411 & \#N/A & $\# N / A$ & 0.95066896 & 4.3878267 & 0.00053252 & 0.00153033 \\
\hline SPBC4C3.03 & thr1 & homoserine kinase Thr1 (predicted) & 0.95028926 & 6.66038645 & $2.51 \mathrm{E}-14$ & $2.82 \mathrm{E}-13$ \\
\hline SPNCRNA.715 & $\# N / A$ & \#N/A & 0.94865002 & 7.36374982 & $3.26 \mathrm{E}-19$ & $5.74 \mathrm{E}-18$ \\
\hline SPBC29B5.03c & rpl26 & $60 S$ ribosomal protein L26 (predicted) & 0.94646711 & 7.42477529 & $1.73 \mathrm{E}-18$ & $2.84 \mathrm{E}-17$ \\
\hline SPNCRNA.1452 & \#N/A & $\# N / A$ & 0.94560809 & 2.23564531 & 0.1585808 & 0.231675047 \\
\hline SPAC343.12 & rds1 & ferritin related conserved fungal protein & 0.94446256 & 8.9841698 & $8.52 \mathrm{E}-15$ & 9.99E-14 \\
\hline SPBC1685.02c & rps1202 & 40S ribosomal protein S12 (predicted) & 0.94382294 & 7.48507537 & $5.88 \mathrm{E}-21$ & $1.25 \mathrm{E}-19$ \\
\hline SPAC2C4.08 & SPAC2C4.08 & conserved fungal protein & 0.94200291 & 4.34960263 & 0.00091718 & 0.002487619 \\
\hline SPCC1450.07c & dao1 & D-amino acid oxidase & 0.94184811 & 6.50651979 & 1.27E-09 & $8.80 \mathrm{E}-09$ \\
\hline SPBC18H10.04c & sce3 & translation initiation factor (predicted) & 0.94109383 & 8.15444962 & $1.44 \mathrm{E}-20$ & $2.91 \mathrm{E}-19$ \\
\hline SPNCRNA.361 & $\# N / A$ & $\# N / A$ & 0.94076658 & 4.09573879 & 0.00157859 & 0.004047594 \\
\hline SPAC17G6.02c & tco1 & RTA1-like protein & 0.93931711 & 7.15524027 & 4.90E-11 & $3.97 \mathrm{E}-10$ \\
\hline SPNCRNA.878 & $\# N / A$ & $\# N / A$ & 0.93697474 & 7.28511415 & $8.88 \mathrm{E}-21$ & $1.85 \mathrm{E}-19$ \\
\hline SPNCRNA.1091 & $\# \mathrm{~N} / \mathrm{A}$ & \#N/A & 0.93629437 & 7.78466246 & $1.72 \mathrm{E}-21$ & $3.81 \mathrm{E}-20$ \\
\hline SPBC1861.01c & cnp3 & CENP-C ortholog Cnp3 & 0.935983 & 7.37976248 & $3.44 \mathrm{E}-17$ & 5.02E-16 \\
\hline SPAC4H3.10c & pyk1 & pyruvate kinase & 0.93567673 & 10.1376113 & $7.71 \mathrm{E}-42$ & $5.64 \mathrm{E}-40$ \\
\hline SPNCRNA.530 & $\# N / A$ & $\# \mathrm{~N} / \mathrm{A}$ & 0.93486532 & 3.63220346 & 0.00855935 & 0.018299214 \\
\hline SPBC21C3.16c & spt4 & DSIF transcription elongation factor complex subunit Spt4 & 0.93369361 & 4.99625307 & $5.98 \mathrm{E}-05$ & 0.000205084 \\
\hline SPAC3A12.16c & $\operatorname{tim} 17$ & TIM23 translocase complex subunit Tim17 (predicted) & 0.93348552 & 5.31887325 & 3.48E-06 & 1.49E-05 \\
\hline SPNCRNA.1346 & \#N/A & \#N/A & 0.93258401 & 4.61060114 & 0.01105096 & 0.023046819 \\
\hline SPBC776.03 & SPBC776.03 & homoserine dehydrogenase (predicted) & 0.93169822 & 8.18145584 & $1.76 \mathrm{E}-27$ & $5.40 \mathrm{E}-26$ \\
\hline SPBTRNAGLU.06 & SPBTRNAGLU.06 & tRNA Glutamic acid & 0.93138301 & 4.54806548 & 0.00015187 & 0.000485868 \\
\hline SPBTRNAGLU.07 & SPBTRNAGLU.07 & tRNA Glutamic acid & 0.93138301 & 4.54806548 & 0.00015187 & 0.000485868 \\
\hline SPAC1002.12c & SPAC1002.12c & succinate-semialdehyde dehydrogenase $[N A D(P)+]$ (predict & 0.93108234 & 8.80131896 & 8.87E-36 & 4.41E-34 \\
\hline SPNCRNA.1117 & $\# \mathrm{~N} / \mathrm{A}$ & \#N/A & 0.93078041 & 5.02783186 & $7.45 \mathrm{E}-05$ & 0.000250835 \\
\hline SPNCRNA.1209 & $\# \mathrm{~N} / \mathrm{A}$ & $\# N / A$ & 0.92792999 & 3.00482829 & 0.0438383 & 0.077445382 \\
\hline SPBC1778.08c & $\operatorname{arc3}$ & ARP2/3 actin-organizing complex subunit Arc21 & 0.92781483 & 6.94189268 & $8.46 \mathrm{E}-16$ & 1.09E-14 \\
\hline SPBC16G5.14c & rps3 & $40 \mathrm{~S}$ ribosomal protein S3 (predicted) & 0.92688722 & 8.29336325 & $8.84 \mathrm{E}-25$ & 2.27E-23 \\
\hline SPBC29A3.13 & pdp1 & PWWP domain protein Pdp1 & 0.92645825 & 7.92733006 & $1.45 \mathrm{E}-23$ & $3.52 \mathrm{E}-22$ \\
\hline SPBC2A9.04c & san1 & sir antagonist, ubiquitin-protein ligase E3 & 0.92299092 & 6.47396805 & $8.85 \mathrm{E}-11$ & $6.93 \mathrm{E}-10$ \\
\hline SPCC1450.08c & wtf16 & wtf element Wtf16 & 0.92283781 & 3.11486739 & 0.0300268 & 0.055782791 \\
\hline SPCP1E11.09c & rpp103 & 605 acidic ribosomal protein Rpp1-3 & 0.92208878 & 6.8357958 & 1.16E-14 & $1.34 \mathrm{E}-13$ \\
\hline
\end{tabular}


SPATRNAGLU.03

SPAC4G9.20c

SPCC645.08C

SPATRNAASP.01

SPBC839.17c

SPCC $31 \mathrm{H} 12.07$

SPAC56F8.05C

SPAC8E11.03C

SPCC1322.04

SPAC8C9.05

SPCC1322.11

SPAC29A4.15

SPCC622.18

SPBC23G7.09

SPAC11D3.03C

SPNCRNA.1455

SPBC18E5.07

SPBC56F2.12

SPAC6F 6.09

SPAC23H4.02

SPBC902.06

SPNCRNA.880

SPNCRNA.703

SPBC16A3.08C

SPAC17A5.15C

SPBC3E7.10

SPNCRNA.565

SPAC222.17

SPAC1071.09c

SPBC1685.01

SPNCRNA.50

SPBC3B8.03

SPNCRNA.1359

SPAC9E9.11

SPNCRNA.1578

SPBC21C3.12C

SPBC713.12

SPBC405.07

SPAC343.20

SPCC4G3.15C

SPCC1682.10

SPAC22A12.16

SPAC31G5.04

SPBC1711.02

SPAC644.15

SPCTRNAASP.08

SPBC800.09 ymc1

snd1

SPATRNAASP.01

fkh1

$\sec 231$

mug64

fyu1

dtd1

rpl2302

srs1

rpl6

mat1-Mc

SPAC11D3.03C

$\# \mathrm{~N} / \mathrm{A}$

$\operatorname{aim} 21$

ilv5

eaf6

ppk9

mto2

$\# N / A$

$\# \mathrm{~N} / \mathrm{A}$

oga1

gus1

fma1

\#N/A

SPAC222.17

SPAC1071.09c pmp1

$\# \mathrm{~N} / \mathrm{A}$

lys9

$\# \mathrm{~N} / \mathrm{A}$

plr1

\#N/A

SPBC21C3.12C

erg1

rpl3602

\#N/A

not2

rpn8

acl2

lys12

mat3-Mc

rpp101

SPCTRNAASP.08

sum2
tRNA Glutamic acid

$0.920764494 .14060308 \quad 0.00140943$

$\begin{array}{llll}\text { mitochondrial carrier, glutamate/glycine (predicted) } & 0.92007742 & 5.67992669 & 5.93 \mathrm{E}-06 \\ \text { RNA-binding protein, involved in chromatin silencing by sm: } & 0.91903972 & 8.09960011 & 1.49 \mathrm{E}-16\end{array}$

tRNA Aspartic acid

FKBP-type peptidyl-prolyl cis-trans isomerase Fkh1

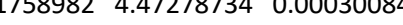

$\begin{array}{llll}0.91572296 & 6.76653997 & 2.90 \mathrm{E}-11\end{array}$

$\begin{array}{lll}0.91467578 & 8.52291434 & 3.00 \mathrm{E}-27\end{array}$

$\begin{array}{lll}0.91333268 & 7.3956145 & 1.12 \mathrm{E}-17\end{array}$

$0.9121835 \quad 5.58296035 \quad 9.47 \mathrm{E}-06$

$0.91211792 \quad 9.20194817 \quad 9.37 \mathrm{E}-19$

$0.91122564 \quad 6.58389413 \quad 8.64 \mathrm{E}-10$

$0.910273417 .56080105 \quad 1.09 \mathrm{E}-20$

$0.9086581 \quad 7.5062761 \quad 2.76 \mathrm{E}-19$

$0.907540398 .42686882 \quad 1.85 \mathrm{E}-27$

$0.906824725 .66828461 \quad 8.20 \mathrm{E}-08$

cytoplasmic serine-tRNA ligase Srs1 (predicted)

60S ribosomal protein L6 (predicted)

$M$-specific trancription factor Mc

$$
\# \mathrm{~N} / \mathrm{A}
$$

$\begin{array}{lll}0.90460996 & 1.73095018 & 0.39495456\end{array}$

$0.90285548 \quad 8.09827006 \quad 9.85 \mathrm{E}-22$

$\begin{array}{lrrr}\text { acetohydroxyacid reductoisomerase (predicted) } & 0.90284801 & 9.12199123 & 2.33 \mathrm{E}-39 \\ \text { Mst2/NuA4 histone acetyltransferase complex subunit Eaff } & 0.9022789 & 4.46734827 & 0.00313113\end{array}$

$\begin{array}{lrrrr}\text { Mst2/NuA4 histone acetyltransferase complex subunit Eaft } & 0.9022789 & 4.46734827 & 0.00313113 \\ \text { serine/threonine protein kinase Ppk9 (predicted) } & 0.90222617 & 7.14193063 & 6.30 \mathrm{E}-13\end{array}$

$\begin{array}{llll}\text { gamma tubulin complex linker Mto2 } & 0.90190364 & 5.92895357 & 7.23 \mathrm{E}-05\end{array}$

$0.90145634 \quad 4.85477296 \quad 0.00024691$

$\begin{array}{lll}0.9010358 & 4.48786041 & 0.0036389\end{array}$

$0.90035635 \quad 8.77763499 \quad 1.31 \mathrm{E}-30$

$0.899094439 .03778602 \quad 1.59 \mathrm{E}-32$

$\begin{array}{lll}0.89889413 & 7.286858 & 4.88 \mathrm{E}-16\end{array}$

$\begin{array}{lll}0.89878955 & 4.81455893 & 0.0061648\end{array}$

$0.89848176 \quad 4.25816215 \quad 0.00171603$

cytoplasmic glutamate-tRNA ligase Gus1 (predicted)

$$
\# \text { N/A }
$$

conserved fungal protein

nucleolar DNAJ domain protein, DNAJC9 family, implicated $0.89789082 \quad 7.09079519 \quad 6.66 \mathrm{E}-14$

dual-specificity MAP kinase phosphatase Pmp1

saccharopine dehydrogenase

$$
\# N / A
$$

pyridoxal reductase PIr1

$$
\text { \#N/A }
$$

thioredoxin family protein, peroxidase

squalene monooxygenase Erg1 (predicted)

$60 S$ ribosomal protein $\mathrm{L} 36$

$$
\text { \#N/A }
$$

CCR4-Not complex NOT box subunit Not2

$19 S$ proteasome regulatory subunit Rpn8

ATP-citrate synthase subunit 2 (predicted)

homoisocitrate dehydrogenase Lys 12

$\begin{array}{llll}0.89766487 & 7.73167091 & 1.99 \mathrm{E}-19\end{array}$

$\begin{array}{llll}0.89740713 & 2.38982198 & 0.12822098\end{array}$

$\begin{array}{llll}0.89724461 & 8.81697419 & 1.19 \mathrm{E}-24\end{array}$

$\begin{array}{llll}0.89688122 & 4.09451762 & 0.00606035\end{array}$

$\begin{array}{llll}0.89643436 & 8.23410377 \quad 4.54 \mathrm{E}-23\end{array}$

$0.89516456 \quad 6.67184159$

$0.89380833 \quad 5.97792427 \quad 6.60 \mathrm{E}-06$

$0.89367098 \quad 8.25508243 \quad 5.93 \mathrm{E}-16$

$0.89150186 \quad 6.736293$

$0.89144229 \quad 8.94681863 \quad 9.02 \mathrm{E}-15$

$\begin{array}{lll}0.89108525 & 6.22744153 \quad 3.10 \mathrm{E}-10\end{array}$

$0.89008923 \quad 7.4086467 \quad 3.78 \mathrm{E}-18$

$\begin{array}{lll}0.88989551 & 8.56482732 \quad 3.35 \mathrm{E}-29\end{array}$

$\begin{array}{lll}0.88947788 & 8.01827531 & 1.74 \mathrm{E}-24\end{array}$

mating type M-specific HMG-box transcription factor Mc at $0.88818992 \quad 5.67427144$

$60 S$ acidic ribosomal protein $A 1$

$0.8877228 \quad 6.8542963 \quad 2.09 \mathrm{E}-15$

$\begin{array}{lll}0.88465794 & 4.47305286 & 0.00046572\end{array}$

$0.88462637 \quad 7.35868463 \quad 5.03 \mathrm{E}-16$ translation initiation inhibitor (predicted)
0.0036496

$2.45 \mathrm{E}-05$

$2.05 \mathrm{E}-15$

0.000909867

$2.44 \mathrm{E}-10$

8.99E-26

$1.69 \mathrm{E}-16$

3.76E-05

$1.58 \mathrm{E}-17$

6.07E-09

2.24E-19

4.92E-18

$5.60 \mathrm{E}-26$

4.40E-07

$6.53 \mathrm{E}-11$

0.490271034

$2.21 \mathrm{E}-20$

$1.50 \mathrm{E}-37$

0.007492022

$6.35 \mathrm{E}-12$

0.000244017

0.000760968

0.008532983

4.86E-29

$6.76 \mathrm{E}-31$

$6.41 \mathrm{E}-15$

0.013700672

0.00437199

7.20E-13

$3.61 \mathrm{E}-18$

0.193898615

$3.03 \mathrm{E}-23$

0.013506769

1.07E-21

8.35E-12

2.70E-05

$7.75 \mathrm{E}-15$

2.13E-12

1.05E-13

2.30E-09

6.04E-17

1.12E-27

4.37E-23

$6.51 \mathrm{E}-07$

2.60E-14

0.00135843

$6.60 \mathrm{E}-15$ 


\begin{tabular}{|c|c|c|c|c|c|c|}
\hline SPNCRNA.579 & $\# \mathrm{~N} / \mathrm{A}$ & $\# \mathrm{~N} / \mathrm{A}$ & 0.88459618 & 5.02712244 & 0.00015651 & 0.000500019 \\
\hline SPNCRNA.668 & $\# N / A$ & $\# N / A$ & 0.88290768 & 4.89086144 & 7.16E-05 & 0.000242105 \\
\hline SPCC576.01c & xan1 & alpha-ketoglutarate-dependent xanthine dioxygenase Xan1 & 0.8812039 & 6.0739163 & 1.09E-09 & 7.56E-09 \\
\hline SPBC19F5.03 & sac11 & inositol polyphosphate phosphatase (predicted) & 0.88038099 & 7.98863903 & $5.72 \mathrm{E}-25$ & $1.48 \mathrm{E}-23$ \\
\hline SPAC3G9.06 & frs2 & cytoplasmic phenylalanine-tRNA ligase alpha subunit Frs2 ( & 0.88034504 & 8.17494776 & $1.08 \mathrm{E}-20$ & 2.22E-19 \\
\hline SPBC685.07c & rpl2701 & $60 S$ ribosomal protein $\mathrm{L} 27$ & 0.87925982 & 7.48868103 & 1.37E-19 & $2.53 \mathrm{E}-18$ \\
\hline SPATRNAGLU.02 & SPATRNAGLU.02 & tRNA Glutamic acid & 0.87871904 & 4.53478668 & 0.00034233 & 0.001026231 \\
\hline SPNCRNA.955 & $\# N / A$ & $\# \mathrm{~N} / \mathrm{A}$ & 0.87437576 & 5.29839516 & 3.43E-06 & 1.47E-05 \\
\hline SPNCRNA.686 & $\# N / A$ & $\# N / A$ & 0.87316598 & 6.92749097 & $4.89 \mathrm{E}-11$ & $3.96 \mathrm{E}-10$ \\
\hline SPBC18H10.12c & rpl701 & RNase MRP subunit, ribosomal protein L7-like Rpl701 & 0.87299772 & 8.43672009 & $3.57 E-23$ & $8.45 \mathrm{E}-22$ \\
\hline SPBC29A3.04 & rpl8 & $60 S$ ribosomal protein L7a/L8 (predicted) & 0.87240981 & 8.58562639 & $1.61 \mathrm{E}-29$ & $5.62 \mathrm{E}-28$ \\
\hline SPAC16C9.02c & SPAC16C9.02c & S-methyl-5-thioadenosine phosphorylase (predicted) & 0.87115121 & 6.92254547 & $4.78 \mathrm{E}-11$ & $3.89 \mathrm{E}-10$ \\
\hline SPNCRNA.1562 & \#N/A & \#N/A & 0.86882705 & 4.33575321 & 0.00132501 & 0.003458637 \\
\hline SPATRNAGLU.01 & SPATRNAGLU.01 & tRNA Glutamic acid & 0.86784928 & 4.52855282 & 0.00044831 & 0.001311571 \\
\hline SPBC19C7.11 & SPBC19C7.11 & plasma membrane CIC chloride channel (predicted) & 0.86502601 & 7.07537326 & 3.15E-11 & $2.63 \mathrm{E}-10$ \\
\hline SPBC16D10.02 & $\operatorname{trm} 11$ & tRNA (guanine-N2-)-methyltransferase catalytic subunit Trı & 0.86396384 & 5.3822916 & $4.22 \mathrm{E}-06$ & $1.78 \mathrm{E}-05$ \\
\hline SPAC589.06c & snd302 & SRP-independent ER targeting protein Snd3b (predicted) & 0.86293876 & 7.54629349 & $9.34 \mathrm{E}-18$ & $1.43 \mathrm{E}-16$ \\
\hline SPCC1442.16c & zta1 & NADPH quinone oxidoreductase/ARE-binding protein (predi & 0.8621959 & 7.11389654 & $5.04 \mathrm{E}-14$ & $5.53 \mathrm{E}-13$ \\
\hline SPNCRNA.1470 & $\# \mathrm{~N} / \mathrm{A}$ & \#N/A & 0.86010365 & 7.04298533 & $3.01 E-11$ & $2.53 \mathrm{E}-10$ \\
\hline SPBC1198.12 & mfr1 & meiotic APC activator Mfr1 & 0.85987716 & 5.56776895 & 7.14E-07 & $3.36 \mathrm{E}-06$ \\
\hline SPAC22A12.17c & SPAC22A12.17c & short chain dehydrogenase (predicted) & 0.85774353 & 8.62067464 & $8.12 E-27$ & $2.37 \mathrm{E}-25$ \\
\hline SPBP8B7.06 & rpp201 & $60 S$ acidic ribosomal protein $\mathrm{A} 2$ & 0.85644341 & 7.0913484 & $3.26 \mathrm{E}-15$ & $3.98 \mathrm{E}-14$ \\
\hline SPBC13G1.08c & ash2 & Ash2-trithorax family protein & 0.85567944 & 7.00895088 & $1.68 \mathrm{E}-09$ & 1.14E-08 \\
\hline SPBC887.06c & snx3 & sorting nexin Snx3 (predicted) & 0.85363551 & 5.03766299 & 0.00011603 & 0.000378331 \\
\hline SPBC1105.05 & exg1 & cell wall glucan 1,6-beta-glucosidase Exg1 & 0.85297515 & 7.70653569 & 3.56E-16 & 4.73E-15 \\
\hline SPAC27F1.06c & ani2 & CENP-A amino terminus domain (NTD) isomerase Ani2 & 0.85291038 & 6.45746903 & $9.42 \mathrm{E}-10$ & $6.59 \mathrm{E}-09$ \\
\hline SPBC15D4.15 & pho2 & 4-phosphoerythronate phosphastase/2-phosphoglycolate pr & 0.85168997 & 6.69508572 & $1.56 \mathrm{E}-11$ & $1.35 \mathrm{E}-10$ \\
\hline SPBC106.18 & rpl2501 & $60 \mathrm{~S}$ ribosomal protein L25 (predicted) & 0.85104242 & 7.60952606 & $1.58 \mathrm{E}-15$ & $1.99 \mathrm{E}-14$ \\
\hline SPATRNAGLU.04 & SPATRNAGLU.04 & tRNA Glutamic acid & 0.84963624 & 4.14959207 & 0.00331427 & 0.007872102 \\
\hline SPBTRNAGLU.05 & SPBTRNAGLU.05 & tRNA Glutamic acid & 0.84963624 & 4.14959207 & 0.00331427 & 0.007872102 \\
\hline SPCC70.08c & SPCC70.08c & methyltransferase (predicted) & 0.84855096 & 6.10539353 & 5.36E-07 & 2.57E-06 \\
\hline SPCC663.11 & saf1 & splicing associated factor Saf1 & 0.84802591 & 4.72182693 & 0.00081277 & 0.002231113 \\
\hline SPRRNA.18 & SPRRNA.18 & $5 S$ rRNA & 0.84801457 & 4.81202095 & 0.00027589 & 0.000841151 \\
\hline SPCC191.03c & SPCC191.03c & Schizosaccharomyces pombe specific protein & 0.84209927 & 6.01687583 & 4.42E-07 & $2.14 \mathrm{E}-06$ \\
\hline SPNCRNA.635 & $\# N / A$ & \#N/A & 0.84199196 & 5.54856854 & $8.49 \mathrm{E}-06$ & $3.41 \mathrm{E}-05$ \\
\hline SPCC4G3.11 & nur1 & Lem2-Nur1 complex subunit Nur1 & 0.84128148 & 4.57329597 & 0.00145953 & 0.003765018 \\
\hline SPNCRNA.1465 & $\# N / A$ & \#N/A & 0.84027731 & 7.88155398 & 1.10E-21 & $2.45 \mathrm{E}-20$ \\
\hline SPCC364.03 & rpl1702 & 60S ribosomal protein L17 (predicted) & 0.84024058 & 7.90459784 & $5.03 E-21$ & $1.09 \mathrm{E}-19$ \\
\hline SPCC306.09c & cap1 & adenylyl cyclase-associated protein Cap1 & 0.83924649 & 8.67182915 & $1.83 \mathrm{E}-28$ & $5.95 \mathrm{E}-27$ \\
\hline SPAC343.02 & img1 & mitochondrial ribosomal protein subunit L19 (predicted) & 0.83786904 & 5.50623662 & 2.09E-06 & 9.25E-06 \\
\hline SPAC1039.02 & SPAC1039.02 & extracellular 5'-nucleotidase, human NT5E family (predicte & 0.83772039 & 5.7847411 & $6.87 \mathrm{E}-05$ & 0.000233103 \\
\hline SPAC27E2.02 & yih1 & IMPACT homolog, cytoplasmic translational regulator Yih1। & 0.83760179 & 5.33366494 & 7.32E-06 & $2.98 \mathrm{E}-05$ \\
\hline SPBC18H10.14 & rps1601 & $40 S$ ribosomal protein S16 (predicted) & 0.83729107 & 7.87777197 & $1.63 E-21$ & $3.61 \mathrm{E}-20$ \\
\hline SPAC8C9.09c & mug129 & Schizosaccharomyces specific protein Mug129 & 0.83713036 & 5.33937398 & $2.66 \mathrm{E}-05$ & $9.76 \mathrm{E}-05$ \\
\hline SPCC1739.13 & ssa2 & heat shock protein Ssa2 & 0.83680988 & 10.1919938 & $6.79 \mathrm{E}-30$ & $2.46 \mathrm{E}-28$ \\
\hline SPBC21C3.13 & rps1901 & 40S ribosomal protein S19 (predicted) & 0.83669219 & 7.69448917 & $9.42 \mathrm{E}-19$ & $1.59 \mathrm{E}-17$ \\
\hline SPAC29A4.02c & SPAC29A4.02c & translation elongation factor EF-1 gamma subunit & 0.83586672 & 9.0229084 & $3.16 \mathrm{E}-29$ & $1.06 \mathrm{E}-27$ \\
\hline
\end{tabular}


SPAC2F7.13C

SPBC6B1.08C

SPBC18E5.04

SPBC530.09C

SPCC1183.08c

SPNCRNA.1246

SPCC613.05C

SPAC17A2.01

SPNCRNA.904

SPCC338.14

SPAC3G9.04

SPAC959.07

SPNCRNA.136

SPNCRNA.601

SPAC1F8.08

SPBC1539.06

SPAC144.03

SPBC28F2.03

SPAC8E11.08C

SPNCRNA.1454

SPBC106.16

SPAC57A7.12

SPBC1347.13C

SPRRNA.40

SPAC212.06c

SPAC3C7.11C

SPNCRNA.707

SPCC1682.14

SPAC1250.05

SPAPB24D3.07C

SPBC11C11.02

SPCTRNAHIS.03

SPCC126.03

SPAC140.01

SPNCRNA.446

SPBC4B4.04

SPAC1002.07C

SPCC4G3.02

SPAC31G5.02

SPAC $186.07 \mathrm{C}$

SPAC222.08C

SPBC14F5.06

SPBC17G9.07

SPBC23E6.05

SPAC21E11.07

SPNCRNA.1161

SPBC14F5.04C acb1

ade2

ppi1

$\# \mathrm{~N} / \mathrm{A}$

$\# N / A$

pre6

ssz1

mrm1

SPRRNA.40

SPAC212.06c cnx1

\#N/A

rpl1902

rpl3002

SPAPB24D3.07C

imp2

SPCTRNAHIS.03

pus1

sdh2

\#N/A

eif21

SPAC1002.07c

aph1

rot1

SPAC186.07C

sno1

rli1

rps 2402

arx1

iba57

\#N/A cytoplasmic tryptophan-tRNA ligase Wrs1 (predicted)

HIF prolyl-3,4-dihydroxylase Ofd1

$60 \mathrm{~S}$ ribosomal protein $\mathrm{L} 10$

0.835860737 .45355198

0.835799727 .36973407

0.834562458 .47216538

cation dependent mannose-6-phosphate cargo receptor Mrl $0.83391406 \quad 6.0304585$

$60 S$ ribosomal protein 110

$$
\# \mathrm{~N} / \mathrm{A}
$$

$0.8337095 \quad 8.36150803$

$0.833564 \quad 4.29125785$ 0.832936417 .53517791

$60 S$ ribosomal protein $L 35$

finity imp

$$
\# \mathrm{~N} / \mathrm{A}
$$

adenosine kinase (predicted)

protein phosphatase Ssu72

$40 S$ ribosomal protein $\mathrm{S} 4$ (predicted)

$$
\# N / A
$$$$
\# \mathrm{~N} / \mathrm{A}
$$

Schizosaccharomyces pombe specific protein

fatty-acyl-CoA binding protein Acb1 (predicted)

adenylosuccinate synthetase Ade2

cyclophilin family peptidyl-prolyl cis-trans isomerase Cyp2

$$
\text { \#N/A }
$$

$20 \mathrm{~S}$ proteasome complex subunit alpha 4 Pre6

heat shock protein Ssz1 (predicted)

mitochondrial ribose methyltransferase Mrm1 (predicted) 5S rRNA

DNA helicase in rearranged telomeric region, truncated

calnexin $\mathrm{Cnx1}$

$60 S$ ribosomal protein $L 19$

$\# N / A$

$60 S$ ribosomal protein L30 (predicted)

Schizosaccharomyces pombe specific protein

contractile ring protein Imp2

tRNA Histidine

832847235.66684755

0.832021225 .73507973

$0.83163395 \quad 8.07588826$

$0.82997295 \quad 8.33624648 \quad 3.37 \mathrm{E}-24$

$\begin{array}{lll}0.82990147 & 4.83406522 & 0.00074853\end{array}$

$\begin{array}{llll}0.82990147 & 4.83406522 & 0.00074853\end{array}$

$0.82958359 \quad 5.01079998 \quad 4.74 \mathrm{E}-05$

$\begin{array}{lll}0.82662676 & 6.18464278 & 2.59 \mathrm{E}-07\end{array}$

$\begin{array}{lll}0.82625713 & 8.37735444 \quad 7.21 \mathrm{E}-24\end{array}$

$0.825981117 .93144938 \quad 7.13 \mathrm{E}-20$

$\begin{array}{lll}0.82453261 & 2.44711585 & 0.18880807\end{array}$

$\begin{array}{lll}0.82453051 & 6.30795066 \quad 1.83 \mathrm{E}-06\end{array}$

$0.82443008 \quad 7.02308738 \quad 4.68 \mathrm{E}-13$

$0.82287242 \quad 8.48116335 \quad 3.41 \mathrm{E}-24$

$\begin{array}{llll}0.82187683 & 4.32921675 & 0.00320934\end{array}$ $\begin{array}{llll}0.82027435 & 4.52262953 & 0.00189449\end{array}$

$\begin{array}{llll}0.81918029 & 2.16817958 & 0.23474194\end{array}$ $\begin{array}{lll}0.81903583 & 9.09681196 \quad 2.84 \mathrm{E}-30\end{array}$

$0.81652826 .40100575 \quad 1.36 \mathrm{E}-09$

$0.81567585 \quad 7.78892005 \quad 6.74 \mathrm{E}-18$

$\begin{array}{lll}0.81466327 & 5.21724675 & 0.0006899\end{array}$

$0.81381448 \quad 9.20553246 \quad 4.02 \mathrm{E}-21$

$0.81250838 \quad 7.33593947 \quad 5.75 \mathrm{E}-12$

$\begin{array}{lll}0.81208656 & 1.82003344 & 0.3083086\end{array}$

TruA family tRNA/ U2 snRNA pseudouridine synthase Lsp1 $0.81184566 \quad 6.31841103 \quad 4.30 \mathrm{E}-09$ succinate dehydrogenase (ubiquinone) iron-sulfur protein sı $0.81166274 \quad 7.44454389 \quad 7.53 \mathrm{E}-16$

$$
\# \mathrm{~N} / \mathrm{A}
$$

$0.81070206 \quad 4.29647121 \quad 0.00284186$

translation initiation factor elF2A (predicted)

spermidine/spermine N1-acetyltransferase (predicted)

bis(5'-nucleosidyl)-tetraphosphatase

$0.80983207 \quad 7.92282652 \quad 6.88 \mathrm{E}-20$

$0.80912126 \quad 6.43551557 \quad 5.47 \mathrm{E}-09$

$0.809092415 .02968769 \quad 0.00029176$

$\begin{array}{lrrr}\text { ER chaperone Rot1 (predicted) } & 0.80859726 & 6.40370255 & 8.47 \mathrm{E}-10 \\ \text { hydroxyacid dehydrogenase, implicated in cellular detoxifici } & 0.80815518 & 1.68352635 & 0.2877378\end{array}$ glutamine aminotransferase subunit Sno1 (predicted) $\quad \begin{array}{lll}0.80728834 & 6.74974344 & \text { 9.49E-09 }\end{array}$

iron-sulfur ATPase involved in ribosome biogenesis and trar $\begin{array}{llll}0.8066652 & 8.03566151 & 2.48 \mathrm{E}-19\end{array}$ $\begin{array}{lllll}\text { 40S ribosomal protein S24 (predicted) } & 0.80652917 & 7.7244817 & 8.86 \mathrm{E}-18\end{array}$ ribosomal export complex protein Arx1, peptidase family ( $p \quad 0.8065111 \quad 6.54702846 \quad 2.19 \mathrm{E}-09$ mitochondrial [4Fe-4S] cluster asssembly protein lba57 (pri $0.80600341 \quad 4.813805910 .00257688$ \#N/A

$\begin{array}{lllll}0.80596715 & 2.9839961 & 0.08355893\end{array}$

phosphoglycerate kinase Pgk1 (predicted) $\begin{array}{rrr}0.80587799 & 9.93012281 & 9.02 \mathrm{E}-29\end{array}$
7.12E-16

$2.16 \mathrm{E}-15$

$3.31 \mathrm{E}-24$

2.60E-06

$6.36 \mathrm{E}-25$

0.01241721

$7.92 \mathrm{E}-18$

$1.16 \mathrm{E}-05$

4.96E-05

5.69E-19

0.007537419

$8.31 \mathrm{E}-23$

0.002079076

0.002079076

0.000165628

1.29E-06

$1.75 \mathrm{E}-22$

$135 \mathrm{E}-18$

0.267563286

8.16E-06

4.76E-12

$8.39 \mathrm{E}-23$

0.007660303

0.004776588

0.32212521

$1.04 \mathrm{E}-28$

9.35E-09

$1.04 \mathrm{E}-16$

0.001936796

8.74E-20

$5.24 \mathrm{E}-11$

0.401692512

2.76E-08

9.74E-15

0.006869309

$1.31 \mathrm{E}-18$

3.47E-08

0.000883594

5.95E-09

0.380513323

5.81E-08

4.45E-18

$1.36 \mathrm{E}-16$

1.45E-08

0.006278439

0.134520589

$2.98 \mathrm{E}-27$ 


\begin{tabular}{|c|c|c|c|c|c|c|}
\hline SPBC2G5.05 & SPBC2G5.05 & transketolase (predicted) & 0.80465807 & 9.98232663 & 4.36E-28 & 1.37E-26 \\
\hline SPBC32H8.06 & mug93 & TPR repeat protein, meiotically spliced & 0.80398316 & 3.64340946 & 0.06055471 & 0.101923028 \\
\hline SPAC227.16c & psf3 & GINS complex subunit Psf3 & 0.80279444 & 5.01927941 & 0.00013157 & 0.000425343 \\
\hline SPAC30C2.02 & $\mathrm{mmd} 1$ & deoxyhypusine hydroxylase & 0.80180834 & 6.27311958 & $1.21 \mathrm{E}-08$ & 7.30E-08 \\
\hline SPBC30D10.18c & rpl102 & $60 S$ ribosomal protein $\mathrm{L} 10 \mathrm{a}$ & 0.80103266 & 6.94993856 & $2.53 \mathrm{E}-12$ & 2.40E-11 \\
\hline SPBC2G2.03c & sbh1 & translocon beta subunit Sbh1 (predicted) & 0.80091521 & 6.07438556 & 4.86E-06 & 2.04E-05 \\
\hline SPAC22A12.05 & rpc11 & DNA-directed RNA polymerase III complex subunit Rpc11 & 0.8008631 & 4.87623718 & 0.00090729 & 0.00246373 \\
\hline SPRRNA.35 & SPRRNA.35 & $5 S$ rRNA & 0.80037927 & 5.02057174 & 7.79E-05 & 0.000261244 \\
\hline SPNCRNA.1097 & $\# N / A$ & \#N/A & 0.80032559 & 5.13747523 & $6.84 \mathrm{E}-05$ & 0.000232139 \\
\hline SPBC4.07c & rpt2 & $19 S$ proteasome base subcomplex ATPase subunit Rpt2 & 0.79978951 & 7.29605144 & $2.03 \mathrm{E}-12$ & $1.95 \mathrm{E}-11$ \\
\hline SPAC26A3.04 & rpl2002 & 605 ribosomal protein L20 (predicted) & 0.79976407 & 7.92955899 & $1.58 \mathrm{E}-20$ & $3.18 \mathrm{E}-19$ \\
\hline SPCC191.02c & acs1 & acetyl-CoA ligase (predicted) & 0.79873922 & 8.84445875 & $2.66 \mathrm{E}-16$ & $3.59 \mathrm{E}-15$ \\
\hline SPNCRNA.88 & \#N/A & \#N/A & 0.79840387 & 1.68294966 & 0.36288329 & 0.458403715 \\
\hline SPCC576.03c & tpx1 & thioredoxin peroxidase Tpx1 & 0.79752652 & 8.38246137 & $6.62 \mathrm{E}-21$ & $1.39 \mathrm{E}-19$ \\
\hline SPAC11D3.05 & $\mathrm{mfs} 2$ & transmembrane transporter Mfs2 (predicted) & 0.79747761 & 6.19690416 & $1.52 \mathrm{E}-08$ & $9.01 \mathrm{E}-08$ \\
\hline SPBC336.10c & tif512 & translation elongation and termination factor elF5A (predic & 0.79637379 & 8.06417858 & $5.40 \mathrm{E}-20$ & $1.03 \mathrm{E}-18$ \\
\hline SPBTRNAASP.04 & SPBTRNAASP.04 & tRNA Aspartic acid & 0.79635465 & 4.16613978 & 0.0037675 & 0.00880723 \\
\hline SPAC186.03 & SPAC186.03 & L-asparaginase (predicted) & 0.79586779 & 6.02021394 & $1.08 \mathrm{E}-07$ & 5.71E-07 \\
\hline SPAC19D5.06c & $\operatorname{din} 1$ & RNA pyrophosphohydrolase Din1 & 0.7947088 & 5.42188261 & 0.00071626 & 0.002002125 \\
\hline SPAC3H5.10 & rpl3202 & $60 S$ ribosomal protein L32 (predicted) & 0.79456441 & 7.37143924 & $2.99 \mathrm{E}-16$ & $4.01 \mathrm{E}-15$ \\
\hline SPAC23H $3.05 \mathrm{c}$ & swd1 & Set1C complex subunit Swd1 & 0.79272294 & 6.27801923 & 3.31E-07 & $1.64 \mathrm{E}-06$ \\
\hline SPBC19C2.10 & SPBC19C2.10 & BAR adaptor protein, human endophilin-A2 ortholog, implic & 0.79175926 & 6.37073676 & $3.08 \mathrm{E}-06$ & $1.33 \mathrm{E}-05$ \\
\hline SPAC1805.11c & $\operatorname{rps} 2602$ & 40 S ribosomal protein S26 (predicted) & 0.79174551 & 7.73617383 & $2.32 \mathrm{E}-19$ & $4.19 \mathrm{E}-18$ \\
\hline SPNCRNA.702 & $\# \mathrm{~N} / \mathrm{A}$ & $\# N / A$ & 0.79120514 & 6.78585652 & $2.53 E-10$ & $1.90 \mathrm{E}-09$ \\
\hline SPAC1687.03c & rfc4 & DNA replication factor $\mathrm{C}$ complex subunit Rfc 4 & 0.79079055 & 6.93046861 & 4.67E-12 & $4.28 \mathrm{E}-11$ \\
\hline SPBTRNALEU.09 & SPBTRNALEU.09 & tRNA Leucine & 0.78993163 & 1.36079823 & 0.57086208 & 0.659208633 \\
\hline SPNCRNA.699 & \#N/A & \#N/A & 0.78919947 & 3.77495165 & 0.1013338 & 0.15874229 \\
\hline SPNCRNA.842 & $\# \mathrm{~N} / \mathrm{A}$ & $\# \mathrm{~N} / \mathrm{A}$ & 0.78687924 & 5.33513089 & 6.61E-05 & 0.000224679 \\
\hline SPNCRNA.1595 & $\# N / A$ & \#N/A & 0.78604363 & 4.72823179 & 0.00649855 & 0.014357989 \\
\hline SPBC3H7.09 & erf2 & palmitoyltransferase Erf2 & 0.78581416 & 5.13388756 & 0.00074801 & 0.002079076 \\
\hline SPAC9E9.03 & leu2 & 3-isopropylmalate dehydratase Leu2 & 0.78509946 & 9.01967238 & 5.19E-21 & $1.12 \mathrm{E}-19$ \\
\hline SPCC285.13c & nup60 & nucleoporin Nup60 & 0.78501832 & 7.41190711 & $1.43 \mathrm{E}-15$ & $1.81 \mathrm{E}-14$ \\
\hline SPAC18B11.08c & SPAC18B11.08c & UPF0139 family conserved fungal ER membrane protein & 0.78486901 & 4.6547228 & 0.00835297 & 0.017902988 \\
\hline SPBC800.05c & atb2 & tubulin alpha 2 & 0.78462277 & 7.94324297 & 1.39E-17 & $2.10 \mathrm{E}-16$ \\
\hline SPCC1223.08c & dfr1 & dihydrofolate reductase/ serine hydrolase family fusion pro & 0.78461075 & 8.5749766 & 4.17E-24 & $1.02 \mathrm{E}-22$ \\
\hline SPBC115.03 & SPBC115.03 & gfo/idh/mocA family oxidoreductase (predicted) & 0.78381598 & 6.43485147 & 4.60E-07 & $2.23 \mathrm{E}-06$ \\
\hline SPCC1223.04c & set11 & ribosomal protein lysine methyltransferase Set11 & 0.78350095 & 4.86611801 & 0.00095505 & 0.002575935 \\
\hline SPAC25G10.09c & pan1 & actin cortical patch component, with EF hand and WH2 mo1 & 0.78345651 & 8.8031304 & 1.39E-18 & $2.30 \mathrm{E}-17$ \\
\hline SPAC328.05 & hrb1 & RNA-binding protein involved in export of mRNAs Hrb1 (pre & 0.78243382 & 6.26748189 & $1.41 \mathrm{E}-08$ & $8.41 \mathrm{E}-08$ \\
\hline SPCC16C4.03 & pin1 & peptidyl-prolyl cis-trans isomerase Pin1 & 0.78206904 & 5.59088933 & 3.11E-05 & 0.000112158 \\
\hline SPAC869.02c & yhb1 & nitric oxide dioxygenase Yhb1 & 0.78197512 & 8.89036824 & $7.63 \mathrm{E}-26$ & 2.07E-24 \\
\hline SPCC24B10.05 & $\operatorname{tim} 9$ & Tim9-Tim10 complex subunit Tim9 (predicted) & 0.7815195 & 6.24757116 & 2.93E-08 & $1.69 \mathrm{E}-07$ \\
\hline SPBC1773.04 & SPBC1773.04 & methylglyoxyl reductase (NADPH-dependent) (predicted) & 0.78138259 & 7.79116345 & $1.55 \mathrm{E}-18$ & 2.57E-17 \\
\hline SPRRNA.34 & SPRRNA.34 & SS rRNA & 0.78082813 & 4.13276617 & 0.00866071 & 0.018504287 \\
\hline SPBP4H10.16c & SPBP4H10.16c & G-patch RNA-binding protein, involved in splicing (predictec & 0.78034052 & 5.42952507 & 4.09E-05 & 0.00014474 \\
\hline SPCC191.04c & SPCC191.04c & Schizosaccharomyces pombe specific protein & 0.77985104 & 2.65902699 & 0.11216584 & 0.172846135 \\
\hline SPNCRNA.1419 & \#N/A & \#N/A & 0.77937774 & 3.7520296 & 0.02406469 & 0.046012979 \\
\hline
\end{tabular}


SPBC3B9.01

SPNCRNA.865

SPAC637.05C

SPCC1450.06c

SPAC1805.16c

SPBC365.04C

SPAC7D4.08

SPCC1393.03

SPNCRNA.402

SPAC637.07

SPNCRNA.1222

SPAC22A12.10

SPBC1347.02

SPBC11G11.05

SPCC777.10c

SPNCRNA.213

SPAC959.02

SPNCRNA.1597

SPNCRNA.135

SPAC17G6.15C

SPNCRNA.812

SPAC13A11.06

SPAC2F $3.03 \mathrm{C}$

SPAC1952.11C

SPNCRNA.1149

SPNCRNA.1041

SPNCRNA.625

SPNCRNA.741

SPAC5H10.01

SPAPJ691.03

SPAC3G6.13C

SPAC24C9.03

SPBC16E9.13

SPAC26F1.13C

SPNCRNA.1332

SPCTRNAGLU.09

SPAC6F 12.07

SPBC337.15C

SPNCRNA.1292

SPAC16E8.14C

SPBC215.06c

SPAC2H10.01

SPCC1494.09C

SPAC27E2.10C

SPBC21B10.04C

SPCC1827.06c

SPAC56E4.02C fes1

\#N/A

vma2

grx3

SPAC1805.16c

SPBC365.04C

SPAC7D4.08

rps1501

$\# N / A$

moe1

\#N/A

ept1

ani1

rpa34

ubc12

\#N/A

$\sec 17$

$\# N / A$

\#N/A

fsf1

$\#$ N/A

pdc202

rpa49

ure2

$\# N / A$

$\# N / A$

$\# N / A$

$\# \mathrm{~N} / \mathrm{A}$

dgc1

mic10

rpl4101

mvd1

ksp1

Irs1

$\# \mathrm{~N} / \mathrm{A}$

SPCTRNAGLU.09

tom 20

coq7

$\# \mathrm{~N} / \mathrm{A}$

tae1

SPBC215.06c

SPAC2 10.01

tfb6

rfc3

nrf1

SPCC1827.06c

alg13
Hsp70 nucleotide exchange factor Fes1 (predicted)

$$
\# N / A
$$

V-type ATPase V1 subunit B

CIA machinery monothiol glutaredoxin Grx3

purine nucleoside phosphorylase (predicted)

RNA-binding protein, involved in ribosome biogenesis (pred $0.77577621 \quad 5.487977450 .00049589$ $\begin{array}{llllll}\text { Schizosaccharomyces pombe specific protein } & 0.77566422 & 4.31992414 & 0.00351963\end{array}$

$40 \mathrm{~S}$ ribosomal protein S15 (predicted)

$$
\text { \#N/A }
$$

translation initiation factor elF3d Moe1

$$
\text { \#N/A }
$$

$0.77366892 \quad 4.54841674 \quad 0.00308339$

$\begin{array}{lll}0.77153028 & 8.48318747 \quad 1.12 \mathrm{E}-20\end{array}$

$\begin{array}{llll}0.77128982 & 3.32978062 & 0.05546552\end{array}$

diacylglycerol cholinephosphotransferase/ diacylglycerol etl $0.77096156 \quad 8.26844735 \quad 6.18 \mathrm{E}-23$

$\begin{array}{llll}\text { CENP-A amino terminus domain (NTD) isomerase Ani1 } & 0.77006438 & 7.42320865 & 2.23 \mathrm{E}-12\end{array}$

DNA-directed RNA polymerase I complex subunit Rpa34 (pr $0.768713356 .69233525 \quad 1.96 \mathrm{E}-09$

NEDD8-conjugating enzyme Ubc12

$\begin{array}{lll}0.76750625 & 5.02479629 & 0.00054347\end{array}$

$\begin{array}{lll}0.76596829 & 1.16850296 & 0.52950427\end{array}$

$0.76511677 \quad 7.1706058 \quad 4.41 \mathrm{E}-13$

$0.76429931 \quad 9.20370255-3.62 \mathrm{E}-25$

$0.763940346 .07319629 \quad 2.19 \mathrm{E}-05$

$0.763702496 .30065783 \quad 1.06 \mathrm{E}-08$

$\begin{array}{crrr}\text { mitochondrial carrier, serine Fsf1 (predicted) } & 0.76370249 & 6.30065783 & 1.06 \mathrm{E}-08 \\ \text { \#N/A } & 0.7635614 & 6.99278623 & 4.77 \mathrm{E}-10 \\ \text { pyruvate decarboxylase (predicted) } & 0.76239916 & 5.98025231 & 2.99 \mathrm{E}-06\end{array}$

$\begin{array}{crrr}\text { mitochondrial carrier, serine Fsf1 (predicted) } & 0.76370249 & 6.30065783 & 1.06 \mathrm{E}-08 \\ \text { \#N/A } & 0.7635614 & 6.99278623 & 4.77 \mathrm{E}-10 \\ \text { pyruvate decarboxylase (predicted) } & 0.76239916 & 5.98025231 & 2.99 \mathrm{E}-06\end{array}$

DNA-directed RNA polymerase I complex subunit Rpa49 (pr $0.759930376 .96436943 \quad 5.17 \mathrm{E}-09$ nickel-dependent urease Ure2

\section{$\# N / A$}

$\# N / A$

\#N/A

0.759650398 .25842148

$0.75781877 \quad 6.12232208 \quad 3.63 \mathrm{E}-06$

$\begin{array}{llll}0.75750578 & 8.25842672 & 9.19 \mathrm{E}-16\end{array}$

$\begin{array}{lll}0.75745043 & 6.88280319 & 1.31 \mathrm{E}-09\end{array}$

$0.75689494 \quad 7.39273389$

$0.75683915 \quad 6.68431596 \quad 4.54 \mathrm{E}-10$

$0.75646583 \quad 6.00371906 \quad 7.80 \mathrm{E}-07$

$0.756273316 .75406805 \quad 3.43 \mathrm{E}-09$

$0.756117127 .65716706 \quad 8.95 \mathrm{E}-17$

$\begin{array}{lll}0.75535678 & 8.34127548 \quad 2.15 \mathrm{E}-15\end{array}$

$0.75486843 \quad 9.34621908 \quad 2.75 \mathrm{E}-23$

$0.75436086 \quad 6.77347935 \quad 1.89 \mathrm{E}-09$

$\begin{array}{llll}0.75381673 & 4.16708616 & 0.00719134\end{array}$

$0.7535467 \quad 5.99912794 \quad 3.65 \mathrm{E}-06$

$0.7528506 \quad 6.59299415 \quad 2.36 \mathrm{E}-08$

$0.7527413 \quad 6.19629081 \quad 9.90 \mathrm{E}-06$

mitochondrial TOM complex subunit Tom20 (predicted)

ubiquinone biosynthesis protein Coq7

$$
\# \mathrm{~N} / \mathrm{A}
$$

0.752041915 .85671413

$6.32 \mathrm{E}-06$

nucleolar RNA-binding protein, human LYAR homolog, impl $\begin{array}{lllll}0.751802 & 5.09414498 & 0.00050749\end{array}$

transcription factor, zf-fungal binuclear cluster type (predic $0.75179046 \quad 5.63534978 \quad 0.00030642$ holo TFIIH complex subunit Tfb6

$\begin{array}{llll}0.75030348 & 4.46947287 & 0.04304717\end{array}$

DNA replication factor $\mathrm{C}$ complex subunit $\mathrm{Rfc} 3$

$0.750138 \quad 5.8627694 \quad 1.31 \mathrm{E}-05$

$\begin{array}{llll}0.750138 & 5.8627694 & 1.31 E-05\end{array}$

$\begin{array}{lllll}\text { aspartate semialdehyde dehydrogenase (predicted) } & 0.74771215 & 7.85415092 & 4.51 \mathrm{E}-16\end{array}$

$\mathrm{N}$-acetylglucosaminyldiphosphodolichol N-acetylglucosamir $0.74771049 \quad 5.32668541 \quad 0.00120868$
3.19E-06

$5.99 \mathrm{E}-19$

$1.05 \mathrm{E}-16$

0.001082948

2.35E-06

0.001439158

0.008307689

$1.01 \mathrm{E}-13$

0.007390791

$2.30 \mathrm{E}-19$

0.094758013

1.44E-21

$2.12 \mathrm{E}-11$

1.31E-08

0.001557186

0.620933408

$4.50 \mathrm{E}-12$

$9.48 \mathrm{E}-24$

8.15E-05

6.42E-08

3.45E-09

1.30E-05

3.29E-08

$8.94 \mathrm{E}-15$

1.55E-05

$1.18 \mathrm{E}-14$

9.03E-09

1.65E-12

$3.31 \mathrm{E}-09$

3.64E-06

2.22E-08

1.25E-15

2.67E-14

$6.55 \mathrm{E}-22$

$1.27 \mathrm{E}-08$

0.015679928

1.56E-05

1.37E-07

3.92E-05

2.60E-05

0.001470815

0.000926328

0.076265557

5.06E-05

0.000543045

5.95E-15

0.003181844 


\begin{tabular}{|c|c|c|c|c|c|c|}
\hline SPCC613.06 & rpl902 & $60 S$ ribosomal protein $L 9$ & 0.74720633 & 8.08894128 & $1.94 \mathrm{E}-19$ & $3.56 \mathrm{E}-18$ \\
\hline SPBC800.06 & brx1 & ribosome biogenesis protein Brx1 (predicted) & 0.74679347 & 6.7151024 & 4.21E-09 & 2.71E-08 \\
\hline SPBC14C8.06 & $\operatorname{arc1}$ & ARP2/3 actin-organizing complex subunit Sop2 & 0.74628372 & 8.42327793 & $3.34 \mathrm{E}-21$ & $7.31 \mathrm{E}-20$ \\
\hline SPNCRNA.1122 & \#N/A & \#N/A & 0.74560128 & 3.26718324 & 0.07697284 & 0.125131432 \\
\hline SPCC297.05 & SPCC297.05 & DENN domain Rab GDP-GTP exchange factor, implicated in & 0.74547342 & 7.00940578 & 2.53E-11 & $2.16 \mathrm{E}-10$ \\
\hline SPAC1687.21 & SPAC1687.21 & fructose-2,6-bisphosphatase, human TIGAR ortholog (predi & 0.74523097 & 7.12492536 & $6.82 \mathrm{E}-13$ & $6.86 \mathrm{E}-12$ \\
\hline SPBC947.04 & $\mathrm{pfl3}$ & cell surface glycoprotein, flocculin Pf|3, DIPSY family & 0.74517265 & 7.46420439 & $4.65 \mathrm{E}-15$ & $5.58 \mathrm{E}-14$ \\
\hline SPAC1687.05 & pli1 & SUMO E3 ligase Pli1 & 0.74489779 & 8.23790262 & $5.44 \mathrm{E}-19$ & $9.40 \mathrm{E}-18$ \\
\hline SPBC3H7.02 & SPBC3H7.02 & sulfate transmembrane transporter (predicted) & 0.74390195 & 7.24674379 & $4.98 \mathrm{E}-11$ & $4.03 E-10$ \\
\hline SPAC20G8.05c & cdc15 & extended Fer/CIP4 (EFC) domain protein Cdc15 & 0.74373229 & 8.19811807 & $1.12 \mathrm{E}-11$ & 9.90E-11 \\
\hline SPAC2C4.16c & rps801 & $40 \mathrm{~S}$ ribosomal protein $\mathrm{S} 8$ (predicted) & 0.74317571 & 8.06680865 & 1.11E-19 & $2.07 \mathrm{E}-18$ \\
\hline SPNCRNA.366 & $\# N / A$ & $\# N / A$ & 0.74284091 & 0.94610028 & 0.69607586 & 0.768570505 \\
\hline SPAC8E11.02c & $\operatorname{rad} 24$ & 14-3-3 protein Rad24 & 0.7427109 & 8.84826081 & $9.38 \mathrm{E}-25$ & $2.40 \mathrm{E}-23$ \\
\hline SPNCRNA.612 & \#N/A & \#N/A & 0.74269392 & 6.70528727 & $5.26 \mathrm{E}-10$ & 3.79E-09 \\
\hline SPBC582.09 & pex11 & peroxisomal biogenesis factor 11 (predicted) & 0.74144849 & 6.26630135 & 3.31E-05 & 0.000118593 \\
\hline SPAC1783.08c & rpl1502 & $60 S$ ribosomal protein L15b (predicted) & 0.74134332 & 7.75033595 & $2.01 \mathrm{E}-16$ & $2.73 \mathrm{E}-15$ \\
\hline SPBC8D2.23 & new15 & mitochondrial protein, ribosomal subunit L35 (predicted) & 0.74104987 & 3.6570729 & 0.06403986 & 0.107074015 \\
\hline SPCP1E11.10 & SPCP1E11.10 & ankyrin repeat protein, unknown biological role & 0.74095118 & 5.62269421 & 1.06E-05 & 4.18E-05 \\
\hline SPAC2C4.04c & $\operatorname{aim} 29$ & DUF2340, human C2orf76 ortholog, implicated in mitochon & 0.74088918 & 5.6225415 & $4.20 \mathrm{E}-05$ & 0.000147555 \\
\hline SPNCRNA.575 & $\# N / A$ & \#N/A & 0.74067789 & 5.14312168 & 0.0001893 & 0.000595288 \\
\hline SPAC186.08c & SPAC186.08c & L-lactate dehydrogenase (predicted) & 0.74067684 & 0.94641462 & 0.69617265 & 0.768570505 \\
\hline SPAC186.09 & pdc102 & pyruvate decarboxylase (predicted) & 0.74067684 & 0.94641462 & 0.69617265 & 0.768570505 \\
\hline SPNCRNA.46 & $\# N / A$ & $\# N / A$ & 0.74067684 & 0.94641462 & 0.69617265 & 0.768570505 \\
\hline SPAC19D5.05c & imp3 & U3 snoRNP-associated protein Imp3 (predicted) & 0.74049735 & 5.12930861 & 0.0003304 & 0.000994426 \\
\hline SPCC584.15c & SPCC584.15c & arrestin involved in ubiquitin-dependent endocytosis & 0.74020185 & 7.65113143 & $3.62 \mathrm{E}-13$ & $3.74 \mathrm{E}-12$ \\
\hline SPBC3B8.07c & $\mathrm{ds} d 1$ & dihydroceramide delta- 4 desaturase & 0.7401865 & 7.86692578 & $4.45 \mathrm{E}-11$ & $3.64 \mathrm{E}-10$ \\
\hline SPBC19F8.08 & $\operatorname{rps} 401$ & $40 \mathrm{~S}$ ribosomal protein $\mathrm{S} 4$ (predicted) & 0.73912187 & 8.4507124 & $1.16 \mathrm{E}-21$ & $2.57 \mathrm{E}-20$ \\
\hline SPNCRNA.117 & $\# \mathrm{~N} / \mathrm{A}$ & \#N/A & 0.73847833 & 0.94629987 & 0.69615475 & 0.768570505 \\
\hline SPNCRNA.1540 & $\# N / A$ & $\# N / A$ & 0.73847833 & 0.94629987 & 0.69615475 & 0.768570505 \\
\hline SPCC550.06c & hsp10 & mitochondrial heat shock protein Hsp10 (predicted) & 0.7379282 & 7.46427161 & 3.97E-14 & 4.39E-13 \\
\hline SPBC776.01 & rpl29 & $60 S$ ribosomal protein $\mathrm{L} 29$ & 0.73779658 & 6.59846461 & $3.23 \mathrm{E}-07$ & 1.60E-06 \\
\hline SPBC557.02c & SPBC557.02c & DUF2458 conserved fungal protein & 0.73717828 & 6.02120686 & $1.65 \mathrm{E}-06$ & 7.39E-06 \\
\hline SPNCRNA.435 & $\# N / A$ & $\# N / A$ & 0.73637207 & 0.94685974 & 0.69634877 & 0.768570505 \\
\hline SPAC19A8.04 & erg5 & C-22 sterol desaturase Erg5 & 0.73611905 & 8.91260997 & 1.17E-14 & $1.35 \mathrm{E}-13$ \\
\hline SPAC1805.12c & uep1 & ribosomal-ubiquitin fusion protein Ubi2 & 0.73530083 & 7.68307483 & $3.48 \mathrm{E}-15$ & 4.23E-14 \\
\hline SPBC530.13 & Isc1 & Lsk1 associated cyclin & 0.73524875 & 5.09547381 & 0.00160552 & 0.004107351 \\
\hline SPAC22E12.04 & $\operatorname{ccs} 1$ & superoxide dismutase copper chaperone Ccs1 & 0.73512979 & 6.36367413 & 4.91E-07 & 2.37E-06 \\
\hline SPCC4G3.17 & hdd1 & HD domain protein phosphoric ester hydrolase family (pred & 0.73395397 & 6.30237975 & $1.26 \mathrm{E}-06$ & 5.76E-06 \\
\hline SPAC3H5.07 & rpl702 & $60 \mathrm{~S}$ ribosomal protein $\mathrm{L7b}$ involved in cytoplasmic translatic & 0.73307352 & 7.77505361 & $1.46 \mathrm{E}-15$ & $1.85 \mathrm{E}-14$ \\
\hline SPBC1709.02c & vrs1 & cytoplasmic valine-tRNA ligase Vrs1/Vas1 & 0.73274992 & 9.17204822 & 2.69E-24 & $6.68 \mathrm{E}-23$ \\
\hline SPAC3A12.10 & rpl2001 & 60S ribosomal protein L20a (predicted) & 0.73184274 & 8.04029443 & $1.76 \mathrm{E}-16$ & $2.40 \mathrm{E}-15$ \\
\hline SPCC5E4.07 & $\mathrm{rpl} 2802$ & $60 S$ ribosomal protein $\mathrm{L} 27 / \mathrm{L} 28$ & 0.73123111 & 7.92877288 & $2.83 \mathrm{E}-16$ & $3.82 \mathrm{E}-15$ \\
\hline SPAC13C5.05c & SPAC13C5.05c & $\mathrm{N}$-acetylglucosamine-phosphate mutase (predicted) & 0.7311091 & 8.27543508 & $3.15 \mathrm{E}-13$ & $3.28 \mathrm{E}-12$ \\
\hline SPAC1687.02 & rce1 & CAAX prenyl protease (predicted) & 0.730895 & 7.23889135 & $3.33 \mathrm{E}-13$ & $3.45 \mathrm{E}-12$ \\
\hline SPAC26F1.06 & gpm1 & monomeric 2,3-bisphosphoglycerate (BPG)-dependent phos & 0.7298411 & 8.9280706 & $5.55 E-23$ & 1.30E-21 \\
\hline SPBC1815.01 & eno101 & enolase (predicted) & 0.72982241 & 10.0091927 & $5.36 \mathrm{E}-27$ & $1.59 \mathrm{E}-25$ \\
\hline SPAC17A5.03 & rpl301 & $60 S$ ribosomal protein $\mathrm{L} 3$ & 0.72965634 & 9.31596575 & $5.39 E-21$ & 1.15E-19 \\
\hline
\end{tabular}




\begin{tabular}{|c|c|c|c|c|c|c|}
\hline SPAC890.07c & rmt1 & type I protein arginine $\mathrm{N}$-methyltransferase $\mathrm{Rmt1}$ & 0.72962207 & 6.9417597 & 9.19E-10 & $6.44 \mathrm{E}-09$ \\
\hline SPAPB1A10.15 & arv1 & Arv1-like family protein Arv1 (predicted) & 0.72827388 & 4.8107808 & 0.00257436 & 0.006274532 \\
\hline SPAC4G9.04c & pcf11 & mRNA cleavage and polyadenylation specificity factor (prec & 0.72799921 & 8.22662045 & 3.65E-19 & $6.41 \mathrm{E}-18$ \\
\hline SPAC3F10.03 & grs1 & mitochondrial and cytoplasmic glycine-tRNA ligase Grs1 & 0.72764253 & 8.74599028 & $3.46 \mathrm{E}-17$ & $5.04 \mathrm{E}-16$ \\
\hline SPAC144.11 & rps1102 & 40S ribosomal protein S11 (predicted) & 0.72751684 & 6.66574344 & $1.14 \mathrm{E}-07$ & 5.99E-07 \\
\hline SPAC15E1.10 & fub1 & PI31 proteasome inhibitor Fub1 (predicted) & 0.72721235 & 4.84919549 & 0.02077873 & 0.040492337 \\
\hline SPAP32A8.03c & bop1 & ubiquitin-protein ligase E3, human RNF126 ortholog (predic & 0.72626531 & 7.22484246 & 1.47E-12 & $1.44 \mathrm{E}-11$ \\
\hline SPAC2E1P5.02c & mug109 & Rab GTPase binding protein upregulated in meiosis II (pred & 0.72589641 & 4.7611905 & 0.0013412 & 0.003490197 \\
\hline SPNCRNA.371 & $\# N / A$ & \#N/A & 0.72516443 & 2.6340634 & 0.20099147 & 0.281661238 \\
\hline SPBC337.03 & rhn1 & RNA polymerase II transcription termination factor homolo: & 0.72486789 & 5.63842253 & 0.00027123 & 0.000828044 \\
\hline SPCC962.04 & rps1201 & 40S ribosomal protein S12 (predicted) & 0.72471625 & 7.5857953 & 8.47E-15 & $9.96 \mathrm{E}-14$ \\
\hline SPNCRNA.1358 & $\# N / A$ & $\# N / A$ & 0.72460195 & 6.27463613 & $6.84 \mathrm{E}-08$ & 3.71E-07 \\
\hline SPBP8B7.11 & nxt3 & ubiquitin protease cofactor Nxt3 (predicted) & 0.72458459 & 7.47242743 & $7.90 \mathrm{E}-12$ & 7.09E-11 \\
\hline SPCC1442.07c & wss2 & ubiquitin/metalloprotease fusion protein Udp7 & 0.72380705 & 6.22600316 & $6.44 \mathrm{E}-07$ & $3.06 \mathrm{E}-06$ \\
\hline SPAC1002.17c & urg2 & uracil phosphoribosyltransferase (predicted) & 0.72375186 & 5.20678317 & 0.00060033 & 0.001701123 \\
\hline SPNCRNA.639 & $\# N / A$ & \#N/A & 0.72371541 & 9.0769092 & $2.80 \mathrm{E}-22$ & $6.39 \mathrm{E}-21$ \\
\hline SPNCRNA.1639 & $\# N / A$ & $\# N / A$ & 0.72283153 & 5.93417079 & $8.60 \mathrm{E}-06$ & $3.45 \mathrm{E}-05$ \\
\hline SPBC119.10 & asn1 & asparagine synthetase & 0.72244891 & 9.19131957 & $1.85 E-23$ & $4.44 \mathrm{E}-22$ \\
\hline SPNCRNA.1658 & \#N/A & $\# N / A$ & 0.72085698 & 8.89555193 & $3.62 \mathrm{E}-16$ & $4.81 \mathrm{E}-15$ \\
\hline SPCC285.15c & rps2802 & $40 \mathrm{~S}$ ribosomal protein $\mathrm{S} 28, \mathrm{Rps} 2802$ & 0.72058693 & 6.87229395 & $6.68 \mathrm{E}-10$ & 4.75E-09 \\
\hline SPCC1672.03c & gud1 & guanine deaminase Gud1 (predicted) & 0.72027855 & 7.22110331 & $4.31 \mathrm{E}-12$ & $3.98 \mathrm{E}-11$ \\
\hline SPBC1348.10c & SPBC1348.10c & phospholipase (predicted) & 0.72025938 & 8.47882776 & $1.11 \mathrm{E}-14$ & $1.29 \mathrm{E}-13$ \\
\hline SPAC3H5.12c & rpl501 & $60 S$ ribosomal protein L5 (predicted) & 0.72006468 & 8.6389804 & $9.86 \mathrm{E}-21$ & 2.05E-19 \\
\hline SPAC22E12.01 & pet3 & Golgi phosphoenolpyruvate transmembrane transporter Pet & 0.71997165 & 6.10517172 & 4.16E-07 & $2.02 \mathrm{E}-06$ \\
\hline SPAC11D3.02c & SPAC11D3.02c & ELLA family acetyltransferase (predicted) & 0.71980989 & 6.59121615 & $6.01 \mathrm{E}-06$ & $2.48 \mathrm{E}-05$ \\
\hline SPBC30B4.04c & sol1 & SWI/SNF complex subunit Sol1 & 0.71888577 & 6.81223924 & 1.57E-09 & $1.07 \mathrm{E}-08$ \\
\hline SPNCRNA.1300 & $\# N / A$ & \#N/A & 0.71851432 & 4.74568269 & 0.004658 & 0.010676219 \\
\hline SPNCRNA.287 & $\# N / A$ & $\# N / A$ & 0.71851432 & 4.74568269 & 0.004658 & 0.010676219 \\
\hline SPNCRNA.608 & $\# N / A$ & \#N/A & 0.71786752 & 7.83344061 & $5.86 \mathrm{E}-15$ & 6.99E-14 \\
\hline SPBC12D12.05c & SPBC12D12.05c & mitochondrial carrier, ATP:ADP antiporter (predicted) & 0.71756607 & 6.63403538 & $1.72 \mathrm{E}-08$ & $1.02 \mathrm{E}-07$ \\
\hline SPAC186.01 & pfl9 & cell surface glycoprotein, flocculin PfI9, DIPSY family & 0.7174822 & 5.04822632 & 0.00065448 & 0.001844949 \\
\hline SPBC16G5.05c & $\operatorname{scs} 2$ & VAP family protein Scs2 & 0.71672705 & 8.48154771 & 4.85E-18 & 7.67E-17 \\
\hline SPAC16C9.06c & upf1 & ATP-dependent RNA helicase Upf1 & 0.71654899 & 6.88891738 & 1.89E-08 & $1.11 \mathrm{E}-07$ \\
\hline SPCPB16A4.03c & ade10 & bifunctional IMP cyclohydrolase/phosphoribosylaminoimida & 0.71541443 & 8.59877235 & $1.17 \mathrm{E}-18$ & 1.96E-17 \\
\hline SPBC106.03 & SPBC106.03 & mitochondrial Rossman fold DUF1776 family protein & 0.71349719 & 6.83123886 & 1.69E-08 & 9.96E-08 \\
\hline SPBC1604.13c & mrpl32 & mitochondrial ribosomal protein subunit L32 (predicted) & 0.71335565 & 5.0425238 & 0.01430409 & 0.029117353 \\
\hline SPBTRNAGLU.08 & SPBTRNAGLU.08 & tRNA Glutamic acid & 0.71285851 & 3.84121943 & 0.03078162 & 0.056982833 \\
\hline SPBC336.06c & rnh1 & ribonuclease H Rnh1 (predicted) & 0.71213843 & 5.96801995 & 1.97E-06 & 8.73E-06 \\
\hline SPAC3F10.06c & rit1 & initiator methionine tRNA 2'-O-ribosyl phosphate transfera: & 0.71149233 & 6.20906428 & $3.66 \mathrm{E}-06$ & $1.56 \mathrm{E}-05$ \\
\hline SPBC16G5.15c & $\mathrm{fkh} 2$ & forkhead transcription factor Fkh2 & 0.71132487 & 6.58222768 & $1.28 \mathrm{E}-07$ & 6.67E-07 \\
\hline SPCC24B10.21 & tpi1 & triosephosphate isomerase & 0.71094629 & 8.94110685 & 1.19E-19 & $2.21 \mathrm{E}-18$ \\
\hline SPAC977.09c & SPAC977.09c & phospholipase (predicted) & 0.71066215 & 8.50287612 & 3.67E-14 & 4.09E-13 \\
\hline SPCC1322.15 & rpl3402 & $60 S$ ribosomal protein $\mathrm{L} 34$ & 0.71064891 & 5.95282751 & $8.88 \mathrm{E}-05$ & 0.000295211 \\
\hline SPBC887.10 & $\operatorname{mcs} 4$ & response regulator Mcs4 & 0.70982444 & 6.55029903 & $9.27 \mathrm{E}-06$ & 3.70E-05 \\
\hline SPBC21B10.10 & $\operatorname{rps} 402$ & $40 \mathrm{~S}$ ribosomal protein S4 (predicted) & 0.70964695 & 8.14575692 & $6.23 \mathrm{E}-19$ & 1.06E-17 \\
\hline SPBC23G7.15c & rpp202 & $60 S$ acidic ribosomal protein $\mathrm{A} 4$ & 0.70893587 & 7.33715241 & $1.36 \mathrm{E}-11$ & 1.19E-10 \\
\hline SPBP23A10.16 & $\operatorname{tim} 18$ & TIM22 inner membrane protein import complex anchor sub & 0.70892177 & 7.10234399 & $1.08 \mathrm{E}-11$ & $9.53 \mathrm{E}-11$ \\
\hline
\end{tabular}




$\begin{array}{ll}\text { SPAC11G7.04 } & \text { ubi1 } \\ \text { SPAC23C4.14 } & \text { alg1 } \\ \text { SPRRNA.55 } & \text { SPRRNA.55 } \\ \text { SPBC405.01 } & \text { ade1 } \\ \text { SPAC1805.13 } & \text { rpl14 } \\ \text { SPCC777.09c } & \text { arg1 } \\ \text { SPBC11C11.08 } & \text { srp1 } \\ \text { SPAC644.05c } & \text { dut1 } \\ \text { SPBP8B7.10c } & \text { utp16 } \\ \text { SPBC3D6.15 } & \text { rps2501 } \\ \text { SPCC417.08 } & \text { tef3 } \\ \text { SPBP16F5.05c } & \text { yar1 } \\ \text { SPAC4G9.11c } & \text { cmb1 } \\ \text { SPAC20G4.06c } & \text { adf1 } \\ \text { SPBC24C6.05 } & \text { sec28 } \\ \text { SPBC19G7.03c } & \text { rps3002 } \\ \text { SPNCRNA.1103 } & \\ \text { SPCC162.03 } & \text { SPCC162.03 } \\ \text { SPCC1795.04c } & \text { pre10 } \\ \text { SPNCRNA.1434 } & \\ \text { SPCC645.06c } & \text { rgf3 } \\ \text { SPAC5H10.06c } & \text { adh4 } \\ \text { SPBC2G2.04c } & \text { mmf1 } \\ \text { SPCC126.12 } & \text { SPCC126.12 } \\ \text { SPAC1805.06c } & \text { hem2 } \\ \text { SPAC12B10.02c } & \text { SPAC12B10.02c } \\ \text { SPCC1739.05 } & \text { set5 } \\ \text { SPBC17A3.04c } & \text { rar1 } \\ \text { SPNCRNA.1143 } & \\ \text { SPBC2D10.12 } & \text { rhp23 } \\ \text { SPBC215.15 } & \text { sec13 } \\ \text { SPAC139.03 } & \text { toe2 } \\ \text { SPBC359.01 } & \text { SPBC359.01 } \\ \text { SPBC16E9.08 } & \text { mcp4 } \\ \text { SPAC23A1.08c } & \text { rpl3401 } \\ \text { SPBC14C8.03 } & \text { fma2 } \\ \text { SPAC821.06 } & \text { spn2 } \\ \text { SPAPB24D3.06c } & \text { SPAPB24D3.06c } \\ \text { SPNCRNA.1136 } & \\ \text { SPAC56F8.08 } & \text { mud1 \#N/A } \\ \text { SPAC26A3.03c } & \text { rmi1 } \\ \text { SPBC244.02c } & \text { utp6 } \\ \text { SPCC1840.03 } & \text { sal3 } \\ \text { SPCC825.05c } & \text { pwi1 } \\ \text { SPBC582.08 } & \text { SPBC582.08 } \\ \text { SPAC1142.06 } & \text { get3 } \\ \text { SPBC56F2.10c } & \text { alg5 } \\ & \end{array}$

ribosomal-ubiquitin fusion protein Ubi1 (predicted)

mannosyltransferase complex subunit Alg1 (predicted)

$5 \mathrm{~S}$ rRNA (predicted)

$0.70816151 \quad 7.702743$

0.707877895 .74537827

0.707838134 .16669772

$2.73 \mathrm{E}-15$

4.53E-05

phosphoribosylamine-glycine ligase/phosphoribosylformylg 0.706063568 .54252755

0.705691787 .69780152

60 S ribosomal protein L14 (predicted)

acetylornithine aminotransferase

0.705247458 .60955225

0.704092297 .68275648

deoxyuridine 5'-triphosphate 0.70359085 .67793498

U3 snoRNP-associated protein Utp16 (predicted)

$40 \mathrm{~S}$ ribosomal protein $\mathrm{S} 25$ (predicted)

translation elongation factor eEF3

ribosome biogenesis protein Yar1 (predicted)

cytosine-mismatch binding protein 1

actin depolymerizing factor, cofilin

coatomer epsilon subunit (predicted)

$40 \mathrm{~S}$ ribosomal protein $\mathrm{S30}$ (predicted) \#N/A

short chain dehydrogenase (predicted)

$20 S$ proteasome complex subunit alpha 7, Pre10

\#N/A

RhoGEF Rgf3

alcohol dehydrogenase Adh

$0.70292453 \quad 5.16940436 \quad 0.00040769$

$0.70218974 \quad 6.5854301 \quad 6.66 \mathrm{E}-08$

$0.702144 \quad 10.6431746 \quad 5.82 \mathrm{E}-27$

$0.7018383 \quad 5.42936119 \quad 7.57 \mathrm{E}-05$

$0.701801115 .71188929 \quad 3.51 \mathrm{E}-05$

$0.7016693 \quad 7.50740288 \quad 3.35 \mathrm{E}-12$

$0.70144338 \quad 7.02304783 \quad 6.62 \mathrm{E}-11$

$0.70115957 \quad 6.50768453 \quad 1.46 \mathrm{E}-06$

$0.70114713 \quad 8.12552747 \quad 4.53 \mathrm{E}-18$

$0.70112334 \quad 5.53043753 \quad 0.00010293$

$0.70060193 \quad 6.94907165 \quad 3.51 \mathrm{E}-10$

$\begin{array}{lll}0.70055395 & 3.98357442 & 0.0340075\end{array}$

$0.700166838 .19992236 \quad 2.16 \mathrm{E}-12$

$\begin{array}{lll}0.69998054 & 6.25265568 & 1.75 \mathrm{E}-05\end{array}$

mitochondrial matrix protein, YjgF family protein Mmf1, re: $0.69997437 \quad 7.43712119 \quad 5.07 \mathrm{E}-13$

$0.69966375 .97216822 \quad 1.95 \mathrm{E}-06$

porphobilinogen synthase Hem2 (predicted) $0.69950373 \quad 7.21967497$

$\begin{array}{llll}\text { endoplasmic reticulum resident protein required for packag } & 0.6991465 & 6.28171438 & 1.60 \mathrm{E}-07\end{array}$

histone lysine methyltransferase Set5 (predicted) $\quad \begin{array}{llll}0.69895533 & 5.59736902 & 2.72 \mathrm{E}-05\end{array}$

cytoplasmic methionine-tRNA ligase Mrs1 (predicted) $\quad 0.698332428 .71303958 \quad 2.96 \mathrm{E}-19$

$0.69762277 \quad 7.23249948 \quad 2.89 \mathrm{E}-11$

$0.69628517 \quad 6.98771613 \quad 6.42 \mathrm{E}-08$

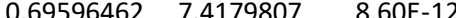

COPII-coated vesicle component Sec13

transcription factor, zf-fungal binuclear cluster type (predic $\begin{array}{lllll}0.6957945 & 5.53009907 & 0.00026691\end{array}$ $\begin{array}{lllll}\text { amino acid transmembrane transporter (predicted) } & 0.69556508 & 6.05886602 & 7.20 \mathrm{E}-06\end{array}$ prospore membrane protein Mcp4/Mug101 $\quad 0.694689494 .50073346 \quad 0.00994977$

$60 \mathrm{~S}$ ribosomal protein $\mathrm{L} 34$

methionine aminopeptidase Fma2 (predicted)

mitotic and meiotic (sporulation) septin Spn2

Alpha/Beta hydrolase fold, DUF1749 family protein

0.694659347 .36826528

$0.69417107 \quad 8.09243679 \quad 6.72 \mathrm{E}-17$

$0.692323596 .90638218 \quad 2.61 \mathrm{E}-09$

$0.69198499 \quad 6.78642641 \quad 1.99 \mathrm{E}-08$

$0.69140118 \quad 5.53122273 \quad 0.00025589$

UBA domain protein Mud1

$$
\# \text { N/A }
$$

0.691329517 .20569853

$1.60 \mathrm{E}-08$

$\begin{array}{lll}\text { RecQ mediated genome instability protein Rmi1 (predicted } 0.69078073 & 8.41725543 & 2.28 \mathrm{E}-19\end{array}$

U3 snoRNP-associated protein Utp6 (predicted)

$0.68978773-5.78406138 \quad 0.00013715$

karyopherin/importin beta family nuclear import signal rec $0.68926508 \quad 8.54209333 \quad 5.81 \mathrm{E}-19$ $\begin{array}{llll}\text { splicing coactivator Srm160 (predicted) } & 0.68857909 & 5.8908523 & 2.05 \mathrm{E}-05\end{array}$ $\begin{array}{llll}\text { alanine aminotransferase (predicted) } & 0.68855841 & 7.48291778 & 2.18 \mathrm{E}-12\end{array}$ GET complex (ER membrane insertion) subunit Get3 (predi $0.688358926 .98812261 \quad 1.81 \mathrm{E}-08$ dolichyl-phosphate beta-glucosyltransferase Alg5 $\quad 0.68820017 \quad 5.3815166 \quad 0.00156386$
3.34E-14

0.00015872

0.034265084

$5.98 \mathrm{E}-18$

4.92E-13

1.85E-19

$7.20 \mathrm{E}-13$

$6.55 \mathrm{E}-05$

0.001201014

3.63E-07

$1.71 \mathrm{E}-25$

0.000254441

0.000125571

3.13E-11

$5.26 \mathrm{E}-10$

6.59E-06

$7.18 \mathrm{E}-17$

0.000338523

2.59E-09

0.0622198

2.07E-11

6.64E-05

$5.14 \mathrm{E}-12$

8.66E-06

4.61E-10

8.20E-07

9.97E-05

5.24E-18

2.43E-10

3.50E-07

$7.68 \mathrm{E}-11$

0.000816321

2.93E-05

0.021007813

6.07E-12

9.53E-16

1.71E-08

$1.17 \mathrm{E}-07$

0.000785788

9.46E-08

4.13E-18

0.000442103

$9.99 \mathrm{E}-18$

7.66E-05

2.08E-11

1.07E-07

0.004012847 
SPBC1677.03C SPAC22F3.07c SPAC3G9.03 SPNCRNA.1104

SPNCRNA.415

SPAC26A3.11

SPAC926.04C

SPCC550.04c

SPAC19D5.10c

SPNCRNA.1233

SPNCRNA.553

SPBC365.16

SPAC13G6.07c

SPCC63.12c

SPAC1565.02c

SPAC139.01C

SPBC83.18c

A.1063

SPBC36B7.04

SPAPB1A10.05

SPNCRNA.927

SPAC12G12.04

SPAC1F3.07C

SPAC6F6.07c

SPAC20G4.07c

SPBC17G9.12C

SPBC27.08C

SPNCRNA.860

SPNCRNA.78

SPAC22E12.13C

SPAC343.05

SPAC12G12.07c

SPBC19C7.05

SPBC56F2.09c

SPAC1399.02

SPNCRNA.697

SPCC1795.02C

SPCC16C4.17

SPAC17G6.14C

SPBC16E9.14C

SPBC17D1.01

SPAC589.10c

SPAC1687.19c

SPBC17G9.03C

SPCC1450.15

SPBC21C3.08C

SPRRNA.04 rpl2301

$\# \mathrm{~N} / \mathrm{A}$

SPAC26A3.11

hsp90

gpi2

$\# N / A$

$\# N / A$

$\# \mathrm{~N} / \mathrm{A}$

SPBC365.16

rps60

pup3

SPAC1565.02c

ath2

fic1

\#N/A

dus1

SPAPB1A10.05

\#N/A

mcp60

rsc58

rps13

erg4

SPBC17G9.12C sua1

\section{$\# N / A$}

$\# N / A$

rlp24

vma1

SPAC12G12.07C

SPBC19C7.05

arg5

SPAC1399.02

\#N/A

vcx1

mug123

uap56

zrg17

spp41

ubi5

qtr1

krs1

SPCC1450.15

car2

SPRRNA.04 threonine ammonia-lyase Tda1

F1-FO ATP synthase subunit $\mathrm{G}$ (predicted)

$60 S$ ribosomal protein $\mathrm{L} 23$

$\# N / A$

\#N/A

mitochondrial omega-amidase (predicted)

Hsp90 chaperone

pig-C (predicted)

\section{$\# N / A$}

$\#$ \#/A

mitochondrial membrane protein, conserved in yeast and a $0.68214741 \quad 7.62812844 \quad 2.74 \mathrm{E}-07$

$40 \mathrm{~S}$ ribosomal protein $\mathrm{S} 6$

20 proteasome complex subunit beta 3, Pup3

0.681661158 .39516448

0.681552927 .1742286

P 0.68095446 .88900848

Ath1 complex protein Ath2 nuclease, XP-G family

C2 domain protein Fic1

$$
\# \mathrm{~N} / \mathrm{A}
$$

tRNA dihydrouridine synthase Dus1 (predicted)

Schizosaccharomyces specific protein

$$
\text { \#N/A }
$$

mitochondrial heat shock protein Hsp60/Mcp60

RSC complex subunit Rsc58

$40 \mathrm{~S}$ ribosomal protein $\mathrm{S} 13$

C-24(28) sterol reductase Erg4

HAD superfamily hydrolase, unknown role (predicted)

sulfate adenylyltransferase

$$
\# N / A
$$

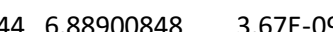

$\begin{array}{lll}0.68051538 & 7.45909172 \quad 4.78 \mathrm{E}-09\end{array}$

$0.68019623 \quad 6.07352215 \quad 2.84 \mathrm{E}-05$

$0.68007458 \quad 4.09556005 \quad 0.02124056$

$0.67973548 \quad 6.14862878 \quad 4.55 \mathrm{E}-06$

$0.679301695 .03553248 \quad 0.00675225$

$0.67922289 \quad 6.8355201 \quad 8.69 \mathrm{E}-08$

$0.67909253 \quad 9.1768145 \quad 5.36 \mathrm{E}-21$

$\begin{array}{lll}0.67901503 & 6.03144635 \quad 8.47 \mathrm{E}-06\end{array}$

$0.67888522 \quad 8.11664451 \quad 3.11 \mathrm{E}-15$

$0.678328526 .91621591 \quad 5.03 E-08$

$\begin{array}{llll}0.67829762 & 5.44794686 & 0.00050863\end{array}$

$\begin{array}{lll}0.67815509 & 8.49284146 \quad 4.38 \mathrm{E}-14\end{array}$

$0.67787809 \quad 5.71685825 \quad 0.00054692$

$\begin{array}{llll}0.67740318 & 3.31407689 & 0.09325179\end{array}$

ribosomal L24-like protein involved in ribosome biogenesis $\begin{array}{lllll}0.67698777 & 6.52241708 & 9.74 \mathrm{E}-06\end{array}$

V-type ATPase V1 domain, subunit A

$0.67649438 \quad 8.63493087 \quad 5.55 \mathrm{E}-19$

$0.67633496 \quad 8.01990399 \quad 6.25 \mathrm{E}-12$

conserved fungal protein

vesicle-mediated transport protein (predicted)

$0.67611698 \quad 6.806641390 .00051916$

$\begin{array}{lll}\text { arginine specific carbamoyl-phosphate synthase subunit Ar£ } 0.67583201 & 8.08643489 & 8.96 \mathrm{E}-14\end{array}$

transmembrane transporter (predicted)

$$
\text { \#N/A }
$$

4.23E-07

$0.67527917 \quad 6.61419339 \quad 2.00 \mathrm{E}-07$

$0.674985497 .38373779 \quad 2.28 \mathrm{E}-10$

(

$0.674907665 .13987445 \quad 0.00099802$

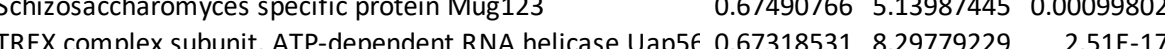

Golgi cation diffusion family zinc transmembrane transport $\begin{array}{llll}0.67314592 & 5.34646965 & 0.00111636\end{array}$ transcriptional regulatory protein Spp41 (predicted) $\quad \begin{array}{lllll}0.67299691 & 8.08778555 & 5.82 \mathrm{E}-13\end{array}$

ribosomal-ubiquitin fusion protein Ubi5 (predicted) $\quad \begin{array}{llll}0.67273366 & 7.87273483 & 1.74 \mathrm{E}-14\end{array}$

queuine tRNA-ribosyltransferase Qtrt1

$0.67235654 \quad 6.60776721 \quad 2.03 \mathrm{E}-07$

$\begin{array}{lll}0.67146165 \quad 8.74937329 & 3.89 \mathrm{E}-18\end{array}$

pig-F/3-ketosphinganine reductase fusion protein (predicte $\quad 0.6700475 \quad 7.38357326 \quad 3.16 \mathrm{E}-11$

$\begin{array}{llll}\text { ornithine transaminase Car2 } & 0.66982202 & 9.06911882 & 2.34 \mathrm{E}-19\end{array}$

$5 S$ rRNA
$1.05 \mathrm{E}-13$

0.000521216

$1.98 \mathrm{E}-11$

$1.65 \mathrm{E}-06$

0.344798307

$5.38 \mathrm{E}-11$

$6.70 \mathrm{E}-19$

0.003515957

0.585199995

4.99E-07

4.80E-05

1.37E-06

$7.87 \mathrm{E}-18$

$6.24 \mathrm{E}-10$

2.37E-08

3.05E-08

0.000103336

0.041274135

$1.91 \mathrm{E}-05$

0.014829784

4.64E-07

1.15E-19

3. $40 \mathrm{E}-05$

3.80E-14

2.79E-07

0.001472852

4.84E-13

0.001565742

0.148023886

3.86E-05

9.56E-18

5.66E-11

0.001496359

$9.60 \mathrm{E}-13$

2.06E-06

$1.01 \mathrm{E}-06$

1.72E-09

0.002675943

$3.71 \mathrm{E}-16$

0.002962888

$5.88 \mathrm{E}-12$

$1.98 \mathrm{E}-13$

1.03E-06

6.19E-17

2.63E-10

4.21E-18

0.000407994 
SPNCRNA.1393

SPBC119.01

SPBC83.02C

SPBC1539.03C

SPAC7D4.10

SPAC1786.04

SPAC1610.02C

SPBC4C3.07

SPAC1039.06

SPAC323.07C

SPNCRNA.1390

SPCC1223.09

SPAC1F3.05

SPAC1565.01

SPAC21E11.03C

SPBC342.03

SPRRNA.05

SPBC12C2.04

SPBC16E9.06C

SPRRNA.20

SPAC2F 3.02

SPBC17D1.04

SPCC330.12C

SPCC132.01C

SPBC428.14

SPAC3G6.11

SPNCRNA.1278

SPCC1259.01c

SPCC584.12

SPAC22A12.09c

SPBP4H10.20

SPAC1783.04C

SPCC18.17c

SPNCRNA.824

SPCC18.02

SPRRNA.11

SPBC25B2.07C

SPAC22G7.01C

SPCC74.01

SPAC18G6.15

SPRRNA.38

SPAC1556.07

SPCC757.12

SPBC1709.05

SPAC4G9.10

SPAC8C9.08

SPAC607.03C rpn3

rpl4302

$\arg 41$

SPAC1786.04

mrpl1

eif6

SPAC1039.06

SPAC323.07C

$$
\# \text { N/A }
$$

uro1

gga21

rcf2

pcr1

gas4

SPRRNA.05

SPBC12C2.04

uvi31

SPRRNA. 20

SPAC2F3.02

acr1

dh3

mtr1

SPBC428.14

chl1

\#N/A

rps1802

mug42

sap114

nhm1

SPCC18.17c

\#N/A

SPCC18.02

SPRRNA.11

$\mathrm{mmb} 1$

fra1

sly1

mal3

SPRRNA.38

pmm1

SPCC757.12

sks2

arg 3

rps5

snu13
\#N/A

$19 S$ proteasome regulatory subunit Rpn3

605 ribosomal protein L37a (predicted)

argininosuccinate lyase (predicted)

V-type ATPase V1 subunit $\mathrm{H}$ (predicted)

Schizosaccharomyces pombe specific protein

mitochondrial ribosomal protein subunit L1 (predicted)

translation initiation factor elF3

D-serine ammonia-lyase activity (predicted)

MatE family transmembrane transporter (predicted)

\#N/A

uricase Uro1

(N)

Golgi localized Arf binding gamma-adaptin ortholog Gga21

cytochrome c oxidase assembly protein Rcf2

transcription factor Pcr1

spore wall 1,3-beta-glucanosyltransferase Gas4

$5 S$ rRNA

NAD binding dehydrogenase family protein

mitochondrial [4Fe-4S] cluster transfer protein Uvi31 (pred

5 S rRNA

ER protein translocation subcomplex subunit (predicted)

RNA polymerase I upstream activation factor complex subu $0.65766877 \quad 6.27673481 \quad 0.00010364$

$\begin{array}{llll}\text { succinate dehydrogenase (ubiquinone) cytochrome b subuni } & 0.65706689 & 7.09440398 & 1.88 \mathrm{E}-07\end{array}$

microtubule regulator Mtr1

$0.656609447 .39663376 \quad 2.54 \mathrm{E}-08$

$0.6558326 \quad 7.28589188 \quad 4.87 \mathrm{E}-11$

$0.65550306 \quad 7.44531384 \quad 8.09 \mathrm{E}-12$

$\begin{array}{lll}0.65503359 & 4.71649958 & 0.00893414\end{array}$

$0.653981797 .64086559 \quad 1.00 \mathrm{E}-12$

$\begin{array}{lll}0.65309974 & 2.65786133 & 0.33705751\end{array}$

$0.6525793 \quad 5.80788746 \quad 7.57 \mathrm{E}-05$

$0.65148958 \quad 5.30880435 \quad 0.0005791$

$0.65142064 \quad 7.27450458 \quad 1.22 \mathrm{E}-10$

$0.65111807 \quad 6.72061041 \quad 2.80 \mathrm{E}-06$

$0.65082956 \quad 6.86182973 \quad 1.51 \mathrm{E}-06$

$0.64995373 \quad 6.91576299 \quad 2.55 \mathrm{E}-09$

$\begin{array}{lll}0.64942188 & 5.27293986 & 0.0005154\end{array}$

$0.6487412 \quad 6.81019969 \quad 3.88 \mathrm{E}-06$

5 S rRNA

mitochondrial microtubule binder Mmb1

$4.36 \mathrm{E}-12$

$\begin{array}{rrr}0.64829934 & 7.47175355 & 5.09 \mathrm{E}-08 \\ 0.6478668 & 6.78425881 & 1.59 \mathrm{E}-06\end{array}$

$\begin{array}{rrr}0.6478668 & 6.78425881 & 1.59 \mathrm{E}-06 \\ 0.64765284 & 5.66413587 & 0.00011319\end{array}$

$\begin{array}{lll}0.64736492 & 8.68989617 \quad 8.29 \mathrm{E}-16\end{array}$

$0.64703133 \quad 8.12421559 \quad 1.71 \mathrm{E}-12$

phosphomannomutase Pmm1

alpha-amylase homolog (predicted)

4.78E-20

ornithine carbamoyltransferase Arg 3

$40 \mathrm{~S}$ ribosomal protein S5 (predicted)

$1.89 \mathrm{E}-09$

$\begin{array}{lll}0.64661807 & 8.31325781 \quad 1.58 \mathrm{E}-16\end{array}$

$0.64647478 \quad 6.9461868 \quad 3.85 \mathrm{E}-08$
1.53E-05

4.69E-10

$5.83 \mathrm{E}-07$

4.24E-10

$5.40 \mathrm{E}-08$

0.4868762

9.49E-05

1.37E-10

$1.12 \mathrm{E}-10$

$1.45 \mathrm{E}-08$

0.004083932

$8.35 \mathrm{E}-05$

8.77E-10

$1.34 \mathrm{E}-14$

0.003572783

$1.82 \mathrm{E}-10$

0.001042375

2.40E-05

$5.66 \mathrm{E}-15$

0.000356653

$8.48 \mathrm{E}-09$

0.00034054

9.53E-07

1.47E-07

3.96E-10

7.25E-11

0.019022778

9.95E-12

0.431628967

0.000254441

0.001648169

9.39E-10

$1.22 \mathrm{E}-05$

$6.82 \mathrm{E}-06$

1.67E-08

0.001488675

1.65E-05

4.01E-11

2.82E-07

$7.14 \mathrm{E}-06$

0.000369765

1.07E-14

$1.67 \mathrm{E}-11$

9.16E-19

$1.27 \mathrm{E}-08$

2.16E-15

2.18E-07 


\begin{tabular}{|c|c|c|c|c|c|c|}
\hline SPBC215.05 & gpd1 & glycerol-3-phosphate dehydrogenase Gpd1 & 0.6461687 & 9.08241085 & $4.86 \mathrm{E}-16$ & $6.40 \mathrm{E}-15$ \\
\hline SPCC126.11c & SPCC126.11c & RNA-binding protein, rrm type & 0.64607339 & 5.98588907 & 7.27E-05 & 0.000245116 \\
\hline SPAC6B12.05c & ies2 & Ino80 complex subunit les2 & 0.64543436 & 6.74686784 & $2.56 \mathrm{E}-06$ & 1.12E-05 \\
\hline SPBC8D2.06 & irs1 & cytoplasmic isoleucine-tRNA ligase Irs1 (predicted) & 0.64520569 & 8.83473327 & $1.51 \mathrm{E}-15$ & $1.91 \mathrm{E}-14$ \\
\hline SPBC36.04 & cys 11 & cysteine synthase & 0.64448463 & 7.5687692 & $1.58 \mathrm{E}-11$ & $1.36 \mathrm{E}-10$ \\
\hline SPNCRNA.952 & $\# N / A$ & \#N/A & 0.64432991 & 5.73463653 & 0.00059342 & 0.001683297 \\
\hline SPAC24C9.05c & mug70 & CBS and PB1 domain protein, conserved in fungi and plants, & 0.64382083 & 7.52285247 & $5.15 \mathrm{E}-10$ & $3.72 \mathrm{E}-09$ \\
\hline SPNCRNA.1694 & $\# N / A$ & \#N/A & 0.6437641 & 3.93362116 & 0.12898928 & 0.194800716 \\
\hline SPCC1739.11c & cdc11 & SIN component scaffold protein, centriolin ortholog Cdc11 & 0.64269912 & 7.0819412 & 6.37E-10 & 4.54E-09 \\
\hline SPBP8B7.15c & mpe1 & mRNA cleavage ubiquitin-protein ligase E3 Mpe1 (predicter & 0.64250196 & 7.96647165 & $4.33 \mathrm{E}-13$ & 4.44E-12 \\
\hline SPNCRNA.1127 & $\# N / A$ & \#N/A & 0.64244853 & 4.77413911 & 0.00655877 & 0.01448165 \\
\hline SPCC1450.04 & tef5 & translation elongation factor EF-1 beta subunit, guanyl-nucl & 0.64217584 & 8.29281059 & 5.09E-14 & 5.57E-13 \\
\hline SPBC2G2.11 & myr1 & N-myristoyltransferase Myr1 (predicted) & 0.64217357 & 7.17617144 & 1.51E-09 & $1.03 \mathrm{E}-08$ \\
\hline SPAC12G12.06c & rcl1 & rRNA processing protein Rcl1 (predicted) & 0.64199372 & 6.75324162 & $4.82 \mathrm{E}-08$ & 2.69E-07 \\
\hline SPAC3C7.14c & obr1 & $\mathrm{NAD}(\mathrm{P}) \mathrm{H}$ dehydrogenase (quinone) (predicted) & 0.64140119 & 8.20374339 & $3.32 \mathrm{E}-15$ & 4.04E-14 \\
\hline SPAC1783.03 & $\mathrm{fta} 2$ & CENP-P ortholog Fta2 & 0.64119572 & 7.44266711 & $3.51 \mathrm{E}-11$ & 2.91E-10 \\
\hline SPAC17C9.10 & stm1 & vacuolar amino acid transmembrane transporter Stm1 (pre & 0.64099446 & 6.96306509 & $5.51 E-07$ & 2.64E-06 \\
\hline SPCC1183.02 & SPCC1183.02 & glutathione S-transferase, translational elongation factor e & 0.64088506 & 7.12357758 & $3.58 \mathrm{E}-10$ & 2.63E-09 \\
\hline SPAC6G9.08 & ubp6 & ubiquitin C-terminal hydrolase Ubp6 & 0.64028312 & 8.39325772 & $6.44 \mathrm{E}-15$ & $7.66 \mathrm{E}-14$ \\
\hline SPAC10F6.05c & ubc6 & ubiquitin conjugating enzyme E2 Ubc6 (predicted) & 0.64024116 & 5.23326062 & 0.00141627 & 0.003664528 \\
\hline SPAC3H5.05c & rps1401 & $40 \mathrm{~S}$ ribosomal protein S14 (predicted) & 0.63896747 & 6.87375805 & $1.03 \mathrm{E}-07$ & $5.42 \mathrm{E}-07$ \\
\hline SPAC22E12.07 & rna1 & RanGAP GTPase activating protein Rna1 & 0.63729963 & 7.29055568 & 1.95E-09 & $1.30 \mathrm{E}-08$ \\
\hline SPAC19A8.15 & $\operatorname{trp2}$ & tryptophan synthase (predicted) & 0.63694431 & 8.78803573 & $1.32 \mathrm{E}-16$ & 1.83E-15 \\
\hline SPAC15A10.08 & ain1 & alpha-actinin & 0.63693492 & 6.94640109 & $2.26 \mathrm{E}-07$ & 1.14E-06 \\
\hline SPCC16C4.07 & scw1 & RNA-binding protein Scw1 & 0.6365362 & 7.34755919 & 5.59E-11 & $4.50 \mathrm{E}-10$ \\
\hline ScpofMt27 & \#N/A & $\# N / A$ & 0.6345515 & 4.14911392 & 0.06116705 & 0.102801135 \\
\hline SPBC119.18 & mdm35 & mitochondrial phosphatidylserine translocation complex sul & 0.63444329 & 5.08131451 & 0.0043046 & 0.009933126 \\
\hline SPCC16C4.13c & rpl1201 & $60 \mathrm{~S}$ ribosomal protein L12.1/L12A & 0.63360381 & 8.05357968 & $1.99 \mathrm{E}-13$ & $2.10 \mathrm{E}-12$ \\
\hline SPAC15F9.01c & gIm1 & Glomulin, ubiquitin-protein transferase inhibitor GIm1 (pre & 0.63357328 & 1.47717345 & 0.81205525 & 0.863014401 \\
\hline SPBC776.02c & dis2 & serine/threonine protein phosphatase PP1, Dis2 & 0.63331555 & 7.49485448 & $2.82 \mathrm{E}-10$ & $2.12 \mathrm{E}-09$ \\
\hline SPCC4G3.05c & mus81 & Holliday junction resolvase subunit Mus 81 & 0.63258273 & 6.05013278 & 0.00017367 & 0.00054865 \\
\hline SPAC1F7.05 & $\operatorname{cdc} 22$ & ribonucleoside reductase large subunit Cdc22 & 0.63242833 & 9.10047324 & 7.43E-10 & 5.25E-09 \\
\hline SPAC6F6.10c & $\operatorname{arc2}$ & ARP2/3 actin-organizing complex subunit Arc34 & 0.63211057 & 7.4983113 & $1.80 \mathrm{E}-10$ & 1.37E-09 \\
\hline SPAC3C7.09 & set8 & Iysine methyltransferase Set8, unknown specificity (predict & 0.62987408 & 5.00627294 & 0.02110697 & 0.041037986 \\
\hline SPBC30B4.05 & kap109 & karyopherin/importin beta family nuclear export signal rec€ & 0.62890539 & 7.77885499 & 4.67E-11 & $3.81 \mathrm{E}-10$ \\
\hline SPCC1281.03c & emc4 & ER membrane protein complex subunit Emc4 (predicted) & 0.62816871 & 5.90403423 & 0.00076442 & 0.002117164 \\
\hline SPAC14C4.04 & SPAC14C4.04 & Thil domain protein (predicted) & 0.62781916 & 6.52499102 & 5.61E-07 & $2.68 \mathrm{E}-06$ \\
\hline SPAC10F6.01c & sir1 & sulfite reductase beta subunit Sir1 & 0.62716352 & 9.970315 & $6.48 \mathrm{E}-21$ & 1.37E-19 \\
\hline SPBC354.10 & def1 & RNAPII degradation factor Def1 (predicted) & 0.62709938 & 8.88770485 & $2.38 \mathrm{E}-14$ & 2.67E-13 \\
\hline SPCC31H12.03c & mlo1 & RNA binding protein (predicted) & 0.62702625 & 6.52458281 & $1.53 \mathrm{E}-06$ & $6.89 \mathrm{E}-06$ \\
\hline SPCC18B5.07c & nup61 & nucleoporin Nup61 & 0.62643316 & 7.3712994 & $4.71 \mathrm{E}-10$ & $3.42 \mathrm{E}-09$ \\
\hline SPBC21D10.07 & $\mathrm{cmc1}$ & copper-binding protein of the mitochondrial intermembran & 0.62624941 & 5.83977548 & 0.00012131 & 0.00039479 \\
\hline SPAC18G6.07c & mra1 & rRNA (pseudouridine) methyltransferase Mra1 & 0.62597059 & 6.90080596 & 2.79E-07 & 1.39E-06 \\
\hline SPBC11C11.09c & rpl502 & $60 S$ ribosomal protein $L 5$ & 0.62562373 & 8.48430629 & $2.01 \mathrm{E}-15$ & $2.51 \mathrm{E}-14$ \\
\hline SPAC3G6.05 & SPAC3G6.05 & mitochondrial Mpv17/PMP22 family protein 1 (predicted) & 0.62530996 & 5.83441289 & 0.00117821 & 0.003110065 \\
\hline SPNCRNA.444 & $\# N / A$ & $\# N / A$ & 0.62513579 & 3.92349539 & 0.14913915 & 0.220054622 \\
\hline SPAC16.02c & srp2 & splicing factor Srp2 & 0.62389504 & 7.56692023 & 6.93E-09 & 4.33E-08 \\
\hline
\end{tabular}


SPAC19G12.14 SPCPB16A4.05c SPBC1271.03C SPAPB2B 4.05

SPBC19G7.17

SPBC1A4.08C

SPCC1223.13

SPBC839.07

SPNCRNA.986

SPAC688.14

SPNCRNA.662

SPCC24B10.15

SPAC19G12.17

SPCC584.04

SPBC16D10.08C

SPAC6F $12.13 \mathrm{C}$

SPCC1183.03C

SPBPB21E7.09

SPNCRNA.360

SPBC2G2.10C

SPCC1620.06c

SPNCRNA.1072

SPCC550.05

SPNCRNA.1000

SPCP31B10.07

SPAC10F6.13C

SPNCRNA. 255

SPBC1685.07c

SPAPB 8E5.09

SPAC4F8.02C

SPBC1289.12

SPCC61.01C

SPCC622.19

SPCC777.04

SPNCRNA.855

SPBC839.19

SPAC12G12.17

SPAPYUG7.04C

SPRRNA.19

SPAPB8E5.06C

SPBC649.02

SPNCRNA.899

SPRRNA.26

SPBPB8B6.03

SPAC1071.12C

SPAC343.21

SPBC1711.07 vma5

sec6102

cct3

cbf12

ibp1

\#N/A

set13

swt1

erh1

sup35

sp104

fps1

fxn1

SPBPB21E7.09

$\#$ N/A

mug 110

SPCC1620.06c

$\# \mathrm{~N} / \mathrm{A}$

nse1

$\#$ N/A

eft202

caa1

\#N/A

avt5

rvb1

mrpl40

usp109

str2

jmj4

SPCC777.04

\#N/A

new20

SPAC12G12.17

rpb9

SPRRNA.19

rpl302

rps1902

\#N/A

SPRRNA.26

fah1

stp1

\#N/A
SPBC1271.03C

1-phosphatidylinositol-4-phosphate 5-kinase Its3

urease accessory protein UreG

$\mathrm{NLI}$ interacting factor family phosphatase (predicted)

V-type ATPase V1 subunit C (predicted)

translocon subunit Sec61 homolog (predicted)

chaperonin-containing T-complex gamma subunit Cct3

$\mathrm{CBF} 1 / \mathrm{Su}(\mathrm{H}) / \mathrm{LAG}-1$ family transcription factor $\mathrm{Cbf} 12$

Cdc25 family phosphatase Ibp1, unknown role, implicated it $0.6 \%$

$$
\text { \#N/A }
$$

ribosome L32 lysine methyltransferase Set13

\#N/A

RNA endoribonuclease involved in mRNP quality control Sw $0.61811753 \quad 6.44237743$

enhancer of rudimentary homolog Erh1

cytoplasmic translation release factor class II eRF3

heat shock protein $\mathrm{Hsp} 104$

geranyltranstransferase Fps1

mitochondrial [2Fe-2S] cluster assembly frataxin Fxn1

L-asparaginase (predicted)

$\# \mathrm{~N} / \mathrm{A}$

Schizosaccharomyces specific protein Mug110

ribose-phosphate pyrophosphokinase (predicted)

$$
\text { \#N/A }
$$

Smc5-6 complex ubiquitin-protein ligase E3 subunit Nse1 $\# \mathrm{~N} / \mathrm{A}$

translation elongation factor 2 (EF-2) Eft2,B

cytoplasmic aspartate aminotransferase Caa1 (predicted) \#N/A

vacuolar amino acid transmembrane transporter Avt5

ASTRA/Swr1/Ino80 complex AAA family ATPase Rvb1

mitochondrial ribosomal protein subunit $L 40$ (predicted)

U1 snRNP-associated protein Usp109

plasma membrane siderophore-iron transmembrane trans

peptidyl-lysine 3-dioxygenase activity jmj4 (predicted)

amino acid transmembrane transporter (predicted)$$
\# \mathrm{~N} / \mathrm{A}
$$

UPF0428 family, human CXorf56 and C15orf40 ortholog

non-classical export protein 1 (predicted)

DNA-directed RNA polymerase II complex subunit Rpb9

5S rRNA

60S ribosomal protein L3

40S ribosomal protein S19 (predicted)

\section{S rRNA}$$
\text { \#N/A }
$$

fatty acid amide hydrolase Fah1 (predicted)

se Stp1, unknown biological role

protein tyrosine phosphatase

WD repeat protein Rrb1 (predicted)

$0.62371583 \quad 7.51753399 \quad 1.97 \mathrm{E}-08$

$0.623620697 .47717554 \quad 3.26 \mathrm{E}-09$

$\begin{array}{lll}0.62202824 & 6.79429622 & 1.24 \mathrm{E}-07\end{array}$

$0.621426726 .43797293 \quad 5.12 \mathrm{E}-06$

$0.62104461 \quad 7.40290063 \quad 2.57 \mathrm{E}-07$

$\begin{array}{lll}0.62031586 & 8.41253808 & 2.37 \mathrm{E}-15\end{array}$

$0.620161347 .15427563 \quad 1.21 \mathrm{E}-05$

$0.61863867 \quad 7.57014706 \quad 8.95 \mathrm{E}-09$

$\begin{array}{lll}0.61822061 & 5.9219447 & 0.00106977\end{array}$

$\begin{array}{lll}0.61801416 & 5.19445816 & 0.0020026\end{array}$

$0.616818328 .65037711 \quad 7.23 \mathrm{E}-15$

$0.616559118 .76095075 \quad 9.89 \mathrm{E}-06$

$\begin{array}{lll}0.61593276 & 7.78329386 & 1.28 \mathrm{E}-09\end{array}$

$\begin{array}{lll}0.61570563 & 7.44073907 & 7.95 \mathrm{E}-11\end{array}$

$0.61533593 \quad 7.10228701 \quad 3.22 \mathrm{E}-09$

$\begin{array}{lll}0.6146096 & 2.88344725 & 0.21265865\end{array}$

$\begin{array}{lll}0.61380721 & 4.1334136 & 0.09767\end{array}$

$0.613170517 .93026076 \quad 9.14 \mathrm{E}-13$

$\begin{array}{lll}0.6125961 & 8.4684045 & 9.72 \mathrm{E}-15\end{array}$

$\begin{array}{llll}0.61221602 & 7.76974639 & 2.12 \mathrm{E}-12\end{array}$

$\begin{array}{lll}0.61218392 & 2.5034993 & 0.34237811\end{array}$

$0.61217398 \quad 10.327812 \quad 1.63 \mathrm{E}-20$

$0.61061017 \quad 8.33030525 \quad 3.38 \mathrm{E}-11$

$\begin{array}{llll}0.61013853 & 1.29992961 & 0.56601767\end{array}$

$0.609468696 .34304258 \quad 1.54 \mathrm{E}-05$

$0.60922007 \quad 7.85842295 \quad 4.43 \mathrm{E}-11$

$\begin{array}{llll}0.60828238 & 5.91770533 & 0.00040216\end{array}$ $\begin{array}{llll}0.60793689 & 6.10399366 & 0.00099297\end{array}$

$\begin{array}{lll}0.60745634 & 9.09634481 & 1.72 \mathrm{E}-17\end{array}$ $\begin{array}{lll}0.60733373 & 5.99181231 & 6.96 \mathrm{E}-05\end{array}$ $0.6073156 \quad 5.086527530 .00549596$ $\begin{array}{llll}0.6068697 & 1.29973607 & 0.56478636\end{array}$ $\begin{array}{llll}0.60561304 & 4.57512968 & 0.01476433\end{array}$ $\begin{array}{llll}0.60525063 & 4.68219326 & 0.01728256\end{array}$ $0.605025525 .90303565 \quad 0.00041314$ $\begin{array}{llll}0.60487183 & 5.64665148 & 0.00028523\end{array}$ $\begin{array}{lll}0.60453781 & 9.21136249 & 1.53 \mathrm{E}-17\end{array}$ $0.60424368 \quad 7.16988968 \quad 1.84 \mathrm{E}-08$ $\begin{array}{llll}0.60386097 & 3.77415434 & 0.07326888\end{array}$ $\begin{array}{llll}0.60382892 & 2.16820878 & 0.4326589\end{array}$ $\begin{array}{llll}0.60381933 & 4.98172092 & 0.01888386\end{array}$ $\begin{array}{llll}0.60364361 & 3.40635192 & 0.18012898\end{array}$ $0.60362337 .12684643 \quad 1.10 \mathrm{E}-07$
1.16E-07

$212 \mathrm{E}-08$

$6.45 \mathrm{E}-07$

2.14E-05

$1.29 \mathrm{E}-06$

2.93E-14

4.73E-05

0.007012224

5.50E-08

0.00285145

0.000146569

7.22E-05

0.005023098

$8.58 \mathrm{E}-14$

$3.92 \mathrm{E}-05$

8.83E-09

6.24E-10

2.09E-08

0.296120745

0.153710541

9.08E-12

1.13E-13

2.03E-11

0.43704427

3.26E-19

2.81E-10

0.655237593

5.91E-05

3.64E-10

0.001186786

0.002664485

$2.56 \mathrm{E}-16$

0.000235745

0.012351382

0.654079503

0.029919966

0.034438594

0.001214958

0.000866911

$2.28 \mathrm{E}-16$

1.09E-07

0.120114372

0.528305004

0.037356007

0.003465544

0.257516332

5.77E-07 
SPBC2F12.13

SPBC1861.06C

SPNCRNA.1353

SPAC1687.01

SPNCRNA.890

SPBC1604.05

SPBC839.16

SPBC3H7.07C

SPBC31F10.12

SPBPB2B2.08

SPAC13G7.03

SPNCRNA.790

SPCC1450.03

SPBC1711.18

SPAC20H4.08

SPAPB15E9.01c

SPAP27G11.12

SPBC14F5.09c

SPAC $13 A 11.02 \mathrm{C}$

SPBC582.07C

SPAC12B10.11

SPNCRNA.1607

SPBC8D2.20C

SPBC83.15

SPNCRNA.1500

SPAC3H1.06C

SPAC4H3.09

SPBC21H7.07C

SPBC83.07

SPAC1F7.10

SPAC19B12.06C

SPBC1685.06

SPCC18.05C

SPBC11B10.02C

SPBC13G1.01C

SPBC4F6.04

SPAC4A8.15C

SPNCRNA.1198

SPNCRNA.1593

SPBC887.14c

SPCC330.06c

SPCC794.12C

SPNCRNA.1412

SPAC6G10.11C

SPNCRNA.1389

SPAC4G9.19

SPBC1773.05C mug131

$\# N / A$

rpc19

\#N/A

pgi1

thf1

ser2

tma20

SPBPB2B2.08

upf3

$\#$ N/A

utp502

tam 9

whi2

pfl2

SPAP27G11.12

ade8

erg11

rpn7

exg2

$\# \mathrm{~N} / \mathrm{A}$

$\sec 31$ wdr74

\#N/A

SPAC3H1.06C

SPAC4H3.09

his5

jmj3

SPAC1F7.10

rbd4

cid11

rsa4

his3

nam9

rpl2502

cdc3

$\# \mathrm{~N} / \mathrm{A}$

$\# N / A$

pfh1

pmp20

mae2

$\#$ N/A

ubi3

\#N/A

SPAC4G9.19

tms1 kinesin-8 family plus-end directed microtubule motor Klp5 $0.60340105 \quad 7.47982973 \quad 1.97 \mathrm{E}-06$ UPF0300 family protein 4

$$
\# \mathrm{~N} / \mathrm{A}
$$

$0.60247474 \quad 6.26693039 \quad 1.47 \mathrm{E}-05$

$\begin{array}{llll}0.60208726 & 5.59267675 & 0.00038877\end{array}$

$0.60207297 \quad 5.5562642 \quad 0.00084776$

$0.601982045 .93212951 \quad 0.00160329$

$0.601798929 .64666034 \quad 6.21 \mathrm{E}-18$

$$
\text { \#N/A }
$$

glucose-6-phosphate isomerase (predicted)

$\begin{array}{lll}0.60148573 & 9.01813422 & 1.23 \mathrm{E}-14\end{array}$

$\begin{array}{llllll} & 0.60147895 & 7.44411122 & 3.00 \mathrm{E}-09\end{array}$

$0.60114963 \quad 6.15310418 \quad 7.29 \mathrm{E}-05$

$\begin{array}{lll}0.60093357 & 1.30003177 & 0.56928462\end{array}$

$0.60093253 \quad 4.44547054 \quad 0.03545105$

$0.60076403 \quad 5.28318359 \quad 0.00346175$ $\begin{array}{lll}0.60069225 & 5.12141497 & 0.00666653\end{array}$

up-frameshift suppressor 3 family protein (predicted)

$$
\# \mathrm{~N} / \mathrm{A}
$$

ribonucleoprotein (RNP) complex Utp502 (predicted)

$\begin{array}{lll}0.60044856 & 5.63310047 & 0.00057652\end{array}$

G-patch RNA-binding protein, involved in splicing (predictec $0.59989775 \quad 5.22009257 \quad 0.00159246$

cell surface glycoprotein, flocculin Pfl2

$0.59965759 \quad 10.3995716 \quad 5.63 \mathrm{E}-09$

human HID1 ortholog 1, possible Golgi protein (by similarit) $0.599611887 .33259196 \quad 1.53 \mathrm{E}-07$

$0.599262997 .95771795-1.49 \mathrm{E}-10$

sterol 14-demethylase

$19 \mathrm{~S}$ proteasome regulatory subunit Rpn7

cell wall glucan glucosidase Exg2

$$
\text { \#N/A }
$$

$0.59875778 \quad 8.80791524 \quad 3.03 \mathrm{E}-10$

$\begin{array}{lll}0.59776127 & 7.79180052 & 2.94 \mathrm{E}-10\end{array}$

$0.595902295 .63164814 \quad 0.00416438$

$0.59562816 \quad 5.41165954 \quad 0.00381583$

COPII-coated vesicle component Sec31 (predicted) $\quad \begin{array}{llll}0.5951813 & 9.57780322 & 2.55 \mathrm{E}-17\end{array}$

ribosome assembly factor, WD repeat protein Nsa1/Wdr74 $\quad \begin{array}{llll}0.595173 & 6.00008523 & 0.0009514\end{array}$

$$
\text { \#N/A }
$$

$\begin{array}{lrrr}\text { transmembrane transporter (predicted) } & 0.59415069 & 8.3060922 & 2.60 \mathrm{E}-11 \\ \text { mitochondrial [2Fe-2S] cluster assembly and type II fatty ac } 0.59358515 & 5.52546655 & 0.00381819\end{array}$

mitochondrial [2Fe-2S] cluster assembly and type II fatty ac $0.59358515 \quad 5.525466550 .00381819$

Lid2 complex subunit, histone demethylase H3-K36 specific $0.59316897 \quad 6.40271081 \quad 1.68 \mathrm{E}-05$

$\begin{array}{lllll}\text { hydantoin racemase family (predicted) } & 0.59266191 & 5.53138611 & 0.00775571\end{array}$

rhomboid family protease, unknown biological role, associa $0.592129817 .04773899 \quad 1.39 \mathrm{E}-05$

poly(A) polymerase Cid11, terminal uridylyl transferase (pre $0.59181512 \quad 5.35493723 \quad 0.00461851$

notchless-like ribosome biogenesis protein Rsa4 (predicted $0.58989776 \quad 6.42886498 \quad 2.51 \mathrm{E}-05$

histidinol-phosphate aminotransferase imidazole acetol ph $0.58988533 \quad 7.76549041 \quad 3.45 \mathrm{E}-09$

mitochondrial ribosomal protein subunit S4 (predicted) $\quad 0.58973135 \quad 6.20375145 \quad 8.47 \mathrm{E}-05$

$60 S$ ribosomal protein L25 (predicted)

profilin

$\# N / A$

$\# N / A$

5' to 3' DNA helicase Pif1/Pfh1

thioredoxin-related chaperone Pmp20 (predicted)

$0.58904111 \quad 7.26567558 \quad 1.57 \mathrm{E}-08$

$0.58900782 \quad 7.1893728 \quad 5.52 \mathrm{E}-08$

$\begin{array}{llll}0.58896499 & 4.94091577 & 0.02171675\end{array}$

$\begin{array}{lll}0.58888446 \quad 6.04171327 & 0.00204872\end{array}$

$\begin{array}{llll}0.58831979 & 7.39959899 & 3.53 \mathrm{E}-07\end{array}$

$0.588190137 .52973973-9.62 \mathrm{E}-07$

malic enzyme, malate dehydrogenase (oxaloacetate decark $0.58771634 \quad 9.73450648 \quad 5.54 \mathrm{E}-15$

$$
\# \mathrm{~N} / \mathrm{A}
$$

$\begin{array}{lll}0.58747927 & 6.55701288 & 1.04 \mathrm{E}-05\end{array}$

$$
\text { \#N/A }
$$

$0.58706353 \quad 8.04079243 \quad 3.41 \mathrm{E}-12$

$0.58574045 \quad 5.19963101 \quad 0.00292441$

$\begin{array}{lll}0.58556844 & 3.91653142 & 0.15896835\end{array}$

$0.58485663 \quad 6.52686436 \quad 3.07 \mathrm{E}-05$
8.73E-06

5.66E-05

0.001150765

0.002316393

0.004103182

9.70E-17

$1.42 \mathrm{E}-13$

$1.95 \mathrm{E}-08$

0.000245821

0.65816885

0.064400529

0.008196668

0.014674599

0.001642194

0.004078535

3.56E-08

$7.86 \mathrm{E}-07$

$1.14 \mathrm{E}-09$

2.26E-09

2.20E-09

0.009647638

0.008907959

3.75E-16

0.002568889

2.94E-07

2. $20 \mathrm{E}-10$

0.008910412

2.64E-07

6.40E-05

0.016754803

5.36E-05

0.010603554

9.27E-05

2.23E-08

0.000282285

9.28E-08

3.04E-07

0.042115276

0.005121793

1.73E-06

4.45E-06

$6.61 \mathrm{E}-14$

4.12E-05

$3.18 \mathrm{E}-11$

0.007039472

0.232124149

0.000110596 
SPAC23D3.04C SPNCRNA.1125 SPAC32A11.04C SPAC1F5.08C

SPBC1703.05

SPAC1565.08

SPCC188.02

SPBC13G1.02

SPBC106.15

SPCC1393.07C

SPAC13A11.05

SPAC1805.10

SPNCRNA.1202

SPAC57A7.04C

SPAC11G7.05C

SPAC8F $11.05 \mathrm{C}$

SPBC16A3.04

SPNCRNA.1002

SPNCRNA.1064

SPAC19D5.11C

SPAC926.09C

SPBC11G11.06c

SPBC577.02

SPAC664.05

SPBC1539.07c

SPBC1709.07

SPAC22F $3.10 \mathrm{C}$

SPAC17A2.05

SPAC1834.10c

SPAC513.01c

SPAC24H6.07

SPAC4D7.09

SPNCRNA.1498

SPBC21.01

SPBC428.13C

SPAC11D3.06

SPNCRNA.1643

SPBP23A10.11C

SPCC16C4.08C

SPNCRNA.1523

SPAC8C9.04

SPBC9B6.10

SPBPB21E7.07

SPBC56F 2.02

SPAC644.17C

SPBC8D2.19

SPAC4G9.16C gpd2

cdc48

par1

mpg2

idi1

mug4

ysp2

SPAC1805.10

\#N/A

pabp

mct1

mug130

rsm25

$\# N / A$

$\# N / A$

ctf8

fas1

sme1

rpl3801

rpl13

fmd1

erg27

gcs1

osm 1

SPAC1834.10C

eft201

rps901

$\#$ N/A

mis17

SPAC11D3.06

\#N/A

SPBP23A10.11C

skb15

$\#$ N/A

SPAC8C9.04

cdc37

aes1

rpl1901

mrpl9

mde3

rpl901 glycerol-3-phosphate dehydrogenase Gpd2

$$
\# \mathrm{~N} / \mathrm{A}
$$

translation initiation factor elF2 beta subunit (predicted) $\begin{array}{lllll}0.58429647 & 8.21080377 & 2.66 \mathrm{E}-12\end{array}$ plasma membrane stretch-activated calcium ion channel Y: $0.58416463 \quad 5.87966804 \quad 0.0006353$ protein kinase, RIO family Rio2 (predicted)

AAA family ATPase involved in ubiquitin-mediated protein c $0.58284393 \quad 9.73543499 \quad 3.97 \mathrm{E}-12$ protein phosphatase PP2A regulatory subunit B-56 Par1 $\quad 0.579831657 .19493383 \quad 3.63 \mathrm{E}-06$ mannose-1-phosphate guanyltransferase (predicted) $\quad 0.57931793 \quad 8.27826528 \quad 9.93 \mathrm{E}-12$ isopentenyl-diphosphate delta-isomerase Idi1 $\quad 0.57885683 \quad 6.30886398 \quad 2.56 \mathrm{E}-05$ $\begin{array}{lllll}\text { Schizosaccharomyces specific protein, DNAJ domain } & 0.57863891 & 4.3660379 & 0.10533887\end{array}$ peptidase family M17 cytoplasmic leucyl aminopeptidase y: $0.57847443 \quad 7.82722803 \quad 6.90 \mathrm{E}-10$ Schizosaccharomyces specific protein

$$
\# \mathrm{~N} / \mathrm{A}
$$
poly(A) binding Pabp/Pab1 $\quad 0.57783698 \quad 9.52462332 \quad 2.50 \mathrm{E}-11$

mitochondrial [acyl-carrier protein] S-malonyltransferase N $\begin{array}{lll}0.57752321 & 6.52002302 & 3.74 \mathrm{E}-06\end{array}$

Schizosaccharomyces specific protein mitochondrial ribosomal protein subunit Rsm25 (predicted) $\begin{array}{lllll}0.5771394 & 6.18919703 & 0.00016521\end{array}$

$$
\# N / A
$$$$
\# \mathrm{~N} / \mathrm{A}
$$

Ctf18 RFC-like complex subunit Ctf8

fatty acid synthase beta subunit Fas1

Sm snRNP core protein Sme1

$60 S$ ribosomal protein L38 (predicted)

$60 S$ ribosomal protein L13 (predicted)

glutathione-dependent formaldehyde dehydrogenase (pred 0.

3-keto sterol reductase Erg27 (predicted)

glutamate-cysteine ligase Gcs1

mitochondrial proton-transporting ATP synthase complex as $\begin{array}{lll}0.57294815 & 5.57834486 & 0.00142432\end{array}$

translation elongation factor 2 (EF-2) Eft2,A $\quad \begin{array}{llll}0.57282184 & 10.3345178 & 8.05 \mathrm{E}-17\end{array}$

$40 \mathrm{~S}$ ribosomal protein $\mathrm{S9}$

translation initiation factor eIF2B gamma subunit

$$
\# \text { N/A }
$$

CENP-U ortholog Mis17

Sid2-Mob1 kinase complex regulatory subunit Mob1

MatE family transmembrane transporter (predicted) $\# \mathrm{~N} / \mathrm{A}$

circularly permuted 1,3-beta-glucanase (predicted)

p21 activated protein kinase inhibitor Skb15

$$
\# N / A
$$

Schizosaccharomyces specific protein

Hsp90 co-chaperone Cdc37

phenazine biosynthesis PhzF protein family

$60 S$ ribosomal protein $\mathrm{L} 19$

mitochondrial ribosomal protein subunit L9 (predicted)

serine/threonine protein kinase, meiotic Mde3

$60 S$ ribosomal protein $\mathrm{L} 9$
$0.58455471 \quad 7.70612244 \quad 1.21 \mathrm{E}-10$

$\begin{array}{llll}0.58449055 & 2.33066055 & 0.37619155\end{array}$

$0.58341604 \quad 6.06276126 \quad 0.00423747$

0.5779458 $\begin{array}{lll}0.57737788 & 4.13390332 & 0.08447155\end{array}$

$\begin{array}{llll}0.577708268 & 5.62866653 & 0.00511057\end{array}$

$\begin{array}{llll}0.57699276 & 3.81871398 & 0.06754219\end{array}$

$\begin{array}{llll}0.57611139 & 4.34248016 & 0.04288257\end{array}$

$0.57574658 \quad 11.0630916 \quad 1.45 \mathrm{E}-17$

$0.57572318 \quad 7.15577633 \quad 2.09 \mathrm{E}-07$

$\begin{array}{lll}0.57556569 & 6.28024634 & 0.00011593\end{array}$

$\begin{array}{lll}0.57533908 & 8.67767344 & 4.18 \mathrm{E}-11\end{array}$

9.37E-10

0.47188636

$2.50 \mathrm{E}-11$

0.001793127

0.009794834

$3.68 \mathrm{E}-11$

$1.55 \mathrm{E}-05$

$8.80 \mathrm{E}-11$

9.43E-05

0.163585344

4.90E-09

$3.08 \mathrm{E}-14$

$8.46 \mathrm{E}-13$

2.13E-10

$1.59 \mathrm{E}-05$

0.135700865

0.000524739

0.011569333

0.11185802

0.076013509

2.17E-16

$1.05 \mathrm{E}-06$

0.000378173

$3.44 \mathrm{E}-10$

$1.51 \mathrm{E}-09$

6.74E-05

1.16E-09

3.17E-07

0.00368396

$1.13 \mathrm{E}-15$

2.59E-10

$\begin{array}{llll}0.57251025 & 7.61327663 & 1.30 \mathrm{E}-08 & 7.79 \mathrm{E}-08\end{array}$

$\begin{array}{llll}0.5723733 & 5.79294292 & 0.00077559 & 0.002141158\end{array}$

$\begin{array}{llll}0.57117205 & 5.40499945 & 0.007938 & 0.017105149\end{array}$

$\begin{array}{llll}0.57111219 & 5.50037502 & 0.00182779 & 0.00461867\end{array}$

$\begin{array}{llll}0.56978707 & 6.72873098 & 4.16 \mathrm{E}-05 & 0.000146413\end{array}$

$\begin{array}{llll}0.56961408 & 6.93905731 & 1.25 \mathrm{E}-05 & 4.85 \mathrm{E}-05\end{array}$

$0.56926754 \quad 8.88986194 \quad 2.36 \mathrm{E}-07$

$\begin{array}{llll}0.56704553 & 6.02356451 & 9.51 \mathrm{E}-05 & 0.000314818\end{array}$

$\begin{array}{llll}0.56703153 & 5.34503281 & 0.03378561 & 0.061887228\end{array}$

$0.56691012 \quad 8.99617613 \quad 6.95 \mathrm{E}-10$

$0.565935217 .29048691 \quad 1.40 \mathrm{E}-05 \quad 5.39 \mathrm{E}-05$

$\begin{array}{llll}0.56560429 & 7.48792674 & 0.0002005 & 0.000628747\end{array}$

$\begin{array}{llll}0.56559997 & 8.37164927 & 4.74 \mathrm{E}-11 & 3.86 \mathrm{E}-10\end{array}$

$\begin{array}{llll}0.5648525 & 7.93372272 & 1.09 \mathrm{E}-11 & 9.68 \mathrm{E}-11\end{array}$

$\begin{array}{llll}0.56473724 & 6.8313239 & 9.89 \mathrm{E}-06 & 3.92 \mathrm{E}-05\end{array}$

$\begin{array}{llll}0.56389225 & 8.22757691 & 1.48 \mathrm{E}-12 & 1.44 \mathrm{E}-11\end{array}$ 
SPAC18G6.14C

SPNCRNA.1114

SPBC1105.02C

SPCC $31 \mathrm{H} 12.05 \mathrm{C}$

SPBC23E6.10C

SPAC29E6.06C

SPAC890.08

SPAC637.13C

SPRRNA.07

SPAC24B11.13

SPBC29A3.18

SPCC1906.01

SPBC106.12C

SPNCRNA.925

SPCC1322.13

SPCC830.08C

SPBC14F5.08

SPAC23C11.02C

SPAC343.09

SPBP22H7.07

SPNCRNA.1515

SPNCRNA.1289

SPBC17D11.03C

SPAC4F 10.20

SPBC887.01

SPNCRNA.1152

SPAC30C2.04

SPBC106.17c

SPAC3G9.09C

SPCC14G10.04

SPAC11E3.13C

SPBC4F6.17C

SPAPB17E12.13

SPBP23A10.10

SPNCRNA.230

SPBC3D6.09

SPBC16G5.16

SPNCRNA.752

SPBC1773.17C

SPBC336.03
SPAC922.06

pre8

rps7

$$
\text { \#N/A }
$$

lys 4

sds21

SPAC29E6.06C

rpl31

SPRRNA.07

hem 3

cyt1

mpg1

tho4

$$
\# \mathrm{~N} / \mathrm{A}
$$

ade6

yop1

med7

rps23

ubx3

prp5

\section{$\# N / A$ \\ $\# N / A$}

SPBC17D11.03C

grx1

$$
\text { \#N/A }
$$

asc1

cys 2

tif211

SPCC14G10.04

gas5

hsp78

rpl1802

ppk32

$$
\text { \#N/A }
$$

$\mathrm{dpb} 4$

SPBC16G5.16

\#N/A

gor2

efc 25 nuclear telomere cap complex subunit Ten1

orotate phosphoribosyltransferase Ura5

clathrin heavy chain Chc1 (predicted)

60 S ribosomal protein L12.1/L12A

conserved fungal protein

20 S proteasome complex subunit alpha 2, Pre8

$40 \mathrm{~S}$ ribosomal protein S7 (predicted)

$$
\text { \#N/A }
$$

homocitrate synthase 3-oxoacyl-[acyl-carrier-protein] reductase (predicted)

$\begin{array}{lll}0.5636744 & 3.98440636 & 0.11994897\end{array}$ $0.56278105 \quad 6.69003418 \quad 7.96 \mathrm{E}-06$ $\begin{array}{lll}0.56207216 & 10.2063868 & 7.29 \mathrm{E}-13\end{array}$ $\begin{array}{lll}0.5620254 & 8.23797477 & 8.78 \mathrm{E}-10\end{array}$ $\begin{array}{llll}0.56195747 & 5.87223805 & 0.00053665\end{array}$ $\begin{array}{llll}0.56184098 & 6.30928999 & 0.00014033\end{array}$ $\begin{array}{lll}0.56177661 & 7.9105707 & 3.46 \mathrm{E}-10\end{array}$ $\begin{array}{lll}0.56136751 & 8.40788941 & 1.59 \mathrm{E}-11\end{array}$ $\begin{array}{llll}0.56045885 & 2.29839582 & 0.48960502\end{array}$ $\begin{array}{lll}0.56037719 & 8.64559221 & 7.90 \mathrm{E}-10\end{array}$ serine/threonine protein phosphatase PP1 subfamily, Sds21 $0.55921305 \quad 6.60859258 \quad 1.54 \mathrm{E}-05$ methylthioribose-1-phosphate isomerase Mri1 (predicted) $0.55919004 \quad 5.68820688 \quad 0.01327708$ cytoplasmic cysteine-tRNA ligase Crs1 (predicted) $60 S$ ribosomal protein L31 (predicted)

cytoskeletal signaling protein SIm1 (predicted) 5S rRNA

hydroxymethylbilane synthase Hem3 (predicted) cytochrome c1 Cyt1 (predicted)

mannose-1-phosphate guanyltransferase Mpg1

THO complex subunit Tho4 (predicted) $\# \mathrm{~N} / \mathrm{A}$

phosphoribosylaminoimidazole carboxylase Ade6 ER membrane protein DP1/Yop1 mediator complex subunit Med7

$40 S$ ribosomal protein $\mathrm{S} 23$ (predicted)

UBX domain protein Ubx3, Cdc48 cofactor

Prp19 complex WD repeat protein Prp5

$\# N / A$

$\# \mathrm{~N} / \mathrm{A}$

carboxymuconolactone decarboxylase-like protein glutaredoxin Grx1

acireductone dioxygenase family Adi1 (predicted)

$$
\# N / A
$$

$\begin{array}{lll}0.55911867 & 8.25052042 \quad 1.30 \mathrm{E}-08\end{array}$

$\begin{array}{llll}0.55892103 & 7.63945971 \quad 4.69 \mathrm{E}-10\end{array}$

$0.55870362 \quad 7.32556097 \quad 7.82 \mathrm{E}-07$

$\begin{array}{llll}0.55850537 & 5.68305616 & 0.00073585\end{array}$

$\begin{array}{lll}0.55848285 & 6.76780911 & 8.66 \mathrm{E}-07\end{array}$

$\begin{array}{lll}0.55781341 & 7.58883644 & 2.19 \mathrm{E}-08\end{array}$

$\begin{array}{lll}0.55746218 & 9.17602346 \quad 1.80 \mathrm{E}-14\end{array}$

$\begin{array}{llll}0.55712857 & 5.90159334 & 0.00050743\end{array}$

$0.55553252 \quad 9.01699853 \quad 1.91 \mathrm{E}-09$

$\begin{array}{llll}0.55551377 \quad 7.35702401 \quad 3.77 \mathrm{E}-08 & 0\end{array}$

$0.55500788 \quad 7.48744662 \quad 6.73 \mathrm{E}-08$

$0.55451163 \quad 8.01636302 \quad 5.87 \mathrm{E}-09$

$0.55443638 \quad 7.71588578 \quad 5.47 \mathrm{E}-10$

$\begin{array}{lll}0.55390581 & 7.47387817 & 1.30 \mathrm{E}-07\end{array}$

$0.55268215 \quad 7.05207471 \quad 2.86 \mathrm{E}-07$

$0.55245018 \quad 7.83159833 \quad 1.59 \mathrm{E}-10$

$\begin{array}{llll}0.55194667 & 6.23399908 & 0.00197167\end{array}$

$\begin{array}{lll}0.55177398 & 5.29685527 & 0.00317204\end{array}$

$0.55169043 \quad 7.19038636 \quad 1.87 \mathrm{E}-07$

$\begin{array}{llll}0.55153291 & 4.62789735 & 0.07589028\end{array}$

$\begin{array}{lll}0.55134862 & 5.35752926 & 0.00357965\end{array}$

cofactor for cytoplasmic methionyl-and glutamyl-tRNA synt $0.55096253 \quad 8.06585527 \quad 5.73 \mathrm{E}-11$

homoserine $\mathrm{O}$-acetyltransferase (predicted)

translation initiation factor elF2 alpha subunit

$\begin{array}{llll}0.55021021 & 6.39999938 & 0.00039739\end{array}$

$\begin{array}{lll}0.54956536 & 8.16697917 & 6.48 \mathrm{E}-11\end{array}$

$0.549080328 .12195812 \quad 3.58 \mathrm{E}-10$

cell wall protein 1,3-tas (pred 0.54901778 .07989011

0.54857

3.11E-05

serine/threonine protein kinase Ppk32

$$
\text { \#N/A }
$$

$\begin{array}{lll}0.54798172 & 7.06342516 & 2.31 \mathrm{E}-07\end{array}$

$\begin{array}{llll}0.54794751 & 4.58795967 & 0.0266649\end{array}$

$\begin{array}{llll}0.54689115 & 5.3042184 & 0.00347238\end{array}$

DNA polymerase epsilon subunit Dpb4

0.546457717 .14101513

$2.80 \mathrm{E}-07$

$\begin{array}{lll}0.54631569 & 7.47533736 \quad 1.50 E-07\end{array}$

$\begin{array}{lll}0.54579691 & 7.0901477 & 1.54 \mathrm{E}-05\end{array}$

glyoxylate reductase (predicted)

$0.54565156 \quad 7.23205479$

$7.70 \mathrm{E}-07$
0.183427581

$3.22 \mathrm{E}-05$

7.30E-12

$6.16 \mathrm{E}-09$

0.001540243

0.000451296

2.56E-09

$137 \mathrm{E}-10$

0.584417149

5.57E-09

$5.90 \mathrm{E}-05$

0.027205815

7.79E-08

3.41E-09

3.65E-06

0.002051386

4.03E-06

1.27E-07

2.04E-13

0.001470815

$1.28 \mathrm{E}-08$

2.14E-07

3.66E-07

3.70E-08

3.94E-09

$6.76 \mathrm{E}-07$

$1.42 \mathrm{E}-06$

$1.21 \mathrm{E}-09$

0.004960158

0.007579257

9.48E-07

0.123666997

0.008425984

4.60E-10

0.001173716

5.17E-10

2.63E-09

3.91E-08

0.000112033

$1.40 \mathrm{E}-09$

$1.16 \mathrm{E}-06$

0.050306244

0.008218968

1.39E-06

$7.70 \mathrm{E}-07$

5.89E-05

3.60E-06 
SPBC9B6.04C

SPBC9B6.11C

SPCC645.04

SPBC17G9.06C

SPCC320.03

SPAP7G5.04C

SPCC320.10

SPBC119.05C

SPNCRNA.362

SPBC2F12.02C

SPAC13G6.10C

SPBC1711.03

SPNCRNA.472

SPNCRNA.1242

SPCC1223.03C

SPNCRNA.793

SPNCRNA.779

SPCC2H8.02

SPAC694.04c

SPBC725.17C

SPAC1B1.01

SPNCRNA.1531

SPAC2C4.03C

SPCC18B5.04

SPBC1A4.01

SPBC19C2.15C

SPAC27E2.03C

SPAC1399.03

SPBC36.05C

SPCC16C4.14c

SPAP8A3.09c

SPBC354.02C

SPCC663.04

SPAC6F6.11C

SPAC1A6.07

SPAC1006.07

SPBC21C3.15C

SPNCRNA.1079

SPCC645.14C
SPBC9B6.11C

nse3

SPBC17G9.06C SPCC320.03

lys1

srp72

Isb1

$$
\text { \#N/A }
$$

mrpl7

asl1

emc3

$\# N / A$

$\# \mathrm{~N} / \mathrm{A}$

gut2

$\#$ N/A

$\# \mathrm{~N} / \mathrm{A}$

SPCC2H8.02

SPAC694.04C

rrn11

deb1

$$
\# N / A
$$

smd2

rsm18

apc10

phs1

SPAC27E2.03C

fur4

clr6

sfc4

paa1

sec61

rpl39

SPAC6F6.11C

sle1

tif1

SPBC21C3.15C

\#N/A
\#N/A

$\begin{array}{llll}0.54549407 & 3.28406394 & 0.17220735\end{array}$ SRP-independent ER targeting protein Snd3a (predicted) INCENP ortholog Pic1

\section{$\# \mathrm{~N} / \mathrm{A}$}

$2.24 \mathrm{E}-11$

$0.54424532 \quad 6.4938163 \quad 4.03 \mathrm{E}-05$

$0.54415427 \quad 6.77669538 \quad 6.88 \mathrm{E}-05$

$0.543991 \quad 2.39063236 \quad 0.36241554$

mRNP complex (predicted)

$\begin{array}{lll}0.54342214 & 7.90420829 & 2.20 \mathrm{E}-08\end{array}$

sec14 cytosolic factor family, phospholipid-intermembrane $0.54335807 \quad 8.08339573 \quad 7.31 \mathrm{E}-11$

But2 family protein But2, similar to cell surface molecules $0.54301386 \quad 8.75711669 \quad 3.32 \mathrm{E}-06$

mitochondrial translation elongation factor EF-Tu Tuf1 $\quad \begin{array}{llll}0.54286777 & 8.39835474 & 5.72 \mathrm{E}-11\end{array}$

$\begin{array}{llll}\text { CCR4/nocturin family endoribonuclease (predicted) } & 0.54262827 & 8.23309719 & 1.49 \mathrm{E}-08\end{array}$

Smc5-6 complex non-SMC MAGE family subunit Nse3 $\quad 0.54245965 .979305020 .00046712$

$0.542230847 .78289635 \quad 7.39 \mathrm{E}-10$

$0.54136507 \quad 7.54039421 \quad 2.04 \mathrm{E}-07$

$0.54109557 \quad 9.76204078 \quad 2.22 \mathrm{E}-13$

aminoadipate-semialdehyde dehydrogenase

540911237.026907310 .00010885

signal recognition particle subunit Srp72 (predicted)

Wiskott-Aldrich syndrome homolog binding protein Lsb1 (p $0.54080704 \quad 7.77586032 \quad 2.31 \mathrm{E}-09$ $\# \mathrm{~N} / \mathrm{A}$

$\begin{array}{lll}0.54080116 & 4.5474752 & 0.04158223\end{array}$

$0.5406776 \quad 6.18095105 \quad 0.00436747$

$\begin{array}{lrrr}\text { cell wall protein Asl1, predicted O-glucosyl hydrolase } & 0.54024735 & 9.2507876 & 7.73 \mathrm{E}-09 \\ \text { ER membrane protein complex subunit Emc3 (predicted) } & 0.53923407 & 5.73223109 & 0.00104089\end{array}$ $\begin{array}{cllll}\text { ER membrane protein complex subunit Emc3 (predicted) } & 0.53923407 & 5.73223109 & 0.00104089 \\ \text { \#N/A } & 0.53877811 & 2.13294574 & 0.40566212\end{array}$

\#N/A

$\begin{array}{llll}0.53869562 & 3.16681667 & 0.40227922\end{array}$

$\begin{array}{lll}0.53852011 & 6.87807248 & 0.00702102\end{array}$

$\begin{array}{lll}0.53802397 & 8.34446333 & 1.33 \mathrm{E}-11\end{array}$

$0.53658183 \quad 4.46042822 \quad 0.03067264$

$\begin{array}{llrr}\text { plasma membrane inorganic phosphate transmembrane tri } & 0.53560159 & 6.82335645 & 4.12 \mathrm{E}-06 \\ \text { nuclear/mitochondrial metal-dependent protein hydrolase, } & 0.53553363 & 6.65644135 & 0.00026896\end{array}$

RNA polymerase I general transcription initiation factor suk $0.53522597 \quad 4.979501660 .03826499$

transcription factor Deb1/Rdp1$$
\# N / A
$$

Sm snRNP core protein Smd2

$0.53519947 .07954444 \quad 1.78 \mathrm{E}-05$

$\begin{array}{lll}0.53515996 & 6.33120813 & 0.00056507\end{array}$

$0.5349801 \quad 5.58297375 \quad 0.00176035$

mitochondrial ribosomal protein subunit S18 (predicted) $\begin{array}{lllll}0.53480911 & 5.33085308 & 0.01075863\end{array}$ $\begin{array}{lllll}\text { anaphase-promoting complex substrate recognition subunit } & 0.53477452 & 4.07025763 & 0.09798\end{array}$ 3-hydroxyacyl-CoA dehydratase involved in very long-chain f $0.53390497 \quad 6.140734810 .00035641$ $0.533620798 .36559759-1.96 \mathrm{E}-09$

$\begin{array}{lllll}\text { plasma membrane uracil transmembrane transporter } & 0.53344396 & 6.98102024 & 1.41 \mathrm{E}-05\end{array}$ histone deacetylase (class I) Clr6

transcription factor TFIIIC complex subunit Sfc4

protein phosphatase regulatory subunit Paa1

translocon alpha subunit Sec61

$60 \mathrm{~S}$ ribosomal protein $\mathrm{L} 39$

pyridoxine-pyridoxal-pyridoxamine kinase (predicted)

eisosome assembly protein Seg1

$0.533366427 .14403686 \quad 4.86 \mathrm{E}-07$

$0.533161117 .67394061-1.21 \mathrm{E}-08$

$0.53304828 \quad 8.35105009 \quad 4.67 \mathrm{E}-11$

$0.532278398 .17264014 \quad 3.07 \mathrm{E}-10$

$0.53036764 \quad 7.07782654 \quad 3.48 \mathrm{E}-06$

$\begin{array}{lll}0.52999718 & 6.18547282 \quad 0.00064994\end{array}$

$0.529935436 .95430533 \quad 1.00 \mathrm{E}-06$

$0.52965907 \quad 9.2907446 \quad 4.63 \mathrm{E}-13$

aldehyde dehydrogenase, implicated in cellular detoxificatic $0.52964281 \quad 7.80237299 \quad 1.32 \mathrm{E}-09$

$$
\text { \#N/A }
$$

$0.52870157 \quad 5.85338665 \quad 0.00054723$

chaperone activator Sti1 (predicted)
$0.5286505 \quad 7.68910627 \quad 1.48 \mathrm{E}-05$
0.247962551

$1.92 \mathrm{E}-10$

0.00014247

0.000233345

0.457982869

$1.28 \mathrm{E}-07$

5.77E-10

$1.43 \mathrm{E}-05$

4.60E-10

8.89E-08

0.001361414

5.23E-09

$1.03 \mathrm{E}-06$

2.33E-12

0.000356653

$1.52 \mathrm{E}-08$

0.073920351

0.01005775

4.81E-08

0.002779928

0.500912847

0.497729105

0.015357777

$1.16 \mathrm{E}-10$

0.056817887

$1.75 \mathrm{E}-05$

0.00082186

0.06883248

$6.74 \mathrm{E}-05$

0.001611598

0.004468182

0.022519979

0.154091489

0.00106562

1.31E-08

5.42E-05

$2.34 \mathrm{E}-06$

$7.28 \mathrm{E}-08$

3. $81 \mathrm{E}-10$

$2.28 \mathrm{E}-09$

$1.49 \mathrm{E}-05$

0.001832919

4.62E-06

$4.71 \mathrm{E}-12$

9.06E-09

0.001565989

5.68E-05 
SPAC9G1.05

SPCC1442.14C

SPAC31G5.13

SPBC17A3.08

SPBC19C2.03

SPAPB18E9.04C

SPNCRNA.739

SPRRNA.41

SPAC1006.05C

SPBC83.13

SPAC23C11.09

SPAC3A12.06C

SPAP32A8.02

SPCC18.18C

SPBC17D1.06

SPBC9B6.07

SPAC6G10.08

SPATRNAMET.02

SPAPB8E5.10

SPNCRNA.121

SPBC16A3.18

SPAC890.04c

SPAC23C11.17

SPAPB2B4.01C

SPAC9G1.03C

SPAC824.09c

SPCC338.07c

SPAC1783.02c

SPAPB17E12.05

SPAC22H12.04C

SPBC16C6.12C

SPAC25G10.02

SPCC1322.02

SPNCRNA.536

SPNCRNA.1360

SPCC1259.02C

SPAC57A10.02

SPAC29A4.10

SPAC19B12.02C

SPCC338.15

SPAC1B2.02C

SPNCRNA.768

SPAC13D6.02C

SPCC594.02C

SPBC3F6.01c

SPAC821.03C

SPAC343.13 rpc10

SPAPB18E9.04 \#N/A

SPRRNA.41

och1

yhm2

ala1

SPAC3A12.06C

SPAP32A8.02

fum 1

dbp3

nop52

idp1

SPATRNAMET.02

SPAPB8E5.10

$\# \mathrm{~N} / \mathrm{A}$

cip1

ytm1

mdm 28

gpi12

rpl3001

SPAC824.09c

naa15

vps66

rpl3703

rps102

las1

cce1

pxd1

erm 1

cdr2

rrn5

gas1

wbp1

ugo1

\#N/A

byr3

SPCC594.02C

SPBC3F6.01C

sIf1

gta2 actin binding WD repeat protein Aip1

$0.52847412 \quad 8.2585473 \quad 1.14 \mathrm{E}-08$

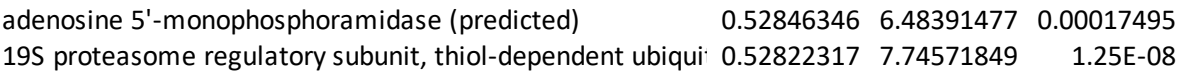
$\begin{array}{lllll}\text { TatD homolog (predicted) } & 0.52808671 & 6.2472013 & 0.00016092\end{array}$

DNA-directed RNA polymerase I, II and III subunit Rpc10 $0.52765496 \quad 5.86209088 \quad 0.00053162$ Schizosaccharomyces pombe specific protein, possible cell : $\begin{array}{llll}0.52722965 & 6.64032406 & 0.04119787\end{array}$ $\# \mathrm{~N} / \mathrm{A}$

$\begin{array}{llll}0.52563379 & 5.39763303 & 0.0052332\end{array}$

$\begin{array}{lll}0.52504337 & 1.09882413 & 0.7363981\end{array}$

5 rRNA

$\begin{array}{llll}\text { alpha-1,6-mannosyltransferase Och1 } & 0.52495794 & 7.60016877 & 1.51 \mathrm{E}-07 \\ \text { mitochondrial carrier, tricarboxylic acid Yhm2 (predicted) } & 0.52404624 & 7.66947579 & 4.63 \mathrm{E}-08\end{array}$

mitochondrial and cytoplasmic alanine-tRNA ligase Ala1 (p 0.523645649 .17757601 6.60E-11

sodium/calcium exchanger (predicted)

$0.52353334 \quad 6.96670302 \quad 6.12 \mathrm{E}-05$

$0.52301823 \quad 7.76766874 \quad 8.71 \mathrm{E}-09$

$\begin{array}{lll}0.52284061 & 8.28871575 & 5.51 \mathrm{E}-07\end{array}$

fumarate hydratase (predicted)

ATP-dependent RNA helicase Dbp3 (predicted)

nucleolar protein Nop52 family Rrp1 (predicted)

isocitrate dehydrogenase Idp1 (predicted)

tRNA Methionine

$0.52279847 \quad 7.14889687 \quad 0.00026647$

$\begin{array}{lll}0.5209739 & 5.36733885 & 0.00885366\end{array}$

$0.52095242 \quad 8.44112312 \quad 1.84 \mathrm{E}-10$

$0.52076671 \quad 1.82042476 \quad 0.53239182$

$0.51942667 \quad 7.2620055 \quad 4.17 \mathrm{E}-07$

$0.51940202 \quad 1.098392850 .73609483$

$0.519398157 .23377852 \quad 1.76 \mathrm{E}-06$

$\# \mathrm{~N} / \mathrm{A}$

ribosome biogenesis WD repeat WDR12/Ytm1 protein (pre $0.51938361 \quad 7.02993836 \quad 6.63 \mathrm{E}-06$

mitochondrial inner membrane protein involved in translati $0.51911173 \quad 7.03294671 \quad 4.12 \mathrm{E}-06$ pig-L, $\mathrm{N}$-acetylglucosaminylphosphatidyl inositoldeacetylası $0.51861097 \quad 6.04320177 \quad 0.00133383$

60S ribosomal protein L30 (predicted) $\quad \begin{array}{lllll}0.51764912 & 7.29030174 & 3.57 \mathrm{E}-07\end{array}$

$\begin{array}{lllll}\text { GTPase activating protein (predicted) } & 0.51723448 & 6.60590161 & 0.00016525\end{array}$

$\begin{array}{lllll}\text { NatA N-acetyltransferase complex regulatory subunit Naa1 } & 0.51709147 & 7.98230523 & 2.62 \mathrm{E}-09\end{array}$

1-acylglycerol-3-phosphate O-acyltransferase Vps66 (predic 0.517066746 .089610460 .00480638

$60 \mathrm{~S}$ ribosomal protein $\mathrm{L} 37$ (predicted)

$40 \mathrm{~S}$ ribosomal protein S3a (predicted)

Las1 pre-rRNA processing protein

mitochondrial cruciform-cutting endonuclease Cce1

$0.5168683 \quad 7.21903982 \quad 1.56 \mathrm{E}-06$

$0.5166566 \quad 8.75452437 \quad 3.10 \mathrm{E}-11$

$\begin{array}{llll}0.51624253 & 6.22357713 & 0.00022136\end{array}$

$\begin{array}{llll}0.51601655 & 4.63289927 & 0.06887561\end{array}$

$\begin{array}{lll}0.51540709 & 5.56097922 & 0.00892791\end{array}$

$0.5150183 \quad 5.21161452 \quad 0.00734895$

$0.51485814 \quad 6.44512824 \quad 0.00032485$

$0.51432954 \quad 8.78371746 \quad 1.21 \mathrm{E}-11$

$\begin{array}{lll}0.51419248 & 7.25619233 & 2.77 \mathrm{E}-05\end{array}$

ER metallopeptidase Erm1 (predicted)

serine/threonine protein kinase Cdr2

00530487

RNA polymerase I upstream activation factor complex subu $0.5141305 \quad 6.267013$ cell wall protein 1,3-beta-glucanosyltransferase Gas1 (pred $0.51402022 \quad 9.22007738 \quad 8.80 \mathrm{E}-09$ dolichyl-di-phosphooligosaccharide-protein glycotransferası $0.51386416 \quad 7.98204121 \quad 3.92 \mathrm{E}-09$ $\begin{array}{llll}\text { mitochondrial fusion protein Ugo1 (predicted) } & 0.51216899 & 7.14974047 & 6.92 \mathrm{E}-06\end{array}$

$$
\# \mathrm{~N} / \mathrm{A}
$$

translational activator, zf-CCHC type zinc finger protein (pre $0.51169028 \quad 7.79630107 \quad 2.05 \mathrm{E}-08$ $\begin{array}{llll}\text { DUF2456 family conserved fungal protein } & 0.51085519 & 8.42264095 & 1.01 \mathrm{E}-08\end{array}$

$\begin{array}{lllll}\text { TPR repeat serine/threonine protein phosphatase (predicte } & 0.5106802 & 7.11627877 & \text { 7.64E-06 }\end{array}$ cell cortex node protein SIf1

$0.510661096 .79825325 \quad 6.49 \mathrm{E}-06$

mitochondrial glutamyl-tRNA amidotransferase beta subun $0.510528125 .71638305 \quad 0.01512872$
$6.88 \mathrm{E}-08$

0.000552458

7.50E-08

0.000512412

0.001529019

0.073332862

0.01182334

0.803332654

7.75E-07

2.59E-07

5.26E-10

0.000209213

5.37E-08

2.64E-06

0.000815334

0.018875052

1.40E-09

0.623889503

2.03E-06

0.803130563

$7.88 \mathrm{E}-06$

2.72E-05

$1.75 \mathrm{E}-05$

0.003473677

1.75E-06

0.000524739

1.72E-08

0.010983005

7.02E-06

$2.59 \mathrm{E}-10$

0.000689104

0.113789397

0.019015471

0.016003064

0.00097901

1.07E-10

0.000101196

0.011957485

5.41E-08

2.53E-08

2.82E-05

0.233861673

1.20E-07

$6.15 \mathrm{E}-08$

3.10E-05

2.67E-05

0.030567434 
SPBC1D7.03

SPCC576.19c

SPNCRNA.1392

SPAC10F6.10

SPAC16A10.04

SPBC2G2.13C

SPNCRNA.1432

SPBC651.07

SPBPB7E8.02

SPBC1271.14

SPBC3F6.03

SPBC3B9.11C

SPNCRNA.1235

SPAC18G6.13

SPCC970.03

SPAC9E9.13

SPNCRNA.1011

SPCC1672.12C

SPAC806.04c

SPBC2F12.14C

SPAC14C4.06C

SPAC3G9.16C

SPAC8E11.07C

SPAC6G9.14

SPBC947.11C

SPNCRNA.1335

SPNCRNA.1322

SPAC664.04c

SPBC1105.03C

SPCC24B10.04

SPCC584.11C

SPNCRNA.240

SPBC3B9.19

SPBC31F10.10c

SPBC1861.08c

SPCC18.13

SPAC328.09

ScpofMt28

SPNCRNA.902

SPAC6F6.19

SPBC2G 2.12

SPAC6G10.03C

SPAPB1E7.07

SPAC16E8.01

SPNCRNA.1049

SPAC26A3.17C

SPBC1711.13 clg1

$\# \mathrm{~N} / \mathrm{A}$

$\# \mathrm{~N} / \mathrm{A}$

rio1

rho4

dcd1

\#N/A

SPBC651.07

SPBPB7E8.02

SPBC1271.14

trr1

$\# \mathrm{~N} / \mathrm{A}$

SPAC18G6.13

cbr1

wos 2

\#N/A

get4

SPAC806.04c

gua1

nab2

bet5

alp31

puf4

elg1

$\# N / A$

rps1602

mrpl1601

SPCC24B10.04

svf1

\#N/A

mge1

SPBC31F10.10C

ea1

trm82

SPAC328.09

$\# \mathrm{~N} / \mathrm{A}$

$\# N / A$

SPAC6F6.19

hrs1

cld1

glt1

shd1

\#N/A

rmt2

his2 cyclin-like protein involved in autophagy Clg1 (predicted)

$\# \mathrm{~N} / \mathrm{A}$

$\# \mathrm{~N} / \mathrm{A}$

protein kinase, RIO family Rio1 (predicted)

Rho family GTPase Rho4

deoxycytidylate deaminase (predicted)

$\#$ N/A

Schizosaccharomyces specific protein

PSP1 family protein

acetyl-CoA:L-glutamate $\mathrm{N}$-acetyltransferase (predicted)

thioredoxin reductase Trr1

mRNA cleavage and polyadenylation specificity factor comr$$
\# \mathrm{~N} / \mathrm{A}
$$

Schizosaccharomyces specific protein

$\mathrm{NADH}$-dependent reductase for Dph3, Cbr1 (predicted)

p23 homolog, predicted co-chaperone Wos2

$$
\text { \#N/A }
$$

$\begin{array}{lll}0.50851315 & 9.03304506 \quad 3.15 E-09\end{array}$ $\begin{array}{llll}0.50850273 & 3.04298573 & 0.2612899\end{array}$ $\begin{array}{llll}0.50837811 & 2.70641833 & 0.33244871\end{array}$ $\begin{array}{llll}0.50815724 & 5.6343964 & 0.00333596\end{array}$ $\begin{array}{lll}0.50802465 & 6.04306597 \quad 0.00074699\end{array}$ $0.50779348 .53567951 \quad 1.10 \mathrm{E}-08$ $0.50739075 \quad 3.69444918 \quad 0.16894552$ $0.50655528 \quad 6.02314947 \quad 0.00355462$ $0.506260868 .68133373 \quad 9.32 \mathrm{E}-09$ $\begin{array}{lll}0.50605847 & 7.98989019 & 3.37 \mathrm{E}-09\end{array}$ $0.50592426 \quad 7.4390879 \quad 3.86 \mathrm{E}-06$

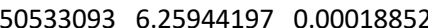
$0.5050356 \quad 6.95684122 \quad 0.00675658$ $0.5045312 \quad 4.58240678 \quad 0.04052013$ $0.504104497 .11547827 \quad 8.74 \mathrm{E}-05$ $\begin{array}{lll}0.50381881 & 8.30467464 & 5.59 \mathrm{E}-10\end{array}$ $0.50326722 \quad 6.08754588 \quad 0.00061648$

GET complex (ER membrane insertion) subunit Get4 (predi $0.50286465 \quad 6.97968543 \quad 1.42 \mathrm{E}-05$ metal-dependent phosphatase involved in cellular detoxific $\quad 0.5023795 \quad 7.2957883 \quad 1.35 \mathrm{E}-06$ IMP dehydrogenase Gua1

poly(A) binding protein Nab2 (predicted)

TRAPP complex subunit Bet5 (predicted)

tubulin specific chaperone cofactor A, Alp31

pumilio family RNA-binding protein Puf4 (predicted)

DNA replication factor $\mathrm{C}$ complex subunit Elg1

$$
\# \mathrm{~N} / \mathrm{A}
$$$$
\# N / A
$$

40 S ribosomal protein S16 (predicted)

mitochondrial ribosomal protein subunit L16 (predicted)

Schizosaccharomyces specific protein

Svf1 family protein, lipocalin superfamily Svf1

$$
\text { \#N/A }
$$

$\begin{array}{llll}0.50177186 & 8.57621841 & 2.88 \mathrm{E}-10\end{array}$

$\begin{array}{lll}0.50149267 & 6.23075089 & 0.0007401\end{array}$

$0.50039709 \quad 5.29625883 \quad 0.03028602$

$\begin{array}{llll}0.49980558 & 5.70366744 & 0.00488249\end{array}$

$0.499636137 .51849991 \quad 1.20 \mathrm{E}-06$

$0.49963162 \quad 6.61830452 \quad 0.00239632$

$\begin{array}{lll}0.4994138 & 4.58268293 & 0.05482546\end{array}$

$\begin{array}{lll}0.4990992 & 7.23386382 & 1.27 \mathrm{E}-05\end{array}$

$0.49795078 \quad 7.33891197 \quad 4.80 \mathrm{E}-07$

$\begin{array}{lll}0.49788366 & 6.29643241 & 0.00060917\end{array}$

$\begin{array}{lll}0.49757786 & 4.92458828 & 0.03421774\end{array}$

$0.4974212 \quad 7.64639645 \quad 1.43 \mathrm{E}-07$

$0.49676322 \quad 2.7757412 \quad 0.36126488$

mitochondrial [2Fe-2S] cluster assembly and protein impor $0.49656494 \quad 7.11110333 \quad 1.00 \mathrm{E}-05$

Armadillo-type fold protein, zf-MYND type zinc finger prote $0.49652688 \quad 7.62932844 \quad$ 7.83E-06

U2 snRNP-associated protein Lea1 (predicted) $\quad 0.496485696 .54148809 \quad 0.00050835$

tRNA (guanine-N7-)-methyltransferase WD repeat subunit 0.496280065 .989918890 .00160672

mitochondrial carrier, 2-oxoadipate/2-oxoglutarate (predict $0.49619711 \quad 6.71498504 \quad 3.13 \mathrm{E}-05$

\#N/A $\quad 0.49592917 \quad 4.365566 \quad 0.13965859$

$\begin{array}{llll}\text { \#N/A } & 0.49513656 & 6.40923017 & 0.0009874\end{array}$

RNA-binding protein, G-patch type, human GPATCH11 orthc 0.495116753 .883109310 .11243972 mitochondrial and cytoplasmic histidine-tRNA ligase Hrs1 ( $0.494953878 .11376763 \quad 3.56 \mathrm{E}-07$ mitochondrial cardiolipin-specific phospholipase Cld1 (pred $0.49490582 \quad 7.57560388 \quad 1.89 \mathrm{E}-07$ $\begin{array}{lllll}\text { glutamate synthase Glt1 } & 0.49421323 & 10.1869478 & 1.61 \mathrm{E}-11\end{array}$ cytoskeletal protein binding protein Sla1 family, Shd1 (pred $0.494194569 .46074136 \quad 1.10 \mathrm{E}-10$

$$
\# \mathrm{~N} / \mathrm{A}
$$

$0.49386653 \quad 6.27501134 \quad 0.00769844$

$\begin{array}{llll}0.49379812 & 6.47336484 & 0.00094661\end{array}$

$\mathrm{N}$-methyltransferase (predicted)

$\begin{array}{lll}0.49373657 & 7.92979941 \quad 2.08 \mathrm{E}-09\end{array}$
2.05E-08

0.351923919

0.426780113

0.007918112

0.002077352

6.67E-08

0.24419589

0.00837841

$5.72 \mathrm{E}-08$

$2.18 \mathrm{E}-08$

1.64E-05

0.000593085

0.014831782

0.072296664

0.000290681

4.01E-09

0.001743613

$5.48 \mathrm{E}-05$

6.15E-06

2.16E-09

0.002060724

0.056157046

0.011138239

5.49E-06

0.005904042

0.09376408

4.92E-05

$2.32 \mathrm{E}-06$

0.001724362

0.062527308

7.40E-07

0.456953063

3.95E-05

3.17E-05

0.001472673

0.004108877

0.000112733

0.208320114

0.002651628

0.173111385

1.75E-06

9.59E-07

1.39E-10

$8.52 \mathrm{E}-10$

0.016647768

0.002557917

1.38E-08 
SPBC2G2.08

SPAC4A8.11C

SPNCRNA.1373

SPNCRNA.1690

SPCC162.11C

SPAC17C9.09C

SPAC5D6.01

SPAC1B3.01C

SPAC1A6.10

SPCC126.06

SPAC1F12.10c

SPAC20G4.01

SPAC23A1.07

SPBC15D4.02

SPAC13G6.02C

SPBC28E12.03

SPNCRNA.1160

SPNCRNA.488

SPBC1289.16C

SPCC736.12C

SPAC27D7.07c

SPAC17G8.06C

SPNCRNA.637

SPAC750.07C

SPBC19C7.06

SPAC6B12.15

SPAC56E4.05

SPAPJ696.02

SPAPB18E9.01

SPAP7G5.03

SPAC3G6.06C

SPBC1685.09

SPAC3A11.12C

SPBC1105.01

SPAC30D11.12

SPAC9G1.13C

SPAC607.04

SPNCRNA.789

SPCC24B10.20

SPAC9.12C

SPAC1527.02

SPBC16D10.11C

SPAC2F3.09

SPAC212.02

SPCC126.04C

SPAC56F8.07

SPAC227.09 urk1

tim13

rps2202

SPAC1B3.01C

tcd1

twf1

SPAC1F12.10C

caf16

SPAC23A1.07

gsf1

rps101

rga4

$\# \mathrm{~N} / \mathrm{A}$

$\# N / A$

cao2

mmi1

smd1

SPAC17G8.06c

$\# \mathrm{~N} / \mathrm{A}$

SPAC750.07C

prs1

cpc2

snd2

Isb4

trm5

prm 1

rad2

rps29

rpt5

SPBC1105.01

rpl3802

swc4

$\arg 82$

$\#$ N/A

SPCC24B10.20

atp12

sft2

rps1801

hem1

SPAC212.02

sgf73

SPAC56F8.07

fol3 $\begin{array}{llll}\text { C-1-tetrahydrofolatesynthase/methylenetetrahydrof olated } 0.49370356 & 7.8609022 & 1.15 \mathrm{E}-07\end{array}$ fatty acid synthase alpha subunit Fas2

$$
\# N / A
$$
$0.49335385 \quad 10.5358863 \quad 9.73 \mathrm{E}-12$ $\begin{array}{llll}0.49264721 & 5.23198293 & 0.01538698\end{array}$ $0.492476897 .70517578 \quad 0.00249946$

uridine kinase/uracil phosphoribosyltransferase (predicted) $0.4920066 \quad 6.393736490 .00116046$ Tim8-Tim13 mitochondrial intermembrane space protein tr $0.49180782 \quad 5.98199678 \quad 0.00204789$ $\begin{array}{lllll}40 \mathrm{~S} \text { ribosomal protein S15a (predicted) } & 0.49142931 & 6.83991858 & 7.17 \mathrm{E}-05\end{array}$ $\begin{array}{llll}\text { uracil phosphoribosyltransferase (predicted) } & 0.49119829 & 7.36257932 & 6.21 \mathrm{E}-07\end{array}$ tRNA threonylcarbamoyladenosine dehydratase Tcd1 (predi $0.49095169 \quad 7.03545579 \quad 1.45 \mathrm{E}-05$ twinfilin (predicted)

NADPH-hemoprotein reductase (predicted)

CCR4-Not complex subunit Caf16 (predicted)

ubiquitin-protein ligase E3 (predicted)

$40 \mathrm{~S}$ ribosomal protein $\$ 3$

RhoGAP, GTPase activating protein Rga4

$$
\# N / A
$$

$\# \mathrm{~N} / \mathrm{A}$

copper amine oxidase-like protein Cao2

$\begin{array}{rrr}0.49095169 & 7.03545579 & 1.45 \mathrm{E}-05 \\ 0.49085878 & 6.02151178 & 0.00527771\end{array}$

$\begin{array}{llll}0.49010978 & 5.83649828 & 0.00286633\end{array}$

$\begin{array}{llll}0.4900373 \quad 6.38711337 & 0.0007874\end{array}$ $\begin{array}{lll}0.48969136 & 5.06465846 & 0.01567951\end{array}$ $\begin{array}{lll}0.4894471 & 8.56845508 & 3.63 \mathrm{E}-10\end{array}$ $\begin{array}{lll}0.48867767 & 7.94736434 & 2.28 \mathrm{E}-08\end{array}$ $\begin{array}{llll}0.48846257 & 6.41528808 & 0.00032508\end{array}$ $\begin{array}{llll}0.48805872 & 1.90504182 & 0.60086429\end{array}$ $\begin{array}{llll}0.48745995 & 7.71327092 & 0.00274382\end{array}$ nucleus specific RNA binding exosome specificity factor Mn $0.48728571 \quad 7.48348206 \quad 4.36 \mathrm{E}-05$ Sm snRNP core protein Smd1

dihydroxy-acid dehydratase (predicted) $\#$ N/A

S. pombe specific protein

cytoplasmic proline-tRNA ligase Prs1 (predicted)

RACK1 ortholog Cpc2

SRP-independent ER targeting protein Snd2 (predicted)

actin cortical patch component Lsb4 (predicted)

tRNA (guanine(37)-N(1))-methyltransferase activity Trm5 ( conjugation protein Prm1

FEN-1 endonuclease Rad2

40 S ribosomal protein $\mathrm{S} 29$ (predicted)

$19 \mathrm{~S}$ proteasome base subcomplex ATPase subunit Rpt5

rRNA processing protein Rrp12-like (predicted)

$60 S$ ribosomal protein L38 (predicted)

Swr1 complex subunit Swc4

inositol polyphosphate kinase Arg82 (predicted)

$$
\text { \#N/A }
$$

$\begin{array}{llll}0.48726284 & 6.10108211 & 0.00127708\end{array}$

$0.48658851 \quad 8.9772779 \quad 4.59 \mathrm{E}-10$

$\begin{array}{lll}0.4863741 & 5.67105973 & 0.00323507\end{array}$

$\begin{array}{llll}0.48617991 & 4.29892916 & 0.06312016\end{array}$

$0.48527063 \quad 9.03049258 \quad 5.84 \mathrm{E}-12$

$\begin{array}{lll}0.48490222 & 9.30971944 & 2.42 \mathrm{E}-12\end{array}$

$\begin{array}{lll}0.48470022 & 5.34751464 & 0.01987345\end{array}$

$0.48368946 \quad 7.38349832 \quad 4.95 \mathrm{E}-07$

$\begin{array}{lll}0.48272404 & 5.90872614 & 0.00798021\end{array}$ $\begin{array}{lll}0.48250909 & 9.8948368 & 1.38 \mathrm{E}-11\end{array}$

$\begin{array}{llll}0.48193717 & 6.06801248 & 0.00076024\end{array}$

$\begin{array}{llll}0.48167708 & 6.16539907 & 0.00239464\end{array}$ $\begin{array}{lll}0.48126914 & 8.27560499 \quad 1.08 \mathrm{E}-08\end{array}$ $0.48066056 \quad 7.18463475 \quad 6.69 \mathrm{E}-06$ $0.48021777 \quad 6.74928238 \quad 0.00010168$ $\begin{array}{lll}0.48012987 & 6.07415949 & 0.00097385\end{array}$ $0.48007125 \quad 4.43334398 \quad 0.15242022$ $0.48007125 \quad 4.43334398 \quad 0.15242022$ short chain dehydrogenase, unknown specificity (predicted) $0.47990827 \quad 4.516954290 .15760616$ mitochondrial F1-FO ATP synthase chaperone Atp12 (predic $0.47972726 \quad 5.86047033 \quad 0.0024455$ Golgi transport protein Sft2 (predicted) $\quad 0.47908311 \quad 5.277093290 .05874119$ $\begin{array}{lllll}40 \mathrm{~S} \text { ribosomal protein S18 (predicted) } & 0.47907588 & 7.53288284 & 1.11 \mathrm{E}-06\end{array}$ $\begin{array}{lllll}5 \text {-aminolevulinate synthase Hem1 } & 0.47883246 & 8.29444725 & 4.78 \mathrm{E}-07\end{array}$ $\begin{array}{llllll}\text { Schizosaccharomyces pombe specific protein } & 0.47872466 & 5.87189171 & 0.00223974\end{array}$ SAGA complex subunit Sgf73 $\quad \begin{array}{llllll}0.47851931 & 5.34408901 & 0.02003381\end{array}$ ER membrane integral protein, implicated in sterol metabo 0.478141155 .940859420 .00256463 folylpolyglutamate synthase Fol3 (predicted) $\quad \begin{array}{llll}0.47809911 & 6.32654586 & 0.00098447\end{array}$
$6.01 \mathrm{E}-07$

$8.64 \mathrm{E}-11$

0.031015518

0.006129307

0.003067947

0.005121616

0.000242429

2.96E-06

$5.58 \mathrm{E}-05$

0.011912042

0.006924172

0.002170202

0.031521141

0.004470824

2.66E-09

$1.32 \mathrm{E}-07$

0.000979277

0.684102642

0.006658939

0.000153107

0.003347653

3.34E-09

0.007713622

0.105744133

5.31E-11

2.30E-11

0.039007447

$238 \mathrm{E}-06$

0.017181163

$1.20 \mathrm{E}-10$

0.002108157

0.005902042

6.54E-08

2.74E-05

0.00033492

0.002621486

0.224166758

0.224166758

0.230597701

0.006012145

0.099373793

5.09E-06

2.31E-06

0.005564589

0.039276892

0.006257543

0.00264482 
SPBC14C8.05C

SPCC18.16C

SPCC1393.09c

SPAC1F7.02C

SPAC5H10.10

SPBC29A10.08

SPBC18E5.12C

SPCC11E10.03

SPAC27F1.03C

SPBC25H2.06C

SPBC32F12.02

SPNCRNA.195

SPAC10F6.03C

SPBC1198.08

SPBC19C2.14

SPCC16C4.10

SPBC15C4.04C

SPBC16D10.06

SPBP35G2.11C

SPNCRNA.1258

SPNCRNA.652

SPBC354.13

SPNCRNA.1488

SPAC31G5.01

SPNCRNA.1648

SPAC18G6.12C

SPBC725.12

SPAC6G9.05

SPCC1393.10

SPBC839.03C

SPBC713.03

SPAC17H9.11

SPAC2OH4.03C

SPAC23A1.17

SPAC4D7.08C

SPCC70.03C

SPBC25B2.09C

SPNCRNA.1494

SPBC1A4.07C

SPBC29A10.09c

SPBC25H2.02

SPNCRNA.1406

SPBC14C8.04

SPAC1006.08

SPAC27E2.05 fwf

meu17

fmn1

gir2

has1

SPAC5H10.10

gas2

mas2

jnm1

uch1

rec14

$\# \mathrm{~N} / \mathrm{A}$

cts1

dug1

smd3

SPCC16C4.10

SPBC15C4.04C

zrt1

$\# N / A$

$\# \mathrm{~N} / \mathrm{A}$

rga6

\#N/A sap49

\#N/A

SPAC18G6.12C

$\mathrm{nbl} 1$

pcd1

ctr4

den1

dld2

gmf1

tfs1

bbc1

ade4

put1

$\# N / A$

sof1

tri1

trs1

\#N/A

ilv6

etd1

cdc1
\#N/A

$0.47801693 \quad 7.36106313 \quad 1.09 E-06$ $0.47775485 \quad 8.59875089 \quad 3.37 \mathrm{E}-09$ $\begin{array}{llll}0.47771915 & 5.18993838 & 0.01910466\end{array}$ $\begin{array}{llll}0.47765991 & 5.34665361 & 0.02385799\end{array}$

glucose-6-phosphate 1-dehydrogenase Zwf1 glucan-alpha-1,4-glucosidase

\begin{tabular}{lr|rrr} 
RWD domain protein, involved in cytoplasmic translation Gi & 0.47754277 & 6.42254435 & 0.00042949 \\
ATP-dependent RNA helicase Has1 (predicted) & 0.47696696 & 6.79193102 & 0.00294443
\end{tabular} $\begin{array}{lll}0.47696696 & 6.79193102 & 0.00294443 \\ 0.47671234 & 6.04121126 & 0.00146482\end{array}$ cell wall $130.0 .47671234 \quad 6.04121126 \quad 0.00146482$ mitochondrial processing peptidase (MPP) complex alpha s $0.47574957 \quad 7.02249556 \quad 1.59 \mathrm{E}-05$ $\begin{array}{lllll}\text { dynactin complex subunit, dynamitin Jnm1 } & 0.47568531 & 5.11997773 & 0.05080779\end{array}$ ubiquitin C-terminal hydrolase Uch1

COPII-coated vesicle component Hrf1 (predicted)

$0.47392316 \quad 6.17282694 \quad 0.00239082$

$0.47362083 \quad 7.4816154 \quad 4.53 \mathrm{E}-06$ $0.472954195 .69597591 \quad 0.02194976$ $\begin{array}{llll}0.47287268 & 2.50413633 & 0.41263285\end{array}$ $0.47277534 \quad 8.72003176 \quad 4.19 \mathrm{E}-09$ $0.47261107 \quad 8.71278112 \quad 4.40 \mathrm{E}-09$ $0.472319616 .91558006 \quad 1.66 \mathrm{E}-05$ $0.47218557 \quad 7.59380097 \quad 5.89 \mathrm{E}-06$ $\begin{array}{lrrr}\text { amino acid transmembrane transporter (predicted) } & 0.47201426 & 6.72035359 & 0.00022382 \\ \text { plasma membrane ZIP zinc transmembrane transporter Zrt } & 0.47162679 & 7.88392113 & 5.17 \mathrm{E}-05\end{array}$

CTP synthase Cts1 (predicted) dipeptidase Dug1 (predicted) Sm snRNP core protein Smd3

6 -phosphogluconolactonase (predicted) cargo receptor for selective autophagy pathway $\quad \begin{array}{lllll}0.47075063 & 6.1296735 & 0.01021329\end{array}$

$0.47059053 \quad 4.63309235 \quad 0.06601545$ $0.4705154 \quad 7.80181399 \quad 7.78 \mathrm{E}-07$ $0.47042007 \quad 7.61315792 \quad 3.28 \mathrm{E}-06$ $0.46986152 \quad 5.42447369 \quad 0.00985183$ 0.469355795 .988090290 .00204143 $0.46903494 \quad 7.65790119 \quad 1.02 \mathrm{E}-07$ $0.468971565 .71391256 \quad 0.00627645$ $\begin{array}{lrrr}\text { Borealin homolog Nbl1 } & 0.46888613 & 3.67059018 & 0.17468193 \\ \text { coenzyme A diphosphatase (predicted) } & 0.46835023 & 5.2348404 & 0.02424516\end{array}$ $\begin{array}{lrrr}\text { Borealin homolog Nbl1 } & 0.46888613 & 3.67059018 & 0.17468193 \\ \text { coenzyme A diphosphatase (predicted) } & 0.46835023 & 5.2348404 & 0.02424516\end{array}$ plasma membrane copper transporter complex subunit Ctr $0.46771848 \quad 6.99351568 \quad 0.00012731$ neddylation protein Dcn1 (predicted) $\quad 0.46769438 \quad 5.248184540 .02099306$ mitochondrial D-lactate dehydrogenase, cytochrome Dld2 (| $0.46756172 \quad 7.413823 \quad 3.27 \mathrm{E}-05$ cofilin/tropomyosin family Glia Maturation Factor homolog 0.467560055 .829356640 .00352515 general transcription elongation factor TFIIS

WIP family cytoskeletal protein Bbc1 (predicted) amidophosphoribosyltransferase Ade4

proline dehydrogenase Put1 (predicted)

$\begin{array}{lll}0.46722961 & 6.63728982 & 0.00014457\end{array}$ $0.46722454 \quad 8.84125421 \quad 5.62 \mathrm{E}-09$ $0.46678978 .31064678 \quad 1.71 \mathrm{E}-09$ $0.46661604 \quad 9.89836641 \quad 5.79 \mathrm{E}-11$ mitochondrial and cytoplasmic arginine-tRNA ligase Rrs1/ $0.46645018 \quad 8.70310221 \quad 7.13 \mathrm{E}-10$

$$
\# \mathrm{~N} / \mathrm{A}
$$

$\begin{array}{llll}0.46637909 & 5.21497381 & 0.02236299\end{array}$ $\begin{array}{lll}0.46607868 & 6.57811847 & 0.00035853\end{array}$ $0.46598458 \quad 9.73473306 \quad 2.60 \mathrm{E}-12$ $0.46590493 \quad 9.18364532 \quad 2.43 \mathrm{E}-09$ $0.4658328 \quad 4.70650299 \quad 0.07100831$ $0.464552947 .86183161 \quad 4.11 \mathrm{E}-08$ $0.46391622 \quad 6.13484745 \quad 0.01054937$ $0.46378242 \quad 6.87027118 \quad 9.14 \mathrm{E}-05$
Spg1-binding protein Etd1

DNA polymerase delta small subunit Cdc1
5.02E-06

$2.18 \mathrm{E}-08$

0.037726987

0.045669099

0.001260319

0.007077657

0.003775785

$7.42 \mathrm{E}-11$

$6.08 \mathrm{E}-05$

0.087886885

0.005896896

$1.91 \mathrm{E}-05$

0.042518687

0.507677914

2.70E-08

$2.82 \mathrm{E}-08$

6.31E-05

$2.44 \mathrm{E}-05$

0.000696101

0.000179198

0.021497496

0.109783751

3.64E-06

$1.41 \mathrm{E}-05$

0.02080747

0.005109208

5.40E-07

0.013921516

0.251052118

0.046306003

0.000412552

0.040858677

0.000117167

0.008314944

0.000464289

3.56E-08

1.16E-08

4.64E-10

5.05E-09

0.043220717

0.001071035

$2.46 \mathrm{E}-11$

1.60E-08

0.116717766

2.32E-07

0.022122782

0.000303075

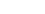


SPAC24H6.11C

SPNCRNA.1468 SPAC22F 3.02

SPBCPT2R1.02 SPBP35G2.14 SPNCRNA.892

SPBC215.07c

SPCC1840.08C

SPBC4F6.13C

SPBC12C2.13C

SPBP8B7.01C

SPAC17G8.08C

SPBC1289.05C

SPAC8C9.11

SPNCRNA.1081

SPAC823.11

SPCC18.11C

SPCC285.04

SPAC890.03

ScpofMt13

SPBC27B12.10C

SPAC22F 8.08

SPCC757.05c

SPCC613.04c

SPAC19G12.15C

SPNCRNA.719

SPNCRNA.19

SPCC4G3.07C

SPCC584.05
SPCC777.12c

ubc14

SPBC1861.05

pre3

$\# N / A$

spc24

spo4

$\# N / A$

$\# \mathrm{~N} / \mathrm{A}$

pga2

$\# \mathrm{~N} / \mathrm{A}$

SPAC24H6.11C

$\# \mathrm{~N} / \mathrm{A}$

atf31

SPBCPT2R1.02

puf2

$\#$ N/A

pdp2

pdi5

erb1

fnx1

pop7

gdt2

vma10

sgp1

sdc1

SPCC285.04

ppk16

tom7

$\sec 24$

SPCC757.05C

rng3

tpp1

$\# \mathrm{~N} / \mathrm{A}$

$\# \mathrm{~N} / \mathrm{A}$

phf1

sec1

plasma membrane urea transmembrane transporter (predi $0.46352884 \quad 5.43885205 \quad 0.01057448$ mitochondrial carrier, thiamine pyrophosphate (predicted) $0.46321676 \quad 5.55291243 \quad 0.01310823$ two-component GAP Cdc16 $0.4631517 \quad 5.80733951 \quad 0.00427791$

plasma membrane malate/succinate:proton symporter Ma! $0.46307412 \quad 8.44033535 \quad 5.95 \mathrm{E}-06$ ER stress response thioredoxin family membrane disulfide , $0.46303004 \quad 6.85267605 \quad 0.00020235$ ubiquitin conjugating enzyme E2 for HECT-type and RBR far $0.46223226 \quad 6.196685920 .00134065$ glyceraldehyde-3-phosphate dehydrogenase Tdh1 $\quad 0.46215467 \quad 10.064004 \quad 6.30 \mathrm{E}-10$ bifunctional pseudouridylate synthase/pseudouridine kinas $0.46212444 \quad 7.72376307 \quad 7.53 \mathrm{E}-07$ 20S proteasome complex subunit beta 1 Pre3 $\quad 0.4618228 \quad 7.17809982 \quad 4.10 \mathrm{E}-05$ \#N/A $\quad 0.461764124 .41909192 \quad 0.06571343$

NMS complex subunit Spci

$0.4614616 \quad 8.59954782 \quad 1.32 \mathrm{E}-08$ $\begin{array}{lll}0.46140371 & 4.89574335 & 0.10122071\end{array}$ $\begin{array}{llll}0.46103716 & 3.76422904 & 0.2031872\end{array}$ $0.46099257 \quad 5.59286385 \quad 0.02110628$ $\begin{array}{lll}0.46066405 & 5.57224051 & 0.01447687\end{array}$ $\begin{array}{llll}0.46066405 & 5.57224051 & 0.01447687\end{array}$ $\begin{array}{llll}0.45948046 & 6.54368698 & 0.00087107\end{array}$ $\begin{array}{lll}0.45933901 & 7.90848822 & 5.95 \mathrm{E}-07\end{array}$ $0.45908241 \quad 5.4350098 \quad 0.01554048$ $\begin{array}{llll}0.45860429 & 6.11453889 & 0.00174169\end{array}$ $\begin{array}{llll}0.45859882 & 5.83615888 & 0.00474736\end{array}$ $0.45858918 .97003886 \quad 1.05 \mathrm{E}-07$ $\begin{array}{lll}0.45814248 & 6.96028727 & 4.15 \mathrm{E}-05\end{array}$ $\begin{array}{llll}0.45791645 & 7.35665085 & 0.00011529\end{array}$ $\begin{array}{llll}0.45729569 & 5.91983502 & 0.00330644\end{array}$ $0.456843527 .46958329 \quad 4.69 \mathrm{E}-05$ $\begin{array}{llll}0.45544822 & 6.36975791 & 0.00054549\end{array}$ $\begin{array}{llll}0.45532541 & 5.79365478 & 0.03178856\end{array}$ $0.45510941 \quad 7.11744983 \quad 4.14 \mathrm{E}-05$ $0.45444529 \quad 6.52087113 \quad 0.00046714$ $\begin{array}{llll}0.45429278 & 6.50838072 & 0.00164448\end{array}$ $\begin{array}{llll}0.45426913 & 5.18746289 & 0.02639872\end{array}$ $\begin{array}{llll}0.4537507 & 6.66864368 & 0.00310733\end{array}$ $0.45244357 \quad 6.9413891 \quad 5.30 \mathrm{E}-05$ $\begin{array}{llll}0.45206021 & 6.27147012 & 0.00109888\end{array}$ $\begin{array}{lll}0.451252 & 6.30780037 & 0.00708796\end{array}$ $\begin{array}{llll}0.45027071 & 3.6333526 & 0.25000166\end{array}$ $\begin{array}{llll}0.45016044 & 6.28303325 & 0.00242864\end{array}$ $\begin{array}{lrrr}\text { coincidence detector Sec24/Sfb2 subunit } & 0.44864907 & 8.76725006 & 6.05 \mathrm{E}-08 \\ \text { peptidase family M20 protein involved in glutathione catabı } 0.44863264 & 6.9126059 & 0.00049091\end{array}$ $\begin{array}{lrrrr}\text { peptidase family M20 protein involved in glutathione catab } & 0.48863264 & 6.9126059 & 0.00049091\end{array}$ UCS-domain protein Rng

trehalose-6-phosphate phosphatase Tpp1 $\# N / A$ $\begin{array}{rrr}0.44850892 & 6.24084009 & 0.0010594 \\ 0.44829226 & 9.01990296 & 9.06 \mathrm{E}-08\end{array}$ $0.448073596 .70559913 \quad 0.00238405$ $\begin{array}{llll}0.44794054 & 4.53561248 & 0.09371566\end{array}$

$\begin{array}{rrr}0.4476145 & 5.87562156 & 0.01956983 \\ 0.44665314 & 8.14258788 & 3.80 \mathrm{E}-08\end{array}$ PHD finger protein Phf1
1.07E-05

0.022161782 0.026876013 0.009874879

2.46E-05 0.000633964 0.003490093

$4.50 \mathrm{E}-09$

3.53E-06

0.00014476

0.109385979

7.86E-08

0.15860164

0.284153082

0.041037986

0.029381179

0.029381179

0.002372002

2.84E-06

0.031297119

0.004429074

0.01086273

5.56E-07

0.000146413

0.000376459

0.007861754

0.000164082

0.001562309

0.058576002

0.000145971

0.001361414

0.004195971

0.049910719

0.007440316

0.00018345

0.002923323

0.015474346

0.339912353

0.005977175

3.32E-07

0.001425798

0.002824933

4.83E-07

0.005882339

0.148621546

0.038466974

2.16E-07 
SPBC15C4.05

SPBC18H10.20c

SPAC27F1.02C

SPBC16G5.08

SPCC663.09c

SPAC1B1.03C

SPBC16A3.05C

SPCC613.10

SPBC646.09c

SPNCRNA.1527

SPAC1786.03

SPAC21E11.05C

SPBC3B9.13C

SPAC10F6.16

SPBC1709.03

SPAC105.02C

SPAC7D4.07C

ScpofMt20

SPCC965.07c

SPCC1753.01C

SPAC29A4.20

SPBC354.12

SPAC4G8.03C

SPCC550.14

SPAPB24D3.08

SPCC1322.03

SPNCRNA.623

SPBC19G7.15

SPCC18.07

SPBC1921.05

SPAC959.08

SPNCRNA.142

SPBC428.15

SPBC3H7.11

SPAC29A4.18

SPBC16C6.07c

SPNCRNA.1380

SPNCRNA.322

SPAC630.03

SPNCRNA. 827

SPNCRNA.562

SPCC1223.15c

SPNCRNA.679

SPBP8B7.21

SPAC26A3.15C

SPAC6G 10.07

SPBC4B4.10c trp4

PCC663.09c

kap95

rae1

qcr2

int6

ut11

rpp102

igo1

PBC1709.03

SPAC105.02C

trx1

\#N/A

st2

ssb2

elp3

gpd3

puf5

vgl1

SPAPB24D3.08C

trp1322

\#N/A

nup44

rpc53

ape2

rpl2102

\#N/A

SPBC428.15

trm141

prw1

$\# N / A$

$\# \mathrm{~N} / \mathrm{A}$

arp3

$\# \mathrm{~N} / \mathrm{A}$

$\# N / A$

spc19

$\#$ N/A

ubp3

nsp1

cbc1

$\operatorname{atg} 5$
ATP-dependent RNA helicase, human DHX29 ortholog (pred $0.44646427 \quad 7.1960867 \quad 0.00021456$ arrestin-related endocytic adaptor Any1

tropomyosin phosphoribosylanthranilate transferase Trp4 short chain dehydrogenase, unknown specificity (predicted) 0.445486546 .879778610 .00017042 karyopherin/importin beta family nuclear import signal rect $0.44453629 \quad 8.61322668 \quad 7.87 \mathrm{E}-09$ RNA export factor, nucleoporin Rae1 ubiquinol-cytochrome-c reductase complex core protein OcI 0.444482217 .80514041 elF3e subunit Int6

\section{$\# \mathrm{~N} / \mathrm{A}$}

spindle pole body docking protein Cut11

cyclophilin family peptidyl-prolyl cis-trans isomerase Cyp8 $0.44176122 \quad 7.37309873 \quad 3.58 \mathrm{E}-05$ $\begin{array}{lllll}60 S \text { acidic ribosomal protein A3 } & 0.44169177 & 7.21258512 & 4.20 \mathrm{E}-05\end{array}$ endosulfine (ENSA) serine/threonine protein kinase Igo1 $0.441676357 .56946181 \quad 4.87 \mathrm{E}-06$ DUF3844 family transmembrane protein, conserved in fung $0.441067297 .38969885 \quad 6.57 \mathrm{E}-05$ ankyrin repeat protein, protein phosphatase regulatory subı $0.44033015 \quad 7.48007554 \quad 1.78 \mathrm{E}-05$ cytosolic thioredoxin Trx1

\section{$\# N / A$}

glutathione S-transferase Gst2

single-stranded DNA binding protein Ssb2

elongator complex subunit Elp3

glyceraldehyde 3-phosphate dehydrogenase Gpd3

pumilio family RNA-binding protein Puf5 (predicted)

$\mathrm{KH}$ domain RNA binding protein, vigilin (predicted)

$0.439621417 .11795518 \quad 1.47 \mathrm{E}-05$

NADP-dependent oxidoreductase, implicated in cellular det $0.43653276 \quad 7.81873846 \quad 3.25 \mathrm{E}-06$

plasma membrane TRP-like calcium ion channel Trp1322 $\begin{array}{lllll}0.43637677 & 6.07099769 & 0.01948253\end{array}$

\#N/A

DNA-directed RNA polymerase III complex subunit Rpc53 (f $0.43604512 \quad 5.922978 \quad 0.01168335$

M1 aminopeptidase Ape2

$60 S$ ribosomal protein L21 (predicted)

$$
\text { \#N/A }
$$

Obg-like ATPase, human OLA1 ortholog (predicted) serine tRNA C32 methyl transferase Trm141

Ir6 histone deacetylase complex subunit Prw1

$19 S$ proteasome base subcomplex ATPase subunit Rpt1

$$
\# N / A
$$

Arp2/3 protein complex, actin-like protein subunit Arp3

$$
\# N / A
$$

DASH complex subunit Spc1

$$
\# N / A
$$$$
\# \mathrm{~N} / \mathrm{A}
$$

ubiquitin C-terminal hydrolase Ubp3

nucleoporin Nsp1

nuclear cap-binding complex large subunit (predicted)

autophagy associated protein Atg5 $0.44619682 \quad 6.86086726 \quad 0.00036006$ $\begin{array}{llll}0.44452218 & 7.18658707 & 0.00011644\end{array}$

$0.44336615 \quad 8.6269206 \quad 131 \mathrm{E}-08$ $\begin{array}{llll}0.44316234 & 4.03352564 & 0.15905877\end{array}$ $\begin{array}{llll}0.44305473 & 6.24737337 & 0.00493594\end{array}$

$\begin{array}{lll}0.43909567 & 5.1355923 & 0.02597359\end{array}$

$0.43909486 \quad 7.84337438 \quad 1.88 \mathrm{E}-06$

$\begin{array}{lll}0.43898945 & 8.06709166 \quad 1.95 \mathrm{E}-07\end{array}$

$0.43809107 \quad 6.85483259 \quad 0.00129705$

$0.43802385 \quad 9.68910811 \quad 8.71 \mathrm{E}-08$

$\begin{array}{lll}0.43711926 & 6.31527303 & 0.01140766\end{array}$

$0.43676028 \quad 9.54365362 \quad 1.61 \mathrm{E}-08$

$\begin{array}{llll}0.43632563 & 6.0335474 & 0.0384394\end{array}$

$\begin{array}{llll}0.43623061 & 6.42803192 & 0.00111812\end{array}$

0.000669438

0.001074192

2.45E-06

0.00337912

0.000539401

$4.88 \mathrm{E}-08$

0.000379298

$8.04 \mathrm{E}-06$

$7.81 \mathrm{E}-08$

0.232124149

0.011230051

0.000127827

0.000147555

$2.04 \mathrm{E}-05$

0.000223714

$6.74 \mathrm{E}-05$

5.66E-05

0.049165576

8.37E-06

9.89E-07

0.003392171

4.65E-07

0.023739791

9.53E-08

$1.40 \mathrm{E}-05$

0.038328606

0.069109676

0.002966385

0.024239394

$3.11 \mathrm{E}-10$

2.83E-06

$0.43526817 \quad 7.9602355 \quad 5.92 \mathrm{E}-07$

$\begin{array}{llll}0.4348544 & 4.96484429 & 0.07454153\end{array}$

$\begin{array}{lll}0.43472243 & 6.88813706 & 0.0001493\end{array}$

$\begin{array}{lll}0.43466144 & 5.889259 & 0.00818551\end{array}$

$\begin{array}{lll}0.43438673 & 7.06757131 & 4.72 \mathrm{E}-05\end{array}$

$0.43335405 \quad 8.08251674 \quad 3.71 \mathrm{E}-06$

$\begin{array}{llll}0.43334881 & 4.96412256 & 0.06043264\end{array}$

$\begin{array}{llll}0.43334881 & 4.96412256 & 0.06043264\end{array}$

$\begin{array}{llll}0.43315448 & 8.43167345 \quad 5.67 \mathrm{E}-06\end{array}$

$\begin{array}{llll}0.43295682 & 3.95463731 & 0.18078789\end{array}$

$\begin{array}{llll}0.432744 & 5.66779184 & 0.0078611\end{array}$

$\begin{array}{llll}0.4325323 & 4.28166998 & 0.24787052\end{array}$

$\begin{array}{llll}0.43225796 & 4.30755441 & 0.12596434\end{array}$

$\begin{array}{llll}0.43222223 & 6.65194029 & 0.00602904\end{array}$

$0.43136314 \quad 8.39894624 \quad 1.27 \mathrm{E}-07$

$\begin{array}{lll}0.43073211 & 7.62977452 & 1.75 \mathrm{E}-05\end{array}$

$\begin{array}{llll}0.43055465 & 5.20981731 & 0.03004671\end{array}$
0.121731485

0.000478545

0.017571733

0.000165135

$1.58 \mathrm{E}-05$

0.101786933

0.101786933

2.36E-05

0.258349761

0.016960935

0.337351776

0.191037967

0.013451683

$6.62 \mathrm{E}-07$

6.63E-05

0.055804549 
SPCC1235.06

SPCC1682.13

SPAC29B12.05C

SPCC14G10.01

SPBC106.01

SPNCRNA.1399

SPNCRNA.334

SPCC757.09C

SPAC869.03C

SPNCRNA.1430

SPAC4G9.09C

SPAC15F9.02

SPAC18B11.10

SPBC651.06

SPBC1703.13C

SPNCRNA.1231

SPAC31G5.07

SPBC18E5.01

SPCP1E11.02

SPCPJ732.01

SPBC4B4.09

SPCC338.05C

SPRRNA.37

SPBC530.03C

SPBC17A3.07

SPBC17D11.08

SPAC1705.03C

SPAC14C4.10C

SPBC4B4.03 gta3

cpd1

hor1

psi1

slt1

rrp42

rnc1

SPAC869.03C

$\# \mathrm{~N} / \mathrm{A}$

$\arg 11$

seh1

tup11

mug166

SPBC1703.13C

\#N/A

dni1

SPBC18E5.01

ppk38

vps5

usp105

mms2

SPRRNA.37

bag102

pgr1

dca7

ecm33

SPAC14C4.10c rsc1
Ino80 complex subunit lec 3

Schizosaccharomyces pombe specific protein

nicotinamide-nucleotide adenylyltransferase (predicted) mitochondrial metalloendopeptidase (predicted)

Clr6 histone deacetylase complex subunit Mug165

alanine racemase Alr2 (predicted)

ADP-ribose diphosphatase, NudF subfamily Ysa1 (predicted $0.42939858 \quad 6.807308610 .00044782$ COPII-coated vesicle component Erv46 (predicted) $\quad \begin{array}{lllll}0.42911727 & 7.04044112 & 0.00031413\end{array}$

$0.43043919 \quad 4.69954606 \quad 0.12188699$ $0.43034608 \quad 6.54067091 \quad 0.0019899$ $\begin{array}{lll}0.42998616 & 6.9893352 & 0.00257166\end{array}$ $\begin{array}{lll}0.42987954 & 8.46196444 & 0.0009097\end{array}$ $0.42951722 \quad 4.52479508 \quad 0.18753536$ $\begin{array}{lll}0.42945285 & 5.95727219 & 0.01031506\end{array}$ $\begin{array}{lllllll}0.42911727 & 7.04044112 & 0.00031413\end{array}$ mitochondrial ribosomal protein subunit Mrp10 (predicted) $\begin{array}{lllll}0.4283861 & 5.11717185 & 0.03668134\end{array}$ 6-phosphofructo-2-kinase/fructose-2,6-bisphosphate 2-pho: $0.42735534 \quad 8.9589864 \quad 5.68 \mathrm{E}-08$ mitochondrial glutamyl-tRNA amidotransferase complex st $0.42674203 \quad 6.878078590 .00051882$ tRNA (m1A) methyltransferase complex catalytic subunit C $0.42631993 \quad 7.11749521 \quad 0.00091798$ translation initiation factor elF3j (p35) $\quad 0.42605715 \quad 7.13230872 \quad 8.57 \mathrm{E}-05$ $\begin{array}{lllll}\text { DNAJ domain protein Psi1 } & 0.42558817 & 8.06072431 & 0.0012152\end{array}$ $\begin{array}{lllll}\text { Schizosaccharomyces specific protein Slt1 } & 0.42547053 & 7.54330527 & 0.00180567\end{array}$ exosome subunit Rrp42

$$
\text { \#N/A }
$$

$\begin{array}{lll}0.42547053 & 7.54330527 & 0.00180567 \\ 0.42494069 & 6.95763323 & 0.00309903\end{array}$ $0.42480525 \quad 8.34910896 \quad 9.09 \mathrm{E}-08$ $\begin{array}{llrrr}\text { GET complex (ER membrane insertion) subunit Get2 } & 0.42477502 & 5.60880429 & 0.009956 \\ \text { Clr6 associated factor 2, Laf2 } & 0.42474077 & 5.51966919 & 0.04252677\end{array}$ mitochondrial N(5)-glutamine methyltransferase (predictec $0.42461975 \quad 5.233324320 .03700331$ dephospho-CoA kinase (predicted) $\quad 0.42433827 \quad 5.27383494 \quad 0.02038778$ dual specificity protein kinase Mph1

$$
\# N / A
$$

$$
\# N / A
$$

$\begin{array}{lll}0.42431378 & 6.82665293 & 0.00431924\end{array}$ $\begin{array}{llll}0.42402659 & 3.29826748 & 0.30025666\end{array}$ $\begin{array}{llll}0.42402659 & 3.29826748 & 0.30025666\end{array}$ $\begin{array}{lll}0.42400162 & 7.27004959 & 0.00191361 \\ 0.42376261 & 4.06941561 & 0.15691438\end{array}$

KH domain RNA-binding protein Rnc1 $\begin{array}{lrrrr}\text { plasma membrane urea transmembrane transporter (predi } & 0.42376261 & 4.06941561 & 0.15691438 \\ \text { \#N/A } & 0.42351148 & 3.2984677 & 0.27645161\end{array}$ $\mathrm{N}$-acetyl-gamma-glutamyl-phosphate reductase/acetylglut: $0.42303818 \quad 8.81771513 \quad 5.20 \mathrm{E}-09$ $\begin{array}{lllll}\text { SEA complex subunit Seh1 } & 0.42299921 & 6.69348852 & 0.00380241\end{array}$ transcriptional corepressor Tup11

Schizosaccharomyces specific protein Mug166

mitochondrial carrier, inorganic phosphate (predicted) $\# \mathrm{~N} / \mathrm{A}$

tetraspan protein, claudin Dni1

$0.42287497 \quad 7.13489146 \quad 4.35 \mathrm{E}-05$ $0.42266503 \quad 4.68914058 \quad 0.18424813$ $\begin{array}{llr}0.42218273 & 8.26224833 \quad 9.82 \mathrm{E}-08\end{array}$ $\begin{array}{llll}0.4213347 & 5.67780501 & 0.01040305\end{array}$ $\begin{array}{lll}0.42132608 & 4.3082617 & 0.25101819\end{array}$ unknown with similarity to bacterial carboxy-cis,cis-muconz $\begin{array}{lllll}0.42118788 & 7.49712181 & 0.00018485\end{array}$ $\begin{array}{lllll}\text { Ark1/Prk1 family protein kinase Ppk38 } & 0.4211129 & 6.83418453 & 0.0022397\end{array}$ retromer complex subunit Vps5 $\quad 0.42068064 \quad 6.679729780 .00197086$ U1 snRNP-associated protein Usp105 $\quad 0.42028937 \quad 6.84197154 \quad 0.00131246$ $\begin{array}{lllll}\text { ubiquitin conjugating enzyme E2 Mms2 } & 0.41956075 & 6.52396548 & 0.00134646\end{array}$ 5S rRNA

molecular chaperone regulator Bag102 WD repeat protein, DDB1 and CUL4-associated factor Dca7 $0.41702954 \quad 8.09676279 \quad 1.34 \mathrm{E}-05$ extracellular leucine-rich repeat domain, receptor $L$ domain $0.41685741 \quad 9.20207937 \quad 3.72 \mathrm{E}-08$ Nudix family hydrolase $0.41683121 \quad 3.6319554 \quad 0.25182587$ RSC complex subunit Rsc1
0.185806672

0.004998611 0.006270204 0.002469286

0.266036715 0.021659921 0.001310728 0.000948808 0.003473677 0.066334443

3.12E-07

0.001495995 0.002487786 0.000285317

0.0031953

0.00456788 0.007425661

4.83E-07

0.021014439 0.075481127 0.066792443 0.039798867 0.009963527

0.39322179

0.39322179

0.004821227

0.22963492

0.368584093

3.30E-08 0.008885786 0.000152624 0.262357918 5.20E-07 0.021829412

0.340749801 0.000582364 0.005564589 0.004959954 0.003431162 0.003499876 0.047287741 0.020518098 6.45E-06 5.18E-05

2.11E-07 0.341778032 0.000136227 
SPBC428.18

SPNCRNA.799

SPAC3G9.14

SPBC19F5.04

SPAC23A1.14C

SPCC162.09c

SPBC3B9.18C

SPAC664.11

SPBC13G1.07

SPBC23G7.08C

SPAC22A12.14C

SPBC16C6.04

SPBC1718.03

SPBC557.04

SPBC36.11

SPACUNK4.16c

SPBC428.02C

SPAC3A11.07

SPBC29A10.16C

SPBC27B12.06

SPBC3B8.06

SPAC323.02C

SPBC3B9.12

SPAC17C9.03

SPAC4F8.06

SPAC14C4.12C

SPBC3H7.14

SPBC947.01

SPAC6B12.16 hm1 1

mep33

pma1

SPAP14E8.05C

SPAC9E9.06C

\#N/A

SPBC30D10.05C

$\mathrm{cdb} 4$

SPBC26H8.11C

SPAC2F3.05c

tif35

hrk1

ent1

$$
\# N / A
$$

sak1

SPBC19F5.04

SPAC23A1.14C

hmg1

vma7

ssc1

swf1

rga7

SPAC22A12.14c

dbl6

ker1

ppk29

SPBC36.11

tps3

eca39

nde2

SPBC29A10.16C

gpi13

SPBC3B8.06

pup2

trs23

tif471

mrps12

laf1

mug176

knk1

meu26
CBF/Mak21 family Noc4 (predicted)

translation initiation factor elF3b (p84)

trans-aconitate 3-methyltransferase (predicted) pyridoxamine 5'-phosphate oxidase Pdx3 (predicted)

serine hydroxymethyltransferase Shm1 (predicted)

translation machinery associated protein Mep33

$0.4167248 \quad 6.56994857 \quad 0.00278545$ $0.41648345 \quad 9.25448963 \quad 5.02 \mathrm{E}-08$ $\begin{array}{lll}0.41624049 & 7.25936901 & 1.75 \mathrm{E}-05\end{array}$ $0.41590726 \quad 6.8950402 \quad 0.00051971$ $0.41575722 \quad 8.98313473 \quad 1.66 \mathrm{E}-07$ $\begin{array}{lll}0.41574059 & 4.61506517 & 0.0907409\end{array}$ plasma membrane P-type proton exporting ATPase, P3-typє $0.41520688 \quad 10.6646606 \quad 9.85 \mathrm{E}-07$ mitochondrial TMEM14 family protein, implicated in heme $0.41508968 \quad 5.08016816 \quad 0.0619502$ threonine synthase (predicted)

$\# \mathrm{~N} / \mathrm{A}$

sepiapterin reductase (predicted)

endonuclease $\mathrm{Cdb} 4$ (predicted)

2-isopropylmalate synthase Leu3

acyl-coenzyme A thioesterase

xylose and arabinose reductase (predicted)

translation initiation factor elF3g

haspin related kinase Hrk1

replication licensing factor $\mathrm{Cdt} 1$

$$
\# \mathrm{~N} / \mathrm{A}
$$

transcriptional activator Sak

aspartate kinase (predicted)

$0.41485481 \quad 8.38443334 \quad 2.87 \mathrm{E}-07$

$\begin{array}{lll}0.41475559 & 8.3729147 & 7.43 \mathrm{E}-07\end{array}$

$0.41453725 \quad 7.43693436 \quad 6.28 \mathrm{E}-05$

$0.41442618 \quad 8.01527448 \quad 1.63 \mathrm{E}-06$

$0.41442047 \quad 9.31383371 \quad 5.09 \mathrm{E}-09$

$0.41355051 \quad 7.69589185 \quad 1.96 \mathrm{E}-06$

$\begin{array}{llll}0.41325749 & 6.2078768 & 0.07230635\end{array}$

$\begin{array}{lll}0.41321724 & 7.86460349 & 5.40 \mathrm{E}-06\end{array}$

$\begin{array}{lll}0.41319251 & 7.75493622 & 2.64 \mathrm{E}-05\end{array}$

$0.41316635 \quad 8.74523357 \quad 2.02 E-07$

$\begin{array}{llll}0.41260712 & 5.29378524 & 0.04264106\end{array}$

$\begin{array}{lll}0.41240098 & 8.01714568 & 1.52 \mathrm{E}-05\end{array}$

$0.41239498 .14736274 \quad 7.23 \mathrm{E}-07$

$0.41213823 \quad 8.23832496 \quad 3.33 \mathrm{E}-06$

pyridoxal phosphate-dependent transferase, unknown speci $0.412094716 .85968853 \quad 0.00049593$

3-hydroxy-3-methylglutaryl-CoA reductase Hmg1 $\quad 0.41194898 \quad 8.55430665 \quad 1.85 \mathrm{E}-05$

V-type ATPase V1 subunit F (predicted) $\quad \begin{array}{lllll}0.41175472 & 6.25073511 & 0.00366142\end{array}$

mitochondrial (2Fe-2S) cluster assembly chaperone Ssc1 $\begin{array}{llll}0.41136054 & 9.97979229 & 8.32 \mathrm{E}-10\end{array}$

palmitoyltransferase Swf1 (predicted)

RhoGAP, GTPase activating protein Rga7

$\begin{array}{rrr}0.41136054 & 9.97979229 & 8.32 \mathrm{E}-10 \\ 0.41121306 & 5.38727528 & 0.0229567\end{array}$

$0.410893696 .34743551 \quad 0.02525975$

$0.41071934 \quad 6.74949981 \quad 0.00509596$

$\begin{array}{lll}0.4104853 & 5.03952537 & 0.06161131\end{array}$

$\begin{array}{lll}0.41035271 & 5.7599431 & 0.01301655\end{array}$

$\begin{array}{lrrr}\text { DNA-directed RNA polymerase I complex subunit Ker1 } & 0.41035271 & 5.7599431 & 0.01301655 \\ \text { Ark1/Prk1 family protein kinase Ppk29 } & 0.41024895 & 8.69316475 & 5.02 \mathrm{E}-08 \\ \text { Schizosaccharomyces specific protein } & 0.41007841 & 6.43009248 & 0.00290356 \\ \text { alpha,alpha-trehalose-phosphate synthase (predicted) } & 0.40927843 & 8.91794106 & 0.00151226 \\ \text { branched chain amino acid aminotransferase Eca39 } & 0.4092757 & 9.06672987 & 5.13 \mathrm{E}-08 \\ \text { mitochondrial NADH dehydrogenase (ubiquinone) Nde2 (pri } 0.40925606 & 9.06343387 & 6.49 \mathrm{E}-06\end{array}$

$\begin{array}{lrrr}\text { DNA-directed RNA polymerase I complex subunit Ker1 } & 0.41035271 & 5.7599431 & 0.01301655 \\ \text { Ark1/Prk1 family protein kinase Ppk29 } & 0.41024895 & 8.69316475 & 5.02 \mathrm{E}-08 \\ \text { Schizosaccharomyces specific protein } & 0.41007841 & 6.43009248 & 0.00290356 \\ \text { alpha,alpha-trehalose-phosphate synthase (predicted) } & 0.40927843 & 8.91794106 & 0.00151226 \\ \text { branched chain amino acid aminotransferase Eca39 } & 0.4092757 & 9.06672987 & 5.13 \mathrm{E}-08 \\ \text { mitochondrial NADH dehydrogenase (ubiquinone) Nde2 (pri } 0.40925606 & 9.06343387 & 6.49 \mathrm{E}-06\end{array}$

$\begin{array}{lrrr}\text { DNA-directed RNA polymerase I complex subunit Ker1 } & 0.41035271 & 5.7599431 & 0.01301655 \\ \text { Ark1/Prk1 family protein kinase Ppk29 } & 0.41024895 & 8.69316475 & 5.02 \mathrm{E}-08 \\ \text { Schizosaccharomyces specific protein } & 0.41007841 & 6.43009248 & 0.00290356 \\ \text { alpha,alpha-trehalose-phosphate synthase (predicted) } & 0.40927843 & 8.91794106 & 0.00151226 \\ \text { branched chain amino acid aminotransferase Eca39 } & 0.4092757 & 9.06672987 & 5.13 \mathrm{E}-08 \\ \text { mitochondrial NADH dehydrogenase (ubiquinone) Nde2 (pri } 0.40925606 & 9.06343387 & 6.49 \mathrm{E}-06\end{array}$

$\begin{array}{lrrr}\text { DNA-directed RNA polymerase I complex subunit Ker1 } & 0.41035271 & 5.7599431 & 0.01301655 \\ \text { Ark1/Prk1 family protein kinase Ppk29 } & 0.41024895 & 8.69316475 & 5.02 \mathrm{E}-08 \\ \text { Schizosaccharomyces specific protein } & 0.41007841 & 6.43009248 & 0.00290356 \\ \text { alpha,alpha-trehalose-phosphate synthase (predicted) } & 0.40927843 & 8.91794106 & 0.00151226 \\ \text { branched chain amino acid aminotransferase Eca39 } & 0.4092757 & 9.06672987 & 5.13 \mathrm{E}-08 \\ \text { mitochondrial NADH dehydrogenase (ubiquinone) Nde2 (pri } 0.40925606 & 9.06343387 & 6.49 \mathrm{E}-06\end{array}$

cytochrome b5 (predicted)

$\begin{array}{lll}0.4091582 & 6.95058598 & 0.00141027\end{array}$

pig-O (predicted)

DUF2427 family conserved fungal protein

$20 \mathrm{~S}$ proteasome complex subunit alpha 5, Pup2

TRAPP complex subunit Trs23 (predicted)

translation initiation factor eIF4G

$\begin{array}{llll}0.40899545 & 6.7394286 & 0.00082436\end{array}$

$0.408857627 .21630314 \quad 0.00105707$

$0.408727737 .65710901 \quad 1.69 \mathrm{E}-05$

0.408408715 .050013990 .05902596

$0.40789049 \quad 9.8549761 \quad 3.58 \mathrm{E}-08$

mitochondrial ribosomal protein subunit MrpS12 (predicted $0.40780906 \quad 4.38981403 \quad 0.12550133$ Clr6 L associated factor 1 Laf1 $\quad 0.407799315 .64837057 \quad 0.04282741$

BRCT domain protein

$\begin{array}{lll}0.40769406 & 5.74680279 & 0.02779817\end{array}$

$0.407102837 .58641107 \quad 2.40 \mathrm{E}-05$

$\begin{array}{lll}0.40698737 & 3.86206189 & 0.22068211\end{array}$
0.006743158

$2.79 \mathrm{E}-07$

$6.64 \mathrm{E}-05$

0.001496653

$8.46 \mathrm{E}-07$

0.144645536

4.55E-06

0.103988919

$1.42 \mathrm{E}-06$

$3.49 \mathrm{E}-06$

0.000214202

7.34E-06

$3.24 \mathrm{E}-08$

8.67E-06

0.118679382

2.25E-05

9.72E-05

$1.02 \mathrm{E}-06$

0.075664249

5.83E-05

3.40E-06

1.43E-05

0.001439158

$6.98 \mathrm{E}-05$

0.008579868

5.86E-09

0.044192459

0.048028106

0.0115401

0.103496654

0.026704146

2.79E-07

0.006994232

0.003892174

2.84E-07

2.66E-05

0.003650405

0.002259261

0.002819822

$6.44 \mathrm{E}-05$

0.099744645

2.04E-07

0.190378181

0.075935521

0.052111993

8.90E-05

0.305461923 
SPBC418.01C

SPAC3A12.14

SPBC16G5.11C

SPAC24H6.04

SPAC19B12.04

SPCC16A11.06C

SPAC1071.11

SPCC965.12

SPNCRNA.1514

SPCC24B10.13

SPNCRNA.1173

SPNCRNA.1309

SPBC11G11.04

SPAC4G8.07C

SPNCRNA.252

SPBC651.01C

SPAC1420.02C

SPAC3C7.08c

SPCC7777.13

SPBC16D10.09

SPBC1289.10C

SPAC13D6.03C

SPNCRNA.805

SPAC926.03

SPAC1805.17

SPBC31F10.07

SPBC30D10.13C

SPAC4G9.08C

SPAC3A12.07

SPNCRNA.318

SPAC15A10.04C

SPNCRNA.782

SPNCRNA.442

SPBC211.02C

SPBC354.14c

SPAC821.11

SPCC285.17

SPAC1687.13C

SPBC30D10.06

SPCC74.02C

SPCC1795.11

SPCPB1C11.01

SPBC32H8.05

SPAC26A3.02

SPBC30D10.07C

SPAPB1A10.03

SPCC645.09 imidazoleglycerol-phosphate synthase His4

calmodulin Cam1

BAG family molecular chaperone regulator Bag101 (predict 0.406560816 .597430240 .00325828 hexokinase 1

$40 \mathrm{~S}$ ribosomal protein S30 (predicted)

$0.40641309 \quad 9.39311384 \quad 6.52 \mathrm{E}-09$

$0.40593326 \quad 6.17728256 \quad 0.00641409$ $\begin{array}{lrrr}\text { pig-B (predicted) } & 0.40589886 & 6.65890704 & 0.0028995 \\ \text { NADH-dependent flavin oxidoreductase (predicted), possibl. } & 0.40513563 & 6.10108148 & 0.01291142\end{array}$ NADH-dependent flavin oxidoreductase (predicted), possibl $0.40513563 \quad 6.101081480 .01291142$ $\begin{array}{lllllll}\text { dipeptidyl peptidase, unknown specificity, implicated inglu } & 0.40457648 & 6.35205216 & 0.00328989 \\ \text { \#N/A } & 0.40447247 & 4.95386223 & 0.06685149\end{array}$ protein kinase activator $\mathrm{Skb5}$

\#N/A

$\begin{array}{lll}0.40447247 & 4.95386223 & 0.06685149 \\ 0.40430751 & 6.00900139 & 0.01375434\end{array}$ $\begin{array}{llll}0.40385053 & 6.26904305 & 0.01652376\end{array}$ $\begin{array}{llll}0.40371326 & 5.96763507 & 0.01501196\end{array}$ $\begin{array}{lll}0.40282568 & 5.05983557 & 0.05335077\end{array}$ $\begin{array}{llll}0.402804 & 6.30942203 & 0.01003093\end{array}$ TRAPP complex subunit Trs20 (predicted) tRNA (m5U54) methyltransferase Trm2 (predicted)

$$
\text { \#N/A }
$$

ribosome export GTP binding protein Nog1 (predicted) chaperonin-containing T-complex epsilon subunit Cct5 AAA family ATPase Elf1

retromer complex subunit Vps35

PCNA

transcription factor Adn2 (predicted)

tRNA (uridine) methyltransferase Trm9 (predicted)

$$
\# N / A
$$

myosin II regulatory light chain RIc1

$\begin{array}{llll}0.40217868 & 1.23618088 & 0.76995387\end{array}$

$0.40201205 \quad 7.9787779 \quad 3.79 \mathrm{E}-05$

$\begin{array}{lll}0.40196817 & 8.57662831 \quad 2.47 \mathrm{E}-07\end{array}$

$\begin{array}{lll}0.40156868 & 8.70742004 & 3.04 \mathrm{E}-06\end{array}$

$0.40118604 \quad 7.80651393 \quad 3.76 \mathrm{E}-05$

$\begin{array}{lll}0.39965811 & 7.72394616 & 3.25 \mathrm{E}-05\end{array}$

$\begin{array}{lll}0.39946614 & 6.76813821 & 0.00288573\end{array}$

$0.39921646 \quad 5.09280308 \quad 0.05436334$

$\begin{array}{llll}0.39896693 & 6.97194177 & 0.00031988\end{array}$ $0.39869235 \quad 4.98976951 \quad 0.05815953$

karyopherin/importin beta family nuclear export signal rece $\begin{array}{lll}0.39852668 & 8.59359875 \quad \text { 4.27E-06 }\end{array}$ $\begin{array}{lllll}\text { actin cortical patch component Lsb5 (predicted) } & 0.39848329 & 6.13831574 & 0.01499585\end{array}$ $\begin{array}{lllll}\text { pyruvate dehydrogenase e1 component beta subunit Pdb1 } & 0.39845915 & 7.84774054 & \text { 4.23E-06 }\end{array}$ DNA-directed RNA polymerase III complex subunit Rpc2 $\quad 0.39831905 \quad 7.85620843 \quad 6.96 \mathrm{E}-06$ RNA polymerase II complex subunit Rpb11

$$
\# \mathrm{~N} / \mathrm{A}
$$

EF-1 alpha binding zinc finger protein Zpr1 (predicted)

$\begin{array}{llll}0.39826161 & 6.08507427 & 0.01663878\end{array}$ $\begin{array}{llll}0.39825663 & 1.23573102 & 0.76272761\end{array}$ $\begin{array}{lll}0.39817852 & 6.87150273 & 0.00086438\end{array}$ $\begin{array}{llll}0.39796418 & 3.43341664 & 0.31949304\end{array}$ $\begin{array}{lll}0.39792379 & 4.29892317 & 0.27602088\end{array}$ $0.39789982 \quad 7.23529848 \quad 0.00085434$ $\begin{array}{lll}0.39765668 & 6.44705001 & 0.00311206\end{array}$

Prp19 complex subunit Cwf3 \#N/A vacuolar protein Vac8 (predicted)

gamma-glutamyl phosphate reductase Pro1 $0.397220826 .89654365 \quad 0.00503439$ RNA polymerase I upstream activation factor complex subu $0.39688945 \quad 7.81241657 \quad 4.88 \mathrm{E}-05$ COP9/signalosome complex protease subunit Csn5 $\quad \begin{array}{lllll}0.39679766 & 4.97476043 & 0.05954352\end{array}$ $\begin{array}{llll}\text { Lsm2-8 complex subunit Lsm4 } & 0.39607043 & 7.86397845 & 2.74 \mathrm{E}-05\end{array}$ mRNA cleavage and polyadenylation specificity factor comr $\begin{array}{lll}0.39592413 & 7.20479192 & 0.00032993\end{array}$ translation initiation RNA helicase Sum3 $\quad 0.395878059 .88923721 \quad 5.64 \mathrm{E}-09$ plasma membrane ammonium transmembrane transportel $0.39559054 \quad 8.1809234 \quad 0.00051204$ $\begin{array}{lllll}\text { UPF0642 conserved fungal protein } & 0.39492626 & 5.58483013 & 0.03402202\end{array}$ adenine DNA glycosylase Myh1

biotin-protein ligase Bpl1 (predicted)

mRNA export receptor Nxt1 $\begin{array}{lll}0.3947809 & 6.8591321 & 0.00114911\end{array}$ $0.39454243 \quad 7.86462516 \quad 3.12 \mathrm{E}-05$ $\begin{array}{llll}0.3943768 & 3.73187634 & 0.34079324\end{array}$ mitochondrial ribosomal protein subunit L37 (predicted) $\begin{array}{llll}0.39397255 & 4.11470618 & 0.22474618\end{array}$
0.014180635

0.006989416

0.02650444

0.007827871

0.110957167

0.028073875

0.033071828

0.030358517

0.091576586

0.021159482

0.829171714

0.000134449

$1.24 \mathrm{E}-06$

$1.32 \mathrm{E}-05$

0.000133436

0.000116752

0.006958675

0.093067307

0.000965306

0.098549697

$1.80 \mathrm{E}-05$

0.030334937

1.79E-05

$2.84 \mathrm{E}-05$

0.033253127

0.822955581

0.00235662

0.413887331

0.368081926

0.002332043

0.007449015

0.011423486

0.000170015

0.100469532

0.000100355

0.00099343

3.57E-08

0.001479591

0.0622198

0.003040312

0.000112529

0.435511362

0.310163568 
SPBC1711.05

SPAC1B1.04C

SPBC409.09C

SPBC17G9.11C

SPAC17G8.07

SPAC21E11.06

SPBC800.12C

SPNCRNA.1069

SPBC1685.03

SPBC211.05

SPBC $32 \mathrm{H} 8.01 \mathrm{C}$

SPAC23H4.10C

SPBC30D10.17C

SPBC9B6.06

SPAC1071.06

SPBC30D10.16

SPAC1142.07c

SPCC 338.10C

SPCC162.05 ost1

SPCC553.12c

SPBC16H5.12C

erv25

lys2

\#N/A

phf2

\#N/A

elp2

SPAC323.04

efm2

dph6

nuc1

fap2

pmk1

SPBC800.10c

PCC622.14

iwr1

tsr2

grn1

SPAC1B3.02C

sty1

imp1

cdc4

tif221

ifa38

srp40

pan3

mis13

pyr1

yaf9

tif224

SPBC800.12C $\# \mathrm{~N} / \mathrm{A}$

$\sec 11$

sap10

SPBC32H8.01c

thi4

smi1

mrpl10

arp9

pha2

vps32

cox5

coq3 dolichyl-diphospho-oligosaccharide-protein glycosyltransfer $0.39394766 \quad 8.23852116 \quad 4.57 \mathrm{E}-06$ transmembrane transporter (predicted) $\quad 0.39391599 \quad 7.6145565 \quad 5.55 \mathrm{E}-05$ DUF2433 metallo phosphatase superfamily conserved fung: $\begin{array}{llll}0.39341536 & 8.75720527 & 2.32 \mathrm{E}-06\end{array}$ COPII-coated vesicle component Erv25 (predicted) $\quad \begin{array}{llll}0.39300045 & 6.8243335 & 0.00552405\end{array}$

homoaconitate hydratase Lys2 \#N/A

Lsd1/2 complex PHD finger protein Phf2

$$
\# \mathrm{~N} / \mathrm{A}
$$

elongator complex WD repeat protein Elp2 (predicted)

$0.39283397 \quad 8.66598623 \quad 1.45 \mathrm{E}-07$ $\begin{array}{lll}0.39278692 & 6.93694065 & 0.00051723\end{array}$ $\begin{array}{llll}0.39268781 & 6.50407315 & 0.00355575\end{array}$ $0.39264114 .90988859 \quad 0.07024155$ $0.39222136 \quad 7.57861643 \quad 0.00016793$ $\begin{array}{lrrr}\text { mitochondrial ATPase (predicted) } & 0.39212747 & 7.73995448 & 8.25 \mathrm{E}-06 \\ \text { elongation factor EF2/EF3 methyltransferase lysine methyl } & 0.39168261 & 6.22915066 & 0.01347305\end{array}$ $\begin{array}{llllll}\text { diphthamide synthetase Dph6 (predicted) } & 0.39164506 & 6.72241416 & 0.00447411\end{array}$ DNA-directed RNA polymerase I complex large subunit Nuc $0.39163085 \quad 9.19222946 \quad 5.93 \mathrm{E}-08$ $\begin{array}{lllll}\text { L-saccharopine oxidase } & 0.39148015 & 7.08140008 & 0.0002261\end{array}$ MAP kinase Pmk1 $\quad \begin{array}{lllll}0.39141875 & 6.46200787 & 0.00416528\end{array}$ transcriptional coactivator, multiprotein bridging factor Mb $\begin{array}{lllll}0.39132644 & 7.24693115 & 0.00050147\end{array}$ EPS15 repeat family actin cortical patch component (predic $0.39130236 \quad 8.78996178 \quad 4.08 \mathrm{E}-06$ $\begin{array}{llll}\text { GTPase activating protein (predicted) } & 0.39127124 & 7.34043866 & 9.56 \mathrm{E}-05\end{array}$ RNA polymerase II nuclear import protein Iwr1 (predicted) $0.39081176 \quad 5.5503977 \quad 0.03404135$ $\begin{array}{lllll}\text { rRNA processing protein Tsr2 (predicted) } & 0.39072268 & 6.26900025 & 0.00683596\end{array}$ $\begin{array}{llll}0.3905619 & 7.2245429 & 0.00054973\end{array}$ transcription elongation factor, Elf1 family (predicted) $\quad \begin{array}{lll}0.39050022 & 6.99085851 & 0.00058042\end{array}$ MAP kinase Sty1 $\begin{array}{rrr}0.39050022 & 6.99085851 & 0.00058042 \\ 0.39033802 & 7.64024058 & 1.38 \mathrm{E}-05\end{array}$ $\begin{array}{lll}0.38925456 & 5.04713287 & 0.0993843\end{array}$

importin alpha family nuclear import signal receptor adapt، $\begin{array}{lll}0.38918184 & 7.24616285 & 0.00094344\end{array}$ $\begin{array}{lllll}\text { myosin II light chain } & 0.38892061 & 8.22849629 & 3.81 \mathrm{E}-06\end{array}$ translation initiation factor elF2B alpha subunit $\quad \begin{array}{lllll}0.38890582 & 7.28441474 & 0.00021062\end{array}$ $\begin{array}{lllll}\text { ketoreductase involved in fatty acid elongation (predicted) } & 0.38872708 & 7.48956206 & 8.89 \mathrm{E}-05\end{array}$ nucleocytoplasmic transport chaperone Srp40 (predicted) $\begin{array}{lllll}0.3886644 & 7.34365887 & 0.00014995\end{array}$ PAN complex protein phosphotransferase subunit Pan3 (pre $\begin{array}{lllll}0.38840295 & 6.90682583 & 0.00095341\end{array}$ NMS complex subunit Mis13/Dsn1

pyruvate carboxylase Pyr1 YEATS family histone acetyltransferase subunit Yaf9 $\quad \begin{array}{lllll}0.38815659 & 5.63060522 & 0.03023918\end{array}$ $\begin{array}{lllll}\text { translation initiation factor elF2B delta subunit } & 0.38784291 & 7.44220978 & 7.33 \mathrm{E}-05\end{array}$ ubiquitin family protein (predicted) \#N/A

$0.387762 \quad 4.64591489 \quad 0.13168859$ $\begin{array}{llll}0.38730947 & 4.97059784 & 0.10793482\end{array}$ $\begin{array}{lll}0.38721657 & 6.26173054 & 0.01165085\end{array}$ $\begin{array}{lll}0.38574199 & 4.1589127 & 0.19309597\end{array}$ $\begin{array}{rrr}0.38526917 & 3.6338324 & 0.34889944 \\ 0.3846079 & 8.40062656 & 1.91 \mathrm{E}-06\end{array}$ plicing factor $3 B$ subunit Sec11 (predicted) endocytic trafficking protein (predicted) \begin{tabular}{lllll} 
bifunctional thiamine-phosphate dipyrophosphorylase/hydr & 0.3846079 & 8.40062656 & $1.91 \mathrm{E}-06$ \\
\hline cell wall biosynthesis/ cell cycle regulator (predicted) & 0.38349841 & 7.49896558 & $3.00 \mathrm{E}-05$
\end{tabular} mitochondrial ribosomal protein subunit L15 (predicted) $\begin{array}{lllll}0.38339279 & 5.62987847 & 0.03674437\end{array}$ SWI/SNF and RSC complex subunit Arp9 $\quad \begin{array}{lllll}0.38325141 & 7.34996882 & 0.00036085\end{array}$ phrenate dehydratase

ESCRT III complex subunit Vps32

cytochrome c oxidase subunit $\mathrm{V}$ (predicted) $\begin{array}{llll}0.38238361 & 5.95262516 & 0.02085197\end{array}$ $\begin{array}{lll}0.38181191 & 6.45565863 & 0.0040154\end{array}$ $\begin{array}{lll}0.38175095 & 7.15088289 & 0.00016457\end{array}$ $\begin{array}{llll}0.38134896 & 6.72210747 & 0.00136312\end{array}$
1.92E-05 0.000191225 $1.02 \mathrm{E}-05$ 0.012410427

7.47E-07 0.001492048 0.00837841

0.115680962 0.000532742 3.32E-05 0.027541039 0.010289385 3.25E-07 0.000701925 0.009647638 0.001454598

1.73E-05

0.000316123

0.062238417

0.014986693

0.001572015

0.00165123

5.34E-05

0.156191719

0.002551718

1.62E-05

0.000657775

0.000295345

0.000480191

0.002572553

0.115880575

5.03E-07

0.056085483

0.000247061

0.198436851

0.167082906

0.024179339

0.272787645

0.44416153

8.47E-06

0.000108298

0.066413128

0.001075583

0.040611815

0.009327282

0.000523065

0.003537767 
SPCC794.06

SPNCRNA.730

SPCC825.03C

SPAC19B12.09

SPNCRNA.745

SPCC1322.05C

SPAC9G1.06C

SPCC1442.10c

SPBC11C11.06c

SPCC794.07

SPAC1A6.02

SPNCRNA.913

SPBC24C6.11

SPBC8D2.10c

SPNCRNA.1230

SPBC83.01

SPBC1773.12

SPBC17D1.05

SPBC1734.06

SPAC3H8.05C

SPAC18B11.07C

SPAC823.07

SPBC27.06C

SPAC25G10.01

SPAC2G11.07c

SPAC29A4.07

SPAC25B8.17

SPNCRNA.734

SPCC4B 3.07

SPAC1687.07

SPAPB1A10.12C

SPNCRNA.615

SPBC409.16c

SPAC19G12.11

SPAC1071.05

SPAC30D11.04C

SPAC821.05

SPBC543.02C

SPAC3F 10.04

\section{$\# \mathrm{~N} / \mathrm{A}$}

SPCC794.06

\#N/A

psy1

srp14

$\# N / A$

lap2

cyk3

rpb3

SPBC11C11.06C

lat1

wdr55

\#N/A

cwf14

rmt3

$$
\# N / A
$$

ucp8

SPBC1773.12

SPBC17D1.05

rhp18

ms

rhp6

pga3

mgr2

SPAC25G10.01

ptc3

srb6

ypf1

\#N/A

nro1

SPAC1687.07

alo1

$\# \mathrm{~N} / \mathrm{A}$

saw1

coq9

hpm 1

nup124

tif38

SPBC543.02C

gsa1 thioredoxin fold protein Plp1 (predicted)

anthranilate synthase component I Trp3

coatomer alpha subunit Cop1 (predicted)

2-hydroxyacyl-CoA lyase Hac1 (predicted)

$40 S$ ribosomal protein S28 (predicted)

uroporphyrinogen decarboxylase Hem12 (predicted)

Brix domain ribosome biogenesis protein $\mathrm{Rpf} 2$

$$
\text { \#N/A }
$$

transmembrane transporter (predicted)

$$
\# \text { N/A }
$$

plasma membrane SNARE Psy

signal recognition particle subunit Srp14

$$
\# \mathrm{~N} / \mathrm{A}
$$

vacuolar aminopeptidase Lap2

Nebulin-family actin filament anchoring protein Cyk3

RNA polymerase II subunit 3

Schizosaccharomyces specific protein

dihydrolipoamide S-acetyltransferase E2, Lat1 (predicted)

WD repeat protein, human WDR55 family, involved in ribos

$$
\# N / A
$$

G10 protein

type I ribosomal protein arginine $\mathrm{N}$-methyltransferase $\mathrm{Rm}$$$
\# \mathrm{~N} / \mathrm{A}
$$

$\begin{array}{lllll}\text { \#N/A } & 0.37361659 & 6.06799605 & 0.0192619\end{array}$

$\begin{array}{llll}\text { UBA/EH/EF hand domain protein Ucp8 } & 0.37355218 & 8.34945786 & 5.38 \mathrm{E}-05\end{array}$

transcription factor, zf-fungal binuclear cluster type (predic $0.37351579 \quad 4.36655307 \quad 0.18143343$

$\begin{array}{lllll}\text { Schizosaccharomyces specific protein } & 0.37349444 & 8.14680266 & 2.03 \mathrm{E}-05\end{array}$

$\begin{array}{lllll}\text { Rad18 homolog ubiquitin protein ligase E3, Rhp18 } & 0.37335125 & 6.39755855 & 0.00522647\end{array}$

Cul8-RING ubiquitin ligase complex subunit Mms1 (predicte $0.3732151 \quad 7.37508606 \quad 0.00021045$

Rad6 homolog, ubiquitin conjugating enzyme E2 Rhp6 $\quad \begin{array}{lllll}0.37318258 & 6.71462912 & 0.00491862\end{array}$

GPI-phospholipase A2 activity regulator Pga3 (predicted) $\begin{array}{lllll}0.37230129 & 6.05285454 & 0.03273165\end{array}$

TIM23 translocase complex subunit Mgr2 (predicted) $\quad \begin{array}{llll}0.37185863 & 6.20685848 & 0.00944248\end{array}$

$\begin{array}{llllll}\text { RNA-binding protein involved in histone acetylation } & 0.37181341 & 7.30880971 & 0.00011638\end{array}$

MAP kinase threonine phosphatase, protein phosphatase 2c $0.37127803 \quad 8.72709517 \quad 3.49 \mathrm{E}-07$ mediator complex subunit Med22 $\quad \begin{array}{llllll}0.37119089 & 7.48170167 & 0.00019225\end{array}$

intramembrane aspartyl protease of the perinuclear ER me $0.37017635 \quad 5.69704043 \quad 0.02919486$ \#N/A $\quad 0.369742136 .94922718 \quad 0.00110736$ $\begin{array}{llr}0.36974213 & 6.94922718 & 0.00110736 \\ 0.36973594 & 7.69589406 & 7.83 \mathrm{E}-05\end{array}$ $0.36952813 \quad 5.61573693 \quad 0.03228138$ $0.36937787 \quad 7.56618074 \quad 0.00145524$ $\begin{array}{llll}0.36927197 & 6.17729199 & 0.01439134\end{array}$ $\begin{array}{lll}0.36877975 & 5.99211669 & 0.01447224\end{array}$ $\begin{array}{llll}0.36874177 & 7.02181152 & 0.00067221\end{array}$ ER membrane DUF2015 family conserved fungal protein D-arabinono-1,4-lactone oxidase (predicted)

$$
\# \text { N/A }
$$

recombination protein Saw1 (predicted)

$\begin{array}{rrr}0.36874177 & 7.02181152 & 0.00067221 \\ 0.36831988 & 6.5214373 & 0.0048379\end{array}$

ubiquinone biosynthesis protein Coq9 (predicted)

$0.368028597 .92957404 \quad 0.00062276$ nucleoporin Nup124

translation initiation factor elF3h (p40)

TPR domain protein, DNAJC7 family

$\begin{array}{lll}0.3676799 \quad 8.02469579 & 2.73 \mathrm{E}-05\end{array}$

$\begin{array}{lll}0.36637065 & 7.67503802 & 0.0003592\end{array}$

$0.3657561 \quad 8.56356531 \quad 6.89 \mathrm{E}-06$
0.004391724

9.82E-05

8.87E-08

0.011628305

0.003465544

0.000841151

0.008263492

0.126481322

0.045588823

4.37E-06

0.002568889

0.112155206

0.674316514

0.002618889

2.29E-05

1.44E-05

0.030315641

4.67E-05

0.001075646

0.000265067

0.00134169

0.002144347

0.037982386

0.000185672

0.259109027

7.61E-05

0.011812073

0.000657528

0.0112065

0.06006982

0.020023716

0.000379289

1.71E-06

0.000603714

0.054430422

0.002942448

0.000262506

0.05930745

0.003756793

0.029259961

0.029381179

0.001891017

0.011047628

0.001759906

1.00E-04

0.00107208

2.82E-05 
SPCC1620.05 SPAC29B12.04 SPBP8B7.31 SPAC23C11.01 SPBC337.09 SPBC543.09 SPNCRNA.1015 SPBC11G11.07 SPAC13G7.08C SPCC550.15C SPAC16C9.03

SPAC9E9.02

SPCC777.05

SPCC645.07

SPBC409.18

SPBC725.06C

SPNCRNA.602

SPNCRNA.1351

SPAC22E12.17C

ScpofMt34

SPBC428.12C

SPCC794.09c

SPBC577.09

SPBC4B4.07C

SPAC17G6.11C

SPAC9.08C

SPAC16E8.15

SPBC20F10.04C

SPCC1494.05C

SPAC212.12

SPAC24H6.10c

SPAC30D11.07

SPBC582.03

SPBC30D10.14

SPNCRNA.1247

SPAC17H9.14C

SPBC26H8.06

SPAC17D4.01

SPAP7G5.02c

SPAC8F11.04

SPBC1D7.04

SPBC3B9.07C

SPBC18E5.06

SPCC306.02C

SPBC19C2.13C

SPAPB17E12.08

SPAC31G5.11 tef101

ckn1

usp102

SPAC17G6.11C

SPAC9.08C

tif45

nse4

ubp12

SPAC212.12

SPAC24H6.10c

nth1

cdc13

SPBC30D10.14

\#N/A

pdi2

grx4

pex7

gua2

SPAC8F11.04

mlo3

rpa43

rps21

yip3

ctu2

eos 1

pac2
Rab geranylgeranyltransferase alpha subunit Bet4 (predict $0.365697918 .42874054 \quad 4.76 \mathrm{E}-06$ $\begin{array}{lllll}\text { pyridoxine biosynthesis protein } & 0.36567034 & 8.65214942 & 5.31 \mathrm{E}-05\end{array}$ HAD superfamily hydrolase, unknown role $\quad \begin{array}{lllll}0.36539002 & 4.65125526 & 0.17381488\end{array}$ ER membrane protein involved triglyceride mobilization ICE $0.36535454 \quad 8.25872824 \quad 5.27 \mathrm{E}-06$ Erg28 protein (predicted)

mitochondrial m-AAA protease Yta12 (predicted) $\# \mathrm{~N} / \mathrm{A}$ $\begin{array}{llll}0.36524008 & 7.1296706 & 0.00112128\end{array}$ $\begin{array}{lll}0.36486553 & 8.32141045 \quad 3.48 \mathrm{E}-06\end{array}$ $0.36484334 \quad 6.99779225 \quad 0.00327188$

karyopherin/importin-beta family nuclear import receptor $\uparrow \begin{array}{llll}0.36447822 & 7.00254952 & 0.00099139\end{array}$ Rix1 complex WD repeat subunit Crb3

ribosome biogenesis protein Rei1 (predicted)

export adaptor $\mathrm{Nmd3}$ (predicted)

$$
\# \text { N/A }
$$

Gtr1/RagA G protein Gtr2

RhoGEF for Rho1, Rgf1

diacylglycerol diphosphate phosphatase (predicted)

serine/threonine protein kinase Ppk31

ARF GTPase activating protein (predicted)

$$
\# N / A
$$

peptidyl-prolyl cis-trans isomerase $\mathrm{E}$ (predicted)

translation elongation factor EF-1 alpha Ef1a-a

ERCC-8 DNA repair homolog

U1 snRNP-associated protein Usp102

sphingolipid biosynthesis protein (predicted)

steroid reductase (predicted)

translation initiation factor elF4E, 4F complex subunit

Smc5-6 complex non-SMC delta-kleisin subunit Nse4

$\mathrm{CSN}$-associated deubiquitinating enzyme Ubp12

$\mathrm{S}$. pombe specific $\mathrm{GPI}$ anchored protein family 1

$\begin{array}{llll}0.36421552 & 6.78223764 & 0.00240234\end{array}$

$\begin{array}{llll}0.3641385 & 7.3067609 & 0.00035828\end{array}$

$\begin{array}{llll}0.36391579 & 7.22003945 & 0.0010877\end{array}$

$\begin{array}{llll}0.36384058 & 4.5367916 & 0.14570373\end{array}$

$\begin{array}{llll}0.36354009 & 5.87129145 & 0.02795617\end{array}$

$\begin{array}{lll}0.36350433 & 7.83385969 & 3.34 \mathrm{E}-05\end{array}$

$\begin{array}{llll}0.36332186 & 7.1559796 & 0.00034168\end{array}$

$\begin{array}{llll}0.36306921 & 5.93370782 & 0.03484632\end{array}$

$\begin{array}{lll}0.36232883 & 4.70531821 & 0.1519012\end{array}$

$0.36212482 \quad 5.45730998 \quad 0.07051583$

$0.36190084 \quad 8.165259 \quad 1.09 \mathrm{E}-05$

$\begin{array}{llll}0.36122416 & 4.69449387 & 0.16970925\end{array}$

$\begin{array}{llll}0.36115992 & 5.49320248 & 0.07622957\end{array}$

$0.36113826 \quad 10.1170289 \quad 5.47 \mathrm{E}-08$

$\begin{array}{llll}0.36101029 & 5.81308897 & 0.03676787\end{array}$

$0.36059548 \quad 5.96561306 \quad 0.02929638$

$0.359997627 .95291585 \quad 0.00020604$

$\begin{array}{lll}0.35985216 & 6.12686261 & 0.02708994\end{array}$

$\begin{array}{llll}0.35926971 & 7.40379117 & 0.00016551\end{array}$

$\begin{array}{llll}0.35922817 & 5.64221197 & 0.05831463\end{array}$

$0.35900404 \quad 7.72158998 \quad 0.00026849$

$\begin{array}{llll}0.35859597 & 4.36024237 & 0.17333699\end{array}$

phospho-2-dehydro-3-deoxyheptonate aldolase (predicted) $0.35842098 \quad 8.08273107 \quad 0.00011534$

DNA endonuclease III

G2/M B-type cyclin Cdc13

dienelactone hydrolase family (predicted)

$$
\text { \#N/A }
$$

ER protein disulfide isomerase Pdi2

$\mathrm{CIA}$ machinery monothiol glutaredoxin Grx4

peroxin-7 (predicted)

$\begin{array}{llll}0.35811462 & 4.53016515 & 0.20674458\end{array}$

$\begin{array}{llll}0.35738958 & 7.85672012 & 0.0008206\end{array}$

$\begin{array}{llll}0.35737752 & 6.39176857 & 0.0607074\end{array}$

$\begin{array}{llll}0.35696946 & 3.62273707 & 0.34988816\end{array}$

$0.35622032 \quad 8.43327565 \quad 3.04 \mathrm{E}-06$

$\begin{array}{llll}0.35489393 & 7.23238745 & 0.00099547\end{array}$

$0.354734018 .81717571 \quad 1.76 \mathrm{E}-06$

GMP synthase [glutamine-hydrolyzing] Gua2 (predicted) $\quad 0.35469106 \quad 8.39647723 \quad 1.11 \mathrm{E}-05$

U3 snoRNP-associated protein Cic1/Utp30 family (predicter $\begin{array}{lllll}0.35439671 & 7.25190296 & 0.00048058\end{array}$ RNA binding protein Mlo3

$\begin{array}{llll}0.35424966 & 7.58089615 & 0.00018741\end{array}$

DNA-directed RNA polymerase I complex subunit Rpa43 $\quad \begin{array}{lllll}0.35356693 & 6.40790922 & 0.00869765\end{array}$

$40 \mathrm{~S}$ ribosomal protein $\mathrm{S} 21$

$\begin{array}{lll}0.3528267 & 7.41397563 & 0.00041857\end{array}$

Rab GTPase binding involved in ER to Golgi vesicle transpor $0.3526025 \quad 7.14005348 \quad 0.01952238$

cytosolic thiouridylase subunit Ctu2

$\mathrm{N}$-glycosylation protein Eos1 (predicted)

cAMP-independent regulatory protein Pac2
$2.00 \mathrm{E}-05$

0.000183462

0.250012734

2.19E-05

0.002972692

$1.49 \mathrm{E}-05$

0.007790459

0.002661308

0.005914588

0.001070755

0.002896991

0.215687156

0.052336102

0.000119568

0.001024723

0.063488412

0.223548375

0.116048419

4.26E-05

0.244987581

0.124160418

3.02E-07

0.066437954

0.054589768

0.000644356

0.051023252

0.000525325

0.098763324

0.000820795

0.249430796

0.000376459

0.288712679

0.002250773

0.102129503

0.445294361

$1.32 \mathrm{E}-05$

0.002670153

7.86E-06

4.36E-05

0.001398185

0.000589874

0.018577373

0.001229325

0.038384794

0.048271005

0.130940392

0.000249547 
SPAC6C3.05

SPBC26H8.07c

SPAC12G12.08

SPBP22H7.09C

SPAC27D7.05c

SPAC1296.02

SPBC1734.15

SPBC1778.06c

SPCC622.15C

SPBC16A3.15C

SPAC31A2.11C

SPAC17D4.04

SPBC16G5.12C

SPNCRNA.600

SPBC36.02C

SPBC530.11C

SPBC19F8.05

SPAC1556.02C

SPBC1604.14c

SPAC4D7.06C

SPNCRNA.1137

SPNCRNA.1675

SPCC16C4.18C

SPAC12B10.06C

SPAC3G6.04

SPNCRNA.788

SPBC365.07C

SPCC285.12

SPAC1782.06c

SPNCRNA.856

SPCC1183.06

SPBC211.01

SPAC19D5.02C

SPAC11D3.04C

SPCC126.10

SPCC $31 \mathrm{H} 12.08 \mathrm{C}$

SPAC29A4.08C

SPAC11E3.12

SPCC1442.08c

SPAC22E12.10C

SPAPJ695.01C

SPNCRNA.746

SPAC1D4.05C

SPAC24C9.10C spo12

ost3

meu43

mrpl1602

mis15

apc14

$\operatorname{cox} 4$

rsc4

fim 1

SPCC622.15c

nda2

cuf1

rm401

top3

SPNCRNA.600

SPBC36.02C

SPBC530.11C

SPBC19F8.05

sdh1

shk1

met8

$\#$ N/A

$\# \mathrm{~N} / \mathrm{A}$

taf6

emi5

rnp24

\#N/A

tmf1

Ism7

phb1

\#N/A

ung 1

rsm10

pex22

SPAC11D3.04c

iah1

ccr4

prp19

SPAC11E3.12

cox12

etp1

SPAPJ695.01C

\#N/A

SPAC1D4.05C

mrp4
\#N/A

$0.35197929 \quad 6.96712490 .00161009$ $\begin{array}{llll}0.35188546 & 7.82415668 & 0.00028886\end{array}$

Spo12 family nuclear protein

oligosaccharyltransferase gamma subunit Ost3 (predicted) $0.35187955 \quad 7.35779562 \quad 0.00177013$ Schizosaccharomyces specific protein Meu43 $\quad 0.351791165 .18194599 \quad 0.14195877$

$\begin{array}{lllll} & 0.35173265 & 8.70741289 & 3.01 \mathrm{E}-06\end{array}$ mitochondrial ribosomal protein subunit L16 (predicted) $\begin{array}{lllll}0.35131655 & 6.74377599 & 0.00529729\end{array}$ $\begin{array}{llll}0.35121506 & 4.02217263 & 0.26894815\end{array}$ anaphase-promoting complex subunit Apc14 $\quad 0.35105952 \quad 5.20532983 \quad 0.17468505$ $\begin{array}{lllll}\text { cytochrome c oxidase subunit IV (predicted) } & 0.35103844 & 7.12933501 & 0.00436514\end{array}$ $\begin{array}{lllll}\text { RSC complex subunit Rsc4 } & 0.35060684 & 6.5683317 & 0.00686182\end{array}$ $\begin{array}{lll}0.3505324 & 9.33912681 & 8.68 \mathrm{E}-07\end{array}$

$\begin{array}{lllll}\text { Schizosaccharomyces specific protein } & 0.35013299 & 7.51006048 & 0.00319559\end{array}$ $\begin{array}{lllll}\text { tubulin alpha } 1 & 0.35009684 & 8.49073102 & 8.62 \mathrm{E}-06\end{array}$ nutritional copper sensing transcription factor Cuf1 $\quad \begin{array}{lllll}0.34961033 & 5.69101901 & 0.04458215\end{array}$ tRNA (cytosine-5-)-methyltransferase (predicted) $\quad \begin{array}{lllll}0.34945648 & 7.19933241 & 0.00054066\end{array}$ DNA topoisomerase III $\begin{array}{rrr}0.34937046 & 6.04091647 & 0.0237757 \\ 0.34931025 & 7.60621474 & 0.00465104\end{array}$ spermidine family transmembrane transporter (predicted) $0.34920278 \quad 7.01614657 \quad 0.00174899$ transcription factor, zf-fungal binuclear cluster type (predic $0.349005097 .81306081 \quad 9.13 \mathrm{E}-05$ $\begin{array}{lllll}\text { Schizosaccharomyces specific protein } & 0.34859888 & 7.54495131 & 0.0018704\end{array}$ $\begin{array}{lllll}\text { succinate dehydrogenase Sdh1 (predicted) } & 0.34854926 & 9.41387649 & 1.21 \mathrm{E}-07\end{array}$ PAK-related kinase Shk1 siroheme synthase Met8 (predicted) $\# \mathrm{~N} / \mathrm{A}$

$\begin{array}{lll}0.34835936 & 7.56834177 & 0.00075517\end{array}$ $\begin{array}{lll}0.34830832 & 6.7934069 & 0.00623715\end{array}$ $\begin{array}{llll}0.34799866 & 6.44828167 & 0.01257118\end{array}$ $\begin{array}{llll}0.34764772 & 7.01774191 & 0.00388506\end{array}$ SAGA complex/transcription factor TFIID complex histone + 0.347238097 .659828310 .00011143 succinate dehydrogenase complex assembly protein Emi5 (| 0.346785356 .038816910 .02409978 RNA-binding protein Rnp24 $\# N / A$

Golgi coiled-coil protein Tmf1 (predicted) Lsm2-8 complex Lsm7 (predicted) prohibitin Phb1 (predicted)

\section{$\# \mathrm{~N} / \mathrm{A}$}

uracil DNA N-glycosylase Ung1

mitochondrial ribosomal protein subunit S10 (predicted) peroxisomal membrane protein Pex22 (predicted) polyketide cyclase SnoaL-like domain protein isoamyl acetate hydrolytic enzyme lah1 (predicted) $\begin{array}{llll}0.34612015 & 5.96029281 & 0.02987951\end{array}$ $\begin{array}{llll}0.34597795 & 5.40371125 & 0.07451885\end{array}$ $\begin{array}{lll}0.34565328 & 5.95805944 & 0.03961276\end{array}$ $0.34536004 \quad 6.40024544 \quad 0.00744319$ $\begin{array}{llll}0.34515588 & 6.62929828 & 0.01188114\end{array}$ $\begin{array}{lll}0.34506891 & 2.9047581 & 0.47991918\end{array}$ $\begin{array}{llll}0.34475593 & 5.92518475 & 0.04409539\end{array}$ $\begin{array}{llll}0.34460073 & 7.11668594 & 0.00219852\end{array}$ $\begin{array}{llll}0.34457831 & 5.80826186 & 0.04860662\end{array}$ $\begin{array}{llll}0.34444842 & 6.66058402 & 0.00418465\end{array}$ $\begin{array}{llll}0.34389964 & 5.7287007 & 0.03687811\end{array}$ $\begin{array}{lrrr}\text { CCR4-Not complex 3'-5'-exoribonuclease subunit Ccr4 } & 0.34357884 & 7.86498222 & 0.00037522 \\ \text { Prp19 complex subunit, ubiquitin-protein ligase E4 Prp19 } & 0.3430905 & 7.53084631 & 0.00083086\end{array}$ mitochondrial thioredoxin family protein, implicated in sulf $\begin{array}{llll}0.34307038 & 8.40536632 & 1.73 \mathrm{E}-05\end{array}$ cytochrome c oxidase assembly protein Vlb (predicted) $\quad \begin{array}{llll}0.34258205 & 6.61442578 & 0.00614851\end{array}$ mitochondrial [2Fe-2S] cluster assembly ferredoxin Etp1/ c' $0.34234457 \quad 8.46475306 \quad 7.77 \mathrm{E}-06$

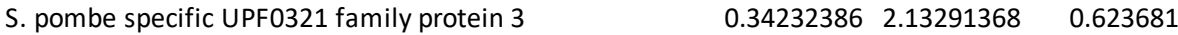
$0.34225332 \quad 3.92376877 \quad 0.32147668$ $0.34214871 \quad 8.86078612 \quad 3.32 \mathrm{E}-06$ Erd1 homolog (predicted)$$
\# \mathrm{~N} / \mathrm{A}
$$

mitochondrial ribosomal protein subunit S2 (predicted)
0.004114395

0.000876235

0.004489663

0.211374798

$1.31 \mathrm{E}-05$

0.011944351

0.360344985

0.251052118

0.010055777

0.015038547

4.04E-06

0.007630183

3.45E-05

0.07833244

0.001549791

0.045562834

0.010673946

0.004444313

0.000302854

0.004721102

$6.34 \mathrm{E}-07$

0.002096655

0.013847892

0.025876244

0.009047863

0.000364569

0.046067136

0.05553948

0.121723653

0.070956777

0.016172072

0.02459725

0.574973597

0.077684038

0.005470172

0.084508962

0.009689189

0.06660178

0.001114994

0.00227618

$6.58 \mathrm{E}-05$

0.013668923

3.15E-05

0.705518045

0.415665749

1.43E-05

0.137436539 
SPAC1F7.04

SPAC821.07C

SPCC895.07

SPAC3H8.07c

SPNCRNA.1059

SPBC1773.14

SPBC365.06

SPBC365.14C

SPAC222.07c

SPCC1393.08

SPBC2G2.15C

SPBC4F6.16C

SPBP4H10.15

SPAC15A10.07

SPCC24B 10.16C

SPNCRNA.1208

SPCC338.16

SPNCRNA.1506

SPNCRNA.688

SPAC664.09

SPBC1198.03C

SPAC1F7.14c

SPBC1271.07C

SPNCRNA.112

SPBC1198.04C

SPNCRNA.1299

SPAC2C4.14c

SPAC1142.08

SPNCRNA.853

SPCC70.07c

SPAC1687.11

SPNCRNA.1179

SPCC1902.02

SPBC20F10.09

SPAC20G8.07c

SPAC16C9.04c

SPBC29A3.09C

SPCC24B10.19C

SPAC7D4.14C

SPBC16H5.11C

SPBC21C3.17C

SPAC17H9.16

SPBC16G5.01 $0.34174953 \quad 8.14842366 \quad 3.27 \mathrm{E}-05$

mitochondrial Cox 1 translation regulator Ppr

ppr4

fex1

erj5

ho1

moc3

alp14

pac10

$\# \mathrm{~N} / \mathrm{A}$

$\arg 7$

pmt3

uge1

hri2

fil1

$\mathrm{mrm} 2$

ero11

acO2

SPAC15A10.07

SPCC24B10.16 $\# \mathrm{~N} / \mathrm{A}$

pof3

$\# N / A$

$\# \mathrm{~N} / \mathrm{A}$

ggt1

SPBC1198.03C

tam6

SPBC1271.07C

$\#$ N/A

zas1

$\#$ N/A

ppk11

$$
\# \mathrm{~N} / \mathrm{A}
$$

tmp1

$\# N / A$

mug72

Ism5

erg2

mot2

gen20

nts1

iss 10

skb1

SPBC21C3.17c

tom 22

rpn12 $\begin{array}{lll}0.34125576 & 6.25634768 & 0.01684953\end{array}$

plasma membrane fluoride efflux channel Fex

$\begin{array}{llll}0.34117475 & 7.40832859 & 0.00084544\end{array}$

Rho family GTPase Rho

$\begin{array}{llll}0.34094645 & 8.50657369 & 3.23 \mathrm{E}-05\end{array}$

$\begin{array}{llll}\text { transcription factor Moc3 } & 0.34080703 & 6.69224705 & 0.00638844 \\ \text { TOG/XMAP215 microtubule plus end tracking polymerase A } 0.34021513 & 6.86715393 & 0.01020049\end{array}$

prefoldin subunit 3 Pac10 (predicted)

$$
\text { \#N/A }
$$

argininosuccinate lyase

ubiquitin-like protein modifier SUMO

UDP-glucose 4-epimerase Uge1

elF2 alpha kinase Hri2

transcription factor, zf-GATA type

$\begin{array}{llll}0.33996757 & 5.75147908 \quad 0.04095169\end{array}$

$\begin{array}{lll}0.33989517 & 2.90524602 & 0.4696252\end{array}$

$\begin{array}{lll}0.33967285 & 5.94615804 & 0.02726135\end{array}$

$0.33945715 \quad 7.34064888 \quad 0.00373026$

$0.33927004 \quad 8.50786287 \quad 1.34 \mathrm{E}-05$

$\begin{array}{lll}0.33911258 & 6.21726992 & 0.02793702\end{array}$

$\begin{array}{llll}0.33870279 & 9.09477993 & 0.00116557\end{array}$

mitochondrial 2' O-ribose methyltransferase Mrm2 (predic1 $0.33810626 \quad 6.11839180 .04389393$

ER oxidoreductin Erola

$0.337517127 .85310782 \quad 0.0005113$

aconitate hydratase/mitochondrial ribosomal protein subur $0.33680608 \quad 9.52075331 \quad 1.23 \mathrm{E}-06$

Schizosaccharomyces specific protein

proteasome assembly chaperone Pac4

$\begin{array}{llll}0.33670101 & 5.49923651 & 0.07346398\end{array}$

$\begin{array}{llll}0.33667445 & 6.94595014 & 0.00667307\end{array}$

$0.33648055 \quad 3.90481533 \quad 0.30661919$

$\begin{array}{llll}0.33581126 & 5.8000538 & 0.0430799\end{array}$

$\begin{array}{lllll}0.33520633 & 2.29942271 & 0.65878048\end{array}$

$\begin{array}{lll}0.33517567 & 7.03181908 & 0.00277596\end{array}$

$\begin{array}{llll}0.33496091 & 6.80661253 & 0.00599288\end{array}$

$\begin{array}{llll}0.33421245 & 5.51372086 & 0.10405677\end{array}$

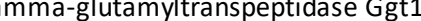

mitochondrial DUF4536, human DMAC1 ortholog, possibly r $\begin{array}{llll}0.33419996 & 3.5704888 & 0.40730302\end{array}$

$\mathrm{N}$-acetyltransferase (predicted)

$$
\# N / A
$$

transcription regulator Zas1

\#N/A

PAK-related GC kinase Ppk11

$\begin{array}{llll}0.33406732 & 7.10016125 & 0.02178347\end{array}$

$\begin{array}{llll}0.33377212 & 5.85574573 & 0.02881711\end{array}$

$\begin{array}{llll}0.33372141 & 6.03826039 & 0.05879287\end{array}$

$\begin{array}{lll}0.3329612 & 2.68420928 & 0.58345328\end{array}$

$\begin{array}{lll}0.33284495 & 7.53354705 & 0.000419\end{array}$

$\begin{array}{llll}0.33281038 & 7.4018318 & 0.00040304\end{array}$

$\begin{array}{llll}0.33186577 & 6.38421306 & 0.01588038\end{array}$

$\begin{array}{llll}0.33158111 & 6.20568446 & 0.06509047\end{array}$

$\begin{array}{llll}0.3297525 & 7.78704943 & 0.00038671\end{array}$ $\begin{array}{llll}0.32940523 & 6.05675971 & 0.02537759\end{array}$ $\begin{array}{llll}0.32928786 & 7.29120151 & 0.00293861\end{array}$

$\begin{array}{llll}0.32880094 & 5.64764796 & 0.04409896\end{array}$

$\begin{array}{rrr}0.3281179 & 6.89297973 & 0.00774983 \\ 0.32795058 & 6.53036995 & 0.05072424\end{array}$

Lsm2-8 complex subunit Lsm5

C-8 sterol isomerase Erg2

$\begin{array}{llll}0.32765904 & 8.0663315 & 0.00010545\end{array}$

$\begin{array}{llll}0.32762507 & 5.91469847 & 0.04199111\end{array}$

$\begin{array}{llll}0.32755789 & 6.08481663 & 0.02956456\end{array}$

$0.3273581 \quad 7.411008290 .00092355$

$\begin{array}{llll}0.32689123 & 5.97671365 & 0.11111479\end{array}$

$\begin{array}{llll}0.32668958 & 6.90658009 & 0.00741283\end{array}$

$\begin{array}{llll}0.32667815 & 7.57728142 & 0.00656552\end{array}$ mitochondrial TOM complex subunit Tom22 (predicted)

$19 \mathrm{~S}$ proteasome regulatory subunit Rpn12
0.085928648

0.00011722

0.03363481

0.002311527

0.000115957

0.014137662

0.021480508

0.072970991

0.564429439

0.051317704

0.008723161

5.19E-05

0.052329037

0.003079072

0.077468482

0.001478073

$5.60 \mathrm{E}-06$

0.120347235

0.014681612

0.400180972

0.076283811

0.736487785

0.006724955

0.013379772

0.162000981

0.502574266

0.042220603

0.053799841

0.099400117

0.66977051

0.001230053

0.001188338

0.031877873

0.10847457

0.001146148

0.048211747

0.007071167

0.077684038

0.016747401

0.08779913

0.000346143

0.074608219

0.055020829

0.002501906

0.171498329

0.016111247

0.014491227 
SPAC1952.14c

SPBC31F10.14C

SPAC323.01C

SPAC16E8.06C

SPBC725.13C

SPAC1B3.12C

SPCC320.04C

SPAC1B3.21

SPAC25H1.09

SPBC337.05C

SPAC1006.06

SPNCRNA.755

SPBC2G2.07C

SPCC1682.16

SPBC887.19

ScpofMt24

SPAC110.03

SPCC1682.12C

SPBC146.07

SPAC23A1.10

SPNCRNA.690

SPBC19G7.01C

SPAC26F1.05

SPCC825.02

SPBC1539.02

SPBC12C2.11

SPAC824.04

SPNCRNA.1060

SPBC211.08c

SPAC890.06

SPAC12G12.05c

SPAC24B11.09

SPNCRNA.1085

SPAC12B10.09

SPAC824.07

SPNCRNA.871

SPCC285.08

SPNCRNA.1199

SPBC725.08

SPCC553.11C

$$
\text { \#N/A }
$$

SPBC11C11.05

pct1

mrpl25

hip3

pos5

nop12

psf2

rpb10

gem 1

coa3

mde5

cct8

cdc42

ubp16

tef102

$\# \mathrm{~N} / \mathrm{A}$

msh2

mug106

gbs1

SPBC1539.02

gfa1

swd22

$$
\# \text { N/A }
$$

mtr3

nup155

taf9

$\mathrm{mpc} 2$

HIRA interacting protein Hip3

GINS complex subunit Psf2

alpha-amylase homolog Mde5

RhoGEF Rgf2

$$
\text { \#N/A }
$$

$$
\# \text { N/A }
$$

Rho family GTPase Cdc42

ubiquitin C-terminal hydrolase Ubp16

U2AF large subunit (U2AF-59)

$$
\# N / A
$$

MutS protein homolog 2

glucosidase II beta subunit

$$
\text { \#N/A }
$$

作

Holliday junction resolvase subunit Eme1

mitochondrial ribosomal protein subunit L25 (predicted) $\quad 0.32499444 \quad 5.319260940 .14213812$

mitochondrial NADH kinase Pos5 (predicted)

RNA-binding protein Nop12 (predicted)

DNA-directed RNA polymerase I, II, and III subunit Rpb10

ERMES complex GTPase subunit Gem1 (predicted)

cytochrome c oxidase assembly protein Coa3 (predicted)

chaperonin-containing T-complex theta subunit Cct8

$0.32466551 \quad 8.20772526 \quad 0.00028031$

$\begin{array}{llll}0.32466229 & 5.81733422 & 0.08514755\end{array}$

$\begin{array}{lll}0.32457374 & 6.78410179 & 0.01269299\end{array}$

$0.32457333 \quad 6.26055904 \quad 0.02308827$

$\begin{array}{llll}0.32440069 & 6.7019922 & 0.0165747\end{array}$

$\begin{array}{lll}0.32399794 & 6.38546452 & 0.01874926\end{array}$

$\begin{array}{lll}0.32367837 & 4.60845879 & 0.18135957\end{array}$

$\begin{array}{lll}0.32356113 & 6.98132954 & 0.00302067\end{array}$

$0.32353276 \quad 8.50395875 \quad 5.84 \mathrm{E}-05$

$0.32350705 \quad 8.20803195 \quad 0.00013884$

$\begin{array}{llll}0.32344687 & 6.2824736 & 0.03907364\end{array}$

mitochondrial ribosomal protein subunit L51-b (predicted) $\quad 0.3232759 \quad 5.4719908 \quad 0.14111774$

19S proteasome base subcomplex ATPase subunit Rpt4 $\quad \begin{array}{lllll}0.32325018 & 7.81037572 & 0.00120184\end{array}$

ER Man5GIcNac2-PP-Dol translocation protein Rft1 (predict $0.32323283 \quad 8.44750581 \quad 2.92 \mathrm{E}-05$

translation elongation factor EF-1 alpha Ef1a-b

Schizosaccharomyces pombe specific protein Mug106

$\begin{array}{rrrr}0.32309285 & 3.81873403 & 0.37930114\end{array}$

$\begin{array}{llll}0.32267706 & 7.56714476 & 0.00604679\end{array}$

$\begin{array}{llll}0.32255841 & 6.50010574 & 0.01222411\end{array}$

$\begin{array}{llll}0.32247823 & 7.2057748 & 0.00331116\end{array}$

$\begin{array}{llr}0.32223651 & 10.0024687 & 1.73 \mathrm{E}-06\end{array}$

$0.32212619 \quad 6.85297242 \quad 0.00625608$

$\begin{array}{llll}0.32188733 & 8.42622165 & 0.00233438\end{array}$

$\begin{array}{llll}0.32172677 & 4.76360169 & 0.16581217\end{array}$

$0.32171895 \quad 7.61520223 \quad 0.00135435$

nuclear protein, human IK ortholog implicated in meiotic cl 0.321591228 .400013640 .00051955 glutamine-fructose-6-phosphate transaminase Gfa1 (predic $0.32151067 \quad 9.32602179 \quad 3.01 \mathrm{E}-06$ mRNA cleavage and polyadenylation specificity factor comr $\begin{array}{lll}0.32140207 & 6.26278129 & 0.04100201\end{array}$

$\begin{array}{lll}0.32058052 & 4.1399912 & 0.29739261\end{array}$ $0.32027743 \quad 7.01012824 \quad 0.00264633$ $0.3201436 \quad 8.81522247 \quad 2.05 \mathrm{E}-05$ $0.32006252 \quad 6.42102897 \quad 0.03251638$ $\begin{array}{llll}0.31910659 & 7.13595082 & 0.04307616\end{array}$ $\begin{array}{lll}0.31872188 & 7.83258402 & 0.00103256\end{array}$ $\begin{array}{lll}0.31866561 & 5.51305063 & 0.0923688\end{array}$ $0.318562467 .05142897 \quad 0.06915459$ $\begin{array}{lll}0.31840556 & 4.88492889 & 0.23011495\end{array}$ $\begin{array}{llll}0.31807684 & 7.26205935 & 0.00134383\end{array}$ $\begin{array}{llll}0.31776308 & 5.80616851 & 0.06419734\end{array}$ $\begin{array}{crrrr}\text { NURS complex subunit, zf-C2H2 type zinc finger protein } & 0.31727111 & 7.21656507 & 0.00708199 \\ \text { transcription factor TFIIA complex small subunit Toa2 (pred } & 0.31681691 & 5.92464149 & 0.04485065\end{array}$
0.000101648

0.007075874

0.807662133

0.000103819

0.016808686

0.0622198

0.014110148

0.211491942

0.00085308

0.136561183

0.026095414

0.044420601

0.03314582

0.037132919

0.259057908

0.007245564

0.000200846

0.000446911

0.070066879

0.21022465

0.003166555

0.00010603

0.475017676

0.013486863

0.025230529

0.007870223

7.73E-06

0.013885399

0.005774421

0.240432941

0.003517682

0.001496653

1.31E-05

0.07304153

0.390385171

0.006433827

7.66E-05

0.05969084

0.076283811

0.002759838

0.146965096

0.114056472

0.316669808

0.003494358

0.107258255

0.015466276

0.078743202 
SPBC27B12.11C SPBC800.11 SPAC5H10.09c SPCC553.04

SPAC17H9.04C

SPCC613.11C

SPBP22H7.06

SPBC776.18c

SPBC2A9.07C

SPBPJ4664.05

SPBC21C3.09c

SPAC1D4.04

SPAC630.04C

SPBC685.09

SPCC16A11.16c

SPCC18.20

SPAC2F 3.11

SPCC11E10.05C

SPAC18B11.02C

SPCC338.17C

SPCC1795.12c

SPCC1672.05C

SPCC576.14

SPAC328.10c

SPNCRNA.1345

SPNCRNA.568

SPAC18B11.03C

SPBC646.10C

SPAC9G1.15c

SPNCRNA.1156

SPCC736.03C

SPBC1105.15c

SPCC $1259.15 \mathrm{C}$

SPAC1142.05

SPCP1E11.11

SPNCRNA.1329

SPCC790.03

SPBC725.01

SPAC1782.11

SPCC1020.09

SPBPB8B6.06C

SPBC19C2.12

SPCC338.04

SPBC32H8.09

SPAC222.11

SPAC1751.03

SPAC15E1.03 meu23

nrk1

mh1

hpz1

SPBPJ4664.05

oaa1

cct2

SPAC630.04c

orc2

rpn1302

ppx1

pus9

$\operatorname{rad} 21$

SPCC1795.12c

yrs1

dph5

rps502

$\# N / A$

$\# \mathrm{~N} / \mathrm{A}$

SPAC18B11.03C

nop56

mzt1

\#N/A

$\mathrm{msf1}$

htd2

ubc11

ctr5

puf6

rbd2

maa1

met14

gnr1

fex2

mrpl51

cid2

wdr8

hem13

tif313

rpl42 transcription factor $\mathrm{Pho}$

$0.31666208 \quad 7.49055097 \quad 0.00363393$ inosine-uridine preferring nucleoside hydrolase (predicted) $0.31651706 \quad 6.56776438 \quad 0.02234038$ 3-methyl-2-oxobutanoatehydroxymethyltransferase Ecm31 $0.31650454 \quad 5.5393397 \quad 0.14312716$ WD repeat containing cyclophilin family peptidyl-prolyl cis-1 $0.31608574 \quad 6.60372017 \quad 0.01882295$ nucleolar RNA-binding protein, human TEX13A and TEX13B $0.31608199 \quad 7.86627385 \quad 0.00231239$ mug2/mug135/meu2 family

$\begin{array}{llll}0.31589547 & 4.38107276 & 0.3774115\end{array}$ nicotinamide riboside kinase Nrk1 (predicted) $\quad \begin{array}{lllll}0.3155954 & 5.72642898 & 0.06401386\end{array}$ transcription factor TFIIK complex ubiquitin-protein ligase E $0.31552423 \quad 5.88157949 \quad 0.05311289$

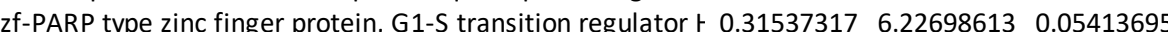
endomembrane system protein, FAR-17a/AIG1-like family $\mid \begin{array}{lllll}0.3153022 & 6.07544143 & 0.0796541\end{array}$ mitochondrial acylpyruvase Oaa1 (predicted) $\quad 0.31520823 \quad 6.58900728 \quad 0.01761047$ chaperonin-containing T-complex beta subunit Cct2 $\quad \begin{array}{lllll}0.3148561 & 8.69571409 & 2.45 \mathrm{E}-05\end{array}$ $\begin{array}{llllll}\text { Schizosaccharomyces specific protein } & 0.31481855 & 8.76089761 & 0.00038056\end{array}$ origin recognition complex subunit Orc2

$19 S$ proteasome regulatory subunit Rpn13b

$$
\text { \#N/A }
$$

exopolyphosphatase, prune Ppx1 (predicted)

nucleoside diphosphatase Ynd1

tRNA pseudouridine synthase Pus9 (predicted)

$\begin{array}{llll}0.31455724 & 6.25734301 & 0.04387849\end{array}$

$\begin{array}{llll}0.31424546 & 6.85303262 & 0.04617475\end{array}$

$\begin{array}{llll}0.31366033 & 4.24230921 & 0.31707334\end{array}$

$\begin{array}{lll}0.31348726 & 7.28353766 & 0.00485939\end{array}$

$\begin{array}{llll}0.31316998 & 6.43361669 & 0.01716429\end{array}$

$\begin{array}{llll}0.31295056 & 6.71512637 & 0.00682536\end{array}$

mitotic cohesin complex, non-SMC subunit Rad21 (kleisin) 0.312642037 .456791410 .00240171

Schizosaccharomyces specific protein

cytoplasmic tyrosine-tRNA ligase Yrs1 (predicted)

diphthine synthase Dph5 (predicted)

$40 \mathrm{~S}$ ribosomal protein S5 (predicted)

$$
\# \mathrm{~N} / \mathrm{A}
$$

$\# \mathrm{~N} / \mathrm{A}$

$\mathrm{N}$-acetyltransferase (predicted)

U3 snoRNP protein Nop56 (predicted)

mitotic spindle organizing protein Mzt1

$$
\# \text { N/A }
$$

$\begin{array}{llll}0.31188933 & 4.21213801 & 0.46121497\end{array}$

$\begin{array}{lllll}0.31096971 & 8.33712689 & 0.00011196\end{array}$

$0.31084718 \quad 7.02975625 \quad 0.00439171$

$\begin{array}{lll}0.31063457 & 8.35594755 & 5.82 \mathrm{E}-05\end{array}$

$\begin{array}{llll}0.31043847 & 6.87036423 & 0.02251318\end{array}$

$\begin{array}{lll}0.31031394 & 7.26088758 & 0.00577727\end{array}$

$\begin{array}{llll}0.31018898 & 7.15733408 & 0.00611363\end{array}$

$\begin{array}{llll}0.31003971 & 8.53358259 & 0.00024609\end{array}$

$\begin{array}{llll}0.30980206 & 4.02220448 & 0.32519687\end{array}$ $\begin{array}{lll}0.3097375 & 2.94634632 & 0.57708577\end{array}$

mitochondrial phenylalanyl-tRNA synthetase Msf1 (predicte $0.30965502 \quad 6.057543940 .05851185$ 3-hydroxyacyl-ACP dehydratase Htd2 (predicted) $\quad \begin{array}{lllll}0.30950509 & 4.33873901 & 0.28755995\end{array}$ $\begin{array}{llllll}\text { ubiquitin conjugating enzyme E2, Ubc11/UbcP4 } & 0.30948675 & 6.34147448 & 0.02154633\end{array}$ plasma membrane copper transporter complex subunit Ctr? $0.30888553 \quad 6.99872957 \quad 0.0101371$ pumilio family RNA-binding protein Puf6 (predicted)

$$
\# \mathrm{~N} / \mathrm{A}
$$

Golgi rhomboid protease Rbd2

$0.308687157 .89785541-0.00053107$

$0.30857912 \quad 5.33897624 \quad 0.10178858$ $\begin{array}{lll}0.30785785 & 4.53060624 & 0.32726421\end{array}$

mitochondrial aspartate aminotransferase Maa1 (predictec 0.307799018 .302804940 .00038711 adenylyl-sulfate kinase (predicted)

$\begin{array}{llll}0.3077111 & 6.88842514 & 0.0100893\end{array}$

heterotrimeric G protein beta (WD repeat) subunit Gnr1 $\begin{array}{lllll}0.30735755 & 4.67663557 & 0.20240007\end{array}$ plasma membrane fluoride export channel Fex2 $\quad \begin{array}{lllll}0.30699843 & 6.37685215 & 0.02588979\end{array}$

mitochondrial ribosomal protein subunit L51 (predicted) $\quad \begin{array}{lllll}0.30690176 & 5.29109865 & 0.1691939\end{array}$ $\begin{array}{lllll}\text { mitochondrial eukaryotic conserved protein Cid2 } & 0.30662776 & 5.37977366 & 0.11331457\end{array}$ mitosis-specific spindle pole body WD repeat protein Wdr8 $0.306607325 .43000966 \quad 0.1138978$ coproporphyrinogen III oxidase Hem13 (predicted) $\quad 0.306554696 .98199107 \quad 0.0120095$ translation initiation factor elF3m

60 S ribosomal protein $\mathrm{L} 36 / \mathrm{L} 42$
0.008524269

0.043189284

0.212566639

0.037268015

0.005726272

0.473329388

0.107072222

0.091280675

0.092726392

0.128905145

0.03504071

9.05E-05

0.001128919

0.077468482

0.080817681

0.411300821

0.01109298

0.034232989

0.014968289

0.005914588

0.55648367

0.00036595

0.010110139

0.000200094

0.043461612

0.012936598

0.01360027

0.000759144

0.419758093

0.664478628

0.099072684

0.380413192

0.041808603

0.021363572

0.001528092

0.15927139

0.421946367

0.001146841

0.021269414

0.283401738

0.049027013

0.244362879

0.174182436

0.17503941

0.024810147

0.000204218

0.003961604 
SPBC106.07c

SPNCRNA.1682

SPNCRNA.1678

SPBC8E4.04

SPAC19A8.07c

SPAC8C9.10c

SPBC16C6.13C

SPBC1271.02

SPCC16A11.02

SPAC4G9.06C

SPBC1703.07

SPAC664.13

SPBP23A10.07

SPBC16H5.06

SPBC83.04

SPAC19A8.13

SPCC24B10.08C

SPNCRNA.128

SPCC126.01C

SPAC6B12.04C

SPAC9G1.04

SPAC222.06

SPNCRNA.787

SPBC577.08C

SPCC1020.01c

SPAC144.01

SPNCRNA.1009

SPAC17C9.12

SPBC1734.12C

SPAC1805.09c

SPAC2F7.07C

SPNCRNA.545

SPBC106.06

SPBC1685.10

SPAC5H10.12C

SPNCRNA.67

SPAC977.15

SPBC23G7.10c

SPBC12C2.06

SPCC1494.04c

SPNCRNA.1644

SPAPB1E7.02C

SPNCRNA.759

SPBC19G7.02 nat2

$\# N / A$

$\# N / A$

SPBC8E4.04

imp4

rrp14

sec27

stt3

utp13

chz1

acl1

SPAC664.13

rpa2

rip1

apc15

usp101

ada2

rrk1

SPCC126.01c

SPAC6B12.04c

oxa101

mak16

\#N/A

txl1

pma2

SPAC144.01

$\# \mathrm{~N} / \mathrm{A}$

$\operatorname{scs} 22$

alg12

fmt1

cph2

\#N/A

cct4

rps 27

otg1

\#N/A

SPAC977.15

SPBC23G7.10c

dbp5

tyr1

\#N/A

$\mathrm{mcl} 1$

\#N/A

$a b z 2$
\#N/A

$0.30524 \quad 7.52911857 \quad 0.00098259$ mitochondrial DUF3818 and PXA domain conserved fungal । $0.30497814 \quad 7.403352360 .00899848$ phosphoribosylamidoimidazolesuccinocarboxamide synthas $\begin{array}{lllll}0.30497255 & 7.33043723 & 0.00525007\end{array}$ $\mathrm{N}$ alpha-acetylation related protein Nat2 (predicted) $\quad \begin{array}{llll}0.30473849 & 8.58600238 & 0.00033203\end{array}$

$$
\# N / A
$$

$$
\# N / A
$$

alditol NADP+ 1-oxidoreductase activity (predicted)

U3 snoRNP-associated protein Imp4 (predicted)

ribosome biogenesis protein Rrp14 (predicted)

coatomer beta' subunit (predicted)

oligosaccharyltransferase subunit Stt3

U3 snoRNP-associated protein Utp13 (predicted)

histone H2A-H2B dimer chaperone Chz1 (predicted)

ATP citrate synthase subunit 1 (predicted)

Schizosaccharomyces pombe specific protein

$\begin{array}{llll}0.30420736 & 5.93969068 \quad 0.06510068\end{array}$

$\begin{array}{lll}0.30409493 & 7.17102102 & 0.01337\end{array}$

$\begin{array}{lll}0.30399085 & 8.39877941 & 0.00057706\end{array}$

$\begin{array}{lll}0.30397355 & 6.2416851 & 0.02649195\end{array}$

$0.30386519 \quad 6.41072085 \quad 0.05190413$

$\begin{array}{lll}0.3034953 & 8.77780883 & 0.00012487\end{array}$

$0.30334194 \quad 9.1954497 \quad 8.22 \mathrm{E}-06$

$0.30252785 \quad 7.09608339 \quad 0.00658804$

$0.30245403 \quad 7.30842982 \quad 0.0074514$

$0.30190188 \quad 9.03016 \quad 9.85 \mathrm{E}-05$

$0.30177615 \quad 3.93627425 \quad 0.32101102$

$\begin{array}{llllr}\text { DNA-directed RNA polymerase I complex subunit Rpa2 } & 0.30167073 & 8.80122631 & 6.07 \mathrm{E}-05 \\ \text { ubiquinol-cytochrome-c reductase complex subunit 5, Rip1 } & 0.30067366 & 7.90684667 & 0.00188638\end{array}$

anaphase-promoting -c reductase complex subcomplex scaffol $0.30060326 \quad 5.03154045 \quad 0.18605632$

$\begin{array}{llllll}\text { U1 snRNP-associated protein Usp101 } & 0.30015711 & 6.70386609 & 0.01521569\end{array}$

$\begin{array}{lllll}\text { SAGA complex subunit Ada2 } & 0.29971372 & 5.85312695 & 0.06882801\end{array}$

$\begin{array}{lllll}\text { RNase P K-RNA } & 0.29969753 & 7.78527656 & 0.00155531\end{array}$

WD repeat protein $\quad \begin{array}{lllll}0.29959643 & 6.00188423 & 0.07011826\end{array}$

2-aminoadipate transaminase/kynurenine-oxoglutarate tra। $\begin{array}{lllll}0.29928692 & 7.40866361 & 0.00851156\end{array}$ $\begin{array}{lllll}\text { mitochondrial inner membrane insertase Oxa101 } & 0.29900694 & 6.22822445 & 0.0386185\end{array}$

nuclear HMG-like acidic protein Mak16 (predicted) $\quad \begin{array}{llll}0.29897036 & 5.8884308 & 0.05646623\end{array}$ \#N/A $\quad 0.298642596 .32586277 \quad 0.14051408$

thioredoxin-like I protein Txl1

P-type proton ATPase, P3-type Pma2

Schizosaccharomyces specific protein \#N/A

$\begin{array}{lll}0.29864259 & 6.32586277 & 0.14051408 \\ 0.29802788 & 7.13877942 & 0.01091575\end{array}$

$0.29757668 \quad 7.35072122 \quad 0.00174631$

$\begin{array}{llll}0.29738587 & 4.83121747 & 0.18444584\end{array}$

$\begin{array}{llll}0.29738587 & 4.83121747 & 0.18444584\end{array}$

$0.29711111 \quad 8.41276663 \quad 0.00024902$

dolichyly pyrotein mitochondrial methionyl-tRNA formyltransferase Fmt1 (prє $0.29703428 \quad 5.25614076 \quad 0.14705408$ $\begin{array}{llllll}\text { Clr6 histone deacetylase associated PHD protein Cph2 } & 0.29648364 & 7.59745298 & 0.00349435\end{array}$ $\# \mathrm{~N} / \mathrm{A}$

$0.29632574 \quad 2.20278455 \quad 0.74453954$ $\begin{array}{lll}0.29627409 & 8.39064241 \quad 0.00072158\end{array}$ $\begin{array}{llll}0.29621763 & 7.26297034 & 0.00352234\end{array}$ $0.29618752 \quad 6.19765411 \quad 0.05052572$ $\begin{array}{llll}0.29567707 & 5.89066307 & 0.12620785\end{array}$ $40 \mathrm{~S}$ ribosomal protein $\mathrm{S} 27$ (predicted)

alpha-1,3-galactosyltransferase (predicted)

$$
\text { \#N/A }
$$

$\begin{array}{lll}0.29521355 & 4.91406033 & 0.23892289\end{array}$

$\mathrm{NADH}$-dependent flavin oxidoreductase, implicated in cellul $0.29509671 \quad 6.423801610 .11005311$

cytoplasmic ATP-dependent RNA helicase Dbp5 (predicted) $0.29496016 \quad 8.01630648 \quad 0.00058544$ prephenate dehydrogenase Tyr1

$$
\text { \#N/A }
$$

DNA polymerase alpha accessory factor Mcl1

$\# \mathrm{~N} / \mathrm{A}$

$\begin{array}{llll}0.29488279 & 6.55244326 & 0.0442739\end{array}$

$\begin{array}{llll}0.29406404 & 5.06773942 & 0.30696276\end{array}$

$\begin{array}{llll}0.29355315 & 7.24720909 & 0.01197934\end{array}$

$\begin{array}{llll}0.29346612 & 4.99046574 & 0.22586211\end{array}$

$0.29344108 \quad 6.37159935 \quad 0.05290633$
0.002640804

0.019147795

0.011857521

0.000998882

0.10847457

0.027363264

0.001643056

0.050021566

0.089487618

0.000405216

3.32E-05

0.014527406

0.016184737

0.000325459

0.415356293

0.000207522

0.00475791

0.264213944

0.03071574

0.113738365

0.003992418

0.115505873

0.01820847

0.069340072

0.096111235

0.209462933

0.022792776

0.004439156

0.262456954

0.262456954

0.000766766

0.01641406

0.217402351

0.008262364

0.809729744

0.002015755

0.008311212

0.087577051

0.191265755

0.327136878

0.170091171

0.001664136

0.0778596

0.400444696

0.024762888

0.311573412

0.091007974 
SPAC22F $3.03 \mathrm{C}$

SPNCRNA.806

SPNCRNA.1158

SPAC644.08

SPAC6F6.17

SPACUNK4.13C

SPBC19G7.10C

SPAC16E8.10C

SPCC1494.08C

SPBC1711.16

SPAC26F1.03

SPBC1A4.09

SPBC1198.05

SPAC1002.13c

SPCC4G3.16

SPAC31F12.01

SPBC25D12.05

SPAC23A1.05

SPNCRNA.957
SPCC594.03

$$
\# N / A
$$

suc22

psh3

\#N/A

etf1

$\operatorname{cox} 24$

spf38

SPBC1703.08C

kms2

SPAC2F $3.18 \mathrm{C}$

shy1

mtg2

$\# N / A$

$\# \mathrm{~N} / \mathrm{A}$

tif33

SPBC26H8.13C

\#N/A

sss 1

tts1

taf12

$\# \mathrm{~N} / \mathrm{A}$

tim54

SPBC2G5.01

ypt1

map3

SPBC27B12.12C

erv14

rdh54

$\# \mathrm{~N} / \mathrm{A}$

$\# \mathrm{~N} / \mathrm{A}$

utr4

rif1

SPACUNK4.13C

pdc2

rsm7

SPCC1494.08C

pwp1

pda1

pus7

SPBC1198.05

psu1

rib2

zds1

trm1

SPAC23A1.05

$\# \mathrm{~N} / \mathrm{A}$

Schizosaccharomyces pombe specific protein

$$
\text { \#N/A }
$$

ribonucleotide reductase small subunit Suc22

ER chaperone SHR3 homologue Psh3

$$
\text { \#N/A }
$$

$\begin{array}{lll}0.41108198 & 0.26783541\end{array}$ $\begin{array}{llll}0.29337711 & 3.82976396 & 0.42403481\end{array}$ $0.29292355 \quad 8.25624482 \quad 0.00035889$ $0.29274713 \quad 7.11418194 \quad 0.04725438$ $0.29265595 \quad 6.32620281 \quad 0.07343636$ electron transfer flavoprotein alpha subunit EtfA (predicted $0.29193527 \quad 7.3199986 \quad 0.00286974$ mitochondrial mRNA processing protein Cox24/Pet20 (pred 0.291852255 .512475960 .10790965 U5 snRNP complex subunit Spf38 $\quad \begin{array}{llllll}0.29181295 & 6.32946176 & 0.02740629\end{array}$ 5-formyltetrahydrofolate cyclo-ligase (predicted) $\quad \begin{array}{lllll}0.29165472 & 7.98754595 & 0.00181335\end{array}$ mitotic and meiotic spindle pole body KASH domain protein $\begin{array}{lllll}0.29130183 & 5.04657475 & 0.20286121\end{array}$ small endoribonuclease with 2 transmembrane domains ( $p \quad 0.29114842 \quad 6.28683413 \quad 0.04156837$ cytochrome c oxidase assembly protein Shy1 (predicted) $\quad \begin{array}{lllll}0.29100523 & 8.62128572 & 0.0007898\end{array}$ mitochondrial translation factor (GTPase) Mtg2 (predicted) $0.29070712 \quad 4.94898192 \quad 0.17053591$

$$
\# N / A
$$

$$
\# \mathrm{~N} / \mathrm{A}
$$

$0.290584493 .73042128 \quad 0.44919562$

$0.29042129 \quad 4.63893829 \quad 0.32112642$

translation initiation factor elF3c $0.290400399 .31357276 \quad 9.88 \mathrm{E}-05$ Siva family protein, human apoptosis-inducing factor orthol $0.29013809 \quad 5.34485721 \quad 0.17190465$ $\# \mathrm{~N} / \mathrm{A}$

$0.29012927 \quad 5.30490891 \quad 0.21359299$ $\begin{array}{lrrr}\text { translocon gamma subunit Sss1 (predicted) } & 0.29005554 & 6.30514236 & 0.05634712 \\ \text { tetra spanning protein 1, Tts1 } & 0.28990534 & 6.9778757 & 0.02208309\end{array}$ transcription factor TFIID complex subunit A/ SAGA comple $0.28985781 \quad 7.2477156 \quad 0.01193364$ \#N/A $\begin{array}{llll}0.28960366 & 1.73054283 & 0.82385026\end{array}$ TIM22 inner membrane protein insertion complex subunit T $\begin{array}{lllll}0.28915949 & 6.32427963 & 0.07627221\end{array}$ $\begin{array}{llllll}\text { ER protein involved in ER-nucleus signaling (predicted) } & 0.28873568 & 7.40831074 & 0.00282369\end{array}$ GTPase Ypt1

pheromone M-factor receptor Map3

$\begin{array}{llll}0.28852078 & 7.4413374 & 0.01255794\end{array}$ $\begin{array}{lll}0.28849426 & 6.9894633 & 0.01369667\end{array}$ CorA family magnesium ion transmembrane transporter ( $\mathrm{p}$. $0.28837782 \quad 8.82878106 \quad 4.87 \mathrm{E}-05$ cornichon family protein Erv14 (predicted) $\quad 0.2881988 \quad 5.51192274 \quad 0.17258097$ ATP-dependent DNA helicase Rdh54 $0.28816553 \quad 6.47575694 \quad 0.02779577$ $\begin{array}{lll}0.28778521 & 4.9347689 & 0.16511837\end{array}$ $\begin{array}{lll}0.28749881 & 4.694764 & 0.24995311\end{array}$

$\begin{array}{llll}\text { \#N/A } & 0.28778521 & 4.934789 & 0.16511837 \\ \text { \#N/A } & 0.28749881 & 4.694764 & 0.24995311\end{array}$

methionine salvage haloacid dehalogenase-like hydrolase L $0.28723344 \quad 6.13310674 \quad 0.09221889$ $\begin{array}{llllll}\text { telomere length regulator protein Rif1 } & 0.28720374 & 8.01384423 & 0.00505633\end{array}$ mitochondrial NTPase Obg family, human OLA1 ortholog, ir $\begin{array}{llll}0.28718166 & 5.88498313 & 0.10594922\end{array}$ topoisomerase II-associated deadenylation-dependent mRR $\begin{array}{lll}0.28714742 & 8.43693098 & 0.00039904\end{array}$ mitochondrial ribosomal protein subunit S7, Rsm7(predicte $0.28669067 \quad 6.088033 \quad 0.06801005$ cortical variant C2 domain protein, human FAM102A and F/ $0.28603175 \quad 5.62226484 \quad 0.13691189$ WD repeat protein Pwp1 (predicted) $\quad 0.286001765 .506482620 .13843042$ pyruvate dehydrogenase e1 component alpha subunit Pda1 $0.28527668 \quad 8.922020390 .00021899$ tRNA/snRNA/rRNA pseudouridine synthase Pus7 (predictec $0.2849281 \quad 8.01722646 \quad 0.0020765$ $\begin{array}{lllll}\text { guanylate kinase (predicted) } & 0.28449239 & 7.73237078 & 0.00132487\end{array}$ cell wall beta-glucosidase Psu1 (predicted) $\quad \begin{array}{llll}0.28388551 & 9.374978 & 0.00329349\end{array}$ CMP deaminase family/ methyltransferase bifunctional enz $0.28387274 \quad 5.650311120 .09368469$ zds family protein phosphatase type A regulator Zds1 (pred $0.28380284 \quad 7.90449559 \quad 0.0017787$ tRNA (guanine-N2-)-methyltransferase Trm1 serine palmitoyltransferase subunit $A$ (predicted) $0.28315567 \quad 6.677670$ $\begin{array}{llll}0.28288798 & 6.08874009 & 0.16444943\end{array}$ $\# \mathrm{~N} / \mathrm{A}$
0.359136936 0.519047528 0.001071634 0.082452835

0.120330945 0.006927487 0.167081954 0.051547764 0.004583897 0.283872206 0.073915013 0.002174196 0.245815894 0.544782781 0.415370712 0.000326162 0.247579078 0.297239323 0.095932491 0.04276387 0.024683407 0.872983651 0.124200141 0.006828435

0.025856809 0.027964557 0.000169902 0.248447952 0.052111993 0.239580071 0.339912353 0.146795218 0.011461809 0.164383136 0.001178087 0.112523318 0.204946203 0.206673756 0.000682336 0.00518174 0.003458637 0.007833686 0.148607071 0.004506349 0.073682583 0.238915105 0.000232139 
SPCC18B5.05C

SPBC776.04

SPAC30C2.08

SPCC830.03

SPAC22G7.06C

SPBC32F12.10

SPAC144.18

SPBC16E9.10C

SPBC1773.06C

SPNCRNA.1211

SPCC970.08

SPCC1739.10

SPBC12D12.03

SPAC18G6.03

SPCC320.09

SPBC146.14c

SPCC70.02C

SPAC26A3.08

SPNCRNA.56

SPBC2F12.03C

SPBC2A9.08C

SPAC1B2.03C

SPAC27D7.04

SPBC530.15C

SPAC23H3.07C

SPAC4G9.05

SPBC17G9.08C

SPBC106.08c

SPNCRNA.1692

SPAC22H10.04

SPAC18G6.04C

SPNCRNA.1349

SPNCRNA.1192

SPBC4F6.18c

SPBC1289.15

SPNCRNA.726

SPAC1805.14

SPCC1620.13

SPAC105.03C ecl 1

$$
\# \text { N/A }
$$

SPCC18B5.05c $\sec 2302$

SPAC30C2.08

grc3

ura 1

SPBC32F12.10

vrg4

x7

adh8

\#N/A

kcs1

mug33

cct1

ypt3

hem 15

$\sec 26$

inh1

smb1

bs1

sec 22

elo2

omt2

SPBC530.15c

mrp2

$\mathrm{mpf} 1$

cnt5

mug2

ppa3

shm2

arf1

pfl5

SPAC1805.14 SPCC1620.13 histone H3-H4 chaperone Cia1

$\begin{array}{llll}0.28254492 & 6.67048 & 0.02700479\end{array}$

Hrd1 ubiquitin ligase complex ubiquitin conjugating enzymє 0.282053196 .407071150 .04739223

F1-FO ATP synthase subunit F (predicted) $\quad \begin{array}{lllll}0.28189586 & 6.47757332 & 0.04814394\end{array}$ snoRNA biogenesis protein Bcd1 (predicted) $\quad 0.281682285 .414169520 .16028332$

$\begin{array}{llllll}\mathrm{N} a t \mathrm{~B} & \mathrm{~N} \text {-acetyltransferase complex regulatory subunit Naa2 } & 0.28144514 & 8.28220843 & 0.00268591\end{array}$

S. pombe specific GPI anchored protein family $1 \quad 0.28087373 \quad 3.79916895 \quad 0.39735861$ $\#$ N/A

$\begin{array}{rrr}0.28087373 & 3.79916895 & 0.39735861 \\ 0.2807783 & 4.97278159 & 0.26740628\end{array}$

$0.28066458 \quad 6.6212502 \quad 0.1959831$

extender of the chronological lifespan protein Ecl1

phosphomethylpyrimidine kinase (predicted)

COPII cargo receptor subunit Sec23b (predicted)

UPF0662 family conserved fungal protein

$0.28065063 \quad 4.37377298 \quad 0.3793161$

$\begin{array}{lll}0.28058719 & 7.6506727 & 0.00198373\end{array}$

$\begin{array}{lll}0.28037238 & 7.93465388 & 0.00366762\end{array}$

polynucleotide 5'-hydroxyl-kinase Grc3 (predicted) $\quad 0.28022347 \quad \begin{array}{llll}7.04024426 & 0.02026448\end{array}$

carbamoyl-phosphate synthase (glutamine hydrolyzing), as| $0.28001939 \quad 10.5046632 \quad 7.84 \mathrm{E}-05$

$\begin{array}{llll}0.28001183 & 9.35620468 & 0.00087731\end{array}$

Golgi GDP-mannose transmembrane transporter Vrg4 $\quad \begin{array}{lllll}0.27988156 & 7.39251923 & 0.00796239\end{array}$

ribosome assembly ATPase Rix7 (predicted)

alcohol dehydrogenase (predicted)

$$
\text { \#N/A }
$$

$\begin{array}{llll}0.27938817 & 6.70809261 & 0.02138753\end{array}$

$\begin{array}{lll}0.27895158 & 6.77259638 & 0.04731091\end{array}$

$0.27866538 \quad 7.14331172 \quad 0.00629933$

$\begin{array}{lll}0.27842333 & 7.9561891 & 0.00444025\end{array}$

$\begin{array}{lll}0.27791754 & 7.37018631 & 0.02465153\end{array}$

$\begin{array}{llll}0.27682927 & 8.60534428 & 0.00055497\end{array}$

$0.27676304 \quad 7.72788188 \quad 0.00709655$

$0.27657078 \quad 5.84535778 \quad 0.15951216$

$0.27556718 \quad 9.23536102 \quad 0.00121324$

Rab11 family GTPase Ypt3

coatomer beta subunit (predicted)

$\begin{array}{lllllll}\text { Sm snRNP core protein Smb1 } & 0.27474787 & 6.60992697 & 0.09265709\end{array}$ $\# \mathrm{~N} / \mathrm{A}$

$0.27471137 \quad 5.47496158 \quad 0.1415885$

EST1 family nonsense-mediated mRNA decay (NMD) pathn 0.274148898 .063126020 .01073769 SNARE Sec22 (predicted)

fatty acid elongase Elo2 (predicted) $\quad 0.27339365 \quad 8.33720821 \quad 0.02014769$

4-alpha-hydroxytetrahydrobiopterin dehydratase (predicted $0.27290917 \quad 5.74390019 \quad 0.24360828$ spermidine family transmembrane transporter (predicted) $0.27198121 \quad 8.14925768 \quad 0.00361632$ mitochondrial ribosomal protein subunit S14 (predicted) $\begin{array}{llllll}0.27187471 & 6.88092803 & 0.01471954\end{array}$ meiotic pumilio family RNA-binding protein Mpf1 (predicte 0.271320737 .326814630 .01904256 Centaurin Cnt5

mug2/mug135/meu2 family$$
\# N / A
$$

protein phosphatase type 2A Ppa1

serine hydroxymethyltransferase Shm2 (predicted)

$\# N / A$

\#N/A

ADP-ribosylation factor, Arf family Arf1

cell surface glycoprotein, flocculin Pfl5 $\# \mathrm{~N} / \mathrm{A}$

Schizosaccharomyces specific protein

$\begin{array}{llll}0.27075663 & 7.61305955 & 0.00473939\end{array}$ $\begin{array}{llll}0.27023772 & 5.55938349 & 0.16310694\end{array}$ $\begin{array}{lll}0.27023031 & 6.76667573 & 0.03170817\end{array}$ $\begin{array}{llll}0.26999026 & 5.30975148 & 0.16702331\end{array}$ $\begin{array}{llll}0.26982885 & 7.93816752 & 0.00426926\end{array}$ $\begin{array}{llll}0.2696156 & 6.8306017 & 0.01787798\end{array}$ $\begin{array}{llll}0.26826072 & 3.50394356 & 0.46236506\end{array}$ $\begin{array}{llll}0.26812672 & 9.28033659 & 0.00011908\end{array}$ $\begin{array}{lll}0.26792572 & 7.14904584 & 0.02650317\end{array}$ $\begin{array}{llll}0.26788516 & 4.37746224 & 0.41581075\end{array}$ $\begin{array}{llll}0.26781574 & 6.35778415 & 0.06770189\end{array}$ phosphoglycerate mutase/6-phosphofructo-2-kinase family $0.26779427 \quad 5.28169293 \quad 0.16238276$ transcription factor (predicted)
0.050876963

0.082629798

0.083790148

0.233760826

0.006523072

0.49271599

0.358702886

0.276349977

0.475017676

0.004984972

0.008591437

0.03962638

0.000262663

0.002387086

0.01715229

0.041535997

0.082530315

0.013958621

0.010214981

0.046976659

0.001584788

0.015483175

0.232735903

0.003192613

0.098204092

0.147320442

0.210879791

0.022483078

0.112533491

0.039443339

0.332882395

0.00849449

0.029838083

0.037618266

0.010851793

0.237167246

0.058459517

0.242034424

0.009858255

0.035525143

0.557251355

0.000387716

0.050025551

0.510848917

0.112067952

0.236316233

0.036166024 


\begin{tabular}{|c|c|c|c|c|c|c|}
\hline SPNCRNA.1655 & \#N/A & $\# N / A$ & 0.26729056 & 6.45322535 & 0.04648291 & 0.081273434 \\
\hline SPNCRNA.1325 & $\# N / A$ & $\# N / A$ & 0.26688117 & 5.64051756 & 0.17106132 & 0.246468835 \\
\hline SPNCRNA.822 & $\# N / A$ & $\# N / A$ & 0.26673294 & 5.5768298 & 0.1181231 & 0.181042277 \\
\hline SPBC660.12c & egt2 & Ergothioneine biosynthesis protein Egt2 & 0.26634875 & 8.21089178 & 0.00121375 & 0.003192726 \\
\hline SPNCRNA.1188 & $\# N / A$ & \#N/A & 0.26578146 & 6.34950144 & 0.06258626 & 0.1050048 \\
\hline SPAC1250.07 & sfc7 & transcription factor TFIIIC subunit Sfc7 (predicted) & 0.26555703 & 5.29053125 & 0.15637141 & 0.228938776 \\
\hline SPNCRNA.778 & \#N/A & $\# N / A$ & 0.26519161 & 4.13256787 & 0.38879237 & 0.484300218 \\
\hline SPNCRNA.867 & $\# N / A$ & $\# N / A$ & 0.26502237 & 6.65727832 & 0.03899409 & 0.069959082 \\
\hline SPAC11D3.13 & hsp3104 & ThiJ domain protein, implicated in cellular detoxification & 0.26492276 & 4.87320917 & 0.25191612 & 0.341799911 \\
\hline SPAC6F6.15 & ypt5 & GTPase Ypt5 & 0.26439357 & 6.79432528 & 0.02276738 & 0.043852813 \\
\hline SPBC4F6.11c & asn2 & asparagine synthase (predicted) & 0.26408663 & 7.28455682 & 0.02686838 & 0.050648018 \\
\hline SPAC1142.02c & SPAC1142.02c & TPR repeat protein, SGT2 family (predicted) & 0.26406884 & 7.70311902 & 0.00639242 & 0.014141869 \\
\hline SPBC1347.05c & SPBC1347.05c & DNAJ domain protein Scj1 (predicted) & 0.26393022 & 7.09054477 & 0.03867167 & 0.069417219 \\
\hline SPBC13G1.09 & enp1 & bystin family U3 and U14 snoRNA associated protein Enp1 & 0.26335897 & 6.74607778 & 0.02421136 & 0.046254444 \\
\hline SPCC126.15c & $\sec 65$ & signal recognition particle subunit Sec65 (predicted) & 0.26317072 & 6.47667549 & 0.0439887 & 0.077591715 \\
\hline SPAC11E3.06 & map1 & MADS-box transcription factor Map1 & 0.26315457 & 5.71376979 & 0.14460762 & 0.214338578 \\
\hline SPAC23A1.03 & apt1 & adenine phosphoribosyltransferase (APRT) Apt1 & 0.26260069 & 6.23416388 & 0.05672309 & 0.096461422 \\
\hline SPCC663.14c & $\operatorname{trp} 663$ & plasma membrane TRP-like ion channel (predicted) & 0.26253408 & 5.24311246 & 0.17811373 & 0.255225086 \\
\hline SPAC1952.12c & $\operatorname{csn} 71$ & COP9/signalosome complex subunit 7a (predicted) & 0.26160848 & 5.08585928 & 0.24962956 & 0.339542107 \\
\hline SPCC1259.03 & rpa12 & DNA-directed RNA polymerase complex I subunit Rpa12 & 0.26148125 & 5.10661176 & 0.2682121 & 0.359500359 \\
\hline SPAC17G8.03c & bur6 & transcription regulator complex subunit Bur6 (predicted) & 0.26143064 & 5.44865582 & 0.15820811 & 0.23137894 \\
\hline SPAC139.02c & oac1 & mitochondrial carrier, ocaloacetate family anion (predicted. & 0.26130138 & 7.04244758 & 0.02219211 & 0.042927032 \\
\hline SPNCRNA.1241 & \#N/A & \#N/A & 0.26085288 & 3.31455335 & 0.70613527 & 0.776668905 \\
\hline SPBP19A11.01 & gcv3 & glycine decarboxylase complex subunit $\mathrm{H}$ (predicted) & 0.26071419 & 6.22797053 & 0.15210765 & 0.223803789 \\
\hline SPCC290.03c & nup186 & nucleoporin Nup186 & 0.26036781 & 8.69164828 & 0.00117935 & 0.003111867 \\
\hline SPAC24H6.05 & $\operatorname{cdc} 25$ & M phase inducer tyrosine phosphatase $\mathrm{Cdc} 25$ & 0.26021384 & 7.27014219 & 0.05940826 & 0.100291054 \\
\hline SPAC4F $10.05 \mathrm{c}$ & lip2 & mitochondrial lipoate-protein ligase Lip2 & 0.26010703 & 5.25182613 & 0.22686831 & 0.312707895 \\
\hline SPAC4C5.01 & SPAC4C5.01 & pseudouridine-5'-phosphatase (predicted) & 0.25948643 & 6.81205212 & 0.0349985 & 0.063707261 \\
\hline SPAC140.03 & arb1 & argonaute inhibitor protein 1 & 0.25928535 & 6.96259237 & 0.038524 & 0.069188657 \\
\hline SPBC25D12.03c & $\mathrm{mcm} 7$ & MCM complex subunit Mcm7 & 0.25743062 & 7.46718207 & 0.00880555 & 0.018790141 \\
\hline SPAC1834.01 & erf1 & cytoplasmic translation release factor class I eRF1 & 0.2568986 & 7.94310581 & 0.01280101 & 0.026293652 \\
\hline SPNCRNA.1586 & $\# N / A$ & \#N/A & 0.25672743 & 7.68217589 & 0.00451452 & 0.010375323 \\
\hline SPAC1805.08 & dlc1 & dynein light chain Dlc1 & 0.25639309 & 5.00962016 & 0.30699779 & 0.400444696 \\
\hline SPNCRNA.747 & $\# N / A$ & $\# N / A$ & 0.25622037 & 6.37125336 & 0.05573869 & 0.09513464 \\
\hline SPBC685.03 & SPBC685.03 & Schizosaccharomyces specific protein & 0.25618191 & 5.51680884 & 0.14279128 & 0.212160406 \\
\hline SPAC17A5.08 & erp2 & COPII-coated vesicle component Erp2/3/4 (predicted) & 0.25599952 & 7.1241313 & 0.02530314 & 0.048097171 \\
\hline SPAC29A4.19c & $\operatorname{cta} 5$ & $\mathrm{Ca} 2+/ \mathrm{Mn} 2+$ transporting P-type ATPase P5 type Cta5 & 0.25551994 & 7.93664564 & 0.00605516 & 0.013501111 \\
\hline SPCC622.12c & $\operatorname{gdh} 1$ & NADP-specific glutamate dehydrogenase Gdh1 (predicted) & 0.25548625 & 9.54176618 & 0.00028836 & 0.000875243 \\
\hline SPCC576.10c & rpt3 & 19S proteasome base subcomplex ATPase subunit Rpt3 & 0.25534203 & 7.99492202 & 0.00300896 & 0.007220022 \\
\hline SPAC1002.11 & gaa1 & GPI-anchor transamidase complex subunit Gaa1 (predicted) & 0.25523671 & 5.63541385 & 0.19691627 & 0.277430217 \\
\hline SPAC1399.04c & uck2 & uracil phosphoribosyltransferase Uck2 & 0.25510786 & 4.80979988 & 0.29003004 & 0.382665054 \\
\hline SPCC1183.01 & $\sec 15$ & exocyst complex subunit Sec15 & 0.25448716 & 7.45257604 & 0.00792058 & 0.017073027 \\
\hline SPAC6G10.04c & pre5 & 205 proteasome complex subunit alpha 6 Pre5 & 0.25444513 & 7.78645141 & 0.00441312 & 0.010156003 \\
\hline SPAC3F10.05c & mug113 & GIY-YIGT nuclease superfamily protein & 0.25443124 & 6.64810541 & 0.04019595 & 0.071789989 \\
\hline SPNCRNA.1478 & $\# N / A$ & \#N/A & 0.25430609 & 6.91251581 & 0.02956814 & 0.055020829 \\
\hline SPBC19F8.06c & meu22 & amino acid transmembrane transporter Meu22 (predicted) & 0.25401311 & 7.28069126 & 0.02806323 & 0.052507634 \\
\hline SPCC777.06c & SPCC777.06c & hydrolase, conserved in fungi, bacteria, plants, protazoa (pr & 0.25388922 & 7.18207977 & 0.01146807 & 0.023836353 \\
\hline
\end{tabular}


SPNCRNA.1040

SPCC4G3.10C

SPAC10F6.14C

SPNCRNA.1017

SPBC16H5.08C

SPAC18G6.05c

SPAC2G11.10c

SPBC17G9.13C

SPCC23B6.04C

SPNCRNA.611

SPAC328.03

SPNCRNA.829

SPBC106.05C

SPBC725.14

SPAC23C4.09c

SPAC1834.11C

SPNCRNA.1405

SPNCRNA.640

SPAC1527.03

SPAPB2B 4.07

SPAC1D4.09C

SPCC569.08C

SPAC22E12.03C

SPAC5H 10.07

SPCC1235.02

SPAC3H5.04

SPCC576.15C

SPAC607.10

SPBC1773.07c

SPAC1039.08

SPBC2A9.12

SPAC6F12.10c

SPAC9.11

SPNCRNA.1252

SPCC736.11

SPBC337.14

SPBC17A3.03C

SPAC15E1.07C

SPBC3D6.06C

SPNCRNA.999

SPCC622.06c

SPCC4B3.03C

SPBC1271.13

SPNCRNA.1180

SPBC17G9.04C uba42

SPBC17G9.13C pdr16

$\#$ N/A

tps1

tim11

arg6

SPAC23C4.09c

sec18

$\# N / A$

$\# N / A$

lar1

SPAPB2B4.07

rtf2

ade5

dj1

SPAC5H10.07

bio2

aar2

ksg1

spo3

sbp1

SPAC1039.08

orc6

ade3

SPAC9.11

\#N/A

rpb4

(

SPBC17A3.03C

moa1

prs5

\#N/A

$\# \mathrm{~N} / \mathrm{A}$

mam301

mrpl8

\#N/A

nup85 thymidylate synthase / phosphopantothenoylcysteine decar $0.25363878 \quad 7.6160055 \quad 0.00607561$ $\begin{array}{llllll}\text { \#N/A } & 0.25324398 & 6.23088247 & 0.09084996\end{array}$ $\begin{array}{llll}0.25301868 & 6.75258046 & 0.04467903\end{array}$ mitochondrial ABC1 kinase family protein, human ADCK5 or $0.25296355 \quad 5.31215205 \quad 0.2970023$ $\# \mathrm{~N} / \mathrm{A}$ $\begin{array}{lll}0.25286293 & 7.92944622 & 0.01558491\end{array}$ ribosome biogenesis ATPase, Arb family ABCF2-like (predic $0.25284677 \quad 8.718307180 .00070707$ translation initiation regulator Gcn1 $\begin{array}{lll}0.25281601 & 9.70715562 & 0.00086807\end{array}$ thiosulfate sulfurtransferase, URM1 activating enzyme E1-1 $0.25249023 \quad 5.36282475 \quad 0.25926978$ $\begin{array}{llllll}\text { mitochondrial conserved fungal protein } & 0.25235523 & 4.48912783 & 0.32722854\end{array}$ meiotic sec14 cytosolic factor family, phospholipid-interme $\begin{array}{lllll}0.25180633 & 8.70058364 & 0.00137783\end{array}$

$$
\text { \#N/A }
$$$$
0.2517958
$$
alpha,alpha-trehalose-phosphate synthase [UDP-forming] $\begin{array}{lllll}0.25159435 & 8.75375599 & 0.05097044\end{array}$ \#N/A

1-FO ATP synthase subunit $E$ (predicted)

acetylglutamate synthase Arg6

$\begin{array}{llll}0.25155421 & 7.92941508 & 0.01173126\end{array}$

$0.25121944 \quad 6.53463475 \quad 0.06170792$ $\begin{array}{lll}0.25121371 & 7.24535957 & 0.02022558\end{array}$ DNA-binding TFAR19-related protein, human Programmed 0.251015917 .494505940 .03454644 secretory pathway protein Sec18 (predicted)

$$
\text { \#N/A }
$$

RNA-binding protein, LARP1 family Lar1 (predicted)

ubiquitin family protein, human UBTD1 homolog

replication termination factor Rtf2

phosphoribosylglycinamide formyltransferase

glyoxylase III sdj1

Schizosaccharomyces pombe specific protein

biotin synthase

U5 snRNP-associated protein Aar2 (predicted)

serine/threonine protein kinase Ksg1

sporulation protein $\mathrm{Spo3}$

Ran GTPase binding protein Sbp1

serine acetyltransferase (predicted)

origin recognition complex subunit Orc6

phosphoribosylformylglycinamidine synthase Ade3

Schizosaccharomyces specific protein

$$
\text { \#N/A }
$$

35642 argonaute

DNA-directed RNA polymerase II complex subunit Rpb4

phosphoprotein phosphatase (predicted)

meiotic kinetochore protein (Meikin) Moa1

ribose-phosphate pyrophosphokinase Prs5 (predicted)

$$
\text { \#N/A }
$$

$\begin{array}{llll}0.25094257 & 8.79613064 & 0.0102425\end{array}$

$\begin{array}{llll}0.25071296 & 6.02947734 & 0.14423209\end{array}$

$\begin{array}{llll}0.25070618 & 4.98986553 & 0.28943619\end{array}$

$\begin{array}{llll}0.25064172 & 6.92699521 & 0.07696689\end{array}$

$\begin{array}{llll}0.25026858 & 6.55419716 & 0.05058386\end{array}$ $\begin{array}{llll}0.24939749 & 5.93693201 & 0.0936792\end{array}$ $\begin{array}{llll}0.24930872 & 6.76428321 & 0.05253301\end{array}$

$\begin{array}{llll}0.24906423 & 7.2516439 & 0.04310621\end{array}$ $\begin{array}{llll}0.24807111 & 3.16935799 & 0.6597174\end{array}$ $\begin{array}{llll}0.24718663 & 8.35598876 & 0.01007352\end{array}$ $\begin{array}{llll}0.24648434 & 7.83213624 & 0.00597712\end{array}$ $\begin{array}{llll}0.24647659 & 6.53646176 & 0.06389233\end{array}$ $\begin{array}{llll}0.24644085 & 7.11097107 & 0.02761108\end{array}$ $0.2462745 \quad 7.87207277 \quad 0.00328102$ $\begin{array}{lll}0.24614624 & 6.81922862 & 0.07064284\end{array}$ $\begin{array}{llll}0.24585903 & 5.20297834 & 0.26964299\end{array}$ $0.24573895 \quad 9.42230655 \quad 0.00103694$ $\begin{array}{lll}0.24564615 & 6.27470755 & 0.11847434\end{array}$ $\begin{array}{lll}0.24561511 & 8.00909795 & 0.02218577\end{array}$ $\begin{array}{llll}0.24554813 & 7.09357907 & 0.05261727\end{array}$ $0.24439348 \quad 6.551686 \quad 0.05707455$ $\begin{array}{llll}0.24434908 & 6.53556632 & 0.10208835\end{array}$ $\begin{array}{llll}0.24430286 & 1.47685623 & 0.80202743\end{array}$ $\begin{array}{llll}0.24423008 & 6.89103473 & 0.04024258\end{array}$ $\begin{array}{llll}0.24394771 & 7.02606448 & 0.02459071\end{array}$ $\begin{array}{llll}0.24371871 & 5.70822332 & 0.15028308\end{array}$ multi-pass membrane and CBS domain protein, implicated $0.24344306 \quad 8.95855686 \quad 0.00102437$ mitochondrial ribosomal protein subunit $L 8$ (predicted)

$\begin{array}{llll}0.24335093 & 5.92119428 & 0.10388745\end{array}$ $\begin{array}{llll}0.24303805 & 3.92671035 & 0.47865995\end{array}$ $\begin{array}{llll}0.24300574 & 7.48592428 & 0.01195103\end{array}$
0.013528975

0.00435103

0.144785481

0.078462139

0.38999401

0.031377305

0.001978444

0.002365721

0.349782135

0.421946367

0.003573217

0.045179168

0.088101045

0.024323958

0.103633373

0.039561684

0.063060217

0.021545662

0.213880876

0.382004119

0.125131432

0.087633184

0.148607071

0.090470049

0.076310551

0.737292967

0.021242737

0.013348968

0.106906141

0.051832666

0.007809489

0.116188915

0.361133748

0.002770474

0.181458001

0.042926973

0.090556525

0.096928449

0.159630329

0.855185942

0.071839126

0.046887021

0.221454389

0.002741196

0.161885534

0.573666947

0.024711862 
SPAC13G7.13C SPAC1A6.03C SPAC11G7.02 SPCC1442.03

SPNCRNA.1066 SPBC23G7.12C SPCC18.10

SPAC29E6.09

SPBC4F6.05C

SPBTRNAALA.07

SPBC28E12.01C

SPAC2F $3.17 \mathrm{C}$

SPAC23D3.09

SPAC19G12.12

SPBC776.07

SPNCRNA.1688

SPAC5D6.05

SPAC1486.06

ScpofMt36

SPNCRNA.963

SPNCRNA.1076

SPAC1556.04C

SPAC23D3.08

SPNCRNA.704

SPNCRNA.804

SPBC1685.11

SPCC162.02C

SPBP23A10.14c

SPAC607.05

SPAC2H10.02C

SPCC2 $38.05 \mathrm{C}$

SPBC776.09

SPCC1450.14

SPAC513.06C

SPNCRNA.525

SPAC2G11.06

SPAC323.03C

SPAC750.08c

SPBC17D11.07C

SPAC8E11.04C

SPAC3F 10.13

SPBC29A3.16

SPBC1604.21C

SPAC1783.06c

SPAC959.05C

SPBC17A3.09c

SPBC16H5.10C mme1

$$
\text { \#N/A }
$$

rpt

SPCC18.10

SPAC29E6.09

emp46

SPBTRNAALA.07

apc13

Ism6

arp42

dlp1

mam33

med18

npt1

$\# \mathrm{~N} / \mathrm{A}$

$\# \mathrm{~N} / \mathrm{A}$

$\# N / A$

cdd1

usp108

$\# \mathrm{~N} / \mathrm{A}$

$\# \mathrm{~N} / \mathrm{A}$

rlp1

SPCC162.02C

ell1

rpn9

nas2

$\mathrm{db} 1$

ste13

ero12

dhd1

\#N/A

vps 4

SPAC323.03C

SPAC750.08c

rpn2

SPAC8E11.04C

ucp6

rrs1

ptr3

$\operatorname{atg} 12$

pdi4

aim22

prp43
RNA-binding protein Msa1

ysophospholipase (predicted)

HECT-type ubiquitin-protein ligase E3 Pub1

mitochondrial carrier, magnesium ion Mme1 (predicted)

$$
\# \mathrm{~N} / \mathrm{A}
$$

$19 \mathrm{~S}$ proteasome base subcomplex ATPase subunit Rpt6 pyridoxine-pyridoxal-pyridoxamine kinase (predicted)

Schizosaccharomyces specific protein

lectin family glycoprotein receptor Emp46 (predicted)

tRNA Alanine

$\begin{array}{llll}0.24299891 & 6.4887357 & 0.11969438\end{array}$

$\begin{array}{llll}0.24276804 & 8.27429363 & 0.00231009\end{array}$

$\begin{array}{llll}0.24229135 & 6.75544574 & 0.04425036\end{array}$

$\begin{array}{llll}0.24217039 & 5.62385334 & 0.24512763\end{array}$

$\begin{array}{llll}0.24216409 & 7.9511455 & 0.02643556\end{array}$

$\begin{array}{llll}0.24205778 & 7.23779149 & 0.01471481\end{array}$

$\begin{array}{llll}0.24203777 & 5.11900793 & 0.23779352\end{array}$

$\begin{array}{llll}0.24151251 & 5.3095413 & 0.21636476\end{array}$

$\begin{array}{llll}0.24137545 & 1.47697837 & 0.80424039\end{array}$

anaphase-promoting complex TPR lobe accessory factor Ap $0.24133323 \quad 4.18515346 \quad 0.42950185$

Lsm2-8 complex subunit Lsm6

SWI/SNF and RSC complex subunit Arp42

$0.24128829 \quad 6.03373992 \quad 0.13065472$

$\begin{array}{llll}0.24110807 & 7.47637588 & 0.0192083\end{array}$

$0.2409191 \quad 6.85072914 \quad 0.02926833$

mitochondrial Cox1 translation regulator Mam33 (predictec $0.24038014 \quad 7.17923665 \quad 0.03203476$

$$
\text { \#N/A }
$$

mediator complex subunit Med18

nicotinate phosphoribosyltransferase Npt1 (predicted)

$$
\begin{aligned}
& \# N / A \\
& \# N / A \\
& \# N / A
\end{aligned}
$$

cytidine deaminase Ccd1 (predicted)

U1 snRNP-associated protein Usp108

$$
\# \mathrm{~N} / \mathrm{A}
$$

RecA family ATPase RIp1

$$
\# N / A
$$

AMP-binding dehydrogenase (predicted)

RNA polymerase II transcription elongation factor Ell1

$19 S$ proteasome regulatory subunit Rpn

$\begin{array}{llll}0.23970147 & 4.37544196 & 0.49377645\end{array}$

$\begin{array}{llll}0.23955518 & 5.91340685 & 0.18457446\end{array}$

$\begin{array}{llll}0.23933341 & 7.26145758 & 0.0297907\end{array}$

$\begin{array}{llll}0.23915308 & 3.90445495 & 0.54465606\end{array}$

$\begin{array}{llll}0.23902201 & 1.47691843 & 0.79964616\end{array}$

$\begin{array}{llll}0.23895461 & 6.87841247 & 0.04105624\end{array}$

$\begin{array}{llll}0.23893512 & 7.23003176 & 0.06024521\end{array}$

$\begin{array}{llll}0.23850604 & 7.46698685 & 0.01996707\end{array}$

$\begin{array}{llll}0.23837853 & 7.14173084 & 0.03047178\end{array}$

$\begin{array}{llll}0.23780387 & 3.76329645 & 0.5405496\end{array}$

$\begin{array}{llll}0.23770187 & 6.15145154 & 0.10395418\end{array}$

$\begin{array}{lllll}0.23765347 & 6.80064941 & 0.05380349\end{array}$

$\begin{array}{llll}0.23686657 & 6.61587722 & 0.07775191\end{array}$

$\begin{array}{llll}0.23664589 & 7.99861638 & 0.00622272\end{array}$

265 proteasome regulatory particle assembly protein Nas2 $0.23661495 \quad 5.67971253 \quad 0.26056274$ double strand break localizing Dbl1

ATP-dependent RNA helicase Ste13

ER protein folding oxidoreductin Ero1b

D-xylose 1-dehydrogenase (NADP+) (predicted)

$$
\# N / A
$$

$\begin{array}{lll}0.23650062 & 6.4137123 & 0.11132175\end{array}$

$\begin{array}{llll}0.23640983 & 8.91922123 & 0.00132344\end{array}$

$\begin{array}{llll}0.23585478 & 8.21301626 & 0.0106763\end{array}$

$\begin{array}{llll}0.23562504 & 9.16337908 & 0.00236177\end{array}$

$\begin{array}{llll}0.23502714 & 3.04358266 & 0.66043995\end{array}$

$\begin{array}{lllll}0.23497411 & 7.07072714 & 0.02719068\end{array}$

$\begin{array}{llll}0.23488213 & 5.73912727 & 0.1645215\end{array}$

0.234416761 .47736074

$0.23405957 \quad 9.6029301$

$\begin{array}{lll}0.23372268 & 6.50780468 & 0.09457754\end{array}$

$\begin{array}{llll}0.23358661 & 7.66217378 & 0.02173757\end{array}$

$\begin{array}{llll}0.23318368 & 5.66568158 & 0.17672007\end{array}$

$\begin{array}{lll}0.2331075 & 9.55706137 & 0.00370166\end{array}$

ribosome biogenesis protein Rrs1

ubiquitin activating enzyme E1

$\begin{array}{llll}0.23304724 & 3.78575371 & 0.54618357\end{array}$

ER membrane protein disulfide isomerase Pdi4 (predicted) $\begin{array}{lllll}0.23293718 & 5.67527579 & 0.21992368\end{array}$

lipoate-protein ligase A (predicted)

ATP-dependent RNA helicase Prp43

$0.23252283 \quad 6.92459652 \quad 0.03076144$

$\begin{array}{llll}0.23238767 & 7.51483453 & 0.01570953\end{array}$
0.183079395

0.008613595

0.005722671

0.077849967

0.334488368

0.04995667

0.029837383

0.32585278

0.300482083

0.857142657

0.524920988

0.19714088

0.037887666

0.054552444

0.058886148

0.588364343

0.262493035

0.05538954

0.635416506

0.853315371

0.073119006

0.101577966

0.039177782

0.056470708

0.631610742

0.161915358

0.092248107

0.126156829

0.013820373

0.351114741

0.171700929

0.003458015

0.0223683

0.005833693

0.73797928

0.051198829

0.238920322

0.011456839

0.149744105

0.042143637

0.253460842

0.008662248

0.636761893

0.304678473

0.056960981

0.031562838 
rp41

utp7

SPAC959.03C

SPBC19G7.14C

SPBC83.14C

SPNCRNA.835

SPAC8C9.15C

SPBC19C7.12C

SPNCRNA.924

SPAC1B3.16C

SPNCRNA.1676

SPBC28F2.09

SPCC4B3.08

SPBC3H7.06C

SPBP26C9.03C

SPCC4B3.17

SPAC4F8.15

SPBC8D2.07c

SPAC1F5.02

SPAC3F10.09

SPNCRNA.1028

SPAC8C9.14

SPAC3G6.01

SPAC4H3.01

SPAC4G9.17C

SPAC1610.01

SPBC28F2.04C

SPNCRNA.1376

SPAC17H9.13C

SPAC14C4.05C

SPBC713.10

SPAC3G9.01

SPBC4C3.06

SPBC14C8.14C

SPAC1834.05

SPAC17H9.02

SPAC9G1.10C

SPAC630.14c

SPBC3H7.04

SPBC17D11.06

SPNCRNA.689

SPCC16A11.07

SPNCRNA.1058

SPBC119.02

SPBC30B4.03C

SPNCRNA.03

SPBC2F12.12C $\operatorname{cog} 5$

toa1

Isg1

pof9

cbp3

itr1

pdi1

SPAC3F10.09

\#N/A

prr1

hrp3

SPAC4H3.01

mrp5

saf5

cwf7

$\# \mathrm{~N} / \mathrm{A}$

pro2

man1

tim16

nsk1

syp1

pol5

alg9

$\mathrm{mtl} 1$

inp53

tup12

SPBC3H7.04

spp2

\#N/A

coq10

\#N/A exosome subunit Rrp41

U3 snoRNP-associated protein Utp7 (predicted) Golgi transport complex subunit $\operatorname{Cog} 5$ (predicted)

DNA replication factor $\mathrm{C}$ complex subunit $\mathrm{Rfc} 5$

$$
\# \mathrm{~N} / \mathrm{A}
$$

translation initiation factor elF2B epsilon subunit

alpha-1,2-mannosyltransferase Omh1

$$
\text { \#N/A }
$$

plasma membrane vitamin $\mathrm{H}$ transmembrane transporter

\#N/A

$0.2323359 \quad 3.3613744 \quad 0.67147214$ 0.231979 $\begin{array}{llll}0.23176518 & 7.14211786 & 0.03608886\end{array}$ $\begin{array}{llll}0.23165547 & 6.68585892 & 0.07400032\end{array}$ $\begin{array}{llll}0.23154713 & 6.67988096 & 0.06658376\end{array}$ $\begin{array}{lll}0.2311495 & 3.89400876 & 0.5090176\end{array}$ $\begin{array}{llll}0.23100129 & 8.28518289 & 0.00665114\end{array}$ $\begin{array}{llll}0.23099349 & 8.70175325 & 0.01598664\end{array}$ $\begin{array}{llll}0.23052232 & 4.95742304 & 0.31205148\end{array}$ $\begin{array}{llll}0.22981833 & 2.0992342 & 0.87133262\end{array}$ transcription factor TFIIA complex large subunit Toa1 (pred $0.22916948 \quad 7.60473041 \quad 0.0173305$ $\begin{array}{llllll}\text { Lsk1 complex gamma subunit Lsg1 } & 0.22909255 & 6.20558046 & 0.11834333\end{array}$ F-box protein Pof9 $0.228889547 .86925232 \quad 0.02002365$

plasma membrane iron/zinc ion transmembrane transporte $0.22864484 \quad 5.309183290 .30199257$ mitochondrial Cbp3-Cbp6 complex Cbp3 (predicted) $\quad \begin{array}{llll}0.22842058 & 6.17203652 & 0.16984845\end{array}$ $\begin{array}{lllll}\text { myo-inositol transmembrane transporter Itr1 } & 0.22836032 & 7.3509206 & 0.0264644\end{array}$ transcription factor TFIIIC complex subunit Sfc9 (predicted) $0.22829864 \quad 7.05086486 \quad 0.0806456$ $\begin{array}{llll}\text { ER protein disulfide isomerase (predicted) } & 0.22824973 & 9.32505978 & 0.00246649\end{array}$ 1-(5-phosphoribosyl)-5-[(5-phosphoribosylamino) methylide $\begin{array}{llll}0.22757887 & 7.4172856 & 0.03191425\end{array}$ $\# \mathrm{~N} / \mathrm{A}$

transcription factor Prr1

ATP-dependent DNA helicase Hrp3

DNAJ domain protein Caj1/Djp1 type (predicted)

mitochondrial ribosomal protein subunit S5 (predicted)

splicing factor Saf5

Prp19 complex subunit Cwf7

glutamate 5-kinase Pro2

$$
\text { \#N/A }
$$

LEM domain nuclear inner membrane protein Man1, Sad1 i TIM23 translocase complex subunit Tim16 (predicted)

Clp1-interacting, microtubule plus-end binding Nsk1

F-BAR domain protein Syp1 (predicted)

$\begin{array}{llll}0.22730113 & 5.21537554 & 0.29682289\end{array}$

$\begin{array}{lll}0.22724913 & 8.42026234 & 0.0091081\end{array}$

$\begin{array}{lll}0.22678574 & 8.51691879 & 0.0097825\end{array}$

$\begin{array}{llll}0.22674091 & 7.74269298 & 0.02484952\end{array}$

$\begin{array}{llll}0.22660598 & 6.37820261 & 0.14347373\end{array}$

$\begin{array}{llll}0.22659554 & 5.123926 & 0.28562804\end{array}$

$\begin{array}{llll}0.22659505 & 6.71547739 & 0.06424756\end{array}$

$\begin{array}{llll}0.22657547 & 8.4994917 & 0.00477096\end{array}$

$\begin{array}{llll}0.22644141 & 7.19399749 & 0.02650876\end{array}$

$\begin{array}{llll}0.22622533 & 7.3694219 & 0.07892645\end{array}$

$0.22615374 \quad 5.83693286 \quad 0.15883616$

$\begin{array}{llll}0.22614087 & 4.84012907 & 0.48833046\end{array}$ $\begin{array}{lll}0.22606756 & 7.41266205 & 0.03134029\end{array}$

polymerase phi, polymerase-related transcriptional regulat $0.22534284 \quad 7.83181854 \quad 0.01498883$ mannosyltransferase complex subunit Alg9 (predicted) $\quad \begin{array}{lllll}0.22517415 & 7.27586576 & 0.03177497\end{array}$ TRAMP/MTREC complex subunit, ATP-dependent RNA helic 0.225084187 .137029870 .04389612 inositol polyphosphate phosphatase Inp53 (predicted) $\quad \begin{array}{lllll}0.22464929 & 8.38773631 & 0.01200719\end{array}$ $\begin{array}{llllll}\text { transcriptional corepressor Tup12 } & 0.22456764 & 7.8882297 & 0.0109135\end{array}$ superoxide dismutase, mitochondrial ribosomal protein sub $0.22456433 \quad 6.56284015 \quad 0.12084318$ DNA primase large subunit Spp2

$$
\text { \#N/A }
$$

$\begin{array}{lll}0.2244983 & 6.51373418 & 0.12415579\end{array}$ $0.22430581 \quad 5.32107306 \quad 0.32820978$ $\begin{array}{lll}0.22421292 & 6.25729129 & 0.13499665\end{array}$ $\begin{array}{lll}0.22386525 & 2.94630309 & 0.7249127\end{array}$ mitochondrial ubiquinone binding protein Coq10

$$
\# \text { N/A }
$$

$0.2237703 \quad 8.25327817 \quad 0.00666379$ ubiquitin conjugating enzyme E2 for APC and SCF Ubc4/Ubc $\begin{array}{ccccc}0.2237703 & 8.25327817 & 0.00666379\end{array}$ $\begin{array}{crrr}\text { \#N/A } & 0.22351934 & 7.7807623 & 0.08884459 \\ \text { cactin, spliceosome complex subunit } & 0.22293031 & 5.26929957 & 0.29230633\end{array}$
0.747728342

0.218499678

0.065436902

0.120992827

0.110539759

0.601365407

0.014652316

0.032081707

0.405714613

0.105477103

0.912639445

0.034513912

0.181298146

0.039268281

0.39505179

0.24503258

0.049993506

0.130354706

0.006055004

0.058743976

0.38999401

0.019350811

0.020667469

0.047287741

0.213034859

0.378740998

0.107315822

0.010913065

0.050025551

0.127840824

0.2319983

0.583254585

0.057922506

0.030329766

0.05856682

0.077468482

0.024810147

0.022792776

0.184529725

0.188673552

0.422925119

0.202435484

0.793219318

0.014674599

0.268628981

0.142088706

0.385045401 
SPCC1906.02C

SPAC732.01

SPAC16.03C

SPAC6F12.05C

SPBC215.11C

SPNCRNA.372

SPBC119.09c

SPBC2F12.11C

SPBC216.02

SPAC26H5.11

SPAC513.05

SPBC691.05C

SPBC354.07C

SPBC25B2.05

SPNCRNA.566

SPAC222.19

SPCC1620.04c

SPBC32F $12.08 \mathrm{C}$

SPBC16E9.09C

SPCC63.08C

SPBC14C8.13

SPBC3E7.06C

SPBC19C2.11C

SPBC359.03C

SPAC17H9.12C

SPAC22F8.06

SPAC13G7.12C

SPNCRNA.1590

SPAC926.06C

SPAC9E9.05

SPBC428.07

SPAC4F10.19c

SPNCRNA.983

SPAC11D3.18C

SPAC227.05

SPBC1685.15C

SPAC17C9.11C

SPAC806.11

SPBC1709.12
SPBC215.11C

orm 1

rep2

num1

mug56

ams1

ist2

SPBC354.07c

mis3

$\# N / A$

duo1

erp5

atg1

ber1

fnx2

mdm 34

aat1

cyc2

pam1

eki1

bsp1

meu6

hit1

\#N/A

SPAC11D3.18c

$\operatorname{gim} 3$

klp6

SPAC17C9.11C

\#N/A
NatC N-acetyltransferase complex subunit Mak10 (predicte 0.222773428 .403137910 .01553221 translation initiation factor elF3i

CENP-W ortholog

P-TEFb-associated cyclin-dependent protein kinase Lsk1

$$
\text { \#N/A }
$$

membrane-tethered transcription factor (predicted)

CIA machinery iron hydrogenase Nar1 (predicted)

glucan endo-1,3-alpha-glucosidase Agn2

CUE domain protein Cue3, human ASCC2 ortholog, activatin 0.220384696 .940628690 .05085429

V-type ATPase Vo proteolipid subunit (predicted) $\quad 0.21976655 \quad 5.97064185 \quad 0.13738882$

dihydroorotase Ura2

$0.21976159 \quad 6.32172368 \quad 0.11123297$

thiamine diphosphokinase Tnr3/ Nudix hydrolase fusion pro $0.2196527 \quad 8.11810032 \quad 0.01151071$ aldo/keto reductase, unknown biological role $\# \mathrm{~N} / \mathrm{A}$

ORMDL family protein Orm1 (predicted)

MBF transcription factor activator Rep2

cortical anchoring factor for dynein Mcp5/Num1

spore wall assembly protein Mug56 (predicted)

alpha-mannosidase Ams1 (predicted)

$\begin{array}{llll}0.2196301 & 7.84582569 & 0.04427872\end{array}$

$0.2195739 \quad 4.25065956 \quad 0.49044443$

$\begin{array}{llll}0.21951036 & 7.35497576 & 0.09598125\end{array}$

$\begin{array}{lll}0.2192849 & 4.08748487 & 0.49586617\end{array}$

$0.2192195 \quad 6.52449133 \quad 0.11962624$

$\begin{array}{llll}0.21895359 & 8.01319235 & 0.02522199\end{array}$

$0.218898119 .17470435 \quad 0.00518102$

anoctamin calcium-activated chloride channel OR Ca(2+)-ar $0.21850841 \quad 6.91527645 \quad 0.06809237$ sterol intermembrane transfer protein (predicted) $\quad \begin{array}{lllll}0.21817304 & 7.79943042 & 0.01725516\end{array}$ rRNA processing protein Mis3

$$
\# \text { N/A }
$$

Ragulator complex subunit, human LAMTOR3 ortholog

meiotic fizzy-related APC coactivator Fzr3

DASH complex subunit Duo1

COPII vesicle coat component Erp5/Erp6 (predicted)

autophagy serine/threonine protein kinase Atg1

$0340885 \quad 0.12059404$

$0.217323263 .34401682 \quad 0.69344387$

$\begin{array}{llll}0.21675242 & 4.98697999 & 0.42122193\end{array}$

$\begin{array}{llll}0.21674773 & 5.35352535 & 0.34585142\end{array}$

$\begin{array}{llll}0.21670165 & 4.47772292 & 0.47817398\end{array}$

$\begin{array}{llll}0.21661208 & 7.34486227 & 0.03135422\end{array}$

$0.21660604 \quad 6.54237349 \quad 0.100049$

SRR1 family protein involved in microtubule stabilization B\& $0.21606021 \quad 7.88501362 \quad 0.0194234$

$\begin{array}{lllll}\text { vacuolar amino acid transmembrane transporter Fnx2 } & 0.21605099 & 6.89619555 & 0.08932379\end{array}$

ERMES complex subunit Mdm34 (predicted) $\quad 0.21581521 \quad 6.99959876 \quad 0.08495107$

plasma membrane amino acid transmembrane transporter $0.21572882 \quad 7.74671865 \quad 0.01633636$ mitochondrial cytochrome c-heme linkage protein Cyc2 (pre $0.21490771 \quad 6.82175961 \quad 0.1127858$ 20S proteasome complex subunit beta 6 Pam $1 \quad 0.21489412 \quad 7.2516052 \quad 0.0331609$ choline/ethanolamine kinase Eki1 (predicted) $\quad 0.2145796 \quad 7.33932128 \quad 0.06896897$ $\begin{array}{lll}0.21447891 & 3.62136478 & 0.58394382\end{array}$ $\begin{array}{llll}0.21447891 & 3.62136478 & 0.58394382 \\ 0.21432097 & 6.81079058 & 0.06620432\end{array}$

$$
\# N / A
$$

$\begin{array}{lll}0.21432097 & 6.81079058 & 0.06620432\end{array}$

synaptojanin to cortical actin cytoskeletal adaptor Bsp1 (pre 0.214269317 .693146820 .01844375 pleckstrin homology domain protein, implicted in meiotic cl $0.214216826 .69417906 \quad 0.13494552$ zf-HIT family C/D snoRNP assembly protein Hit1 (predicted) $0.21377605 \quad 4.6513275 \quad 0.57359449$

$$
\text { \#N/A }
$$

$\begin{array}{llll}0.21321803 & 5.15596069 & 0.36687792\end{array}$ carboxylic acid transmembrane transporter (predicted) $\quad \begin{array}{lllll}0.21282885 & 7.14543571 & 0.05261004\end{array}$ prefoldin subunit 4, Gim3 (predicted) $\begin{array}{lll}0.2126075 & 6.16847979 & 0.16544097\end{array}$ kinesin-8 family plus-end directed microtubule motor Klp6 $\quad 0.21253897 \quad 6.76662251 \quad 0.06584654$ zf- $\mathrm{C} 2 \mathrm{H} 2$ type zinc finger protein/UBA domain protein $\quad \begin{array}{lllll}0.21246841 & 6.57496148 & 0.1178056\end{array}$ $\begin{array}{lrl}0.21242114 & 7.7906564 & 0.10430539\end{array}$ $\begin{array}{llll}0.21240391 & 8.20419097 & 0.02512065\end{array}$
0.031289723

0.012936598

0.599825561

0.149023691

0.016680369

0.050641835

0.250243405

0.159229072

0.087931122

0.205524499

0.17160289

0.023917681

0.0778596

0.585199995

0.151473194

0.589757617

0.183016308

0.047969722

0.011713241

0.112604751

0.034394071

0.184248422

0.766675143

0.516471209

0.440733876

0.573286459

0.057932516

0.157091239

0.038245435

0.142720913

0.136310314

0.032715978

0.173604927

0.060824768

0.113890856

0.670220564

0.110017195

0.036570433

0.2024034

0.66141

0.462248514

0.090556525

0.239945818

0.1095564

0.180596331

0.162243155

0.047790338 
SPBC3H7.10

SPACUNK4.15

SPNCRNA.1599

SPAC4D7.12C

SPAC57A7.07C

SPCC18.03

SPBC354.06

SPACUNK4.07c

SPNCRNA.771

SPBC1A4.10C

SPAC8F11.08C

SPBC21C3.19

SPNCRNA.1632

SPBC32H8.11

SPCC4B3.18

SPAC2F3.04C

SPNCRNA.1568

SPBC28F2.11

SPCC4G3.18

SPBC32H8.12C

SPAC6F6.16C

SPAC3H1.09c

SPBC354.01

SPNCRNA.578

SPBC2G5.06C

SPCC $330.07 \mathrm{C}$

SPAC25B8.19C

SPAC1D4.08

SPCC1442.05c

SPCC1919.09

SPCC1281.02C

SPAC25G10.03

SPCC63.05

SPBC1105.17

SPBC713.05

SPNCRNA.852

SPAC $22 \mathrm{H} 10.05 \mathrm{C}$

SPBC2A9.10

SPAC12G12.03

SPNCRNA.1207

SPBC649.04

SPCC1682.15

SPAC23C11.15

SPAC2F7.05C

SPAC4G8.04

SPBC4.04C

SPBC14F5.11C elp6

elongator complex subunit Elp6

2',3'-cyclic-nucleotide 3'-phosphodiesterase (predicted)

$$
\# \mathrm{~N} / \mathrm{A}
$$

$0.2123648 \quad 6.58773289 \quad 0.07697092$

$\begin{array}{llll}0.21194761 & 6.4984985 & 0.16649174\end{array}$ $\begin{array}{llll}0.21113447 & 7.94541713 & 0.03747568\end{array}$ fet5

SPAC57A7.07c

SPCC18.03

mrps16

cta4

$\#$ N/A

med14

SPAC8F11.08c

rtc

\#N/A

mei4

ppc1

$\operatorname{rim} 1$

$\# N / A$

nmo1

rix1

act1

tpz1

avt3

gtp1

\#N/A

hmt2

SPCC330.07c

loz1

pis1

mic26

tif6

spf30

zip1

tap42

cnp1

wdr8s

\#N/A

SPAC22H 10.05

SPBC2A9.10

cip2

$\# \mathrm{~N} / \mathrm{A}$

uvi15

mug122

pst2

tif5

PAC4G8.04

$\mathrm{mcm} 2$

snx41 predicted GTPAse with a role in RNA polymerase localizatic 0.210970196 .330779680 .13312756

homocysteine methyltransferase (predicted)

$0.21070049 \quad 5.78779181 \quad 0.22163718$

shuttle craft like transcriptional repressor/ubiquitin-protein $\begin{array}{lllll}0.2106892 & 6.67891299 & 0.09509774\end{array}$

mitochondrial ribosomal protein subunit S16 (predicted) $\quad \begin{array}{lllll}0.2104874 & 6.55354432 & 0.0989514\end{array}$

P-type ATPase, calcium transporting Cta4

$0.20995192 \quad 8.69243893 \quad 0.00755161$

$\begin{array}{llll}0.20956892 & 6.98673912 & 0.09233241\end{array}$

$\begin{array}{lll}0.20948729 & 7.33211861 & 0.05245848\end{array}$

$0.20896257 \quad 7.35215794 \quad 0.04700712$

$\begin{array}{llll}0.20859784 & 6.62596559 & 0.18703117\end{array}$

$\begin{array}{lll}0.20844907 & 6.9489994 & 0.09584162\end{array}$

$0.2083685 \quad 5.50196058 \quad 0.26273955$

$0.20830233 \quad 6.57336318 \quad 0.18313132$

$\begin{array}{lll}0.20828027 & 5.63962151 & 0.21291203\end{array}$

$\begin{array}{llll}0.20818045 & 6.03713651 & 0.19148402\end{array}$

$\begin{array}{llll}0.20814379 & 7.74986963 & 0.02028081\end{array}$

$\begin{array}{llll}0.2079161 & 7.58410388 & 0.04280836\end{array}$

$0.20763366 \quad 9.95757096 \quad 0.00389112$

$\begin{array}{llll}0.2075249 & 7.48793509 & 0.03971055\end{array}$

$\begin{array}{llll}0.20724205 & 7.64297818 & 0.03465627\end{array}$

actin Act1

vacuolar amino acid transmembrane transporter Avt3

$\begin{array}{crrrr}\text { cytoplasmic translation associated GTP binding protein Gtp } & 0.20708973 & 6.58717446 & 0.13445318 \\ \text { \#N/A } & 0.20683516 & 7.2291775 & 0.12866631\end{array}$

$\begin{array}{llll}0.20679703 & 8.03280158 & 0.0472401\end{array}$

$\begin{array}{llll}0.20644531 & 7.22197397 & 0.03625521\end{array}$

$0.206382996 .48500276 \quad 0.19757495$

transmembrane transporter (predicted)

transcription factor $\mathrm{zf}-\mathrm{C} 2 \mathrm{H} 2$ type

$\begin{array}{llll}0.20633447 & 6.73191282 & 0.24019625\end{array}$

MICOS complex subunit Mic23/26/27 (predicted) $\quad 0.20553554 \quad 5.81336785 \quad 0.25567725$

translation initiation factor-like ribosome biogenesis protei $\begin{array}{llll}0.20547929 & 7.47755789 & 0.02950127\end{array}$

splicing factor Spf30 (predicted) $\quad 0.20536522 \quad 5.097690890 .35988028$

transcription factor Zip1 $\quad \begin{array}{llllll}0.20519954 & 7.7846371 & 0.04621039\end{array}$

TAP42 family protein involved in TOR signalling Tap42 (prer $0.20450163 \quad 5.66955079 \quad 0.21776974$ centromere-specific histone H3 CENP-A $\begin{array}{lll}0.20431405 & 5.93800767 & 0.20123366\end{array}$

WD repeat protein, human MAPK organizer 1 (MORG1) fan $0.2042612 \quad 4.95098573 \quad 0.35826304$

$$
\# N / A
$$

$\begin{array}{lll}0.20405608 & 6.77627416 & 0.1031317\end{array}$

mRNA cleavage and polyadenylation specificity factor com $0.20394647 \quad 7.551094330 .05158654$

Bin3 family, 7SK RNA methyltransferase (predicted) $\quad 0.20380056 \quad 5.747087250 .23111946$

RNA-binding protein Cip2

\section{$\# N / A$}

tail anchored plasma membrane protein Uvi15

PX/PXA domain protein

Clr6 histone deacetylase complex subunit Pst2

translation initiation factor elF5, Tif5(predicted)

GTPase activating protein (predicted)

MCM complex subunit Mcm2

PX domain sorting nexin Snx41

$\begin{array}{llll}0.20325875 & 8.47443909 & 0.01563799\end{array}$

0.20318851 .90608024

$\begin{array}{llll}0.20213765 & 7.21425124 & 0.10078784\end{array}$

$\begin{array}{llll}0.20207562 & 7.11276441 & 0.10042537\end{array}$

$\begin{array}{llll}0.20197071 & 7.47190258 & 0.06277023\end{array}$

$\begin{array}{llll}0.2017565 & 9.02483084 & 0.01286159\end{array}$

$\begin{array}{llll}0.2012964 \quad 7.61065536 & 0.04971066\end{array}$

$\begin{array}{llll}0.201284 & 6.9684803 & 0.08590209\end{array}$

$\begin{array}{llll}0.20118872 & 7.27602939 & 0.06657467\end{array}$

0.125131432

0.241366906

0.067537606

0.200294729

0.306428483

0.150427731

0.155583245

0.01637625

0.146941537

0.090374668

0.082126679

0.26537679

0.151287918

0.35362789

0.261204916

0.296412924

0.270791507

0.039646918

0.075921528

0.009058858

0.071057134

0.063209847

0.201845725

0.194399257

0.082449058

0.065668533

0.277961042

0.328748087

0.345901322

0.054941479

0.455694705

0.080859266

0.302124994

0.281942568

0.453924523

0.160855196

0.089007709

0.317666714

0.031465549

1
0.158068973

0.15760946

0.105235624

0.026410119

0.086252176

0.137706415

0.110539759 
SPAC29E6.05C

SPNCRNA.1474

SPAC10F6.11C

SPAC23C4.17

SPBC3B8.02

SPNCRNA.1444

SPBC1709.17

SPAC1556.03

SPBC2D10.03C

SPCC553.06

SPAC30D11.03

SPCC4B3.16

SPNCRNA.1502

SPAC5D6.06C

SPCC645.13

SPNCRNA.749

SPBC23G7.05

SPAC30D11.02C

SPCC613.03

SPAC3A11.02

SPBC106.10

SPBC16H5.07C

SPAC17A5.04C

SPAC1039.07c

SPAC $23 \mathrm{H} 3.12 \mathrm{C}$

SPBC18H10.17C

SPBC16C6.08

SPBC577.03C

SPBC83.10

SPCC1235.07

SPCC1840.05c

SPNCRNA.35

SPBC13E7.06

SPAPB1A10.16

SPBC32F12.07C

ScpofMt18

SPCC965.03

SPAC31G5.19

SPCC330.10 tas 3

rpm 1

dre2

tom5 $\mathrm{m} \times \mathrm{r} 1$

g17

trm402

php5

met7

azr1

ess1

swp1

dd $\times 27$

tip41

alg14

bye1

\#N/A

sui1

SPAC30D11.02

SPCC613.03

cps3

pka1

ppa2

mde10

SPAC1039.07c

SPAC23H3.12C

SPBC18H10.17C

qcr6

SPBC577.03C

ecm7

fta7

SPCC $1840.05 C$

$\# \mathrm{~N} / \mathrm{A}$

msd1

dpc13

SPBC32F12.07C

\#N/A

vma8

abo1

pcm1
\#N/A $\begin{array}{lllll}\text { rRNA (guanine-N7-)-methyltransferase Bud23 (predicted) } & 0.20087032 & 5.59821059 & 0.25391877 \\ \text { phenazine biosynthesis PhzF protein family } & 0.20069967 & 1.58369085 & 0.81720783\end{array}$ conserved fungal protein, possibly DNA J domain Hua1 $\quad 0.199688825 .16542407 \quad 0.45673985$ RITS complex subunit 3

mitochondrial 3'-5' exonuclease for RNA 3' ss-tail

CIA machinery anamorsin Dre2 (predicted)

mitochondrial TOM complex subunit Tom5 (predicted)

peptide-methionine (S)-S-oxide reductase MsrA

$$
\# N / A
$$

autophagy associated protein kinase activator Atg17

TRNA (cytosine-5-)-methyltransferase (predicted)

CCAAT-binding factor complex subunit Php5 \#N/A

folylpolyglutamate synthase Met7 (predicted)

serine/threonine protein phosphatase Azr1

DUF866 family protein, human C1orf123 ortholog

oligosaccharyltransferase delta subunit Swp1 (predicted)

ATP-dependent RNA helicase Ddx27/Drs1 (predicted)

TIP41-like type 2a phosphatase regulator Tip41

$$
\# \mathrm{~N} / \mathrm{A}
$$

UDP-GICNAC transferase associated protein Alg14 (predicte $0.19589693 \quad 4.969123110 .42408586$

transcription elongation regulator Bye1 (predicted) $\quad \begin{array}{lllll}0.19587321 & 6.93430188 & 0.06698819\end{array}$ \#N/A

translation initiation factor elF1

endoplasmic reticulum EF hand protein (predicted) $\quad \begin{array}{llll}0.19517939 & 7.34813545 & 0.08039455\end{array}$

zf-CCCH type zinc finger protein, unknown biological role $\mathrm{Cp} 0.194960096 .809203580 .12715659$

cAMP-dependent protein kinase catalytic subunit Pka1 $\quad \begin{array}{lllll}0.19488203 & 7.60509579 & 0.0614526\end{array}$

$\begin{array}{llllll}\text { serine/threonine protein phosphatase Ppa2 } & 0.19474573 & 8.70724403 & 0.01786707\end{array}$

spore wall assembly ADAM family peptidase Mde10 $\quad \begin{array}{lllll}0.19446736 & 6.19678224 & 0.2891651\end{array}$

aminotransferase class-III, possible transaminase, unknowr $0.19355528 \quad 8.08445491 \quad 0.02556076$

mitochondrial hydrogen/potassium transport system protei $\begin{array}{lllll}0.19336301 & 3.83174446 & 0.58272781\end{array}$

mitochondrial recombinase Mhr1 (predicted) $\quad 0.19327844 \quad 6.04844863 \quad 0.22321158$

ubiquinol-cytochrome-c reductase complex subunit 8 , hinge $0.19324523 \quad 7.776597950 .04064208$

$\mathrm{N}$-acetyltransferase (predicted)

(predicted)

microtubule-anchoring factor Msd

$\begin{array}{crrrr}\text { membrane associated ubiquitin-protein ligase E3, MARCH f } & 0.19167472 & 7.6596653 & 0.07442949 \\ \text { \#N/A } & 0.19153988 & 1.58411855 & 0.81420791\end{array}$ \#N/A

-type ATPase V1 subunit D (predicted)

ATPase with bromodomain protein

P-TEFb-cap methyltransferase Pcm1 $\begin{array}{lll}0.20117078 \quad 6.50830357 & 0.11885676\end{array}$

$\begin{array}{llll}0.19949877 & 6.22975601 & 0.19403821\end{array}$

$\begin{array}{llll}0.19945532 & 7.95750625 & 0.03616321\end{array}$

$0.19876985 \quad 5.77960835 \quad 0.255149$

$\begin{array}{llll}0.19846131 & 6.69028252 & 0.13801426\end{array}$

$\begin{array}{llll}0.19841664 & 4.55822488 & 0.44535516\end{array}$

$\begin{array}{llll}0.19813906 & 7.60161935 & 0.06378727\end{array}$

$\begin{array}{llll}0.19796205 & 7.11220116 & 0.06546187\end{array}$

$\begin{array}{llll}0.19787617 & 7.64657625 & 0.06572803\end{array}$

$\begin{array}{llll}0.19738691 & 2.84291301 & 0.81953371\end{array}$

$\begin{array}{llll}0.1971746 & 7.74304817 & 0.03850355\end{array}$

$\begin{array}{llll}0.19699651 & 7.65184803 & 0.09303071\end{array}$

$\begin{array}{llll}0.19695871 & 5.54378607 & 0.26756168\end{array}$

$\begin{array}{llll}0.19694853 & 7.4509986 & 0.04738405\end{array}$

$\begin{array}{llll}0.19691066 & 7.13829944 & 0.06898723\end{array}$

$\begin{array}{llll}0.19649777 & 5.77535775 & 0.2767946\end{array}$

$\begin{array}{llll}0.19632694 & 3.81011862 & 0.59447699\end{array}$

$\begin{array}{llll}0.19561847 & 5.7551525 & 0.31197159\end{array}$

$\begin{array}{llll}0.19533346 & 7.33535818 & 0.06102923\end{array}$

$\begin{array}{llll}0.19532936 & 1.58398852 & 0.81304932\end{array}$

$\begin{array}{llll}0.19313122 & 7.47479013 & 0.03966067\end{array}$

$\begin{array}{llll}0.19256232 & 6.45622619 & 0.14560543\end{array}$

$\begin{array}{llll}0.19250076 & 8.16174603 & 0.02541438\end{array}$

$\begin{array}{llll}0.19220719 & 2.70719798 & 0.8609173\end{array}$

$\begin{array}{llll}0.19218293 & 4.14328565 & 0.59925698\end{array}$

$\begin{array}{llll}0.19204639 & 5.71761974 & 0.22600276\end{array}$

0.181920889

0.344195074

0.867295586

0.551866955

0.422925119

0.273948137

0.065536796

0.345388712

0.206188151

0.541282701

0.106756604

0.109023012

0.109385979

0.869086317

0.069170174

0.147776369

0.358840601

0.082629798

0.113890856

0.368824607

0.678305821

0.519047528

0.111129843

0.405688263

0.102594847

0.863825232

0.130010665

0.192545753

0.103255534

0.03552014

0.381868528

0.048492037

0.669163473

0.308354613

0.072495251

0.07098655

0.459815663

0.215588542

0.0482547

0.904375152

0.68261529

0.311704251

0.121636097

0.864786147

0.115986273

0.124870334

$\begin{array}{llll}0.19090071 & 8.39004573 & 0.07670213\end{array}$

$\begin{array}{llll}0.19031445 & 5.90532336 & 0.3315185\end{array}$

0.426059191 
SPCC285.03

SPBC1718.05

SPAC4F10.03C

SPBC17G9.02C

SPBC17A3.02

SPCC16A11.17

SPBC32F12.06

SPAP27G11.07C

SPNCRNA.1397

SPCC1322.12C

SPAC23H4.12

SPAC1F7.11C

SPNCRNA.1290

SPAC4F10.07c

SPCC1742.01

SPNCRNA.1132

SPCC24B10.22

SPNCRNA.82

SPAC1F3.04C

SPBC2A9.06C

SPAC23C11.14

SPAC1142.01

SPCC16C4.11

SPAC2F 7.03C

SPCC417.09c

SPBC13E7.11

SPCC1223.01

SPAC23D3.02

SPBC646.08C

SPBC8D2.04

SPCC1322.09

SPBC1861.09

SPCC970.06

SPBC21B10.03C

SPAC1A6.01C

SPAC57A10.10C

SPBC660.11

SPAC26A3.01

SPNCRNA.1407

SPBC1539.09c

SPAC637.10c

SPBC25B2.03

SPAC17G6.10

SPNCRNA.1354

SPAC13G7.05

SPBC106.04

SPCP1E11.04C pog1

mrp1

tsr3

nus1

zhf1

rqc1

pef1

pom1

SPCC417.09c

rbd1

SPCC1223.01

rfc2

SPBC646.08C hht2

SPCC1322.09

ppk22

erv29

ath1

SPAC1A6.01c

sla1

$\operatorname{tcg} 1$

sxa1

rpn10

SPBC25B2.03 ssr1

\#N/A

are1

ada1

pal1
ATP-dependent RNA helicase Dbp6 (predicted)

TRAPP complex subunit Trs 31 (predicted)

tRNA 2'-O-ribose methyltransferase Trm7 (predicted)

$\begin{array}{lll}0.18935041 & 7.7674341 & 0.03673011\end{array}$

$0.1892373 \quad 5.39031501 \quad 0.33573722$

$\begin{array}{llll}0.18899199 & 5.83207099 & 0.25055863\end{array}$

RNA polymerase II accessory factor, Cdc73 family (predicter 0.188938546 .813955330 .09561867

mitochondrial conserved fungal membrane protein Aim19 $\quad \begin{array}{llll}0.18861 & 5.2861319 & 0.39797726\end{array}$

MCM complex subunit Mcm4/Cdc21

P-TEFB associated cyclin, cyclin T Pch1

$\begin{array}{lll}0.1881901 & 7.35268203 & 0.05157909\end{array}$ $\begin{array}{lll}0.18755755 & 7.71750335 & 0.06983014\end{array}$

EKC/KEOPS complex associated ATPase Bud32 (predicted) $0.18742797 \quad 5.788996890 .30806975$

$$
\text { \#N/A }
$$

$0.18740308 \quad 6.54152461 \quad 0.17659905$

$\begin{array}{llll}0.18701896 & 6.92519337 & 0.1342096\end{array}$

MRG family Clr6 histone deacetylase complex subunit Alp1 $0.18697236 \quad 7.2503718 \quad 0.07621399$

transcription factor, zf-fungal binuclear cluster type (predic $0.1865681 \quad 6.90222602 \quad 0.12394492$$$
\# \mathrm{~N} / \mathrm{A}
$$

$\begin{array}{llll}0.18649469 & 6.76137939 & 0.27778855\end{array}$

autophagy associated protein kinase regulatory subunit Atg $0.18638864 \quad 6.80789469 \quad 0.12490403$ cell surface glycoprotein, galactose-specific flocculin Gsf2 $\quad 0.18604567 \quad 7.99477047 \quad 0.07502136$ \#N/A

mitochondrial DNA polymerase gamma Pog1

RNAse MRP

SSU-rRNA maturation protein Tsr3 (predicted)

di-trans, poly-cis-decaprenylcistransferase Nus 1

ER zinc ion transmembrane transporter Zhf1

$\begin{array}{llll}0.18598806 & 7.25507889 & 0.17113702\end{array}$

$\begin{array}{llll}0.18591233 & 7.26653388 & 0.08244768\end{array}$

$\begin{array}{lll}0.18589068 & 7.93014426 & 0.05085921\end{array}$

$\begin{array}{lll}0.18587648 & 6.71695942 & 0.1552662\end{array}$

$\begin{array}{lll}0.1856162 & 5.79165963 & 0.2990688\end{array}$

$0.18524397 \quad 7.40084051 \quad 0.14636449$

ribosome quality control complex (RQC) complex subunit Rs $0.18477151 \quad 7.310456150 .08506128$

$\begin{array}{lllll}\text { Pho85/PhoA-like cyclin-dependent kinase Pef1 } & 0.18475222 & 5.8996156 & 0.38697699\end{array}$

$\begin{array}{lllll}\text { DYRK family cell polarity protein kinase Pom } 1 & 0.18473033 & 7.852596 & 0.02895188\end{array}$

transcription factor (predicted)

mitochondrial rhomboid protease (predicted)

$\begin{array}{llll}0.18444404 & 5.73206243 & 0.31028824\end{array}$

ubiquitin-protein ligase E3 involved in rescue of stalled ribo $0.18404687 \quad 6.32910106 \quad 0.19468544$

DNA replication factor $C$ complex subunit $\mathrm{Rfc} 2$

sterol intermembrane transfer protein (predicted)

$\begin{array}{llll}0.18377775 & 5.96802771 & 0.29273421\end{array}$

$\begin{array}{llll}0.1837229 & 8.07587101 & 0.06723401\end{array}$

0.183026637 .393899110 .10428952

Golgi protein, associated with COP vesicles (predicted), DU 0.182773348 .142159380 .05187758 serine/threonine protein kinase Ppk22 (predicted) $\quad \begin{array}{lllll}0.18266162 & 6.48160122 & 0.21917487\end{array}$ COP II adaptor Erv29 (predicted)

ataxin-2 homolog

$\begin{array}{lll}0.18206426 & 7.30441476 & 0.07512318\end{array}$

$0.18183437 \quad 9.48087682 \quad 0.01102384$

human thyroid receptor interacting protein homolog, transc $0.18181229 \quad 7.54872546 \quad 0.05772557$

La protein homolog

single-stranded telomeric binding protein Tgc1

aspartic protease Sxa1

\#N/A

$0.18137393 \quad 7.23735174 \quad 0.11328889$

$\begin{array}{llll}0.18129325 & 7.96872572 & 0.07926584\end{array}$

$\begin{array}{llll}0.18091507 & 9.3721808 & 0.01175084\end{array}$

$\begin{array}{llll}0.18082212 & 7.14477575 & 0.09308384\end{array}$

anthranilate synthase component II, multifunctional enzym $0.17988777 \quad 8.714600350 .02478245$

19S proteasome regulatory subunit Rpn10 $\quad \begin{array}{llllll}0.17984857 & 7.40409351 & 0.05582543\end{array}$

zf-C3HC4 type zinc finger

$\begin{array}{llll}0.17962315 & 7.1354318 & 0.10724794\end{array}$

$\begin{array}{lll}0.17953322 & 7.30720055 & 0.10682877\end{array}$

$0.17947273 \quad 6.92217651 \quad 0.17411259$

$\begin{array}{lllll}\text { acyl-coA-sterol acyltransferase Are1 (predicted) } & 0.17939047 & 5.81189299 & 0.28569071 \\ \text { adenosine deaminase Ada1 } & 0.17898516 & 8.27544931 & 0.04381933 \\ \text { membrane associated protein Pal1 } & 0.17893018 & 7.67172222 & 0.07149758\end{array}$

$\begin{array}{lllll}\text { acyl-coA-sterol acyltransferase Are1 (predicted) } & 0.17939047 & 5.81189299 & 0.28569071 \\ \text { adenosine deaminase Ada1 } & 0.17898516 & 8.27544931 & 0.04381933 \\ \text { membrane associated protein Pal1 } & 0.17893018 & 7.67172222 & 0.07149758\end{array}$
0.066404997

0.430181096

0.340397576

0.1510411

0.493393197

0.089007709

0.115086965

0.401458191

0.253428199

0.201621676

0.124160418

0.188437263

0.369859332

0.189641218

0.122397595

0.246525704

0.133014803

0.087931122

0.227614501

0.391980582

0.21647693

0.136454977

0.482719965

0.054021811

0.107380337

0.403885126

0.274679138

0.385459805

0.111483286

0.162243155

0.089464508

0.303826636

0.12253434

0.022997315

0.097960716

0.174182314

0.128337857

0.024357134

0.147826266

0.047186508

0.095234902

0.166170815

0.165634495

0.25033513

0.378750295

0.077431974

0.117436893 
SPCC825.01

SPBC1289.04C SPAC3F10.07C

SPCC622.16c

SPAC17A5.12

SPAC19E9.02

SPAC5H $10.13 \mathrm{C}$

SPNCRNA.922

SPAC664.02C

SPAC15E1.05C

SPBC1539.01C

SPCC1322.06

SPBC800.07C

SPBC12D12.02C

SPAC8C9.12C

SPAPYUG 7.05

SPAC806.02C

SPBC800.13

SPAC6G9.01C

SPBC1D7.05 not3

SPCC1672.09

rna4

brr2

ssr4

sif3

toe4

rpb5

pet1

ams2

SPCC825.01

pob1

erf4

epe1

ucp7

fin1

gmh2

arp8

ect1

mrp15

kap113

tsf1

cdm1

mrs3

SPAPYUG7.05

SPAC806.02C

cnp20

SPAC6G9.01c byr2
\#N/A

methionine synthase reductase (predicted) metacaspase Pca1

1-phosphatidylinositol 4-kinase Stt4

Golgi serine carboxypeptidase (predicted) $\begin{array}{llll}0.17878451 & 6.55053022 & 0.14463523 \\ 0.17761716 & 7.29040376 \quad 0.09316059\end{array}$ $\begin{array}{lll}0.177613 & 8.49997898 & 0.0227759\end{array}$ $\begin{array}{lll}0.17722063 & 9.10449487 & 0.0646112\end{array}$ $\begin{array}{lll}0.17715734 & 6.80642225 & 0.13782254\end{array}$ mitochondrial [2Fe-2S] cluster asssembly NADPH-ferredoxi $\begin{array}{lllll}0.17676132 & 7.74781945 & 0.1107497\end{array}$ $\begin{array}{lllll}\text { NAD-dependent glutamate dehydrogenase Gdh2 } & 0.1753145 & 8.80826328 & 0.0587627\end{array}$ rRNA processing protein Rrp15 (predicted) $\quad 0.17530506 \quad 4.97050542 \quad 0.45556663$ dol-P-Man:Man(5)GIcNAc(2)-PP-Dol alpha-1,3-mannosyltra $0.17491317 \quad 5.953726 \quad 0.26821056$ $\begin{array}{lllll}\text { ESCRT II complex subunit Vps25 } & 0.17467959 & 6.62885646 & 0.18931229\end{array}$ Schizosaccharomyces pombe specific protein, predicted GP $0.17453282 \quad 5.69704218 \quad 0.4590115$ $\begin{array}{llll}\text { mitochondrial tyrosine-tRNA ligase (predicted) } & 0.1740415 & 5.95419706 & 0.2808085\end{array}$ $\begin{array}{lllll}\text { SNARE associated Golgi protein (predicted) } & 0.17397762 & 5.93117825 & 0.28722146\end{array}$ DUF3807 family conserved fungal protein, domain associatı $\begin{array}{llll}0.17361156 & 4.449456 & 0.53397024\end{array}$ WD repeat protein, human WDR44 family $\quad \begin{array}{lllll}0.17332844 & 7.5095712 & 0.11427621\end{array}$ $\begin{array}{llllll}19 S & \text { proteasome regulatory subunit Rpn6 } & 0.17320497 & 8.36776747 & 0.0258073\end{array}$ transcription factor Gaf1

CCR4-Not complex NOT box subunit Not3/5

$0.17304235 \quad 8.54051911 \quad 0.07979299$

$\begin{array}{lll}0.1729601 & 7.71050282 & 0.08116821\end{array}$ triglyceride lipase-cholesterol esterase (predicted) $\quad \begin{array}{llll}0.17267744 & 6.5458757 & 0.31128467\end{array}$ U4/U6 x U5 tri-snRNP complex WD repeat subunit Rna4 $\begin{array}{rrrr}0.1723022 & 6.16308796 & 0.27544199\end{array}$ U5 snRNP complex subunit Brr2

SWI/SNF and RSC complex subunit Ssr4

$0.17225873 \quad 9.31033556 \quad 0.01073145$ $0.17162185 \quad 7.34609002 \quad 0.11042239$ mitochondrial protein, involved in mitochondrial gene expre $\begin{array}{lllll}0.17154052 & 6.3298119 & 0.26016042\end{array}$ transcription factor, zf-fungal binuclear cluster type(predict $0.17145543 \quad 7.751092260 .10700378$ DNA-directed RNA polymerase I, II and III subunit Rpb5 $\quad \begin{array}{lllll}0.17126776 & 6.72327175 & 0.15026829\end{array}$ Golgi phosphoenolpyruvate transmembrane transporter Pet $\begin{array}{llll}0.17114902 & 8.17935683 & 0.03414313\end{array}$ cell cycle regulated GATA-type transcription factor Ams2 $\quad \begin{array}{llll}0.17083873 & 8.0821011 & 0.10617077\end{array}$ ATPase, involved in cytoplasmic translational initiation (pre $0.17070211 \quad 8.40673186 \quad 0.031801$ Boi family protein

palmitoyltransferase complex subunit Erf4

Imjc domain chromatin associated protein Epe1

UBA/TPR/DNAJ domain protein Ucp7

serine/threonine protein kinase, NIMA related Fin1 alpha-1,2-galactosyltransferase $\mathrm{Gmh} 2$

$$
\# \mathrm{~N} / \mathrm{A}
$$

Ino80 complex actin-like protein Arp8

$0.17068628 \quad 8.59809057 \quad 0.05896037$ $\begin{array}{lll}0.17036911 & 4.89466836 & 0.44458575\end{array}$ $\begin{array}{lll}0.16999845 & 7.62146541 & 0.07142177\end{array}$ $\begin{array}{lll}0.16958131 & 6.89550931 & 0.13886615\end{array}$ $\begin{array}{lll}0.16939897 & 7.5626768 & 0.1051985\end{array}$ $0.16939317 \quad 7.91230106 \quad 0.05706138$ $0.16918429 \quad 6.53487752 \quad 0.17300697$ $\begin{array}{lll}0.16916531 & 6.72296376 & 0.16196543\end{array}$ ethanolamine-phosphate cytidylyltransferase (predicted) $\quad \begin{array}{lllll}0.16913817 & 6.91921767 & 0.1336845\end{array}$ mitochondrial ribosomal protein subunit L15 Mrp15 (predic 0.168719536 .229352830 .30603679 karyopherin/importin beta family nuclear import signal rec $0.16791094 \quad 6.63900695 \quad 0.20928064$ mitochondrial translation elongation factor EF-Ts Tsf1 $\quad \begin{array}{lllll}0.16764308 & 7.51068378 & 0.10202124\end{array}$ DNA polymerase delta subunit Cdm1

mitochondrial carrier, iron ion Mrs3 (predicted)

delta-1-pyrroline-5-carboxylate reductase (predicted) CIA machinery CIA1/CFD1 fusion protein (predicted)

$\begin{array}{llll}0.16756014 & 6.33462469 & 0.33748796\end{array}$ $\begin{array}{lll}0.16688864 & 6.22909155 & 0.30916102\end{array}$ $0.16684827 .26211986 \quad 0.10275437$ $\begin{array}{rrr}0.1663463 & 5.78474157 & 0.35025013\end{array}$ MAP kinase kinase kinase Byr2
0.214338578 0.147913633 0.043856824 0.107790937

0.205946941 0.171051233 0.099373793

0.550644923

0.359500359

0.268221974

0.554218105

0.373441833

0.380039285

0.625308467

0.175581307

0.048891616

0.129099208

0.131074915

0.405027185

0.367474597

0.022476926

0.170623156

0.350711507

0.165868039

0.221454389

0.062407743

0.164651803

0.058583061

0.09965854

0.540540647

0.117340685

0.207233365

0.163468956

0.096928449

0.249008553

0.23586021

0.200999344

0.399650812

0.291894807

0.159562063

0.432017606

0.4025719

0.16040308

0.333795222

0.66360855

0.44558836

0.398611798 
SPAC22H10.08

SPCC613.01

SPAC227.07c

SPAC1486.01

SPNCRNA.742

SPAC17A5.02C

SPAC1F12.02C

SPAC9E9.15

SPBC17D11.01

SPNCRNA.705

SPBC25D12.02C

SPAC13G7.10

SPAC9G1.09

SPCC1672.10

SPAC4C5.02C

SPNCRNA.1464

SPBC106.14c

SPNCRNA.905

SPNCRNA.761

SPBC725.16

SPAC26A3.12C

SPCC320.11C

SPAC9.06c

SPAC3H5.06c

SPAC139.05

SPAC26A3.16

SPBC2D10.11C

SPAC2C4.13

SPBC660.06

SPAC11E3.15

SPAC22E12.11C

SPBC2G 2.05

SPNCRNA.1051

SPAPB24D3.02C

SPAC1635.01

SPNCRNA.1565

SPCC16A11.01

SPNCRNA.315

SPAC29A4.04C
SPAC22H10.08

SPCC613.01

pab1

sod2

dbr1

\#N/A

tma19

SPAC9E9.15

nep1

\#N/A

dnt

teb1

sid1

mis16

ryh1

sda1

res1

dhp1

nip7

SPAC9.06c

pol1

SPAC139.05

dsk2

nap2

vma16

SPBC660.06

rpl22

set3

rpl1603

\#N/A

SPAPB24D3.02C por1

\#N/A

sfk1

\#N/A
\#N/A

$0.16595646 \quad 7.35236604 \quad 0.1187157$ $\begin{array}{lllll}\text { TIM23 translocase complex subunit Tim50 (predicted) } & 0.16574958 & 7.21550256 & 0.10831102 \\ \text { inorganic phosphate transmembrane transporter (predictec } & 0.16569784 & 10.0346376 & 0.01341735\end{array}$ $\begin{array}{llllll}\text { Armadillo repeat protein, human ATXN10 ortholog } & & 0.16497183 & 5.29770668 & 0.38183082\end{array}$ $0.16486433 \quad 6.65774598 \quad 0.21578884$ mitochondrial threonine-tRNA ligase (predicted) $\quad \begin{array}{lllll}0.16474945 & 6.9721912 & 0.22474894\end{array}$ calcium transport regulatory factor (predicted) $\quad \begin{array}{lllll}0.16457326 & 5.9809989 & 0.31177431\end{array}$ cleavage factor two Cft2/polyadenylation factor CPSF-73 ( $\mathrm{p} \quad 0.16432194 \quad 7.35140456 \quad 0.12261008$ DUF2009 family protein, conserved in yeast and apicomple) $0.16401096 .37059125 \quad 0.22743854$ $\begin{array}{lllll}\text { transmembrane transporter (predicted) } & 0.16392416 & 6.48317988 & 0.23789412\end{array}$ protein phosphatase PP2A regulatory subunit B-55 Pab1 $\begin{array}{llllll}0.16392341 & 7.7119888 & 0.08453617\end{array}$ mitochondrial superoxide dismutase Sod2

$$
\# N / A
$$

$0.1639188 \quad 7.80860724 \quad 0.06867401$ $\begin{array}{lll}0.16390569 & 6.31582601 & 0.28430744\end{array}$ $\begin{array}{llll}0.16384709 & 6.12143264 & 0.36644504\end{array}$ $\begin{array}{lllllll} & 0.16384709 & 6.12143264 & 0.36648504\end{array}$ mitochondrial protein complex assembly protein (predicted $0.16293595 \quad 5.78710911 \quad 0.37996155$ NEDD8 protease Nep1

$$
\text { \#N/A }
$$

$0.162913526 .87044452 \quad 0.1991955$

nucleolar protein Dnt1

$\begin{array}{llll}0.1629085 & 5.20612464 & 0.41379491\end{array}$ $\begin{array}{llll}0.16268524 & 7.19370361 & 0.10885172\end{array}$ $\begin{array}{lll}0.16260098 & 6.4970241 & 0.26423885\end{array}$ $\begin{array}{llll}0.16197177 & 8.5057166 & 0.05811055\end{array}$ $\begin{array}{llll}0.16189789 & 6.81426716 & 0.15521093\end{array}$ $0.161273897 .67779073 \quad 0.13421693$ $\begin{array}{llll}0.16125458 & 5.29839975 & 0.44176825\end{array}$ $\begin{array}{llll}0.16092499 & 7.01835162 & 0.20052727\end{array}$ $\begin{array}{llll}0.16092471 & 6.41002439 & 0.27188358\end{array}$ $\begin{array}{llll}0.16083685 & 6.92024853 & 0.16215084\end{array}$ $\begin{array}{llll}0.16075216 & 6.85763884 & 0.18502408\end{array}$ $\begin{array}{lllll}5 '-3 ' \text { exoribonuclease Dhp1 } & 0.16010746 & 8.76690742 & 0.04498938 \\ \text { RNA-binding ribosome biogenesis protein Nip7 (predicted) } & 0.15978835 & 6.01124869 & 0.40696553\end{array}$ $\begin{array}{llllll}\text { RNA-binding ribosome biogenesis protein Nip7 (predicted) } & 0.15978835 & 6.01124869 & 0.40696553\end{array}$ DNA polymerase alpha catalytic subunit

$\begin{array}{lllll}\text { succinate-semialdehyde dehydrogenase (predicted) } & 0.15926824 & 6.32737144 & 0.3813433\end{array}$ UBA domain protein Dph1/Dsk2

histone H2A-H2B chaperone Nap2

V-type ATPase V0 subunit c" (predicted)

WW domain containing conserved fungal protein

$60 S$ ribosomal protein L22 (predicted)

histone lysine methyltransferase Set3

$60 S$ ribosomal protein L13/L16 (predicted)

$$
\text { \#N/A }
$$

$\begin{array}{llll}0.15875784 & 7.9129743 & 0.07469566\end{array}$

$\begin{array}{llll}0.15822887 & 7.70622752 & 0.13541252\end{array}$

$\begin{array}{llll}0.15789438 & 8.01328815 & 0.0632644\end{array}$ $\begin{array}{lll}0.15784166 & 8.60377944 & 0.03936524\end{array}$ $\begin{array}{llll}0.15774351 & 8.06842522 & 0.05055014\end{array}$ $\begin{array}{lllll}0.15771772 & 8.15114588 & 0.07062918\end{array}$ $0.1574197 \quad 7.871901840 .09006371$ $\begin{array}{lll}0.15741863 & 8.74195162 & 0.034255\end{array}$ $\begin{array}{lllll}\text { amino acid transmembrane transporter (predicted) } & 0.15731751 & 7.43152547 & 0.14918124 \\ \text { mitochondrial outer membrane voltage-dependent anion-sı } & 0.15723223 & 8.44246673 & 0.11291303\end{array}$ $\# \mathrm{~N} / \mathrm{A}$ $\begin{array}{llll}0.1571772 & 5.77766692 & 0.33472578\end{array}$ plasma membrane protein involved in inositol lipid-mediat؛ $\begin{array}{llll}0.15708398 & 7.17526448 & 0.18976269\end{array}$ $\# \mathrm{~N} / \mathrm{A}$ $0.15693384 \quad 3.40410974 \quad 0.73177647$

0.18174586 0.167627129 0.027443666 0.477463932

0.299865843 0.310163568 0.405509203

0.186658069 0.313430385

0.325925

0.135772623

0.113511456 0.377210394 0.461788608

0.065653634 0.475527853 0.279951542 0.508831652

0.16842563

0.355331135 0.098491227

0.227582498 0.201621676 0.537787651

0.28124239

0.363438678

0.236079692

0.2629676

0.078966399 0.502248905 0.432958975

0.185348656 0.476941951

0.121953919

0.202969643 0.105959693 0.070569133 0.087597074

0.116188915

0.143700716

0.062578588

0.220069013

0.173722179

0.428965894

0.268692281

0.799171801 pseudouridine synthase Cbf5 (predicted)

0.064153795 
SPAC343.17c

SPBTRNAPHE.03

SPBC19F5.05

SPBC1E8.02

SPCC63.10c

SPAC13G6.06C

SPAC26F 1.02

SPBC11G11.03

SPAC10F6.06

SPNCRNA.837

SPAC1556.08c

SPBC947.03C

SPBC146.04

SPBC543.04

SPBC15D4.10c

SPNCRNA.1225

SPBC1734.05C

SPBC29A3.02C

SPAC26F1.12C

SPBC409.19C

SPCC1223.06

SPNCRNA.1435

SPBC13E7.08C

SPCC1494.07

SPNCRNA.811

SPBC13E7.07

SPBC27.02C

SPAC607.06C

SPCC1682.09c

SPNCRNA.674

SPAC1D4.13

SPCC126.14

SPBC21H7.06C

SPAC6G9.04

SPNCRNA.1476

SPAC4G8.13C

SPAC24C9.02C

SPCC645.10

SPNCRNA.284

SPAC10F6.08C

SPAC23A1.19c

SPAC30.04

SPAC1952.08C

SPAP27G11.16
SPBTRNAPHE.03

ppp1

SPBC1E8.02

sec59

gcv2

pnn1

mrt4

$\# N / A$

cbs 2

naa38

erv2

npr3

amo1

$$
\# N / A
$$

spf31

his7

hgh1

$\mathrm{mtx} 2$

tea1

leo1 trm72

\section{$\# \mathrm{~N} / \mathrm{A}$}

SPBC13E7.07

ask1

SPAC607.06c

ggc1

\#N/A

byr1

prp18

opi10

spo7

\#N/A

prz1

cyt2

cca2

\#N/A

nht1

hrq1

abc4

SPAC1952.08c

SPAP27G11.16 mitochondrial F1-FO ATP synthase chaperone Atp11 (predic 0.155812396 .357753230 .26041687 DDB1-CUL4-associated factor (DCAF), Wdr70 $\quad \begin{array}{lllll}0.15561311 & 9.28388013 & 0.02729817\end{array}$

tRNA Phenylalanine

pescadillo-family BRCT domain protein Ppp1 (predicted) $\begin{array}{lllll}0.15527698 & 7.56405876 & 0.12085951\end{array}$ ER ubiquitin family protein (predicted)

dolichol kinase Sec59 (predicted)

$\begin{array}{llll}0.15500737 & 5.52567523 & 0.47495549\end{array}$

glycine cleavage complex subunit P (predicted)

splicing factor, pinin ortholog Pnn1

$\begin{array}{lll}0.15512201 & 6.28008797 & 0.29310998\end{array}$

$\begin{array}{llll}0.15485332 & 8.81330886 & 0.04314904\end{array}$

$\begin{array}{lll}0.15470525 & 4.28862041 & 0.71297419\end{array}$

mRNA turnover and ribosome assembly protein Mrt4 (pred 0.154332297 .104556940 .14267043

RNA-binding protein Vip1

$\begin{array}{llll}0.15419039 & 8.22599045 & 0.07734741\end{array}$

$\begin{array}{lll}0.15409975 & 7.42828572 & 0.18817881\end{array}$

$0.15381323 \quad 7.43696549 \quad 0.13766031$

AMP-activated protein kinase gamma subunit Cbs2

$\begin{array}{lllllll}\mathrm{NatC} N \text {-acetyltransferase non-catalytic Sm-like domain sub } & 0.15358794 & 8.32833116 & 0.08952871 \\ \text { ER thiol oxidase Erv2 (predicted) } & 0.15340453 & 5.39767849 & 0.42342148\end{array}$

ER thiol oxidase Erv2 (predicted)

$\mathrm{SEA} / \mathrm{Im} 1 / \mathrm{Npr} 2 / 3$ complex subunit Npr3

nuclear rim protein Amo1

\#N/A

$\begin{array}{llll}0.15333729 & 6.68913284 & 0.25662774\end{array}$

$0.15332073 \quad 6.40890266 \quad 0.31533686$

$\begin{array}{lll}0.15323254 & 5.7217848 & 0.38485961\end{array}$

$\begin{array}{lrrrr}\text { DNAJ protein, splicing factor Spf31 (predicted) } & 0.15317723 & 5.4618857 & 0.44564954 \\ \text { phosphoribosyl-AMP cyclohydrolase/phosphoribosyl- ATP p) } & 0.15299533 & 8.18196266 & 0.09905583\end{array}$

phosphoribosyl-AMP cyclohydrolase/phosphoribosyl-ATP p) $0.15295330 .186010136 \quad 0.26537383$

$\begin{array}{llllll}\text { metaxin } 2 \text { Mtx2 (predicted) } & 0.15286425 & 7.95512675 & 0.07290182\end{array}$ cell end marker Tea 1

\#N/A

$0.152755637 .89939674 \quad 0.12040753$

$\begin{array}{llll}0.15274348 & 4.58943417 & 0.56184724\end{array}$

RNA polymerase II associated Paf1 complex subunit Leo1 $\quad \begin{array}{lllll}0.15260053 & 7.60500041 & 0.10258299\end{array}$ tRNA 2'-O-methylase subunit Trm72 (predicted) $\quad \begin{array}{lllll}0.15258655 & 8.13353679 & 0.06087489\end{array}$

$\begin{array}{lll}0.15246003 & 2.60958557 & 0.7872634\end{array}$

$0.15228897 .60652155 \quad 0.10273837$

$0.15214195 \quad 5.06581517 \quad 0.53579905$

$0.15194475 \quad 6.9929818 \quad 0.24745831$

DASH complex subunit Ask1

mitochondrial carrier, guanine nucleotide Ggc1 (predicted) $0.15191456 \quad 6.20660395 \quad 0.3035962$

$\begin{array}{lll}0.15177617 & 8.05825065 & 0.09400567\end{array}$

$0.151528428 .05940724 \quad 0.09480553$

$0.15136337 \quad 7.06621398 \quad 0.16868538$

$0.15100481 \quad 5.04051614 \quad 0.59917416$

$\begin{array}{lll}0.15093804 & 6.66883161 & 0.26707853\end{array}$

$\begin{array}{llll}0.15069697 & 5.35447983 & 0.57080805\end{array}$

$\begin{array}{llll}0.15061133 & 9.10988891 & 0.08459978\end{array}$

$\begin{array}{lll}0.15028534 & 6.93990132 & 0.21223513\end{array}$

$\begin{array}{llll}0.15013539 & 6.89016865 & 0.21631307\end{array}$

$\begin{array}{lll}0.14973152 & 2.36190802 & 0.881994\end{array}$

$\begin{array}{lll}0.14972102 & 5.77398739 & 0.43086319\end{array}$

$\begin{array}{lll}0.14954301 & 7.26295793 & 0.13455917\end{array}$

3157

vacuolar phytochelatin and glutathione $S$-conjugate $A B C$ far 0.1

pyridoxamine 5'-phosphate oxidase (predicted) $\quad \begin{array}{lllll}0.14912311 & 7.61582715 & 0.15834848\end{array}$

Schizosaccharomyces pombe specific protein

$\begin{array}{lll}0.1487841 & 3.20270768 & 0.75979879\end{array}$
0.350987684

0.051372805

0.184529725

0.385805301

0.569604229

0.076366508

0.7821682

0.212027145

0.125648744

0.266782661

0.205794906

0.142981187

0.51842086

0.346916831

0.409240131

0.480544892

0.541491617

0.155711466

0.356398045

0.119570237

0.184004765

0.651229852

0.160219696

0.102385967

0.843142503

0.16040308

0.626802908

0.337127889

0.396920678

0.148977319

0.150046862

0.243975351

0.68261529

0.35833387

0.659208633

0.135810692

0.295591499

0.300471614

0.91998301

0.526367309

0.201912939

0.071575103

0.231484724

0.821485666 
SPNCRNA.731

SPAC23C4.18C

SPAC11E3.10

SPNCRNA.1067

SPAC4F10.14c

SPNCRNA.80

SPBC2D10.08C

SPAC4F10.04

SPNCRNA.1575

SPBC1E8.03C

SPAC17G6.17

SPAC1687.09

SPAC2F7.15

SPCC550.10

SPAC23C11.11

SPAC4H3.02C

SPCC1739.01

SPNCRNA.1450

SPNCRNA.614

SPAP27G11.09c

SPCC736.14

SPCC1494.10

SPAC3H1.02c

SPBC1347.07

SPBC13A2.01C

SPAC31A2.04C

SPCC188.06C

ScpofMt14

SPAC343.10

SPAC4A8.06C

SPBC25H2.12C

SPAC23H3.06

SPBC2A9.11C

SPBC19C7.04C

SPBC2A9.02

SPAC26H5.06

$\mathrm{SPBC} 2 \mathrm{H} 8.03$

SPBC32H8.04C

SPBC776.17

SPAC22G7.03

SPBC1198.10c

SPCC18B5.10C

SPAC22E12.05C

SPAC2C4.11C

myosin type I

$\begin{array}{llll}0.14841694 & 4.84260707 & 0.54058219\end{array}$ $\begin{array}{lllll}0.1484074 & 9.55778183 & 0.05620144\end{array}$ $\begin{array}{llll}0.1477513 & 6.9866982 & 0.32084476\end{array}$

\#N/A

SPBC2D10.08C ypa1

$\# \mathrm{~N} / \mathrm{A}$

SPBC1E8.03C pof8

rsm24

atd3

cka1

swc3

SPCC1739.01

\#N/A

rib1

dis1

adn3

sdd3

rex2

cbc2

pre1

srp54

\#N/A

met11

SPAC4A8.06c

cct7

apl6

iss9

SPBC19C7.04C

SPBC2A9.02

pot1

bem 46

utp24

SPAC22G7.03

slm5

tex1

rer1

pno1 $\begin{array}{lll}0.14721509 & 7.6506558 & 0.19476514\end{array}$ $\begin{array}{llll}0.14682885 & 7.5910086 & 0.15335634\end{array}$ $\begin{array}{llll}0.14672427 & 6.75724978 & 0.25544208\end{array}$ $0.14628482 \quad 4.65330554 \quad 0.59241345$ $0.14626242 \quad 8.15757377 \quad 0.06906226$ 0.145767061 .16931716

complex beta subunit

$\# N / A$

BRCT domain protein Rad

Golgi multispanning membrane VanZ-like family protein $\begin{array}{crrrr}\text { mitochondrial ribosomal protein subunit Yml6 (predicted) } & 0.1454924 & 6.26680201 & 0.32651351 \\ \text { protein } & 0.14576706 & 1.16931716 & 1 \\ \end{array}$ protein phosphatase type 2A regulator, PTPA family Ypa1 $0.14539793 \quad 5.71609157 \quad 0.38206241$ ER membrane protein, conserved in fungi plants and protoz $0.14521658 \quad 5.90533017 \quad 0.45939455$ $\begin{array}{lllll}\text { LARP7 family RNA-binding protein Pof8/Lar7 } & 0.14520342 & 5.38154828 & 0.48111052\end{array}$ autophagy/CVT pathway ENTH/VHS domain protein Irs4 (pr $0.14510874 \quad 8.17873501 \quad 0.15313199$ mitochondrial ribosomal protein subunit S24 (predicted) $\begin{array}{lllll}0.14506743 & 6.09075713 & 0.40428462\end{array}$ $\begin{array}{llllll}\text { betaine-aldehyde dehydrogenase Atd3 (predicted) } & 0.14430698 & 6.28758693 & 0.29703732\end{array}$ serine/threonine protein kinase Cka1 $\quad 0.14402316 \quad 8.08975207 \quad 0.08421975$ Swr1 complex subunit Swc3

zf-CCCH type zinc finger protein

$$
\# N / A
$$

$0.14378567 \quad 5.70796709 \quad 0.44958657$ $\begin{array}{lll}0.14352235 & 8.33933573 & 0.15842651\end{array}$ $0.14333197 \quad 5.62594702 \quad 0.50826688$ $0.14328972 \quad 5.64265194 \quad 0.48851369$ $\begin{array}{lll}0.14298924 & 7.43053788 & 0.18009304\end{array}$ GTP cyclohydrolase Rib1 (predicted)

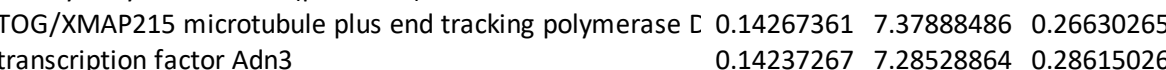
transcription factor Adn3

mitochondrial protease Sdd3 (predicted)

RNA exonuclease (predicted)

nuclear cap-binding complex small subunit (predicted)

205 proteasome complex subunit beta 4 Pre1

signal recognition particle subunit Srp54

$$
\text { \#N/A }
$$

methylenetetrahydrofolate reductase Met11

$0.14225131 \quad 7.741501110 .16877266$

$\begin{array}{llll}0.14212797 & 6.92809912 & 0.25748185\end{array}$

$0.14188247 \quad 7.82172935 \quad 0.16979554$ $\begin{array}{lll}0.14137291 & 6.41946433 & 0.33245575\end{array}$ $\begin{array}{lll}0.14135127 & 8.05622077 & 0.10430769\end{array}$ $0.14129762 \quad 2.36210963 \quad 0.88387225$ $\begin{array}{lll}0.14126941 & 6.27393458 & 0.33293328\end{array}$ esterase/lipase, implicated in fatty acid biosynthetic proces $\begin{array}{lllll}0.14113019 & 7.43914259 & 0.23864596\end{array}$ $\begin{array}{lllll}\text { chaperonin-containing T-complex eta subunit Cct7 } & 0.14089048 & 8.51037558 & 0.08312233\end{array}$ AP-3 adaptor complex subunit Apl6 (predicted) $\quad 0.14080165 \quad 7.90074803 \quad 0.10962479$ SAC3/GANP/THP3 family protein, human LENG8 ortholog $\begin{array}{lllll}0.14077416 & 6.21846123 & 0.3529018\end{array}$ $\begin{array}{llllll}\text { DUF2406 family conserved fungal protein } & 0.14045099 & 8.45532597 & 0.20789586\end{array}$ NADH-dependent glycolaldehyde/furfural/butyraldehyde/pr 0.140351997 .751132990 .16001512 shelterin complex subunit Pot1 esterase/lipase, human ABHD13 ortholog (predicted) $18 \mathrm{~S}$ rRNA endonuclease Utp24

rRNA processing protein Rrp7 (predicted)

Schizosaccharomyces specific protein mitochondrial asparagine-tRNA ligase SIm5 (predicted)

REX complex subunit Tex1 (predicted)

Rer1 family protein (predicted)

KH domain RNA-binding protein Pno1 (predicted)
0.631610742

0.0957084

0.415322162

0.274689483

0.225202829

0.34565189

0.676763897

0.11395943

0.421138061

0.47757801

0.432872562

0.554582212

0.576096614

0.224970468

0.499383474

0.38999401

0.135392246

0.545126

0.231549055

0.600687064

0.583319118

0.257516332

0.357363341

0.379137946

0.243997866

0.347927714

0.24503258

0.426780113

0.162243155

0.921229937

0.427312512

0.326823459

0.133976186

0.169506175

0.448382828

0.290141784

0.233419696

0.162000981

0.499066921

0.365778274

0.56915459

0.709677498

0.1449319

0.453924523

0.252630021

0.513887665 
SPBC1347.10

SPAC1B3.14

SPBC17F3.02

SPCC553.08C

SPNCRNA.1608

SPNCRNA.727

SPAC19D5.03

SPBC1A4.04

SPBC25H2.15

SPBC1539.08

SPBC2G2.06c

SPBC13G1.13

SPAC18G6.06

SPBC1709.16c

SPBC1703.12

SPAC521.03

SPAP8A3.06

SPAC1687.15

SPBC12D12.01

SPBC839.15C

SPBC13G1.11

SPNCRNA.551

SPNCRNA.1495

SPBC651.04

SPNCRNA.958

SPAC22H10.12C

SPBC1734.07C

SPAC4G8.05

SPAC57A7.10c

SPNCRNA.1567

SPNCRNA.1171

ScPofMt32

SPBC1289.07c

SPBC29A3.06

SPCC1450.05C

SPNCRNA.1377

SPAC8E11.01

SPAC823.14

SPCC18.15
SPAC922.05c

emc1

SPCP20C8.01c

SPBC354.04

bx 2

alg2

cdc23

vma3

nak1

$\# N / A$

$\# N / A$

cid1

SPBC1A4.04

trs401

apl1

tfb2

utp11

SPBC1709.16c

ubp9

SPAC521.03

uaf2

gsk3

sad1

tef103

ykt6

\section{$\# \mathrm{~N} / \mathrm{A}$}

$\# \mathrm{~N} / \mathrm{A}$

SPBC651.04

\#N/A

gdi1

trs8502

ppk14

sec21

suc2

ptf1

dph7 transmembrane transporter (predicted)

$0.13753117 .68094083 \quad 0.14215491$

ER membrane protein complex subunit Emc1 (predicted) $\begin{array}{lllll}0.13752623 & 8.20118801 & 0.08973674\end{array}$

mug $2 /$ mug $135 /$ meu2 family

Schizosaccharomyces specific protein

vacuolar SNARE VsI1/Vam7

UBX domain protein Ubx2

mannosyltransferase complex subunit Alg2 (predicted)

Lst4-Lst7 complex subunit Lst4

MCM-associated protein $\mathrm{Mcm} 10$

V-type ATPase VO subunit c (proteolipid subunit)

PAK-related GC kinase Nak1

GTPase Ria1 (predicted)

\section{$\# N / A$}

$$
\# N / A
$$

terminal uridylyltransferase Cid1

Schizosaccharomyces specific protein

$\begin{array}{llll}0.13742062 & 6.36591021 & 0.33121414\end{array}$

$0.137346725 .81208396 \quad 0.39547863$

$\begin{array}{llll}0.13711499 & 5.52414931 & 0.49993816\end{array}$

$\begin{array}{llll}0.13675341 & 6.83496084 & 0.31851861\end{array}$

$\begin{array}{llll}0.13658027 & 8.61472857 & 0.07956085\end{array}$

$\begin{array}{llll}0.13657702 & 7.37825923 & 0.23319506\end{array}$

$\begin{array}{llll}0.13636294 & 6.64377403 & 0.35085797\end{array}$

$\begin{array}{llll}0.13634069 & 7.42462362 & 0.30479375\end{array}$

$\begin{array}{llll}0.13600571 & 7.21980074 & 0.16813554\end{array}$

$\begin{array}{llll}0.13556924 & 8.14992062 & 0.15151144\end{array}$

$\begin{array}{llll}0.13551516 & 6.78231129 & 0.27545745\end{array}$

$\begin{array}{llll}0.135422 & 8.00415202 & 0.14900492\end{array}$

$\begin{array}{llll}0.13527069 & 4.83842188 & 0.62476485\end{array}$

$\begin{array}{lll}0.13507561 & 5.98642206 & 0.4742801\end{array}$

SSU-rRNA maturation protein Tsr4 homolog 1 Tsr401 (pred $0.134815796 .84373148 \quad 0.30753718$

ADP-ribosylation factor, Arf family Arf6

AP-2 adaptor complex beta subunit Apl1 (predicted)

transcription factor TFIIH complex subunit Tfb2

U3 snoRNP-associated protein Utp11 (predicted)

aromatic ring-opening dioxygenase (predicted)

$0.13427922 \quad 7.94952512 \quad 0.15034479$

$\begin{array}{llll}0.13399607 & 6.79974913 & 0.25040559\end{array}$

$0.13386793 \quad 5.81996784 \quad 0.43702808$

$0.13343009 \quad 5.8967686 \quad 0.39812201$

$\begin{array}{llll}0.13314877 & 7.24169617 & 0.1943664\end{array}$

short chain dehydrogenase, human DHRS7 family(predicted $0.13290323 \quad 7.930034360 .11959203$

U2AF small subunit, U2AF-23

serine/threonine protein kinase Gsk3

spindle pole body SUN domain protein Sad1

translation elongation factor EF-1 alpha Ef1a-c

SNARE Ykt6 (predicted)

\section{$\# N / A$}

$\# N / A$

Schizosaccharomyces specific protein

$$
\# N / A
$$

GDP dissociation inhibitor Gdi1 (predicted)

TRAPP complex subunit Trs85b (predicted)

serine/threonine protein kinase Ppk14 (predicted)

coatomer gamma subunit Sec21 (predicted)

$$
\begin{aligned}
& \# \mathrm{~N} / \mathrm{A} \\
& \# \mathrm{~N} / \mathrm{A} \\
& \# \mathrm{~N} / \mathrm{A}
\end{aligned}
$$

DNA-directed RNA polymerase I and III subunit Rpc40

CGI-48 family Utp18 (predicted)

mediator complex subunit Med19

$$
\# \mathrm{~N} / \mathrm{A}
$$

$\begin{array}{llll}0.13281398 & 5.78787967 & 0.48525289\end{array}$

$\begin{array}{lll}0.13272384 & 7.25251186 & 0.2847583\end{array}$

$\begin{array}{lll}0.13269446 & 7.69799195 & 0.35466213\end{array}$

$\begin{array}{lll}0.13260714 & 10.2867409 & 0.04918849\end{array}$

$\begin{array}{lll}0.13249121 & 6.58189499 & 0.384502\end{array}$

$0.13244052 \quad 5.16631378 \quad 0.54889877$

$\begin{array}{llll}0.13183302 & 5.68475351 & 0.49624045\end{array}$

$\begin{array}{llll}0.13163663 & 6.51836143 & 0.31721371\end{array}$

$\begin{array}{llll}0.1314315 & 4.93856823 & 0.57119252\end{array}$

$\begin{array}{llll}0.13096081 & 8.00560507 & 0.22071363\end{array}$

$\begin{array}{llll}0.13085762 & 7.03137941 & 0.23668658\end{array}$

$\begin{array}{llll}0.13066044 & 7.09367192 & 0.23590916\end{array}$

$\begin{array}{lll}0.13062445 & 9.27952103 & 0.06535088\end{array}$

$\begin{array}{llll}0.13050469 & 6.67140005 & 0.31998577\end{array}$

$\begin{array}{llll}0.13019822 & 5.82018719 & 0.43511402\end{array}$

$0.13011873 \quad 5.181312790 .51955154$

$\begin{array}{llll}0.13000985 & 6.76152091 & 0.28936407\end{array}$

$\begin{array}{llll}0.12980833 & 6.59224119 & 0.38008704\end{array}$

$\begin{array}{llll}0.12976175 & 5.72185795 & 0.46232527\end{array}$

$\begin{array}{llll}0.12952083 & 6.8317281 & 0.27666377\end{array}$

$\begin{array}{lrrr}\text { sucrose alpha-glucosidase Suc2 (predicted) } & 0.1290895 & 6.16819652 & 0.45180462 \\ \text { Mst2 histone acetytransferase acytyltransferase complex, } \mathrm{k} & 0.12856268 & 7.12647592 & 0.32964997\end{array}$ $\begin{array}{lllll}\text { Mst2 histone acetytransferase acytyltransferase complex, } \mathrm{F} & 0.12856268 & 7.12647592 & 0.32964997 \\ \text { diphthamide biosynthesis complex WD repeat protein subu } & 0.12838187 & 6.81888943 & 0.34893278\end{array}$
0.211491942

0.143246194

0.42574844

0.490832043

0.593368332

0.412860789

0.128784867

0.320131603

0.446111451

0.398409807

0.243387007

0.223119527

0.367474597

0.219946365

0.706233354

0.56901906

0.40084093

0.461329127

0.221497353

0.340325551

0.532684229

0.493412525

0.274297666

0.183005111

0.580341991

0.377734933

0.450023458

0.08544406

0.480186472

0.639051516

0.589860427

0.411326091

0.659366424

0.305461923

0.324466591

0.323531199

0.108864802

0.414446732

0.530921801

0.610946647

0.381983027

0.475545556

0.557251355

0.368722476

0.547069472

0.424138411

0.44416153 
SPAC17G6.06

SPBC1711.10C

SPBC1921.02

SPAC1A6.08C

SPAC9.09

SPCC330.13

SPNCRNA.1496

SPAC1D4.06C

SPAC21E11.08

SPAC824.03C

SPCC965.11c

SPCC1259.16

SPAC25B8.05

SPCC1827.04

SPAC890.02C

SPAPB2C8.01

SPAC17A2.04C

SPCC364.06

SPBC1289.08
Schizosaccharomyces specific protein Mug35

medi

ser3

$\operatorname{los} 1$

nop4

ste24 SPCC61.04c

isa2

SPCC622.07

rrp46

ymr31

SPAC688.16

oms1

dga1

rps2401

npl4

rad60

mug125

met26 rpc37

csk1

Icb2

SPAC824.03C

agp3

SPCC1259.16

deg1

vms1

alp7

SPAPB2C8.01

cns1

nap1

uap1

Rab GTPase binding Yip5 (predicted)

reticulon Rtn1 \#N/A \#N/A mediator complex subunit Med1 RNA-binding protein Nop4 (predicted)

$$
\text { \#N/A }
$$

CAAX prenyl protease (predicted)

Rab GTPase binding (predicted) diacylglycerol O-acyltransferase Dga1

40 S ribosomal protein S24 (predicted)

DNA repair protein, SUMO-related Rad60

homocysteine methyltransferase Met26

$$
\text { \#N/A }
$$
Schizosaccharomyces specific protein
$0.12825467 \quad 6.54055322 \quad 0.41347092$ $\begin{array}{llll}0.12736269 & 8.52417136 & 0.16875269\end{array}$ $\begin{array}{llll}0.12704163 & 6.61347275 & 0.33955355\end{array}$ $\begin{array}{llll}0.12670368 & 8.05875735 & 0.28711264\end{array}$

sec14 cytosolic factor family, phospholipid-intermembrane $0.12666336 \quad 7.87780775 \quad 0.13720627$

$\begin{array}{llll}0.12633802 & 6.33515058 & 0.35796569\end{array}$ $0.125735197 .73150235 \quad 0.17968862$ pumilio family RNA-binding protein Puf1 (predicted) mitochondrial membrane-associated ribosome receptor Ml $0.12541903 \quad 6.559280940 .36821282$ $\begin{array}{lrllll}\text { DUF221 family protein implicated in Golgi to plasma meml } & 0.12523197 & 8.33834549 & 0.10395064 \\ \text { 60S ribosomal protein L41 (predicted) } & 0.1249983 & 6.00433878 & 0.43091731\end{array}$ Gtr1/RagA G protein Gtr1 $\quad \begin{array}{llll}0.12490904 & 6.267633 & 0.38724001\end{array}$ U3-containing 90S preribosome complex subunit Cms1 (pre 0.124755244 .958697330 .61087619 $\begin{array}{lll}0.12426593 & 8.13324707 & 0.18512235\end{array}$ cyclophilin family peptidyl-prolyl cis-trans isomerase Cyp3 $\quad 0.12402727 \quad 5.94844117 \quad 0.50389134$ $0.12323192 \quad 5.40557123 \quad 0.5156063$ $\begin{array}{lll}0.1230072 & 7.85708497 & 0.34437314\end{array}$ $0.12285368 \quad 5.51831686 \quad 0.58538835$ mitochondrial [4Fe-4S] cluster assembly and transfer prote $\begin{array}{llll}0.12256734 & 5.12972216 & 0.57167679\end{array}$ $\begin{array}{llllll}\text { Schizosaccharomyces pombe specific protein } & 0.12210237 & 4.61248562 & 0.66211188\end{array}$ $\begin{array}{llll}0.12117086 & 6.39597884 & 0.46455292\end{array}$ mitochondrial ribosomal protein Ymr1 (predicted) $\quad \begin{array}{lllll}0.12091573 & 4.78554986 & 0.66616911\end{array}$ human TMEM254 ortholog $\quad \begin{array}{lllll}0.12058298 & 5.41767952 & 0.58212439\end{array}$ mitochondrial methyltransferase Oms1 (predicted) $\quad \begin{array}{lllll}0.11951729 & 5.32604778 & 0.53476904\end{array}$

Hrd1p ubiquitin ligase complex Npl4 (predicted)

Schizosaccharomyces specific protein Mug125

$0.11906467 \quad 6.52325496 \quad 0.34120022$ $0.11878858 \quad 7.90051044 \quad 0.17485222$ $0.1187151 \quad 6.394248190 .45338895$ $\begin{array}{llll}0.11857791 & 6.18330223 & 0.46492532\end{array}$ $\begin{array}{llll}0.11809992 & 3.65728591 & 0.82157972\end{array}$ $\begin{array}{lll}0.1180082 & 10.2890509 & 0.09251885\end{array}$

cyclin-dependent kinase activating kinase Csk1

$0.11756322 \quad 5.41959663 \quad 0.56110367$

$0.11740822 \quad 5.55251805 \quad 0.50783027$ $\begin{array}{lll}0.11733098 & 8.62863642 & 0.13978306\end{array}$

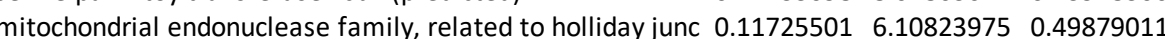
plasma membrane leucine transmembrane transporter Agr $0.11695622 \quad 8.29758215 \quad 0.14650561$ $\begin{array}{lll}0.11692961 & 5.79050549 & 0.53893609\end{array}$ tRNA-pseudouridine synthase Deg1 (predicted) $\quad \begin{array}{lllll}0.11684733 & 6.05165301 & 0.48581811\end{array}$ Cdc48p-Npl4p-Vms1p AAA ATPase complex subunit involvel $0.11622004 \quad 6.76038282 \quad 0.35290793$ $\begin{array}{llllll}\text { TACC protein Alp7 } & 0.11584873 & 5.93564793 & 0.49352313\end{array}$ cell surface glycoprotein, adhesion molecule (predicted) $\quad 0.115527348 .962762980 .15158306$ HSP chaperone complex subunit Cns1 (predicted) $\quad 0.1153553 \quad 6.11326012 \quad 0.45710493$ histone H2A-H2B chaperone Nap1 $\quad 0.11481188 \quad 8.714227690 .12110285$ UDP-N-acetylglucosamine diphosphorylase Uap1/Qri1(pred $0.11477997 \quad 6.097809540 .50024228$
0.508525147

0.243997866

0.434090163

0.37996918

0.205341685 0.453707675

0.257102822

0.463501334

0.161915358

0.526367309

0.482719965

0.693413258

0.26305232

0.597383369

0.659366424

0.204328514

0.370724927

0.295591499

0.606936668

0.439261337

0.671425542

0.659813529

0.739361884

0.55932106

0.74279548

0.668809126

0.626028443

0.43587033

0.251239302

0.548366356

0.559670411

0.870984574

0.147169412

0.705518045

0.650811393

0.60048392

0.208418871

0.592471061

0.21663857

0.630039531

0.580813813

0.448382828

0.588265034

0.223176697

0.552210018

0.184859784

0.593470839 
SPBC21B10.05C

SPAC1952.10C

SPBC19C7.01

SPBPB8B6.04C

SPBC1683.05

SPNCRNA.841

SPBC2D10.09

SPBC29A10.12

SPNCRNA.1239

SPAC3A12.17C

SPBC25H2.07

SPNCRNA. 680

SPAC22E12.02

SPAC19G12.08

SPCC285.14

SPAC26H5.02C

SPNCRNA.1321

SPAC664.01C

SPCC162.01C

SPCC24B10.17

SPAC56F8.03

SPBC4B 4.01C

SPBC646.11

SPBC25B2.06C

SPAC19A8.09

SPCC663.13C

SPCC1672.08c

SPNCRNA.1596

SPAC23G3.12C

SPNCRNA.971

SPAC688.03C

SPAC1834.02

SPAC29A4.05

SPAP14E8.02

SPNCRNA.1654

SPAC1002.02

SPBC342.02

SPBC9B6.09C

SPNCRNA.1467

SPAPB24D3.03

SPAC25B8.16

SPBC215.12 pop3

SPAC1952.10c

mni1

grt1

SPBC1683.05

\#N/A

snr1

oxs1

$$
\text { \#N/A }
$$

cys 12

tif11

\#N/A

SPAC22E12.02

ScS 7

$\operatorname{trs} 130$

mgs1

\#N/A

swi6

snp27

emp24

tif52

ptk1

cct6

btb2

yos 1

naa50

tfa2

htr12

$\#$ N/A

SPAC688.03C

aro1

cam2

\#N/A

pom34

qrs1

mdl1

$\#$ N/A

SPAPB24D3.03

pop100

cwf10 mitochondrial aminoacyl-tRNA hydrolase Pth2 (predicted)

histone $\mathrm{H} 2 \mathrm{~A}$ dioxygenase $\mathrm{Ofd} 2$

COPI-coated vesicle associated protein (predicted)

$0.11450063 \quad 6.27684003 \quad 0.413285$

$0.11441177 \quad 5.09719913 \quad 0.66255626$

$\begin{array}{lll}0.11429112 & 7.24062127 & 0.31242074\end{array}$

domain RNA-binding protein, involved in splicing (predic $0.11423103 \quad 7.258970250 .30651488$

1-acylglycerol-3-phosphate O-acyltransferase SIc1 (predicte $0.11397914 \quad 7.18445015 \quad 0.25046626$

WD repeat protein Pop3

$0.11394479 \quad 6.67251895 \quad 0.41789533$

$\begin{array}{lll}0.11392311 & 5.00499343 & 0.64271443\end{array}$

exon-exon junction complex disassembly factor, human par $\begin{array}{lllll}0.11368618 & 5.01512301 & 0.62538677\end{array}$

transcription factor Grt1 (predicted) $\quad \begin{array}{llll}0.11355012 & 6.19056541 & 0.52305937\end{array}$

plasma membrane uricil/uridine transmembrane transport $0.11338519 \quad 7.44628919 \quad 0.3292364$ \#N/A

3-hydroxyisobutyryl-CoA hydrolase snr1

oxidative stress transcription coactivator Oxs1 $\# \mathrm{~N} / \mathrm{A}$

cysteine synthase-like protein Cys12

translation initiation factor elF $1 \mathrm{~A}$

$\#$ N/A

pplicing factor, WW domain -binding Rbm42 (predicted)

ER sphingosine hydroxylase Scs7

TRAPP complex subunit Trs130 (predicted)

DNA replication ATPase Mgs1 (predicted)

$$
\text { \#N/A }
$$

$\begin{array}{llll}0.11286071 & 7.11734519 & 0.35572185\end{array}$

$\begin{array}{llll}0.11282059 & 6.39914452 & 0.49624555\end{array}$

$\begin{array}{lll}0.11273287 & 6.57070632 & 0.46282805\end{array}$

$\begin{array}{llll}0.11233334 & 5.6481734 & 0.51689622\end{array}$

$\begin{array}{llll}0.1121209 & 5.79548925 & 0.51110383\end{array}$

$\begin{array}{llll}0.11205805 & 7.53283704 & 0.27578743\end{array}$

$\begin{array}{llll}0.1120346 & 3.97539569 & 0.78403621\end{array}$

$\begin{array}{llll}0.1120126 & 7.51826226 & 0.3239787\end{array}$

$\begin{array}{llll}0.11181 & 8.3368437 & 0.28699058\end{array}$

$\begin{array}{llll}0.11174365 & 8.51013621 & 0.17366678\end{array}$

$0.11146255 \quad 5.94448903 \quad 0.49438253$

$0.11075741 \quad 5.22693904 \quad 0.63445988$

heterochromatin (HP1) family chromodomain protein Swi6 $0.11054851 \quad 6.97435182 \quad 0.31345173$

U4/U6 x U5 tri-snRNP complex subunit (predicted) $\quad 0.10985785 \quad 3.65942929 \quad 0.8198599$

COPII-coated vesicle component Emp24 (predicted) $\quad 0.10962818 \quad 7.17818904 \quad 0.34246285$

translation initiation factor elF5B Tif52 (predicted) $\quad \begin{array}{lllll}0.10941815 & 9.16677421 & 0.12975262\end{array}$

fumble family pantothenate kinase (predicted)

chaperonin-containing T-complex zeta subunit Cct6

$\mathrm{BTB} / \mathrm{POZ}$ domain protein Btb2

ER to Golgi transport protein Yos1 (predicted)

NatA N-acetyltransferase subunit Naa50 (predicted)

transcription factor TFIIE beta subunit, TFIIEB, Tfa2

$$
\# \text { N/A }
$$

serine protease, involved in lipid metabolism (predicted)

$$
\text { \#N/A }
$$

human AMMECR1 homolog

pentafunctional aromatic polypeptide Aro1 (predicted)

myosin I light chain Cam 2

chromatin binding FHA domain protein Tos4 (predicted)

$$
\# \text { N/A }
$$

nucleoporin Pom34

cytoplasmic glutaminyl-tRNA ligase Ors1 (predicted)

mitochondrial peptide-transporting ATPase

$$
\# \mathrm{~N} / \mathrm{A}
$$

gmatinase (predicted)

RNase $P$ and RNase MRP subunit Pop100

U5 snRNP GTPase subunit Cwf10
0.508388363

0.739736727

0.406117126

0.400180972

0.340340031

0.51303946

0.722085467

0.706818964

0.6146467

0.423799069

0.451199849

0.589860427

0.557564862

0.608034361

0.602981181

0.367842698

0.840744784

0.418344409

0.37988755

0.249852527

0.588777229

0.714581179

0.407301703

0.869296763

0.437070465

0.195866587

0.369811031

0.277575512

0.624875038

0.894525167

0.68261529

0.511104513

0.859762188

0.280080015

0.682934715

0.650872429

0.178566099

0.49518081

0.505919368

0.675256182

0.657724265

0.352631157

0.382665054

0.383557598

0.439938595

0.557251355

0.327745038 
SPBC3F6.04C SPNCRNA.1250 SPBC13E7.09 SPAC29B12.10C SPAPJ696.01c SPAC1093.01

SPCC1393.13

SPNCRNA.291

SPAC10F6.17C

SPNCRNA.1513 SPAC12B10.14C

SPAC1B3.15C

SPCC285.16C

SPBC4.06

SPBP23A10.17

SPNCRNA.1613

SPBC21D10.12

SPCC1281.06c

SPAC323.05C

SPBC29A3.15C

SPAC3A11.03

SPAC2F3.01

SPCC70.10

SPAC26H5.07c

SPAC24B11.10c

SPNCRNA.1334

SPCC24B10.06

SPBC405.02C

SPAC16E8.07C

SPAC3A11.13

SPAC17C9.13C

SPNCRNA.1489

SPCC70.06

SPBC29A10.17

SPBC6B1.12C

SPAC1142.04

SPBC1105.18C

SPCC550.11

SPAC11G7.06c

SPAC167.04

SPAC31G5.21

SPAC31G5.05c

SPAC29A4.16

SPAC14C4.14

SPBC36.12C

SPNCRNA.764

SPBC36.07 nop14

$$
\# \mathrm{~N} / \mathrm{A}
$$

vrp1

pgt1

vps17

ppr5

SPCC1393.13

\#N/A

SPAC10F6.17C

\#N/A

tea5

SPAC1B3.15C

msh6

SPBC4.06

SPBP23A10.17

\#N/A

hob1

ole1

mtq2

rsm23

efm 3

SPCC70.10

SPAC26H5.07c

\#N/A

SPCC24B10.06

SPBC405.02C

vph1

gim 1

cut8

$\# N / A$

sac32

lam1

sus1

noc201

pth3

nmd5

mug132

pam17

SPAC31G5.21

SPAC31G5.05c

hal4

atp1

git7

\#N/A

elp1
U3 snoRNP protein Nop14 (predicted)

\#N/A

$106055697.04821297 \quad 0.33628689$

$\begin{array}{llll}0.10586125 & 6.03755751 & 0.56504592\end{array}$

$\begin{array}{lll}0.10575333 & 7.35620139 & 0.32294416\end{array}$

verprolin

$\begin{array}{llll}0.1056273 & 7.94269095 & 0.21963417\end{array}$

$\begin{array}{llll}0.10529968 & 8.9159313 & 0.26431833\end{array}$

metal-dependent phosphatase involved in cellular detoxific $\begin{array}{lllll}0.10522493 & 7.76857874 & 0.27065899\end{array}$

\#N/A

$0.10478048 \quad 2.98576243 \quad 0.91811858$

mitochondrial pyruvate dehydrogenase (lipoamide) phosphi $0.10459902 \quad 7.8874879 \quad 0.28172548$

\#N/A

$\begin{array}{llll}0.10405162 & 5.13399288 & 0.67410736\end{array}$

$\begin{array}{llll}0.10394278 & 7.11446789 & 0.36109667\end{array}$

$\begin{array}{llll}0.10386022 & 6.42619521 & 0.48319468\end{array}$

$\begin{array}{llll}0.10368945 & 8.40137332 & 0.31924517\end{array}$

$\begin{array}{llll}0.10341478 & 7.9145477 & 0.24770008\end{array}$

$\begin{array}{llll}0.10325099 & 7.12687347 & 0.38703802\end{array}$

$\begin{array}{lll}0.10296005 & 5.5537855 & 0.58770279\end{array}$

$\begin{array}{llll}0.10245636 & 8.42746577 & 0.21356136\end{array}$

$\begin{array}{lll}0.10222042 & 9.6175397 & 0.2047775\end{array}$

$\begin{array}{llll}0.10217793 & 7.60623215 & 0.26985352\end{array}$

$\begin{array}{lllll}\text { mitochondrial ribosomal protein subunit S23 (predicted) } & 0.10187687 & 6.57740186 & 0.4951559 \\ \text { elongation factor EF2 methyltransferase Efm3 (predicted) } & 0.10167311 & 7.13576783 & 0.35010962\end{array}$

acyl-coA desaturase (predicted)

eRF1 methyltransferase Mtq2 (predicted)

etongation factor EF2 methyltransferase Efm3 (predicted)

mannosyltransferase Imt1

Schizosaccharomyces specific protein

seven transmembrane receptor protein (predicted)

SEL1/TPR repeat protein Cfh1 (predicted)

$10157055 \quad 7.65672062 \quad 0.25467661$

$0.10121477 \quad 5.66355027 \quad 0.60521808$

$\begin{array}{llll}0.1007401 & 8.65258375 & 0.29382631\end{array}$

$\begin{array}{llll}0.10061264 & 7.3994523 & 0.33375533\end{array}$ $\begin{array}{llll}0.10060321 & 4.83692906 & 0.71032832\end{array}$

$$
\text { \#N/A }
$$

$\begin{array}{llll}0.10060121 & 6.23724405 & 0.47501896\end{array}$

$\begin{array}{llll}0.10045872 & 5.28530342 & 0.68798954\end{array}$

$\begin{array}{llll}0.10034632 & 8.9460322 & 0.16328927\end{array}$

$0.10031785 \quad 5.588630990 .60817713$

$\begin{array}{llll}0.10031051 & 7.79761279 & 0.26580809\end{array}$

$\begin{array}{llll}0.10029719 & 6.06989937 & 0.51585144\end{array}$

$\begin{array}{llll}0.10013135 & 6.83652169 & 0.47042999\end{array}$

$\begin{array}{llll}0.0999392 & 7.42486128 & 0.38592618\end{array}$

$\begin{array}{llll}0.09959862 & 4.77405145 & 0.72721789\end{array}$

$\begin{array}{llll}0.09942387 & 7.66541262 & 0.27132556\end{array}$

Ragulator complex subunit, human LAMTOR1 ortholog

SAGA complex subunit Sus 1

Noc complex subunit Noc201 (predicted)

$\begin{array}{llll}0.09929105 & 5.27584784 & 0.62885978\end{array}$

export sig $0.09905844 \quad 8.5280229 \quad 0.19371376$

UPF0300 family protein $3 \quad 0.09903465 \quad 5.461776640 .57976258$

TIM23 translocase complex-associated motor subunit Pam $10.09895583 \quad 6.63014943 \quad 0.41849736$

DUF1754 family, human FAM32A homolog, implicated in $\mathrm{n} \quad 0.098881954 .86430556 \quad 0.69032863$

ribulose phosphate 3-epimerase (predicted)

serine/threonine protein kinase Hal4

F1-FO ATP synthase alpha subunit

SGT1-like protein Git7

$\# \mathrm{~N} / \mathrm{A}$

$0.09871113 \quad 8.06120078 \quad 0.22827835$ $\begin{array}{llll}0.09837122 & 8.85254673 & 0.20536095\end{array}$ $0.09834464 \quad 9.55557456 \quad 0.17906291$ $0.0983358 \quad 5.55311512 \quad 0.58517516$ $\begin{array}{llll}0.09830412 & 5.80266423 & 0.70234801\end{array}$ $\begin{array}{llll}0.09830363 & 8.22545369 & 0.27818177\end{array}$
0.430804269

0.654268757

0.417246063

0.379363298

0.304401327

0.355331135

0.362280566

0.947640971

0.374441777

0.749558924

0.456825221

0.578286979

0.413644968

0.337210122

0.482719965

0.673739468

0.297239323

0.286259419

0.361344594

0.589285747

0.445492889

0.344959063

0.688368549

0.386523883

0.427987749

0.780020045

0.569604229

0.762503061

0.237381645

0.691387468

0.356910817

0.607120214

0.565197044

0.481699864

0.795358042

0.362958607

0.709670528

0.273546824

0.666771064

0.513593244

0.764472955

0.314460354

0.286957135

0.256369264

0.67140734

0.773503511

0.370310433 
SPNCRNA.576

SPAC17A2.08C

SPNCRNA.1383

SPBP18G5.03

SPCC1919.07

SPBC651.09C

SPAC1B9.03C

SPAC2G11.11C

SPNCRNA.324

SPAC212.03

SPNCRNA.1680

SPAC926.07c

SPAC977.16c

SPNCRNA.693

SPNCRNA.874

SPNCRNA.1129

SPAC31A2.13C

SPBC1711.04

SPAC19E9.01C

SPNCRNA.94

SPAC24B11.11C

SPAC57A10.11C

SPBC577.07

SPCC1494.06C

SPCC1739.02c

SPBC4F6.10

SPBC146.01

SPCC162.12

SPBC887.04C

SPAC19A8.06

SPNCRNA.574

SPNCRNA.706

SPBC11B10.07C

SPAC24B11.07C

SPNCRNA.582

SPAPB1A10.13

SPBC16E9.02C

SPAC17G6.07C

SPCC553.07C

SPCC777.02

SPAC2C4.09

SPNCRNA.1112

SPBC337.06C

SPBC16G5.17

SPBC1306.02

SPAC25B8.14

SPNCRNA.1411
\#N/A

$0.09807588 \quad 8.36231921 \quad 0.30337324$ spliceosome complex disassembly protein Ntr2 (predicted) $0.09765287 \quad 5.8454494 \quad 0.55710718$

$$
\text { \#N/A }
$$

$0.0976108 \quad 6.32606789 \quad 0.54841418$

$\begin{array}{lll}0.09761041 & 6.77984258 & 0.40435018\end{array}$

$0.097572 \quad 5.91274208 \quad 0.54278449$

Schizosaccharomyces specific protein

$\begin{array}{lllll}\text { RNA polymerase II associated Paf1 complex (predicted) } & 0.09751298 & 7.87422552 & 0.30713375 \\ \text { RNA-binding protein involved in ribosomal large subunit as: } & 0.09748288 & 6.69203762 & 0.42446672\end{array}$

ATP-depend protein involved in ribosomal large subunit as! $0.0974813127 .02799415 \quad 0.38585416$

SPAC1B9.03c

prh1

\#N/A

SPAC212.03

\#N/A

dak2

$\# N / A$

$\# N / A$

$\# \mathrm{~N} / \mathrm{A}$

sft1

mtd1

nup40

sid2

mia40

ubp10

dbp9

mrpl22

vps901

med15

tco89

lub1

pbr1

$\# \mathrm{~N} / \mathrm{A}$

$\# \mathrm{~N} / \mathrm{A}$

vn1

SPAC24B11.07C

$\# \mathrm{~N} / \mathrm{A}$

SPAPB1A10.13

SPBC16E9.02C

use1

kpa1

SPCC777.02

SPAC2C4.09

\#N/A

cwf15

SPBC16G5.17

rtt10

mal2

$\# \mathrm{~N} / \mathrm{A}$

\#N/A

$\# \mathrm{~N} / \mathrm{A}$

edicted)

dynein light chain Dlc2 (predicted)

dihydroxyacetone kinase Dak2

$\# N / A$

SNARE Sft1 (predicted)

$\# N / A$

methylenetetrahydrofolate reductase Mtd1 (predicted)

nucleoporin Nup40$$
\# \text { \#/A }
$$

$0.09658437 \quad 1.30013051$

0.096549191 .29967241

$0.09653224 \quad 5.00438988 \quad 0.67252718$

$\begin{array}{llll}0.09623316 & 5.70095035 & 0.65426913\end{array}$

$\begin{array}{llll}0.09581322 & 7.67398848 & 0.30497453\end{array}$

$\begin{array}{llll}0.09575427 & 5.54658273 & 0.62036986\end{array}$

$\begin{array}{llll}0.09551064 & 4.6224348 & 0.75039251\end{array}$

$\begin{array}{llll}0.09544994 & 3.86157796 & 0.88121888\end{array}$

$\begin{array}{llll}0.09492453 & 5.24581524 & 0.66479016\end{array}$

$\begin{array}{llll}0.09486121 & 6.8527855 & 0.43550044\end{array}$

$\begin{array}{llll}0.09432774 & 6.74997984 & 0.42484889\end{array}$

$\begin{array}{llll}0.0940583 & 3.59686835 & 0.8661821\end{array}$

$\begin{array}{llll}0.09372918 & 7.2882975 & 0.48126643\end{array}$

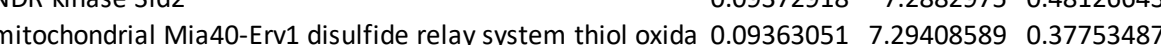

$\begin{array}{lllll}\text { ubiquitin C-terminal hydrolase Ubp10 (predicted) } & 0.09362378 & 5.34028976 & 0.69253229\end{array}$

ATP-dependent RNA helicase Dbp9 (predicted) $\quad \begin{array}{llll}0.09324248 & 6.15460159 & 0.63265059\end{array}$

mitochondrial ribosomal protein subunit L22 (predicted) $\quad \begin{array}{lllll}0.09317329 & 5.75685435 & 0.58117293\end{array}$

guanyl-nucleotide exchange factor Vps902 $\quad \begin{array}{lllll} & 0.09305405 & 8.32077615 & 0.29868811\end{array}$

mediator complex subunit Med15 $\quad \begin{array}{lllll}0.09303975 & 7.66763583 & 0.32832673\end{array}$

TORC1 subunit Tco89

WD repeat protein Lub1

$0.09288206 \quad 6.78671686 \quad 0.45252164$

$\begin{array}{llll}0.09206767 & 7.97642391 & 0.38937311\end{array}$

ER oxidoreductase Pbr1, implicated in lipid metabolism (pr $0.09199433 \quad 6.67191594 \quad 0.47268775$ $\# N / A$

\#N/A

$\begin{array}{llll}0.09175552 & 6.83286463 & 0.45108888\end{array}$

$\begin{array}{llll}0.09172887 & 6.62532931 & 0.55874215\end{array}$

plasma membrane phospholipid-translocating ATPase com| $\begin{array}{lllll}0.09172775 & 7.22964928 & 0.40326581\end{array}$ oxidoreductase (predicted)

$\# N / A$

Schizosaccharomyces specific protein

CUE domain protein, human TOLLIP ortholog

SNARE Use1 (predicted)

DinB translesion DNA repair polymerase, pol kappa

transcription factor (predicted)

mitochondrial calcium uniporter regulator (predicted)

$$
\text { \#N/A }
$$

$\begin{array}{llll}0.09166295 & 6.13103524 & 0.63873566\end{array}$

$\begin{array}{llll}0.09153066 & 3.62224523 & 0.87626688\end{array}$

$\begin{array}{llll}0.09139321 & 7.64385376 & 0.43679429\end{array}$

$\begin{array}{llll}0.09118639 & 8.36413716 & 0.33046613\end{array}$

$\begin{array}{llll}0.09106019 & 6.39617699 & 0.55051865\end{array}$

$\begin{array}{llll}0.09101156 & 5.95931354 & 0.58148961\end{array}$

$\begin{array}{llll}0.09093886 & 7.27850683 & 0.43377868\end{array}$

$\begin{array}{llll}0.09082831 & 6.7163863 & 0.53196006\end{array}$

$\begin{array}{llll}0.0906778 & 4.85124287 & 0.70165461\end{array}$ $\begin{array}{llll}0.09023039 & 6.29109388 & 0.63527875\end{array}$

Prp19 complex subunit Cwf15

$\begin{array}{llll}0.09014682 & 5.92147076 & 0.54755643\end{array}$

$\begin{array}{llllll}\text { WD repeat protein, human WDR6 family, involved in endoc } & 0.0901102 & 7.26311952 & 0.4637082\end{array}$ CENP-O ortholog Mal2

$0.09000246 \quad 6.30859686 \quad 0.54065137$

$\begin{array}{llll}0.08990693 & 4.90104061 & 0.74510241\end{array}$
0.396705397

0.646947844

0.638596593

0.499383474

0.633602954

0.400545259

0.519420254

0.481698318

1
1

0.748413316

0.732217772

0.398569557

0.702665928

0.814421215

0.919456377

0.74162247

0.531202812

0.519794441

0.907803663

0.576181918

0.473396866

0.766165216

0.713133548

0.668167225

0.391679662

0.422995724

0.547645342

0.484846109

0.567472603

0.546397268

0.648735767

0.498633063

0.718445649

0.915658598

0.532494648

0.425027782

0.640499129

0.668418374

0.529387353

0.623598311

0.772990065

0.715148365

0.637706916

0.55850164

0.631610742

0.80997155 
SPAC12B10.03

SPBC119.03

SPBC17A3.05C

SPBC18H10.08C

SPCC777.08c

SPAC1250.02

SPCC417.10

SPAC6G9.15C

SPBC13G1.04C

SPBC660.16

SPBC582.05C

SPAC19B12.11C

SPNCRNA.685

SPAC30D11.13

SPBC409.13

SPACUNK4.14

SPNCRNA.859

SPAC823.10c

SPBC119.12

SPAC17H9.07

SPNCRNA.1263

SPAC22H10.02

SPBC651.11C

SPCC1223.02

SPAC57A10.03

SPNCRNA.313

SPCC1259.06

SPAC959.04C

SPBC19F5.02C

SPAC664.14

SPBC19C2.02

SPNCRNA.281

SPBC31F10.17C

SPNCRNA.1196

SPNCRNA.114

SPNCRNA.622

SPAC6G9.03C

SPBC2D10.20

SPCC4B3.11C

SPAC1093.03

SPBC83.08

SPBC21H7.02

SPNCRNA.1487

SPNCRNA.569

SPAC9.07c

SPCP1E11.05C

SPBP23A10.06 bun62

SPBC119.03

SPBC17A3.05C

ubp4

bit61

mug95

dal51

ebp1

abh1

gnd1

brc1

bud20

hus5

$\mathrm{mdb} 1$

$\#$ N/A

hem 25

rud3

srp21

$\# \mathrm{~N} / \mathrm{A}$

SPAC22H10.02

apm3

nmt1

cyp1

taf8

$\#$ N/A

omh6

utp4

amt2

pmt1

$\# \mathrm{~N} / \mathrm{A}$

SPBC31F10.17

$\# N / A$

$\# N / A$

$\# N / A$

mug183

ubc1

fra3

fig 4

rvb2

taf10

$\# N / A$

$\# N / A$

SPAC9.07C

are2

$\mathrm{mtm} 1$
WD repeat protein $W d r 20$

$\begin{array}{llll}0.08961072 & 6.28121056 & 0.60768493\end{array}$

O-methyltransferase, human COMT catechol homolog $1 \quad 0.08906251 \quad 8.418253090 .27189806$

DNAJ/DUF1977, human DNAJB12 homolog, Hsp70 co-chapє $0.08864716 \quad 6.495829370 .50750955$ ubiquitin C-terminal hydrolase Ubp4 $\quad \begin{array}{llllll}0.0886258 & 7.25296927 & 0.39260933\end{array}$

Protor homolog, Bit61

Schizosaccharomyces specific protein Mug95

enticted

all binding protein Ebp1

tRNA demethylase (predicted)

phosphogluconate dehydrogenase, decarboxylating

BRCT domain protein Brc1

zinc finger ribosome biogenesis protein Bud20 (predicted) \#N/A

SUMO conjugating enzyme E2 Hus5

6,7-dimethyl-8-ribityllumazine synthase

BRCT domain protein Mdb1

$\# \mathrm{~N} / \mathrm{A}$

mitochondrial carrier, glycine Hem 25 (predicted)

Golgi matrix protein Rud3 (predicted)

signal recognition particle subunit Srp21 (predicted) $\# N / A$

DUF4452 family conserved fungal protein

AP-3 adaptor complex subunit Apm3 (predicted)

$\begin{array}{llll}0.08842826 & 6.50064073 & 0.49598572\end{array}$

$\begin{array}{llll}0.08815856 & 4.04185218 & 0.84194139\end{array}$

$\begin{array}{llll}0.08795909 & 6.86037635 & 0.48837394\end{array}$

$\begin{array}{llll}0.08792366 & 6.22339144 & 0.59137831\end{array}$

$\begin{array}{llll}0.08736763 & 6.20507069 & 0.56240987\end{array}$

$\begin{array}{llll}0.08714275 & 9.76517176 & 0.18789552\end{array}$

$\begin{array}{llll}0.08673492 & 6.90074596 & 0.51399635\end{array}$

$\begin{array}{llll}0.08651591 & 6.1987534 & 0.61989169\end{array}$

$\begin{array}{llll}0.08627796 & 6.4539372 & 0.59095965\end{array}$

$\begin{array}{llll}0.08623625 & 5.74661056 & 0.64201737\end{array}$

$\begin{array}{llll}0.08618641 & 6.61269631 & 0.52024898\end{array}$

$\begin{array}{llll}0.08603675 & 6.33558374 & 0.59174858\end{array}$

$\begin{array}{llll}0.08557193 & 5.83588132 & 0.65028167\end{array}$

$\begin{array}{llll}0.08545066 & 4.82059146 & 0.79100107\end{array}$

$\begin{array}{llll}0.08537942 & 6.64398099 & 0.54516467\end{array}$

$\begin{array}{llll}0.08494561 & 6.78330824 & 0.50080216\end{array}$

$\begin{array}{llll}0.08482799 & 7.74132879 & 0.3720327\end{array}$

$\begin{array}{llll}0.08461017 & 5.05808013 & 0.78662046\end{array}$

$\begin{array}{llll}0.08439836 & 6.98883323 & 0.45343975\end{array}$

4-amino-5-hydroxymethyl-2-methylpyrimidine phosphate s! $0.08435925 \quad 5.58816502 \quad 0.67030811$

cyclophilin family peptidyl-prolyl cis-trans isomerase Cyp1 $0.0839473 \quad 5.98786948 \quad 0.58748379$

$$
\# N / A
$$

$0.08383661 \quad 1.94706239$

$0.08359739 \quad 5.5987016 \quad 0.67159402$

$0.08354646 \quad 6.52184599 \quad 0.55351875$ alpha-1,2-mannosyltransferase Omh6 (predicted)

U3 snoRNP-associated WD repeat protein Utp4 (predicted) $0.08300313 \quad 7.07515541 \quad 0.49593761$

plasma membrane ammonium transmembrane transportel $0.08297374 \quad 7.57000998 \quad 0.42631793$ tRNA (cytosine-5-)-methyltransferase Pmt1

$$
\text { \#N/A }
$$

Schizosaccharomyces pombe specific protein

$\# N / A$

$\# N / A$

$\# \mathrm{~N} / \mathrm{A}$

$\begin{array}{lll}0.08289501 & 5.88650238 & 0.6539329\end{array}$

0.082839961 .94603187

$0.08253734 \quad 6.52528346 \quad 0.55642741$

$0.08242232 \quad 7.0362776 \quad 0.46586889$

$0.08201932 \quad 3.55719253 \quad 0.86326878$ $\begin{array}{lll}0.08187785 & 7.98661391 & 0.32105424\end{array}$

histone H3.3 H4 heterotetramer chaperone Rtt106-like (pre 0.081503115 .506551390 .70771956 ubiquitin conjugating enzyme E2 Ubc1 (predicted)

$0.08147115 \quad 7.01725373 \quad 0.50692091$

mitochondrial [4Fe-4S] cluster transfer protein Fra3 (predic $0.0812157 \quad 5.12536435 \quad 0.7125677$

inositol polyphosphate phosphatase Fig4 (predicted) $\quad \begin{array}{lllll}0.08100442 & 7.63206646 & 0.48723933\end{array}$

ASTRA/Swr1/Ino80 complex AAA family ATPase Rvb2 $\quad \begin{array}{lllll}0.08086627 & 7.97211892 & 0.38215972\end{array}$

SAGA complex/transcription factor TFIID complex subunit T $0.080740796 .51647574 \quad 0.64886703$

$$
\# \mathrm{~N} / \mathrm{A}
$$

$\begin{array}{lll}0.08073775 & 5.76280421 & 0.70452092\end{array}$

$0.08054896 \quad 6.39686058 \quad 0.61309427$

GTase Obg family, involved in cytoplasmic translation Rbg $0.08046264 \quad 7.505983190 .40394719$

acyl-coA-sterol acyltransferase Are2 (predicted)

$0.079693857 .58853303 \quad 0.44859432$

mitochondrial carrier, manganese ion Mtm1 (predicted) $\begin{array}{lllll}0.07956817 & 6.39181998 & 0.57653193\end{array}$
0.690943345

0.363438678

0.600239497

0.487893805

0.589757617

0.888969924

0.583254585

0.676260978

0.651659995

0.266436551

0.605705181

0.702323926

0.676220017

0.721421434

0.611555415

0.676440297

0.728782402

0.845814307

0.635791871

0.594031491

0.467318228

0.842719174

0.548366356

0.746676494

0.673601889

1

0.747741705

0.643329645

0.589757617

0.521216945

0.732030493

0.646379061

0.560707077

0.905983208

0.415356293

0.778034186

0.599825561

0.781848311

0.582308334

0.477611923

0.727611467

0.775269219

0.695698933

0.499066921

0.544441648

0.664049896 
SPCC550.13

SPAC19A8.03

SPAC1B3.06C

SPBC18H10.15

SPAC24C9.08

SPBP8B7.18C

SPAC328.06

SPNCRNA.1145

SPNCRNA.1471

SPBC119.11C

SPCC794.08

SPBC12D12.06

SPAC23C11.13C

SPAC652.01

SPAC644.09

SPBC36.08C

SPCC4E9.02

SPBC691.04

SPAC1002.09c

SPAC1782.12C

SPNCRNA.909

SPCC16A11.10C

SPAC25B8.06C

SPCC1259.10

SPNCRNA.750

SPAC1805.04

SPBC32F12.12C

SPAC23D3.07

SPBC21H7.05

SPAC4G9.14

SPCP31B10.04

SPAC26F1.14c

SPBC29A10.01

SPCC61.05

SPBC6B1.07

SPAC12B10.15C

SPAC31A2.02

SPNCRNA.643

SPBC3E7.15C
SPBC1683.12 SPAC3G6.03C gcs2

kin1

hsf1

emp70

mde1

dfp1

ymr1

SPAC1B3.06C

cdk11

cps1

SPBP8B7.18C

ubp2

\#N/A

\#N/A

pac1

efr3

srb11

hpt1

SPAC652.01

SPAC644.09

$\operatorname{cog} 2$

cig1

mss116

dld1

SPAC1782.12C

\#N/A

oca 8

dia4

pgp1

nup132

$\# \mathrm{~N} / \mathrm{A}$

SPBC32F12.12C

pup1

sfc6

sym 1

SPCP31B10.04

aif1

ccr1

SPCC61.05

prp1

SPAC12B10.15C

trm112

\#N/A carboxylic acid transmembrane transporter (predicted) $\quad \begin{array}{llll}0.07948122 & 8.03039542 & 0.38913601\end{array}$ Maf-like protein, nucleoside-triphosphate diphosphatase, h $0.07935503 \quad 5.53540193 \quad 0.68661461$ glutamate-cysteine ligase regulatory subunit Gcs2 (predicte $0.0782652 \quad 7.145405790 .46908783$ microtubule affinity-regulating kinase Kin1

$\begin{array}{lll}0.077844 & 8.52361225 & 0.3925128\end{array}$

transcription factor Hsf 1

EMP70 family endosomal transport protein Emp70

$\begin{array}{llll}0.0778833 & 7.78223507 & 0.46626851\end{array}$ $\begin{array}{lll}0.07741531 & 7.07813667 & 0.46977983\end{array}$

5'-methylthioribulose-1-phosphate dehydratase, adducin M $0.07729587 \quad 7.00268864 \quad 0.51057206$

$$
\# \mathrm{~N} / \mathrm{A}
$$

$\begin{array}{llll}0.07695609 & 7.51027142 & 0.42198282\end{array}$

Hsk1-Dfp1 kinase complex regulatory subunit Dfp1 $\quad \begin{array}{lllll}0.07684253 & 7.4338928 & 0.49452957\end{array}$ phosphatidylinositol-3-phosphatase, myotubularin family (f $\begin{array}{lllll}0.07672957 & 7.6522613 & 0.42587723\end{array}$ UbiE family methyltransferase (predicted) $\quad 0.07668992 \quad 7.45810897 \quad 0.42085565$ $\begin{array}{lllll}\text { vacuolar carboxypeptidase (predicted) } & 0.07643085 & 8.71223042 & 0.34562497\end{array}$ phosphomethylpyrimidine kinase (predicted) $\quad \begin{array}{lllll}0.07639396 & 6.41392829 & 0.59149234\end{array}$ ubiquitin C-terminal hydrolase Ubp2 $\quad 0.0761767 \quad 8.59678684 \quad 0.39200971$ $\begin{array}{lrl}0.0759243 & 5.923557 & 0.71065865\end{array}$ $\begin{array}{lll}0.07591908 & 8.20511457 & 0.37025912\end{array}$

$0.07586107 \quad 6.11738386 \quad 0.62779095$

double-strand-specific ribonuclease Pac1

$$
\# N / A
$$

phosphatidylinositol-4 kinase plasma membrane scaffold E. $0.07567335 \quad 8.20145394 \quad 0.41426589$ cyclin CycC, Srb mediator subunit Srb11 $\quad 0.07563783 \quad 5.82829004 \quad 0.70880136$ guanine/xanthine/hypoxanthine phosphoribosyltransferase $0.07557366 \quad 6.39322229 \quad 0.59192934$ BC10 family small membrane protein, unknown biological r $0.075402486 .38588846 \quad 0.68925128$ pyridoxal phosphate homeostasis protein (predicted) $\quad \begin{array}{lllll}0.07488051 & 6.28746055 & 0.62854431\end{array}$ Golgi transport complex subunit Cog2 (predicted) $\quad \begin{array}{lllll}0.07481348 & 8.42492411 & 0.38020519\end{array}$ $0.07459023 \quad 6.75238379 \quad 0.57232647$ mitochondrial ATP-dependent RNA helicase Mss116 (predic 0.074159696 .958273290 .50921519 $\begin{array}{llllll}\text { dihydrolipoamide dehydrogenase Dld1 } & 0.07383811 & 8.99384097 & 0.30157953\end{array}$ ER transmembrane protein, DUF423 family protein, human $0.073834757 .44407126 \quad 0.46422059$

$$
\# N / A
$$

cytochrome b5 (predicted)

mitochondrial serine-tRNA ligase (predicted)

$0.07343695 \quad 6.35181897 \quad 0.60892298$ $0.07326837 \quad 7.40024105 \quad 0.49248361$ $0.072866647 .03659139 \quad 0.58996389$ mitochondrial metallopeptidase, tRNA N6-threonyl-carbam $0.07284954 \quad 6.367782350 .62617066$ $\#$ N/A $0.07280956 \quad 5.27007052 \quad 0.78784219$ $0.07217103 \quad 7.91539024 \quad 0.39813787$

nucleoporin, WD repeat Nup132 $0.07206528 \quad 6.80454892$

Golgi membrane protein involved in vesicle-mediated trans 20S proteasome complex subunit beta 2 Pup1 $\quad 0.07172057 \quad 7.73491811 \quad 0.51212655$ transcription factor TFIIIC complex subunit Sfc6 $\quad \begin{array}{lllll}0.07140894 & 6.68621239 & 0.6099041\end{array}$ mitochondrial Mpv17/PMP22 family protein 2 (predicted) $0.07135668 \quad 5.75308303 \quad 0.69079583$ DUF4448 family conserved fungal membrane protein, simil $0.07094763 \quad 6.94770849 \quad 0.58081597$ mitochondrial inner membrane anchored oxidoreductase, a $\begin{array}{lllll}0.07074327 & 8.05826522 & 0.59242947\end{array}$ NADPH-cytochrome p450 reductase $\quad \begin{array}{llllll}0.07063761 & 8.36448951 & 0.35846364\end{array}$ Schizosaccharomyces specific multicopy membrane protein $0.07053514 \quad 6.29528047 \quad 0.66763107$ U4/U6 x U5 tri-snRNP complex subunit Prp1 $\quad 0.07017037 \quad 7.448214840 .53868928$

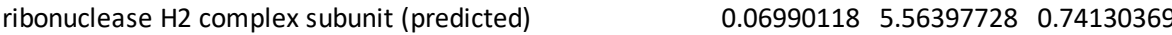
eRF1 methyltransferase complex and tRNA (m2G10) methı $0.06958513 \quad 5.2464125 \quad 0.75710074$ $\# \mathrm{~N} / \mathrm{A}$

$\begin{array}{llll}0.06958513 & 5.2464125 & 0.75710074\end{array}$ sphingosine $\mathrm{N}$-acyltransferase Lac1
0.484639564

0.761475295

0.563883185

0.487862935

0.56089044

0.564515579

0.602574204

0.517217779

0.588849297

0.520865304

0.516115103

0.707646392

0.440527802

0.676260978

0.487415689

0.780256874

0.465620502

0.708947468

0.509134803

0.778720253

0.676533281

0.763523215

0.709562716

0.475605939

0.660227438

0.601369707

0.394587327

0.559019837

0.691888609

0.587296249

0.675307703

0.707587403

0.843582702

0.493412525

0.681824816

0.603780516

0.692772057

0.764741433

0.667869666

0.676763897

0.454085088

0.744181728

0.629859198

0.807389818

0.819740739

0.819740739

0.54456573 
SPAC1B3.13

SPAC15A10.16

SPCC5E4.03C

SPBC14F5.03C

SPAC1039.10

SPAC29B12.06C

SPNCRNA.758

SPBC29A3.10c

SPAC1039.03

SPNCRNA.847

SPBC16D10.03

SPNCRNA.1661

SPAC1F5.04c

SPNCRNA.528

SPAC1B3.20

SPAC2E1P3.02C

SPAC26H5.05

SPCC584.03C

SPAC1687.17c

SPCC330.21

SPBC29A10.07

SPAC6G9.12

SPAC18B11.06

SPBC32H8.10

SPBC646.12C

SPAC1486.10

SPAC3G6.10c

SPAC3G9.08

SPAC19G12.07C

SPCC4G3.04c

SPAC17A5.09C

SPAC26F1.01

SPBP8B7.12C

SPCC757.08

SPBC30D10.15

SPAC9E9.04

SPAC1F3.08c

SPAC6F $6.03 \mathrm{C}$

SPAC2OH4.04

SPNCRNA.754

SPAC25H1.06

SPAC1486.05

SPAC23H4.21 rpb6

SPCC757.13

$\# \mathrm{~N} / \mathrm{A}$

$\# \mathrm{~N} / \mathrm{A}$

nan1

bud6

taf5

kap123

$\mathrm{mmf2}$

rcd1

$\# N / A$

atp14

SPAC1039.03

\#N/A

kae1

$\#$ N/A

cdc12

$\#$ N/A

SPAC1B3.20

amt3

mga2

SPCC584.03c

SPAC1687.17c

new 25

pom152

cfr1

Icp5

cdk9

gap1

thi1

vps51

png1

rsd1

coq5

glc8

$\sec 74$

fta3

rp45

SPBC30D10.15

SPAC9E9.04

\#N/A

nog2

$\mathrm{fml} 2$

\#N/A

pcf3

nup189

SPAC23H4.21
DNA-directed RNA polymerase I, II and III subunit Rpb6

dipeptide transmembrane transporter (predicted)

$$
\# \mathrm{~N} / \mathrm{A}
$$$$
\# \mathrm{~N} / \mathrm{A}
$$

U3 snoRNP-associated protein Nan1 (predicted)

nucleation-promoting factor Bud6

$\begin{array}{lll}6.89329889 & 0.57061198\end{array}$

$0.06912437 \quad 6.05620066 \quad 0.6410749$

$0.06829052 \quad 5.63275048 \quad 0.73667831$

$\begin{array}{lll}0.06805962 & 8.04683813 & 0.52608381\end{array}$

$0.06778324 \quad 7.88050498 \quad 0.49946565$ $\begin{array}{lll}0.06776465 & 8.10955705 & 0.43808639\end{array}$ SAGA complex subunit/TATA-binding protein associated far $0.06775417 \quad 7.713352240 .49670258$ karyopherin/importin beta family nuclear import signal rect $0.06772076 \quad 8.65787913 \quad 0.36575557$ mitochondrial matrix protein, YjgF family protein Mmf2, re: 0.067524146 .318090620 .62905696 CCR4-Not complex RNA-binding protein subunit Rcd1

$0.067293 \quad 6.26663484 \quad 0.72153897$$$
\# \mathrm{~N} / \mathrm{A}
$$

$0.0671013 \quad 7.50403713 \quad 0.52590661$

F1-FO ATP synthase subunit $\mathrm{H}$ (predicted)

esterase/lipase, implicated in cellular detoxification (predic $0.06677553 \quad 6.80611108 \quad 0.56997531$ $\# \mathrm{~N} / \mathrm{A}$ $\begin{array}{llll}0.06668395 & 7.87172649 & 0.44872449\end{array}$

EKC/KEOPS complex N(6)-L-threonylcarbamoyladenine synt $\begin{array}{lllll}0.06666106 & 6.66253131 & 0.58972018\end{array}$ \#N/A

$0.06648729 \quad 7.21157943 \quad 0.51532791$

$0.06643028 \quad 8.29176542 \quad 0.40341323$

$0.065908394 .63969964 \quad 0.81881346$

$\begin{array}{lrrr}\text { Schizosaccharomyces specific protein } & 0.06590257 & 5.58980231 & 0.7283216 \\ \text { plasma membrane ammonium transmembrane transporte } & 0.0657648 & 5.88879112 & 0.69304333\end{array}$ $\begin{array}{llllll}\text { plasma membrane ammonium transmembrane transporte| } & 0.0657648 & 5.88879112 & 0.69304333\end{array}$ IPT/TIG ankyrin repeat gene-specific transcription coactivat $\begin{array}{llllll}0.06565183 & 8.3424207 & 0.45250442\end{array}$ GTPase regulator (predicted)

Der1-like (degradation in the ER) family (predicted) $\begin{array}{rrr}0.06561992 & 6.06482063 & 0.69064452 \\ 0.0654225 & 6.1747045 & 0.70187849\end{array}$ Schizosaccharomyces specific SAP domain containing prote 0.065168395 .285007550 .79183468 nucleoporin Pom152

exomer complex BRCT domain subunit Cfr1

U3 snoRNP-associated protein Lcp5 (predicted)

P-TEFb-associated cyclin-dependent protein kinase Cdk9

GTPase activating protein Gap1

transcription factor Thi1

GARP complex subunit Vps51 (predicted)

ING family homolog Png1

RNA-binding protein Rsd1 (predicted)

C-methyltransferase (predicted)

protein phosphatase regulatory subunit Glc8 (predicted)

guanyl-nucleotide exchange factor Sec74 (predicted)

CENP-H ortholog Fta3

exosome subunit Rrp45

snoRNP assembly factor Naf1 (predicted)

$\begin{array}{llll}0.06121625 & 6.39730739 & 0.69697677\end{array}$ $\begin{array}{ccccc}\text { \#N/A } & 0.06089062 & 2.02449831 & 0.67582458\end{array}$

ribosome export GTPase Nog2 (predicted)

ATP-dependent 3' to 5' DNA helicase (predicted)

$\# \mathrm{~N} / \mathrm{A}$

CAF assembly factor (CAF-1) complex subunit C, Pcf3

nucleoporin Nup98 and Nup96

DUF4050 family protein, conserved in fungi and plants
0.061496 .50365750 .649

$\begin{array}{llll}0.06059067 & 4.86191393 & 0.84856875\end{array}$
0.659208633

0.720481369

0.80337476

0.617135036

0.592962969

0.533687408

0.59030057

0.461175964

0.709670528

0.789908732

0.617033528

0.822111854

0.658855438

0.544502522

0.675256182

0.606713916

0.498678838

0.868513631

0.796181284

0.766585408

0.547645342

0.76469833

0.773111541

0.846439684

0.538874285

0.551568382

0.801536461

0.605705181

0.492296885

0.693413258

0.822955581

0.829732409

0.664049896

0.834043243

0.738219657

0.775269219

0.768832369

0.781289133

0.768832369

0.750977505

0.58081381

0.702323926

0.727611467

0.676220017

0.633602954

0.894440886 
SPBC1105.04C SPCC569.01C

SPNCRNA.1629

SPAC23G3.05C

SPAC29B12.08

SPAC10F6.07C

SPBC1198.09

SPNCRNA.1266

SPCC16C4.06C

SPAC4A8.02c

SPBC21.07c

SPAC2F7.14C

SPNCRNA.820

SPAC3G9.15C

SPAP14E8.04

SPAC $14 C 4.15 \mathrm{C}$

SPAC959.10

SPAC23A1.04c

SPACUNK4.12C

SPBC428.19C

SPAC11H11.06

SPNCRNA.1588

SPAC19G12.13C

SPBC16D10.01C

SPNCRNA.928

SPCC962.02C

SPBC25H2.04C

SPAC823.03

SPAC17C9.06

SPAC6G10.10c

SPACUNK4.11C

SPBC1289.13C

SPAC4G8.06C

SPNCRNA.1185

SPAC1093.04C

SPAC16.05c

SPAC6G9.07C

SPNCRNA.645

SPBC1604.03C

SPCC737.05

SPBC1198.13C

SPAC17D4.02

SPBC23E6.09

SPNCRNA.1277

SPAPB1E7.01C

SPBP19A11.03C

SPAC16E8.11C cbp1

SPCC569.01C

$\# N / A$

SPAC23G3.05C

clr5

mug94

ubc16

\#N/A

pus3

SPAC4A8.02C

ppk24

rrp4

$\# N / A$

fcf2

oma1

dpp1

sen15

$\mathrm{mnl} 1$

iph1

utp15

arp2

$$
\text { \#N/A }
$$

poz1

SPBC16D10.01c

\#N/A

bir1

tim22

ppk15

sam50

SPAC6G10.10c

mpp6

gmh6

trm12

$$
\# \text { N/A }
$$

cca1

sfp1

$\operatorname{arc4}$

$\#$ N/A

SPBC1604.03C

SPCC737.05

tfg2

cdc45

ssn6

$\# \mathrm{~N} / \mathrm{A}$

SPAPB1E7.01c

$\mathrm{mts} 4$

tfb1
CENP-B homolog

mug2/mug135/meu2 family

\#N/A

$0.06054597 \quad 7.05499229 \quad 0.57776422$

$\begin{array}{llll}0.06050496 & 6.42607383 & 0.70365664\end{array}$

0.060472696 .421287490 .66314138

conserved endomembrane protein, regulator of G-protein s 0.060425394 .202153080 .89717743

Clr5 protein

Schizosaccharomyces pombe specific protein Mug94

ubiquitin conjugating enzyme E2 Ubc16 (predicted)

$$
\text { \#N/A }
$$

tRNA pseudouridine synthase Pus3 (predicted)

conserved protein, UPF0047 family

serine/threonine protein kinase Ppk24

exosome subunit Rrp4

\section{$\# \mathrm{~N} / \mathrm{A}$}

RNA processing protein Fcf2 (predicted)

metallopeptidase Oma1 (predicted)

vacuolar dipeptidyl peptidase (predicted)

tRNA-splicing endonuclease subunit Sen15 (predicted)

alpha mannosidase Mnl1 (predicted)

insulinase pombe homologue 1

$\begin{array}{llll}0.06039258 & 8.26808156 & 0.55309527\end{array}$

$\begin{array}{llll}0.06003802 & 4.08883056 & 0.84848745\end{array}$

$\begin{array}{llll}0.06003398 & 7.73010347 & 0.50705174\end{array}$

$\begin{array}{llll}0.05963826 & 4.47044804 & 0.86404168\end{array}$

$\begin{array}{lllll}0.05950523 & 6.15018412 & 0.73658963\end{array}$

$\begin{array}{llll}0.0593528 & 7.37369865 & 0.58337153\end{array}$

$\begin{array}{llll}0.0593003 & 4.66926328 & 0.89248289\end{array}$

$\begin{array}{llll}0.05929058 & 5.90840305 & 0.75455546\end{array}$

$\begin{array}{llll}0.05907695 & 5.66939814 & 0.74693727\end{array}$

$\begin{array}{llll}0.05894481 & 6.52097509 & 0.63383315\end{array}$

$\begin{array}{llll}0.05882847 & 5.77936648 & 0.73888387\end{array}$

$\begin{array}{llll}0.05849257 & 6.75979544 & 0.6392665\end{array}$

$\begin{array}{llll}0.05835326 & 5.66099471 & 0.73699771\end{array}$

$\begin{array}{llll}0.0581886 & 7.00179679 & 0.62791439\end{array}$

$\begin{array}{llll}0.05787107 & 7.74188868 & 0.5455233\end{array}$

U3 snoRNP-associated WD repeat protein Utp15 (predicted $0.05773871 \quad 7.260776610 .59322101$

ARP2/3 actin-organizing complex subunit Arp2 $\quad 0.057733148 .61794343 \quad 0.45039061$

$$
\# \mathrm{~N} / \mathrm{A}
$$

shelterin complex subunit Poz1

TPR repeat protein, conserved fungal protein

$\# \mathrm{~N} / \mathrm{A}$

$0.0575594 \quad 5.24678321 \quad 0.77084422$

$\begin{array}{lll}0.05708914 & 7.46300033 & 0.57855527\end{array}$

$\begin{array}{lll}0.05681278 & 7.36928882 & 0.65367405\end{array}$

$0.05673966 \quad 8.19641955 \quad 0.49924203$

$\begin{array}{lll}0.056643 & 6.13662968 & 0.71921629\end{array}$

survivin, Bir1

$\begin{array}{lrr}0.05642676 & 5.6197194 & 0.80876778\end{array}$

serine/threonine protein kinase Ppk15 (predicted) $\quad \begin{array}{llll}0.05623502 & 8.11449276 & 0.5662104\end{array}$

mitochondrial sorting and assembly machinery complex sut $0.05621721 \quad 7.26135938 \quad 0.64825356$

human mmtag2 ortholog, implicated in splicing $\quad \begin{array}{llll}0.05616876 & 4.95236096 & 0.84458285\end{array}$

nuclear exosome-associated RNA binding protein Mpp6 $\quad 0.05589222 \quad 4.86183763 \quad 0.8225578$

alpha-1,2-galactosyltransferase Gmh6

tRNA 4-demethylwyosine alpha-amino-alpha-carboxypropy $0.05573966 \quad 6.32041392 \quad 0.77692875$ $\# \mathrm{~N} / \mathrm{A}$

$\begin{array}{llll}0.05570107 & 5.90268109 & 0.76877161\end{array}$

ATP(CTP) tRNA nucleotidyltransferase Cca1 (predicted)

transcription factor Sfp1 (predicted)

ARP2/3 actin-organizing complex subunit Arc4$$
\# \mathrm{~N} / \mathrm{A}
$$

$19.0350593 \quad 0.51815251$

$\begin{array}{lll}0.05514831 & 7.79437072 & 0.61792731\end{array}$

$\begin{array}{lll}0.05477466 & 7.04616763 & 0.64565074\end{array}$

$0.05469277 \quad 6.2690062 \quad 0.76023489$

conserved fungal protein implicated in vesicle trafficking ol $\begin{array}{llll}0.05457779 & 6.00462043 \quad 0.75800188\end{array}$

peroxin Pex28/29 (predicted)

$0.05425568 \quad 7.51261076 \quad 0.57212892$

transcription factor TFIIF complex beta subunit Tfg2 (predic $0.05422718 \quad 7.58250448 \quad 0.59272883$

DNA replication pre-initiation complex subunit $\mathrm{Cdc} 45$

$\begin{array}{llll}0.0539501 & 6.42306822 & 0.70649211\end{array}$

$\begin{array}{llll}0.05382957 & 8.16461447 & 0.61657318\end{array}$

$0.05378465 \quad 6.73116694 \quad 0.66719736$

$\# \mathrm{~N} / \mathrm{A}$

19S proteasome regulatory subunit Rpn1/Mts $4 \quad 0.053$ plant fal $0.05341867 \quad 6.3238615 \quad 0.77558154$

transcription factor TFIIH complex subunit Tfb1 (predicted) $\begin{array}{lllll}0.05321504 & 6.31890999 & 0.74364398\end{array}$
0.665147275

0.774819328

0.740250464

0.931110635

0.64294727

0.894440886

0.599876056

0.906258271

0.80337476

0.66977051

0.927511618

0.817934271

0.811577213

0.714111684

0.805269817

0.718805107

0.80347155

0.708969206

0.636101105

0.677099798

0.545695988

0.829736622

0.665832677

0.732030493

0.592904362

0.787619381

0.860485422

0.655282616

0.72734797

0.891067639

0.871749853

0.795607543

0.834043243

0.828410559

0.609406819

0.700599022

0.724786088

0.821826596

0.820324875

0.660111449

0.676934725

0.77693581

0.699296753

0.743820122

0.833122714

0.577428394

0.808903015 
SPCC1259.09C SPBC1734.14C SPNCRNA.1308 SPBP35G2.08C SPNCRNA.906 SPBC36B7.03 SPBC21D10.05C SPBC1778.07

SPCC1672.07

SPAC12G12.02 SPCC14G10.02

SPBC1683.11C

SPNCRNA.923

SPCC584.13

SPAC20G8.04C

SPAC3A11.10C

SPNCRNA.1229

SPAC8F11.06

SPAC823.15

SPBC25H2.09

SPCC188.09C

SPBC14C8.19

SPAC6F $6.02 \mathrm{C}$

SPNCRNA.866

SPBC15C4.03

SPBC16G5.04

SPAC13C5.04

SPAC1F5.06

SPAC4F 10.18

SPAPB8E5.07C

SPBC4.02C

SPAC17H9.17c

SPBC106.09

SPAC2C4.12C

SPNCRNA.1503

SPAC22G7.02

SPBC1709.06

SPBC651.10

SPNCRNA.677

SPAC664.03

SPBPB8B6.05C

SPNCRNA.784

SPCC1795.03

SPCC18.12C

SPBC646.07c

SPCC1682.02C

SPAC12B10.07 utp21

efg1

urb1

SPBC1683.11C \#N/A

SPCC584.13

SPAC20G8.04c dpe1

\section{\#N/A}

brr6

ppa1

mic19

pfl4

tam 10

pof5

mrs6

mrpl23

SPAC13C5.04

sh1

nup37

rrp12

SPBC4.02C

$\mathrm{mdm} 10$

cut4

tpt1

kap111

dus2

nse5

paf1

SPBPB8B6.05C \#N/A

gms1

utp23

tsc13

$\mathrm{mcm} 3$

acp1 pyruvate dehydrogenase protein x component, Pdx1 (predic 0.053028147 .582301980 .59726689 cyclin-dependent protein kinase regulatory subunit Suc1 \#N/A

TRAMP complex zinc knuckle subunit Air1

$$
\text { \#N/A }
$$

ER protein translocation subcomplex subunit Sec63 (predict $0.05214278 \quad 7.99807544 \quad 0.57074956$ GTPase activating protein Ucp3

rRNA processing protein Efg1 (predicted)

ribosome biogenesis protein Urb1 (predicted)

isocitrate lyase (predicted)

$$
\# \text { N/A }
$$

mitochondrial electron transfer flavoprotein-ubiquinone oxi $\begin{array}{lllll}0.0491937 & 8.18825504 & 0.55137896\end{array}$ dipeptidyl peptidase, unknown specificity, implicated in glu $0.04898851 \quad 8.72985772 \quad 0.5825942$ $\# \mathrm{~N} / \mathrm{A}$

nuclear envelope protein $\mathrm{Brr6} / \mathrm{Br} 1$ MICOS complex subunit Mic19/25

cell surface glycoprotein, flocculin Pf|4

F-box protein Pof5

$$
\text { \#N/A }
$$

Rab geranylgeranyltransferase escort protein Mrs6 (predict $0.04715525 \quad 6.809514990 .69197561$ mitochondrial ribosomal protein subunit L13 (predicted) $\quad \begin{array}{lllll}0.04672239 & 5.89360125 & 0.76891335\end{array}$ class I glutamine amidotransferase family protein, conserve $0.04620927 \quad 6.273018750 .79673303$ ER heat shock protein Lsh1 (predicted)

nucleoporin, WD repeat Nup37

rRNA processing protein Rrp12 (predicted)

rRNA 2'-O-methyltransferase fibrillarin-like (predicted)

ERMES complex subunit Mdm10 (predicted) anaphase-promoting complex, platform subcomplex scaffol $0.04500591 \quad 7.51139363 \quad 0.71114459$ tRNA 2'-phosphotransferase Tpt1/ ClA machinery involved i $0.044925915 .83002296 \quad 0.79064331$

$$
\text { \#N/A } \quad 0.044638498 .15702098 \quad 0.59421121
$$

karyopherin/importin beta family nuclear import signal rec $\begin{array}{lllll}0.04427695 & 7.42032707 & 0.63905511\end{array}$ tRNA dihydrouridine synthase Dus2 (predicted)

Smc5-6 complex non-SMC subunit Nse5

$$
\text { \#N/A }
$$

\section{RNA polymerase II associated Paf1 complex (predicted)} L-asparaginase (predicted)

$$
\text { \#N/A }
$$

Golgi UDP-galactose transmembrane transporter Gms1 rRNA processing protein Utp23 (predicted)

enoyl reductase (predicted)

MCM complex subunit Mcm3

F-actin capping protein alpha subunit $\begin{array}{llll}0.05273624 & 6.16391231 & 0.7744242\end{array}$ 0.05231883 .00533472

0.76240312
203

$\begin{array}{llll}0.05215968 & 7.7050651 & 0.57934412\end{array}$ $\begin{array}{llll}0.05161945 & 7.88002654 & 0.55918978\end{array}$ $\begin{array}{llll}0.05008618 & 6.72517659 & 0.73569056\end{array}$ $0.0499634 \quad 7.80701342 \quad 0.5727246$ $\begin{array}{llll}0.04986656 & 6.58555861 & 0.70517645\end{array}$ $\begin{array}{llll}0.04946618 & 7.75293497 & 0.72824531\end{array}$ $\begin{array}{llll}0.04942457 & 7.22310971 & 0.63497215\end{array}$ $\begin{array}{llll}0.04938563 & 5.95744836 & 0.77251255\end{array}$ $\begin{array}{llll}0.04923733 & 5.19047636 & 0.87291715\end{array}$ $\begin{array}{llll}0.04896781 & 5.14570971 & 0.83227441\end{array}$ $\begin{array}{lll}0.04890241 & 5.22360853 & 0.85876111\end{array}$ $\begin{array}{llll}0.04866689 & 6.60340362 & 0.74343608\end{array}$ $\begin{array}{llll}0.04797313 & 5.65626323 & 0.8022387\end{array}$ $0.04764017 \quad 6.64175584 \quad 0.74974145$

$\begin{array}{llll}0.04754197 & 5.26361489 & 0.85593108\end{array}$ $0.04733595 \quad 6.90265435 \quad 0.70436556$

$\begin{array}{llll}0.04558414 & 9.07004348 & 0.56304244\end{array}$

$\begin{array}{llll}0.04543578 & 6.79860981 & 0.74008406\end{array}$

$\begin{array}{llll}0.04511398 & 8.5269948 & 0.61560488\end{array}$

$\begin{array}{llll}0.04510281 & 7.3741036 & 0.71388518\end{array}$

$\begin{array}{llll}0.04507633 & 5.66802709 & 0.81053598\end{array}$

0.6811465

0.832405094

1
-5581

0.666515085

0.659208633

0.649144735

0.802818192

0.660574725

0.775865119

0.796181284

0.714945904

0.83126943

0.913455517

0.641280774

0.669122962

0.880128574

0.902388715

0.808806162

0.855277193

0.814104123

0.892772896

0.899970798

0.775269219

0.765673826

0.828410559

0.851024111

0.652170855

0.806190498

0.698314925

0.782915174

0.86209711

0.780664448

0.845670538

0.678116259

0.718686175

0.822041022

0.906278141

0.786258899

0.735950792

0.77261392 $\begin{array}{llll}0.04329746 & 6.95594111 & 0.70119964\end{array}$ $\begin{array}{lll}0.04286131 & 2.90481446\end{array}$

$\begin{array}{llll}0.04268296 & 7.55340999 & 0.69744309\end{array}$ $\begin{array}{llll}0.04210477 & 6.12974707 & 0.84454413\end{array}$ $\begin{array}{llll}0.04186197 & 6.64313939 & 0.80174086\end{array}$ $\begin{array}{llll}0.04184596 & 8.33364238 & 0.59696631\end{array}$ $\begin{array}{llll}0.04180598 & 7.81503918 & 0.63542664\end{array}$
0.76897282

0.891067639

0.855014347

0.680917828

0.71519654 
SPAC959.11

SPNCRNA.1520

SPBP4H10.03

SPNCRNA.967

SPBC16G5.18

SPBC12C2.09c

SPAC22A12.12C

SPCC1739.14

SPCC320.05

SPCC794.10

SPAC16A10.07C

SPAC31A2.09C

SPBC1778.10c

SPAC23D3.06C

SPAC2OH4.01

SPBC16A3.14

SPBC119.06

SPBC1861.04C

SPBP4H10.18C

SPBC3H7.12

SPBC106.19

SPBC13E7.03C

SPAC1782.09c

SPBC902.04

SPNCRNA.166

SPCC364.02C

SPAC13G6.04

SPAC22F3.06C

SPNCRNA.814

SPCC1281.05

SPAC6G9.02c

SPAC11D3.15

SPBP4H10.05C

SPAP27G11.06c

SPAC1952.07

SPBP4H10.07

SPBC16E9.03c

SPBC23E6.07C

SPNCRNA.299 naa30

spn1

SPBPB7E8.01

SPAC959.11

\#N/A

oxa102

$\# N / A$

erg24

izh2

rrp40

npp106

SPCC320.05

ugp1

apm4

ppk21

nup146

utp5

SPBC16A3.14

sco1

prp24

SPBP4H $10.18 \mathrm{c}$

rav2

ppr10

vts1

clp1

rmn1

$\# \mathrm{~N} / \mathrm{A}$

bis1

tim8

lon1

rsc7

nop9

SPAC11D3.15

spe2

vas2

rad1

SPBP4H10.07

coa1

rfc1
RNA export factor Rsm 1

serine/threonine protein kinase Nnk1 (predicted)

$\# \mathrm{~N} / \mathrm{A}$

$\# \mathrm{~N} / \mathrm{A}$

NatC $\mathrm{N}$-acetyltransferase complex catalytic subunit Naa30

mitotic septin Spn1

Schizosaccharomyces specific protein, predicted GPI anchor 0.

dynamin family protein $\mathrm{Vps} 1$

Schizosaccharomyces specific protein

\#N/A

mitochondrial inner membrane insertase Oxa102

\#N/A

C-14 sterol reductase Erg24 (predicted)

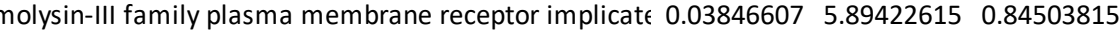

exosome subunit Rrp40

nucleoporin Npp106

sulfate transmembrane transporter (predicted)

UTP-glucose-1-phosphate uridylyltransferase-like Ugp1

shelterin complex subunit Taz1

AP-2 adaptor complex mu subunit Apm4 (predicted)

serine/threonine protein kinase Ppk21 (predicted)

nucleoporin, WD repeat Nup146

U3 snoRNP-associated protein Utp5 (predicted)

$\begin{array}{lll}0.04148936 & 7.22748277 \quad 0.69651349\end{array}$

$\begin{array}{llll}0.04146336 & 8.33020728 & 0.65340165\end{array}$

$\begin{array}{llll}0.04106793 & 3.99408091 & 0.94894458\end{array}$

$\begin{array}{llll}0.04060046 & 5.01610107 & 0.88729164\end{array}$ $\begin{array}{llll}0.03982414 & 9.41362844 & 0.59304229\end{array}$

$\begin{array}{llll}0.03960414 & 8.87836708 & 0.61819235\end{array}$

$\begin{array}{llll}0.03924232 & 5.32659005 & 0.87850068\end{array}$

$\begin{array}{llll}0.03912455 & 8.08728225 & 0.63326344\end{array}$

$0.0389687 \quad 5.96528488 \quad 0.87520856$

$\begin{array}{llll}0.0388346 & 7.44281426 & 0.69093195\end{array}$

$0.03785786 \quad 6.15356131 \quad 0.82980015$

$\begin{array}{llll}0.03757215 & 7.83582677 & 0.67472509\end{array}$

$\begin{array}{llll}0.03753525 & 6.93606146 & 0.80492222\end{array}$

$\begin{array}{llll}0.03743904 & 6.89188122 & 0.7703392\end{array}$

$\begin{array}{llll}0.03726599 & 6.87239339 & 0.77373123\end{array}$

$\begin{array}{lll}0.0372624 & 7.09717702 & 0.75476907\end{array}$

$0.03725838 \quad 7.05055225 \quad 0.73176603$

$\begin{array}{llll}0.03686812 & 8.45282236 & 0.69651009\end{array}$

$\begin{array}{llll}0.03670075 & 7.11454581 & 0.80046712\end{array}$

superoxide dismutase, mitochondrial ribosomal protein sub 0.036486646 .615673320 .79674866

mitochondrial copper chaperone for cytochrome c oxidase $\subseteq \begin{array}{llll}0.03628285 & 6.48811608 & 0.79725618\end{array}$

RNA-binding protein Prp24

Schizosaccharomyces specific protein

RAVE complex subunit Rav2

mitochondrial PPR repeat protein Ppr10

Smaug family RNA-binding protein Vts1 (predicted)

Cdc14-related protein phosphatase Clp1/Flp1

RNA-binding protein

$$
\text { \#N/A }
$$

$0.03620506 \quad 7.74535271 \quad 0.70672953$

$\begin{array}{llll}0.03613284 & 6.15995572 & 0.80758265\end{array}$

$0.03607037 \quad 6.1934196 \quad 0.82707447$

$\begin{array}{llll}0.03516655 & 6.32967103 & 0.83478997\end{array}$

$\begin{array}{llll}0.03484338 & 7.81615522 & 0.69316672\end{array}$

$0.03473025 \quad 7.10944252 \quad 0.77725213$

$\begin{array}{llll}0.03453742 & 7.73387648 & 0.74202846\end{array}$

$0.03438995 \quad 1.53106549$

$\begin{array}{lll}0.03434736 & 5.46003605 & 0.920965\end{array}$

mitochondrial matrix Lon protease $\# \mathrm{~N} / \mathrm{A}$

RSC complex subunit Rsc7

pumilio family RNA-binding protein Nop9 (predicted)

5-oxoprolinase (ATP-hydrolizing) (predicted)

S-adenosylmethionine decarboxylase proenzyme Spe2

AP-1 adaptor complex sigma subunit Aps 1

checkpoint clamp complex protein Rad1

$\begin{array}{llll}0.03417736 & 9.14611334 & 0.68472115\end{array}$

$\begin{array}{llll}0.03395388 & 6.13925562 & 0.82737409\end{array}$

$\begin{array}{llll}0.03386021 & 7.14027198 & 0.78426077\end{array}$

$\begin{array}{llll}0.03378996 & 7.40819657 & 0.78680488\end{array}$

$\begin{array}{llll}0.03377218 & 8.94705631 & 0.73199674\end{array}$

$\begin{array}{llll}0.03337937 & 7.36164706 & 0.73910008\end{array}$

$\begin{array}{llll}0.03293842 & 6.50496174 & 0.86476175\end{array}$

$\begin{array}{llll}0.03244528 & 7.11889647 & 0.78059326\end{array}$

ubiquitin-protein ligase E3, unknown specificity (predicted) $0.0322293 \quad 6.777189160 .78589629$

cytochrome c oxidase assembly protein Coa1 (predicted)

DNA replication factor $C$ complex subunit $\mathrm{RfC} 1$

$\# N / A$

$\begin{array}{llll}0.03162922 & 7.42493196 & 0.7690036\end{array}$

$0.03141544 \quad 5.00951917 \quad 0.9322208$
0.768570505

0.731917399

0.969604787

0.92338578

0.888391686

0.805282051

0.677099798

0.70078277

0.917323476

0.713598452

0.91529034

0.764767719

0.832405094

0.891409802

0.877784866

0.750000686

0.857734996

0.829455415

0.832054517

0.817997054

0.799171801

0.768570505

0.854057498

0.851024111

0.85129896

0.77707133

0.859762188

0.875854611

0.882240369

0.766585408

0.83425879

0.807662133

0.949426874

0.898418502

0.759746849

0.875899296

0.840853085

0.842784075

0.799171801

0.805282051

0.906594312

0.837316699

0.842341195

0.905824493

0.828410559

0.957693623 
SPAC16E8.04C

SPNCRNA.192

SPBC1347.14C

SPNCRNA.1422

ScPofMt25

SPAC17A2.12

SPNCRNA.563

SPAC22A12.13

SPAC18G6.11C

SPBC646.04

SPBP35G2.09

SPCC736.08

SPAC13G7.02C

SPBC1105.06

SPCC417.12

SPBP4H10.12

SPNCRNA.1035

SPAC8F11.09C

SPBC3D6.12

SPBC36B7.08C

SPAC1782.10C

SPBC83.11

SPNCRNA.1574

SPCC23B6.01C

SPBC887.22

SPBC36B7.07

SPBP8B7.14C

SPBC409.17C

SPBC365.10

SPAC17G6.04C

SPBC651.08C

SPBC11B10.03

SPAC222.03C

SPAC12G12.14c

SPCC1442.17C

SPCC1620.12C

SPBC19F8.02

SPBC15D4.09C

SPAC1705.02

SPBC16A3.12C

SPAC11E3.07

SPNCRNA.1018 pmc4 SPCC417.12

SPBP4H10.12

$\# \mathrm{~N} / \mathrm{A}$

nnt1

dip2

ccp1

nhp2 pet2

SPBC409.17c

arp5

сpp1

rpc1

$\operatorname{cog} 8$

$\operatorname{tim} 10$

pfs2

ist1

SPCC1620.12c

nud3

met3

SPAC1705.02

SPBC16A3.12C vma4

\#N/A
RCC1 family Ran GDP/GTP exchange factor contractile ring myosin $\mathrm{V}$ regulator Rng8 prohibitin Phb2 (predicted)

pumilio family RNA-binding protein Puf3 (predicted)

$$
\# \mathrm{~N} / \mathrm{A}
$$

$$
\# N / A
$$

$\# N / A$

$\# \mathrm{~N} / \mathrm{A}$ chorismate mutase Aro7 (predicted)

$\begin{array}{llll}0.03140086 & 7.90497421 & 0.71405786\end{array}$ $\begin{array}{llll}0.03128824 & 5.74480023 & 0.89654924\end{array}$ $\begin{array}{lll}0.03073771 & 7.50034387 & 0.74685448\end{array}$ $\begin{array}{llll}0.0298742 & 8.10649526 & 0.77507156\end{array}$ $\begin{array}{llll}0.02935366 & 5.32939229 & 0.93603367\end{array}$ $\begin{array}{llll}0.02883968 & 6.83128147 & 0.8407702\end{array}$ 0.028805553 .25261136

$0.02879512 \quad 5.790073690 .92032318$ $\begin{array}{llll}0.02876144 & 6.31040534 & 0.84919612\end{array}$ 0.028396814 .131749760 .95861102 ATP-dependent DNA helicase/ ubiquitin-protein ligase E3 (k $0.02833004 \quad 6.434898330 .90084116$ $\# \mathrm{~N} / \mathrm{A}$

$0.02822215 \quad 6.51200408 \quad 0.86432699$ $\begin{array}{llll}0.02807415 & 5.57148144 & 0.87229474\end{array}$ RNA polymerase I general transcription inititation factor su $\begin{array}{llll}0.02805783 & 7.22365909 & 0.84378762\end{array}$ poly(A) polymerase Pla

U1 snRNP-associated protein Usp103

$\mathrm{CBF} 1 / \mathrm{Su}(\mathrm{H}) / \mathrm{LAG}-1$ family transcription factor Cbf11

heat shock protein Ssa1 (predicted)

mediator complex subunit Med4

carboxylesterase, type B family protein

$0.02784487 \quad 7.2335098 \quad 0.81119999$

$\begin{array}{lll}0.02769704 & 4.94958598 & 0.93059049\end{array}$

$\begin{array}{lll}0.02766717 & 8.02146262 & 0.75015389\end{array}$

$0.02759575 \quad 8.14461253 \quad 0.8387838$

$0.0275925 \quad 6.37546311 \quad 0.87664245$ $0.027590695 .74350913 \quad 0.95427181$

protein with a role in ER insertion of tail-anchored membra $0.027462326 .58658487 \quad 0.84763592$

$$
\text { \#N/A }
$$

nicotinamide $\mathrm{N}$-methyltransferase Nnt1 (predicted)

U3 snoRNA associated protein Dip2 (predicted)

0.026993143 .39090162

$\begin{array}{lll}0.02684336 & 6.18352517 & 0.8810995\end{array}$ $0.02656896 \quad 7.61169517 \quad 0.79118555$

$\begin{array}{llllll}\text { histone chaperone, CENP-A nucleosome disassembly Ccp1 } & 0.02632342 & 7.38671934 & 0.7958731\end{array}$ box H/ACA snoRNP complex subunit Nhp2 $\begin{array}{lll}0.02607826 & 7.4011517 & 0.81581737\end{array}$ Golgi phosphoenolpyruvate transmembrane transporter Pet 0.025872647 .675336540 .79589131 \#N/A

terol intermembrane transfer protein (predicted) signal peptidase complex subunit Spc1 (predicted) SNARE TIg1 (predicted)

DNA polymerase epsilon catalytic subunit B, Dpb2 DUF1769 family protein

Ino80 complex actin-like protein Arp5

$\begin{array}{llll}0.02551008 & 7.64427962 & 0.80857097\end{array}$ $\begin{array}{lllll}0.02545725 & 8.59890746 & 0.77762573\end{array}$ $\begin{array}{lll}0.02491667 & 5.88423696 & 0.92077834\end{array}$ $\begin{array}{lll}0.02483884 & 7.38407075 & 0.8107552\end{array}$ $0.02478327 \quad 6.67174582 \quad 0.8727106$ $\begin{array}{llll}0.02450047 & 5.73020994 & 0.89332513\end{array}$ $\begin{array}{llll}0.02446565 & 7.58124342 & 0.80366283\end{array}$ $\begin{array}{llll}0.024193 & 8.29024896 & 0.7890923\end{array}$ protein farnesyltransferase beta subunit Cpp1 $\begin{array}{lllll}\text { DNA-directed RNA polymerase III complex large subunit Rp } & 0.02386195 & 8.47636427 & 0.7712952\end{array}$ Golgi transport complex subunit Cog8 (predicted) $\quad \begin{array}{llllll}0.02370427 & 7.55091831 & 0.81093087\end{array}$ Tim9-Tim10 complex subunit Tim10 (predicted) $\quad \begin{array}{llllll}0.02364118 & 6.51907001 & 0.89970539\end{array}$ mRNA cleavage and polyadenylation specificity factor comf $0.02362013 \quad 6.44186733 \quad 0.8728236$ $\begin{array}{lllll}\text { MVB sorting pathway protein Ist1 (predicted) } & 0.02347005 & 5.44483627 & 0.93506561\end{array}$ $\begin{array}{llll}0.02301225 & 8.48314113 & 0.78129551\end{array}$ nuclear distribution protein NUDC homolog $\quad \begin{array}{lllll}0.02298247 & 5.36789884 & 0.91668384\end{array}$ $\begin{array}{lllll}\text { cystathionine gamma-synthase Met3 } & 0.02263759 & 7.64125602 & 0.82965511\end{array}$ SERF family protein, DUF, human 4F5S homolog, implicate 0.022548625 .850112270 .92009165 triglyceride lipase-cholesterol esterase (predicted) $\quad \begin{array}{lllll}0.02240412 & 7.12474911 & 0.83647553\end{array}$ V-type ATPase V1 subunit E (predicted) $0.022269547 .69269671 \quad 0.8253082$ $0.022084515 .90055438 \quad 0.91922698$
0.782978361

0.930742571

0.811577213

0.832706345

0.960016445

0.888008882

0.949196613

0.894824962

0.977746094

0.93391592

0.906278141

0.9134019

0.890504765

0.862398984

0.956596422

0.814293588

0.88604839

0.915804709

0.974316524

0.893734493

0.919456377

0.845878661

0.850375307

0.866138327

0.850375307

0.860485422

0.834528166

0.949378274

0.862195525

0.913455517

0.928103439

0.85666127

0.844436724

0.830090755

0.862247592

0.932956877

0.913455517

0.95916815

0.837937891

0.946558828

0.877767885

0.94910166

0.88388447

0.874256277

0.948353489 
SPNCRNA.950 SPNCRNA.1101 SPCC338.02

SPCC188.13C

SPBC1703.02

SPAC22A12.15C

SPNCRNA.1369

SPAC1639.01C

SPAC227.17c

SPBC19G7.06

SPAC3F10.11C

SPNCRNA.1119

SPAC1006.02

SPAC25B8.15C

SPBC16H5.09C

SPAC5H10.08C

SPNCRNA.1511

SPAC1687.04

SPNCRNA.1068

SPAC22A12.06

SPCC285.09c

SPAC23D3.10c

SPNCRNA.849

SPAPB1A10.06c

SPAC16C9.01C

SPBC405.06

SPNCRNA.541

SPAC23G3.06

SPCC1827.01C

SPAC227.14

SPNCRNA.1582

SPBC18E5.05C

SPAC2E1P3.01

SPBC16E9.18

SPNCRNA.915

SPBC2G2.16

SPNCRNA.1402

SPNCRNA.1269

SPAC9.05

SPBC21D10.11C

SPBC839.11C

SPAC4F10.13C

SPBC12D12.09

SPAC11G7.01

SPCC1235.05c

SPAC17A5.05C

SPAC13C5.01C mug112

dcr1

rsc9

bip1

elo1

\#N/A

SPAC227.17c

$\mathrm{mbx} 1$

$a b c 2$

\#N/A

asa1

tyw3

omh2

pan6

mcb1

fsh2

cgs 2

eng2

$\# \mathrm{~N} / \mathrm{A}$

SPAPB1A10.06C

SPAC16C9.01C

$x \mathrm{dj}$

$\# N / A$

nop58

utp25

yfh7

$\# \mathrm{~N} / \mathrm{A}$

elp5

SPAC2E1P3.01 psd1

$\# \mathrm{~N} / \mathrm{A}$

mpi1

$\#$ N/A

$\# \mathrm{~N} / \mathrm{A}$

fml1

nfs1

hut1

$\mathrm{mpd} 2$

rev7

$\mathrm{mtl} 2$

fft2

SPAC17A5.05C

pre9
$\# N / A$

$\# \mathrm{~N} / \mathrm{A}$

Schizosaccharomyces pombe specific protein Mug112

dicer

RSC complex subunit Rsc9

ER heat shock protein BiP

$\# \mathrm{~N} / \mathrm{A}$

fatty acid elongase Elo1 (predicted)

DUF3128 family, human c22orf39 ortholog

MADS-box transcription factor Mbx1

vacuolar phytochelatin and glutathione S-conjugate $A B C$ far 0.0203

$$
\text { \#N/A }
$$

ASTRA complex WD repeat subunit Asa1

wybutosine biosynthesis protein Tyw3 (predicted)

alpha-1,2-mannosyltransferase Omh2 (predicted)

pantoate-beta-alanine ligase

\#N/A

MCM binding protein homolog Mcb1

\#N/A

0.021972631 .53134485

$0.021918695 .66185896 \quad 0.9379566$

0.021776183 .55850003

$0.02173598 \quad 7.78658737 \quad 0.81215933$

$\begin{array}{llll}0.02132224 & 7.1835008 & 0.87615447\end{array}$

$\begin{array}{llll}0.02126369 & 10.0931966 & 0.75068632\end{array}$

$0.02124722 \quad 4.21643559$

$\begin{array}{llll}0.02107056 & 8.07426467 \quad 0.854196\end{array}$

$\begin{array}{llll}0.02067121 & 7.89895674 & 0.89615293\end{array}$

0.020162662 .6584093

$0.0201056 \quad 4.42757812 \quad 0.95667434$

$\begin{array}{llll}0.01984325 & 6.36262789 & 0.91426703\end{array}$

$\begin{array}{llll}0.01963997 & 5.45798952 & 0.96826403\end{array}$

$\begin{array}{llll}0.01946108 & 6.81458019 & 0.87790672\end{array}$

$\begin{array}{llll}0.01936071 & 5.63943978 & 0.97034552\end{array}$

$\begin{array}{llll}0.01882755 & 6.49203509 & 0.93403255\end{array}$

$\begin{array}{llll}0.0179541 & 5.6766407 & 0.94713869\end{array}$

serine hydrolase-like, human TSTD2 and OVCA2 ortholog, u $0.017782567 .09695423 \quad 0.88706114$

cAMP-specific phosphodiesterase Cgs2

cell wall and ascospore endo-1,3-beta-glucanase Eng2

$$
\text { \#N/A }
$$

$0.01772457 \quad 7.19810436 \quad 0.8825643$

$\begin{array}{llll}0.01769363 & 7.32037306 & 0.88080426\end{array}$

$\begin{array}{lll}0.01761107 & 4.87403893 & 0.96005194\end{array}$

$\begin{array}{llll}0.01759245 & 7.48788148 & 0.8834793\end{array}$

$\begin{array}{llll}0.01749229 & 7.16475532 & 0.89768434\end{array}$

$\begin{array}{llll}0.01708998 & 6.60624675 & 0.89415978\end{array}$

$\begin{array}{llll}0.01708998 & 6.60624675 & 0.89415978\end{array}$

$0.01689736 \quad 7.20252244 \quad 0.89109278$

$0.01627444 \quad 6.60883494 \quad 0.9378581$

$\begin{array}{lll}0.01623003 & 6.28079344 & 0.9093664\end{array}$

$0.01614164 \quad 6.61544496 \quad 0.91679195$

$0.01598139 \quad 5.8653201 \quad 0.9734334$

$0.015422557 .81702566 \quad 0.90466946$

$\begin{array}{lll}0.01520522 & 7.5025922 & 0.88494179\end{array}$

0.014821632 .36051452

$\begin{array}{lll}0.01477379 & 8.06148774 & 0.88400169\end{array}$

$0.01445327 \quad 5.10189218$

$0.01439496 \quad 5.4060697$

merase Mpi1 (predicted)

\#N/A

ATP-dependent 3' to 5' DNA helicase, FANCM ortholog Fml $0.01417084 \quad 8.09996002 \quad 0.87554315$ mitochondrial [2Fe-2S] cluster asssembly and tRNA modific $0.01378526 \quad 8.40570668 \quad 0.88033601$ ER uridine diphosphate-glucose transmembrane transporte $0.01363774 \quad 7.75023563 \quad 0.91614371$ GYF domain protein

$\begin{array}{llll}0.01332602 & 8.33299746 & 0.87541124\end{array}$ $0.01305444 \quad 5.21492089$

DNA polymerase zeta Rev7 (predicted)

$\begin{array}{llll}0.013014 & 7.13282127 & 0.93271343\end{array}$

$0.01273266 \quad 8.23605403 \quad 0.87414866$ SMARCAD1 family ATP-dependent DNA helicase Fft2 (predi methyltransferase (predicted)

$\begin{array}{llll}0.01247892 & 5.38257909 & 0.96698437\end{array}$

$20 S$ proteasome complex subunit alpha 3 Pre9

$\begin{array}{llll}0.01231152 & 7.69146861 & 0.90657891\end{array}$
0.863014401

0.915658598

0.81461017

1
885

0.898701885

0.918002009

0.930473103

0.851562196

0.976330069

0.944955523

0.985256113

0.916843958

0.986878082

0.958686504

0.968341166

0.923287101

0.920295749

0.919305676

0.978602971

0.921056628

0.931276203

0.92868701

0.92868701

0.92646268

0.960974845

0.940600854

0.946558828

0.989279311

0.936743107

0.921602286

0.921229937

1

0.91535890

0.918957898

0.946176646

0.915358905

0.957775599

0.914603568

0.984489618

0.938149249 
SPAC16E8.13 SPCC13B11.03C SPBC1289.06C SPAC10F6.04

SPBC3F6.02C SPBC2A9.09 SPCC553.02

SPCC576.17c

SPNCRNA. 242

SPNCRNA.583

SPBC26H8.16

SPBC13G1.06C

SPAC7D4.09C

SPAC9E9.07C

SPBC8E4.12C

SPBC13G1.10C

SPBP35G2.07

SPNCRNA.86

SPBC1703.01C

SPNCRNA.633

SPBC16A3.13

SPAC1805.05

SPBC14C8.12

SPBC2G2.14

SPAC589.05C

SPCC1450.02

SPAC644.10

SPBC1683.10c

SPBP4G3.03

SPBC660.13C

SPBP4H10.11C

SPNCRNA.1539

SPAC821.04c

SPBP4H10.04

SPCC830.11C

SPNCRNA.796

SPNCRNA.965

SPAC1B3.08

SPCC1235.16

SPCC4B3.13

SPAC1687.16C

SPAC27D7.08c

SPNCRNA.618

SPNCRNA.269

SPBC16G5.03

SPBC23G7.11

SPBC1709.18
SPAC16E8.13 SPCC13B11.03C

ppr8

ats1

erg 26

plp2

qns1

SPCC576.17c

\#N/A

$\# \mathrm{~N} / \mathrm{A}$

sdh8

isd11

$\operatorname{dfg} 10$

ypt2

ecl3

slh1

ilv1

\#N/A

pop4

\#N/A

meu7

cki3

rpb8

csi1

qtr3

bdf1

med11

pcl1

fub2

$\mathrm{ssb} 1$

Icf2

\#N/A

cid13

ppb1

fap7

$\# \mathrm{~N} / \mathrm{A}$

$\# N / A$

SPAC1B3.08

vma21

SPCC4B3.13

erg31

$\mathrm{mt} 16$

$\# N / A$

$\# \mathrm{~N} / \mathrm{A}$

mrz1

mag2

tif452 ubiquitin-protein ligase E3, human BRAP ortholog (predicte $0.01161947 \quad 7.151251490 .92520838$ hydroxyacylglutathione hydrolase (predicted)

mitochondrial PPR repeat protein Ppr8

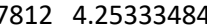

$\begin{array}{llll}0.01149933 & 7.5573399 & 0.94240844\end{array}$ $0.01118498 \quad 7.52044632 \quad 0.92465249$

$\begin{array}{lllll}3 \text { beta-hydroxysteroid dehydrogenase/delta } 5-->4 \text {-isomeras } 0.01109131 & 8.109099 & 0.92576127\end{array}$ phosducin family protein Plp2 (predicted) $\quad \begin{array}{lllll}0.01108878 & 6.80167035 & 0.94602414\end{array}$ glutamine-dependent NAD(+) synthetase Qns1 (predicted) $0.01091185 \quad 8.45958793 \quad 0.89672537$ pyridoxamine/pyridoxine/pyridoxal transmembrane transpc $0.01075507 \quad 6.7224646 \quad 0.94423563$

$$
\# \mathrm{~N} / \mathrm{A}
$$

0.010541225 .77779022

\#N/A $\quad 0.01032057 \quad 7.56982320 .93306608$

mitochondrial respiratory chain complex II assembly factor $0.01020636 \quad 6.17050819 \quad 0.9525734$ mitochondrial [2Fe-2S] cluster assembly protein Isd11 (prer $0.0099065 \quad 6.10913852 \quad 0.97947243$ 3-oxo-5-alpha-steroid 4-dehydrogenase (predicted) $\quad \begin{array}{lllll}0.00979228 & 5.53365311 & 0.97460212\end{array}$ GTPase Ypt2

$0.00966645 \quad 7.66725179 \quad 0.92859989$ 0.009179983 .63391517

extender of the chronological lifespan protein Ecl3

ATP-dependent RNA helicase SIh1, human ASCC3 ortholog $(0.008967678 .551112990 .92782474$ acetolactate synthase catalytic subunit

$$
\text { \#N/A }
$$

RNase $P$ and RNase MRP subunit Pop4

$$
\# N / A
$$

alpha-amylase homolog Aah4

serine/threonine protein kinase $\mathrm{Cki3}$

DNA-directed RNA polymerase I, II and III subunit Rpb8 mitotic centromere-SPB clustering protein Csi1

RNA queuosine modification protein Qtr3

Swr1 complex bromodomain subunit Bdf1

mediator complex subunit Med11

0.91388562

008743185.81812014

0.008493924 .81239407

$0.008442247 .33393275 \quad 0.95556322$

$0.008163317 .42017727 \quad 0.95878026$

$\begin{array}{llll}0.00813376 & 8.20373422 & 0.93094384\end{array}$

$0.00811323 \quad 6.33679517$

0.007749137 .040923340 .93937435

0.007331675 .78513557

$0.00706293 \quad 8.12321244 \quad 0.93901366$

0.006841965 .65472525

vacuolar ferrous iron/manganese transmembrane transpor $0.00637297 .96320323 \quad 0.96371885$

PI31 proteasome regulator Fub2 (predicted)

DNA replication factor $A$ subunit Ssb1

long-chain-fatty-acid-CoA ligase

$0.00557257 \quad 8.15205169 \quad 0.97612979$

$0.00540128 \quad 7.91506425 \quad 0.95959918$

$0.00504124 \quad 8.4509922 \quad 0.95050606$

$0.00471264 \quad 6.62991356 \quad 0.98245945$

poly(A) polymerase Cid13

$$
\text { \#N/A }
$$

0.00428397 .13620078

calcium-dependent serine/threonine protein phosphatase c 0.004229257 .366672340 .98549557

nucleoside-triphosphatase involved in SSU-rRNA maturatio 0.004184515 .46584745

\#N/A $\quad 0.00403656 \quad 6.922777720 .98273581$

$0.003849046 .00131316 \quad 1$

$0.0038016 \quad 7.1165473 \quad 1$

$0.00366554 \quad 5.38739263$

0.003658267 .452855210 .97167138

complex subunit (predicted)

V-ATPase assembly protein Vma21 (predicted)

MatE family transmembrane transporter (predicted)

C-5 sterol desaturase Erg31

$0.00328681 \quad 6.86340823$

23S rRNA/U6 snRNA (adenine-N(6))-methyltransferase Mt| 0.00307965 .47212431

$0.00296696 \quad 5.73331963$

\#N/A

$0.00275578 \quad 4.44093648$

ubiquitin-protein ligase E3/SUMO transferase, Topors, poss 0.002625816 .29844515

DNA-3-methyladenine glycosidase Mag2

$0.00260535 \quad 4.56547529$

translation initiation factor elF4E, $4 \mathrm{~F}$ complex $\mathrm{E}$ subunit iso $0.00212387 \quad 7.1654805 \quad 0.98560239$
0.952502728

0.964519389

0.952074475

0.952850756

0.967346995

0.930783422

0.96580843

0.957838987

0.972728388

0.994822376

0.99031916

0.954982964

0.95447428

0.944704792

0.975447258

0.977746094

0.956670614

0.962137633

0.961912985

0.981752512

0.991723383

0.978288004

0.970908694

0.997357583

0.999407576

9973925

0.997392568

0.987636102

1

1

1

0.999407576 
SPCC1235.08c SPBC776.08C SPBC14C8.17C SPAC6B12.11

SPAC23C4.12

SPAC26F1.04C

SPAC15A10.09c

SPBC1105.16c

SPAC926.10

SPAC6B12.07C

SPAC1F3.03

SPBC244.01C

SPBC1709.08

SPCC1795.07

SPCC1919.03c

SPAC806.06c

SPNCRNA.729

SPBC12C2.05C

SPAC22A12.07C

SPAC4F 10.22

SPAC3A12.11C

SPAC31G5.18C

SPAC7D4.02C

SPAPB24D3.01

SPBC27B12.13

SPBC4B4.08

SPNCRNA.938

SPAC23H3.14

SPBC3H7.13

SPBC1604.12

SPNCRNA.1633

SPNCRNA.1583

SPNCRNA.1004

SPNCRNA.1030

SPCC1223.11

SPNCRNA.1665

SPBC800.03

SPCC417.06C

SPAC9E9.08

SPBC3D6.08C

SPCC1259.12C

SPAC343.01c

SPBC725.11C

SPAC1687.12C

SPAC29A4.09

SPNCRNA.1482

SPBC1347.08C hhp2

etr1

pun1

pr2

new9

SPAC6B12.07C

sro7

sid4

cft1

sws2

SPAC806.06C

$\# \mathrm{~N} / \mathrm{A}$

bzz1

ogm 1

cmc4

cwf2

sde2

sfp47

toe3

tom 40

ght2

avl9

far10

SPBC1604.12

$\# N / A$

$\# N / A$

$\# \mathrm{~N} / \mathrm{A}$

$\# \mathrm{~N} / \mathrm{A}$

ptc2

\#N/A

clr3

mug27

$\operatorname{rad} 26$

Ism1

gid1

erg 8

php2

coq4

SPAC29A4.09

\#N/A

SPBC1347.08C
Golgi to ER retrograde transport protein (predicted)

0.002012157 .15918995

SAGA complex subunit Spt8

replication preinitiation complex assembly protein

$\begin{array}{llll}0.00150952 & 6.71551309 & 0.98450729\end{array}$

0.0013865 .79454019

$0.00099891 \quad 6.68911227$

$0.00094419 \quad 7.29005003$

$0.00081002 \quad 6.43463218$

$0.00046258 \quad 5.81504471$

$-0.0002021 \quad 3.55740699$

SUR7 family protein Pun1 (predicted)

RNase P subunit Rpr2 (predicted)

Schizosaccharomyces specific protein New 9

$-0.0002518 \quad 7.66399435$

Lgl family protein Sro7 (predicted) $\quad-0.00048828 .25829463$

$\begin{array}{llll}\text { SIN component scaffold protein Sid4 } & -0.0006803 & 7.44988277 \\ \text { mRNA cleavage and polyadenylation specificity factor comr } & -0.0008651 & 8.39040187\end{array}$

mitochondrial ribosomal protein subunit S37 (predicted) $\quad-0.0010483 \quad 8.11662637 \quad 0.99767254$

AMP-activated protein kinase beta subunit Amk2

0.0013767 .50653581

nicotinamide mononucleotide (NMN) adenylyltransferase (| $-\begin{array}{lll}-0.0014582 & 7.34927801\end{array}$ \#N/A

F-BAR domain protein Bzz1

$-0.00164175 .68928656$

$-0.001747 \quad 7.34301404$

protein O-mannosyltransferase Ogm1

$-0.0020357-9.028521170 .98605654$

mitochondrial intermembrane space protein, mature-T-Cell $-0.0021865 \quad 4.14945$

zf-CCCh type zinc finger protein, Prp19 complex subunit, RN -0.0021929 6.61042372

Ubp4 interactor $\mathrm{Sfp} 47$

transcription factor (predicted)

0.00230445 .98311964

$-0.00276497 .33083306$

$0.0027718 \quad 8.50482471 \quad 0.98860447$

mitochondrial TOM complex subunit Tom 40 (predicted) - - -

plasma membrane glucose/fructose:proton symporter Ght_- -0.00277897 .3995171$ $\# \mathrm{~N} / \mathrm{A}$

$-0.0028594 .0799318$

post-Golgi vesicle-mediated transport protein Avl9 (predict -0.00287086 .73122093$

SIP/FAR complex FHA domain subunit Far10/Csc1

Schizosaccharomyces specific phosphoprotein

$\# N / A$

$\# N / A$

$\#$ \#N/A

$0.0029553 \quad 7.0066542 \quad 0.96366987$

$-0.00305317 .89943768$

$\begin{array}{lll}-0.0032414 & 6.99306709 & 0.98161464\end{array}$

$-0.0037403 \quad 4.11507293$

$-0.00374446 .07496294$

$\begin{array}{lll}0.0037839 & 8.26856713 & 0.96583697\end{array}$

A A \#N/A

histone deacetylase (class II) Clr3

$\begin{array}{lll}-0.0043832 & 6.79997157 & 0.98357159\end{array}$

$\begin{array}{lll}-0.004471 & 7.76872058 & 0.98454614\end{array}$

$\begin{array}{lll}-0.0046137 & 7.26255004 & 0.9698283\end{array}$

$\begin{array}{lll}-0.0049888 & 6.12401811 & 0.98022814\end{array}$

$\begin{array}{lll}-0.005 & 6.8752735 & 0.98255477\end{array}$

mRNA decapping complex subunit (predicted)

GID complex subunit, Ran GTPase binding protein Gid1 (pre -0.0050261 6.49670757

phosphomevalonate kinase Erg8 (predicted)

$\begin{array}{llll}-0.0051134 & 7.54271197 & 0.97090294\end{array}$

$\begin{array}{lll}-0.0053008 & 8.39954352 & 0.95922965\end{array}$

$\begin{array}{llll}-0.0058321 & 7.08552472 & 0.97097432\end{array}$

$-0.0058963 \quad 6.3851585$

$-0.0061463 \quad 5.18812439$

$-0.00615767 .05816042$

0.99

0.998634142

42
1

ubiquinone biosynthesis protein Coq4 (predicted)

rRNA exonuclease Rrp17 (predicted)$$
\# \mathrm{~N} / \mathrm{A}
$$

ribonuclease $\mathrm{H} 2$ complex subunit (predicted)

1

(1)

(1)

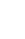

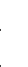

0.999719125

(1)

1

1

0.981752512

0.996700631

1
1

0.983615768

0.998634142

0.998091962

0.998634142

0.986553442

0.995441351

0.997357583

0.987075033

0.978057801

0.987075033

1
1
1

1

1 
SPCC16A11.03C

SPCC1322.10

SPNCRNA.1519

SPBC216.06C

SPCC4G3.19

SPAC23H 4.15

SPCC550.02C

SPAPB17E12.12C

SPCC4B3.09C

SPBC1271.05C

SPAC824.06

SPAC1851.03

SPCC126.08c

SPNCRNA.1033

SPAC630.09c

SPBC1539.10

SPCC4B3.12

SPAP27G11.15

SPBC24C6.13

SPBC336.05C

SPAPB1E7.05

SPAC1071.02

SPNCRNA.1483

SPAC2C4.06C

SPAC6G9.10C

SPAC22E12.16c

SPBC336.04

SPCC1739.12

SPCPB16A4.04C

SPAC17A5.16

SPBC4C3.09

SPAC607.02C

SPAC4A8.07C

SPBC1711.12

SPCC63.06

SPAC23H4.17c

SPAC1805.01c

SPBC11B10.10

SPNCRNA.1116

SPNCRNA.1080

SPBC19C2.05

SPNCRNA.458

SPBC651.02

SPBC1289.09

SPCC794.11C
SPAC806.05

sec72

SPCC16A11.03

SPCC1322.10

\#N/A

wi1

alp16

cwf5

SPAPB17E12.12C

mrpl12

SPBC1271.05c

tim14

ckb1

SPCC126.08c

$\# \mathrm{~N} / \mathrm{A}$

mug58

nop16

set9

coa6

gde1

mms19

\#N/A

$\mathrm{rcm} 1$

sen1

pik1

cdc6

ppe1

trm8

ftp105

otg 3

SPAC607.02C

Icb4

ppp16

SPCC63.06

srb10

ppk6

pht1

\#N/A

$\# \mathrm{~N} / \mathrm{A}$

pat1

\#N/A

nit1

tim 2

ent3 mitochondrial ANC9 family protein

Sec7 domain protein, ARF GEF Sec72

DUF2009 family protein, conserved in yeast and apicomple) $\quad-0.006738 \quad 6.60699743$

conserved fungal cell surface protein, Kre9/Knh1 family $\quad-0.00695627 .710465220 .95864152$

$$
\text { \#N/A }
$$

replication fork protection complex subunit Swi

gamma tubulin complex subunit Alp16

ribosome biogenesis protein Tsr1 (predicted)

Prp19 complex subunit, RNA-binding Cwf5

mitochondrial carrier, 3'-phosphoadenosine 5'-phosphosulfi -0.00882245 .13710185$

mitochondrial ribosomal protein subunit L12 (predicted) $\quad-0.0088907 \quad 7.446034270 .91610946$

zf-AN1 type zinc finger protein, involved in ER membrane ts $-0.0088947 \quad 6.93147178 \quad 0.96156212$

$\begin{array}{lllll}\text { TIM23 translocase complex subunit Tim14 (predicted) } & -0.0089038 & 6.64736584 & 0.9682417\end{array}$

CK2 family regulatory subunit Ckb1

lectin family glycoprotein receptor (predicted)

$$
\# N / A
$$

GLYK family kinase of unknown specificity, implicated in nu $-0.01000897 .03672883 \quad 0.91261235$ ribosome biogenesis protein Nop16 (predicted)

histone lysine $\mathrm{H} 3-\mathrm{K} 20$ methyltransferase Set9

structure-specific endonuclease catalytic subunit SIx1

cytochrome c oxidase assembly protein Coa6 (predicted)

small RNA 2'-O-methyltransferase Hen1 (predicted)

glycerophosphoryl diester phosphodiesterase Gde1 (predict $\quad \begin{array}{llll}-0.0107208 & 8.61778173 & 0.9374218\end{array}$ $\mathrm{ClA}$ machinery protein Mms19

tRNA (cytosine-5-)-methyltransferase Rcm1 (predicted)

ATP-dependent 5' to 3' DNA/RNA helicase Sen1

1-phosphatidylinositol 4-kinase Pik1

DNA polymerase delta catalytic subunit Cdc6

serine/threonine protein phosphatase Ppe1

$\begin{array}{llllll} & 0.07274328\end{array}$

Golgi localized protein, human HID1 ortholog 3, implicated $-0.0123146 \quad 6.965567190 .92437962$ alpha-1,3-galactosyltransferase $\quad \begin{array}{llll}-0.0125429 & 7.20132845 & 0.91738498\end{array}$ conserved fungal protein

sphingoid long chain base kinase (predicted)

serine-type peptidase activity

WD repeat protein, human WDR89 family

cyclin-dependent protein Srb mediator subunit kinase Srb1C $-0.0133309 \quad 5.16209041 \quad 0.96831637$

serine/threonine protein kinase Ppk6 (predicted)

histone H2A variant H2A.Z, Pht1

$\# N / A$

serine/threonine protein kinase Ran1/Pat1

$\# \mathrm{~N} / \mathrm{A}$

bis(5'-adenosyl)-triphosphatase Nit1 (predicted)

TIM23 translocase complex subunit Tim21 (predicted)

ENTH/VHS domain protein Ent3 (predicted)
$-0.0063873 \quad 7.37460203 \quad 0.95246024$ $\begin{array}{llll}-0.0065599 & 8.93276489 & 0.93674185\end{array}$

$-0.00700993 .97567644$

$-0.0078933 \quad 8.85658955 \quad 0.92293159$

$\begin{array}{llll}-0.007935 & 6.7537169 & 0.9614416\end{array}$

$\begin{array}{lll}-0.0081605 & 7.59178203 & 0.94763665\end{array}$

$-0.0082004 \quad 6.26654306$

$\begin{array}{llll}-0.0089204 & 8.01592793 & 0.92395384\end{array}$

$\begin{array}{llll}-0.0094076 & 7.37797736 & 0.94415914\end{array}$ $\begin{array}{llll}-0.0095509 & 7.41827079 & 0.92426793\end{array}$

$\begin{array}{llll}-0.0100135 & 6.11476419 & 0.94761362\end{array}$

$\begin{array}{llll}-0.0102037 & 7.58868145 & 0.93290565\end{array}$

$\begin{array}{llll}-0.0103661 & 7.54377471 & 0.92748063\end{array}$

$\begin{array}{llll}-0.0104405 & 5.5019642 & 0.96801558\end{array}$

$\begin{array}{llll}-0.0104636 & 7.43263865 & 0.89977343\end{array}$

$\begin{array}{llll}-0.0109422 & 7.74256554 & 0.93258409\end{array}$

$0.0110089 \quad 5.5134495$

$\begin{array}{llll}-0.0113129 & 5.72865447 & 0.96988134\end{array}$

$\begin{array}{llll}-0.0116468 & 8.35011784 & 0.88497889\end{array}$

$\begin{array}{llll}-0.0119869 & 7.95058692 & 0.90814813\end{array}$

$\begin{array}{lll}-0.0121155 & 8.21941844 & 0.8879868\end{array}$

$\begin{array}{llll}0.01217 & 8.07498128 & 0.90274328\end{array}$

$\begin{array}{llll}-0.0126772 & 5.49485597 & 0.96655189\end{array}$

$\begin{array}{llll}-0.0130362 & 7.88270057 & 0.90262397\end{array}$

$\begin{array}{llll}-0.0130392 & 7.83265467 & 0.92754847\end{array}$

$\begin{array}{llll}-0.0130538 & 6.89430255 & 0.93466587\end{array}$

0.972728388

0.960453263

0.977746094

0.950878039

0.979848792

0.968413789

1
1

0.946176646

0.979848792

0.985256113

0.951787148

0.96580843

0.95193755

0.943531915

0.968413789

0.957818792

0.954334345

0.985256113

0.932956877

0.960741615

0.957775599

0.986553442

0.921602286

0.939630234

0.923826662

0.935033184

0.970123137

0.95193755

0.947027422

0.984196564

0.935033184

0.954334345

0.95916815

0.985256113

0.921371137

0.95916815

0.977105058 $\begin{array}{llll}0.0137211 & 6.94900854 & 0.95757732\end{array}$ $-0.0138013 \quad 4.46207201$

$\begin{array}{llll}0.0138589 & 7.30766106 & 0.92202266\end{array}$ $\begin{array}{llll}-0.0140277 & 6.95147379 & 0.94348355\end{array}$ $\begin{array}{llll}-0.0142772 & 6.34171026 & 0.97850398\end{array}$ $\begin{array}{lll}-0.0144168 & 6.96631631 & 0.9365003\end{array}$ $\begin{array}{llll}-0.0144562 & 8.30023458 & 0.88908986\end{array}$ 
SPAC1B2.04

SPBC1271.11

SPNCRNA. 25

SPBC21C3.04C SPBC30D10.12C SPAC6F 6.05

SPAC19B12.01

SPCC285.11

SPAC27E2.14

SPAC17G6.16C

SPNCRNA.713

SPBC16H5.03C

SPBC30D10.11

SPNCRNA.1226

SPAC20G8.06

SPCC1450.09C

SPAC1783.05

SPAC10F6.02C

SPNCRNA.653

SPBC646.05C

SPAC17H9.19c

SPAC222.14c

SPAC683.02C

SPNCRNA.1564

SPNCRNA.1331

SPBC1685.08 mitochondriat iron-sensing transcription factor Fep1 $\# N / A$

pst3

snf5

alg6

SPBC1271.10c

dnm1

wis1

drp1

smf1

ini1

$\# \mathrm{~N} / \mathrm{A}$

SIN3 family co-repressor Pst3

SWI/SNF complex subunit Snf5

glucosyltransferase Alg6

SAGA complex bromodomain subunit Spt7

transmembrane transporter (predicted)

mitochondrial dynamin family scission GTPase Dnm1

MAP kinase kinase Wis1

RINT1 family protein (predicted)

Sm snRNP core protein Smf1

RING finger-like protein Ini1

na1

sil1

ubx4

dps1

gf11

pet802

mrx14

rsm27

ost2

SPAC19B12.01

dsc5

SPAC27E2.14

ysh1

$\# \mathrm{~N} / \mathrm{A}$

uba2

gpi1

$\# \mathrm{~N} / \mathrm{A}$

not1

SPCC1450.09c

hrp1

prp22

erg 9

$\# \mathrm{~N} / \mathrm{A}$

cdt2

sey1

SPAC683.02C

$\# \mathrm{~N} / \mathrm{A}$

$\# \mathrm{~N} / \mathrm{A}$

$$
\# \mathrm{~N} / \mathrm{A}
$$

glucosamine-phosphate $\mathrm{N}$-acetyltransferase (predicted) nucleotide exchange factor for the ER lumenal Hsp70 chap $\epsilon$ UBX domain protein Ubx4 (predicted)

decaprenyl diphosphate synthase subunit Dps1

$$
\# \text { N/A }
$$

SAGA complex subunit Sgf11

cytochrome c oxidase subunit VI (predicted)

$\begin{array}{lll}0.0155356 & 7.18999272 & 0.89127935\end{array}$

$\begin{array}{ll}-0.0157117 & 3.3305742\end{array}$

$\begin{array}{lll}0.0161605 & 8.53559779 \quad 0.85410248\end{array}$

$\begin{array}{lll}-0.0166348 & 6.98388568 & 0.91495089\end{array}$

$\begin{array}{llll}-0.0172776 & 6.88718181 & 0.88586775\end{array}$

$\begin{array}{lll}-0.0176452 & 7.43811899 & 0.87711018\end{array}$

$\begin{array}{lll}-0.017729 & 7.64756881 & 0.85329135\end{array}$

$\begin{array}{lll}-0.0179868 & 8.43444058 & 0.82725195\end{array}$

$\begin{array}{llll}-0.0183147 & 8.02156203 & 0.85020475\end{array}$

$\begin{array}{lll}-0.0183494 & 7.11636885 & 0.90172116\end{array}$

$\begin{array}{lll}-0.0186661 & 7.2695121 & 0.87514731\end{array}$

$\begin{array}{llll}-0.0188322 & 5.9015871 & 0.89977898\end{array}$

$\begin{array}{llll}-0.0190367 & 5.41681085 & 0.93732391\end{array}$

$\begin{array}{ll}-0.0191281 & 4.0895282\end{array}$

$\begin{array}{lll}0.0195331 & 5.97701059 & 0.92930689\end{array}$

$\begin{array}{lll}-0.0195905 & 6.2236522 & 0.9095009\end{array}$

$\begin{array}{lll}-0.0197271 & 6.26542048 & 0.91164554\end{array}$

$\begin{array}{llll}-0.0197408 & 5.41622974 & 0.93478645\end{array}$

$\begin{array}{llll}-0.0198273 & 6.39452841 & 0.90340911\end{array}$

mitochondrial carrier, S-adenosylmethionine Pet802 (predic $-0.0200149 \quad 5.663428350 .94070564$ $\# \mathrm{~N} / \mathrm{A}$

$-0.02009867 .15725176 \quad 0.87264496$

mitochondrial ribosomal protein subunit 134, Mrx14 (predic $-0.0205055 \quad 7.14146290 .88503614$ mitochondrial ribosomal protein subunit S27 (predicted) $\quad \begin{array}{lllll}0.0206644 & 6.55575863 & 0.90895191\end{array}$ oligosaccharyltransferase epsilon subunit Ost2 (predicted) $\quad \begin{array}{llll}-0.021279 & 7.59913247 & 0.85674094\end{array}$ TPR repeat protein, human TTC27 ortholog $\quad-0.02141647 .410451920 .81746783$ UBX domain Sre1 cleavage protein

$-0.0217198 \quad 7.20330684 \quad 0.85279423$ $\begin{array}{lll}-0.0218649 & 4.92088071 & 0.9580831\end{array}$ $\begin{array}{llllll}\text { Schizosaccharomyces pombe specific protein } & -0.0218649 & 4.92088071 & 0.9580831\end{array}$

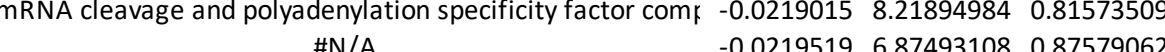
SUMO activating enzyme E1-type Uba2 pig-Q

\#N/A

CCR4-Not complex scaffold subunit Not1

phospholipase (predicted)

ATP-dependent DNA helicase Hrp1

ATP-dependent RNA helicase Prp22

$$
\text { \#N/A }
$$

$\begin{array}{lll}-0.0228196 & 6.81169179 & 0.85250871\end{array}$

$\begin{array}{lll}-0.0230347 & 6.27981208 & 0.91597355\end{array}$

$-0.0233092 \quad 6.85007851 \quad 0.8631487$

$-0.0235655 \quad 9.29466404 \quad 0.75380149$

$\begin{array}{lll}-0.023701 & 6.85284061 & 0.86292947\end{array}$

$\begin{array}{lll}-0.0237465 & 8.30544426 & 0.79725537\end{array}$

$\begin{array}{lll}-0.0238279 & 8.18251044 \quad 0.77779856\end{array}$

$\begin{array}{lll}-0.0240528 & 5.99389653 & 0.92582648\end{array}$

farnesyldiphosphate:farnesyldiphosphatefarnesyltransferas $-\begin{array}{llll}0.0242027 & 8.62155072 \quad 0.79346034\end{array}$

WD repeat protein $\mathrm{Cdt} 2$

$\begin{array}{llll}-0.0243109 & 6.8773261 & 0.88251521\end{array}$

$\begin{array}{lrrrr}\text { GTP binding protein Sey1 (predicted) } & -0.0244684 & 7.74548955 & 0.7980218 \\ \text { zf-CCHC type zinc finger protein, human ZCCHC9 ortholog, ir } & -0.0245556 & 5.74483783 & 0.94747367\end{array}$

$\begin{array}{lll}-0.0245898 & 5.4296929 & 0.97057264\end{array}$

$\# N / A$

$\begin{array}{lll}-0.024657 & 7.79869709 & 0.80141514\end{array}$

histone deacetylase complex ubiquitin-like protein ligase sı $\begin{array}{cccc}0.0247781 & 6.28569031 & 0.94278361\end{array}$

0.896604541

0.983615768

0.92646268

0.898701885

0.945518726

0.922186025

0.916152692

0.898305633

0.875899296

0.895610425

0.934543477

0.91529034

0.932956877

0.960741615

0.925420664

0.955565638

0.940600854

0.942675565

0.95916815

0.935580432

0.963066329

0.913455517

0.921602286

0.9403189

0.90054404

0.867436231

0.897921203

0.977474588

0.866138327

0.915476987

0.897759477

0.946176646

0.905983208

0.817729819

0.905983208

0.85129896

0.83458202

0.952850756

0.848044233

0.920295749

0.851715553

0.968413789

0.986961571

0.854934949

0.96475827 
SPCC613.12c

SPCC1450.11C

SPBC1709.13C

SPBC32C12.03C

SPAC637.08

SPAC6C3.08

SPAC8F11.10C

SPBC3D6.05

SPAC16A10.06C

SPBC2G5.02C

SPAC22G7.04

SPAC19A8.01C

SPAP8A3.03

SPBC29A3.11C

SPNCRNA.556

SPAC19D5.01

SPAC222.10c

SPAC2E12.03C

SPBC428.08C

SPBC1703.03

SPAC688.04c

SPBC1861.07

SPCC794.15

SPAP27G11.13C

SPBC20F10.06

SPBC1683.02

SPAC2C4.10C

SPAC25A8.03C

SPNCRNA.09

SPAC25B8.01

SPBP23A10.08

SPBC342.06c

SPBC1734.03

SPBC32F12.09

SPNCRNA.1462

SPAC1399.05C

SPBC13G1.14C

SPBC1703.11

SPAC227.04

SPCC1620.11

SPAC19B12.05C

SPBC146.08C

SPNCRNA.1254

SPBC691.01

SPCC1235.03

SPNCRNA.722

SPNCRNA.1491 syo2

SPBC1861.07

SPCC794.15

nop10

$\operatorname{mad} 2$

SPBC1683.02

CsC4

SPAC25A8.03C

\#N/A

dap1

alp5

rtt109

fol1

rum1

$\# N / A$

toe1

rns1

opa3

$\operatorname{atg} 10$

nup97

fcp1

tif1102

pfa5

$\# N / A$
CLRC ubiquitin ligase complex WD repeat subunit Raf1/Dos $\quad-0.02482836 .702897250 .90190861$ serine/threonine protein kinase Cek1

ribosomal lysine methyltransferase Set10

serine/threonine protein kinase Ppk25 (predicted)

ClA machinery ATPase Nbp35 (predicted)

proteasome assembly chaperone, gankyrin

Golgi pyruvyltransferase Pvg1

phosphatidate cytidylyltransferase Ptp4 (predicted)

Smc5-6 complex non-SMC SUMO ligase subunit Nse2

CK2 family regulatory subunit Ckb2 (predicted)

(poly(A)-specific ribonuclease) ubiquitin C-ter -(

guanyl-nucleotide exchange factor Sec73 (predicted)

ER ZIP zinc transmembrane transporter Zip3 (predicted)

mitochondrial carrier, ornithine Ort1 (predicted)

$$
\text { \#N/A }
$$

tyrosine phosphatase Pyp2

two-component GAP Byr4

$\begin{array}{llll}-0.0248775 & 8.61626476 & 0.75897877\end{array}$

$\begin{array}{lll}-0.0249315 & 7.01466767 & 0.82226303\end{array}$

$\begin{array}{llll}-0.0255835 & 5.9689144 & 0.89774752\end{array}$

$\begin{array}{llll}-0.0256014 & 6.73037968 & 0.85467545\end{array}$

$\begin{array}{llll}-0.0256168 & 5.76379417 & 0.91919988\end{array}$

$\begin{array}{llll}-0.0257574 & 8.10824312 & 0.80866658\end{array}$

$\begin{array}{llll}-0.0258437 & 6.88735684 & 0.85745445\end{array}$

$\begin{array}{llll}-0.0258828 & 5.52696977 & 0.91583978\end{array}$

$\begin{array}{lll}-0.0260429 & 6.28228321 & 0.87636803\end{array}$

$\begin{array}{lll}-0.0262668 & 7.29298052 & 0.80690952\end{array}$

$\begin{array}{lll}-0.0265159 & 6.79052519 & 0.8493357\end{array}$

$\begin{array}{llll}-0.0266221 & 5.96196786 & 0.89520689\end{array}$

$\begin{array}{llll}-0.0267905 & 5.38655711 & 0.93272284\end{array}$

$\begin{array}{llll}-0.0273132 & 5.34781984 & 0.94200146\end{array}$

$\begin{array}{llll}-0.0273235 & 6.57597405 & 0.85474741\end{array}$

plasma membrane basic amino acid transmembrane transı $-0.0274496 \quad 6.373958520 .88487862$

histone H3 lysine methyltransferase Clr4 $\quad-0.0278455 \quad 7.55690045 \quad 0.83705377$

armadillo repeat protein, involved in nucleocytoplasmic tral $\begin{array}{cccc}-0.0283329 & 7.17472342 & 0.81030053\end{array}$ glutathione S-transferase Gst3

elongin C (predicted)

Schizosaccharomyces specific protein

box H/ACA snoRNP complex protein (predicted)

mitotic spindle checkpoint protein Mad2

adenine deaminase (predicted)

SIP/FAR complex subunit, Csc4

$\begin{array}{lll}-0.0291294 & 7.41508811 & 0.76899113\end{array}$

$-0.0292993 \quad 3.87326944$

$-0.0295214 \quad 5.69943595 \quad 0.91913839$

$-0.0298034 \quad 5.77934374 \quad 0.88356468$

$\begin{array}{lll}-0.0298541 & 5.50967698 & 0.94010188\end{array}$

$\begin{array}{lll}-0.029908 & 5.99807357 \quad 0.88555766\end{array}$

$\begin{array}{lll}-0.0301795 & 6.36061219 & 0.82819398\end{array}$

mitochondrial arginine methyltransferase, human NDUFAF $-0.0302059 \quad 5.36379404 \quad 0.90615821$

$$
\text { \#N/A }
$$

cytochrome P450 regulator Dap1

actin-like protein Arp4

RTT109 family histone lysine acetyltransferase

$-0.0304528 \quad 3.76484183$

$\begin{array}{lll}-0.0309572 & 6.16517607 & 0.82544389\end{array}$

$\begin{array}{lll}-0.0310795 & 7.60624834 & 0.74226829\end{array}$

$\begin{array}{lll}-0.0313893 & 4.79203695 & 0.92129036\end{array}$

trifunctional dihydropteroatesynthase/2-amino-4-hydroxy- $6 \begin{array}{llll}-0.031667 & 7.50849387 & 0.75436949\end{array}$ CDK inhibitor Rum 1

$\begin{array}{lll}0.0321362 & 6.75530462 \quad 0.83362318\end{array}$

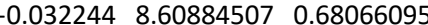
$-0.03234426 .06445552 \quad 0.8973329$

transcription factor, zf-fungal binuclear cluster type $-0.0327613 \quad 5.766929890 .86132873$

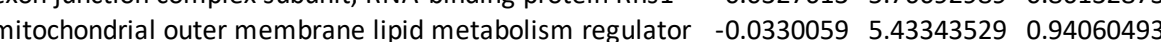
Atg12 conjugating enzyme Atg10

nucleoporin Nic96 homolog

CTD phosphatase Fcp1

translation initiation factor elF1A-like (predicted)

$$
\# N / A
$$

$\begin{array}{lll}0.0341346 & 5.09168391 & 0.89133742\end{array}$

$\begin{array}{lll}-0.0343243 & 8.27349051 & 0.66055641\end{array}$

$-0.0349796 \quad 8.05108055 \quad 0.68396769$

$\begin{array}{lll}-0.0353845 & 6.46827951 & 0.81586285\end{array}$ $\begin{array}{lll}-0.0355709 & 5.97764126 & 0.85546068\end{array}$ vacuolar membrane palmitoyltransferase Pfa5 (predicted) $\quad \begin{array}{llll}-0.0356463 & 5.99944307 & 0.8818441\end{array}$

$$
\# N / A
$$

$\# N / A$
0.934595412

0.820917459

0.871573192

0.931276203

0.899004034

0.948353489

0.860485422

0.901154829

0.946176646

0.915658598

0.845670538

0.859448935

0.894833477

0.929632671

0.957775599

0.964247887

0.899004034

0.921602286

0.884358159

0.861981411

0.828410559

0.948353489

0.921056628

0.962737894

0.922004262

0.876551477

0.937856515

0.874263919

0.807793969

0.949618431

0.817934271

0.881280985

0.755981352

0.931129976

0.904667692

0.963066329

0.92646268

0.737988235

0.75903459

0.866138327

0.89961519

0.919967671

0.935033184

0.818786624

0.890504765 
SPAC1F $5.05 \mathrm{C}$

SPAC4F8.01

SPAC328.01C

SPAC13A11.03

SPNCRNA.249

SPBC2F12.05C

SPBC12C2.12C

SPBC646.14c

SPBC776.15C

SPAC630.15

SPCC1827.05C

SPNCRNA.1408

SPBP8B7.25

SPBC83.16C

SPAC23H3.08c

SPNCRNA.1545

SPCC4B3.14

SPNCRNA.1174

SPAC1782.08c

SPAC18G6.02C

SPCC790.02

SPNCRNA.1240

SPBC2F12.17

SPBC2G2.09C

SPBP23A10.04

SPCC553.03

SPBC9B6.05C

SPAC29A4.06C

SPAC23C4.13

SPBC16C6.05

SPBC530.04

SPBC15D4.04

SPAC227.08C

SPAC23C4.08

SPAC31G5.16C

SPNCRNA.724

SPCC126.05C

SPAC823.12

SPAP27G11.04c

SPAC1834.13

SPBC713.04c

SPAC1F7.12

SPNCRNA.795

SPCC4B3.20

SPCC4G3.12C

SPBC146.10

SPBC3B9.16C exocytic docking protein Mso1

$\begin{array}{llll}-0.0361898 & 5.36879968 & 0.89310223\end{array}$

$\begin{array}{llll}-0.0364396 & 6.8353675 & 0.76702795\end{array}$

orc5

kgd2

mug177

SPCC1827.05C

\#N/A

cyp4

SPBC83.16c

bub3

cwf20

$\# \mathrm{~N} / \mathrm{A}$

\#N/A

rex3

chp1

pep3

$\# N / A$

$\operatorname{cox} 7$

crs1

apc2

pex1

Ism3

SPAC29A4.06c

bet1

tma22

$\bmod 5$

gpt2

th1

rho3

dpm1

mrpl17

vps11

pwp2

$\# N / A$

Cmc2

SPCC4G3.12c

mug 57

nup120

ESCRT III complex subunit Did4

karyopherin/importin beta family nuclear import/export sig $\quad \begin{array}{lllll}-0.037838 & 8.45314063 & 0.66600657\end{array}$ meiosis specific coiled-coil protein Mcp7

$$
\# \mathrm{~N} / \mathrm{A}
$$

$-0.0380268 \quad 1.8219343$

sterol intermembrane transfer protein Osh2 (predicted) glyoxalase I

origin recognition complex subunit Orc5

$\begin{array}{lll}-0.0380525 & 9.15522078 & 0.69712128\end{array}$

$\begin{array}{lll}-0.0380845 & 7.94555353 & 0.75597889\end{array}$

$\begin{array}{llll}-0.038257 & 6.21068095 & 0.85668515\end{array}$

dihydrolipoamide S-succinyltransferase, e2 component of o $-\begin{array}{llll}-0.0384527 & 8.20855373 & 0.66805651\end{array}$

$\begin{array}{lllll}\text { Schizosaccharomyces pombe specific protein } & -0.0384939 & 7.13314425 & 0.70795346\end{array}$

nucleolar RNA-binding protein NIFK (predicted) $\quad \begin{array}{lllll}-0.0387487 & 6.48329863 & 0.80870997\end{array}$

$$
\text { \#N/A }
$$

$\begin{array}{llll}0.0392859 & 7.51856344 & 0.68561778\end{array}$

cyclophilin family peptidyl-prolyl cis-trans isomerase Cyp4 $\begin{array}{lllll}-0.039362 & 8.23996808 & 0.62397807\end{array}$ protein with a role in clearing protein aggregates (predicter $\begin{array}{llll}-0.0395433 & 8.8107617 & 0.60589805\end{array}$ mitotic spindle checkpoint WD repeat protein Bub3 $\quad-0.0395952 \quad 4.80420837 \quad 0.88979852$ $\# \mathrm{~N} / \mathrm{A}$

complexed with Cdc5 protein Cwf20

\#N/A

$\begin{array}{lll}-0.0397035 & 4.80589179 & 0.92074589\end{array}$ $\begin{array}{lll}-0.03975 & 5.13686355 & 0.86338003\end{array}$

$-0.03975 \quad 5.13686355 \quad 0.86338003$

exonuclease Rex3 (predicted)

$\begin{array}{lll}-0.0397661 & 7.31701062 & 0.73391256\end{array}$

heterochromatin (HP1) family chromodomain protein Chp1 $\begin{array}{lllll}-0.0397907 & 7.00488117 & 0.73148051\end{array}$

HOPS/CORVET complex subunit, ubiquitin-protein ligase E3 $\quad \begin{array}{lllll}-0.039811 & 7.66880363 & 0.69377112\end{array}$$$
\text { \#N/A }
$$

cytochrome c oxidase subunit VII Cox7 (predicted)

meiosis specific cyclin Crs1

anaphase-promoting complex cullin family subunit Apc2

AAA family ATPase Pex1 (predicted)

Lsm2-8 complex subunit Lsm3

splicing protein, human NSRP1 ortholog

SNARE Bet1 (predicted)

translation machinery associated protein ortholog Tma22

Tea1 anchoring protein Mod5

$\begin{array}{lll}-0.0398556 & 6.59833654 & 0.7616659\end{array}$

$\begin{array}{lll}-0.0401257 & 6.00601023 & 0.81684928\end{array}$

$\begin{array}{lll}-0.0407224 & 4.04342199 & 0.94586222\end{array}$

$\begin{array}{lll}-0.0407978 & 6.33381596 & 0.82824776\end{array}$

$\begin{array}{lll}-0.0412381 & 6.97684296 & 0.76255556\end{array}$

$\begin{array}{lll}-0.0413599 & 5.93299039 & 0.81844513\end{array}$

$-0.0414078 \quad 5.46742535 \quad 0.87232902$

$\begin{array}{lll}-0.0422528 & 5.67078531 & 0.87097501\end{array}$

UDP-N-acetylglucosamine--dolichyl-phosphate N-acetylgluc $-0.0429085 \quad 6.72048691 \quad 0.77436748$

mRNA cleavage and polyadenylation specificity factor comr $\begin{array}{llll}-0.0429895 & 5.96909438 & 0.78625346\end{array}$

Rho family GTPase Rho3

$\begin{array}{lll}-0.0430578 & 7.07182419 & 0.76304934\end{array}$

dolichyl-phosphate beta-D-mannosyltransferase catalytic st $-0.0431061 \quad 8.09663561 \quad 0.62759538$ \#N/A

$\begin{array}{lll}-0.0431089 & 8.21030747 & 0.59742217\end{array}$

$\begin{array}{lll}-0.0432864 & 5.96438147 & 0.78882272\end{array}$

mitochondrial ribosomal protein subunit L17 (predicted)

HOPs/CORVET complex ubiquitin protein ligase E3 subunit - -0

tRNA specific adenosine deaminase subunit Tad3

$$
\# \mathrm{~N} / \mathrm{A}
$$

$-0.0440732 \quad 5.54627637 \quad 0.86520263$

$-0.04424632 .20189615$

$\begin{array}{lll}-0.0442665 & 7.80063731 & 0.62277837\end{array}$

snoRNP-associated protein Utp1 (predicted)

aldose reductase ARK13 family YakC, implicated in cellular $-\begin{array}{lll}-0.0444612 & 7.7058521 & 0.69946641\end{array}$ $\# \mathrm{~N} / \mathrm{A}$ $-0.0444968 \quad 7.73723985 \quad 0.66323396$ copper binding protein of the mitochondrial inner membrar $-0.0445759 \quad 4.10803457 \quad 0.95566596$ ubiquitin-protein ligase E3 (predicted)

$\begin{array}{llll}-0.0447657 & 7.22945301 & 0.71750442\end{array}$ cell surface fascilin domain protein, implicated in adhesion $\begin{array}{ccc}0.0448789 & 6.41787684 & 0.79074234\end{array}$ $\begin{array}{lllll}\text { nucleoporin, WD repeat Nup120 } & -0.044937 & 7.72754924 & 0.64000725\end{array}$
0.928013544

0.826936823

0.742735936

0.843582702

0.768840872

0.818786624

0.90054404

0.744533992

0.778048298

0.860485422

0.760617701

0.705695653

0.689026781

0.925420664

0.949378274

0.905983208

0.905983208

0.801134905

0.799121025

0.766912354

0.822589496

0.867050279

0.967326785

0.876551477

0.822955581

0.868337875

0.9134019

0.912405313

0.791975855

0.778048298

0.832405094

0.842458637

0.823169109

0.708844241

0.681209423

0.844281001

0.723234666

0.906916808

0.70492462

0.770953829

0.740250464

0.975447258

0.786250813

0.845670538

0.719519142 
SPCC1020.02

SPBC409.21

SPAC644.13C

SPBPB2B2.09c

SPNCRNA.657

SPBC115.02C

SPNCRNA.335

SPBC146.11C

SPBC16E9.12C

SPAC17A5.13

SPNCRNA 698

SPBC839.10

SPBC8D2.18C

SPAC664.10

SPNCRNA.1473

SPNCRNA.665

SPCC1672.01

SPNCRNA.1525

SPBC365.20C

SPAC1296.06

SPBP35G2.05C

SPNCRNA.1110

SPNCRNA.1070

SPNCRNA.72

SPBC685.08

SPAC6F12.08C

SPBC15D4.01C

SPAC6G10.09

SPBC21C3.03

SPBC14F5.02

SPAC17G8.04C

SPAC17C9.07

SPAC694.02

SPAC4C5.04

SPAC9G $1.11 \mathrm{C}$

SPAC1486.02C

SPBC1734.01C

SPAC4F 10.12

SPAC17G8.05 usp107

SPBC8D2.18C

klp2

\#N/A

\#N/A

SPCC1672.01

\#N/A

pnc1

tah18

cki2

$\# \mathrm{~N} / \mathrm{A}$

$\# N / A$

$\# \mathrm{~N} / \mathrm{A}$

SPBC685.08

exo84

gls1

SPBC21C3.03

trs65

arc5

alg8

SPAC694.02

rad31

spn4

dsc2

esf1

fta1

$\operatorname{med} 20$

signal recognition particle subunit (predicted)

mitochondrial TOM complex subunit Tom70 (predicted)

spindle pole body half bridge protein, centrin Cdc31

SIN pathway serine/threonine protein kinase $\mathrm{Cdc} 7$ $\begin{array}{llll}0.0453468 & 9.18155224 & 0.55157772\end{array}$ $\begin{array}{lll}-0.0462555 & 8.35688618 & 0.65729274\end{array}$ $\begin{array}{lll}-0.0463069 & 6.59181866 & 0.75815916\end{array}$ exon junction complex subunit, ATP-dependent RNA helicas - $-0.0463368 \quad 7.38731173 \quad 0.65605922$ Pof6 interacting protein Sip1, predicted AP-1 accessory prot $-\begin{array}{lllll}0.0470234 & 9.12458994 & 0.53578581\end{array}$ serine palmitoyltransferase complex subunit (predicted) $\quad \begin{array}{lllll}-0.0471036 & 8.25638859 & 0.58416774\end{array}$ serine/threonine protein phosphatase Pzh1 $\quad-0.0475901 \quad 8.13276884 \quad 0.57855183$ NMS complex subunit, Spc105/KNL-1 family member, blink $-0.0475906 \quad 7.08517857 \quad 0.71335182$ ER protein translocation subcomplex subunit Sec66 (predict $-\begin{array}{lllll}0.0478621 & 5.96638176 & 0.81133954\end{array}$ Rab GTPase binding (predicted) $\quad-0.0478773 \quad 8.4229036 \quad 0.56220613$

2-dehydropantoate 2-reductase Pan5 (predicted) $\quad-0.0479148 \quad 6.38524562 \quad 0.78988701$ $\begin{array}{lll}-0.0480647 & 5.97153129 & 0.76050494\end{array}$ $-0.0481173 \quad 6.213013150 .80506096$ $-0.04817842 .06156389$

$\begin{array}{lll}-0.0484162 & 5.97131652 & 0.80517589\end{array}$ $\begin{array}{lll}-0.0484434 & 7.35504807 & 0.63327275\end{array}$ $\begin{array}{lll}-0.0486404 & 6.56919527 & 0.72069025\end{array}$ $\begin{array}{lll}-0.0489125 & 4.80379773 & 0.90562336\end{array}$ $\begin{array}{lll}-0.0490811 & 7.31224513 & 0.63850077\end{array}$ $\begin{array}{lll}-0.0494558 & 9.50841708 & 0.46830931\end{array}$

AFG1 family mitochondrial ATPase Afg1 (predicted)

poly(A) binding protein $\mathrm{Pab2}$

GTP cyclohydrolase Fol2 (predicted)

$$
\text { \#N/A }
$$

adenosylhomocysteinase (predicted)

kinesin-14 family minus-end directed microtubule motor $\mathrm{KI}$

$$
\# N / A
$$$$
\# \text { N/A }
$$

histidinol-phosphatase (predicted)

$$
\text { \#N/A }
$$

nicotinamidase Pnc1 (predicted)

CIA machinery NADPH-dependent diflavin oxidoreductase T serine/threonine protein kinase Cki2

$$
\begin{aligned}
& \# N / A \\
& \# N / A \\
& \# N / A
\end{aligned}
$$

Schizosaccharomyces specific protein exocyst complex subunit Exo84 (predicted)

kinesin- 6 family microtubule motor protein Klp9

alpha glucosidase I GIs1 (predicted)

mitochondrial membrane $A B C 1$ kinase family protein, unkn

RAPP complex subunit Trs65 (predicted)

ARP2/3 actin-organizing complex subunit Arc5

$\begin{array}{lll}0.0495377 & 7.13764736 & 0.6893588\end{array}$ $\begin{array}{lll}-0.0500479 & 7.20320116 & 0.67002038\end{array}$ $\begin{array}{llll}-0.0502005 & 6.30205812 & 0.74597894\end{array}$ $\begin{array}{lll}-0.050933 & 9.18901832 & 0.52433216\end{array}$ $\begin{array}{llll}-0.0510505 & 6.15534965 & 0.75420751\end{array}$ $\begin{array}{lll}-0.0511723 & 8.21705532 & 0.54167125\end{array}$ $\begin{array}{lll}0.6937817 & 0.66490232\end{array}$ $\begin{array}{lll}-0.0517499 & 7.75534095 & 0.58818585\end{array}$ $\begin{array}{llll}-0.0518919 & 4.57225028 & 0.86370916\end{array}$ $\begin{array}{llll}-0.0518923 & 5.19838965 & 0.84308295\end{array}$ $\begin{array}{llll}-0.0523016 & 6.18321595 & 0.74476098\end{array}$ $\begin{array}{llll}0.0525251 & 3.75344699 & 0.93744663\end{array}$ $\begin{array}{lll}-0.052606 & 7.01561437 & 0.6436098\end{array}$ $\begin{array}{lll}-0.0527383 & 6.31824356 & 0.77642513\end{array}$ $\begin{array}{llll}-0.0528077 & 8.76567042 & 0.59958737\end{array}$ $\begin{array}{llll}-0.0529221 & 7.13718131 & 0.62433938\end{array}$ $\begin{array}{llll} & -0.05375 & 6.6354343 & 0.76786825\end{array}$ DEAD/DEAH box helicase, human DDL60 and DDL60L ortholc -0.054162910 .05865560 .3941712$ SUMO activating enzyme E1-type Rad31 mitotic septin Spn4

Golgi Dsc E3 ligase complex subunit Dsc2

pre-rRNA processing protein Esf1 (predicted)

CENP-L ortholog Fta1

mediator complex subunit Med20
0.740397206

0.641402319

0.735066081

0.820364617

0.733927826

0.626802908

0.670364498

0.665832677

0.782456343

0.862412617

0.651534861

0.845154257

0.821987952

0.857736679

0.857736679

0.713598452

0.78910654

0.937588154

0.718445649

0.563046792

0.763523215

0.746478169

0.810794876

0.61571706

0.817934271

0.632585014

0.741626011

0.67406613

0.906049152

0.890037155

0.809729744

0.960741615

0.722852727

0.833897251

0.682877329

0.74135653

0.705959821

0.797895217

0.827711616

0.489477188

0.831624903

0.746004864

0.757552705

0.668617991

0.832670738

0.909485941 
SPBC3E7.01

SPBC15D4.08C

SPNCRNA.432

SPAC15A10.11

SPNCRNA.737

SPAC23C4.19

SPNCRNA.270

SPBC21B10.06C

SPAC17C9.08

SPNCRNA.769

SPNCRNA.1228

SPBC776.14

SPBC31F10.16

SPAC637.06

SPBC6B1.10

SPAP8A3.12C

SPAC22H10.03C

SPCC188.03

SPCC306.06c

SPAC922.04

SPAC17C9.15C

SPBC3B9.22C

SPCC548.04

SPCC548.05C

SPAPB24D3.09c

SPAC1D4.01

SPBC216.04c

SPAC1F8.06

SPAC57A7.11

SPAC23C4.10

SPBC1921.03C

SPAC3G6.02

SPBC24C6.03

SPCC18.06C

SPNCRNA.1050

SPBC1271.08C mid1 $\# N / A$

SPAC15A10.06

$\# \mathrm{~N} / \mathrm{A}$

pvg5

SPAC1B1.02c

fab1

SPBC15D4.08C

$\# \mathrm{~N} / \mathrm{A}$

ubr11

$\# N / A$

spt5

\#N/A

inp2

pnu1

$\# N / A$

$\# \mathrm{~N} / \mathrm{A}$

plh1

bch1

gmh5

prp17

tpp2

kap114

cnd3

big1

SPAC922.04

SPAC17C9.15C

dad4

urm 1

dbl5

pdr1

tls1

$\mathrm{m} \times \mathrm{r} 2$

pfl8

mip1

sec2

mex67

rpn15

SPBC24C6.03

caf1

\#N/A

SPBC1271.08C
ER associated protein disulfide isomerase Pdi3

palmitoyltransferase Akr1 (predicted)

RNA-binding protein, rrm type

nuclear pore protein Ely5

mitochondrial ribosomal protein subunit S35

$$
\# \mathrm{~N} / \mathrm{A}
$$

medial ring protein Mid1

CPA1 sodium ion/proton antiporter (predicted)

$$
\# \mathrm{~N} / \mathrm{A}
$$

Golgi 4,6-pyruvylated galactose (PvGal) residue biosynthesi NAD/NADH kinase (predicted)

1-phosphatidylinositol-3-phosphate 5-kinase Fab1

Schizosaccharomyces specific protein \#N/A

UBR ubiquitin-protein ligase E3 Ubr11 \#N/A

DSIF transcription elongation factor complex subunit Spt5

$$
\# \mathrm{~N} / \mathrm{A}
$$

myosin binding vezatin family protein involved in peroxison mitochondrial endodeoxyribonuclease Pnu1

$$
\# N / A
$$$$
\text { \#N/A }
$$

phospholipid-diacylglycerol acyltransferase Plh1 exomer complex ChAPs family (Chs5p-Arf1p-binding) protei

alpha-1,2-galactosyltransferase (predicted)

Prp19 complex WD repeat protein Prp17

tripeptidyl-peptidase II Tpp2

$\begin{array}{lllll}7.9378212 & 0.55991419\end{array}$ condensin complex non-SMC subunit Cnd3

R membrane protein, BIG1 family, implicated in protein g| $\begin{array}{llll}-0.0616365 & 6.98015449 & 0.60485111\end{array}$ $\begin{array}{lllll}\text { Schizosaccharomyces specific protein } & -0.0617057 & 7.93258802 & 0.46284848\end{array}$ $\begin{array}{lllll}\text { Schizosaccharomyces specific protein } & -0.0618516 & 6.62176541 & 0.6205545\end{array}$ DASH complex subunit Dad4 $\quad \begin{array}{llll}-0.06201 & 7.15580118 & 0.58193195\end{array}$ ubiquitin-like protein modifier Urm1 (predicted) ubiquitin-protein ligase $\mathrm{E} 3 \mathrm{Db} 5$

$A B C$ transmembrane transporter Pdr1 splicing factor Tls1

peptide-methionine (R)-S-oxide reductase MsrB

cell surface glycoprotein, flocculin Pfl8

WD repeat protein, Raptor homolog Mip1

guanyl-nucleotide exchange factor Sec2 (predicted)

mRNA export receptor, Tap, nucleoporin Mex67

multifunctional proteasome assembly, lid subcomplex subu $-(-(p)$ mitochondrial proline-tRNA ligase (predicted)

CCR4-Not complex CAF1 family ribonuclease subunit Caf1 \#N/A

Schizosaccharomyces pombe specific protein $\begin{array}{lll}0.0555566 & 8.52056791 \quad 0.51674967\end{array}$ $\begin{array}{llll}-0.0559384 & 6.81321284 & 0.67499403\end{array}$ $\begin{array}{lll}0.0560512 & 4.05395202 & 0.90605159\end{array}$ $\begin{array}{lll}0.0563206 & 7.17055415 & 0.6543154\end{array}$ $\begin{array}{ll}-0.056531 & 2.50335927\end{array}$

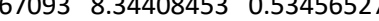
$\begin{array}{lll}0.0569229 & 7.30411436 & 0.59277796\end{array}$ $\begin{array}{llll}0.0571214 & 5.66449134 & 0.86025008\end{array}$ $\begin{array}{lll}-0.0579731 & 6.54989634 & 0.68389897\end{array}$ $\begin{array}{lll}0.0581499 & 9.58893879 & 0.4942016\end{array}$ $\begin{array}{lll}0.0581658 & 4.30032628 & 0.90117696\end{array}$ $\begin{array}{llll}0.0583053 & 5.6407561 & 0.74192172\end{array}$ $\begin{array}{llll}0.0583509 & 9.59360871 & 0.44568619\end{array}$ $\begin{array}{llll}-0.0589342 & 7.44763388 & 0.55074732\end{array}$ $\begin{array}{llll}-0.0591479 & 8.24241736 & 0.54511807\end{array}$ $\begin{array}{llll}0.0596604 & 6.36815642 \quad 0.65208746\end{array}$ $\begin{array}{llll}-0.0603967 & 7.24070445 & 0.64663084\end{array}$ $\begin{array}{llll}-0.0607958 & 5.35800975 & 0.75766436\end{array}$ $\begin{array}{llll}0.0608009 & 8.56591922 & 0.45268509\end{array}$ $\begin{array}{llll}0.5148012 & 0.53382058\end{array}$ $\begin{array}{llll}-0.0611092 & 7.83497531 & 0.52459028\end{array}$ $\begin{array}{llll}-0.0611614 & 9.59161001 & 0.47258637\end{array}$ $\begin{array}{llll}-0.0620669 & 6.86882223 & 0.65388902\end{array}$ $\begin{array}{llll}-0.0620669 & 6.86882223 & 0.65388902\end{array}$ $\begin{array}{llll}-0.0621266 & 7.60568531 & 0.54359516\end{array}$ $\begin{array}{llll}-0.0621582 & 7.11857066 & 0.64866975\end{array}$ $\begin{array}{llll}-0.0625662 & 6.01675446 & 0.72887597\end{array}$ $\begin{array}{llll}-0.062882 & 6.04745617 & 0.71841072\end{array}$ $\begin{array}{llll}-0.0629709 & 8.54587434 & 0.4878672\end{array}$ $\begin{array}{llll}-0.0632256 & 6.08433611 & 0.74903987\end{array}$ $\begin{array}{llll}0.0634307 & 6.99200995 & 0.65917787\end{array}$ $\begin{array}{llll}-0.0638731 & 6.90084331 & 0.62170035\end{array}$ $-0.0641814 \quad 7.59447772 \quad 0.51118272$ $\begin{array}{lll}-0.0642871 & 6.70450429 & 0.60960888\end{array}$ $\begin{array}{llll}-0.0644075 & 5.5051141 & 0.79799805\end{array}$ $\begin{array}{llll}-0.0648063 & 6.67117691 & 0.71834119\end{array}$
0.608034361

0.750177102

0.937856515

0.732217772

0.727611467 1

0.625897573

0.676934725

0.903813765

0.820917459

0.75903459

0.588664774

0.934121736

0.807662133

0.541491617

0.640655642

0.635791871

0.859542268

0.730565549

0.739292882

0.725766599

0.820220482

0.547745736

0.625240796

0.615913879

0.567472603

0.663723962

0.649653209

0.663030585

0.688066166

0.557564862

0.702758095

0.668700927

0.732030493

0.732030493

0.634396336

0.727575017

0.796659323

0.786863826

0.582853986

0.813472124

0.736811006

0.703938581

0.602981181

0.692552328

0.851715553

0.786863826 
SPCC584.16c

SPAC27F1.08

SPAC1420.04C

SPBC1734.13

SPBC1773.01

SPAC31G5.15

SPAC343.19

SPBC18A7.02C

SPCC188.11

SPAC25A8.01C

SPAC13G6.03

SPAC4C5.03

SPNCRNA.701

SPNCRNA.692

SPBC4F6.15C

SPBC1604.10

SPNCRNA.560

SPBP8B7.19

SPCC16A11.05C

SPBC30D10.03C

SPBC1348.03

SPAC22H 12.02

SPNCRNA.98

SPNCRNA.684

SPCC16A11.14

SPCC1795.08C

SPAC521.02

SPAC1F3.09

SPAC1B2.05 spc 25

sap1

$\# \mathrm{~N} / \mathrm{A}$

$\# \mathrm{~N} / \mathrm{A}$

cki1

cbh1

\#N/A

dbp2

$\# \mathrm{~N} / \mathrm{A}$

hem4

$\# N / A$

$\#$ N/A

lag1

mcp1

$\# N / A$

$\operatorname{aim} 41$

SPCC584.16c

pdt1

cox1101

atp3

far8

psd3

SPBC18A7.02C

prp45

gpi7

SPAC4C5.03

$\# N / A$

$\# \mathrm{~N} / \mathrm{A}$

swi10

srb7

$\# N / A$

spt16

$\operatorname{dim} 1$

isn1

ftm6

tfg3

srp7

\#N/A

sfh1

vid21

wss1

mug161
$\#$ N/A

NMS complex subunit Spc25

switch-activating protein Sap1$$
\# \text { N/A }
$$

serine/threonine protein kinase Cki1

CENP-B homolog Cbh1

$\# \mathrm{~N} / \mathrm{A}$

ATP-dependent RNA helicase Dbp2

$\# \mathrm{~N} / \mathrm{A}$

uroporphyrinogen-III synthase Ups

\#N/A

arrestin Aly1 related Aly2

\#N/A

sphingosine $\mathrm{N}$-acyltransferase Lag1

microtubule binding protein Mcp1

\#N/A

$\begin{array}{llllll} & 0.070173 & 4.86910947 & 0.81886605\end{array}$

Schizosaccharomyces specific protein

Nramp family manganese ion transmembrane transporter $\quad-0.0710907 \quad 6.10126912 \quad 0.67124648$ cytochrome c oxidase assembly protein Cox1101/mitochon $-0.0711598 \quad 8.02842481 \quad 0.53320963$

F1-FO ATP synthase gamma subunit (predicted)

$\begin{array}{lll}-0.0712338 & 8.05601663 & 0.39121356\end{array}$

SIP/FAR complex WD repeat (scaffold) subunit, striatin, Fal $-0.0714302 \quad 7.89506948 \quad 0.50810629$ phosphatidylserine decarboxylase Psd3

1-phosphatidylinositol 4-kinase Lsb6 (predicted)

seven transmembrane receptor-like protein (predicted) Prp19 complex subunit Prp45

SMARCAD1 family ATP-dependent DNA helicase Fft3

GPI anchor biosynthesis protein Gpi7 (predicted)

$-0.0714814 \quad 7.26550582 \quad 0.5394311$

$\begin{array}{llll}0.0716941 & 6.78407809 & 0.59314159\end{array}$

$\begin{array}{lll}-0.0718352 & 7.78663015 & 0.42289962\end{array}$

$\begin{array}{lll}-0.0719049 & 6.6876334 & 0.62262782\end{array}$

$\begin{array}{llll}-0.071993 & 8.38356163 & 0.43160293\end{array}$ $\begin{array}{llll}-0.0720228 & 7.06100524 & 0.50753535\end{array}$

multispanning membrane protein, CTNS/PQ domain proteiı $-0.0722475 \quad 7.35564199 \quad 0.5595436$ $\begin{array}{lll}-0.0723472 & 5.57983909 & 0.69721044\end{array}$ $\begin{array}{lll}-0.0724421 & 6.81990767 & 0.57881897\end{array}$ $\begin{array}{lll}-0.0725615 & 5.48684085 & 0.75193189\end{array}$ $\begin{array}{lll}-0.0725951 & 6.24713824 & 0.67293285\end{array}$ $\begin{array}{llll}-0.0730385 & 5.36478051 & 0.75504563\end{array}$

DNA repair endonuclease non-catalytic subunit Swi10 mediator complex subunit Med21

$$
\text { \#N/A }
$$

histone $\mathrm{H} 2 \mathrm{~A}-\mathrm{H} 2 \mathrm{~B}$ chaperone, FACT complex subunit Spt16 U4/U6 x U 5 tri-snRNP complex subunit Dim1

IMP 5'-nucleotidase Isn1 (predicted)

$\begin{array}{llll}-0.0730685 & 8.88573788 & 0.49998149\end{array}$

$\begin{array}{lll}-0.0731687 & 5.51560953 & 0.68895437\end{array}$

$\begin{array}{llll}-0.0732313 & 7.89288338 & 0.44430515\end{array}$

$\begin{array}{llll}-0.0733857 & 6.23503617 & 0.62898429\end{array}$

sub-telomeric $5 \mathrm{Tm}$ protein family Ftm 6

TFIID, TFIIF, Ino80, SWI/SNF, and NuA3 complex subunit Tf: $\begin{array}{lllll}-0.0734579 & 7.93768143 & 0.43543484\end{array}$ 7SL signal recognition particle component

$\begin{array}{llll}-0.0738398 & 7.69462832 & 0.43619499\end{array}$

$\begin{array}{llll}-0.0742891 & 4.74807986 & 0.78539957\end{array}$

$\begin{array}{lll}-0.0745745 & 7.42569569 & 0.45840685\end{array}$

$\begin{array}{llll}-0.0750547 & 8.04546064 & 0.37785655\end{array}$

NuA4 histone acetyltransferase complex subunit Vid21

$\begin{array}{llll}-0.075137 & 6.81857119 & 0.56257608\end{array}$

\begin{tabular}{llllll} 
WLM domain metallopeptidase implicated in DNA repair $h$ & -0.075137 & 6.81857119 & 0.56257608 \\
\hline
\end{tabular}

MCM complex subunit Mcm5 $\begin{array}{lll}-0.075575 & 8.2181695 & 0.34180274\end{array}$
0.76152636

0.640254155

0.560863387

0.956297487

0.780017886

0.632585014

0.674316514

0.832054517

0.600687064

0.956697346

0.496273494

0.812460898

0.655237593

0.834043243

0.599825561

0.769887465

0.868513631

0.898364102

0.617145238

0.747599392

0.624740274

0.486692559

0.600687064

0.630509904

0.677099798

0.518061553

0.704871451

0.52711018

0.600239497

0.649333932

0.768840872

0.666023515

0.815831719

0.748742324

0.818166479

0.593368332

0.763323755

0.540392588

0.709670528

0.531202812

0.531859331

0.841941405

0.55358627

0.473625684

0.651741614

0.637127676

0.436425951 
SPAC5D6.08c

SPNCRNA.1220

SPBC119.15

SPBC660.17c

SPBC83.06C

SPBP23A10.12

SPNCRNA.403

SPAC3A12.15

SPNCRNA.1170

SPCC895.03C

SPNCRNA.961

SPBC839.08C

SPBC2D10.06

SPNCRNA.15

SPCC18.08

SPBC29A3.21

SPAC19B12.13

SPAC6C3.09

SPNCRNA.736

SPBC19G7.05C

SPAC4A8.13C

SPBC19G7.07C

SPCC663.15C

SPCC663.12

SPAC4F $8.05 \mathrm{C}$

SPBC16G5.06

SPBC18H10.16

SPBC3H7.18

SPNCRNA.1634

SPBC6B1.02

SPNCRNA.1006

SPAC31A2.14

SPAC821.12

SPNCRNA.475

SPNCRNA.204

SPBC32F12.03C

SPBC16G5.02C

SPAC23H3.11C

SPCC550.09 npa3

SPBC660.17c SPBC83.06c frg1

rep1

$\# \mathrm{~N} / \mathrm{A}$

SPCC18.08 SPBC29A3.21 cox1102 rpp40

bgs1

pts1

ppr3

SPCC663.15c

cid12

mrpl28

SPBC16G5.06

can1

tam8

k30

\section{$\# \mathrm{~N} / \mathrm{A}$}

bun107

orb6

$\#$ N/A $\# N / A$

gpx1

SPAC23H3.11C pex32
CDP-diacylglycerol-serine O-phosphatidyltransferase Pps1 $\quad-0.0758296 \quad 6.37376817 \quad 0.62958804$ plasma membrane OPT oligopeptide transmembrane trans SNAP-25 homologue, t-SNARE component Sec9

ER farnesyl pyrophosphate synthetase Erg20

transcriptional regulatory protein Rxt3 $\begin{array}{lll}-0.0764852 & 5.73918426 & 0.70546908 \\ -0.0765311 & 6.69390118 & 0.59126522\end{array}$ mitochondrial complex assembly factor Fmc1 (predicted) $\quad \begin{array}{llll}-0.0768132 & 5.67493747 & 0.69325005\end{array}$ aprataxin Hnt3

MBF complex corepressor Nrm1

meiotic APC inhibitor Mes1

$$
\# N / A
$$
$-0.076324 \quad 10.1702321 \quad 0.29115728$ $\begin{array}{lll}-0.0763324 & 8.11809229 & 0.36215378\end{array}$ $\begin{array}{lll}-0.0768132 & 5.67493747 & 0.69325005 \\ -0.0769449 & 6.93591208 & 0.56905609\end{array}$ $\begin{array}{lll}-0.0769947 & 7.69259868 & 0.47274095\end{array}$ $\begin{array}{lll}-0.077382 & 4.84201706 & 0.78616484\end{array}$ $-0.0774557 \quad 3.69509535 \quad 0.86768218$ RNA polymerase II binding AAA family ATPase Npa3 (predic $-\begin{array}{lllll}-0.0774581 & 6.63100307 & 0.56152717\end{array}$ $\begin{array}{llllll}\text { Schizosaccharomyces specific protein } & -0.0775074 & 6.02458732 & 0.6486311\end{array}$ mitochondrial ribosomal protein subunit L36, Rtc6 (predicte $-\begin{array}{llll}0.0775584 & 4.67094863 & 0.83006382\end{array}$ FRG1 family protein, involved in mRNA splicing (predicted) $\quad-0.0775953 \quad 6.088157110 .68820956$

$$
\text { \#N/A }
$$

GARP complex subunit Vps53 (predicted)

$$
\text { \#N/A }
$$

$\begin{array}{llll}-0.0776343 & 4.59537404 & 0.78029656\end{array}$

$\begin{array}{lll}-0.0776717 & 7.0215458 & 0.51489659\end{array}$ $\begin{array}{lll}-0.0776795 & 6.66221872 & 0.58988714\end{array}$

tRNA N6-threonyl-carbamoyl-adenosine (t6A) Sua5 (predict - $-0.0777526 \quad 5.931292740 .71461668$

$$
\# \mathrm{~N} / \mathrm{A}
$$

$\begin{array}{llll}-0.0784473 & 5.55346653 & 0.67311921\end{array}$ $\begin{array}{lll}-0.0786617 & 8.32738377 & 0.40349762\end{array}$ $\begin{array}{lll}-0.0787121 & 4.95842992 & 0.73679087\end{array}$ $\begin{array}{lll}-0.0787924 & 5.28474133 & 0.71198433\end{array}$ $\begin{array}{lll}-0.0788822 & 6.92949146 & 0.56771839\end{array}$ $\begin{array}{lll}-0.0789312 & 5.02006614 & 0.76602264\end{array}$

MBF transcription factor activator Rep1

$$
\text { \#N/A }
$$

mitochondrial lysine-tRNA ligase (predicted)

Schizosaccharomyces pombe specific protein

$0.0794048 \quad 8.02476420 .48076365$

$\begin{array}{lrrrr}\text { cytochrome c oxidase assembly protein Cox1102/ mitochon } & -0.0794048 & 8.0247642 & 0.48076365 \\ \text { RNase P and RNase MRP subunit Rpp40 } & -0.0795014 & 5.90049689 & 0.62916217\end{array}$

$$
\text { \#N/A }
$$

$-0.0796767 \quad 7.53718426 \quad 0.44236397$

primary septum and spore wall linear 1,3-beta-glucan syntt $-0.0797007 \quad 9.90658547 \quad 0.32250006$

$20 \mathrm{~S}$ proteasome complex subunit beta 5

mitochondrial PPR repeat protein Ppr3

DUF3818 and PXA domain conserved fungal protein

poly(A) polymerase Cid12

mitochondrial ribosomal protein subunit L28 (predicted)

Schizosaccharomyces specific protein

plasma membrane arginine/lysine transmembrane transpo Schizosaccharomyces specific protein Tam8 \#N/A

Ark1/Prk1 family protein kinase Ppk30

$$
\# \mathrm{~N} / \mathrm{A}
$$

WD repeat protein, human WDR48 family Bun107 serine/threonine protein kinase Orb6

$$
\# \text { \#/A }
$$

glutathione peroxidase $\mathrm{Gpx} 1$

ribokinase Rbk1 (predicted)

beta-glucan biosynthesis protein (predicted) peroxin Pex32 (predicted)
0.70991659

0.383754657

0.457737083

0.776061607

0.676260978

0.766585408

0.658016425

0.567472603

0.842458637

0.909235788

0.651032329

0.727575017

0.877927303

0.762622713

0.837130391

0.606415935

0.675307703

0.783464882

0.748827237

0.498692573

0.80337476

0.781334194

0.656692777

0.826049184

0.575782588

0.709671563

0.538416533

0.416751408

0.591986975

0.474110816

0.516714328

0.588483954

0.761789817

0.51808823

0.495446221

0.820580899

0.798861065

0.540540647

0.791718329

0.487546095

0.579983573

0.822955581

0.923511358

0.488888884

0.626271391

0.817934271

0.597960599 
SPAC589.03C

SPBC1773.11C

SPAC3A11.11C

SPAC13G7.04C

SPAC9G1.08C

SPBC28E12.05

SPBC4B4.12C

SPCC757.15

SPAPB8E5.04C

SPBC3H7.15

SPAC27E2.13

SPCC553.10

SPCPB16A4.02C

SPNCRNA.510

SPBC887.09C

SPNCRNA.1023

SPBC13E7.10c

SPBC $32 \mathrm{H} 8.08 \mathrm{C}$

SPAC14C4.11

SPAPB1E7.03

SPAC6F12.06

SPAC139.06

SPCC63.02C

SPAC4A8.14

SPAPB1A10.08

SPCC576.02

SPAC977.12

SPBC1773.13

SPAC29B12.07

SPNCRNA.819

SPCC1840.11

SPAPB18E9.05C

SPBC28E12.06C

SPAC1F7.01c

SPNCRNA.990

SPAC26H5.10C

SPBC16G5.13

SPNCRNA.1371

SPNCRNA.1618
SPBC119.16C

atp16

coq6

spc3

spp1

\#N/A

ret3

noc12

mug89

SPAC3A11.11C

mac1

SPAC9G1.08C

esf2

SPBC4B4.12C

Cox14

npc2

hhp1

opy1

\#N/A

sog2

\#N/A

brf1

omh5

vtc2

rpc82

rdi1

hat1

aah3

SPAC4A8.14

SPAPB1A10.08

SPCC576.02

SPAC977.12

SPBC1773.13 $\sec 16$

$\#$ N/A

CSI4

SPAPB18E9.05C

Ivs1

spt6

$\# N / A$

ptf2

$\# N / A$

$\# \mathrm{~N} / \mathrm{A}$

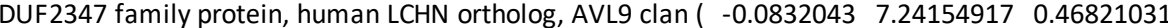
F1-FO ATP synthase delta subunit (predicted)

monooxygenase Coq6 (predicted)

signal peptidase subunit Spc3 (predicted)

DNA primase catalytic subunit Spp1

copper chaperone Atx1

$$
\text { \#N/A }
$$

coatomer zeta subunit (predicted)

CCAAT-binding factor, Noc1 related $\begin{array}{lll}0.0834832 & 7.48593116 & 0.40833436\end{array}$ $\begin{array}{lll}-0.0835502 & 7.32399838 & 0.39710405\end{array}$ $\begin{array}{lll}-0.0838033 & 6.82005915 & 0.49338353\end{array}$ $\begin{array}{lll}-0.0840355 & 6.64872226 & 0.52428877\end{array}$ $\begin{array}{lll}-0.0841425 & 4.13308179 & 0.78829194\end{array}$ $\begin{array}{lll}-0.0841614 & 3.09860709 & 0.92159995\end{array}$ $-0.0843156 \quad 7.80336222 \quad 0.33717906$ $\begin{array}{lll}-0.0843861 & 4.47031137 & 0.77499416\end{array}$ phospholipid-translocating ATPase complex Lem3 family su $-\begin{array}{llll}0.0843933 & 6.70397078 & 0.53609354\end{array}$ oxidoreductase, implicated in vitamin metabolism, or cellul $-\begin{array}{llll}0.0845706 & 7.16564098 & 0.40978315\end{array}$ plasma membrane anchored protein Mac1 palmitoyl-(protein) hydrolase (predicted) U3 snoRNP-associated protein Esf2 (predicted)

Schizosaccharomyces specific protein

cytochrome c oxidase assembly protein Cox14 (predicted) $\quad-0.0859722 \quad 6.51556267 \quad 0.51115761$ Niemann-Pick disease type C2 protein hE1 homolog Npc2 ( $k-0.0860374 \quad 7.350673810 .53506684$ serine/threonine protein kinase Hhp1

$$
\text { \#N/A }
$$

$\begin{array}{lll}-0.0861222 & 8.55206604 & 0.31280208\end{array}$ $\begin{array}{lll}-0.0862472 & 7.24997495 & 0.40330292\end{array}$ conserved fungal cell surface protein, Kre9/Knh1 family (prı $-\begin{array}{llll}-0.0863196 & 8.92583394 & 0.48951816\end{array}$ $\begin{array}{lllll}\text { pleckstrin homology domain protein Opy1 } & -0.0864196 & 6.28068137 & 0.59594149\end{array}$

$-0.0864388 \quad 5.73473803 \quad 0.60882811$ $\begin{array}{lll}-0.0864766 & 6.9369725 & 0.50018135\end{array}$ $\begin{array}{lll}-0.0865945 & 7.39496776 & 0.426697\end{array}$ $\begin{array}{lll}-0.0869966 & 7.07453493 \quad 0.45042974\end{array}$ $\begin{array}{lll}-0.0874915 & 6.61600593 & 0.49253486\end{array}$ \#N/A

transcription factor TFIIIB complex subunit Brf1 alpha-1,2-mannosyltransferase Omh5 (predicted) $\begin{array}{rrr}-0.0875904 & 8.63448454 & 0.29961888\end{array}$

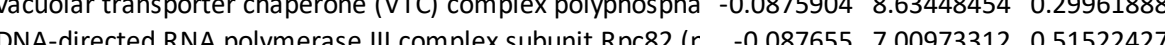
$\begin{array}{lllll}\text { Rho GDP dissociation inhibitor Rdi1 (predicted) } & -0.0878602 & 7.35741092 & 0.4079806\end{array}$ $\begin{array}{lll}-0.0878609 & 6.11847579 & 0.6264385\end{array}$ $\begin{array}{lll}-0.0881866 & 8.35247958 & 0.33210927\end{array}$ $\begin{array}{lrrr}\text { ribose-phosphate pyrophosphokinase (predicted) } & -0.0882755 & 7.45766935 & 0.37093392 \\ \text { conserved fungal protein } & -0.0883089 & 6.54859695 & 0.63863471 \\ \text { hydantoin racemase family, implicated in amino acid, or de } & -0.0890361 & 5.7875764 & 0.6088868\end{array}$ $\begin{array}{lrrr}\text { ribose-phosphate pyrophosphokinase (predicted) } & -0.0882755 & 7.45766935 & 0.37093392 \\ \text { conserved fungal protein } & -0.0883089 & 6.54859695 & 0.63863471 \\ \text { hydantoin racemase family, implicated in amino acid, or de } & -0.0890361 & 5.7875764 & 0.6088868\end{array}$ hydantoin racemase family, implicated in amino acid, or de $\begin{array}{rrrr}-0.0890361 & 5.7875764 & 0.6088868\end{array}$

aromatic aminotransferase (predicted)

multidomain vesicle coat component Sec16 (predicted)

$$
\# \mathrm{~N} / \mathrm{A}
$$

exosome subunit CsI4

Schizosaccharomyces pombe specific protein

beige protein homolog Lvs1

nucleosome remodeling protein Spt6

$$
\# N / A
$$

$\begin{array}{lll}-0.0893584 & 6.17289815 & 0.5467048\end{array}$ $\begin{array}{lll}-0.0895105 & 9.53676581 & 0.27995296\end{array}$ $\begin{array}{lll}-0.0895119 & 3.36185004 & 0.93090166\end{array}$ $\begin{array}{lll}-0.0896117 & 6.48318435 & 0.55395674\end{array}$ $\begin{array}{llll}-0.0896494 & 6.41253014 & 0.61292201\end{array}$ $\begin{array}{lll}-0.0897419 & 9.13913706 & 0.20090871\end{array}$ $\begin{array}{lll}-0.0898506 & 8.81095095 & 0.25863735\end{array}$ $\begin{array}{lll}0.0898566 & 7.06624823 & 0.46138813\end{array}$

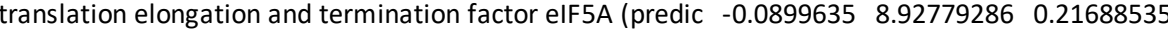
Mst2 histone acetytransferase acytyltransferase complex sı $-0.0901527 \quad 5.27710336 \quad 0.67234891$ $\# \mathrm{~N} / \mathrm{A}$

$\# N / A$
0.563027237

0.50348167

0.492579848

0.588205139

0.61571706

0.843845627

0.949793681

0.431703393

0.832706345

0.627039624

0.504993542

0.450015692

0.478645826

0.363434424

0.709670528

0.602981181

0.62626932

0.406535172

0.498633063

0.584415984

0.679862837

0.691888609

0.593470839

0.521586684

0.545695988

0.587296249

0.392625934

0.606696857

0.503227832

0.707655043

0.426558521

0.466267279

0.718445649

0.691888609

0.482773949

0.637127676

0.372376831

0.956670614

0.643728757

0.695619461

0.281603247

0.349142458

0.556495253

0.301082178

0.74841146

0.621156779

0.70111819 
SPBC16D10.07C

SPAC25B8.03

SPAP8A3.04C

SPBC800.08

SPAC30C2.06C

SPAC1F 7.09C

SPAC1527.01

SPCC4G3.09C

SPNCRNA.607

SPAPB1A10.09

SPBC56F $2.05 \mathrm{C}$

SPBC839.09C

SPAC6F6.01

SPAC22A12.03C

SPBP23A10.02

SPAC12G12.12

SPCC417.03

ScpofMt31

SPAC19G12.10c

SPAC1039.05C

SPAC25B8.04C

SPBC36.09

SPBC887.15C

SPBC16E9.01C

SPAC17G6.03

SPAC1D4.11C

SPNCRNA.18

SPAC24B11.12C

SPBC106.20

SPCC126.13C

SPNCRNA.53

SPACUNK4.19

SPNCRNA.910

SPAC13G7.07

SPAC688.07c

SPNCRNA.1552

SPNCRNA.1518

SPBC1604.07

SPBC18A7.01

SPNCRNA.1232

SPAC27E2.11C

SPNCRNA.1451

SPAC29A4.14C

SPNCRNA.1357

SPNCRNA.1288

SPAC15A10.10

SPAC6C3.06c mrp21

cch1

csn 4

cpy1

klf1

mss51

sap61

sur2

php4

SPAC17G6.03

Ikh1

f 2

exo70

sap18

\#N/A

mug153

\#N/A

arb2

rng10

$\# \mathrm{~N} / \mathrm{A}$

$\# N / A$

atp4

xpa1

$\# \mathrm{~N} / \mathrm{A}$

SPAC27E2.11C

$\# \mathrm{~N} / \mathrm{A}$

pex3

$\# \mathrm{~N} / \mathrm{A}$

$\# \mathrm{~N} / \mathrm{A}$

mde6

neo1
Sirtuin family histone deacetylase Sir2

phosphatidylserine decarboxylase Psd2

$0.09029 \quad 5.98527012 \quad 0.62327114$ $\begin{array}{llll}-0.0904224 & 7.85043304 & 0.34079311\end{array}$ $\begin{array}{lrrr}\text { heat shock protein Hsp9 } & -0.0906233 & 8.4850207 & 0.27107605 \\ \text { tRNA (m1A) methyltransferase non-catalytic subunit Gcd1C } & -0.0912287 & 6.94511143 & 0.44697102\end{array}$ mitochondrial inheritance GTPase, tubulin-like (predicted) $\quad-0.0913513 \quad 6.42610658 \quad 0.55690571$ allantoicase Dal2

alpha-1,3-glucan synthase Mok11

GTPase activating protein Gyp3 (predicted)

$$
\text { \#N/A }
$$

antiparallel microtubule cross-linking factor Ase1

transcription factor (predicted)

$\begin{array}{lll}-0.09166 & 6.66488458 & 0.50855479\end{array}$ $\begin{array}{lll}-0.0916656 & 8.41345842 & 0.31536447\end{array}$

$\begin{array}{lll}-0.091964 & 7.66359299 & 0.31060638\end{array}$ $\begin{array}{lll}-0.0920159 & 6.09750222 & 0.59146188\end{array}$ $\begin{array}{lll}-0.0927622 & 7.67035932 & 0.31351419\end{array}$ $\begin{array}{llll}0.0928416 & 5.42233861 & 0.61464986\end{array}$

mitochondrial ribosomal protein subunit Mrp21 (predicted) $\quad-0.092875 \quad 5.155321640 .71508736$ plasma membrane calcium ion import channel Cch1 $\quad-0.0929146 \quad 8.02922693 \quad 0.29403854$ COP9/signalosome complex subunit Csn4

V-ATPase assembly factor Pkr1 (predicted)

$\begin{array}{lll}-0.0936652 \quad 5.64208559 & 0.63499383\end{array}$ $-0.0939854 \quad 6.47593816 \quad 0.48491175$

Golgi UDP-galactose transmembrane transporter Gms2 (pr $-0.0939983 \quad 6.743367590 .44963883$ \#N/A $\quad-0.09412313 .1161803 \quad 0.92817148$ $\begin{array}{llll}0.0941456 & 3.87588473 & 0.76158044\end{array}$ $\begin{array}{lll}-0.0941723 & 11.127495 & 0.19067733\end{array}$ vacuolar carboxypeptidase $Y$ $\begin{array}{lrrr}\text { transcription factor, zf-fungal binuclear cluster type Klf1 } & -0.0942819 & 7.6685159 & 0.37260777 \\ \text { mitochondrial Cox1 translation regulator Mss51 (predicted) } & -0.094649 & 5.47661594 & 0.65469347\end{array}$ regulator Mss51 (predicted) $\begin{array}{lll}-0.0948948 & 7.15794382 & 0.3739594\end{array}$ 2 snRNP-associated protein sap61

sphingosine hydroxylase/sphingolipid delta-4 desaturase ac $\begin{array}{rllll}0.0952691 & 8.09348489 & 0.44314754\end{array}$ CCAAT-binding factor complex subunit Php4 $\quad-0.0954846 \quad 6.090249680 .57654661$

extracellular 5'-nucleotidase, human NT5E family (predicte $-0.0955211 \quad 8.00146784 \quad 0.24649174$ dual specificity protein kinase Lkh1

\#N/A

$\begin{array}{lll}-0.095608 & 7.98448428 & 0.44829497\end{array}$ $\begin{array}{lll}-0.0961207 & 5.83685191 & 0.60325006\end{array}$

plasma membrane phospholipid-translocating ATPase com| $\begin{array}{lllll}-0.0961988 & 9.86959417 & 0.21375642\end{array}$ exocyst complex subunit Exo70

splicing factor Sap18 (predicted)

$$
\text { \#N/A }
$$

Schizosaccharomyces pombe specific protein

$$
\# \mathrm{~N} / \mathrm{A}
$$

argonaute binding protein 2

coiled-coil protein involved in septum formation Rng10

$$
\# N / A
$$$$
\# N / A
$$

F1-FO ATP synthase subunit (predicted)

$X$-Pro dipeptidase (predicted)

\#N/A

Schizosaccharomyces specific protein

\#N/A

peroxin-3 peroxisome import protein Pex3 (predicted)

$\# N / A$

$\begin{array}{llll}-0.0963153 & 6.35411676 & 0.52338087\end{array}$

$\begin{array}{lll}-0.0963648 & 5.41838629 & 0.63089659\end{array}$

$\begin{array}{lll}-0.0963701 & 10.0539495 & 0.15587356\end{array}$

$\begin{array}{lll}-0.0966404 & 3.65986927 & 0.8137707\end{array}$

$\begin{array}{lll}-0.0968713 & 5.42615156 & 0.64446138\end{array}$

$\begin{array}{lll}-0.0969446 & 4.59564875 & 0.75015525\end{array}$

$\begin{array}{llll}-0.0969813 & 8.5467388 & 0.22884552\end{array}$

$\begin{array}{llll}-0.0969919 & 6.0164246 & 0.51683646\end{array}$

$\begin{array}{lll}-0.0970248 & 7.12642177 & 0.4114177\end{array}$

$\begin{array}{llll}-0.0972082 & 8.01065541 & 0.25705296\end{array}$

$\begin{array}{llll}-0.0972889 & 7.093445 & 0.40218687\end{array}$

$\begin{array}{lll}-0.097365 & 4.25367129 & 0.74466419\end{array}$

$\begin{array}{llll}-0.0974018 & 10.0582665 & 0.14999234\end{array}$

$\begin{array}{llll}-0.0974669 & 8.22266398 & 0.28682543\end{array}$

$\begin{array}{lll}-0.0978165 & 7.01643462 & 0.39036965\end{array}$

$\begin{array}{lll}-0.0978323 & 6.68681722 & 0.46181361\end{array}$

$\begin{array}{llll}-0.0978628 & 4.99401885 & 0.68282354\end{array}$

Muskelin homolog, kelch repeat, expressed during meiotic ( $\begin{array}{lllll}-0.0982121 & 8.39921532 & 0.24423422\end{array}$

$\begin{array}{llllll}\text { Golgi membrane phospholipid translocase (flippase) Neo1। } & -0.0982423 & 8.18164175 & 0.2306727\end{array}$
0.705247769

0.435511362

0.362696125

0.542858786

0.646824264

0.600922965

0.409240131

0.404221921

0.676260978

0.407305093

0.697347835

0.783738047

0.386728313

0.714945904

0.580035934

0.545126

0.954686652

0.822589496

0.269875048

0.467822116

0.732520401

0.469259397

0.539080984

0.664049896

0.336012875

0.544271182

0.686589177

0.297405943

0.614918304

0.711274234

0.228316411

0.864456701

0.723570321

0.814293588

0.315077815

0.608034361

0.506549208

0.347417158

0.497729105

0.809729744

0.221169641

0.379810673

0.48582031

0.556909727

0.758012106

0.333491457

0.317184519 
SPBC1703.06

SPNCRNA.1563

SPAC630.13C

SPCC16C4.04

SPNCRNA.886

SPAC26A3.14C

SPBC887.02

SPAC29A4.13

SPAC57A10.09C

SPAC23H4.07C

SPBC17F3.01C

SPBC1709.20

SPAC23A1.18C

SPCC1827.02C

SPAC6F 12.12

SPBC21B 10.02

SPNCRNA.1646

SPAC1B3.11C

SPBC32F12.15

SPAC4A8.03C

SPAC20G8.09c

SPNCRNA.555

SPNCRNA.1003

SPBC16A3.11

SPAC343.18

SPBC3H7.05C

SPBC3B8.05

SPAC27E2.06C

SPAC637.12C

SPBP4H $10.17 \mathrm{C}$

SPBC543.07

SPAC23G3.10c

SPCC1020.12C

SPAC688.11

SPNCRNA.1479

SPACUNK4.06C

SPAC20G4.08

SPAC644.12

SPBC947.07

SPAPB1A11.03

SPAC31A2.03

SPBC577.04

SPBC19F8.04C

SPNCRNA.1589

SPBC3D6.07

SPCC16C4.15

SPAC23G3.08C pof10

tsc2 SPCC16C4.04

\#N/A

SPAC26A3.14C

SPBC887.02

ure6

nhp6

srp102

rga5

pop8

mrp51

pcy1

SPBC21B10.02

$\# \mathrm{~N} / \mathrm{A}$

ypt4

tfb5

ptc4

nat10

$\# \mathrm{~N} / \mathrm{A}$

$\# \mathrm{~N} / \mathrm{A}$

eso1

rfp2

SPBC3H7.05C

dph1

msm1

SPBP4H10.17c

pek1

ssr3

xap5

end4

$\# N / A$

rpb7

pdc1

cdc5

rrp1402

SPAPB1A11.03

mrp11

tho5

$\mathrm{cl} 3$

\#N/A

gpi3

$\mathrm{rml} 2$

ubp7
F-box/WD repeat protein Pof10

\#N/A

tuberin, GTPase activator Tsc2

Schizosaccharomyces specific protein

$\# \mathrm{~N} / \mathrm{A}$

DUF1748 family protein

CIC chloride channel (predicted)

urease accessory protein UreF

$0.098817 \quad 7.168826380 .50934486$

$\begin{array}{lll}-0.0984076 & 7.84893108 & 0.33859751\end{array}$

$\begin{array}{lll}-0.0984661 & 4.33153088 & 0.75454056\end{array}$

$\begin{array}{lll}0.0986192 & 4.49124192 & 0.73192977\end{array}$

$\begin{array}{lll}-0.0986197 & 5.38974161 & 0.67962266\end{array}$

$\begin{array}{lll}-0.0987418 & 7.76448843 & 0.28159119\end{array}$

$\begin{array}{lll}-0.0990674 & 6.25805591 & 0.53866884\end{array}$

High-mobility group non-histone chromatin protein (predict $-0.0993552 \quad 7.50205778 \quad 0.37095827$

signal recognition particle receptor beta subunit Srp102 (pri $\begin{array}{ccccc}0.0995259 & 6.30833434 & 0.46610859\end{array}$

RhoGAP, GTPase activating protein Rga5

$\begin{array}{llll}0.0997278 & 7.0947524 & 0.41176657\end{array}$

$\begin{array}{llll}-0.0998241 & 3.83104067 & 0.84735907\end{array}$

RNase $P$ and RNase MRP subunit Pop8

$\begin{array}{lll}-0.0999735 & 6.77061685 & 0.4124944\end{array}$

$\begin{array}{lll}-0.1002142 & 7.46039969 & 0.34672891\end{array}$

$\begin{array}{lll}-0.1002412 & 7.04438547 & 0.40680016\end{array}$

$\begin{array}{lll}-0.1006144 & 6.37835993 & 0.44051143\end{array}$

$\begin{array}{lll}-0.1008363 & 4.82716693 & 0.67241553\end{array}$

$\begin{array}{lll}-0.1009922 & 6.21226928 & 0.47267931\end{array}$

transcription factor TFIIH complex subunit Tfb5 (predicted) $-0.1012676 \quad 6.0184191 \quad 0.49478705$

$\begin{array}{llllll}\text { protein phosphatase 2C Ptc4 } & -0.1014096 & 6.40408459 & 0.44510519\end{array}$

rRNA/tRNA cytidine N-acetyltransferase $\quad-0.1014177 \quad 8.11900895 \quad 0.25345028$

$\begin{array}{lll}-0.1014398 & 6.10777607 & 0.49467184\end{array}$ $\begin{array}{lll}-0.1020451 & 5.81044133 & 0.559525\end{array}$

$\# \mathrm{~N} / \mathrm{A}$

$\begin{array}{lll}-0.1022272 & 7.41601359 & 0.38000301\end{array}$

mitotic cohesin N-acetyltransferase/DNA polymerase eta $E-0.1022272 \quad 7.416013590 .38000301$

mitochondrial Membrane Bound O-Acyl Transferase (MBOt $\quad-0.1025997 .78015213 \quad 0.35437734$

diphthamide biosynthesis protein Dph1 (predicted) $\quad \begin{array}{lllll}0.1026959 & 6.09294569 & 0.57364318\end{array}$

mitochondrial methionine-tRNA ligase Msm1 (predicted) $\quad-0.1027268 \quad 6.55806806 \quad 0.46135576$

KAT5 family histone acetyltransferase Mst1 $\quad \begin{array}{lllll}0.102825 & 7.17315471 & 0.32551548\end{array}$

protein repair carboxyl methyl esterase (predicted) $\quad \begin{array}{lllll}-0.1030393 & 6.92331635 & 0.37121338\end{array}$

$\begin{array}{llll}-0.1032902 & 6.83814337 & 0.36802427\end{array}$

SWI/SNF and RSC complex subunit Ssr3 $\quad-0.1036709 \quad 8.08449346 \quad 0.24770597$

xap-5-like protein

Clathrin adaptor End4

\#N/A

$\begin{array}{lll}0.1037789 & 5.31229892 & 0.65724307\end{array}$

$\begin{array}{lll}-0.1047611 & 9.07978308 & 0.18957545\end{array}$

$\begin{array}{lll}-0.1049496 & 4.64699257 & 0.74286598\end{array}$

$\begin{array}{lll}-0.1052987 & 6.95463864 & 0.36378297\end{array}$

$\begin{array}{lll}-0.1054512 \quad 8.26035687 & 0.21536526\end{array}$

$\begin{array}{llll}-0.1055369 & 8.14308666 & 0.22814469\end{array}$

$\begin{array}{lll}-0.1057914 & 5.52456735 & 0.54687182\end{array}$

Prp19 complex subunit $\mathrm{Cdc} 5$

ribosome biogenesis protein Rrp14 (predicted)

$\begin{array}{lll}-0.1060146 & 5.40927077 & 0.58242649\end{array}$

mitochondrial ribosomal protein subunit L11 (predicted) $\quad \begin{array}{lllll}-0.1060688 & 6.54228058 & 0.46011661\end{array}$

human THOC5 ortholog Th05 (predictec

$\begin{array}{llll}0.1064063 & 4.98265381 & 0.63397656\end{array}$

mitochondrial nuclease Lcl3, implicated in DNA repair (prec $-0.1066243 \quad 5.260593990 .61078008$ $\# \mathrm{~N} / \mathrm{A}$

$\begin{array}{lll}0.1066243 \quad 5.26059399 & 0.61078008\end{array}$

pig-A, phosphatidylinositol N-acetylglucosaminyltransferası $-0.1067183 \quad 5.98609168 \quad 0.52139136$

mitochondrial ribosomal protein subunit L2 (predicted)

$\begin{array}{llll}-0.1072599 & 7.13904644 & 0.34150721\end{array}$

$\begin{array}{lll}-0.1085877 & 7.71326787 & 0.223515\end{array}$
0.601369707

601369707

0.433030649

0.817934271

0.799171801

0.754951391

0.374393479

0.629859198

0.466267279

0.560863387

0.506887028

0.893581041

0.507599345

0.441686671

0.502135879

0.536449588

0.74841146

0.567472603

0.588949779

0.541075529

0.343640001

0.588915655

0.649333932

0.475527853

0.671425542

0.449913767

0.66141

0.556495253

0.420010016

0.466501767

0.463349701

0.337210122

0.735066081

0.268538908

0.808315158

0.459284437

0.299460686

0.314339868

0.637127676

0.669043258

0.555256879

0.714155021

0.693413258

0.693413258

0.612792434

0.436178032

0.308711097 
SPAC3F10.16C

SPBP8B7.20C

SPNCRNA.1131

SPBC839.12

SPNCRNA.728

SPBC216.05

SPNCRNA.1521

SPAC26F1.10c

SPBC1105.11C

SPAC26F1.09

SPBC31F10.03

SPNCRNA.888

SPBC582.04C

SPNCRNA.934

SPNCRNA.858

SPAC664.06

SPBC577.10

SPBC405.04C

SPNCRNA.1401

SPCC965.10

SPBC2D10.19c

SPAC29A4.03C

SPBC1734.09

SPCC962.03C

SPBC947.06C

SPCC338.13

SPAC13G6.11C

SPBC31E1.01C

SPBC16A3.01

SPNCRNA.1379

SPAC2F7.11

SPAC7D4.05

SPNCRNA.1557

SPBC1734.10c

SPAC17A2.02C

SPCC24B10.10C

SPAC222.12C

SPBC2G2.02

SPBC1347.12 rpn502

hub1 $\# N / A$

anc1

kap104

dbp7

SPAC3F10.16c nop2

\#N/A

rpc31

$\# N / A$

rad3

\#N/A

pyp1

hht3

gyp51

ggg1

dsh1

\#N/A

$\# \mathrm{~N} / \mathrm{A}$

$\# N / A$

rlp7

pre4

ypt7

\#N/A

SPCC965.10

alb1

mrpss

yea4

cut15

SPBC947.06c

$\operatorname{cog} 4$

erg12

atg2

spn3

\#N/A

nrd1

SPAC7D4.05

$\# \mathrm{~N} / \mathrm{A}$

ipa1

SPAC17A2.02c

yta4

atp2

syj1

arp1 protein O-mannosyltransferase Ogm4

$0.1094013 \quad 8.30634958 \quad 0.15587921$

mitochondrial genome maintenance protein Rrg9 (predicter $-0.096252 \quad 5.29735136 \quad 0.6186943$

19S proteasome regulatory subunit Rpn502 $\quad \begin{array}{lllll}-0.1097284 & 8.64943498 & 0.20156181\end{array}$ $\# \mathrm{~N} / \mathrm{A}$

ubiquitin-like protein modifier Hub1

$\begin{array}{llll}0.322 & 6.33902427 & 0.45751849\end{array}$

$\begin{array}{lll}0.1104982 & 5.70813746 & 0.51147006\end{array}$

$\begin{array}{lll}-0.1106254 & 9.03204623 & 0.20330768\end{array}$

mitochondrial carrier, ATP:ADP antiporter Anc1

$\begin{array}{lll}-0.1106852 & 8.38008509 & 0.18638029\end{array}$

ATP-dependent RNA helicase Dbp7 (predicted) $\quad \begin{array}{llllll}0.110756 & 6.71892941 & 0.37234085\end{array}$

$\begin{array}{llll}-0.1109881 & 7.22453984 & 0.26925935\end{array}$

rRNA (cytosine-C5-)-methyltransferase activity Nop2 (predi $\quad-0.111018 \quad 9.540568810 .18519716$

$\begin{array}{lll}-0.1110699 & 6.26993705 & 0.42289\end{array}$

DNA-directed RNA polymerase III complex subunit Rpc31 $\quad-0.11108896 .49825294 \quad 0.41206033$

$\begin{array}{lll}-0.1112457 & 6.3355512 & 0.42602303\end{array}$

$\begin{array}{lll}-0.1115597 & 9.49802907 & 0.14839099\end{array}$

$\begin{array}{lll}0.1116103 & 5.31529747 & 0.57197752\end{array}$

$\begin{array}{lll}-0.111695 & 7.41937088 & 0.32049567\end{array}$

$\begin{array}{lll}-0.1117265 & 7.70137819 & 0.22173996\end{array}$

$\begin{array}{lll}-0.1119586 & 7.56903762 & 0.33828307\end{array}$

histone H3 h3.3

GTPase activating protein Gyp51 (predicted)

$\begin{array}{lll}-0.1140255 & 7.40438228 & 0.28299596\end{array}$

$\begin{array}{lll}-0.1141349 & 6.57903846 & 0.36179737\end{array}$

$\begin{array}{lll}-0.1146289 & 7.99572622 & 0.19965039\end{array}$

$\begin{array}{lll}-0.1147883 & 7.38702224 & 0.28375912\end{array}$

$\# N / A$

$\begin{array}{lll}-0.1148006 & 7.48178859 & 0.32900607\end{array}$

$\begin{array}{lll}-0.1149318 & 8.0165919 & 0.1813076\end{array}$

$-0.11498347 .92143742 \quad 0.18999832$

$\begin{array}{lll}-0.1151508 & 5.37702315 & 0.56158116\end{array}$

$-0.11517967 .91499545 \quad 0.18363943$

$-0.11520565 .55688554 \quad 0.54725386$

transcription factor (predicted)

pre-60S shuttling factor Alb1 (predicted)

$\begin{array}{lll}-0.1153077 & 7.24300688 & 0.28637395\end{array}$

ER UDP-N-acetylglucosamine transmembrane transporter $\left(\begin{array}{llllll}-0.1156015 & 7.60149387 & 0.21801037\end{array}\right.$

importin alpha family nuclear import signal receptor adapts $-\begin{array}{llllll}0.1156275 & 7.81361161 & 0.23483486\end{array}$

spermidine family transmembrane transporter (predicted) $\quad \begin{array}{llll}0.1163039 & 7.33325501 & 0.23301395\end{array}$

Golgi transport complex subunit $\operatorname{Cog} 4$ (predicted) $\quad \begin{array}{lllll}-0.1164829 & 6.6212428 & 0.34120224\end{array}$

mevalonate kinase Erg12 (predicted) $\quad-0.1165773 \quad 7.14870961 \quad 0.50299028$

autophagy associated protei

mitotic septin Spn3

$\begin{array}{lll}-0.116643 & 7.87771701 & 0.22859932\end{array}$

$\begin{array}{lll}-0.1168485 & 7.13186738 & 0.27044709\end{array}$

$\begin{array}{lll}-0.1169995 & 4.65086666 & 0.69166015\end{array}$

$\begin{array}{lll}-0.1173208 & 6.03296022 & 0.50625773\end{array}$

$\begin{array}{lll}-0.117384 & 6.03385041 & 0.44837388\end{array}$

HAD superfamily hydrolase, unknown role

$\begin{array}{lll}0.1180349 & 6.76219962 & 0.31486796\end{array}$

$\# N / A$

$\begin{array}{lrrr}\text { cleavage and polyadenylation HECT-type ubiquitin-protein li } & -0.1182231 & 7.58517033 & 0.2062974 \\ \text { ER protein, DUF887 family protein, implicated in lipid meta } & -0.1189884 & 7.7200068 & 0.1829943\end{array}$

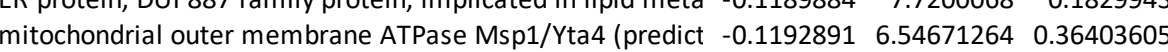
F1-FO ATP synthase beta subunit Atp2 $\quad-0.1206922 \quad 10.0475045 \quad 0.05688369$ inositol-polyphosphate 5-phosphatase, synaptojanin homolı $\begin{array}{ccccc}0.1207396 & 7.5112081 & 0.19346835\end{array}$ dynactin complex subunit, centractin family actin-like prote $\begin{array}{llll}-0.1207663 & 3.92783065 & 0.70829653\end{array}$
0.228316411

0.70111819

0.282344197

0.552611522

0.603111027

0.284263156

0.264508419

0.467573254

0.360690915

0.263103685

0.518061553

0.507156911

0.520949993

0.21914078

0.660048672

0.414949205

0.306508318

0.432872562

0.321946874

0.375836745

0.457371564

0.280295268

0.376703245

0.4237105

0.259038048

0.268969956

0.651032329

0.261819887

0.637463623

0.379360466

0.302397176

0.322187743

0.319947524

0.43587033

0.596419031

0.314838773

0.362068151

0.765449229

0.599438613

0.544271182

0.408829707

0.28814733

0.261064195

0.459433477

0.096652564

0.273256976

0.778291275 
SPBC21.02

SPAC13F5.01C

SPAC56F8.02

SPAC19B12.10

SPNCRNA.935

SPAC824.08

SPBC25D12.06

SPBC365.02C

SPAC664.07c

SPAC3H1.10

SPNCRNA.1338

SPAC3C7.10

SPAC56E4.04C

SPAC3C7.01C

SPBC23E6.04C

SPBC27B12.05

SPBC16A3.16

SPAC4G8.08

SPAC17A5.19

SPNCRNA.1403

SPBC776.12C

SPAC17A2.03C

SPNCRNA.453

SPAC5D6.12

SPBC $3 \mathrm{H} 7.08 \mathrm{C}$

SPBC24C6.07

SPAC13G6.05c

SPAPB1E7.08C

SPAC24C9.06C

SPAC1F5.07c

SPAC6F12.17

SPAC15E1.06

SPAC343.07

SPCC737.02c

SPBC29B5.04C

SPAC19B12.03

SPAC630.12

SPCC320.13c

SPAC227.01C

$S P B C 25 B 2.10$

SPAC24B11.05

SPAC824.02

SPCC1682.11C iws1

\#N/A

pop6

mrps28

rtc5

msh1

SPAC56F8.02

sst2

\#N/A

gda1

SPBC25D12.06

$\operatorname{cox} 10$

$\operatorname{rad} 9$

pcs2

\#N/A

pex13

cut6

sac12

utp10

SPBC27B12.05

coa5

SPAC4G8.08

SPAC17A5.19

\#N/A

hsk1

vma6

$\# N / A$

mtf2

SPBC3H7.08C

cdc14

trs33

SPAPB1E7.08C

aco1

hem14

na14

vps 29

mug28

qcr7

SPBC29B5.04c

bgs 3

SPAC630.12

ark1

SPBC25B2.10

SPAC24B11.05

bst1

ctl1 transcription elongation factor complex subunit Iws1 (predi $-\begin{array}{llll}0.1211989 & 6.67471397 & 0.32932395\end{array}$ $\# \mathrm{~N} / \mathrm{A}$

RNase $\mathrm{P}$ and RNase MRP subunit Pop6/Pop7 ortholog mitochondrial ribosomal protein subunit S28 (predicted)

TLDc domain protein 2

mitochondrial MutS protein Msh1 (predicted)

AMP binding enzyme, human DIP2 family (predicted)

$0.12119896 .67471397 \quad 0.32932395$

$\begin{array}{llll}-0.1212126 & 4.85370665 & 0.61742773\end{array}$

$\begin{array}{lll}-0.1218162 & 6.76642019 & 0.29872409\end{array}$

$\begin{array}{llll}0.1219892 & 6.42157372 & 0.43568883\end{array}$

$\begin{array}{lll}-0.1222375 & 6.323795 & 0.39164801\end{array}$

$\begin{array}{lll}-0.1224535 & 8.0852964 & 0.17964155\end{array}$

human AMSH/STAMBP protein homolog, ubiquitin specific- $-\begin{array}{llll}0.1229055 & 7.21767377 & 0.26264713\end{array}$

$$
\text { \#N/A }
$$

Golgi nucleoside-diphosphatase Gda1

mitochondrial ATP-dependent RNA helicase (predicted)

protoheme IX farnesyltransferase (predicted)

checkpoint clamp complex protein Rad9

phytochelatin synthetase

$$
\text { \#N/A }
$$

peroxin 13 (predicted)

acetyl-CoA/biotin carboxylase

nositol polyphosphate phosphatase (predicted)

U3 snoRNP-associated protein Utp10 (predicted)

cytochrome c oxidase assembly protein Coa5 (predicted)

mitochondrial carrier, iron ion (predicted)

Schizosaccharomyces specific protein

$\# \mathrm{~N} / \mathrm{A}$

bf4(Dfp1)-dependent protein kinase Hsk1

V-type ATPase V0 subunit $d$ (predicted)

$$
\# \mathrm{~N} / \mathrm{A}
$$

mitochondrial translation protein Mtf2 (predicted)

mitochondrial conserved fungal membrane protein

SIN component Cdc14

TRAPP complex subunit Trs33 (predicted)

transmembrane transporter (predicted)

aconitate hydratase Aco1 (predicted)

$\begin{array}{llll}-0.1230499 & 8.06230283 & 0.18451727\end{array}$

$\begin{array}{llll}0.1231875 & 8.47663012 & 0.13247131\end{array}$

$\begin{array}{llll}0.1234615 & 7.12990664 & 0.29839057\end{array}$

$\begin{array}{llll}-0.123501 & 5.98457366 & 0.48768237\end{array}$

$\begin{array}{llll}-0.1240819 & 4.93885009 & 0.58814727\end{array}$

$\begin{array}{llll}0.1252062 & 6.42532192 & 0.40037492\end{array}$

$\begin{array}{llll}0.1253265 & 4.26771763 & 0.7004704\end{array}$

$\begin{array}{llll}-0.1253802 & 6.46230223 & 0.34739991\end{array}$

$\begin{array}{llll}-0.1254088 & 10.478432 & 0.07529876\end{array}$

$\begin{array}{llll}0.1254088 & 10.478432 & 0.07529876\end{array}$

$\begin{array}{lll}-0.1254481 & 8.83572134 & 0.12375145 \\ -0.1256265 & 6.01998603 & 0.44885642\end{array}$

$\begin{array}{llll}-0.1257456 & 6.20651689 & 0.45036321\end{array}$

$\begin{array}{llll}-0.1264917 & 6.33531262 & 0.36849379\end{array}$

$\begin{array}{lll}-0.1265299 & 4.24305459 & 0.75782363\end{array}$

$\begin{array}{llll}-0.1270711 & 5.71886598 & 0.45436906\end{array}$

$\begin{array}{llll}-0.1272787 & 5.90614838 & 0.41669812\end{array}$

$\begin{array}{lll}-0.1283814 & 7.77078692 & 0.16963184\end{array}$

$\begin{array}{llll}-0.128729 & 6.45534486 & 0.33070627\end{array}$

$\begin{array}{llll}-0.1288204 & 6.0544117 & 0.41078562\end{array}$

$\begin{array}{llll}-0.1292414 & 5.78467971 & 0.45952239\end{array}$

$\begin{array}{lll}-0.1296507 & 6.86977175 & 0.25923752\end{array}$

$\begin{array}{llll}0.1299647 & 6.20639601 & 0.43308551\end{array}$

$\begin{array}{llll}-0.1299774 & 7.05354589 & 0.29128298\end{array}$

$\begin{array}{lll}-0.1300832 & 10.3821488 & 0.05938216\end{array}$

$\begin{array}{llll}-0.1301231 & 7.15588974 & 0.28392275\end{array}$

mRNA cleavage and polyadenylation specificity factor comr $-\begin{array}{lllll}-0.1302018 & 7.85902208 & 0.19264227\end{array}$

$\begin{array}{lllll}\text { retromer complex subunit Vps29 } & -0.1307035 & 6.5673595 & 0.29377058\end{array}$

$\begin{array}{rrr}-0.1307035 & 6.5673595 & 0.29377058 \\ -0.1308379 & 6.86579634 & 0.30133962\end{array}$

ubiquinol-cytochrome-c reductase complex subunit 6 (predi $-0.1309609 \quad 7.522934380 .18436214$

phosphatidate phosphatase converting phosphatidate to dic $\begin{array}{llll}-0.1312421 & 7.38517797 & 0.18499019\end{array}$

cell wall 1,3-beta-glucan synthase catalytic subunit Bgs3 $\quad \begin{array}{llll}0.1320685 & 9.49401107 & 0.09481286\end{array}$

GPI-remodelling mannose-ethanolamine phosphate phospt $\begin{array}{lllll}-0.1322803 & 6.6486912 & 0.27769792\end{array}$

aurora-B kinase Ark1

Erd1 homolog (predicted)

Usp (universal stress protein) family protein

pyrimidine 5 '-nucleotidase (predicted)

GPI inositol deacylase Bst1 (predicted)

choline transporter-like, implicated in autophagy $\mathrm{Ct} \mid 1$
0.423799069

0.423799069

0.70014926

0.391679662

0.531337359

0.487054959

0.257089532

0.353573433

0.262466558

0.199483789

0.391393186

0.582735475

0.67406613

0.495823717

0.771935399

0.442458615

0.122791288

0.289643333

0.188185168

0.544565509

0.545695988

0.463718697

0.82026241

0.549294984

0.511754319

0.244927784

0.425175927

0.505954055

0.554638141

0.349782135

0.528703004

0.383845986

0.100271852

0.37677346

0.272259746

0.386523883

0.394349258

0.262456954

0.2629676

0.150046862

0.369811031

0.597960599

0.538496637

0.428739989

0.334634767

0.19016135

0.357307239 
SPCC364.01 SPBC32F12.01C SPBP8B7.05C

SPAC630.08c

SPBC725.10

SPAC1B3.18C

SPBP23A10.15C

SPBC1271.12

SPNCRNA.1323

SPAC17G8.02

SPNCRNA.515

SPBC28F2.10C

SPAC823.06

SPBC336.02

SPBC660.10

SPNCRNA.491

SPBC887.13C

SPNCRNA.659

SPAC664.08c

SPAC29A4.17C

SPAC15A10.05C

SPAC1002.18

SPNCRNA.1083

SPCC1620.14C

SPAC29B12.02C

SPCC1259.13

SPBC530.05

SPBC4B4.02C

SPBC16E9.11C

SPAC1420.03

SPBC16A3.17c

SPNCRNA.808

SPAPB1A11.02

SPBC24C6.08C

SPBC19C2.01

SPNCRNA.1217

SPCC31H12.06

SPCC16C4.19

SPAC17H9.09c

SPBC342.04

SPAC144.16

SPNCRNA.732

SPBC3B9.10

SPCC1620.03

SPNCRNA.850

SPNCRNA.926

SPAC1296.04 ngg1

taf3

cdh1

mef2

set2

chk1

prt1

nca2

pub3

rpn501

SPBC16A3.17C

$$
\# \mathrm{~N} / \mathrm{A}
$$

SPAPB1A11.02

bhd1

cdc28

$\# \mathrm{~N} / \mathrm{A}$

mug111

rpp21

ras1

rpn1301

cia2

\#N/A

vti

mug163

$\# \mathrm{~N} / \mathrm{A}$

$\# \mathrm{~N} / \mathrm{A}$ calnexin independence factor Cif1

inositol phosphosphingolipid phospholipase C, Css1

carbonic anhydrase (predicted)

C-4 methylsterol oxidase Erg25 (predicted)

mitochondrial lipid translocator protein, tspO

mitochondrial ribosomal protein subunit S18 (predicted)

mitochondrial processing peptidase (MPP) complex beta su

sterol intermembrane transfer protein Kes1 (predicted)

$$
\text { \#N/A }
$$

uridine ribohydrolase Urh2 (predicted)

$\# \mathrm{~N} / \mathrm{A}$

SAGA complex subunit Ngg1/Ada3

transcription factor TFIID complex subunit Taf3 (predicted)

$18 \mathrm{~S}$ rRNA dimethylase Cdh1 (predicted)

mitochondrial translation elongation factor G Mef2 (predic

$$
\# \mathrm{~N} / \mathrm{A}
$$

3-oxoacyl-[acyl-carrier-protein]-synthase condensing enzym

$$
\# \mathrm{~N} / \mathrm{A}
$$

traub family protein involved in ribosome biogenesis (predi - $-0.1382325 \quad 6.273306560 .35504605$

mitochondrial FUN14 family protein involved in mitophagy $\quad \begin{array}{lllll}0.138874 & 5.76077697 & 0.45368523\end{array}$ NADHX epimerase (predicted)

$\begin{array}{lll}0.1389041 & 5.98014547 & 0.4181974\end{array}$

DUF1688 family fungal conserved protein, implicated in ura $-0.1389407 \quad 6.099005540 .43719283$$$
\# \mathrm{~N} / \mathrm{A}
$$

ATP-dependent DNA helicase Snf22

histone lysine methyltransferase Set2

Chk1 protein kinase

transcription factor (predicted)

mitochondrial protein $\mathrm{Nca} 2$ (predicted)

HECT-type ubiquitin-protein ligase E3 Pub3 (predicted)

$19 S$ proteasome regulatory subunit Rpn501

transmembrane transporter (predicted)

esterase/lipase (predicted)

$$
\text { \#N/A }
$$

Lst4-Lst7 complex subunit, folliculin Bhd1/Lst7

ATP-dependent RNA helicase Cdc28

$$
\text { \#N/A }
$$

$\begin{array}{lll}-0.1389962 & 5.66814746 & 0.4404189\end{array}$

$\begin{array}{lll}-0.1393561 & 8.8145688 & 0.05817822\end{array}$

$\begin{array}{llll}0.1395241 & 7.56392388 & 0.15771603\end{array}$

$\begin{array}{lll}-0.1396644 & 7.07423038 & 0.18196843\end{array}$

$\begin{array}{lll}-0.1398045 & 7.25623893 & 0.19717855\end{array}$

$\begin{array}{llll}-0.1398326 & 6.80084609 & 0.36001299\end{array}$

$\begin{array}{lll}-0.1400662 & 8.66941245 & 0.11001314\end{array}$

$\begin{array}{llll}-0.1401055 & 8.58084226 & 0.11234186\end{array}$

$\begin{array}{lll}-0.1402886 & 7.39761525 & 0.18447195\end{array}$

$\begin{array}{llll}-0.1404655 & 5.89202164 & 0.36519726\end{array}$

$\begin{array}{lll}-0.1405898 & 2.94617388 & 0.82874455\end{array}$

$\begin{array}{lll}-0.1407462 & 6.87007028 & 0.22642327\end{array}$

$\begin{array}{lll}-0.1408646 & 7.91263114 & 0.15283509\end{array}$

$\begin{array}{lll}-0.1410144 & 7.7638809 & 0.11852521\end{array}$

major facilitator family transmembrane transporter Mug11 $\begin{array}{lllll}0.1411066 & 4.50215137 & 0.72711854\end{array}$

RNase MRP subunit Rpp21

GTPase Ras1

19S proteasome regulatory subunit Rpn13a

ClA machinery protein $\mathrm{Cia} 2$ (predicted)

$$
\text { \#N/A }
$$

SNARE Vti1 (predicted)

mitochondrial protein, human C6orf136 ortholog

$$
\# N / A
$$

$\begin{array}{llll}0.1411066 & 4.50215137 & 0.72711854\end{array}$

$\begin{array}{lll}-0.1420159 & 6.50283282 & 0.36722919\end{array}$

$\begin{array}{lll}-0.14216 & 6.02282477 & 0.41743812\end{array}$

$\begin{array}{llll}-0.1422307 & 7.67391542 & 0.24771689\end{array}$

$\begin{array}{llll}0.1425618 & 4.91685358 & 0.54424751\end{array}$

$-0.1428377 \quad 7.17275992 \quad 0.1965083$

$\begin{array}{llll}-0.1429055 & 6.13882825 & 0.39472287\end{array}$

$\begin{array}{llll}0.14307 & 6.83375909 & 0.2362052\end{array}$

$\begin{array}{lll}-0.1430921 & 6.00218154 & 0.40060043\end{array}$

dysferlin-like membrane trafficking protein Mug65 (predictt $\quad \begin{array}{llll}-0.1432823 & 5.28354103 & 0.480733\end{array}$
0.658946812

0.380513323

0.364670456

0.345388712

0.203569416

0.510409122

0.145185561

0.159229072

0.225431367

0.489454444

0.547322052

0.353939866

0.68261529

0.380996371

0.364670456

0.465620502

0.458800012

0.592962969

0.450426616

0.548565736

0.513317698

0.532789609

0.536432905

0.098556834

0.230708847

0.259764037

0.277575512

0.455708411

0.17006802

0.172999861

0.262456954

0.460642698

0.876940861

0.312157686

0.224631266

0.181495059

0.795358042

0.455694705

0.462605454

0.51257062

0.337210122

0.635048748

0.276975756

0.490072823

0.323871926

0.496012727

0.575782588 
SPAC22E12.09c SPCC1223.10c SPBC582.10C SPBC30B 4.01C SPCC1620.08 ScpofMt26 SPAP27G11.10c SPAC16.04

SPAC869.11

SPAC31G5.09C

SPNCRNA.14

SPBC902.03

SPBC27B12.04C

SPCC4B3.01

SPNCRNA.1685

SPAC3A12.05C

SPBC342.05

SPAC22A12.02C

SPAC11E3.11C

SPAC3C7.06C

SPBP4H 10.08

SPAC4H3.13

SPBC28F2.12

SPAC16E8.17C

SPBPB2B2.18

SPBC646.15C

SPCC1020.08

SPAC167.01

SPAC30.03C

SPAC31A2.08

SPAC19D5.04

SPAPB18E9.02C

SPNCRNA.507

SPNCRNA.893

SPNCRNA.543

SPBC1773.09c

SPBP8B7.02

SPNCRNA.1627

SPAC23C4.07

SPAC26A3.09c

SPAC27F1.09C

SPNCRNA.1273

SPAC24C9.15C

SPAC24C9.16C

SPAC22F3.04

SPNCRNA.1670

SPNCRNA.200 cat1

spk1

far11 tum 1

\#N/A

crb2

mug103

syt22

pit1

qcr10

pcc1

rpb1

SPAC16E8.17C

SPBPB2B2.18

pex16

tyw1

ire1

tsn1

mrp20

ptr1

ppk18

$\# N / A$

$\# \mathrm{~N} / \mathrm{A}$

$\# N / A$

mug184

rng9

\#N/A

tht2

rga2

prp10

$\# N / A$

spn5

cox8

mug62

$\# N / A$

\#N/A kexin $\begin{array}{lrrr}\text { ATP-dependent DNA helicase Rhp16b (predicted) } & -0.143431 & 6.29435533 & 0.32568449 \\ \text { plasma membrane-associated serine-rich cell wall sensor } & -0.143602 & 7.65601581 & 0.1983492\end{array}$ $\begin{array}{lrrrr}\text { plasma membrane-associated serine-rich cell wall sensor V } & -0.143602 & 7.65601581 & 0.1983492 \\ \text { succinate-CoA ligase beta subunit Lsc2 (predicted) } & -0.1440395 & 7.58993489 & 0.16456743\end{array}$

tRNA dihydrouridine synthase Dus3 (predicted) $\quad-\begin{array}{llll}0.1454622 & 7.20741808 & 0.17666725\end{array}$

plasma membrane arginine/lysine amino acid transmembr $\begin{array}{lllll}0.1463798 & 6.71286067 & 0.30069928\end{array}$ MAP kinase Spk1 Nem1-Spo7 complex regulatory subunit (predicted) $\quad-\begin{array}{lllll}0.1469373 & 4.55678896 & 0.71509618\end{array}$ SIP/FAR complex subunit, Far11/Csc2 thiosulfate sulfurtransferase, involved in tRNA wobble posi $-0.14728626 .89757955 \quad 0.29302107$

TATA-binding protein associated factor Taf2 (predicted) DNA repair protein Rad9 homolog Crb2

Schizosaccharomyces specific protein Mug103

guanyl-nucleotide exchange factor Syt22

serine/threonine protein kinase, meiotic Pit1

RNA polymerase II large subunit Rpb1

succinate-CoA ligase alpha subunit (predicted)

conserved fungal plasma membrane protein

$\begin{array}{lllll}\text { Pex16 family peroxisome import protein Pex16 (predicted) } & -0.1496215 & 5.87481957 & 0.35983143\end{array}$ wybutosine biosynthesis protein Tyw1 (predicted) $\quad-\begin{array}{lllll}0.1499003 & 7.87431673 & 0.08831763\end{array}$

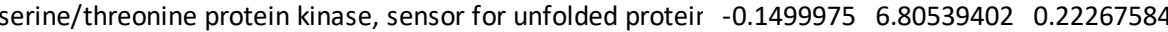
translin

mitochondrial ribosomal protein subunit L23 (predicted)

HECT-type ubiquitin-protein ligase E3 Ptr1

greatwall kinase Ppk18

DNAJ domain protein, exocytosis associated Mug184 (predi $-0.1516524 \quad 6.25728065 \quad 0.28179631$ contractile ring myosin $\mathrm{V}$ regulator Rng9

karyogamy protein Tht2

$\begin{array}{lll}-0.1523582 & 1.09884873 & 1\end{array}$ $\begin{array}{lll}0.1433878 & 8.95658699 & 0.05067743\end{array}$

$\begin{array}{llll}-0.1434185 & 6.39682989 & 0.38711332\end{array}$

$\begin{array}{lll}-0.1442879 & 3.8311352 & 0.75971618\end{array}$ $\begin{array}{lll}-0.1452854 & 8.72745338 & 0.07800775\end{array}$

\#N/A

$\begin{array}{lllll}-0.1468148 & 8.0959238 & 0.07065242\end{array}$ $\begin{array}{llll}-0.1468794 & 5.50939747 & 0.47391892\end{array}$ $\begin{array}{llll}-0.1470674 & 6.28898748 & 0.33639409\end{array}$ $\# \mathrm{~N} / \mathrm{A}$

$\begin{array}{llll}0.1472862 & 6.89757955 & 0.29302107\end{array}$

$\begin{array}{lll}-0.1475946 & 8.17973589 & 0.07791913\end{array}$

$\begin{array}{llll}-0.1479653 & 6.22362949 & 0.28796144\end{array}$

$\begin{array}{llll}-0.1481141 & 2.16917366 & 0.87430464\end{array}$

$\begin{array}{llll}-0.148135 & 7.95795261 & 0.09956184\end{array}$

$\begin{array}{lll}-0.1482747 & 6.44828263 & 0.3171525 \\ -0.1485081 & 5.67788946 & 0.45133668\end{array}$

$\begin{array}{llll}0.1488763 & 5.49955897 & 0.38720077\end{array}$

$\begin{array}{llll}-0.1489853 & 9.37551785 & 0.04210948\end{array}$

$\begin{array}{lll}-0.1491303 & 6.51500174 & 0.29689433\end{array}$

$\begin{array}{llll}-0.149497 & 8.24977168 & 0.11998871\end{array}$

$$
\text { \#N/A }
$$$$
\# \mathrm{~N} / \mathrm{A}
$$

$\begin{array}{llll}-0.150243 & 6.29402798 & 0.36157196\end{array}$

$\begin{array}{llll}-0.1502681 & 6.31388381 & 0.29268808\end{array}$

$\begin{array}{llll}-0.1505 & 10.1369207 & 0.03521485\end{array}$ $\begin{array}{llll}-0.1506355 & 8.63621879 & 0.04420331\end{array}$ $\begin{array}{llll}-0.1509072 & 5.88697568 & 0.33057038\end{array}$ $\begin{array}{llll}-0.1510322 & 5.048125 & 0.47949778\end{array}$ $-0.1516152 \quad 1.77654745$

$$
\text { \#N/A }
$$

$\begin{array}{llll}-0.1518173 & 4.8703231 & 0.50564261\end{array}$

$\begin{array}{llll}-0.1518173 & 4.8703231 & 0.50564261\end{array}$

$\begin{array}{lll}-0.1520141 & 5.5057726 & 0.50632963\end{array}$

$\begin{array}{llll}-0.1520643 & 7.58626959 & 0.10243421\end{array}$

$\begin{array}{llll}-0.1521613 & 7.81469815 & 0.14461306\end{array}$

$\begin{array}{llll}-0.1521695 & 6.66302328 & 0.30681474\end{array}$

$\begin{array}{llll}-0.1521917 & 5.9086 & 0.33213787\end{array}$

$\begin{array}{llll}-0.1522197 & 5.24832581 & 0.47417948\end{array}$

$\begin{array}{llll}-0.15233 & 7.37490576 & 0.15353482\end{array}$

$\begin{array}{lll}-0.1523582 & 1.09884873\end{array}$

0.087772955

0.482719965

0.420148423

0.278877338

0.238933513

0.821485666

0.126481322

0.253460842

0.393662682

0.116188915

0.568786282

0.783738047

0.430860488

0.385762886

0.41936374

0.12639802

0.380574407

0.914626145

0.156434614

0.411325094

0.546600121

0.482719965

0.074799016

0.38999401

0.183447128

0.455694705

0.141312357

0.30773946

0.45717153

0.385459805

0.064022725

0.077787309

0.425081528

0.574569889

0.374462778

0.598937856

0.598937856

0.599438613

0.160024053

0.214338578

0.4003594

0.426558521

0.568998656

0.225367661

$$
\# N / A
$$


SPAC343.08C

SPCC320.08

SPAC3F $10.02 \mathrm{C}$

SPCC962.01

SPBC29A10.13

SPNCRNA.785

SPBC32F12.17

SPCC24B 10.07

SPBC336.13C

SPBC354.03

SPBC21B10.11

SPAC18G6.09C

SPBC609.05

SPAC25B8.09

SPNCRNA.567

SPCC576.13

SPCC61.02

SPNCRNA.998

SPBC16A3.09C

SPBC609.01

SPBC146.02

SPAC1D4.02C

ScpofMt19

SPBC18H10.02

SPAC222.18

SPAC890.05

SPBC530.14c

SPCC5E4.04

SPAC17A5.07C

SPAC1142.03C

SPCC613.02

SPAC2F7.02C

SPNCRNA.641

SPAC20G4.04C

SPNCRNA.1417

SPBC337.16

SPBC1198.07c

SPAC7D4.11C

SPBC543.06C hob3

hrd3

SPBC21.03C

ssb3

mrp17

SPCC320.08

trk1

tcb1

atp7

$\# N / A$

$\# N / A$

gad8

mmp2

swd3

dpm2

edc1

pob3

SPAC25B8.09

\#N/A

swc5

spt3

\#N/A

ufd1

SPBC609.01

SPBC146.02

grh1

\#N/A

Icf1

SPAC222.18

pxr1

dsk1

cut1

ulp2

swi2

SPCC613.02

psr1

\#N/A

hus1

$\# N / A$

cho1

SPBC1198.07c

sec39

$\mathrm{dbp} 8$
\#N/A

mug2/mug135/meu2 family

$$
\text { \#N/A }
$$$$
\# \mathrm{~N} / \mathrm{A}
$$

BAR adaptor protein Hob3

Hrd1 ubiquitin ligase complex subunit (predicted) $\begin{array}{llll} & \end{array}$ DNA replication factor A subunit Ssb3 $\quad-0.15469963 .97747905 \quad 0.65102389$ mitochondrial ribosomal protein subunit Mrp17 (predicted) $-\begin{array}{llll}0.1547475 & 7.23381209 & 0.14525403\end{array}$ $\begin{array}{lllll}\text { transmembrane transporter (predicted) } & -0.1548409 & 7.69772004 & 0.1339322\end{array}$ plasma membrane potassium ion transmembrane transpor $\begin{array}{llll}-0.1549033 & 7.7098894 & 0.0846669\end{array}$ tricalbin, C2 domain protein (phospholipid binding) ER-plası -0.15494269 .474199350 .02093122$ $\begin{array}{lllll}\text { F1-FO ATP synthase subunit D (predicted) } & -0.1550497 & 7.44136235 & 0.12883174\end{array}$ \#N/A $\quad-0.15510496 .44167252 \quad 0.35148434$ $\# N / A$ $\begin{array}{lll}-0.1554178 & 5.04598528 & 0.55463021\end{array}$ $\begin{array}{lll}-0.1555553 & 7.71637307 & 0.10104138\end{array}$

AGC family protein kinase Gad8 $\begin{array}{llll}\text { mitochondrial inner membrane peptidase complex catalytic } & -0.1557302 & 6.37571478 & 0.40966298 \\ \text { WD repeat protein Swd3 } & -0.1557833 & 6.41465673 & 0.27655981\end{array}$ $\begin{array}{lllll}\text { dolichol-phosphate mannosyltransferase regulatory subunit } & -0.156189 & 4.9057713 & 0.50937472\end{array}$ Dcp2-Dcp1 mRNA-decapping complex subunit Edc1 $\quad-0.1561965 \quad 8.15486407 \quad 0.09552595$ histone H2A-H2B chaperone, FACT complex subunit Pob3 $\quad-0.1562554 \quad 7.705441140 .15164514$ $\begin{array}{lllll}\text { trans-aconitate 3-methyltransferase (predicted) } & -0.1562865 & 6.79458923 & 0.2000238\end{array}$ $\begin{array}{lll}-0.1571349 & 6.73019881 & 0.19745805\end{array}$ $\begin{array}{lll}-0.1572601 & 6.24830645 & 0.37206909\end{array}$ $\begin{array}{lll}-0.1572741 & 6.73164924 & 0.23067541\end{array}$ $\begin{array}{lll}-0.1573175 & 7.96099543 & 0.0757327\end{array}$ $\begin{array}{lll}-0.1573322 & 6.47957043 & 0.29695503\end{array}$

Swr1 complex subunit Swc5

$$
\# \text { N/A }
$$

SAGA complex subunit Spt3

$$
\# N / A
$$

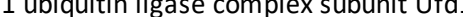

cytoplasmic P body 3'-5'-exoribonuclease, Dis3L2-related ( $\mathrm{p}-0.15734246 .87548812$ Schizosaccharomyces specific protein

human GRASP protein family Golgi protein (predicted) \#N/A

long-chain-fatty-acid-CoA ligase Lcf1

$\begin{array}{lll}-0.1574399 & 5.79573694 & 0.36785022\end{array}$ $\begin{array}{lll}-0.1576479 & 7.40520458 & 0.22054867\end{array}$ $\begin{array}{lll}-0.1580414 & 4.37701449 & 0.57520725\end{array}$ $\begin{array}{lll}-0.1584041 & 9.44468839 & 0.03950574\end{array}$ $\begin{array}{lll}-0.1586613 & 5.69784128 & 0.3698299\end{array}$ Srp1 family splicing factor (predicted) ribosome biogenesis protein, G-patch domain, PINX1 famil! $\begin{array}{llll}0.1586642 & 6.27308523 & 0.26393942\end{array}$ SR protein-specific kinase Dsk1

$\begin{array}{lll}-0.1589324 & 7.54005962 & 0.10445987\end{array}$ $\begin{array}{lll}-0.1590074 & 8.079834 & 0.07762128\end{array}$ $\begin{array}{lll}-0.159078 & 6.95627982 & 0.16821453\end{array}$ $\begin{array}{lll}-0.159274 & 7.11067425 & 0.20076188\end{array}$ $\begin{array}{lll}-0.1600106 & 7.04024686 & 0.19195535\end{array}$ $\begin{array}{lll}-0.1601734 & 6.6105155 & 0.18335811\end{array}$ $-0.1601866 \quad 4.15227727 \quad 0.62307189$ $\begin{array}{lll}-0.1602474 & 5.78747308 & 0.4082742\end{array}$ $\begin{array}{crrr}\text { \#N/A } & -0.1602481 & 6.29962579 & 0.33328277 \\ \text { phosphatidyl-N-dimethylethanolamine N-methyltransferas } & -0.160416 & 6.26315172 & 0.29106548\end{array}$ mannan endo-1,6-alpha-mannosidase (predicted) $\quad-0.16044157 .492349550 .08714654$ $\begin{array}{lllll}\text { secretory pathway protein Sec39 (predicted) } & -0.1606334 & 6.27314042 & 0.25925932\end{array}$ ATP-dependent RNA helicase Dbp8 (predicted)
0.509134803

0.449419173

0.504255969

0.493986351

0.095290187

0.159229072

0.417284019

0.729494085

0.215115056

0.201327287

0.135886387

0.04075449

0.194605996

0.446824379

0.644401324

0.158357112

0.504936896

0.368656128

0.601369707

0.150964726

0.223219801

0.280594095

0.277854018

0.467318228

0.317184519

0.123469339

0.38999401

0.264465872

0.463216269

0.305357825

0.663030585

0.07080236

0.465278698

0.355064163

0.162405615

0.125974909

0.243449563

0.281455416

0.271401649

0.26147358

0.70513957

0.50348167

0.427599787

0.383707985

0.139569829

0.349782135

0.286839717 
SPBC543.03C

SPCC584.02

SPNCRNA.964

SPAC959.09c

SPAC144.09c

SPAC17A2.13C

SPNCRNA.1251

SPAC25B8.20

SPAC521.04C

SPCC188.12

SPNCRNA.792

SPBC16A3.03C

SPAC17G8.14C

SPBC13G1.03C

SPAC17G8.01C

SPAC167.03c

SPAC6G10.05c

SPNCRNA.1477

SPBC11C11.10

SPCC1223.12C

SPBC14C8.10

SPAC23C11.12

SPAC17G6.09

SPAC32A11.01

SPBC26H8.14C

SPAC977.02

SPAC31A2.07C

SPBC947.10

SPAC4G9.02

SPAC23H4.13C

SPBC21D10.10

SPAC19A8.12

SPAC19G12.02C

SPBC25H2.03

SPCC663.01C

SPAC343.15

SPAC1093.07

SPNCRNA.503

SPAC3F10.12C

SPCC4F11.03C

SPAC144.17c

SPNCRNA.1395

SPBP8B7.07C

SPAC22A12.08C

SPCC1235.09

SPCC550.01c

SPAC1952.04C
Ku domain protein Pku80

middle-meiotic transcription factor Cuf2

$\# N / A$

$-0.1614147 .027516250 .19093469$

$\begin{array}{llll}0.1614156 & 4.02435164 & 0.64999765\end{array}$

$\begin{array}{llll}-0.1615376 & 6.39212562 & 0.25823643\end{array}$

anaphase-promoting complex, platform subcomplex scaffol $\begin{array}{lllll}-0.1616909 & 6.01895582 & 0.37297659\end{array}$

transcription factor TFIIIA

14-3-3 protein $\operatorname{Rad} 25$

$\# \mathrm{~N} / \mathrm{A}$

$\begin{array}{llll}-0.1621431 & 6.71726545 & 0.18631\end{array}$

$\begin{array}{lll}-0.1622637 & 8.42643176 & 0.05105653\end{array}$

$\begin{array}{lll}-0.1622853 & 2.0988334 & 0.86363876\end{array}$

$\begin{array}{llll}\text { Schizosaccharomyces specific protein } & -0.1626268 & 5.61622562 & 0.4058632 \\ \text { ER membrane calcium trnamembrane transporter (predict } \epsilon & -0.1627198 & 7.58562263 & 0.10094453\end{array}$

meiotic (sporulation) septin Spn6

\#N/A$$
\# \mathrm{~N} / \mathrm{A}
$$

ppr7

pck1

pex14

trl1

snu66

$\operatorname{trs} 120$

pus4

meu10

mrpl24

$\sec 62$

mug8

$\operatorname{cox} 17$

$\mathrm{ftm} 2$

dbp10

dsc1

rnh201

SPAC23H4.13c

bdc1

dcp2

pms1

vac14

ekc1

tit1

$\# \mathrm{~N} / \mathrm{A}$

$$
\# \mathrm{~N} / \mathrm{A}
$$

SPAC3F10.12C

SPCC4F11.03C

SPAC144.17C

$\# \mathrm{~N} / \mathrm{A}$

set6

crd1

hif2

coa4

SPAC1952.04c

mitochondrial PPR repeat protein Ppr7

protein kinase C (PKC)-like Pck1

peroxisomal docking protein Pex14 (predicted)

tRNA ligase Trl1 (predicted)

U4/U6 x U5 tri-snRNP complex subunit Snu66

TRAPP complex subunit Trs120

$\begin{array}{lll}-0.162847 & 6.25082138 & 0.30657018\end{array}$

$\begin{array}{lll}-0.1629721 & 3.42071432 & 0.64104819\end{array}$

$\begin{array}{lll}-0.1634044 & 6.96286101 & 0.16750494\end{array}$

$\begin{array}{lll}-0.1637774 & 7.89920023 & 0.06431775\end{array}$

$\begin{array}{lll}-0.1638195 & 5.96006342 & 0.28933502\end{array}$

$\begin{array}{lll}-0.1639535 & 7.23800353 & 0.12530861\end{array}$

$\begin{array}{lll}-0.1647583 & 7.93649406 & 0.0591306\end{array}$

$\begin{array}{llll}-0.1647783 & 8.40261351 & 0.05791852\end{array}$

$-0.1654177 \quad 3.1351163 \quad 0.75582191$

$\begin{array}{lll}-0.1656215 & 6.93456649 & 0.19086983\end{array}$

tRNA pseudouridine synthase Pus4 (predicted)

$\begin{array}{llll}-0.1658658 & 6.14667747 & 0.25518992\end{array}$ mitochondrial ribosomal protein subunit L28 (predicted) $\quad \begin{array}{lllll}0.1659989 & 7.3599846 & 0.10004741\end{array}$ anaphase-promoting complex subunit TPR lobe accessory fi $-0.1661052 \quad 4.11794429 \quad 0.59123846$

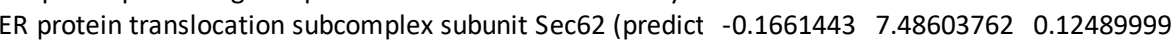
$\begin{array}{llllll}\text { DUF1708 family conserved fungal protein, cell division site } & -0.1662888 & 7.3978198 & 0.13425391\end{array}$ mitochondrial copper chaperone for cytochrome c oxidase C $\begin{array}{ccc}-0.1666414 & 5.61410909 & 0.33848808\end{array}$ sub-telomeric 5Tm protein family Ftm2

ATP-dependent RNA helicase Dbp10 (predicted)

Golgi Dsc E3 ligase complex subunit Dsc1

ribonuclease $\mathrm{H} 2$ complex subunit Rnh201

Schizosaccharomyces specific protein

bromodomain protein Bdc1

mRNA decapping complex catalytic subunit Dcp2

MutL family mismatch-repair protein Pms1

PAS complex subunit, involved in phosphoinositide metabol $-0.1687553 \quad 6.732524690 .16864202$

protein phosphatase regulatory subunit Ekc1

tRNA isopentenyltransferase Tit1

$\# N / A$

transcription factor (predicted)

Schizosaccharomyces specific protein

6-phosphofructo-2-kinase (predicted)

$$
\# \mathrm{~N} / \mathrm{A}
$$

$\begin{array}{lll}0.1667681 & 7.56793868 & 0.0866206\end{array}$

$\begin{array}{lll}-0.1673841 & 7.33746125 & 0.1422558\end{array}$

$\begin{array}{llll}-0.1674782 & 7.3853091 & 0.10569696\end{array}$

$\begin{array}{llll}-0.1675302 & 5.8693036 & 0.33882961\end{array}$

$\begin{array}{lll}-0.1677196 & 6.44508644 & 0.40224534\end{array}$

$\begin{array}{llll}-0.1678808 & 6.45441387 & 0.30024358\end{array}$

$\begin{array}{llll}-0.1680012 & 7.43565205 & 0.13737582\end{array}$

$\begin{array}{llll}0.1687924 & 9.786591 & 0.01025524\end{array}$

$\begin{array}{llll}-0.1688242 & 6.34292209 & 0.23723123\end{array}$

$\begin{array}{lll}-0.1689359 & 6.56626938 & 0.33377384\end{array}$

$\begin{array}{llll}-0.1690527 & 3.50463793 & 0.68147265\end{array}$

$\begin{array}{llll}-0.1692046 & 5.78739322 & 0.38224647\end{array}$

$\begin{array}{lll}-0.1693772 & 7.96950001 & 0.04416952\end{array}$

$\begin{array}{llll}-0.1696831 & 7.75736792 & 0.14618087\end{array}$

$\begin{array}{llll}-0.1702265 & 3.85270814 & 0.74870332\end{array}$

$\begin{array}{lll}-0.170296 & 6.47664767 & 0.1798178\end{array}$

histone lysine methyltransferase Set6 (predicted)

cardiolipin synthase/ hydrolase fusion protein Crd1 (predict $\quad-0.1704105 \quad 7.99701383 \quad 0.11164674$

Set3 complex subunit Hif2

cytochrome c oxidase assembly protein Coa4 (predicted)

$\begin{array}{llll}-0.1705208 & 7.26295118 & 0.14400388\end{array}$

$\begin{array}{lll}-0.1708735 & 4.87476651 & 0.52381003\end{array}$

$\begin{array}{lll}-0.1712366 & 6.23940316 & 0.2786021\end{array}$
0.270126934

0.728584088

0.348739608

0.468198806

0.264465872

0.088227453

0.906049152

0.501070221

0.158241771

0.400180972

0.720481369

0.242577383

0.107380337

0.381983027

0.190170624

0.099871868

0.098239167

0.818786624

0.270091329

0.345388712

0.157091239

0.676260978

0.189641218

0.201632721

0.432972061

0.138792837

0.211503322

0.164066559

0.433246046

0.497729105

0.39322179

0.205524499

0.075782028

0.243964488

0.021565793

0.325147733

0.427987749

0.756759367

0.477632617

0.077747957

0.216258062

0.813236409

0.257233575

0.172085178

0.213682161

0.615316281

0.370724927 
SPAPB1A10.04C SPBC29A3.17 SPAC167.02

SPNCRNA.918

SPBC15C4.01c

SPCC830.05C

SPNCRNA.1007

SPCC16C4.12

SPAC32A11.03C

SPNCRNA.1043

SPBC29A3.08

SPAC1B9.02C

SPAP8A3.10

SPAC212.01C

SPBC211.03C

SPBC1921.04C

SPAPB17E12.10C

SPBC1778.04

SPBC14F5.12C

SPBC1734.08

SPBC11B10.09

SPAC16E8.09

SPCC736.09C

SPAC22F3.12C

SPBC409.11

SPBC28E12.02

SPAC8C9.16C

SPAC3H5.11

SPAC1071.01c

SPNCRNA.1651

SPCC11E10.09C

SPAC30D11.10

SPAC977.17

SPBC1289.01C

SPBC2G5.04C

SPNCRNA.1529

SPRRNA.49

SPCC188.14

SPAC589.04

SPAPB17E12.04C

SPAC56E4.07

SPBC14C8.15

SPAC23H3.10

SPAC1296.01c

SPBC30B4.07C

SPNCRNA.1669

SPBC4.05 sck1

ups1

SPAC212.01c

gea1

\section{\#N/A}

SPAPB17E12.10C

spo6

cbh2

hse1

cdc2

scd1

$\mathrm{fx} 1$

rgs1

meu18

SPBC28E12.02

mug63

SPAC3H5.11

pta1

$\#$ N/A

SPCC11E10.09C

rad52

SPAC977.17

chr4

erv41

\#N/A

SPRRNA.49

SPCC188.14

$\mathrm{mtx} 1$

$\operatorname{csn} 2$

SPAC56E4.07

SPBC14C8.15

ssr2

SPAC1296.01C

tfb4

mlo2

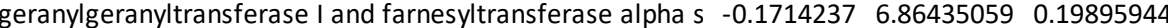
Cdc42/Rho3/Rho4 RhoGEF Gef3

geranylgeranyltransferase II beta subunit Ptb1

$\# \mathrm{~N} / \mathrm{A}$ $\begin{array}{lll}-0.172042 & 5.85854288 \quad 0.39045493\end{array}$ $\begin{array}{lll}-0.1721107 & 8.1913231 & 0.04115679\end{array}$ $-0.1721536 \quad 5.37477713 \quad 0.53237326$

TPR repeat protein Oca3/ER membrane protein complex E NuA4 histone acetyltransferase complex Epl1

$0.1726762 \quad 7.06737378 \quad 0.09711275$

$\begin{array}{lll}-0.1726784 & 5.20050719 & 0.36852178\end{array}$ $\#$ N/A $\begin{array}{lrrrr}\text { NatB N-acetyltransferase complex catalytic subunit Naa20 } & -0.1727881 & 6.24026818 & 0.26445958 \\ \text { stationary phase-specific homeobox transcription factor Ph) } & -0.172989 & 8.24174482 & 0.03067582\end{array}$ $\# \mathrm{~N} / \mathrm{A}$

elongin-A, F-box protein Pof4 (predicted)

$\begin{array}{lll}-0.1731202 & 6.32370206 & 0.22116625\end{array}$

$\begin{array}{llll}-0.173401 & 4.22141144 & 0.54934041\end{array}$

$\begin{array}{llll}-0.1736224 & 8.31532394 & 0.05337145\end{array}$

serine/threonine protein kinase Sck1

$\begin{array}{lll}-0.1737276 & 5.47158407 & 0.37501533\end{array}$

$\begin{array}{lll}-0.1739932 & 5.98452979 & 0.3782307\end{array}$

$\begin{array}{lll}-0.1743089 & 8.48651567 & 0.03814037\end{array}$

$\begin{array}{lll}-0.1743938 & 5.76213704 & 0.28838271\end{array}$

specific DUF999 family protein 2

$\# \mathrm{~N} / \mathrm{A}$

$\begin{array}{llrrr}\text { mitochondrial 2' O-ribose methyltransferase Mrm2-like (pr } & -0.1744074 & 4.70907558 & 0.49756842 \\ \text { Spo4-Spo6 kinase complex regulatory subunit Spo6 } & -0.1745378 & 6.5758446 & 0.24425003\end{array}$

$\begin{array}{llll}-0.1745707 & 6.9882794 & 0.18396383\end{array}$

$\begin{array}{lll}-0.1747246 & 6.47237792 & 0.1649974\end{array}$

$\begin{array}{llll}-0.1747401 & 7.07318444 & 0.10788952\end{array}$

$\begin{array}{llll}-0.1749467 & 7.58178745 & 0.073523\end{array}$

$-0.1751825 \quad 5.42347876 \quad 0.3878681$

$\begin{array}{lll}-0.1752371 & 7.30456049 & 0.13332832\end{array}$

$\begin{array}{llll}-0.1755443 & 6.23926953 & 0.32907307\end{array}$

$\begin{array}{lll}-0.1755678 & 6.98445287 & 0.12622303\end{array}$

Schizosaccharomyces specific protein Meu18

RNA-binding protein

TLDc domain protein 1, implicated in response to oxidative $\quad-0.17580525 .451382790 .34757305$

NAD/NADH kinase (predicted)

$\begin{array}{lll}-0.1758129 & 6.96077063 & 0.15445615\end{array}$

mRNA cleavage and polyadenylation specificity factor comr $-\begin{array}{llll}0.1760199 & 7.87799002 & 0.04831309\end{array}$ \#N/A

$\begin{array}{lll}-0.1764364 & 5.7147031 & 0.30875076\end{array}$ $-0.1765097 \quad 7.50974501 \quad 0.10071768$

alpha-amylase homolog (predicted)

DNA recombination protein, Rad51 mediator Rad52 (previo -0.1765616 7.437878090 .06767023 MIP water channel (predicted)

1,3-beta-glucan synthase regulatory factor $\mathrm{Chf} 3 / \mathrm{Chr} 4$

COPII-coated vesicle component Erv41 (predicted)

$\begin{array}{lll}-0.1767578 & 7.49032572 & 0.1590474\end{array}$

$\begin{array}{llll}-0.1769758 & 7.45090199 & 0.06460577\end{array}$

$\begin{array}{llll}-0.177366 & 6.52909566 & 0.17873982\end{array}$

$\begin{array}{llll}-0.177379 & 7.36609423 & 0.10169452\end{array}$

$\begin{array}{llll}-0.1775939 & 12.0904106 & 0.03499164\end{array}$

$\begin{array}{llll}-0.1778333 & 6.40248324 & 0.22050018\end{array}$

$\begin{array}{llll}-0.1778722 & 5.75829835 & 0.29176794\end{array}$

$\begin{array}{llll}-0.1785463 & 6.6342928 & 0.17000754\end{array}$

$\begin{array}{llll}-0.1787106 & 8.20853794 & 0.04747327\end{array}$

$\begin{array}{llll}-0.178723 & 7.96664076 & 0.06454139\end{array}$

$\begin{array}{llll}-0.1787651 & 6.60893963 & 0.15425838\end{array}$

$\begin{array}{llll}-0.1789666 & 8.12987798 & 0.04122042\end{array}$

$\begin{array}{llll}0.1791597 & 5.67739442 & 0.38646487\end{array}$

$\begin{array}{llll}-0.1792382 & 4.79677934 & 0.51019057\end{array}$

$$
\text { \#N/A }
$$

$\begin{array}{llll}-0.179277 & 6.49043838 & 0.21142985\end{array}$
0.279677541

0.485837597

0.073278903

0.623889503

0.256053021

0.152904275

0.463718697

0.355450812

0.056817887

0.305839532

0.639346929

0.091576586

0.470497666

0.474007344

0.068644604

0.380996371

0.591226315

0.333491457

0.262172542

0.239455601

0.167081954

0.120385947

0.48323735

0.200552429

0.423716598

0.191265755

0.442596295

0.22652459

0.08404155

0.402191595

0.157995342

0.112042802

0.232124149

0.107790937

0.25601441

0.159229072

0.063707261

0.305352807

0.38441061

0.245210108

0.082728714

0.107727282

0.226283317

0.073353813

0.48219535

0.602228416

0.294711214 
SPRRNA.47

SPRRNA.48

$$
\text { \#N/A }
$$

SPCC297.06C

med6

SPCC297.06C

SPAC $1002.15 \mathrm{C}$

SPNCRNA.1512

SPBC32H8.13C

SPNCRNA.1656

SPAC11D3.10

SPNCRNA.1485

SPNCRNA.1271

SPCC1020.06c

SPBC31E1.06

SPAC3F10.17

SPBC1711.17

SPNCRNA.1265

SPCC553.09c

SPAC4A8.12C

SPAC4F10.09c

SPNCRNA.661

SPBC336.12C

SPBC8D2.09c

SPAC23C4.06C

SPAC15A10.17

SPAC222.13C

SPAC23C11.16

SPNCRNA.1301

SPRRNA.43

SPRRNA.44

SPCC16C4.09

SPAC12B10.12C

SPNCRNA.1032

SPBC18E5.11C

SPBC1105.10

SPBC13A2.03

SPAC8C9.17C

SPBC2F12.09C

SPAC23C4.04C

SPAC23C4.11

SPNCRNA.1378

SPAC664.12C

SPNCRNA.1554

SPAC23A1.02C

SPNCRNA.1147

SPBC3E7.11C

SPNCRNA.744

SPCC1739.06C

mok12

$\# \mathrm{~N} / \mathrm{A}$

SPAC11D3.10

$\# N / A$

\#N/A

tal1

bms1

Itv1

prp16

$\# \mathrm{~N} / \mathrm{A}$

spb70

sds22

noc1

cdc10

$\mathrm{msl} 1$

efm 6

coa2

SPAC222.13c

plo1

SPRRNA.44

sts5

rhp41

$$
\# \mathrm{~N} / \mathrm{A}
$$

edc3

rav1

SPBC13A2.03

spc34

atf21

atp18

\#N/A

SPAC664.12C

$\#$ N/A

ted

\#N/A

SPBC3E7.11C

\#N/A

met1
$28 \mathrm{~S}$ ribosomal RNA

$28 \mathrm{~S}$ ribosomal RNA

$\#$ N/A

conserved fungal protein

mediator complex subunit Med6

\#N/A

SPAC23C4.04c

$$
\# \mathrm{~N} / \mathrm{A}
$$

$\# \mathrm{~N} / \mathrm{A}$

$\# N / A$

transaldolase (predicted)

$$
\text { \#N/A }
$$

DNA polymerase alpha B-subunit

$$
\# N / A
$$

Polo kinase Plo1

\#N/A

$18 \mathrm{~S}$ ribosomal RNA

$18 \mathrm{~S}$ ribosomal RNA DNA repair protein $\mathrm{Rhp41}$

$$
\text { \#N/A }
$$

RAVE complex subunit Rav1

DASH complex subunit Spc34

$$
\# \text { N/A }
$$
\#N/A $\# \mathrm{~N} / \mathrm{A}$ nifs homolog, possible cysteine desulfurase

GTP binding protein Bms1 (predicted)

ribosome biogenesis protein Ltv1 (predicted)

ATP-dependent RNA helicase Prp16

protein phosphatase regulatory subunit Sds 22

ribosome biogenesis protein Noc1 (predicted)

MBF transcription factor complex subunit Cdc10

U2 snRNP-associated protein Msl1 (predicted)

elongation factor EF-1 alpha (eEF1A) lysine 390 methylase cytochrome c oxidase assembly protein Coa2 (predicted)

6-phosphofructo-2-kinase (predicted)

cytoplasmic P body 3'-5'-exoribonuclease, Dis3L2-related ( $p$

enhancer of mRNA decapping Edc3

ER phosphatidate cytidylyltransferase

transcription factor, Atf-CREB family Atf21

Schizosaccharomyces pombe specific protein

F1-FO ATP synthase subunit J (predicted)

$\begin{array}{lll}0.1793798 & 12.0923359 & 0.03218819\end{array}$

$\begin{array}{llll}-0.1798403 & 12.0928046 & 0.03164366\end{array}$

$\begin{array}{lll}-0.1798545 & 6.6527305 & 0.1606343\end{array}$

$\begin{array}{lll}-0.1799841 & 5.36660455 & 0.39350171\end{array}$

$\begin{array}{lll}-0.18111 & 6.90568658 & 0.11108626\end{array}$

$\begin{array}{lll}-0.1812273 & 6.18121142 & 0.1922647\end{array}$

$\begin{array}{llll}-0.1814724 & 8.19927392 & 0.05166921\end{array}$

$\begin{array}{lll}-0.181677 & 6.86984448 & 0.12213358\end{array}$

$\begin{array}{llll}-0.1817853 & 7.13402999 & 0.08285999\end{array}$

$\begin{array}{llll}-0.1820517 & 3.43365174 & 0.67461023\end{array}$

$\begin{array}{llll}-0.1823082 & 6.19140246 & 0.22111492\end{array}$

$\begin{array}{llll}-0.1824518 & 9.06824187 & 0.02180148\end{array}$

$\begin{array}{llll}-0.1829083 & 8.44904305 & 0.01936349\end{array}$

$\begin{array}{llll}-0.1832379 & 6.68298318 & 0.16748059\end{array}$

$\begin{array}{llll}-0.183247 & 7.29152826 & 0.10277111\end{array}$

$\begin{array}{lll}-0.1835393 & 5.23992772 & 0.390262\end{array}$

$\begin{array}{lll}-0.1836268 & 6.79729742 & 0.18866065\end{array}$

$\begin{array}{llll}-0.1842654 & 6.26327918 & 0.24756547\end{array}$

$\begin{array}{llll}-0.1843001 & 7.67612846 & 0.07736491\end{array}$

$\begin{array}{lll}-0.1843287 & 5.63508395 & 0.36397878\end{array}$

$\begin{array}{lll}-0.1843912 & 7.49509797 & 0.0776067\end{array}$

$\begin{array}{lll}-0.1848755 & 4.76633748 & 0.42319981\end{array}$

$\begin{array}{lll}-0.1850246 & 6.35372571 & 0.27144479\end{array}$

$\begin{array}{llll}-0.1850949 & 4.46254676 & 0.52540589\end{array}$

$\begin{array}{llll}-0.1857145 & 6.72156616 & 0.12125407\end{array}$

$\begin{array}{lll}-0.1858043 & 7.30091152 & 0.07009769\end{array}$

$\begin{array}{llll}-0.18587 & 4.84774847 & 0.50620008\end{array}$

$\begin{array}{lll}-0.1859639 & 10.958911 & 0.02688751\end{array}$

$-0.1859902 \quad 10.9596904 \quad 0.02681588$

$\begin{array}{lll}-0.186102 & 9.50814911 & 0.01492105\end{array}$

$\begin{array}{lll}-0.1864676 & 6.65423516 & 0.14890074\end{array}$

$\begin{array}{lll}-0.1865796 & 5.29790217 & 0.32784411\end{array}$

$\begin{array}{lll}0.1869994 & 8.28995919 & 0.02880082\end{array}$

$\begin{array}{lll}-0.1870187 & 7.88374591 & 0.0289896\end{array}$

$\begin{array}{lll}-0.1870342 & 6.82069106 & 0.2306128\end{array}$

$\begin{array}{lll}-0.1870348 & 6.07953983 & 0.28588284\end{array}$

$\begin{array}{lll}-0.187088 & 5.94531356 & 0.22604969\end{array}$

$\begin{array}{lll}-0.1872186 & 4.80171893 \quad 0.43807229\end{array}$

$\begin{array}{lll}-0.1874083 & 5.75779157 & 0.27889795\end{array}$

$\begin{array}{lll}-0.1875771 & 7.04370275 & 0.08918821\end{array}$

mitochondrial succinate dehydrogenase assembly factor $1\left(\begin{array}{llll}-0.1876588 & 6.18222294 & 0.26131838\end{array}\right.$

$\begin{array}{llll}-0.1877757 & 5.02127665 & 0.38411335\end{array}$

GPI-remodelling mannose-ethanolamine phosphate phospr $-\begin{array}{lllll}0.1878442 & 6.45539202 & 0.16452324\end{array}$

$\begin{array}{llll}-0.1878978 & 4.53098384 & 0.51967418\end{array}$

$\begin{array}{llll}0.1879098 & 6.63345332 & 0.14393743\end{array}$

$\begin{array}{llll}-0.1883201 & 5.50611101 & 0.28699514\end{array}$

$\begin{array}{llll}-0.1892019 & 6.67924299 & 0.18405558\end{array}$
0.059152207

0.058372223

0.234122173

0.488888884

0.171493192

0.271782568

0.089127753

0.186057528

0.133616647

0.749995521

0.305830693

0.042243475

0.038149555

0.242577383

0.16040308

0.485775178

0.267410059

0.337206365

0.125648744

0.459433477

0.125974909

0.518242697

0.363046735

0.616668232

0.185049098

0.115499938

0.599438613

0.050670029

0.050577062

0.030210569

0.219845857

0.422613976

0.053784196

0.054062538

0.317184519

0.378931195

0.311705807

0.533687408

0.37104605

0.142559272

0.351923919

0.479789166

0.238920322

0.610985279

0.213636183

0.37988755

0.262248365 
SPRRNA.46

SPAC1071.04c

SPAC3G9.05

SPCC4G3.13C

SPBC216.07c

SPNCRNA.1102

SPBC577.11

SPBC18H10.07

SPBC1685.16

SPBC31E1.05

SPCC1259.14C

SPAC57A7.15C

SPAC22G7.10

SPBC56F2.01

SPBC27B12.09c

SPRRNA.42

SPAC227.10

SPAPB17E12.07

SPCC576.05

SPNCRNA.711

SPAC23H4.01C

SPBC651.12C

SPBC18E5.10

SPAC1F3.10C

SPRRNA.45

SPNCRNA.809

SPNCRNA.942

SPAC1565.05

SPBC $32 \mathrm{H} 8.07$

SPAC3H1.11

SPNCRNA.1343

SPBC19C7.08C

SPCC330.02

SPCC1840.06

SPBC32F12.04

SPNCRNA.1480

SPNCRNA.1457

SPAC13G6.01C

SPAC644.14c

SPAC1296.03C

SPBC19F8.01C

SPBC146.06C

SPCC1795.01c

SPCC1840.07c

SPAC823.09C

SPAC13C5.02

SPBC337.08C
SPRRNA.46

spc2

spa2

cue1

\#N/A

SPBC577.11

wbp4

vma9

gle1

meu27

SPAC57A7.15C

iss 1

pof 12

SPRRNA.42

gim4

sen2

sac3

osh3

$\mathrm{dbl7}$

SPBC18E5.10

SPRRNA.45 $\# N / A$

$$
\# N / A
$$

utp8

git5

hsr1

$$
\text { \#N/A }
$$

ppm2

rhp7

atp5

gtb1

$\# N / A$

$\# N / A$

rad8

rad51

sxa2

spn7

fan1

$\operatorname{mad} 3$

SPCC1840.07c

SPAC823.09c

dre4

ubi4
$18 \mathrm{~S}$ ribosomal RNA

signal peptidase subunit Spc2 (predicted)

ARF GTPase-activating protein Spa2

$\begin{array}{llll}0.1899484 & 4.44978034 & 0.52589849\end{array}$

$\begin{array}{lll}-0.1899812 & 6.2916719 & 0.27420642\end{array}$

Hrd1 ubiquitin ligase complex, CUE domain protein Cue1 ( $\mathrm{p}$ - $-0.1901958 \quad 6.601884630 .16262214$

$\begin{array}{lll}-0.1904045 & 9.16804019 & 0.01587561\end{array}$

$\begin{array}{lll}-0.1907007 & 4.27805254 & 0.51442409\end{array}$

$$
\# \mathrm{~N} / \mathrm{A}
$$

$\begin{array}{lll}-0.190919 & 7.02659054 & 0.08962517\end{array}$

$\begin{array}{lllll} & 0.0648611\end{array}$

$\begin{array}{llll}V \text {-type ATPase V0 subunit e (predicted) } & -0.1910161 & 5.97350188 & 0.37336285\end{array}$

RNA export factor, cytoplasmic nucleoporin Gle1 $\quad-0.1911698 \quad 5.98255054 \quad 0.37151665$

UPF0300 family protein $5 \quad-0.19117296 .45550624 \quad 0.14075063$

Schizosaccharomyces specific protein $\quad \begin{array}{llll}0.1914717 & 3.9564182 & 0.60362209\end{array}$

mRNA cleavage and polyadenylation specificity factor comf $-\begin{array}{llll}0.1916062 & 8.06524805 & 0.03610604\end{array}$

$\begin{array}{llll}-0.1920088 & 6.44393135 & 0.25101676\end{array}$

mitochondrial carrier, FAD FIx1 (predicted) $\quad-0.1921443 \quad 6.30452387 \quad 0.18416733$

$\begin{array}{lllll}18 S & \text { ribosomal RNA } & -0.1922088 & 10.4330709 & 0.02325216\end{array}$

prefoldin subunit 2, Gim4(predicted) $\quad \begin{array}{llll}-0.192825 & 7.37881348 & 0.06732943\end{array}$

tRNA-splicing endonuclease subunit catalytic subunit Sen2 $\quad-0.1930609 \quad 5.78997691 \quad 0.23103335$

TREX2 complex sununit Sac3 (predicted)

$$
\text { \#N/A }
$$

$\begin{array}{lll}-0.193293 & 7.50034094 \quad 0.05590061\end{array}$

$\begin{array}{lll}-0.1934757 & 7.13818526 & 0.05482269\end{array}$

$\begin{array}{llll}-0.1937004 & 8.38115908 & 0.02132297\end{array}$

$\begin{array}{llllll} & 0.37928273\end{array}$ mitochondrial iron-sulfur cluster protein (predicted)

35703 mitochondrial intermediate peptidase Oct1 (predicted)

$18 \mathrm{~S}$ ribosomal RNA

\section{$\# N / A$}

$$
\# N / A
$$

-UTP complex subunit Utp8 (predicted)

heterotrimeric $\mathrm{G}$ protein beta (WD repeat) subunit Git5 transcription factor Hsr1

$$
\text { \#N/A }
$$

RNA methyltransferase Ppm2 (predicted)

Rad7 homolog Rhp7

F1-FO ATP synthase delta subunit (predicted)

gamma-tubulin Gtb1

$$
\# N / A
$$$$
\# N / A
$$

$\begin{array}{llll}-0.1940117 & 8.28358331 & 0.02383713\end{array}$

$\begin{array}{lll}-0.1941342 & 8.36298064 & 0.04712531\end{array}$

$\begin{array}{llll}0.1942645 & 10.0917137 & 0.02146361\end{array}$

$\begin{array}{lll}-0.1943671 & 3.51870587 & 0.6043712\end{array}$

$\begin{array}{lll}-0.1945427 & 5.87109934 & 0.21544578\end{array}$

$\begin{array}{llll}0.1945891 & 7.76028561 & 0.04017859\end{array}$

$\begin{array}{llll}-0.1945983 & 6.15899659 & 0.24744792\end{array}$

$\begin{array}{llll}-0.1947808 & 8.50978065 & 0.02398861\end{array}$

$\begin{array}{lll}-0.1954251 & 4.65280705 & 0.46233142\end{array}$

$\begin{array}{llll}-0.1956577 & 6.70082263 & 0.21090662\end{array}$

$\begin{array}{lll}-0.1957599 & 7.12360075 & 0.07707523\end{array}$

$\begin{array}{llll}-0.1961307 & 6.84901249 & 0.08397167\end{array}$

$\begin{array}{lll}-0.1961399 & 7.32136427 & 0.06212461\end{array}$

$\begin{array}{llll}-0.1961518 & 2.9666094 & 0.73854364\end{array}$

$\begin{array}{lll}-0.1963244 & 6.18715378 & 0.16436529\end{array}$

ubiquitin-protein ligase E3/ ATP-dependent DNA helicase R $-\begin{array}{lllll}-0.1964333 & 8.27071287 & 0.0241363\end{array}$

RecA family recombinase Rad51/Rhp51

serine carboxypeptidase Sxa2

meiotic septin Spn 7

Fanconi-associated nuclease Fan

mitotic spindle checkpoint protein Mad3

vacuolar endopolyphosphatase (predicted)

threonine aspartase, unknown biological role (predicted)

splicing associated factor Dre4

protein modifier, ubiquitin
0.04715216

0.617033528

0.365949142

0.23652273

0.031877695

0.606069245

0.143101661

108154813

0.468597234

0.466796682

0.209723675

0.686897713

0.06545061

0.340749801

0.262352667

0.044710629

0.1115517

0.31761251

0.095290187

0.09376408

0.041422444

0.475477134

0.04565484

0.082290927

0.041659971

0.687635181

0.299511448

0.071781394

0.337127889

0.045880407

0.557251355

0.29404211

0.125267955

0.135057245

0.104255966

0.805027951

0.23884386

0.046123981

0.222799558

0.450015692

0.342341211

0.360205219

0.257352576

0.111483286

0.309733635

0.186519788

0.00616907 
SPCC830.10

SPAC343.11c

SPNCRNA.1584

SPAPB1A11.04

SPBC3F 6.05

SPAC23G3.07C

SPCC965.06

SPBC15D4.05

SPBC365.08C

SPBC354.09c

SPBC31E1.02C

SPBC1683.04

SPAC22F $3.05 \mathrm{C}$

SPBC428.06C

SPAC1782.01

SPBC2G5.03

SPAC17H9.20

SPNCRNA.1275

SPBP35G2.02

SPNCRNA.1510

SPNCRNA.1078

SPAC637.11

SPAC144.10c

SPAC1782.02C

SPBC557.05

SPAC1002.01

SPCC1442.19

SPAC23G3.09

SPBC17D1.02

SPAC19G12.03

SPAC24C9.04

SPAPB1E7.14

SPBC337.04

SPNCRNA.1194

SPCC622.08C

SPNCRNA.11

SPCC736.04c

SPNCRNA.1005

SPAC13G6.09 pet117

ham 1

$\mathrm{msc1}$

mca1

rga1

snf30

osr2

SPBC15D4.05

der1

tre1

pmr1

SPBC1683.04

alp41

$x+2$

ecm 29

ctu1

psc3

txc1

\#N/A

\#N/A

rpm2

gwt1

SPAC1782.02C

SPBC557.05

mrx11

mrp49

taf4

dph2

cda1

SPAC24C9.04

iec5

ppk27

\#N/A

hta1 \#N/A

gma12

$\# N / A$

$\operatorname{trs} 402$
\#N/A

F1-FO ATP synthase subunit $K$ (predicted)

RNA-binding protein $\mathrm{Cwf}_{29}$

catalase

SNF2 family ATP-dependent DNA helicase Rhp26

$\# N / A$

$\# N / A$

cytochrome c oxidase assembly protein Pet117 (predicted) nucleoside triphosphatase Ham1 (predicted)

Swr1 complex subunit Msc1

\#N/A

$\begin{array}{lll}0.1993086 & 5.23127406 & 0.350357\end{array}$

$\begin{array}{llll}-0.1995946 & 6.34460716 & 0.14727426\end{array}$

$\begin{array}{lll}-0.1998606 & 6.5400491 & 0.10414206\end{array}$

$\begin{array}{llll}-0.1999307 & 8.57426928 & 0.08119349\end{array}$

$\begin{array}{llll}-0.2002437 & 7.24649014 & 0.11313293\end{array}$

$\begin{array}{lll}-0.2003368 & 6.05636069 & 0.21906001\end{array}$

$\begin{array}{llll}-0.2005766 & 5.86633457 & 0.22990154\end{array}$

$\begin{array}{lll}0.2007604 & 4.93812295 & 0.38732041\end{array}$

$\begin{array}{llll}-0.2007993 & 6.61014721 & 0.14224528\end{array}$

$\begin{array}{lll}-0.200844 & 8.43990871 & 0.01904408\end{array}$

$\begin{array}{lll}-0.2012043 & 4.66643783 & 0.41920155\end{array}$

transcription factor, zf-fungal binuclear cluster type Mca1 $\quad-0.2012118 \quad 7.52618525 \quad 0.02870564$

RhoGAP, GTPase activating protein Rga1 $\quad-0.2012238 \quad 8.52013862 \quad 0.00768526$

SWI/SNF complex subunit Snf30 $\quad-0.2014028 \quad 6.86390262 \quad 0.14223965$

potassium channel, beta subunit, aldo-keto reductase (pred -0.2016096 8.000083540 .05442648

conserved CobW/HypB/UreG nucleotide binding domain prc $-\begin{array}{lllll}0.2016222 & 6.45886403 & 0.14953302\end{array}$

Hrd1 ubiquitin ligase complex (derlin) Der1/Dfm1 (predicte $\quad \begin{array}{lllll}0.201867 & 5.78885693 & 0.35042732\end{array}$

Tre1 family protein, involved in vacuolar protein degradatio $\quad-0.201995 \quad 7.7658142 \quad 0.04326039$

plasma membrane P-type ATPase, calcium transporting Pm $-\begin{array}{llll}0.2020922 & 9.24559971 & 0.00287871\end{array}$

glycosyl hydrolase family 3 (predicted) $\quad \begin{array}{lllll}-0.2024553 & 7.36445907 & 0.05587472\end{array}$

GTP-binding protein involved in beta-tubulin folding Alp41 $\quad-0.20248427 .61898871 \quad 0.03450532$

$\begin{array}{lllll}\text { histone deacetylase complex subunit Rxt2 } & -0.2028537 & 5.98213891 & 0.2989451\end{array}$

cytosolic thiouridylase subunit Ctu1

$\begin{array}{lll}-0.2029141 & 8.83751755 & 0.00587051\end{array}$

$\begin{array}{lll}-0.203087 & 5.64053512 & 0.24571768\end{array}$

$\begin{array}{lll}-0.203128 & 7.23847365 & 0.04437449\end{array}$

$\begin{array}{lll}-0.2036006 & 5.72549677 & 0.28219228\end{array}$

$\begin{array}{lll}-0.2037037 & 7.11823591 & 0.06500313\end{array}$

$\begin{array}{llll}-0.2038384 & 3.92693426 & 0.48989677\end{array}$

$\begin{array}{llll}-0.2038985 & 6.33147238 & 0.13263462\end{array}$

$\begin{array}{lll}-0.2039904 & 6.36310004 & 0.1279667\end{array}$

$\begin{array}{llll}-0.2042948 & 7.79726236 & 0.03195303\end{array}$

$\begin{array}{llll}-0.2043827 & 6.01571117 & 0.25987648\end{array}$

$\begin{array}{lll}-0.2048756 & 7.3447849 & 0.09265169\end{array}$

pig-W

conserved fungal protein

arrestin, implicated in vesicle-mediated transport

$-0.20551523 .98795761 \quad 0.54682743$

mitochondrial ribosomal protein Mrp49 (predicted)

$\begin{array}{llll}0.2059017 & 5.03566306 & 0.34182958\end{array}$

transcription factor TFIID complex subunit Taf4 (predicted) $\quad \begin{array}{lllll}-0.2059904 & 7.48219432 & 0.05421678\end{array}$

$\begin{array}{llllll}\text { diphthamide biosynthesis protein Dph2 (predicted) } & -0.2061097 & 6.46974757 & 0.13575997\end{array}$

chitin deacetylase Cda1

Schizosaccharomyces specific protein

Ino80 complex subunit lec5

$\begin{array}{llll}0.7133411 & 0.02741557\end{array}$

$\begin{array}{llll}-0.2062842 & 6.51865389 & 0.13100936\end{array}$

$\begin{array}{llll}-0.2066426 & 6.77055066 & 0.15423543\end{array}$

calcium/calmodulin-dependent protein kinase Ppk27 (predii $-0.20690995 .33959277 \quad 0.2645261$

$\begin{array}{lll}-0.2071304 & 8.25979049 & 0.03538816\end{array}$

$\begin{array}{llll}-0.2071513 & 8.24651558 & 0.03727142\end{array}$

$\begin{array}{lll}-0.2075766 & 2.53201523 & 0.76062722\end{array}$

$\begin{array}{lll}-0.2076437 & 7.77191569 & 0.02271865\end{array}$

$\begin{array}{lll}0.2079891 & 7.27525679 & 0.04239723\end{array}$

alpha-1,2-galactosyltransferase Gma12

$\# \mathrm{~N} / \mathrm{A}$

$\begin{array}{llll}0.2085141 & 6.24759784 & 0.13909208\end{array}$
0.44564101

0.217680572

0.16209668

0.131084619

0.173981822

0.303729293

0.316440122

0.48273175

0.211503322

0.037618266

0.514179256

0.053621161

0.016634278

0.211503322

0.093151956

0.220540141

0.445647147

0.076543672

0.006944664

0.095290187

0.063018975

0.391893929

0.013141059

0.335091942

0.077999406

0.374915752

0.108365098

0.584662844

0.199685528

0.193600128

0.058799456

0.350398142

0.147320442

0.637127676

0.436425951

0.092839749

0.20344562

0.051550964

0.19750082

0.226283317

0.355470002

0.064303452

0.067205098

0.821989557

0.04377135

0.07527085

0.207525016 
SPAC1610.03C SPBC29B5.01

SPAC343.06c

SPAC1851.04C

SPNCRNA.617

SPNCRNA.1610

SPNCRNA.1573

SPAC4F10.10C

SPNCRNA.883

SPAC144.08

SPAC3G6.09c

SPAC11D3.11C

SPBC26H8.12

SPAC688.13

SPAC3G9.07C

SPNCRNA.471

SPBC409.05

SPCC297.03

SPBC1A4.05

SPAC10F6.15

SPNCRNA.400

SPCC 1259.08

SPBP35G2.16c

SPCC777.14

SPAC4F8.10c

SPBC2D10.07c

SPBC21B10.09

SPAC1B3.09C

SPAC24H6.09

SPAC26H5.13C

SPAC29B12.01

SPBC21D10.09c

SPNCRNA.1365

SPBC1683.13C

SPBPB2B2.17C

SPAC1687.20C

SPBC354.05c

SPCC1682.07

SPBC336.09c exo2

SPCC970.02

spt20

SPCC1494.01

SPAC27F1.10

SPAC30C2.03

SPCC4B3.06C

pep7

rp79

atf1

SPAC343.06c

$\# N / A$

$\# \mathrm{~N} / \mathrm{A}$

$\# \mathrm{~N} / \mathrm{A}$

mnn9

\#N/A

jac1

tps2

SPAC11D3.11C

cyc3

$\mathrm{scn} 3$

hos 2

skp1

ssp1

blt1

SPAC10F6.15

\#N/A

SPCC1259.08

ecl2

prp4

stg1

$\mathrm{mmp1}$

SPBC21B10.09

noc 202

gef1

kei1

ino80

rkr1

$\# N / A$

cha4

ftm7

mis6

sre2

ssl1

rrn7 exonuclease II Exo2

mannan endo-1,6-alpha-mannosidase (predicted)

SAGA complex subunit Spt20

iron/ascorbate oxidoreductase family

chizosaccharomyces pombe specific protein

Schizosaccharomyces specific protein

NADPH-dependent FMN reductase (predicted) poly(A) binding protein Crp79

transcription factor, Atf-CREB family Atf1

$\begin{array}{lll}-0.2090128 & 8.49552817 & 0.02030611\end{array}$ $\begin{array}{llll}0.2090742 & 6.44656792 & 0.23260806\end{array}$ $\begin{array}{lll}-0.2091151 & 6.56789403 & 0.09486392\end{array}$ $\begin{array}{lll}-0.2092003 & 6.49972301 & 0.09284873\end{array}$ $\begin{array}{llll}0.2094268 & 3.86593425 & 0.55201178\end{array}$ $\begin{array}{lll}-0.2094637 & 5.14918945 & 0.3212083\end{array}$ $\begin{array}{lll}0.2097521 & 6.17929827 & 0.18074452\end{array}$ $\begin{array}{lll}-0.2104906 & 8.31627118 & 0.02795242 \\ -0.2108795 & 6.19129328 & 0.1940791\end{array}$ $\begin{array}{llll}0.2110668 & 7.96817927 & 0.00965622\end{array}$ $\begin{array}{lll}-0.2110754 & 8.28556532 & 0.01622051\end{array}$ $\begin{array}{llll}-0.2111442 & 6.62019318 & 0.16906332\end{array}$ $\begin{array}{lll}-0.2123097 & 6.46433088 & 0.12800703\end{array}$

$\begin{array}{llll}\# N / A & -0.2111442 & 6.62019318 & 0.16906332 \\ \text { \#N/A } & -0.2123097 & 6.46433088 & 0.12800703\end{array}$

mannosyltransferase complex subunit, Anp family Mnn9 (p| $-\begin{array}{llll}0.2125964 & 8.44318598 & 0.01201562\end{array}$ $\# \mathrm{~N} / \mathrm{A}$

$\begin{array}{lll}-0.2127601 & 6.06413454 & 0.26611472\end{array}$

mitochondrial (2Fe-2S) cluster assembly co-chaperone Jac1 $-0.2129565 \quad 7.210406840 .05946082$ trehalose-phosphate synthase Tps2 (predicted) $\begin{array}{lll}-0.2132362 & 7.84407205 & 0.09640762\end{array}$ zn(2)-C6 fungal-type DNA-binding transcription factor, trunc $-\begin{array}{llll}-0.2135268 & 6.78197479 & 0.08920167\end{array}$ cytochrome c heme lyase Cyc3 (predicted) $\quad-0.2137266 \quad 7.68503764 \quad 0.0358309$ TatD DNase family Scn1

histone deacetylase (class I) Hos2 \#N/A

SCF ubiquitin ligase complex subunit Skp1

$\begin{array}{llll}0.2138463 & 5.26850635 & 0.29814864\end{array}$ $\begin{array}{lll}-0.2141401 & 6.19790412 & 0.12700137\end{array}$ $\begin{array}{lll}-0.2144064 & 4.11754661 & 0.5113633\end{array}$ $\begin{array}{lll}-0.2145848 & 6.86332465 & 0.11135039\end{array}$

Ca2+/calmodulin-dependent (CaMMK)-like protein kinase S $-0.2147675 \quad 8.70125153 \quad 0.00557785$ ubiquitin domain-like protein Blt1

UPF0300 family protein 1

$\# \mathrm{~N} / \mathrm{A}$

DUF 2457 family conserved fungal protein

extender of chronological lifespan protein Ecl2

serine/threonine protein kinase Prp4

SM22/transgelin-like actin modulating protein Stg1

$\begin{array}{rrr}-0.2150458 & 7.70589949 & 0.0368294\end{array}$

$\begin{array}{lll}-0.2153055 & 4.91619457 & 0.41068311\end{array}$

$\begin{array}{lll}-0.2153363 & 2.70667757 & 0.70436327\end{array}$

$\begin{array}{lll}-0.2153864 & 8.26943116 & 0.00666772\end{array}$

$\begin{array}{llll}-0.2155844 & 8.08540584 & 0.25082475\end{array}$

$\begin{array}{llll}0.2163056 & 6.14271035 & 0.22091372\end{array}$

$\begin{array}{lll}-0.2164874 & 6.82559859 & 0.08085016\end{array}$

mitochondrial inner membrane peptidase complex catalytic $\quad \begin{array}{lllll}-0.2165497 & 6.17425522 & 0.15343069\end{array}$

endomembrane system acetyl-CoA transmembrane transpc $-0.2166591 \quad 7.29707117 \quad 0.03199126$

$\begin{array}{llllll}\text { Noc complex subunit Noc202 (predicted) } & -0.2167637 & 7.22943461 & 0.02819054\end{array}$

Cdc42 RhoGEF Gef1

$\begin{array}{lll}-0.2167637 & 7.22943461 & 0.02819054 \\ -0.216856 & 7.71953348 & 0.04138259\end{array}$

inositol phoshorylceramide synthase regulatory subunit Kei' $-0.2169015 \quad 7.552145990 .02897221$

SNF2 family ATP-dependent 3' to 5' DNA helicase Ino80

RQC complex ubiquitin-protein ligase E3 Rkr1 (predicted) \#N/A

transcription factor Cha4 (predicted)

sub-telomeric $5 \mathrm{Tm}$ protein family Ftm7

CENP-I ortholog Mis6

membrane-tethered transcription factor Sre2

transcription factor TFIIH complex subunit SsI1

$\begin{array}{llll}-0.2169019 & 8.82082434 & 0.00313349\end{array}$

$\begin{array}{lll}-0.2170596 & 8.35309902 & 0.07694506\end{array}$

$\begin{array}{lll}-0.2171428 & 3.13260491 & 0.71022512\end{array}$

$\begin{array}{lll}-0.2173144 & 6.38903811 & 0.16541698\end{array}$

$\begin{array}{lll}-0.2180673 & 6.07452495 & 0.20835786\end{array}$

$\begin{array}{llll}-0.2182725 & 5.07187726 & 0.30243534\end{array}$

$\begin{array}{llll}-0.2187502 & 8.2123507 & 0.02766917\end{array}$

$\begin{array}{llll}-0.2190382 & 6.81705753 & 0.07894007\end{array}$

RNA polymerase I general transcription initiation factor suk $\begin{array}{lllll}-0.2193336 & 6.68324233 & 0.15323438\end{array}$
0.039684974

319454668

0.150092757

0.147521746

0.641797398

0.415397659

0.258342045

0.173614587

0.356398045

0.052336102

0.273949031

0.020419695

0.032503106

0.24431427

0.193618108

0.024815264

0.357252053

0.100354885

0.152005062

0.142559272

0.065003808

0.391151238

0.192396326

0.603089645

0.171706182

0.012518884

0.066531469

0.505919368

0.775269219

0.014674599

0.340623111

0.305676669

0.13065432

0.225263402

0.058837985

0.052716849

0.073623136

0.05404493

0.007495033

0.125131432

0.780020045

0.239945818

0.29072693

0.395554972

0.051927394

0.127840824

0.225072294 
po41

srw1

svp26

SPBC2A9.03

dom34

spo14

$\operatorname{atg} 14$ SPAC11D3.17

snt1

img2

mad1

SPCC757.11c SPAC23D3.03C nup82
SEA complex ubiquitin-protein ligase E3 subunit Sea4 (pred -0.21986227 .727802190 .01784514$ serine/threonine protein kinase Ppk13 (predicted) nucleoporin Nup107

mitochondrial ribosomal protein subunit L39 (predicted)

NMS complex subunit Nnf1

para-aminobenzoate synthase (predicted)

transcription factor Thi5

ribosome biogenesis protein Bmt5 (predicted)

U3 snoRNP protein Utp14 (predicted)

Sirtuin family histone deacetylase Hst2

Arf3/6 docking factor (predicted)

mitochondrial RNA-binding protein (predicted)

GINS complex subunit SId5

RNA polymerase II associated Paf1 complex subunit Tpr1

DASH complex subunit Dad5

rRNA processing protein Ebp2 \#N/A

mitochondrial RNA polymerase Rpo41

truncated $\mathrm{C}$ terminal region of membrane transporter

conserved fungal plasma membrane protein

ATP-dependent DNA replication ligase Cdc17

substrate-specific mitotic G1 APC coactivator Srw1/Ste9

Sed5 Vesicle Protein Svp26 (predicted)

WD40/YVTN repeat-like protein

$\begin{array}{llll}0.2198758 & 6.21972231 & 0.16091809\end{array}$ $\begin{array}{llll}-0.2198957 & 8.13599349 & 0.00638763\end{array}$ $\begin{array}{lll}-0.2200207 & 4.06152955 & 0.5923633\end{array}$ $\begin{array}{lll}-0.2204253 & 5.345883 & 0.2639632\end{array}$ $\begin{array}{lll}-0.2204766 & 6.9354931 & 0.07692998\end{array}$ $\begin{array}{llll}-0.2213336 & 6.01383039 & 0.18768327\end{array}$ $\begin{array}{lll}-0.2215056 & 5.73448512 & 0.24827581\end{array}$ $\begin{array}{llll}-0.221519 & 7.82585224 & 0.01000292\end{array}$ $\begin{array}{llll}-0.2215494 & 6.61969866 & 0.09546852\end{array}$ $\begin{array}{llll}-0.2218394 & 6.34932479 & 0.15900956\end{array}$ $\begin{array}{llll}-0.2220613 & 8.30268295 & 0.04750259\end{array}$ $\begin{array}{llll}-0.2221486 & 6.28798008 & 0.11120129\end{array}$ $\begin{array}{lll}-0.2228678 & 8.46253474 & 0.035103\end{array}$ $\begin{array}{lll}-0.222918 & 6.36160704 & 0.09745273\end{array}$ $\begin{array}{llll}-0.2231061 & 8.2514451 & 0.01958087\end{array}$ $\begin{array}{lll}-0.2233201 & 6.36700939 & 0.09448992\end{array}$ $\begin{array}{lll}-0.2233466 & 8.2373525 & 0.00699209\end{array}$ $\begin{array}{lll}-0.2234302 & 5.5330848 & 0.24210961\end{array}$ $\begin{array}{lll}-0.2234316 & 8.52043412 & 0.01130008\end{array}$ $\begin{array}{lll}-0.2236053 & 7.18465587 & 0.06462799\end{array}$ $\begin{array}{lll}-0.2237345 & 7.01836905 & 0.06624491\end{array}$ $\begin{array}{lll}0.2242026 & 6.54465568 & 0.07349907\end{array}$ $-0.2244175 \quad 5.32916262 \quad 0.29011804$

Dom34-Hbs 1 translation release factor complex subunit, pe $-0.2244576 \quad 6.271949330 .11064055$ GDP/GTP exchange factor, WD repeat protein Spo14 $\quad-0.2244985 \quad 7.10113318 \quad 0.04398954$ $\begin{array}{lll}-0.2245277 & 6.40084263 & 0.11431796\end{array}$ transcription factor, zf-fungal binuclear cluster type $\quad-\begin{array}{llll}-0.2245393 & 7.38725279 & 0.10086776\end{array}$

$$
\text { \#N/A }
$$
mitochondrial ribosomal protein subunit L31 (predicted) $\quad \begin{array}{lllll}-0.2251925 & 5.48806413 & 0.34425005\end{array}$ glutathione-dependent formaldehyde dehydrogenase (predi $-0.2253377 \quad 6.20323323 \quad 0.13097913$ Set3 complex subunit Snt1

$\begin{array}{llll}-0.2254678 & 7.34877991 & 0.02385578\end{array}$ mitochondrial ribosomal protein subunit Mrpl49/Img2 (prer $\quad-0.225506 \quad 5.14344767 \quad 0.39725828$ mitotic spindle checkpoint protein Mad1 $\quad-0.2256983 \quad 5.99531390 .15308213$ transmembrane transporter (predicted) $\quad-\begin{array}{llll}0.2257303 & 6.77219528 & 0.0737827\end{array}$ GTPase activating protein (predicted) $\quad-\begin{array}{llll}-0.2257502 & 7.36045104 & 0.03715081\end{array}$ $\begin{array}{lllll}\text { nucleoporin, WD repeat Nup82 } & -0.2259786 & 7.58408336 & 0.02771063\end{array}$ \#N/A $\# N / A$ $\begin{array}{lll}-0.2260976 & 5.46860775 & 0.21393152\end{array}$ $\begin{array}{lll}-0.2261018 & 8.72999158 & 0.0318739\end{array}$ glucose 1-dehydrogenase (NADP+) (predicted) inosine-containing RNA endoribonuclease (predicted)

$$
\text { \#N/A }
$$

CLIP170 family protein Tip1

$\begin{array}{llll}0.2261445 & 7.14482527 & 0.17608147\end{array}$

$\begin{array}{lll}-0.2264424 & 7.89916056 & 0.01380107\end{array}$ $\begin{array}{llll}-0.2264447 & 7.30586182 & 0.02351395\end{array}$ MEMO1 family, human MEMO1 ortholog, ancient conserver $\quad \begin{array}{lllll}0.2267727 & 4.97127775 & 0.30030472\end{array}$ phosphatidylinositol-3,4,5-trisphosphate3-phosphatase Ptn: $-0.2268092 \quad 4.666537880 .36323229$ $\# \mathrm{~N} / \mathrm{A}$
0.035486893

0.23448557

0.014137662

0.676763897

0.355064163

0.125131432

0.266191045

0.33783581

0.021106929

0.150909014

0.232124149

0.082758605

0.171592926

0.063870572

0.153404078

0.038477568

0.14964019

0.015309094

0.331033929

0.023537515

0.107792525

0.110057759

0.120375731

0.382681389

0.170921446

0.077591715

0.175605797

0.15815786

0.077654692

0.439186646

0.197498995

0.045669099

0.492681353

0.224945772

0.12072403

0.067023136

0.051990858

0.573617688

0.297588717

0.058685568

0.252738753

0.0281608

0.045162981

0.39322179

0.458759414

0.120596788

0.145359438 
SPAC1952.13

SPAC24C9.07C

SPBC29A10.15

SPBC13G1.05

SPAC144.02

SPBC11C11.01

SPCC11E10.04

SPAC4H3.11C

SPAC22F8.10C

SPNCRNA.838

SPBC25H2.10C

SPBC8D2.14C

SPBC1778.03C

SPBC11C11.04C

SPBC8D2.13

SPAC29E6.01

SPAC4D7.03

SPBC28E12.04

SPBC947.14C

SPNCRNA.193

SPAC3H8.06

SPNCRNA.1183

SPNCRNA.1466

SPAC821.10c

SPCC757.04

SPAC13A11.01C

SPBC887.11

SPNCRNA.969

SPAC25B8.02

SPAC3A11.09

SPBC4C3.12

SPAC458.06

SPAC17C9.02C

SPNCRNA.59

SPAC4F8.12C

SPNCRNA.277

SPAC4A8.08C

SPBC36B7.09

SPBC146.09C pck

SPAC57A10.07

not11

ned1

bgs 2

orc1

SPBC13G1.05

iec1

SPBC11C11.01

ppr6

ppc89

sap145

$\tan 1$

sed5

SPBC1778.03c

alp1

shq1

pof11

pop2

SPBC28E12.04 cbp6

$\# N / A$

aur1

$\# \mathrm{~N} / \mathrm{A}$

$\# N / A$

sod1

SPCC757.04

rga8

pus2

\#N/A

sds3

$\operatorname{sod} 22$

$\operatorname{atg} 1803$

lys7

$\# \mathrm{~N} / \mathrm{A}$

spp42

\#N/A

vrs2

gcn2

Isd1
$\# N / A$

$\# N / A$

protein kinase $\mathrm{C}$ (PKC)-like Pck2

conserved membrane protein with Rossmann-like alpha/be

CCR4-Not complex subunit Not11

lipin, phosphatidate phosphatase Ned1

spore wall 1,3-beta-glucan synthase catalytic subunit Bgs2 origin recognition complex subunit Orc1

ER protein folding protein (predicted)

Ino80 complex subunit lec1

U2-associated protein (predicted)

mitochondrial PPR repreat protein Ppr6

spindle pole body protein Ppc89

U2 snRNP-associated protein Sap145

$$
\text { \#N/A }
$$

tRNA acetyltransferase Tan1 (predicted)

SNARE Sed5 (predicted)

NADH pyrophosphatase (predicted)

tubulin specific chaperone cofactor D, Alp1

box H/ACA snoRNP assembly protein Shq1 (predicted)

F-box/WD repeat protein Pof11

F-box/WD repeat protein Pop2

Schizosaccharomyces specific protein

mitochondrial Cob1 translation regulator $\mathrm{Cbp} 6$ (predicted) $\#$ N/A

inositol phosphorylceramide synthase Aur1 (predicted)

superoxide dismutase Sod1

$$
\# \text { \#N/A }
$$

transcription factor (predicted)

RhoGAP, GTPase activating protein Rga8

tRNA pseudouridine synthase Pus2 (predicted)

$$
\# \mathrm{~N} / \mathrm{A}
$$

Clr6 histone deacetylase complex subunit Sds3

plasma membrane sodium ion/proton antiporter Sod22

35673 forkhead transcription factor Sep1

autophagy associated WD repeat protein Atg18c

alpha-aminoadipate reductase phosphopantetheinyl transf

$$
\# \mathrm{~N} / \mathrm{A}
$$

U5 snRNP complex subunit Spp42

\#N/A

mitochondrial valine-tRNA ligase Vrs2/Vas2

elF2 alpha kinase Gcn2

histone demethylase SWIRM1 $\begin{array}{lll}-0.2273395 & 4.9844927 & 0.37970862\end{array}$ $\begin{array}{llll}-0.2276449 & 6.74152607 & 0.12157609\end{array}$ $\begin{array}{llll}-0.2277666 & 5.14002968 & 0.28163413\end{array}$ $\begin{array}{llll}-0.2278755 & 6.08886299 & 0.22043549\end{array}$ $\begin{array}{lll}0.2284234 & 5.56732147 & 0.24040787\end{array}$ $\begin{array}{llll}-0.2291283 & 8.21815693 & 0.00455035\end{array}$ $\begin{array}{llll}-0.2291591 & 6.52916801 & 0.07448601\end{array}$ $\begin{array}{lll}-0.2291911 & 4.83050292 & 0.39009877\end{array}$ $\begin{array}{lll}-0.2291972 & 7.62095257 & 0.09040209\end{array}$ $\begin{array}{llll}-0.2294745 & 7.9424811 & 0.00852738\end{array}$ $\begin{array}{llll}-0.2299035 & 7.4776538 & 0.02759535\end{array}$ $\begin{array}{llll}-0.2300746 & 6.24753583 & 0.25478464\end{array}$ $\begin{array}{llll}-0.2301223 & 6.57958613 & 0.05499104\end{array}$ $\begin{array}{llll}-0.2303065 & 6.17763067 & 0.13664812\end{array}$ $\begin{array}{llll}-0.2303895 & 6.5927051 & 0.08459066\end{array}$ $\begin{array}{llll}-0.2319304 & 7.23738976 & 0.12707748\end{array}$ $\begin{array}{llll}-0.2319315 & 6.37647709 & 0.11830919\end{array}$ $\begin{array}{llll}-0.231968 & 7.13282644 & 0.09650504\end{array}$ $\begin{array}{llll}-0.232142 & 6.38700509 & 0.17005976\end{array}$ $\begin{array}{lll}-0.2323456 & 7.48755832 & 0.02055206\end{array}$ $\begin{array}{llll}-0.2323869 & 6.19198021 & 0.12458547\end{array}$ $\begin{array}{llll}-0.232419 & 6.65264268 & 0.26076809\end{array}$ $\begin{array}{lll}-0.2324668 & 7.48590222 & 0.02036942\end{array}$ $\begin{array}{llll}-0.2324692 & 7.45063893 & 0.03398078\end{array}$ $\begin{array}{llll}-0.2328704 & 7.01685134 & 0.03127677\end{array}$ $\begin{array}{llll}-0.2330904 & 5.55311343 & 0.19786281\end{array}$ $\begin{array}{llll}-0.2331594 & 6.97999464 & 0.03512646\end{array}$ $\begin{array}{llll}-0.2334722 & 4.15209784 & 0.44782021\end{array}$ $\begin{array}{llll}-0.2341142 & 7.67749531 & 0.03849426\end{array}$ $\begin{array}{lll}-0.2341863 & 4.71466533 & 0.40782271\end{array}$ $\begin{array}{llll}-0.2344385 & 4.14366003 & 0.54209182\end{array}$ $\begin{array}{llll}-0.2355097 & 8.80341278 & 0.00177398\end{array}$ $\begin{array}{llll}-0.2355234 & 7.4893819 & 0.01982964\end{array}$ $\begin{array}{llll}-0.2355727 & 8.22796787 & 0.00626501\end{array}$ $\begin{array}{llll}-0.2355731 & 5.83201613 & 0.14290532\end{array}$ $\begin{array}{llll}-0.2359073 & 6.52817949 & 0.10437425\end{array}$ $\begin{array}{llll}-0.2363132 & 5.46368519 & 0.33037476\end{array}$ $\begin{array}{llll}-0.2363787 & 7.23049229 & 0.03089074\end{array}$ $\begin{array}{lll}-0.2363795 & 7.22460585 & 0.0408319\end{array}$ $\begin{array}{llll}-0.2364759 & 4.86138426 & 0.33168179\end{array}$ $\begin{array}{lll}0.2374999 & 3.96669094 & 0.4870422\end{array}$ $\begin{array}{llll}-0.2376524 & 5.11786486 & 0.25605211\end{array}$ $\begin{array}{llll}-0.2377643 & 10.0045094 & 0.00083863\end{array}$ $\begin{array}{llll}-0.2381851 & 3.67153286 & 0.52541402\end{array}$ $\begin{array}{llll}-0.238319 & 7.45958381 & 0.05192161\end{array}$ $\begin{array}{llll}-0.2386011 & 8.7507068 & 0.00231619\end{array}$ $\begin{array}{llll}-0.2388639 & 7.60259166 & 0.03103614\end{array}$
0.475421743

0.185374252

0.374393479

0.305325364

0.32897156

0.010454126

0.121699237

0.485660839

0.14413922

0.018236576

0.051817427

0.345036708

0.094023626

0.204596357

0.135810692

0.192468783

0.181286651

0.152065368

0.245233458

0.040073563

0.189241991

0.351321893

0.039774424

0.062211255

0.05782081

0.278250961

0.063889154

0.543793096

0.069170174

0.503124287

0.63296754

0.004497734

0.038943933

0.013900687

0.212283502

0.162309595

0.424990594

0.057169286

0.072795677

0.42618855

0.582174982

0.346339616

0.002296517

0.616668232

0.089495082

0.005733596

0.057422757 
SPAC1F12.09 SPAPB1A10.10c SPAC3A11.08 SPNCRNA.978 SPAC19G12.01c SPAPB17E12.03 SPCC1840.01C SPCC1235.01

SPAC1952.02 SPBC1709.14 SPBC56F2.11

SPBC16A3.10 SPCC306.10

SPBC2D10.14C

SPBC1921.06c

SPNCRNA.1459

SPAC19A8.05C

SPAC5D6.13

SPAC1327.01C

SPAC3A12.03C

SPCC1322.14C

SPAC1A6.06C

SPNCRNA.710

SPAC13G7.01C

SPBC646.02

SPBC20F10.07

SPAC26A3.10

SPCC736.10c

SPBC685.05

SPCC757.10

SPCC1020.11c

SPCC16A11.13

SPAC17H9.08

SPCC736.06

SPAC2E1P5.05

SPBC19C7.07C

SPBC1604.15

SPBC713.08

SPBC17D11.04c

SPBC1703.15C

SPAC15A10.13

SPNCRNA.840

SPBC16H5.05C

SPAC19A8.11C

SPAC644.11C

SPNCRNA.1657

SPBC36B7.02 $\begin{array}{llll}-0.2395272 & 6.64803886 & 0.05389405\end{array}$ $\begin{array}{llll}0.2397978 & 7.24395003 & 0.06418425\end{array}$ cut20 pex12 $\operatorname{mog} 1$ SPCC1235.01 tma23 ngl1

met6 ale1 wtf8 myo51 pvg3

sst4 $\# \mathrm{~N} / \mathrm{A}$

vps 74

SPAC1327.01C meu34 vtc4 meu31 erg7 cwf11 Itc1 cnt6 mrps8 gpi15 vph2 emc6 luc7 SPAC17H9.08 dar2 rrp9 sen34 gpi16 tom13 nto1 vps33 ppk3

\#N/A $\quad-0.2403352 \quad 3.76735012 \quad 0.49161482$

\#N/A $\quad-0.2403352 \quad 3.76735012 \quad 0.49161482$

anaphase-promoting complex platform subcomplex scaffol $-0.2403385 \quad 6.55379519 \quad 0.05073125$ ubiquitin-protein ligase E3 Pex12 involved in peroxisome or $\begin{array}{lllll}-0.2406416 & 6.77131163 & 0.05309313\end{array}$ Ran GTPase binding protein Mog1

Schizosaccharomyces specific protein

$\begin{array}{lll}-0.240792 & 5.4694738 & 0.16262893\end{array}$ $\begin{array}{lll}-0.241058 & 9.80833631 & 0.00067412\end{array}$ $\begin{array}{lll}-0.2411344 \quad 6.11002596 & 0.10589634\end{array}$ $-0.2412467 \quad 6.88439905 \quad 0.03490486$ $\begin{array}{lll}-0.2412834 \quad 8.11461589 & 0.00253097\end{array}$ peptide $\mathrm{N}$-glycanase $\mathrm{Ngl1}$

homoserine O-acetyltransferase Met6

membrane bound O-acyltransferase, MBOAT Ale1 (predicte $-0.2413337 \quad 7.27251009 \quad 0.01803124$ wtf element Wtf8

myosin type $\mathrm{V}$

$\begin{array}{lll}0.2415046 & 4.40530322 & 0.42985447\end{array}$ $\begin{array}{lll}-0.2419101 & 7.48859033 & 0.01470824\end{array}$

Golgi galactosylxylosylprotein 3-beta-galactosyltransferase $\quad \begin{array}{llll}0.2420477 & 6.55773549 & 0.06904731\end{array}$ $\# \mathrm{~N} / \mathrm{A}$ $\begin{array}{lll}-0.2421083 & 6.10503118 & 0.14422847\end{array}$ sorting receptor for ubiquitinated membrane proteins, ESCF $-0.2422692 \quad 7.645621 \quad 0.01852106$ Golgi phosphoprotein 3 family Vps74 (predicted) $\quad-0.24232347 .69388403 \quad 0.01022463$ transcription factor, zf-fungal binuclear cluster type (predic $\quad \begin{array}{llll}0.2423823 & 7.83269301 & 0.00408417\end{array}$ ubiquitin-protein ligase E3 Meu34, human RNF13 family ho -0.24248925 .519663180 .15824972$ vacuolar transporter chaperone (VTC) complex subunit (pre $-\begin{array}{lllll}-0.2426043 & 8.71567171 & 0.00162573\end{array}$ Schizosaccharomyces specific protein Meu31

$\begin{array}{lll}-0.2431247 & 3.94450081 & 0.67389624\end{array}$ $-0.2431247 \quad 3.94450081 \quad 0.67389624$ $-0.2431555 \quad 6.81837935 \quad 0.05156859$ $\begin{array}{llll}-0.2433795 & 7.5712956 & 0.01952097\end{array}$ $\begin{array}{llll}-0.2439286 & 7.83087446 & 0.02646974\end{array}$ U2-type spliceosomal complex ATPase Cwf11 GRAM domain membrane contact site protein Ltc1 centaurin ADP ribosylation factor GTPase activating protein $\begin{array}{ccc}-0.2440864 & 8.20250917 & 0.00343888\end{array}$ mitochondrial ribosomal protein subunit S8 (predicted) $\quad \begin{array}{llllll}-0.2445111 & 6.51069708 & 0.05911064\end{array}$ pig-H (predicted)

$-0.2447158 \quad 3.76754245 \quad 0.49578471$ endoplasmic reticulum membrane protein involved in asser $\begin{array}{ccccc}-0.2450469 & 5.31976753 & 0.28246191\end{array}$ ER membrane protein complex subunit Emc6 (predicted) $\quad \begin{array}{lll}-0.2450899 & 5.57183486 & 0.3607415\end{array}$ U1 snRNP-associated protein Luc7

mitochondrial carrier, coenzyme A (predicted) mitochondrial aspartate-tRNA ligase Dar2 (predicted) U3 snoRNP-associated protein Rrp9 (predicted) $\begin{array}{lll}-0.2451054 & 6.00584266 & 0.11301398\end{array}$ $\begin{array}{lll}-0.2455294 & 7.25473033 & 0.08360983\end{array}$ $-0.2459553 \quad 7.51943095 \quad 0.02540513$ $\begin{array}{lll}-0.2459694 & 7.06146018 \quad 0.04645115\end{array}$ tRNA-splicing endonuclease catalytic subunit Sen34 (predic $-0.2463039 \quad 6.70793590 .09967273$ pig-T, Gpi16 (predicted) $\quad \begin{array}{lllll}0.24641 & 7.68382439 & 0.01028257\end{array}$ mitochondrial TOM complex assembly protein Tom13 (prec $-0.2470524 \quad 6.13979324 \quad 0.21770225$ histone acetyltransferase complex PHD finger subunit Nto1 $-\begin{array}{llll}0.2470652 & 7.11481875 & 0.04389691\end{array}$ $\begin{array}{llllll}\text { HOPS/CORVET complex subunit, vacuolar sorting protein } V_{k} & -0.2470993 & 7.18030854 & 0.02019847\end{array}$ protein kinase domain and HEAT repeat protein Ppk3 $\quad \begin{array}{llll}-0.2477036 & 7.07333106 & 0.03368726\end{array}$

$$
\text { \#N/A }
$$

$\begin{array}{llll}-0.2477196 & 6.16831741 & 0.13117215\end{array}$ cyclophilin family peptidyl-prolyl cis-trans isomerase Cyp7 ( $\begin{array}{ccccc}-0.2480778 & 6.63695931 & 0.06978624\end{array}$ clathrin coat adaptor Irc6 $\begin{array}{lll}0.2483512 \quad 8.13936653 & 0.00333828\end{array}$ mitochondrial pyruvate dehydrogenase (lipoamide) kinase f $\begin{array}{rllll}-0.2485895 & 7.27286355 & 0.01506224\end{array}$ \#N/A $\begin{array}{lll}-0.2486188 & 4.43615769 & 0.41087349\end{array}$ $\begin{array}{llll}\text { Svf1 family protein, lipocalin superfamily Svf2 } & -0.248643 & 7.3217292 & 0.05399946\end{array}$
0.064914785

0.092380104

0.107258255

0.586404701

0.08779913

0.091280675

0.23652273

0.001895623

0.164338568

0.063578052

0.006195409

0.035804674

0.525257618

0.029832953

0.11395943

0.213880876

0.036713023

0.021514718

0.009479128

0.231390068

0.004152809

0.749446632

0.749446632

0.089007709

0.038384794

0.049993506

0.008151028

0.099862932

0.589757617

0.375200724

0.456545641

0.173838188

0.134570687

0.048250611

0.081238782

0.156572698

0.021616593

0.302092947

0.077468482

0.039520015

0.061723701

0.197702435

0.115042479

0.007920844

0.030451143

0.505970696

0.092514178 
SPBC21B10.12

SPAC458.05

SPAC27E2.12

SPBC29A3.01

SPBC336.14C

SPAC23A1.16c

SPAC2C4.05

SPAC23H4.04

SPCC550.07

SPAC1A6.05C

SPAC1952.16

SPBC23G7.16

SPAC31A2.12

SPBC4F6.07C

SPAC2G11.12

SPAC6B12.14C

SPBC12D12.07C

SPCC306.08C

SPBC83.12

SPNCRNA.1624

SPNCRNA.92

SPNCRNA.1414

SPNCRNA.1214

SPNCRNA.1047

SPAPB1E7.10

SPBC56F2.03

SPAC $1250.04 \mathrm{C}$

SPAC31A2.10

SPBC2D10.10C

SPAC630.10

SPAC6F6.12

SPAC2OH4.06C

SPBC16E9.19

SPAC227.11C

SPCC1020.03

SPBC646.03

SPNCRNA.803

SPBP23A10.13

SPNCRNA.875

SPAC637.09

SPBC36.03C

SPBC25B2.04C

SPBC11C11.03

SPCC191.07

SPNCRNA.669

SPAC13F5.06C

SPCC553.01C rod1

mak5

rqh1

SPAC6B12.14C trx2

mdh1 SPBC83.12

$\# \mathrm{~N} / \mathrm{A}$

$\# N / A$

$\# N / A$

$\# \mathrm{~N} / \mathrm{A}$

$\# N / A$

rpc17

arp10

atl1

SPAC31A2.10

fib1

bmt2

$\operatorname{atg} 24$

SPAC20H4.06C

SPBC16E9.19

yos9

$\mathrm{mmt1}$

gta1

orc4

$\# N / A$

$\#$ N/A

rex1

$\mathrm{mfs} 3$

$\mathrm{mtg} 1$

ndc80

cyc1

$\# \mathrm{~N} / \mathrm{A}$

$\sec 10$

$\mathrm{db} / 2$ meiotic recombination protein Rec6

phosphatidylinositol 3-kinase Pik3

Schizosaccharomyces pombe specific protein

Golgi copper transporting ATPase Ccc2 (predicted)

$\begin{array}{llll}-0.2490334 & 7.17511936 & 0.02753221\end{array}$

$\begin{array}{lll}-0.2492203 & 2.2031052 & 0.74160804\end{array}$

$\begin{array}{lll}0.2494981 & 8.26738288 & 0.0028789\end{array}$

PAN complex protein phosphotransferase subunit Ppk26 (pr -0.2496866 $6.78679755 \quad 0.03562914$ RNA polymerase II CTD phosphatase Rtr1 (predicted) $\quad \begin{array}{lllll}-0.2497637 & 5.12702795 & 0.23205309\end{array}$ cornichon family protein (predicted) $\quad \begin{array}{lllll}-0.2498778 & 6.52501109 & 0.13200236\end{array}$ mitochondrial tRNA-specific 2-thiouridylase SIm3 (predicte $\quad-0.2499408 \quad 6.182710590 .13754187$ fatty-acid amide hydrolase (predicted) $\quad \begin{array}{lllll}-0.2499848 & 6.63379205 & 0.06734084\end{array}$ triacylglycerol lipase ptl3 $\quad \begin{array}{lllll}0.2499985 & 6.87936416 & 0.02823859\end{array}$ RhoGAP, GTPase activating protein Rga9 (predicted) $\quad \begin{array}{llll}-0.2500512 & 7.104198110 .02222482\end{array}$ arrestin/PY protein involved in ubiquitin-mediated endocytc $\begin{array}{cccc}0.2503789 & 7.43714027 & 0.01886746\end{array}$ ATP-dependent RNA helicase Mak5 (predicted) $\quad-0.25055167 .087226780 .05682152$ $\begin{array}{lllll}\text { RecQ type DNA helicase Rqh1 } & -0.2507994 & 7.79272371 & 0.0096875\end{array}$ conserved fungal protein mitochondrial thioredoxin Trx2 malate dehydrogenase Mdh1 (predicted)

Schizosaccharomyces pombe specific protein

$\# N / A$
$\# N / A$
$\# N / A$

$\#$ \#N/A $-0.2509857 \quad 7.35766825 \quad 0.01690159$ $\begin{array}{lll}-0.2511938 & 5.97898108 & 0.09779947\end{array}$ $\begin{array}{lll}-0.2515537 & 8.47134685 & 0.00526237\end{array}$ $\begin{array}{lll}-0.2516433 & 5.23197667 & 0.2058824\end{array}$ $\begin{array}{lll}-0.2517137 & 6.25219496 & 0.09618551\end{array}$ $\begin{array}{lll}-0.2518625 & 6.12665594 & 0.34484862\end{array}$ $\begin{array}{lll}-0.2518684 & 6.92027602 & 0.05360077\end{array}$ $\begin{array}{lll}-0.2519253 & 7.5744007 & 0.00916992\end{array}$ $-0.2520285 \quad 5.53151343 \quad 0.18489245$ DNA-directed RNA polymerase III complex subunit Rpc17 $\quad-0.2520578 \quad 5.43884813 \quad 0.18585117$ dynactin complex actin-like protein Arp10 (predicted) $\quad \begin{array}{llll}-0.252147 & 4.62753894 & 0.37780802\end{array}$ alkyltransferase-like protein Atl1

Ran GTPase binding protein (predicted)

fibrillarin, rRNA methyltransferase

$\begin{array}{rrr}-0.252147 & 4.62753894 & 0.37780802 \\ -0.2521884 & 3.54412046 & 0.57040151\end{array}$ $\begin{array}{lll}-0.2523261 & 6.0212957 & 0.10229299\end{array}$ $-0.25239125 .68730707 \quad 0.18221342$ rRNA (adenine) methyltransferase activity Bmt2 (predicted $\quad \begin{array}{lllll}0.252421 & 5.77247619 & 0.17009973\end{array}$ autophagy associated PX/BAR domain sorting nexin Atg24 $\quad-0.2526701 \quad 7.27569474 \quad 0.0102994$ G-patch RNA-binding protein, involved in splicing (predictec $-0.2531041 \quad 5.73358244 \quad 0.16683596$ $\begin{array}{llllll}\text { proteasome assembly chaperone } 3 \text { (predicted) } & -0.2536264 & 6.75901667 & 0.03468532\end{array}$ sensor for misfolded ER glycoproteins Yos9 (predicted) $\quad \begin{array}{llll}-0.2537213 & 8.12013882 & 0.00381518\end{array}$ mitochondrial iron ion transmembrane transporter Mmt1 ( 1 -0.2540394 5.970985140 .15003599 mitochondrial glutamyl-tRNA amidotransferase alpha subu $\begin{array}{ccccc}-0.2548437 & 6.27071201 & 0.21743354\end{array}$ $\# \mathrm{~N} / \mathrm{A}$

$\begin{array}{llll}-0.2552889 & 7.74848393 & 0.00398212\end{array}$ origin recognition complex subunit Orc4

$$
\# \mathrm{~N} / \mathrm{A}
$$

$\begin{array}{lll}-0.255932 & 7.57207461 & 0.00705491\end{array}$ $\begin{array}{lll}0.2562233 & 5.78573532 & 0.16135351\end{array}$ $-0.2562942 \quad 7.22900954 \quad 0.01306355$ plasma membrane spermidine transmembrane transporter $\begin{array}{ccccc}-0.2570275 & 9.48447541 & 0.000731\end{array}$ mitochondrial translation factor (GTPase) Mtg1 (predicted) $-\begin{array}{llll}-0.2572625 & 5.3632 & 0.23016699\end{array}$ NMS complex subunit Ndc80 cytochrome c

\section{\#N/A}

exocyst complex subunit Sec10

DNA recombination protein Dbl2
0.332436083

0.051727403

0.807592045

0.006944664

0.064706777

0.318756818

0.198865631

0.205684307

0.1115517

0.05279219

0.042978089

0.171829708

0.03733441

0.096571034

0.020479475

0.033728832

0.15387871

0.011881368

0.287626713

0.151760356

0.439785397

0.091946877

0.019476059

0.262890326

0.263977711

0.473625684

0.659124175

0.159896222

0.260059205

0.245239126

0.02163859

0.24181442

0.063237366

0.008907959

0.221186077

0.301781611

0.009254881

0.015420831

0.235069707

0.026792476

0.002038721

0.31667739

0.063237366

0.011511321

0.348923223

0.053992737

0.277826851 
SPCC16C4.05 SPNCRNA.1615 SPBC56F2.04

SPCC576.12C

SPNCRNA.1398

SPBC4C3.04C

SPBP16F5.06

SPBC28F2.05C

SPBC1604.19c

SPBC12D12.08c

SPCC1620.09c

SPAC1B3.17

SPAC30D11.09

SPNCRNA.419

SPCC777.07

SPBC1709.19c

SPAC1783.07C

SPAC24C9.14

SPBC106.13

SPCC1020.07

SPCC1672.11c

SPCC24B10.02C

SPBC8D2.17

SPAC29E6.07

SPBC887.03C

SPNCRNA.712

SPNCRNA.1204

SPAC6F12.09

SPCC1620.07c

SPBC19C7.02

SPNCRNA.1042

SPAC1639.02C

SPBC1773.02C

SPAC1D4.03C

SPAC23D3.17

SPBC14F5.13C

SPBC12C2.10C

SPCC576.04

SPBC1289.14

SPAC23A1.09

SPCC965.04C

SPAC589.02C

SPAC23D3.01

SPBC3B8.09

SPNCRNA.1475

SPBC30B4.06C

SPNCRNA.585 pop23

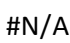

utp20

$\mathrm{mhf} 2$

$\# \mathrm{~N} / \mathrm{A}$

dss4

nop8

SPBC28F $2.05 \mathrm{C}$

trs 8501

ned8

tfg1

cwf19

\#N/A

omh3

nfu1

pap1

gid9

SPCC1020.07

SPCC1672.11c

SPCC24B10.02C

gmh4

SPAC29E6.07

noc3

$\# \mathrm{~N} / \mathrm{A}$

$\# N / A$

rdp1

Inp1

ubr1

\#N/A

bcp1

aut12

SPAC23D3.17

pho8

pst1

bxi1

SPBC1289.14

rbm8

yme1

med13

pdp3

utp3

\#N/A

\#N/A
RNase $\mathrm{P}$ and RNase MRP subunit Pop23

$$
\# \mathrm{~N} / \mathrm{A}
$$

U3 snoRNP protein Utp20 (predicted)

CENP-X ortholog, FANCM-MHF complex subunit Mhf2

$$
\# \mathrm{~N} / \mathrm{A}
$$

guanyl-nucleotide exchange factor (predicted)

ribosome biogenesis protein Nop8 (predicted)

xylose and arabinose reductase (predicted)

TRAPP complex subunit Trs85a (predicted)

ubiquitin-like protein modifier for cullin Ned8

chromatin silencing protein $\mathrm{Cl} 2$

complexed with Cdc5 protein Cwf19

$$
\text { \#N/A }
$$

$\begin{array}{lll}-0.2585705 & 4.89295674 & 0.24147321\end{array}$ $\begin{array}{llll}-0.2588265 & 5.73357266 & 0.12731343\end{array}$ $\begin{array}{lll}-0.2591651 & 8.97197281 & 0.0031421\end{array}$ $\begin{array}{lll}-0.2591885 & 7.36200333 & 0.02589318\end{array}$ $\begin{array}{lll}0.2592244 & 6.26871218 & 0.20899285\end{array}$ $\begin{array}{llll}-0.2596045 & 3.37691196 & 0.59752788\end{array}$ $\begin{array}{llll}-0.2597486 & 6.36547977 & 0.08140003\end{array}$ $\begin{array}{llll}-0.2598854 & 6.45278384 & 0.07870083\end{array}$ $\begin{array}{llll}-0.2600816 & 7.00986986 & 0.05875422\end{array}$ $\begin{array}{llll}-0.2608139 & 7.13352363 & 0.03977828\end{array}$ $\begin{array}{llll}-0.2612589 & 5.9943876 & 0.08914654\end{array}$ $\begin{array}{llll}-0.2613221 & 6.98505518 & 0.01419582\end{array}$ $\begin{array}{llll}-0.2617403 & 5.44406767 & 0.17771477\end{array}$ $\begin{array}{lllll}\text { alpha-1,2-mannosyltransferase Omh3 (predicted) } & -0.2618233 & 7.29565449 & 0.00656737 \\ \text { mitochondrial [4Fe-4S] cluster transfer protein Nfu1 (predic } & -0.2619999 & 6.87483843 & 0.04988276\end{array}$ $\begin{array}{lrrrr}\text { mitochondrial [4Fe-4S] cluster transfer protein Nfu1 (predir } & -0.2619999 & 6.87483843 & 0.04988276 \\ \text { transcription factor Pap1/Caf3 } & -0.2621321 & 7.97767074 & 0.0020972\end{array}$ ubiquitin-specific cysteine protease, OTU family, Otu1 $\quad-0.262386 \quad 7.36330685 \quad 0.01460126$ GID complex subunit Gid9 (predicted)

pseudouridine-5'-phosphatase (predicted)

P-type ATPase P5 type (predicted)

$\mathrm{NAD} / \mathrm{NADH}$ kinase (predicted)

alpha-1,2-galactosyltransferase (predicted)

$0.2623983 \quad 6.17196529 \quad 0.1557338$

$-0.2624157 \quad 6.014279410 .10522636$

$\begin{array}{lll}-0.262681 & 8.02177939 & 0.00299189\end{array}$

$\begin{array}{lll}-0.2627264 & 6.58955355 & 0.03203308\end{array}$

$\begin{array}{lll}-0.2628435 & 7.59042778 & 0.01100049\end{array}$

Schizosaccharomyces pombe specific protein, expressed du $\begin{array}{rlll}-0.2630094 & 2.06196991 & 0.72579753\end{array}$

$\begin{array}{lll}-0.2631365 & 7.23335495 & 0.01946217\end{array}$

$\begin{array}{lll}-0.2638821 & 6.98386408 & 0.01335984\end{array}$

$\begin{array}{lll}-0.264024 & 5.73816898 & 0.12197178\end{array}$

$\begin{array}{lll}-0.2642734 & 7.81450392 \quad 0.00386262\end{array}$

$\begin{array}{lll}-0.2644994 & 6.29051791 & 0.07398842\end{array}$

RNA-directed RNA polymerase Rdp1

lunapark Lnp1

$\mathrm{N}$-end-recognizing protein, UBR ubiquitin-protein ligase E3 \#N/A

$\begin{array}{lll}-0.2645699 & 8.8202739 & 0.00084547\end{array}$

$\begin{array}{llll}-0.265241 & 6.54148172 & 0.0457075\end{array}$

plasma membrane potassium ion transmembrane transpor $-\begin{array}{lllll}0.2653021 & 7.5134847 & 0.00754112\end{array}$

thioredoxin peroxidase $B c p 1$

autophagy associated protein Aut12 (predicted)

protease inhibitor 178 family

vacuolar membrane alkaline phosphatase (predicted)

Clr6 histone deacetylase complex subunit Pst1

BAX inhibitor family protein Bxi1

adducin (predicted)

$\begin{array}{lll}-0.265343 & 6.82677581 & 0.02353591\end{array}$

$-0.2656675 \quad 6.53656534 \quad 0.02825751$

$\begin{array}{llll}-0.2657211 & 5.6807504 & 0.13082165\end{array}$

$\begin{array}{lll}-0.2657226 & 7.82616203 & 0.00696563\end{array}$

$\begin{array}{llll}-0.2657707 & 8.70886607 & 0.00080204\end{array}$

$\begin{array}{llll}-0.2660349 & 6.6482136 & 0.07998379\end{array}$

$-0.2668569 \quad 3.63522092 \quad 0.47178984$

exon junction complex subunit, RNA-binding protein Rbm8 $\quad-0.267315 \quad 5.832559120 .11233572$ mitochondrial inner membrane i-AAA protease complex sul $-\begin{array}{llll}0.2678246 & 8.21891404 & 0.00274946\end{array}$ mediator complex subunit Med13

$\begin{array}{llll}0.2678706 & 6.57545962 & 0.03164006\end{array}$

PWWP domain protein, involved in chromatin remodeling $\left(\mid \begin{array}{lllll}-0.2681162 & 6.04812547 & 0.10711641\end{array}\right.$

U3 snoRNP-associated protein Utp3 (predicted) $\quad \begin{array}{llllll}-0.2686331 & 7.81608471 & 0.00607882\end{array}$

$\# \mathrm{~N} / \mathrm{A}$

$\begin{array}{llll}0.2688559 & 6.67755978 & 0.02463085\end{array}$

mitochondrial GIDA family tRNA uridine 5-carboxymethylar $\begin{array}{rlll}-0.2690605 & 6.74685382 & 0.14236856\end{array}$

\#N/A

$\begin{array}{lll}-0.269189 & 5.0474084 & 0.25624422\end{array}$
0.330296523

192740359

0.007513008

0.049027013

0.291553169

0.681215823

0.131386912

0.127514078

0.099373793

0.416274105

0.071159614

0.142538115

0.028905608

0.254707029

0.014491227

0.086528729

0.005231455

0.0296248

0.228201663

0.163468956

0.007181584

0.058886148

0.022955628

0.794059837

0.038299613

0.027358924

0.185894273

0.00900175

0.120992827

0.002311527

0.0801029

0.01636395

0.04517965

0.052813056

0.19734899

0.015256225

0.002206089

0.129377156

0.566730781

0.172999861

0.006670256

0.058372223

0.166004818

0.013531695

0.046950394

0.211624735

0.346530584 
SPBP8B7.08C

SPNCRNA.1280

SPAC5H10.11

SPAC3A12.04C

SPAC17C9.14

SPNCRNA.1157

SPBC428.10

SPAC23H4.18C

SPNCRNA.1538

SPAC4F8.11

SPNCRNA.1522

SPAC31G5.10

SPNCRNA.678

SPNCRNA.248

SPAC22G7.05

SPNCRNA.1056

SPAC6G10.02C

SPNCRNA.1603

SPCC364.04C

SPCC2H8.04

SPBC1604.20C

SPCC1739.03

SPAC589.07c

SPBC21D10.06c

SPAC458.07

SPNCRNA.648

SPNCRNA.1438

SPCC338.12

SPAC13D6.04C

SPNCRNA.691

SPCC1393.04

SPBC1706.03

SPBC19G7.13

SPNCRNA.580

SPAC1834.06C

SPBC14F5.05C

SPNCRNA.1344

SPBC25B2.01

SPAC222.15 sec6

mti2

rib5

ubp14

ppm1

mh1

pex19

$\# \mathrm{~N} / \mathrm{A}$

SPBC428.10

rbx1

\#N/A

sea2

N/A

eta2

$\# N / A$

$\# N / A$

kri

\#N/A

tea3

\#N/A

coy1

SPCC2H8.04

tea2

hrr1

$\operatorname{atg} 1801$

map4 tfa1

pbi2

btb3

$\mathrm{fta} 4$

fzo1

tbf1

$\# \mathrm{~N} / \mathrm{A}$

pmo25

sam1

hbs1

meu13
DUF4598 family, human C12orf45 ortholog, domain archite $\quad-0.2693197 \quad 5.0802499 \quad 0.21619462$ tubulin specific chaperone cofactor $B$ $\begin{array}{llll}-0.2693725 & 6.8832277 & 0.04949894\end{array}$ ATP-dependent RNA helicase Hca4 (predicted) $\quad \begin{array}{llll}-0.2696983 & 7.5537258 & 0.00613485\end{array}$ transcription factor, zf-fungal binuclear cluster type(predict $\begin{array}{llll}-0.2698359 & 6.59880066 & 0.0587334\end{array}$ $\begin{array}{lllll}\text { exocyst complex subunit Sec6 } & -0.2698981 & 7.11305898 & 0.0129945\end{array}$ mitochondrial translation initiation factor IF-2Mt/Mti2 (pre $\quad \begin{array}{lllll}-0.269903 & 7.21702767 & 0.00669413\end{array}$ riboflavin synthase Rib5 $\quad-0.2700795 \quad 7.16582957 \quad 0.02598628$ Lys48-specific deubiquitinase Ubp14 $\begin{array}{lll}-0.2703009 & 8.09435189 & 0.00491938\end{array}$ leucine carboxyl methyltransferase, involved in regulation o $\quad \begin{array}{ccc}-0.2705285 & 6.44596166 & 0.03399737\end{array}$ $\# \mathrm{~N} / \mathrm{A}$

alpha-1,2-galactosyltransferase Gmh1 (predicted)

$\begin{array}{rrr}-0.2705993 & 8.2215118 & 0.00243926\end{array}$ $\begin{array}{lll}-0.2708164 & 4.53078034 & 0.31458722\end{array}$ $\begin{array}{lll}-0.2711449 & 5.40381016 & 0.22363626\end{array}$ $-0.2718172 \quad 6.23209698 \quad 0.05396618$ $\begin{array}{lll}-0.2718971 & 4.54534466 & 0.29802609\end{array}$

$\begin{array}{lllll}\text { Schizosaccharomyces pombe specific protein } & -0.2719646 & 8.10184147 & 0.00471777 \\ \text { SCF complex, Cul4-RING and CLRC ubiquitin ligase ligase E3 } & -0.2720721 & 6.26668975 & 0.05027011\end{array}$ $\# N / A$ $\begin{array}{crrrr}\text { SCF complex, Cul4-RING and CLRC ubiquitin ligase ligase E } 3 & -0.2720721 & 6.26668975 & 0.05027011 \\ \text { \#N/A } & -0.272351 & 8.09900813 & 0.00421468\end{array}$ SEA complex WD repeat subunit Sea2 (predicted) $\quad-0.2735128 \quad 8.92261476 \quad 0.00032164$ $\# \mathrm{~N} / \mathrm{A}$ $\begin{array}{llll}-0.2738402 & 3.58511539 & 0.43314023\end{array}$ $\begin{array}{lllll}\text { Myb family protein, RNA polymerase I termination factor } \mathrm{E} \cdot & -0.274221 & 7.0807518 & 0.0094946\end{array}$ \#N/A $\quad-0.274245 \quad 5.83660497 \quad 0.09582335$ \#N/A $\quad-0.27445725 .316792350 .14599521$ $\begin{array}{rrr}-0.2746024 & 5.83122845 & 0.0952709\end{array}$ $\begin{array}{lcrrr} & \text { \#N/A } & -0.2746555 & 3.58423141 & 0.44656969 \\ \text { cell end marker Tea3 } & & -0.2749226 & 8.03889979 & 0.00394197 \\ & \text { \#N/A } & -0.2751293 & 6.35708786 & 0.06412259 \\ \text { CASP family protein involved in Golgi vesicle transport Coy1 } & -0.2755969 & 7.50991457 & 0.00813464\end{array}$ $\begin{array}{lcrrr} & \text { \#N/A } & -0.2746555 & 3.58423141 & 0.44656969 \\ \text { cell end marker Tea3 } & & -0.2749226 & 8.03889979 & 0.00394197 \\ & \text { \#N/A } & -0.2751293 & 6.35708786 & 0.06412259 \\ \text { CASP family protein involved in Golgi vesicle transport Coy1 } & -0.2755969 & 7.50991457 & 0.00813464\end{array}$ $\begin{array}{lcrrr} & \text { \#N/A } & -0.2746555 & 3.58423141 & 0.44656969 \\ \text { cell end marker Tea3 } & & -0.2749226 & 8.03889979 & 0.00394197 \\ & \text { \#N/A } & -0.2751293 & 6.35708786 & 0.06412259 \\ \text { CASP family protein involved in Golgi vesicle transport Coy1 } & -0.2755969 & 7.50991457 & 0.00813464\end{array}$ CASP family protein involved in Golgi vesicle transport Coy1 $-0.2755969 \quad 7.50991457 \quad 0.00813464$ kinesin family plus-end directed microtubule motor Tea2 $\quad \begin{array}{lllll}0.275705 & 6.70491962 & 0.0446595\end{array}$ $\begin{array}{lllll}\text { Helicase Required for RNAi-mediated heterochromatin ass } & -0.2758393 & 7.36914275 & 0.00380512\end{array}$ autophagy associated WD repeat protein Atg18a $\quad-0.2758413 \quad 5.70013434 \quad 0.14618473$ cell surface adhesion protein for conjugation Map4 $\quad \begin{array}{lllll}-0.2758838 & 5.3807118 & 0.25194245\end{array}$ transcription factor TFIIE alpha subunit, TFIIEA, Tfa1 $\quad \begin{array}{lllll}-0.2759746 & 6.60735523 & 0.06653731\end{array}$ $\# \mathrm{~N} / \mathrm{A}$$$
\# N / A
$$

vaculoar proteinase $\mathrm{B}$ inhibitor $\mathrm{Pbi2}$

substrate adaptor for cullin 3 ubiquitin ligase Btb3

$$
\text { \#N/A }
$$

Mis6-Sim4 complex subunit Fta4

$\begin{array}{llll}-0.2763498 & 4.55126921 & 0.3610797\end{array}$

$\begin{array}{llll}-0.2763989 & 5.97060273 & 0.07181644\end{array}$

$\begin{array}{llll}-0.2770251 & 6.65536784 & 0.05581013\end{array}$

$\begin{array}{lll}-0.2771304 & 6.79540474 & 0.0158603\end{array}$

$\begin{array}{llll}-0.2772332 & 6.29625032 & 0.03836534\end{array}$

$\begin{array}{llll}-0.2776196 & 5.53162691 & 0.14523876\end{array}$

mitofusin, mitochondrial dynamin family fusion GTPase prc $\quad \begin{array}{lllll}0.2778586 & 7.2496996 & 0.01346249\end{array}$ DNA binding factor Trf1

mo25 family protein $\mathrm{PmO25}$

$\mathrm{S}$-adenosylmethionine synthetase

$\begin{array}{lll}0.2779283 & 7.2530272 & 0.00495612\end{array}$

$\begin{array}{llll}-0.2780209 & 6.29226125 & 0.06732731\end{array}$

$-0.2781996 \quad 6.47893335 \quad 0.13808427$

$\begin{array}{llll}-0.2785209 & 8.56002307 & 0.00039586\end{array}$

$\begin{array}{llll}-0.2788113 & 6.12223928 & 0.13527968\end{array}$

\#N/A

$\begin{array}{lllll}\text { Dom34-Hbs } 1 \text { translation release factor complex subunit } \mathrm{Hb}: & -0.2788605 & 6.50809233 & 0.10323331 \\ \text { Tat binding protein 1(TBP-1)-interacting protein (TBPIP) hol } & -0.2789594 & 4.15273667 & 0.32368078\end{array}$
0.300368396

0.085928648

0.013643026

0.099373793

0.026666932

0.014718439

0.049175927

0.0112065

0.0622198

0.00600114

0.408621122

0.308815894

0.092480452

0.391065854

0.010805934

0.087171131

0.009748768

0.000969799

0.528703004

0.020115138

0.151287918

0.216071642

0.150631612

0.542468178

0.009170965

0.107186003

0.017495647

0.239147923

0.078448083

0.008889057

0.216258062

0.341799911

0.110516564

0.456825221

0.117932167

0.095232675

0.031856348

0.068994742

0.215115056

0.02752772

0.01126466

0.1115517

0.206247464

0.001169691

0.20281521

0.160976779

0.418039036 
SPCC622.03C

SPNCRNA.1577

SPAC144.06

SPAC23D3.16

SPNCRNA.1253

SPAC4A8.10

SPBC530.01

SPNCRNA.953

SPAC1F12.05

SPNCRNA.991

SPAC328.07C

SPBC15D4.14

SPAC20G8.03

SPNCRNA.930

SPBC215.02

SPCC1235.04c

SPAC13D6.01

SPBC1703.14C

SPAC806.08C

SPAC26H5.03

SPAC630.06C

SPAC821.13C

SPNCRNA.1490

SPNCRNA.655

SPBP8B7.13

SPBC31F10.15C

SPAC144.05

SPAC3C7.07C

SPAC8C9.07

SPNCRNA.1449

SPNCRNA.610

SPBC19C7.03

SPNCRNA.1592

SPAC3H1.03

SPCC622.11

SPCC1183.04c

SPBC211.04C

SPNCRNA.1124

SPAC1782.07

SPBC660.07

SPBC11B10.05C mak3

SPCC622.03C

\#N/A

apl5

lam4

$$
\# N / A
$$

$\operatorname{rog} 1$

gyp1

\#N/A

any2

taf73

itr2

bob1

nad1

pof14

top1

$\bmod 21$

pcf2

SPAC630.06c

dnf1

$\#$ N/A

$\# \mathrm{~N} / \mathrm{A}$

vac7

atp15

SPAC144.05

ate1

fyv7

\#N/A

\#N/A

cyr1

\#N/A

mug151

SPCC622.11

pet127

mcm6

$\# \mathrm{~N} / \mathrm{A}$

qcr8

ntp1

rsp1
\#N/A

$\begin{array}{lll}-0.2789831 & 8.53855839 & 0.00112137\end{array}$

\#N/A $\quad-0.2792625 .605070410 .10604897$

human GEMIN7 ortholog

mitochondrial thioredoxin-related protein (predicted)

$\begin{array}{lll}0.2794261 & 4.7945455 & 0.31147136\end{array}$

$\begin{array}{lll}-0.2795925 & 4.62938265 & 0.2419627\end{array}$

$\begin{array}{rrr}-0.2800089 & 9.65454857 \quad 5.07 \mathrm{E}-05\end{array}$

$\begin{array}{llll}-0.2800836 & 6.34736544 & 0.06694137\end{array}$

$\begin{array}{llll}-0.2801856 & 6.74299459 & 0.03023389\end{array}$

$\begin{array}{lll}-0.2804806 & 7.9785192 & 0.0013295\end{array}$

$-0.2805165 \quad 5.28102652 \quad 0.13812111$

$\begin{array}{lll}-0.2805481 & 7.50548989 & 0.00397063\end{array}$

$\begin{array}{lll}-0.2809045 & 7.63288122 & 0.00192864\end{array}$

$\begin{array}{llll}-0.2821048 & 6.93364571 & 0.01675048\end{array}$

$\begin{array}{lll}-0.2823015 & 5.33821154 & 0.17673353\end{array}$

$\begin{array}{lll}-0.2824735 & 7.3282365 & 0.08365201\end{array}$

$\begin{array}{lll}-0.2826325 & 7.71621487 & 0.0017561\end{array}$

arrestin-related endocytic adaptor Any2 (predicted)

$$
\text { \#N/A }
$$

$\begin{array}{lll}-0.2828356 & 6.84894758 & 0.02387445\end{array}$ $\begin{array}{lllll}\text { transcription factor TFIID complex subunit Taf5-like } & -0.2829399 & 7.14826982 & 0.01082752\end{array}$

$\begin{array}{lll}-0.2829743 & 9.78475993 \quad 3.71 \mathrm{E}-05\end{array}$ $\begin{array}{lll}-0.2831194 & 7.70353739 & 0.00244028\end{array}$ $\begin{array}{lll}-0.2831823 & 7.07135603 & 0.01270314\end{array}$ $\begin{array}{lll}-0.283554 & 5.50057915 & 0.1795438\end{array}$ $\begin{array}{lll}0.2839399 & 5.3719138 & 0.12771455\end{array}$ $\begin{array}{lll}-0.284184 & 7.88855332 & 0.00129623\end{array}$ $\begin{array}{lll}-0.2846839 & 5.9678115 & 0.09524002\end{array}$ $\begin{array}{lll}-0.2847299 & 6.08054832 & 0.04918804\end{array}$ $\begin{array}{lll}-0.2850692 & 8.45935837 & 0.00255807\end{array}$ prefoldin subunit 5 (predicted)

FAD synthetase Nad1 (predicted)

gamma tubulin complex subunit Mod21

CAF assembly factor (CAF-1) complex subunit B, Pcf2

DUF2011 family conserved fungal protein

$\begin{array}{lll}-0.2853177 & 9.24054712 \quad 7.05 \mathrm{E}-05\end{array}$

trans-Golgi network aminophospholipid translocase (flippas -0.2853177

$$
\# N / A
$$

$\begin{array}{llll}0.2854264 & 4.59193104 & 0.24593185\end{array}$ $\begin{array}{lll}-0.2862168 & 6.91412002 & 0.04349505\end{array}$

PAS complex phosphatidylinositol phosphate kinase activatı $\begin{array}{cccc}-0.2862527 & 5.75619499 & 0.08372132\end{array}$ F1-FO ATP synthase epsilon subunit (predicted) $\quad-0.2863445 \quad 6.45323062 \quad 0.05467658$ DNA-dependent ATPase/ ubiquitin-protein ligase E3 (predic $-0.2865518 \quad 7.68940793 \quad 0.00234769$ $\begin{array}{lllll}\text { arginine-tRNA protein transferase Ate1 (predicted) } & -0.2871096 & 6.461735 & 0.03111827\end{array}$ rRNA processing protein Fyv7 (predicted) $\quad \begin{array}{lllll}-0.2877849 & 5.85351936 & 0.12615258\end{array}$ $\begin{array}{lll}-0.2878666 & 6.51783136 & 0.04198101\end{array}$ $\begin{array}{lll}-0.2880423 & 7.30151784 & 0.01019812\end{array}$ $\begin{array}{lll}-0.2882923 & 8.69711514 \quad 0.00016836\end{array}$

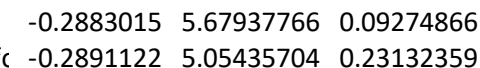

adenylate cyclase

$$
\# N / A
$$

\#N/A $\begin{array}{lllll}\text { mouse transcriptional regulator HCNGP-like, conflicting infc } & -0.2891122 & 5.05435704 & 0.23132359\end{array}$

$\begin{array}{lrlll}\text { mitochondrial RNA 5'-end processing Pet127 } & -0.28925 & 6.61603648 & 0.04547601\end{array}$ $\begin{array}{lll}-0.2897159 & 7.95499594 & 0.00066111\end{array}$ $\begin{array}{lll}-0.2898504 & 2.7991866 & 0.68723405\end{array}$ $-0.2898604 \quad 7.27264105 \quad 0.00497546$ $\begin{array}{lll}-0.2898747 & 9.02321585 & 0.02265596\end{array}$ ubiquinol-cytochrome-c reductase complex subunit 7 alpha,alpha-trehalase Ntp1 $\begin{array}{lll}-0.2899125 & 6.0140092 & 0.10351683\end{array}$
0.002972692

0.157354397

0.164500397

0.405192622

0.330899548

0.000176296

0.111079258

0.056085483

0.003465544

0.206257215

0.009234486

0.004857288

0.033466525

0.253460842

0.134606743

0.004459043

0.045687749

0.022650258

0.000131855

0.006001478

0.026108385

0.257003691

0.193304609

0.003391319

0.150617789

0.08544406

0.006246019

0.000238618

0.335316812

0.076918865

0.134686426

0.093556487

0.005803128

0.057543425

0.191265755

0.074608219

0.021480508

0.000533385

0.147397153

0.317883081

0.281334551

0.079799969

0.001862103

0.761789817

0.011304834

0.043675301

0.161344946 
SPCC736.15 SPAC27E2.04C SPAC4G8.10 SPBC20F10.08C SPAPJ698.03C SPAC22E12.14C SPCC1281.01

SPBC418.02

SPNCRNA.775

SPAC4D7.02C

SPBC713.13

SPAPB2B 4.02

SPCC622.02

SPBC2F12.08C

SPAC25B8.11

SPCC23B6.02C

SPNCRNA.1084

SPCC970.01

SPBC23G7.14

SPBC14C8.07C

SPBC2A9.13

SPAC31G5.14

SPAC3H8.08C

SPAC9.10

SPBC1706.01

SPNCRNA.1436

SPNCRNA.1176

SPCC330.01C

SPBC30D10.21

SPAC1751.02C

SPBC14F5.10C

SPCC306.05c

SPAC1565.06C

SPAC4F 10.06

SPAC12G12.13c

SPBC651.05C

SPBC19C2.06C

SPBC651.03C

SPBC8D2.16C

SPCC1442.13C

SPCC1795.09

SPNCRNA.654

SPNCRNA.1469

SPBC839.02

SPAPB21F2.03

SPBC4B4.11

SPAC56F8.09 pil1

prp12

sck2

ags1

SPBC418.02

\#N/A

pgc1

\#N/A

grx 5

SPCC622.02

ceg1

SPAC25B8.11

SPCC23B6.02C

$\#$ N/A

rad16

SPBC23G7.14

cdc18

$\# \mathrm{~N} / \mathrm{A}$

gcv1

SPAC3H8.08c

thi9

tea4

$\# \mathrm{~N} / \mathrm{A}$

$\# \mathrm{~N} / \mathrm{A}$

rhp16

mzm1

rsm19

SPBC14F5.10C

ins1

spg1

bud22

cid14

dot2

mug124

gyp10

SPBC8D2.16c

sqs2

yps1

$\#$ N/A

$\# N / A$

aly1

slx9

rrp8 eisosome BAR domain protein Pil1

$\# \mathrm{~N} / \mathrm{A}$

SNARE Gos1 (predicted)

RNA polymerase II nuclear import protein Rtp1 (predicted)

U2 snRNP-associated protein Sap130

serine/threonine protein kinase S6K Sck2

cell wall alpha-1,3-glucan synthase Ags1

NatA $\mathrm{N}$-acetyltransferase complex subunit (predicted)

$$
\text { \#N/A }
$$

phosphatidylglycerol phospholipase C Pgc1 (predicted)

\#N/A

Ruanylyltransferase Ceg1

ranscription factor (predicted)

pre-ribosomal factor (predicted) $\# N / A$

DNA repair endonuclease XPF

Schizosaccharomyces specific protein

MCM loader

\#N/A

glycine decarboxylase T subunit (predicted)

transcription factor (predicted)

plasma membrane thiamine transmembrane transporter $\mathrm{T}$

tip elongation aberrant protein Tea4

$$
\# N / A
$$$$
\# N / A
$$

$\begin{array}{lll}0.2900277 & 9.18689843 & 0.00013893\end{array}$ $\begin{array}{llll}-0.290125 & 2.58434669 & 0.67896987\end{array}$ $\begin{array}{llll}0.2902688 & 6.57719695 & 0.01683343\end{array}$ 0.2909436 8.68659177 0.00204548 $\begin{array}{llll}-0.2910066 & 8.21065909 & 0.00032165\end{array}$ $-0.2910356 \quad 9.77956915 \quad 1.02 \mathrm{E}-05$ $\begin{array}{llll}-0.2911916 & 6.29928926 & 0.08329452\end{array}$ $\begin{array}{llll}-0.2913685 & 2.53200273 & 0.68616803\end{array}$ $\begin{array}{llll}-0.2917273 & 7.30143171 & 0.00722582\end{array}$ $\begin{array}{rrr}-0.2922072 & 3.7986455 & 0.5018821 \\ -0.2922499 & 7.02135339 & 0.00659597\end{array}$ $\begin{array}{llll}-0.2923763 & 6.55684999 & 0.03451873\end{array}$ $\begin{array}{llll}-0.2923818 & 4.64711027 & 0.2438021\end{array}$ $\begin{array}{llll}-0.2924377 & 7.45597272 & 0.00349664\end{array}$ $\begin{array}{llll}-0.2926049 & 4.7440244 & 0.29659359\end{array}$ $\begin{array}{llll}-0.2932017 & 5.0430431 & 0.17066747\end{array}$ $\begin{array}{lll}-0.2932736 & 7.19305017 & 0.0090176\end{array}$ $\begin{array}{lll}-0.2932751 & 6.81520844 & 0.0118125\end{array}$ $\begin{array}{llll}-0.2936849 & 6.8557373 & 0.0215663\end{array}$ $\begin{array}{llll}-0.2939103 & 4.67691175 & 0.25074714\end{array}$ $\begin{array}{llll}-0.2941353 & 5.93757957 & 0.06897371\end{array}$ $\begin{array}{llll}-0.2942287 & 6.09627314 & 0.08207613\end{array}$ $\begin{array}{llll}-0.2942974 & 6.77055101 & 0.01431597\end{array}$ $\begin{array}{llll}-0.2943503 & 7.34499174 & 0.00290167\end{array}$ $\begin{array}{llll}-0.2945997 & 6.5267862 & 0.02216789\end{array}$ $\begin{array}{llll}-0.2951446 & 5.8072647 & 0.10201125\end{array}$ Rad16 homolog ATP-dependent DNA helicase/ ubiquitin pre $\quad-0.2953 \quad 8.249898030 .00046248$ mitochondrial respiratory chain complex III assembly protei $\begin{array}{ccc}0.2953376 & 4.37126307 & 0.36508609\end{array}$ mitochondrial ribosomal protein subunit S19 (predicted) $\quad \begin{array}{lllll}-0.2957483 & 6.7156077 & 0.02999218\end{array}$ ubiquitin-protein ligase E3, unknown biological role, implič́c -0.29585147 .019775820 .03754091$ INSIG domain protein

GTPase Spg1

$\begin{array}{llll}0.2958985 & 6.13714641 & 0.09354424\end{array}$ $\begin{array}{lll}-0.2959383 & 5.96945797 & 0.10060713\end{array}$ ribosome small subunit biogenesis protein, BUD22 family $\left(\mid \begin{array}{lllll}-0.2959475 & 6.69520731 & 0.03011813\end{array}\right.$ TRAMP complex poly(A) polymerase subunit Cid14 $\quad-0.2959694 \quad 7.39703258 \quad 0.00321842$ ESCRT II complex subunit Dot2 $\quad \begin{array}{lllll}0.2960736 & 6.20602955 & 0.0324324\end{array}$ $\begin{array}{llllll}\text { Schizosaccharomyces pombe specific protein } & -0.2967431 & 5.71827437 & 0.19681652\end{array}$ GTPase activating protein Gyp10

SPOUT domain containing methyltransferase (predicted) $\quad-\begin{array}{lllll}-0.2970562 & 7.34587111 & 0.00236587\end{array}$ R3H and G-patch domain protein Sqs2 $\quad-0.2971533 \quad 5.51826573 \quad 0.09794146$ aspartic protease, yapsin family, unknown specificity Yps1 $\quad-0.2972805 \quad 6.581655390 .02238281$ $\begin{array}{lll}-0.2978551 & 4.57291846 & 0.21977492\end{array}$ $\begin{array}{lll}-0.2978969 & 7.25561597 & 0.00573881\end{array}$ $\begin{array}{lll}-0.2979981 & 6.68813959 & 0.05027872\end{array}$ $\begin{array}{lll}-0.2983554 & 5.73055214 & 0.07372608\end{array}$ ribosome biogenesis protein Slx9 (predicted) mitochondrial conserved fungal protein, implicated in mitor $-0.29881057 .31894547 \quad 0.01824266$ rRNA methyltransferase Rrp8
0.000447015

0.754349382

0.03361253

0.005117453

0.00297305

0.000969799

4.04E-05

0.134190151

0.76110406

0.015750067

0.595208726

0.014540193

0.063026556

0.333080365

0.008263492

0.389862733

0.245953437

0.019182493

0.024470026

0.041835429

0.340585685

0.113890856

0.132446779

0.029132822

0.006992149

0.042904566

0.159562063

0.001349558

0.460587848

0.055733693

0.067637243

0.14845349

0.157858316

0.055921936

0.007676599

0.059568828

0.277352753

0.00501562

0.005839588

0.154066491

0.043246749

0.30453438

0.01286317

0.087171131

0.120660401

0.036203343

0.088261248 
SPAC823.13C

SPAC23D3.14C

SPAC1006.01

SPBP16F5.02

SPNCRNA.939

SPAC328.04

SPCC1494.03

SPBC21B10.13C

SPBC887.12

SPNCRNA.708

SPAC637.04

SPBC3B 8.08

SPAC4D7.01C

SPCC1183.07

SPAC750.04c

SPBP8B7.24C

SPBC947.13

SPCC663.18

SPCC290.02

SPNCRNA.864

SPAP7G5.06

SPAC30D11.06c

SPNCRNA.261

SPNCRNA.426

SPAPB17E12.02

SPNCRNA.839

SPCC4F11.04C

SPBC577.15C

SPNCRNA.1296

SPNCRNA.1105

SPAC31A2.15C

SPAC22E12.06C

SPCC306.04C

SPBC1773.15

SPCC338.06c

SPAC15A10.03C

SPNCRNA.1536

SPCC1393.06C

SPACUNK12.02

SPAC23G3.02C

SPAC167.09

SPAC29E6.03C

SPAC22F3.13

SPAC2OH4.10

SPAC1002.14

SPBC28F2.06C

SPBC1718.04 SPBC887.12$$
\# \text { N/A }
$$

SPBC3B8.08

sec71

rrp5

tm3

atg8

rba50

SPCC663.18

rpc34

\#N/A

$\# \mathrm{~N} / \mathrm{A}$

yip12

$\# N / A$

imt2

$\operatorname{sim} 3$

\#N/A

$\# N / A$

dcc1

gmh3

set1

dal52

SPCC338.06c

rad54

$\# \mathrm{~N} / \mathrm{A}$

ipi1

cmk1

sib1

pga1

uso1

tsc1

ufd2

$\mathrm{mdm} 12$

sct1 mitochondrial inner membrane protein She9 (predicted)

alpha-amylase homolog Aah2 (predicted)

vacuolar serine protease Psp3 (predicted)

TFIIH complex cyclin Mcs2

$\#$ N/A

microtubule severing ATPase Spg4 (predicted)

human RAP1 GTPase-GDP dissociation stimulator ortholog,

MBF complex corepressor Yox1

P-type ATPase (predicted)

$\# \mathrm{~N} / \mathrm{A}$

$\begin{array}{lll}-0.2989521 & 5.36512564 & 0.22296758\end{array}$ $\begin{array}{llll}-0.2992351 & 6.67319164 & 0.01361831\end{array}$ $\begin{array}{llll}-0.2993155 & 8.85725097 & 0.00068587\end{array}$ $\begin{array}{lll}-0.2995506 & 5.15413406 & 0.27748333\end{array}$ $\begin{array}{llll}0.2996233 & 5.23895951 & 0.14418144\end{array}$ $\begin{array}{lll}-0.2997168 & 6.53894556 & 0.02782358\end{array}$ $\begin{array}{llll}-0.2997818 & 6.09682452 & 0.05617211\end{array}$ $\begin{array}{llll}-0.2998226 & 6.66469824 & 0.07280264\end{array}$ $\begin{array}{llll}-0.2999597 & 8.42155371 & 0.00071641\end{array}$ $\begin{array}{llll}-0.3001024 & 5.99274948 & 0.04718904\end{array}$ phosphatidylinositol-4 kinase plasma membrane scaffold E| $\begin{array}{lllll}-0.3006036 & 7.37522827 & 0.00297993\end{array}$ Sjogren's syndrome/scleroderma autoantigen 1 family, imF $-0.3007542 \quad 5.21214896 \quad 0.21054976$ Sec7 domain protein, ARF GEF (predicted)

U3 snoRNP-associated protein Rrp5 (predicted)

sub-telomeric $5 \mathrm{Tm}$ protein family Ftm3

autophagy associated protein Atg8

RNA polymerase II associated protein (predicted)

$\begin{array}{llll}-0.3014206 & 8.78204883 & 0.00026951\end{array}$

$\begin{array}{lll}-0.3014397 & 8.33485465 & 0.00015851\end{array}$

$\begin{array}{llll}-0.3017785 & 6.0703534 & 0.07492293\end{array}$

$-0.30203826 .10692666 \quad 0.04016433$

$\begin{array}{lll}-0.3022311 & 6.22772607 & 0.0482763\end{array}$

(a)

DNA-directed RNA polymerase III complex subunit Rpc34 (k $-\begin{array}{lllll}0.3023407 & 6.2503701 & 0.03573607\end{array}$

$$
\text { \#N/A }
$$

$\begin{array}{lll}-0.302404 & 5.51444619 & 0.07761469\end{array}$

plasma membrane amino acid transmembrane transporter $-\begin{array}{llll}-0.3025991 & 8.37618126 & 0.00066016\end{array}$ organic solute transmembrane transporter (predicted)

$$
\# N / A
$$$$
\# N / A
$$

SMN family protein Yip12

$$
\# N / A
$$

mannosyltransferase Imt2

NASP family CENP-A chaperone

$\# \mathrm{~N} / \mathrm{A}$

$$
\# N / A
$$

Ctf18 RFC-like complex subunit Dcc1

alpha-1,2-galactosyltransferase $\mathrm{Gmh3}$

histone lysine methyltransferase Set1

dipeptide transmembrane transporter Dal5h2 (predicted)

heat shock protein $\mathrm{Hsp2O}$ family (predicted)

DNA-dependent ATPase Rad54/Rhp54

$$
\# \mathrm{~N} / \mathrm{A}
$$

Rix1 complex, Armadillo-type fold Ipi1

calcium/calmodulin-dependent protein kinase $\mathrm{Cmk}$

ferrichrome synthetase Sib1 $\begin{array}{lll}-0.3027925 & 1.73057478 & 0.83382643\end{array}$ $\begin{array}{lll}-0.3029347 & 6.04137693 & 0.06041946\end{array}$ $\begin{array}{llll}-0.3030035 & 6.42220078 & 0.10561551\end{array}$ $\begin{array}{lll}-0.3036661 & 7.27247511 & 0.00420899\end{array}$ $\begin{array}{lll}-0.3039024 & 7.93413743 & 0.00055125\end{array}$ $\begin{array}{llll}-0.3040565 & 6.36385378 & 0.05516337\end{array}$ $\begin{array}{lll}-0.3040752 & 4.05306158 & 0.33345782\end{array}$ $\begin{array}{lll}-0.3046153 & 7.85572095 & 0.00097658\end{array}$ $\begin{array}{llll}-0.3047487 & 7.21305448 & 0.00600649\end{array}$ $\begin{array}{llll}-0.3047536 & 6.91750713 & 0.01564743\end{array}$ $\begin{array}{lll}0.3052516 & 7.77218701 & 0.00361309\end{array}$ $\begin{array}{llll}-0.3053741 & 6.12710348 & 0.04416559\end{array}$ $\begin{array}{lll}-0.3055339 & 6.46066706 & 0.01676297\end{array}$ $\begin{array}{lll}-0.305554 & 6.0371156 & 0.10765429\end{array}$ $\begin{array}{lll}-0.3059617 & 3.46345844 & 0.49360666\end{array}$ $\begin{array}{llll}-0.3060573 & 6.33242671 & 0.05367628\end{array}$ $\begin{array}{lll}-0.3061613 & 7.22285598 & 0.00213196\end{array}$ $-0.3064515 \quad 10.2349111 \quad 1.39 \mathrm{E}-06$ GPI-mannosyltransferase II complex subunit Pga1 (predicte $-\begin{array}{lllll}0.3069284 & 6.39247016 & 0.03108837\end{array}$ ER to Golgi tether Uso1 (predicted)

hamartin $\begin{array}{lll}-0.3072356 & 8.73751492 \quad 3.82 \mathrm{E}-05\end{array}$ $\begin{array}{lll}-0.3072427 & 8.03824528 & 0.00029021\end{array}$

ubiquitin-protein ligase 3 involved in regulation of cytoplas $\begin{array}{cccc}-0.3073829 & 6.65995637 & 0.01112105\end{array}$ ERMES complex subunit Mdm12 $\quad-0.30763415 .568202390 .09657938$ glycerol-3-phosphate O-acyltransferase Sct1/Gpt1 (predicte $-\begin{array}{llll}0.3077559 & 7.64318918 & 0.00110315\end{array}$
0.308080074

0.027812903

0.001926258

0.369669937

0.213880876

0.052145268

0.095682409

0.119436345

0.002002125

0.082381076

0.007155398

0.293604738

0.000823177

0.000505232

0.122282135

0.071774749

0.083999033

0.002789966

0.064883657

0.125974909

0.001860201

0.003166372

0.881358936

0.101786933

0.163977536

0.009738923

0.001575483

0.09429459

0.427743725

0.00262673

0.013405766

0.031475242

0.008490002

0.077747957

0.03348165

0.166762449

0.588265034

0.092053204

0.005316225

6.29E-06

0.05750376

0.002211092

0.000135572

0.000879675

0.023180704

0.152134885

0.002933538 
SPBP35G2.03C SPAC4G8.11C

SPNCRNA.1086

SPAC22E12.08

SPNCRNA.1146

SPAC16A10.05C

SPCP31B10.05

SPNCRNA.1553

SPBP23A10.03C

SPBC19C2.09

SPAC15A10.15

SPAC1834.08

SPBC27B12.03C

SPNCRNA.667

SPAC19A8.10

SPAC7D4.15C

SPAC688.02C

SPAC17A5.18C

SPNCRNA.929

SPAC12B10.16C

SPBC21C3.20C

SPAC19E9.03

SPAC1782.05

SPBC215.03C

SPBC23E6.01C

SPBC8D2.01

SPNCRNA.1640

SPAC2G11.14

SPCC569.06

SPCC191.08

SPBC3B9.08C

SPBC16A3.06

SPBP8B7.28C

SPNCRNA.631

SPAC167.07c

SPBC8D2.11

SPCC1672.04c

SPBC530.06C

SPAC8F11.07C

SPNCRNA.1178

SPAC31A2.16

SPNCRNA.1687

SPNCRNA.1264

SPAC1F7.03

SPNCRNA.514

SPBC30D10.10C

SPNCRNA.586 dad1

hul5 meiotic inner centromere protein, shugoshin, Sgo1

$\begin{array}{lll}-0.3079566 & 4.60867574 & 0.30752651\end{array}$ mitochondrial F1-FO ATPase assembly protein (predicted) $\# \mathrm{~N} / \mathrm{A}$

$079857 \quad 6.59334195 \quad 0.01089116$ $\begin{array}{lll}-0.3081061 & 7.48504402 & 0.00423958\end{array}$ RNA polymerase I upstream activation factor complex subu $\begin{array}{llll}-0.3085718 & 6.63558783 & 0.01328907\end{array}$ $\#$ N/A

$\begin{array}{llll}-0.3087913 & 4.70737492 & 0.21828227\end{array}$ $\begin{array}{lll}-0.3088346 & 3.77543643 & 0.41408812\end{array}$ $\begin{array}{lll}-0.3091087 & 6.40383083 & 0.02573435\end{array}$ $-0.3092697 \quad 5.56747836 \quad 0.07576698$ tyrosyl-DNA phosphodiesterase Tdp1

$$
\# \mathrm{~N} / \mathrm{A}
$$
mitochondrial respiratory chain complex II assembly ACN9 I $-\begin{array}{lllll}-0.309288 & 6.60655051 & 0.01142203\end{array}$ sterol regulatory element binding protein, transcription fact $\begin{array}{lllll}-0.3096718 & 8.02999558 & 0.00157519\end{array}$ $\begin{array}{llll}\text { inner centromere protein, shugoshin Sgo2 } & -0.3097036 & 6.72948191 & 0.007889\end{array}$ histidine kinase Mak1

C-5 sterol desaturase Erg32

$$
\text { \#N/A }
$$

SMO-targeted ubiquitin-protein ligase subunit Rfp1 oligosaccharyltransferase subunit Ost4 (predicted) NMS complex subunit Mis14/Nsl1

meiotic recombination protein $\operatorname{Rec} 25$

$$
\# \mathrm{~N} / \mathrm{A}
$$

alpha-mannosidase GH125 family Mug157 (predicted) C2 domain protein Git1

cyclin Pas1

protein phosphatase type $2 \mathrm{~A}$ regulator, PTPA family Ypa2 COP9/signalosome complex subunit Csn1

splicing factor Cxr1

serine/threonine protein kinase Gsk31 (predicted) \#N/A $\begin{array}{llll}-0.3099211 & 8.79554594 \quad 2.13 E-05\end{array}$ $\begin{array}{lll}-0.3104245 & 6.57585846 & 0.02264832\end{array}$ $\begin{array}{llll}-0.3105497 & 3.68396009 & 0.37895727\end{array}$ $\begin{array}{llll}-0.3105604 & 6.75428866 & 0.01190745\end{array}$ $\begin{array}{llll}-0.3106647 & 4.72586378 & 0.28342674\end{array}$ $\begin{array}{lll}-0.3110264 & 5.6074367 & 0.08401845\end{array}$ $\begin{array}{llll}-0.3110358 & 6.75924108 & 0.00790963\end{array}$ $\begin{array}{llll}-0.3110687 & 4.07087704 & 0.39566091\end{array}$ $\begin{array}{lll}-0.3111705 & 7.93778263 & 0.00075754\end{array}$ $\begin{array}{llll}-0.3112796 & 8.05396944 & 0.00015769\end{array}$ $\begin{array}{llll}-0.3123386 & 8.41712403 & 0.04610181\end{array}$ $\begin{array}{lll}-0.3123809 & 6.36899451 & 0.02245304\end{array}$ $\begin{array}{llll}-0.3129177 & 7.28115056 & 0.00284261\end{array}$ $\begin{array}{llll}-0.3129533 & 7.50246693 & 0.02531671\end{array}$ $\begin{array}{lll}-0.3130368 & 7.43161097 & 0.00214817\end{array}$ $\begin{array}{lll}-0.3131692 & 7.11270548 & 0.00483566\end{array}$ $\begin{array}{lrrr}\text { transcription factor TFIID complex subunit Taf111 } & -0.3131857 & 8.42095238 & 4.23 \mathrm{E}-05 \\ \text { Schizosaccharomyces specific multicopy membrane protein } & -0.3131953 & 7.16068056 & 0.00628544\end{array}$ $\begin{array}{lrrrr}\text { Schizosaccharomyces specific multicopy membrane protein } & -0.3131953 & 7.16068056 & 0.00628544 \\ \text { CIA machinery involved in ribosome biogenesis protein Lto1 } & -0.3131971 & 5.100968 & 0.1439415\end{array}$ exon junction complex subunit, Mago-nashi homolog Mnh1 $\quad-0.3133211 \quad 6.66163790 .01164905$ $-0.31349346 .36596623 \quad 0.05672867$ CLRC ubiquitin ligase complex subunit, LIM-like Stc1 $\# \mathrm{~N} / \mathrm{A}$

HECT-type ubiquitin-protein ligase E3 (predicted)

$\begin{array}{lll}-0.3137882 & 4.55777734 & 0.35699024\end{array}$ $\begin{array}{lll}-0.3146047 & 5.3484651 & 0.16920469\end{array}$ $\begin{array}{lll}-0.314628 & 7.36172153 & 0.00230189\end{array}$ $\begin{array}{lrrrr}\text { Schizosaccharomyces specific protein } & -0.3150711 & 6.71058172 & 0.0065717 \\ \text { mitochondrial copper chaperone for cytochrome c oxidase } C & -0.315448 & 4.95715146 & 0.22886549\end{array}$ translation initiation factor 3 complex subunit Clu1 (predict $\quad-0.3160927 \quad 7.57692323 \quad 0.0004753$ DNA replication protein $\mathrm{Cdc} 24$ $\#$ N/A

RhoGEF Gef2

$\# N / A$ $\# \mathrm{~N} / \mathrm{A}$

plasma membrane TRP-like calcium ion channel Pkd2 \#N/A

serine/threonine protein kinase Tor

\#N/A
0.40084093

0.022762383 0.009796378 0.027222189

0.302712627 0.509100214 0.048794158

0.123495638

0.023755169

0.004040397

0.017015732

7.94E-05

0.043672951

0.474743042

0.024636733

0.376335409

0.135100558

0.017054833

0.490968719

0.002102387

0.000503079

0.080710781

0.043357804

0.006869309

0.048109524

0.005354682

0.011046206

0.000148531

0.013932386

0.213636183

0.024179339

0.096461422

0.452555623

0.244362879

0.005704425

0.014496072

0.315077815

0.001384009

0.160696512

0.040858677

0.014307973

0.001666268

0.054970097

7.25E-05

0.277575512

0.001530143

0.096461422 
SPCC895.04c

SPAC1F $7.07 \mathrm{C}$

SPCC126.09

SPNCRNA.77

SPBC16C6.06

SPBC6B1.04

SPCC16C4.20c

SPCC188.08C

SPCC1620.10

SPAC1006.03C

SPNCRNA.656

SPBC31F10.02

SPAC630.05

SPAC2OH4.02

SPCC306.03C

SPNCRNA.1099

SPCC1739.04c

SPNCRNA.169

SPAC $1002.03 \mathrm{C}$

SPAC2F7.16c

SPBP23A10.09

SPBC20F10.01

SPAC6F12.11C

SPNCRNA.807

SPAC144.07C

SPBC725.07

SPAC17G6.12

SPCC1840.10

SPBC21H7.03C

SPAC1F5.03C

SPAC8C9.19

SPBC902.02C

SPAC23E2.02
SPAC13G7.09c

$\# \mathrm{~N} / \mathrm{A}$

$\# N / A$

ufe1

fip1

zip2

vps 10

mde4

hap2

ubp5

cwf26

red1

\#N/A

SPBC31F10.02

gyp7

cnd2

dms1

\section{$\#$ N/A}

\#N/A

gls2

pld1

psf1

gar1

sfc1

\#N/A

gpn2

pex5

cul1

Ism8

SPBC21H7.03C

SPAC1F5.03C

SPAC8C9.19

ctf18

Isd2

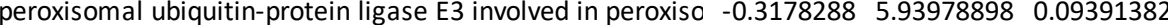
mitochondrial conserved fungal membrane protein Mug99 Lid2 complex PHD finger and jmjC subunit Lid2 Cul4-RING E3 adaptor Ddb1

$$
\# \mathrm{~N} / \mathrm{A}
$$

tRNA specific adenosine deaminase subunit Tad2

Schizosaccharomyces specific protein

$$
\# N / A
$$

$\mathrm{N} / \mathrm{A}$

NADHX dehydratase (predicted)

kinesin-like protein Klp8

conserved fungal protein

$$
\text { \#N/A }
$$

SNARE Ufe1 (predicted)

plasma membrane iron transmembrane transporter Fip1 vacuolar zinc exporter (predicted)

$$
\text { \#N/A }
$$

sorting receptor for vacuolar proteins, Vps10

microtubule-site clamp monopolin complex subunit Mde Ino80 complex, HMG box protein Hap2

ubiquitin C-terminal hydrolase Ubp5

complexed with $\mathrm{Cdc} 5$ protein Cwf26

RNA elimination defective protein Red1

$$
\# \mathrm{~N} / \mathrm{A}
$$

acyl-CoA hydrolase (predicted)

GTPase activating protein Gyp7 (predicted)

Golgi Dsc E3 ligase complex subunit Dsc3

condensin complex non-SMC subunit Cnd2

$$
\text { \#N/A }
$$

meiotic spindle pole body protein Dms1

$$
\text { \#N/A }
$$

glucosidase II alpha subunit Gls2

phospholipase D, PId1

GINS complex subunit Psf1

box H/ACA snoRNP complex subunit Gar1 $\begin{array}{llll}-0.3178335 & 6.6394812 & 0.06328243\end{array}$ $\begin{array}{lll}-0.3179445 & 7.45299207 & 0.00077492\end{array}$ $\begin{array}{lll}0.3179513 & 8.22684722 & 0.00010771\end{array}$ $\begin{array}{llll}-0.3179633 & 5.8998715 & 0.05797842\end{array}$ $\begin{array}{llll}-0.3182405 & 7.02790537 & 0.01175485\end{array}$ $\begin{array}{llll}-0.3182748 & 3.88612485 & 0.46809103\end{array}$ $\begin{array}{lll}-0.3186702 & 5.2751838 & 0.08774687\end{array}$ $\begin{array}{llll}-0.3186704 & 5.12196214 & 0.11705849\end{array}$ $\begin{array}{llll}-0.3187466 & 7.42292953 & 0.00245977\end{array}$ $\begin{array}{lll}-0.3188002 & 7.38860857 & 0.00091798\end{array}$ $\begin{array}{llll}-0.319033 & 4.65432384 & 0.22111179\end{array}$ $\begin{array}{llll}-0.3193427 & 7.92778468 & 0.00102822\end{array}$ $\begin{array}{llll}-0.3198669 & 3.65946433 & 0.34672288\end{array}$ $\begin{array}{llll}-0.3199566 & 6.36467674 & 0.01525895\end{array}$ $\begin{array}{llll}-0.3202516 & 6.63884341 & 0.07696562\end{array}$ $\begin{array}{llll}-0.3203446 & 7.12247464 & 0.00932378\end{array}$ $\begin{array}{llll}-0.3208612 & 5.04532194 & 0.12857234\end{array}$ $\begin{array}{llll}-0.3211616 & 9.29658516 & 0.00020465\end{array}$ $\begin{array}{llll}-0.3212196 & 6.7075596 & 0.01240211\end{array}$ $\begin{array}{llll}-0.32129 & 5.55683008 & 0.12033797\end{array}$ $\begin{array}{llll}-0.3216373 & 8.30540885 & 0.00113126\end{array}$ $\begin{array}{lllll}-0.3218871 & 5.98342209 & 0.06067108\end{array}$ $\begin{array}{llll}-0.3222922 & 7.43136754 & 0.00250735\end{array}$ $\begin{array}{llll}-0.3226146 & 3.43527673 & 0.36551347\end{array}$ $\begin{array}{llll}-0.3226764 & 6.72046486 & 0.00815003\end{array}$ $\begin{array}{llll}-0.3232168 & 8.37138911 & 0.00061285\end{array}$ $\begin{array}{llll}-0.3233605 & 6.9987674 & 0.00294038\end{array}$ $\begin{array}{llll}-0.3239119 & 6.95859378 & 0.00736189\end{array}$ $\begin{array}{llll}-0.3239543 & 4.37881225 & 0.21569708\end{array}$ $\begin{array}{llll}-0.3240806 & 5.802459 & 0.06589146\end{array}$ $\begin{array}{llll}-0.3244286 & 2.23569279 & 0.64805872\end{array}$ $\begin{array}{llll}-0.3249292 & 8.36393509 & 0.00061853\end{array}$ $\begin{array}{lll}-0.3254131 & 9.20935218 & 8.09 \mathrm{E}-06\end{array}$ $\begin{array}{lll}-0.3255817 & 5.2377779 & 0.1052399\end{array}$ $\begin{array}{llll}-0.3256735 & 4.02618408 & 0.2885451\end{array}$ transcription factor TFIIIC complex A box associated subuni1 $\begin{array}{llll}-0.3262064 & 7.14094517 & 0.00233675\end{array}$ $\# \mathrm{~N} / \mathrm{A}$

conserved GTPase Gpn2 (predicted)

$\begin{array}{llll}-0.3263109 & 5.39397544 & 0.07308347\end{array}$ $\begin{array}{lll}-0.3264666 & 6.79462896 & 0.00503157\end{array}$ $\begin{array}{lll}-0.3267208 & 7.1925659 & 0.0084121\end{array}$ $\begin{array}{llll}-0.3267963 & 7.32930341 & 0.00333497\end{array}$ $\begin{array}{llll}-0.3268206 & 5.34724103 & 0.08941711\end{array}$ cullin 1

Lsm2-8 complex Lsm8 (predicted) $\begin{array}{lllll}\text { multiple inositol polyphosphate phosphatase (predicted) } & -0.3270773 & 6.78456403 & 0.00641411 \\ \text { FAD-dependent oxidoreductase involved in late endosome t } & -0.3276161 & 7.43807498 & 0.00043846\end{array}$ DUF5310 family conserved fungal membrane protein $\quad \begin{array}{lllll}-0.3276326 & 3.2040057 & 0.44111441\end{array}$ Ctf18 RFC-like complex subunit Ctf18 $\begin{array}{rrr}-0.3285848 & 6.772149 & 0.02453867\end{array}$ histone demethylase SWIRM2 (predicted)
0.148866425

0.10596382

0.002141025

0.000353241

0.09831626

0.024358029

0.562983286

0.140465174

0.17949144

0.006042855

0.002487786

0.305830693

0.002750418

0.441686671

0.030784799

0.125131432

0.019784323

0.194300418

0.000640583

0.02556691

0.183939778

0.002996589

0.102093652

0.006144215

0.460956103

0.017517683

0.001734071

0.007072912

0.016026114

0.299799549

0.109604343

0.727249276

0.001748702

3.27E-05

0.163468956

0.381123714

0.005778193

0.119839284

0.011420895

0.01801836

0.007918112

0.142836478

0.014180635

0.001284982

0.537087768

0.046806722

0.00141122 
SPAC3H5.09c

SPNCRNA.1544

SPBC16H5.14C

SPBPB10D8.04

SPBC3H7.03C

SPNCRNA.1144

SPAC926.02

SPAC24H6.03

SPNCRNA.976

SPAC2G11.13

SPAC8F11.03

SPBC1921.07C

SPAP27G11.02

SPCC962.05

SPAP27G11.03

SPNCRNA.1550

SPNCRNA.1418

ScpofMt30

SPBC19C2.08

SPAC6G9.11

SPNCRNA.1293

SPCP31B10.02

SPAC8C9.03

SPNCRNA.895

SPAP14E8.03

SPBC13G1.12

SPNCRNA.1635

SPNCRNA.709

SPBPJ4664.06

SPAC3A12.08

SPBC28F 2.07

SPAC589.09

SPBC11B 10.08

SPAC3H8.09c

SPCC777.17c

SPNCRNA.1089

SPCC622.01C

SPBC336.11

SPCC622.10c

SPBC405.03C

SPBC19C7.10

SPBC800.02

SPCC1235.11 sei1

SPAC1B3.10C

SPBC800.14C

SPAC3H5.09c

$\# \mathrm{~N} / \mathrm{A}$

SPBC16H5.14C

SPBPB10D8.04c

kgd1

\#N/A

SPAC926.02

cul3

\#N/A

atg22

msh3

sgf29

mgr3

ast1

cdc123

prp38

syb1

$\# \mathrm{~N} / \mathrm{A}$

SPCP31B10.02 cgs1

\#N/A

bos 1

did2

$\# \mathrm{~N} / \mathrm{A}$

\#N/A

gpt1

SPAC3A12.08

sfr1

csr101

SPBC11B10.08

nab3

SPCC777.17c

$\# \mathrm{~N} / \mathrm{A}$

SPCC622.01C

vps52

sec5

SPBC405.03C

bqt4

whi5

$\mathrm{mpc} 1$ seipin, lipid droplet associated protein Sei1 (predicted)

SEL1 repeat protein, ERAD E3 ligase adaptor subunit (predis $-0.329883 \quad 6.857140360 .00594207$

mitochondrial tRNA(Ile)-lysidine synthetase Til1 (predicted) $\begin{array}{cllll}0.3301776 & 5.77903981 & 0.04402156\end{array}$

mitochondrial DUF1772 family protein, multimembrane spi $-\begin{array}{lllll}0.3303413 & 6.08324763 & 0.03159427\end{array}$

mitochondrial protein, human KIAA0100 ortholog (predictec $-0.3303821 \quad 8.71300798 \quad 0.00023023$

$$
\# \mathrm{~N} / \mathrm{A}
$$

short chain dehydrogenase DHRS3 family, implicated in lipi $\quad \begin{array}{cccc}-0.3306382 & 6.73645816 & 0.01141283\end{array}$

transmembrane transporter (predicted)

2-oxoglutarate dehydrogenase (lipoamide) (e1 component, $-0.3307173 \quad 9.81666887 \quad 7.83 \mathrm{E}-05$$$
\# \mathrm{~N} / \mathrm{A}
$$

MutS protein homolog

SAGA complex subunit Sgf29

TPR repeat protein, involved in mitochondrial protein turnol $\begin{array}{lllll}-0.3324083 & 6.48447942 & 0.03186589\end{array}$

nuclease, XP-G family protein Ast1

translation initiation factor elF2 assembly protein

$$
\# N / A
$$

$\# N / A$

$\# \mathrm{~N} / \mathrm{A}$

U4/U6 x U5 tri-snRNP complex subunit Prp38 SNAP receptor, synaptobrevin family

$$
\# \mathrm{~N} / \mathrm{A}
$$

mitochondrial oxidoreductase-like protein, human OXLD1 ol -0.33395875 .525015610 .06911126$ $\begin{array}{lllll}\text { cAMP-dependent protein kinase regulatory subunit Cgs1 } & -0.3340224 & 8.87087523 & 0.00067129\end{array}$

$$
\text { \#N/A }
$$

SNARE Bos1 (predicted)

ESCRT III complex subunit Did2 (predicted)

$$
\# N / A
$$

$\# \mathrm{~N} / \mathrm{A}$

UDP-glucose-glycoprotein glucosyltransferase Gpt1 acyl-coenzyme A thioesterase

Swi five-dependent recombination mediator $\mathrm{Sfr} 1$

sec14 cytosolic factor family, phospholipid-intermembrane WW domain containing conserved fungal protein

poly(A) binding protein Nab3 (predicted)

mitochondrial ribosomal protein subunit $L 9$ (predicted)

$$
\# \text { N/A }
$$

Schizosaccharomyces pombe specific protein

GARP complex subunit Vps52 (predicted)

exocyst complex subunit Sec5 (predicted)

transmembrane transporter (predicted)

bouquet formation protein Bqt4

cell cycle transcriptional repressor Whi5 (predicted)

mitochondrial carrier, pyruvate Mpc1 (predicted) $\begin{array}{lll}-0.3298462 & 6.32996744 \quad 0.03965128\end{array}$

$\begin{array}{llll}-0.3305374 & 6.32085753 & 0.02358847\end{array}$

$\begin{array}{lll}0.3313724 & 6.89317285 & 0.00851117\end{array}$

$\begin{array}{lll}-0.3315559 & 6.47339687 & 0.0081793\end{array}$

$\begin{array}{llll}0.3316201 & 7.04785356 & 0.00704977\end{array}$

$\begin{array}{llll}-0.3320639 & 2.53328561 & 0.64350918\end{array}$

$\begin{array}{lll}-0.3321374 & 7.25301258 & 0.00310696\end{array}$

$\begin{array}{lll}-0.3321739 & 7.05115485 & 0.00699427\end{array}$

$\begin{array}{lll}-0.33233 & 6.02977973 & 0.05679856\end{array}$

$\begin{array}{lll}-0.3325009 & 5.23805153 & 0.07493262\end{array}$

$\begin{array}{llll}-0.3326437 & 5.88628838 & 0.05124371\end{array}$

$\begin{array}{lll}-0.3332042 & 4.77343997 & 0.17682393\end{array}$

$\begin{array}{llll}-0.3333915 & 5.83118922 & 0.04438115\end{array}$

$\begin{array}{lll}-0.33369 & 2.7546035 & 0.45326021\end{array}$

$\begin{array}{lll}-0.3337159 & 4.98317297 & 0.15269876\end{array}$

$\begin{array}{llll}-0.3338389 & 7.73397992 & 0.01352722\end{array}$

$\begin{array}{llll}-0.3338957 & 3.34672502 & 0.44976151\end{array}$

0.07098655

0.013286398

0.077622802

0.058328539

0.000713761

0.045267769

0.023743301

0.076749177

0.000262506

0.01820847

0.017563938

0.015415709

0.722852727

0.007440316

0.015309094

0.09655613

0.058685568

0.122282135

0.088483493

0.253511474

0.077999406

0.548344124

0.224479375

0.027643457

0.545177606

0.114012643

0.00188923

0.091280675

$\begin{array}{lllll}-0.3343216 & 6.53477558 & 0.01442899 & 0.02932774\end{array}$

$\begin{array}{lllll}-0.3345801 & 6.44223259 & 0.02368228 & 0.045396603\end{array}$

$\begin{array}{lll}-0.3345801 & 6.44223259 & 0.02368228\end{array}$

$\begin{array}{llll}-0.3350714 & 3.58433304 & 0.35684714 & 0.452458507\end{array}$

$\begin{array}{llll}-0.3365681 & 8.3531957 & 1.78 \mathrm{E}-05 & 6.74 \mathrm{E}-05\end{array}$

$\begin{array}{lll}-0.3367364 & 5.76573289 & 0.08347766\end{array}$

$\begin{array}{lll}-0.3368104 & 5.17154352 & 0.08294443\end{array}$

0.031465549

0.04635281

0.048800805

0.100528072

0.408681992

0.022923245

0.014829784

0.000402739

0.151082695

0.003166555

0.172976007

0.003267743 
SPAC56F8.10

SPNCRNA.1423

SPAC1687.18C

SPCC5E4.06

SPAC17G8.10c

SPBP8B7.32

SPNCRNA.670

SPBC1773.08c

SPAC19B12.12C

SPAC144.04C

SPNCRNA.1556

SPBC25H2.13C

SPNCRNA.1061

SPBC56F2.07C

SPNCRNA.493

SPAC13F5.02C

SPNCRNA.1501

SPAC323.06c

SPNCRNA.1626

SPBC8D2.12C

SPNCRNA.763

SPNCRNA.254

SPBC14F5.07

SPAC1751.01C

SPAC22H10.11C

SPCC18B5.01C

SPBC16A3.19

SPAC11E3.01C

SPNCRNA.757

SPBC947.08C

SPAPB21F 2.02

SPBPB10D8.05

SPAC4F8.04

SPNCRNA.321

SPNCRNA.1542

SPCC1919.14C

SPNCRNA.436

SPAC3H1.01C

SРBC3B9.03

SPCC663.10

SPBPB10D8.06C

SPAC6B12.13

SPCPB1C11.03

SPAC323.08 spe1

cdc20 \#N/A SPBC56F2.07C $\# \mathrm{~N} / \mathrm{A}$

taf7 $\# \mathrm{~N} / \mathrm{A}$

uba5

doa10

gti1

crf1

bfr1

eaf7

swr1

$$
\text { \#N/A }
$$

hip4

dop1

SPBPB10D8.05C

SPAC4F8.04

$\# \mathrm{~N} / \mathrm{A}$

$\# N / A$

bdp1

$\#$ N/A

orc3

srp101

SPBPB10D8.06C

SPAC6B12.13

SPCPB1C11.03

rmp1 carboxylic acid transmembrane transporter (predicted)

$\begin{array}{lll}-0.3390898 & 6.11246939 & 0.05266823\end{array}$

$\begin{array}{llll}0.3391231 & 4.45999377 & 0.24398301\end{array}$

$$
\# \mathrm{~N} / \mathrm{A}
$$

methylenetetrahydrofolate reductase Met 9

$$
\# N / A
$$

cohesin loading factor $\mathrm{Ssl} 3$

Smc5-6 complex SMC P-loop ATPase subunit Smc6

mitotic spindle checkpoint ubiquitin ligase $\mathrm{Dma1}$

$$
\# \mathrm{~N} / \mathrm{A}
$$$$
\# \mathrm{~N} / \mathrm{A}
$$

alpha-1,2-mannosyltransferase Omh4 (predicted)

SMN family protein Yip11

ornithine decarboxylase Spe1 (predicted)

$$
\text { \#N/A }
$$

DNA polymerase epsilon catalytic subunit Pol2

$$
\text { \#N/A }
$$

ribosome biogenesis factor recycling AAA family ATPase ( $p$

$$
\# \mathrm{~N} / \mathrm{A}
$$

transcription factor TFIID complex subunit Taf7$$
\# N / A
$$

NEDD8 activating enzyme E1-type Uba5 (predicted)

$$
\text { \#N/A }
$$

mitochondrial Cox1 translational activator Tac1 (predicted)

$$
\# N / A
$$

ER ubiquitin-protein ligase E3 Doa10 (predicted)

gluconate transmembrane transporter inducer Gti1

transcriptional corepressor for ribosomal proteins via TOR

plasma membrane brefeldin A efflux transporter Bfr1

histone acetyltransferase complex subunit Eaf7

SNF2 family ATP-dependent DNA helicase Swr1

$$
\text { \#N/A }
$$

histone promoter control protein Hip4

Dopey family protein Dop1

transmembrane transporter (predicted)

Brix domain protein Rpf1 (predicted)

$$
\# N / A
$$

$$
\# \mathrm{~N} / \mathrm{A}
$$

transcription factor TFIIIB complex subunit Bdp1 (predicted

$$
\# N / A
$$

origin recognition complex subunit Orc3

signal recognition particle receptor alpha subunit Srp101 (

tRNA (uracil) methyltransferase Trm44 (predicted)

transmembrane transporter (predicted)

protein phosphatase type I regulatory subunit (predicted)

cysteine transmembrane transporter (predicted)

RNase MRP subunit Rmp1 $\begin{array}{lll}-0.3393012 & 7.58672257 & 0.00028895\end{array}$

$\begin{array}{lll}-0.3397181 & 7.39934823 & 0.0008686\end{array}$

$\begin{array}{lll}0.3397369 & 1.53150008\end{array}$

$\begin{array}{lll}-0.3404815 & 7.33351976 & 0.00267601\end{array}$

$\begin{array}{llll}-0.3409961 & 7.58596617 & 0.00026574\end{array}$

$\begin{array}{llll}-0.3414151 & 6.8125369 & 0.00262123\end{array}$

$\begin{array}{llll}-0.3415271 & 8.00912391 & 0.00419776\end{array}$

$\begin{array}{lll}-0.3416332 & 3.90521961 & 0.36145762\end{array}$

$\begin{array}{llll}-0.3419345 & 5.99254695 & 0.03138627\end{array}$

$\begin{array}{llll}-0.3431833 & 5.95532333 & 0.05316015\end{array}$

$\begin{array}{lll}-0.3434478 & 8.63623042 & 1.96 \mathrm{E}-05\end{array}$

$\begin{array}{lll}-0.3437434 & 3.7207914 & 0.3224545\end{array}$

$\begin{array}{lll}-0.3437684 & 8.50507868 & 0.0015506\end{array}$

$\begin{array}{lll}-0.3438266 & 6.84880405 & 0.00738746\end{array}$

$\begin{array}{lll}6.8659767 & 0.00226945\end{array}$

$\begin{array}{lll}0.3447376 & 1.53158592\end{array}$

$\begin{array}{llll}-0.3448909 & 7.0370222 & 0.00219434\end{array}$

$\begin{array}{llll}-0.3451317 & 4.23986586 & 0.27327612\end{array}$

$\begin{array}{llll}0.3457943 & 6.01417021 & 0.03347902\end{array}$

$\begin{array}{lll}-0.3460369 & 8.81655243 \quad 9.46 \mathrm{E}-06\end{array}$

$\begin{array}{llll}-0.3469692 & 6.4044485 & 0.01385437\end{array}$

$\begin{array}{llll}-0.3470185 & 7.39569804 & 0.00044987\end{array}$

$\begin{array}{llll}-0.3470251 & 5.58751065 & 0.03786056\end{array}$

$\begin{array}{llll}-0.347151 & 8.25762889 & 0.00025341\end{array}$

$\begin{array}{llll}-0.3473094 & 7.3494739 & 0.00084793\end{array}$

$\begin{array}{lll}-0.347425 & 8.20711704 & 2.42 \mathrm{E}-05\end{array}$

$\begin{array}{lll}-0.3476966 & 8.46802491 \quad 2.77 \mathrm{E}-05\end{array}$

$\begin{array}{lll}-0.3477302 & 4.93483653 & 0.21195716\end{array}$

$\begin{array}{lll}-0.3480602 & 8.28012204 & 5.16 \mathrm{E}-05\end{array}$

$\begin{array}{llll}-0.3484386 & 3.95603879 & 0.30530575\end{array}$

$\begin{array}{lll}-0.3488457 & 6.04579233 & 0.01896532\end{array}$

$\begin{array}{lll}-0.3493076 & 8.93851354 & 2.59 \mathrm{E}-05\end{array}$

$\begin{array}{llll}-0.3496341 & 7.83256456 & 0.04087565\end{array}$

$\begin{array}{llll}-0.3496894 & 6.42522754 & 0.01654803\end{array}$

$\begin{array}{llll}-0.3499107 & 3.95519002 & 0.34307746\end{array}$

$\begin{array}{llll}-0.3500988 & 5.42070985 & 0.16158832\end{array}$

$\begin{array}{llll}-0.3503272 & 6.37406809 & 0.03812687\end{array}$

$\begin{array}{llll}-0.3503436 & 4.34817517 & 0.21676902\end{array}$ $\begin{array}{llll}-0.3523376 & 7.24676893 & 0.00365976\end{array}$ $\begin{array}{llll}0.3528583 & 7.24130937 & 0.00474657\end{array}$ $\begin{array}{lll}-0.3529258 & 7.98291973 \quad 8.10 \mathrm{E}-05\end{array}$ $\begin{array}{lll}-0.3529341 & 7.84016662 & 0.03815127\end{array}$ $\begin{array}{llll}-0.3530151 & 6.96115443 & 0.00180427\end{array}$ $\begin{array}{llll}-0.3530848 & 6.86793676 & 0.00143395\end{array}$ $\begin{array}{llll}-0.3533318 & 4.91546935 & 0.14823212\end{array}$
0.090621317

0.333260615

0.000876235

0.002366216

0.006501335

0.000813486

0.006377358

0.009716244

0.457111898

0.057975996

0.091329156

7.36E-05

0.416751408

0.003981826

0.016061226

0.005632252

0.005461776

0.364850695

0.06135866

3.76E-05

0.028261078

0.001315024

0.068177055

0.000778532

0.002316393

$8.95 \mathrm{E}-05$

0.000101066

0.295325236

0.000178739

0.398849227

0.037484463

9.53E-05

0.072854581

0.033110655

0.437772762

0.235361408

0.068638443

0.300982082

0.008578939

0.01086273

0.000270587

0.06864605

0.004567728

0.003707449

0.218953676 
SPAC11E3.09

SPAC630.07c

SPAC17H9.03C

SPAC750.06c

SPCC126.02C

SPAC3G9.02

SPNCRNA.783

SPCC1840.12

SPCC18B5.08C

SPAC17A5.01

SPBC409.23

SPAC823.08c

SPNCRNA.1088

SPBC1778.05C

SPCC24B10.12

SPBC1A4.11C

SPNCRNA.1045

SPNCRNA.1020

SPNCRNA.120

SPBC16E9.07

SPNCRNA.851

SPNCRNA.498

SPBC3E7.04C

ScpofMt33

SPBC17D11.02C

SPBC725.05C

SPBC16H5.13

SPAC3H1.12C

SPAC57A7.09

SPNCRNA.797

SPBC609.03

SPNCRNA.1671

SPNCRNA. 271

SPBC215.01

SPAC1296.05c

SPCC1450.16c

SPNCRNA.767

SPAC24H6.01C

SPNCRNA.1106

SPNCRNA.872

SPBC18H10.19

SPNCRNA.501

SPAC18B11.05

SPBC4.03C

SPBC8D2.15

SPAC13C5.06C

SPBC1685.17 рур3

SPAC630.07c

rdl1

SPAC750.06c

ku70

oar2

\#N/A

opt3

ism 1

pex6

$\operatorname{mim} 2$

$\# N / A$

lam2

cgi121

SPBC1A4.11C

$\# N / A$

$\# N / A$

$\# \mathrm{~N} / \mathrm{A}$

mug100

$\# N / A$

\#N/A

SPBC3E7.04C

$\#$ N/A

hrd1

npp1

wdr7

snt2

SPAC57A7.09

$\# \mathrm{~N} / \mathrm{A}$

iqw1

$\#$ N/A

$\# N / A$

SPBC215.01

Icp1

ptl1

\#N/A

gup1

\#N/A

$\# \mathrm{~N} / \mathrm{A}$

\#N/A

gpi18

sfb3

lip5

mug121

SPBC1685.17 protein-tyrosine phosphatase Pyp3

Schizosaccharomyces specific protein

RAD51D-like protein 1

S. pombe specific DUF999 protein family 4

Ku domain protein Pku70

3-oxoacyl-[acyl-carrier-protein] reductase Oar2 (predicted) $\# N / A$

$\begin{array}{lll}0.3544501 & 6.17990158 & 0.03021743\end{array}$ $\begin{array}{lll}-0.3550838 & 5.82685363 & 0.0275762\end{array}$ $\begin{array}{lll}-0.3552424 & 5.20986883 & 0.13771539\end{array}$ $\begin{array}{lll}-0.3554212 & 5.62050093 & 0.06029233\end{array}$ $\begin{array}{lll}-0.3554567 & 6.62646235 & 0.00459492\end{array}$ $\begin{array}{llll}0.3557289 & 5.74486925 & 0.05254043\end{array}$ $\begin{array}{llll}-0.3557653 & 6.01021747 & 0.02858625\end{array}$ OPT oligopeptide transmembrane transporter family protei $-\begin{array}{lllll}-0.355906 & 6.47506208 & 0.01133889\end{array}$ mitochondrial isoleucine-tRNA ligase (predicted) $\quad-\begin{array}{llll}0.3559174 & 7.43217653 & 0.00115418\end{array}$ peroxin-6 (predicted) $\quad \begin{array}{lllll}-0.356326 & 7.03259741 & 0.00149591\end{array}$ mitochondrial outer membrane translocase complex assem $\quad \begin{array}{lllll}0.3564195 & 5.26036209 & 0.08339523\end{array}$ ATP-dependent RNA helicase Rrp3 (predicted)

$$
\# N / A
$$

Ragulator complex subunit, human LAMTOR2 ortholog

EKC/KEOPS complex subunit Cgi121 (predicted)

Schizosaccharomyces specific protein

$$
\# N / A
$$

N/A

Schizosaccharomyces pombe specific protein Mug100

$\# N / A$

Ric8 family guanine nucleotide exchange factor, human sy \#N/A

$\begin{array}{lll}-0.3567975 & 6.74332789 & 0.00780878\end{array}$ $\begin{array}{lll}-0.356837 & 3.59711573 & 0.31875093\end{array}$ $\begin{array}{lll}-0.3573833 & 7.07641077 \quad 0.00286958\end{array}$ $\begin{array}{lll}0.3574118 & 5.62110026 & 0.04296328\end{array}$ $\begin{array}{llll}-0.3574974 & 3.98513828 & 0.28490286\end{array}$ $\begin{array}{lll}-0.3575273 & 4.52289702 & 0.25447\end{array}$ $\begin{array}{lll}-0.3576263 & 3.91882474 & 0.31750211\end{array}$ $\begin{array}{llll}-0.3580728 & 4.09892431 & 0.25819793\end{array}$ $\begin{array}{lll}-0.3581728 & 5.50819418 & 0.08247694\end{array}$ $\begin{array}{llll}-0.3582522 & 5.53066933 & 0.04015008\end{array}$ $\begin{array}{lll}-0.3584 & 5.23647777 & 0.07343613\end{array}$ $\begin{array}{llll}-0.3588961 & 5.33883416 & 0.18286387\end{array}$ $\begin{array}{llll}-0.3588983 & 4.15293679 & 0.24305779\end{array}$ $\begin{array}{llll}-0.359336 & 7.38415517 & 0.00014946\end{array}$ $\begin{array}{lll}-0.3593751 & 7.08170576 & 0.00362438\end{array}$ $\begin{array}{lll}-0.3594812 & 7.84013323 & 0.00041311\end{array}$ $\begin{array}{llll}-0.3598655 & 7.59059046 & 0.00025215\end{array}$

Hrd1 complex ubiquitin-protein ligase E3 subunit, $\mathrm{Hrd} 1$ nucleotide pyrophosphatase Npp1 (predicted) WD repeat protein, human WDR7 ortholog

Lid2 complex PHD finger subunit Snt2

ubiquitin-protein ligase E3, human RNF13 family homolog, $-\begin{array}{lll}-0.3602128 & 6.74592775 & 0.00717202\end{array}$ $\begin{array}{lll}-0.3607664 & 6.16202902 & 0.01226302\end{array}$ $-0.36077347 .64746592 \quad 0.00018986$ $\begin{array}{llll}0.3609546 & 4.94225915 & 0.12546688\end{array}$ $\begin{array}{lll}-0.361583 & 2.09845583 & 0.62441913\end{array}$ $\begin{array}{lll}-0.3617589 & 8.29203741 & 6.30 \mathrm{E}-05\end{array}$ $\begin{array}{lll}0.3617857 & 6.57236751 & 0.00569107\end{array}$ $\begin{array}{lll}-0.3618739 & 6.72143176 & 0.01436495\end{array}$ $\begin{array}{lll}-0.3619191 & 4.30143334 & 0.16984275\end{array}$

family cyclin

triacylglycerol lipase ptl1

$$
\# \mathrm{~N} / \mathrm{A}
$$

$\begin{array}{llll}0.3623594 & 6.22211408 & 0.03196409\end{array}$

membrane bound O-acyltransferase, MBOAT Gup1 (predict - 0.3623594

$\# N / A$

$\begin{array}{llll}0.3624387 & 4.66522221 & 0.24130547\end{array}$ $\begin{array}{llll}-0.362731 & 7.38787794 & 0.00027775\end{array}$ phophatidylinositol 3-kinase complex subunit Vps38 $\quad-0.3637601 \quad 6.10515065 \quad 0.02449744$

$$
\# \mathrm{~N} / \mathrm{A}
$$

$-0.363844 \quad 4.59051762$ $\begin{array}{lll}-0.3640435 & 6.87062955 & 0.00478208\end{array}$ $\begin{array}{llll}-0.3642564 & 4.76520576 & 0.25663179\end{array}$ $\begin{array}{lll}-0.3645598 & 7.11531599 & 0.00035511\end{array}$ $\begin{array}{llll}-0.3652227 & 6.64984589 & 0.00671051\end{array}$ $\begin{array}{lll}-0.3661003 & 4.99977692 & 0.08676663\end{array}$
0.056075704

0.051795765

0.205832024

0.101632226

0.010552962

0.090470049

0.053412806

0.023611112

0.003052531

0.00385302

0.1343206

0.016858739

0.413083239

0.006927487

0.076136755

0.377853043

0.344798307

0.411621619

0.348739608

0.133030478

0.071768128

0.120330945

0.260932817

0.332263617

0.000478835

0.008505028

0.001214958

0.000775363

0.01564281

0.025295497

0.000596773

0.190368372

0.705959821

0.000214878

0.012768788

0.029223768

0.24503258

0.058803905

0.330133434

0.000845691

0.046748484

0.236354483

0.010934832

0.346916831

0.001062203

0.014744923

0.138994097 
SPAC6F 6.13C SPAC25B8.13C SPBC1604.16C SPAC57A7.13

SPBC2F12.15C SPBC1683.06C SPBC24C6.10c SPCC1682.03C SPNCRNA.1667 SPBC23E6.03C SPAC14C4.07

SPAC4F8.03

SPNCRNA.1504

SPAC31G5.12C

SPNCRNA.907

SPBC146.05C

SPCC965.13

SPNCRNA.845

SPBC31F10.11C

SPAC1039.09

SPNCRNA.846

SPRRNA.50

SPBC1718.07C

SPBC17D1.03C

SPBC902.05C

SPAC30D11.05

SPNCRNA.1555

SPCC330.19c

SPCC11E10.02C

SPAC343.04C

SPCC63.13

SPNCRNA.1641

SPBC11G11.02

SPAC140.02

SPNCRNA.682

SPNCRNA.87

SPBC337.02C

SPAC17H9.06C

SPBC713.09

SPCC1672.06C

SPNCRNA.380

SPAC17C9.01c

SPNCRNA.1243

SPCC320.12

SPBC660.15

SPBC19G7.18C

SPBC21C3.07C
SPAC6F $6.13 \mathrm{C}$

isp 7

SPBC1604.16C

SPAC57A7.13

pfa3

rh1

dip1

mug174

\#N/A

nta1

SPAC14C4.07

sdo1

$\# N / A$

maf1

\#N/A

cwf25

SPCC965.13

\#N/A

cwf4

isp5

$\# \mathrm{~N} / \mathrm{A}$

SPRRNA. 50

zfs1

rrp43

aps3

\#N/A

SPCC330.19c

gpi8

gid7

SPCC63.13

$\# \mathrm{~N} / \mathrm{A}$

end3

gar2

$\# \mathrm{~N} / \mathrm{A}$

$\# \mathrm{~N} / \mathrm{A}$

SPBC337.02C

SPAC17H9.06c

SPBC713.09

asp1

\#N/A

nuc2

\#N/A

atp23

msi2

SPBC19G7.18c

trm140
Golgi localized Alpha/Beta hydrolase fold, DUF726 family p $-0.3665772 \quad 8.14575788 \quad 0.00207405$ 2-OG-Fe(II) oxygenase superfamily protein $\quad-0.3666659 \quad 8.63186633 \quad 9.89 \mathrm{E}-07$ RNA-binding protein, G-patch type, human GPANK1 ortholo $-0.3671678 \quad 6.51610548 \quad 0.00347521$ RNA-binding protein, involved in splicing (predicted) $\quad-0.36729616 .536013210 .00410708$ palmitoyltransferase Pfa3 (predicted)

uridine ribohydrolase Urh1 (predicted)

WISH/DIP/SPIN90 ortholog, endocytosis protein Dip1

Schizosaccharomyces specific protein Mug174

$$
\text { \#N/A }
$$

protein $\mathrm{N}$-terminal amidase $\mathrm{Nta1}$ (predicted)

transmembrane transporter (predicted)

SBDS family ribosome assembly protein Sdo1 (predicted)

$$
\# \mathrm{~N} / \mathrm{A}
$$

repressor of RNA polymerase III Maf1

$$
\text { \#N/A }
$$

complexed with $\mathrm{Cdc} 5$ protein $\mathrm{Cwf} 25$

plasma membrane pyridoxal family transmembrane transp $\# N / A$

Prp19 complex subunit Cwf

amino acid transmembrane transporter Isp5

$$
\text { \#N/A }
$$

$\begin{array}{lll}-0.367513 & 6.52894047 & 0.0052922\end{array}$

$\begin{array}{lll}-0.3685081 & 7.24348779 & 0.00019135\end{array}$

$\begin{array}{llll}-0.3687532 & 6.33027938 & 0.01339669\end{array}$

$\begin{array}{llll}-0.3688109 & 5.93949195 & 0.04076024\end{array}$

$\begin{array}{llll}-0.3692991 & 6.87383396 & 0.00110583\end{array}$

$-0.3693396 \quad 7.94645136 \quad 1.81 \mathrm{E}-05$

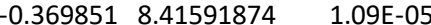

$\begin{array}{llll}-0.3698892 & 6.50890638 & 0.00590857\end{array}$

$\begin{array}{lll}-0.3703244 & 5.59875433 & 0.08844976\end{array}$

$\begin{array}{llll}-0.3704771 & 5.68589038 & 0.05861389\end{array}$

$\begin{array}{llll}-0.3708998 & 3.77970592 & 0.3209637\end{array}$

$\begin{array}{lll}-0.3710551 & 6.86925448 & 0.01615119\end{array}$

$\begin{array}{lll}-0.3710955 & 7.66769499 & 2.56 \mathrm{E}-05\end{array}$

$\begin{array}{llll}-0.3715499 & 2.02502067 & 0.58702848\end{array}$

$\begin{array}{lll}-0.3716922 & 7.17784891 & 0.0011369\end{array}$

$-0.37198419 .56004671 \quad 2.50 \mathrm{E}-08$

$\begin{array}{lll}-0.3720397 & 6.7097749 & 0.00175493\end{array}$

$\begin{array}{lll}-0.3724148 & 7.8586579 & 3.93 \mathrm{E}-05\end{array}$

$\begin{array}{lllll}5.8 S & \text { ribosomal RNA } & -0.3720397 & 6.7097749 & 0.00175493 \\ \text { zf-CCCH tandem zinc finger protein, human Tristetraprolin } \mathrm{r} & -0.3724278 & 8.2170788 & 0.00029638\end{array}$ $\begin{array}{lllll}-0.3733356 & 6.14069174 & 0.01137402\end{array}$

isocitrate dehydrogenase (NAD+) subunit 2

AP-3 adaptor complex subunit Aps3 (predicted) $\#$ N/A

Schizosaccharomyces pombe specific protein

pig-K

GID complex subunit Gid7 (predicted)

DNAJ domain protein

$$
\# \mathrm{~N} / \mathrm{A}
$$

actin cortical patch component End3 (predicted)

nucleolar protein required for rRNA processing

$$
\# \text { \#/A }
$$

mug2/mug135/meu2 family

DUF4196 and DUF4211, human CCDC82 ortholog

Schizosaccharomyces specific protein

inositol hexakisphosphate kinase/inositol pyrophosphate sy

$$
\# \mathrm{~N} / \mathrm{A}
$$

$0.3734592-8.5$

$\begin{array}{lll}-0.3735651 & 7.16590138 & 0.00018678\end{array}$

$\begin{array}{lll}-0.3739696 & 3.96774938 & 0.19695303\end{array}$

$\begin{array}{llll}-0.3744273 & 4.67117279 & 0.22567145\end{array}$

$\begin{array}{llll}-0.3744398 & 6.89445253 & 0.00324631\end{array}$

$\begin{array}{lll}-0.374478 & 7.73065204 & 0.00236036\end{array}$

$\begin{array}{lll}-0.3745933 & 5.57406636 & 0.06090424\end{array}$

$\begin{array}{llll}-0.3746508 & 6.32035523 & 0.01935165\end{array}$

$\begin{array}{lll}-0.37504 & 7.78351728 & 0.00022392\end{array}$

$\begin{array}{lll}-0.3752688 & 8.7915337 & 2.31 \mathrm{E}-06\end{array}$

$-0.3753857 \quad 5.21774214 \quad 0.06175783$

$\begin{array}{lll}-0.3762897 & 4.76620793 & 0.09679788\end{array}$

$\begin{array}{lll}-0.3766686 & 7.1328411 & 0.00205452\end{array}$

$-0.37666997 .96605769 \quad 5.78 \mathrm{E}-06$

$\begin{array}{lll}-0.3769828 & 7.32818782 & 0.00215301\end{array}$

$\begin{array}{llll}\text { \#N/A } & -0.3772724 & 2.30100398 & 0.63867495\end{array}$

$-0.3773352 \quad 6.538687950 .0017$

$\begin{array}{ccccc}\text { anaphase-promoting complex TPR lobe subcomplex subunit } & -0.3773352 & 6.53868795 & 0.00177504 \\ \text { \#N/A } & -0.3775216 & 4.66535797 & 0.18424356\end{array}$

mitochondrial inner membrane peptidase Atp23 (predicted -0.37798315 .554996640 .04652652$ mRNA cleavage factor complex subunit Msi2

Schizosaccharomyces specific protein

tRNA (cytosine) methyltransferase Trm140 (predicted)

$\begin{array}{lll}-0.3785972 & 8.1648118 \quad 3.77 \mathrm{E}-05\end{array}$

$\begin{array}{lll}-0.3786476 & 6.58424769 & 0.00384436\end{array}$

$\begin{array}{lll}-0.3787299 & 6.81992851 & 0.0015482\end{array}$
0.005179421

4.57E-06

0.008219961

0.009522538

0.011936829

0.000601156

0.02740965

0.072686956

0.002939513

6.83E-05

4.26E-05

0.013221898

0.141490511

0.099220773

0.415356293

0.032392832

9.45E-05

0.673193258

0.003009184

$1.45 \mathrm{E}-07$

0.004457736

0.000139086

0.00089678

0.023677026

$1.43 \mathrm{E}-05$

0.000588175

0.277430217

0.311373523

0.007734995

0.005832335

0.102410016

0.038137268

0.000696101

1.02E-05

0.103691596

0.152443779

0.005134422

2.40E-05

0.005364796

8.46E-05

0.718445649

0.004498755

0.262357918

0.081328794

0.000133965

0.008962267

0.003977169 
SPBC23E6.02

SPAC6F12.16C

SPNCRNA.810

SPNCRNA.160

SPAC12B10.04

SPAC959.06C

SPBC776.13

SPAC31A2.06

SPNCRNA.1337

SPAC13G6.12C

SPAC9G1.07

SPAC2F7.09C

SPBC26H8.10

SPCC550.03C

SPCC126.07c

SPBC1271.01c

SPBC1198.11C

SPBC1703.09

SPBC21.04

SPAC4H3.16

SPAC22F8.12C

SPNCRNA.523

SPBC3B9.14C

SPNCRNA.1316

SPAP8A3.05

SPBC29A3.07C

SPCC594.07c

SPBC428.16C

SPBPB10D8.07c syf 2

SPBC16G5.07C

$\# \mathrm{~N} / \mathrm{A}$

$\# N / A$

$\# N / A$

$\# \mathrm{~N} / \mathrm{A}$

pmp31

cut12

SPBC20F10.02 \#N/A

ubp15 $\# \mathrm{~N} / \mathrm{A}$

nob1

SPRRNA.51

$\# \mathrm{~N} / \mathrm{A}$

$\# \mathrm{~N} / \mathrm{A}$

rer2

rrp2

$\mathrm{mtr} 4$

$\# N / A$

$\# \mathrm{~N} / \mathrm{A}$

pby1

SPAC959.06c

cnd1

atp25

chs1

\#N/A

SPAC9G1.07

gep3

dis3

ski2

asr1

pof13

reb1

SPBC1703.09

med8

SPAC4H3.16

shf1

mrpl3

\#N/A

$\# \mathrm{~N} / \mathrm{A}$

ski7
sap14

bqt3

SPBPB10D8.07C
Prp19 complex subunit Syf2

stomatin (predicted)

$$
\begin{aligned}
& \# N / A \\
& \# N / A \\
& \# N / A \\
& \# N / A
\end{aligned}
$$

plasma membrane proteolipid Pmp31

spindle pole body protein Cut12

DUF1741 family protein, human C10orf76 ortholog \#N/A

ubiquitin C-terminal hydrolase Ubp15

$$
\# \text { N/A }
$$

ribosome biogenesis protein Nob1 (predicted)

$5.8 \mathrm{~S}$ ribosomal RNA

\section{$\# N / A$}

$\# \mathrm{~N} / \mathrm{A}$

cis-prenyltransferase

ATP-dependent DNA helicase/ ubiquitin-protein ligase E3 ( $k-0.38223097 .95491686 \quad 1.01 \mathrm{E}-05$

TRAMP complex ATP-dependent RNA helicase subunit Mtr4 $-0.3829402 \quad 8.37548136 \quad 4.48 \mathrm{E}-06$ $\# N / A$

$$
\text { \#N/A }
$$

tubulin-tyrosine ligase Pby1 (predicted)

alpha/beta hydrolase fold

condensin complex non-SMC subunit Cnd1

mitochondrial polynucleotide adenylyltransferase Atp25 (pr \#N/A

chitin synthase I

Schizosaccharomyces specific protein

mitochondrial GTPase related protein Gep3 (prediected)

exosome 3'-5' exoribonuclease subunit Dis3

Ski complex RNA helicase Ski2 (predicted)

ubiquitin-protein ligase E3 Asr1 (predicted)

F-box protein Pof13

RNA polymerase I transcription termination factor/ RNA po

Schizosaccharomyces specific protein

mediator complex subunit Med8

Schizosaccharomyces specific protein

small histone ubiquitination factor Shf1

$$
\text { \#N/A }
$$

mitochondrial ribosomal protein subunit L3 (predicted)

$$
\text { \#N/A }
$$

Ski complex interacting GTPase Ski7

U2 snRNP-associated protein SF3B14 Sap14

bouquet formation protein Bqt3

Rheb GTPase Rhb1

transmembrane transporter (predicted) $\begin{array}{lll}0.3787341 & 4.49872111 & 0.12271757\end{array}$ $\begin{array}{llll}-0.3787986 & 6.04171718 & 0.02005821\end{array}$ $\begin{array}{llll}-0.3789843 & 6.72745471 & 0.00125292\end{array}$ $\begin{array}{lll}-0.3793184 & 5.90781903 & 0.014435\end{array}$ $\begin{array}{llll}0.3794548 & 3.96897007 & 0.26430099\end{array}$ $\begin{array}{llll}0.3797127 & 6.15083885 & 0.00907122\end{array}$ $\begin{array}{llll}-0.3798496 & 3.63531233 & 0.27259143\end{array}$ $\begin{array}{llll}-0.380008 & 7.08856527 & 0.00167295\end{array}$ $\begin{array}{llll}-0.38024 & 6.46117003 & 0.00617401\end{array}$ $\begin{array}{llll}-0.3802826 & 5.63340749 & 0.04236567\end{array}$ $-0.3803283 \quad 8.43830212 \quad 1.01 \mathrm{E}-06$ $\begin{array}{lll}-0.3806619 & 9.70781888 \quad 8.37 E-05\end{array}$ $\begin{array}{llll}-0.3806707 & 6.72027301 & 0.0057218\end{array}$ $\begin{array}{llll}-0.3811504 & 6.58829163 & 0.00261838\end{array}$ $-0.38123257 .86352023 \quad 2.71 \mathrm{E}-05$ $\begin{array}{lll}-0.3812549 & 8.36599936 & 9.56 \mathrm{E}-06\end{array}$ $\begin{array}{llll}-0.3813777 & 6.5557798 & 0.00345527\end{array}$ $\begin{array}{llll}-0.3821072 & 6.64730328 & 0.00236394\end{array}$ $\begin{array}{llll}-0.3829871 & 5.0993905 & 0.14903346\end{array}$ $\begin{array}{llll}-0.3830099 & 2.39085013 & 0.56105684\end{array}$ $\begin{array}{llll}-0.3833236 & 6.43492034 & 0.00410677\end{array}$ $\begin{array}{llll}-0.3835974 & 4.17133303 & 0.18157844\end{array}$ $\begin{array}{lll}-0.3840032 & 7.78447633 & 0.00012887\end{array}$ $\begin{array}{lll}-0.3843697 & 8.05287926 & 3.87 \mathrm{E}-05\end{array}$ $\begin{array}{lll}-0.3844683 & 6.05042988 & 0.017932\end{array}$ $\begin{array}{lll}-0.3845662 & 6.22528476 & 0.00888889\end{array}$ $\begin{array}{llll}-0.3846428 & 7.06263483 & 0.00047575\end{array}$ $\begin{array}{llll}-0.3850445 & 6.07880048 & 0.01944069\end{array}$ $\begin{array}{lll}-0.3867035 & 8.54053438 \quad 4.04 \mathrm{E}-06\end{array}$ $\begin{array}{lll}-0.3869032 & 7.74411212 & 0.00134209\end{array}$ $\begin{array}{llll}0.3871261 & 6.73467801 & 0.00109212\end{array}$ $\begin{array}{llll}0.3873147 & 6.04086794 & 0.00883766\end{array}$ $\begin{array}{lll}6.9802522 & 0.00037565\end{array}$ $\begin{array}{llll}-0.3886929 & 4.18028713 & 0.30079912\end{array}$ $\begin{array}{llll}-0.3887704 & 7.53494821 & 0.00034487\end{array}$ $\begin{array}{llll}-0.3893074 & 6.85864995 & 0.00092576\end{array}$ $\begin{array}{lll}-0.3907466 & 7.87088328 & 1.05 E-05\end{array}$ $\begin{array}{llll}-0.3910127 & 3.20295691 & 0.36757741\end{array}$ $\begin{array}{llll}-0.3910884 & 6.7059148 & 0.00140328\end{array}$ $\begin{array}{llll}-0.3911873 & 6.04878309 & 0.02324431\end{array}$ $\begin{array}{llll}-0.3913777 & 5.77776865 & 0.02054822\end{array}$ $\begin{array}{llll}-0.3914194 & 5.35373021 & 0.03960286\end{array}$ $\begin{array}{lll}-0.39171 & 6.98781833 & 0.00319\end{array}$ $\begin{array}{llll}-0.3918284 & 6.84376154 & 0.00147421\end{array}$ $\begin{array}{llll}-0.3923055 & 7.89068101 & 0.01855503\end{array}$
0.186779935

0.039307772

0.003286851

0.029331202

0.355331135

0.019284494

0.364293932

0.004267031

0.01371667

0.075234439

4.66E-06

0.000279027

0.012833496

0.00637269

9.92E-05

3.79E-05

0.008187015

0.00583693

3.99E-05

1.89E-05

0.219946365

0.650811393

0.009522538

0.259261704

0.000417216

0.000137166

0.035617995

0.018938292

0.001384734

0.038268411

1.72E-05

0.003491185

0.00290762

0.018852741

0.001115803

0.393717645

0.001032948

0.002505889

4.15E-05

0.462958412

0.003635647

0.04470818

0.040073563

0.070956777

0.007619506

0.003798554

0.036769651 
SPBC2A9.14

SPNCRNA.663

SPBC23G7.06C

SPAC644.07

SPNCRNA.1215

SPBC2G2.01C

SPAC7D4.12C

SPNCRNA.1535

SPBC3B9.15C

SPCC622.13C

SPAC29E6.08

SPCC645.03C

SPAC3G $9.13 \mathrm{C}$

SPNCRNA.27

SPAC13D1.01C

SPAC26H5.08C

SPCC736.02

SPBC2D10.13

SPAC227.03C

SPAC2OH4.07

SPAC16E8.16

SPBC409.04c

SPAC4F8.07C

SPCP1E11.06

SPBP16F5.03C

SPBC1348.01

SPAC24H6.02C

SPAC110.02

SPAC17A5.06

SPBC1539.05

SPBC29A10.03C

SPCC1682.01

SPCC4E9.01C

SPAPB2B4.03

SPBCPT2R1.01C

SPAC1805.07c

SPBP22H7.03

SPBC1685.13

SPAC1250.01

SPCC1919.11

SPAC22F8.11

SPAC3H1.04C

SPAC12B10.13

SPAC31A2.05C

SPBC11B10.06

SPNCRNA.216

SPNCRNA.1189
SPBC2A9.14

$\# \mathrm{~N} / \mathrm{A}$

nvj2

bcs1

\#N/A

liz1

SPAC7D4.12C

\#N/A

scp1

tti1

tbp1

isa1

msw1

bg 12

SPCC736.02

est1

yea 6

rad57

sua7

mis12

hxk2

apl4

SPBC1348.01

tim15

pds5

ptr8

$\operatorname{cog} 3$

pcf1

qcr9

rec11

cig2

SPBCPT2R1.01C

dad2

sbg1

fhn1

snf21

mug137

plc1

mdm31

gid8

mis4

sws1

\#N/A

$\# N / A$
Schizosaccharomyces pombe specific protein

$$
\text { \#N/A }
$$

nucleus-vacuole junction protein $\mathrm{Nvj2}$

mitochondrial Rieske ISP assembly ATPase Bcs1 (predicted)

$$
\text { \#N/A }
$$
plasma membrane ThrE amino acid transmembrane transp $-\begin{array}{llll}0.3943888 & 6.87942707 & 0.00433913\end{array}$

$$
\# \mathrm{~N} / \mathrm{A}
$$

Sre1 cleavage activating protein, Scap Scp1

ASTRA complex subunit, Armadillo-type fold TTi1

TATA-binding protein (TBP)

$\begin{array}{lll}0.3943856 & 4.77851471 \quad 0.12301594\end{array}$

$-0.39445178 .38192442 \quad 6.98 \mathrm{E}-06$

$-0.39472118 .48050617 \quad 4.74 \mathrm{E}-06$

$\begin{array}{lll}-0.3951178 & 8.492846 \quad 1.32 \mathrm{E}-06\end{array}$

mitochondrial [4Fe-4S] cluster asssembly and transfer prot $-0.3952116 \quad 6.931592390 .00069929$

mitochondrial tryptophan-tRNA ligase Msw1 (predicted) $\quad \begin{array}{llll}-0.3952295 & 7.84692951 & 8.36 \mathrm{E}-06\end{array}$ \#N/A

retrotransposable element/transposon Tf2-type

glucan beta-glucosidase Bgl2 (predicted)

Schizosaccharomyces specific protein

telomerase regulator Est1

mitochondrial carrier, NAD Yea6 (predicted)

RecA family ATPase Rad57/Rhp57

transcription factor TFIIB

NMS complex subunit Mis12

hexokinase 2

AP-1 adaptor complex gamma subunit Apl4

SAGA complex phosphatidylinositol pseudokinase Tra1

S. pombe specific DUF999 protein family 5

TIM23 translocase complex subunit Tim15 (predicted)

mitotic and meiotic cohesin loader subunit Pds 5

$\begin{array}{lll}-0.3960549 & 2.68387686 & 0.5127184\end{array}$

$\begin{array}{lll}-0.3960806 & 6.51994733 & 0.00832645\end{array}$

$\begin{array}{lll}-0.3962125 & 7.37367669 & 0.00261174\end{array}$

$\begin{array}{lll}-0.3964227 & 5.15749644 & 0.06618455\end{array}$

$\begin{array}{llll}-0.3964619 & 4.77849593 & 0.06953794\end{array}$

$\begin{array}{llll}-0.3967758 & 6.29447259 & 0.00798085\end{array}$

$\begin{array}{lll}-0.3972361 & 6.6435611 & 0.00705665\end{array}$

$\begin{array}{lll}-0.3974212 & 7.49639063 & 0.0001345\end{array}$

$\begin{array}{lll}-0.3980108 & 6.92170851 & 0.00059211\end{array}$

$-0.3980711 \quad 9.8421206 \quad 1.78 \mathrm{E}-08$

$-0.39810478 .52599531 \quad 6.90 \mathrm{E}-07$

$\begin{array}{lll}-0.3981157 & 9.65210294 \quad 6.87 E-09\end{array}$

$\begin{array}{lll}-0.3985365 & 6.07013394 & 0.02019009\end{array}$

$\begin{array}{lll}-0.3987376 & 5.65003175 & 0.01914652\end{array}$

$\begin{array}{lll}-0.3999312 & 7.4348308 & 0.00084003\end{array}$

transcription factor TFIIH complex DNA helicase (ERCC-3) sI $-0.4000687 \quad 7.90935856 \quad 2.77 \mathrm{E}-05$

$\begin{array}{llllll}\text { Golgi transport complex subunit } \operatorname{Cog} 3 \text { (predicted) } & -0.4000979 & 6.24141523 & 0.00825699\end{array}$

CAF assembly factor (CAF-1) complex large subunit Pcf1 $\quad \begin{array}{llll}-0.4005503 & 7.06161826 & 0.00861701\end{array}$

ubiquinol-cytochrome-c reductase complex subunit 9 (predi $-0.4008861 \quad 7.11776875 \quad 7.09 \mathrm{E}-05$

meiotic cohesin complex subunit Rec11

G1/S-specific B-type cyclin Cig2

. pombe specific DUF999 protein family 9

DASH complex subunit Dad2

plasma membrane-actinomyosin ring linker protein Sbg1

eisosome assembly protein Fhn

RSC-type complex ATP-dependent DNA helicase Snf21

BAR adaptor protein, involved in endocytosis (predicted)

phosphoinositide phospholipase C PIc1

mitochondrial inner membrane protein Mdm31

GID complex subunit Gid8 (predicted)

cohesin loading factor (adherin) Mis4

Srs2 interacting SWIM domain protein Srs1

$\# N / A$

$\# N / A$ $\begin{array}{lll}-0.401091 & 5.16105362 & 0.10297246\end{array}$

$\begin{array}{lll}-0.401513 \quad 8.37991772 & 3.23 \mathrm{E}-05\end{array}$

$\begin{array}{lll}-0.4020204 & 6.06129955 & 0.01437776\end{array}$

$\begin{array}{llll}-0.402423 & 4.54496732 & 0.23143376\end{array}$

$\begin{array}{lll}-0.403182 & 6.36703955 & 0.00678563\end{array}$

$-0.4036265 \quad 9.07988589 \quad 6.98 \mathrm{E}-07$

$-0.403908 \quad 8.50298454 \quad 1.70 \mathrm{E}-06$

$\begin{array}{lll}-0.4042042 & 6.93133533 & 0.00187906\end{array}$

$\begin{array}{lll}-0.404231 & 8.04613979 \quad 4.41 \mathrm{E}-06\end{array}$

$\begin{array}{llll}-0.404426 & 7.60289679 & 3.60 \mathrm{E}-05\end{array}$

$\begin{array}{llll}-0.4045596 & 6.32053525 & 0.00815729\end{array}$

$\begin{array}{llll}-0.4049443 & 7.15965911 & 0.00172241\end{array}$

$\begin{array}{llll}-0.4049787 & 5.6955832 & 0.02258594\end{array}$

$\begin{array}{lll}-0.4050654 & 4.96569297 & 0.11586477\end{array}$

$-0.405467 \quad 7.48036677 \quad 1.15 \mathrm{E}-05$
0.130172869

201912939

0.00497051

0.059598257

0.028716458

0.014437668

0.009999243

0.187192196

2.85E-05

1.99E-05

6.01E-06

0.001961857

3.36E-05

0.604373591

0.017851766

0.006361085

0.1100112

0.114660936

0.017181163

0.015420831

0.000434402

0.001680274

$1.05 \mathrm{E}-07$

3.26E-06

4.30E-08

0.039514977

0.037798689

0.002299425

0.000101097

0.017708412

0.018416694

0.000239769

0.160680486

0.000116062

0.029241072

0.317970272

0.014885954

3.29E-06

$7.62 \mathrm{E}-06$

0.004741196

1.86E-05

0.000128307

0.017522213

0.004386603

0.043577355

0.177821323

4.50E-05 
SPAC1D4.12

SPNCRNA.1386

SPCC1183.05

SPAC167.05

SPAC1F5.09c

SPNCRNA.558

SPBC12C2.02C

SPAC140.04

SPBC365.09c

SPBC27B12.01C

SPAC29E6.02

SPBC21.05C

SPAC2E1P5.01C

SPNCRNA.1140

SPAPB1A10.07c

SPNCRNA.619

SPNCRNA.1095

SPBC6B1.03C

SPAC2C4.07C

SPBC25H2.18

SPNCRNA.975

SPAPB2B 4.04C

SPCC645.11C

SPBC4B4.05

SPNCRNA.1543

SPNCRNA.634

SPNCRNA.794

SPAP4C9.02

SPCC162.06C

SPNCRNA.1027

SPBC26H8.03

SPBC18H $10.06 \mathrm{C}$

SPAC4D7.07C

SPCC18.19C

SPAC4D7.13

SPNCRNA.1244

SPNCRNA.836

SPNCRNA.984

SPCC364.05

SPAC9G 1.14

SPBCPT2R1.04C

SPBP19A11.07C

SPAC688.09

SPCC1020.13C

SPAC3A12.02

SPCC622.09

SPBC8D2.02C $\operatorname{rad} 15$

\#N/A

lig4

dbs2

shk2

$\# N / A$

ste20

ctr1

kin17

$\mathrm{mmm} 1$

prp3

ral2

mns1

$\# N / A$

SPAPB1A10.07

$\# N / A$

$\# \mathrm{~N} / \mathrm{A}$

SPBC6B1.03C

dis32

$\operatorname{cox} 20$

$\# N / A$

pmc1

mug117

smg1

$\# \mathrm{~N} / \mathrm{A}$

$\# N / A$

$\# N / A$

emc5

vps60

$\# N / A$

cho2

swd2

csi2

ost5

usp104

$\# N / A$

$\# N / A$

$\# N / A$

vps3

$\# \mathrm{~N} / \mathrm{A}$

SPBCPT2R1.04C

SPBP19A11.07C

rim2

SPCC1020.13C

PAC3A12.02

htb1

vps68 transcription factor TFIIH complex DNA helicase subunit Ral $-0.40617817 .66273244 \quad 5.16 \mathrm{E}-06$ $\# \mathrm{~N} / \mathrm{A}$ $\begin{array}{lll}0.4073972 & 7.21928469 & 0.00048327\end{array}$ $\begin{array}{llll}-0.4076651 & 7.5688829 & 0.00068526\end{array}$ $\begin{array}{lll}-0.4080661 & 8.09892785 & 4.10 \mathrm{E}-05\end{array}$ $-0.4086532 \quad 5.23468508 \quad 0.02792218$ $-0.40887394 .40874311 \quad 0.1155143$ $-0.4091033 \quad 8.39017132 \quad 5.08 \mathrm{E}-05$ $\begin{array}{lll}-0.4097108 & 5.70866229 & 0.01617298\end{array}$ $\begin{array}{lll}-0.4097856 & 6.27554299 & 0.0027568\end{array}$ $\begin{array}{lll}-0.4098594 & 6.7754747 & 0.00203207\end{array}$ $-0.4099859 .00228633 \quad 3.49 \mathrm{E}-07$ $\begin{array}{llll}-0.4101368 & 7.48363809 & 0.00014893\end{array}$ $\begin{array}{lll}-0.410468 & 6.59918104 & 0.00576023\end{array}$ $\begin{array}{lll}-0.4104723 & 6.14923308 & 0.03480394\end{array}$ $\begin{array}{lll}-0.4105236 & 6.00014422 & 0.0085068\end{array}$ $\begin{array}{lll}-0.4108353 & 6.12721808 & 0.00701142\end{array}$ $\begin{array}{lll}-0.4119563 & 8.22905382 & 1.13 \mathrm{E}-05\end{array}$ $\begin{array}{lll}-0.41235 & 4.05177773 & 0.27325853\end{array}$ $\begin{array}{lll}-0.4127378 & 6.8609907 & 0.0007391\end{array}$

$\begin{array}{cccc}\text { cytochrome c oxidase assembly protein Cox20 (predicted) } & -0.4128784 & 5.73958764 & 0.0274506 \\ \text { \#N/A } & -0.4130211 & 6.36967183 & 0.00549554\end{array}$

$\begin{array}{cccc}\text { cytochrome c oxidase assembly protein Cox20 (predicted) } & -0.4128784 & 5.73958764 & 0.0274506 \\ \text { \#N/A } & -0.4130211 & 6.36967183 & 0.00549554\end{array}$ 3'-5'-exoribonuclease activity Dis3L2

vacuolar calcium transporting P-type ATPase P2 type, Pmc1 $\quad-0.413173 \quad 9.38858779 \quad 4.63 \mathrm{E}-07$ $\begin{array}{lllll}\text { conserved fungal protein Mug117 } & -0.4132516 & 5.47707946 & 0.01663578\end{array}$ Sm snRNP core protein Smg1

$$
\# N / A
$$$$
\# N / A
$$

$-0.4135993 \quad 5.82742782 \quad 0.01614999$

$\begin{array}{lll}0.4138415 & 6.93546218 & 0.00023761\end{array}$ $\begin{array}{lll}-0.4139771 & 5.38329443 & 0.01917797\end{array}$ $\begin{array}{lll}-0.4150045 & 4.9123082 & 0.09270983\end{array}$ $\begin{array}{lll}-0.4150758 & 5.0533417 & 0.07115014\end{array}$ $\begin{array}{lll}-0.4157571 & 6.33059543 & 0.00254059\end{array}$ $\begin{array}{lll}-0.415878 & 7.51246025 & 0.00010283\end{array}$

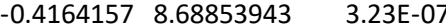
$\begin{array}{lll}-0.4167573 \quad 5.20136531 & 0.03768836\end{array}$ $\begin{array}{lll}-0.417252 & 6.54780452 & 0.02011242\end{array}$ Set1C complex subunit Swd21

mitotic chromosome segregation protein $\mathrm{Csi} 2$

$\begin{array}{lll}-0.4176054 & 3.25306071 & 0.32007875\end{array}$

U1 snRNP-associated protein Usp104

$$
\begin{array}{lrrr}
\text { \#N/A } & -0.4178246 & 2.6342297 & 0.48418752 \\
\text { \#N/A } & -0.4180182 & 8.95310668 & 1.06 E-05
\end{array}
$$$$
\# \text { \#/A }
$$

$\begin{array}{lll}0.4176992 & 8.07595296 \quad 3.08 \mathrm{E}-07\end{array}$

$\begin{array}{lll}-0.4180267 & 4.68841355 & 0.20000633\end{array}$

CORVET complex subunit, GTPase regulator Vps3 (predicter $\quad-0.41804 \quad 6.7341738 \quad 0.00077088$ $\# \mathrm{~N} / \mathrm{A}$

$-0.418617 \quad 4.10929311 \quad 0.16231433$ $\begin{array}{lllll}\text { S. pombe specific DUF999 protein family } 10 & -0.4187774 & 6.20226244 & 0.01398844\end{array}$ mitochondrial carrier, pyrimidine nucleotide Rim2 (predicte $\begin{array}{llll}-0.4189429 & 6.90690305 & 0.00280759\end{array}$ DDHD family phospholipase (predicted) $-0.419193 \quad 7.82842546 \quad 7.22 \mathrm{E}-05$ mitochondrial inorganic diphosphatase (predicted) $\quad \begin{array}{llll}-0.4197192 & 5.62181129 & 0.02257842\end{array}$ histone $\mathrm{H} 2 \mathrm{~B}$ Htb1

vacuolar sorting protein Vps68 (predicted)
$2.15 \mathrm{E}-05$

0.001405409 0.001925335 0.000144761

0.052315642

0.177323436

0.000176768

0.032417423

0.006683303

0.005087652

$1.71 \mathrm{E}-06$

0.000477591

0.012906931

0.063428167

0.01820847

0.015341715

$4.41 \mathrm{E}-05$

0.364850695

0.002059616

0.051602569

0.012351382

2.24E-06

0.033253127

0.032392832

0.000734982

0.037849784

0.147369871

0.116922659

0.006214486

0.000338456

$1.59 \mathrm{E}-06$

0.067884944

0.039385609

0.414488282

$1.53 \mathrm{E}-06$

0.579373317

4.17E-05

0.280594095

0.002131866

0.236267171

0.02851748

0.017282684

0.006791921

0.000243912

0.043575196

$1.02 \mathrm{E}-06$

0.058372223 
SPAC1565.04C

SPBC3E7.09

SPNCRNA.1082

SPBC146.03C

SPAP19A11.05c

SPCC16C4.22

SPAC1565.03

SPAC25H1.03

SPCP1E11.08

SPNCRNA.504

SPBC24C6.02

SPAC25B8.07C

SPAPB17E12.06

SPBC577.14C

SPBC36B7.06C

SPCC16A11.12c

SPAC24H6.12C

SPBC365.15

SPAC6B12.18

SPNCRNA.1653

SPCC4B3.04C

SPCC330.11

SPNCRNA.564

SPAC1952.06c

SPAC1486.03C

SPAC12B10.05

SPNCRNA.1001

SPNCRNA.1528

SPCC794.01C

SPNCRNA.735

SPAC222.16c

SPNCRNA.1453

SPAC13G6.08

SPAC212.04C

SPNCRNA.1367

SPAC17G8.11C

SPCC1919.01

SPBC31F10.04C

SPAPYUK71.03C

SPCC1919.05

SPCC895.08C

SPAC16E8.05C sat1

SPBC1348.05

SPBC20F10.03

\#N/A

hot13

ste4

SPBC3E7.09

$\#$ N/A

cut3

mrp7

dpb3

SPAC1565.03

$\operatorname{atg} 101$

nsa2

\#N/A

spb4

rcf1

sos 7

spa1

mug20

ubp1

uba3

alp4

gon7

\#N/A

nte1

btb1

\#N/A

SPAC1952.06C

ntr1

icp55

$\# N / A$

$\# \mathrm{~N} / \mathrm{A}$

$\operatorname{gcd} 1$

\#N/A

\#N/A

fzr2

SPAC212.04C

$\# \mathrm{~N} / \mathrm{A}$

imt3

ckk2

srb4

tcb3

ski3

SPCC895.08C

SPAC16E8.05C
Golgi membrane exchange factor subunit Sat1 (predicted) $\quad-0.4205387 \quad 6.83243487 \quad 0.02035536$ $\begin{array}{lll}-0.4206844 & 5.57300507 \quad 0.02746004\end{array}$ transmembrane transporter (predicted)

$\begin{array}{llll}0.4206844 & 5.57300507 & 0.02746004 \\ -0.4207092 & 5.95597445 & 0.01258682\end{array}$ $\begin{array}{ccccc}\text { Armadillo-type fold protein, human IFRD1 ortholog, implica } & -0.4207092 & 5.95597445 & 0.01258682 \\ \text { \#N/A } & -0.4212239 & 5.62574121 & 0.01920118\end{array}$

helper of TIM Hot13 (predicted)

$\begin{array}{llll}0.4212239 & 5.62574121 & 0.01920118\end{array}$

$\begin{array}{rrr}-0.4219983 & 6.62474322 & 0.00173393 \\ -0.4222914 & 7.29856361 & 2.93 \mathrm{E}-05\end{array}$

adaptor protein Ste4

$-0.4224432 \quad 8.69784366 \quad 8.63 \mathrm{E}-06$

$-0.42285217 .98381195 \quad 1.86 \mathrm{E}-06$

$\begin{array}{lll}-0.4232367 & 5.94395696 & 0.01357596\end{array}$

$-0.423421 \quad 4.95407434 \quad 0.05547626$

$\begin{array}{lll}-0.4240686 & 4.21180201 & 0.14485331\end{array}$

$-0.4241865 \quad 5.43701902 \quad 0.0188408$

$-0.4243985 \quad 7.36738471 \quad 1.18 \mathrm{E}-05$

$\begin{array}{lll}-0.4244795 & 3.49008252 & 0.27104494\end{array}$

$\begin{array}{lll}-0.4251967 & 6.71117853 & 0.00100229\end{array}$

$\begin{array}{lll}-0.4258237 & 5.86856241 & 0.02945621\end{array}$

$\begin{array}{lll}-0.4258562 & 6.03207264 & 0.00492919\end{array}$

cytochrome c oxidase assembly protein Rcf1

NMS complex subunit Sos 7

ornithine decarboxylase antizyme with +1 programmed ribc $-0.4261014 \quad 7.01157112 \quad 5.11 \mathrm{E}-05$

$\begin{array}{lllll}\text { Schizosaccharomyces specific protein Mug20 } & -0.4262585 & 3.74252625 & 0.33215777\end{array}$

ubiquitin C-terminal hydrolase Ubp1 $\quad-0.4263863 \quad 7.08983198 \quad 0.00010144$

NEDD8 activating enzyme E1-type Uba3 $\quad-0.4264144 \quad 6.74788556 \quad 0.00097386$

gamma tubulin complex Spc97/GCP2 subunit Alp4 $\quad \begin{array}{llll}-0.4265358 & 8.15540631 & 4.13 \mathrm{E}-05\end{array}$

EKC/KEOPS complex subunit Gon7 (predicted) $\quad-0.42730055 .763147880 .00709065$

$\begin{array}{lll}-0.4278408 & 6.63707971 & 0.00144476\end{array}$

$-0.4279965 \quad 8.40120968 \quad 5.12 \mathrm{E}-07$

$\begin{array}{lll}-0.4280433 & 7.98243991 & 9.70 \mathrm{E}-07\end{array}$

$\begin{array}{lll}-0.4289907 & 6.68486072 & 0.00025732\end{array}$

$\begin{array}{llll}-0.4292929 & 6.5719908 & 0.00132375\end{array}$

$\begin{array}{lrr}-0.4294546 & 7.49703806 & 6.73 \mathrm{E}-05\end{array}$

spliceosomal complex subunit (predicted)

RNA-binding splicing factor Ntr1 (predicted)

$-0.429744 \quad 6.92795211 \quad 0.00024589$

mitochondrial intermediate cleavage peptidase Icp55 (pred

$\# N / A$

$\# N / A$

glucose dehydrogenase $\mathrm{Gcd} 1$

\#N/A

COP9/signalosome complex subunit Csn3 (predicted)

$\# \mathrm{~N} / \mathrm{A}$

meiotic fizzy-related APC coactivator Fzr2 (predicted)

S. pombe specific DUF999 family protein 1 $\#$ N/A

mannosyltransferase Imt3

calmodulin-dependent kinase kinase 2

mediator complex subunit Med17

$\begin{array}{llll}-0.4300152 & 6.46424997 & 0.00109413\end{array}$

$\begin{array}{llll}-0.4300153 & 4.7989492 & 0.07275547\end{array}$

$\begin{array}{llll}-0.4304779 & 6.20997507 & 0.00488814\end{array}$

$\begin{array}{lll}-0.4309812 & 7.00267366 & 0.00039562\end{array}$

$\begin{array}{lll}-0.431026 & 5.60027004 & 0.02359512\end{array}$

$\begin{array}{llll}-0.4311789 & 5.59302791 & 0.10232568\end{array}$

$\begin{array}{llll}-0.4312371 & 5.78791004 & 0.01521166\end{array}$

$\begin{array}{llll}-0.4317979 & 5.56384367 & 0.02808315\end{array}$

$\begin{array}{llll}-0.4319451 & 6.07279377 & 0.03890126\end{array}$

$\begin{array}{lll}-0.4322858 & 7.15733728 & 0.00294729\end{array}$

$\begin{array}{lll}-0.4323799 & 6.5181146 & 0.00085896\end{array}$ $\begin{array}{llll}-0.4324322 & 6.7593735 & 0.00078824\end{array}$

$\begin{array}{lllll}\text { Ski complex TPR repeat subunit Ski3 (predicted) } & -0.4331426 & 8.45133239 & 1.61 \mathrm{E}-07\end{array}$

Schizosaccharomyces specific protein

Schizosaccharomyces specific protein Mde1

$\begin{array}{lll}0.4337284 & 4.58420133 & 0.09651291\end{array}$
0.039758391

051606061

0.025900617

0.037884611

0.253511474

0.004410988

0.00010624

$3.45 \mathrm{E}-05$

$8.30 \mathrm{E}-06$

0.027734744

0.094758013

0.214615009

0.037292517

4.62E-05

0.362696125

0.002686334

0.054872573

0.011218447

0.000177413

0.426558521

0.000334279

0.002621486

0.000145661

0.015475258

0.003733995

2.46E-06

4.49E-06

0.00078983

0.003458015

0.000228641

0.000758858

0.002911818

0.119387746

0.011147386

0.001169503

0.045267776

0.159896222

0.03071574

0.052530463

0.069810935

0.007082034

0.002342772

0.00217165

4.33E-08

8.24E-07

0.006033038

0.152065368 
slu7

peg1

$\operatorname{mrps} 17$

cwf28

gyp2

SPAC20H4.09

$\mathrm{mtf1}$

wtf21

uap2

sec3

nup211

rad13

SPBP4H10.19c

atm1

SPAC11D3.16C

SPAPJ691.02

exg3

$\# N / A$

eri1 \#N/A

coq11

raf2

nem 1
$\# \mathrm{~N} / \mathrm{A}$

transmembrane transporter (predicted)

chromatin binding protein ortholog

$-0.4337284 \quad 4.58420133 \quad 0.09651291$ $\begin{array}{lll}-0.4337424 & 6.06899114 & 0.00349848\end{array}$ $\begin{array}{llll} & \end{array}$ mitochondrial 3'-tRNA processing endonuclease tRNAse $Z, \quad-0.4348183 \quad 6.548963320 .00070376$ hexaprenyldihydroxybenzoate methyltransferase, Coq3 vari; $-\begin{array}{lllll}-0.4350765 & 7.09685461 & 0.00010286\end{array}$ $\begin{array}{llll}\text { AMP-activated protein serine/threonine kinase alpha subun } & -0.4351877 \quad 8.47124908 & 2.18 \mathrm{E}-08\end{array}$ $\begin{array}{lllll}\text { dihydroxyacetone kinase Dak1 } & -0.4353412 & 9.15960969 & 1.02 \mathrm{E}-05\end{array}$ CCAAT-binding factor complex subunit Php3 $\# \mathrm{~N} / \mathrm{A}$ $\begin{array}{lll}-0.4354624 & 5.31103093 & 0.0655142\end{array}$ $\begin{array}{lll}-0.4356235 & 6.66450167 & 0.02048292\end{array}$ $-0.4359795 \quad 5.55919003 \quad 0.01657535$ transmembrane transporter (predicted)

O-methyltransferase, human COMT catechol homolog $2 \quad-0.436462 \quad 8.954824 \quad 1.03 \mathrm{E}-08$ rRNA processing protein Faf1 (predicted)

$$
\text { \#N/A }
$$

mitochondrial translational activator, Mpa1 centromere localized protein $\mathrm{Cnl} 2$

histone acetyltransferase Mst2

\section{$\# N / A$} $\begin{array}{rrr}-0.436462 & 8.954824 & 1.03 E-08 \\ -0.4366591 & 5.97806352 & 0.00662242 \\ -0.4370742 & 2.20384326 & 0.51472137\end{array}$ $\begin{array}{lll}-0.4370742 & 2.20384326 & 0.51472137\end{array}$ $\begin{array}{lll}-0.4374113 & 3.1517049 & 0.29653776\end{array}$ $-0.4377592 \quad 6.83897375 \quad 0.00059194$ $\begin{array}{lll}-0.4379956 & 5.49180219 & 0.01190603\end{array}$ $\begin{array}{lll}-0.4381743 & 8.55824087 & 2.68 \mathrm{E}-07\end{array}$ $\begin{array}{lll}-0.4384672 & 3.39351343 & 0.3561512\end{array}$ $\begin{array}{lll}-0.4385895 & 4.94435953 & 0.04797017\end{array}$ short chain dehydrogenase, unknown specificity (predicted) $\quad-\begin{array}{lllll}0.438957 & 5.62806098 & 0.02799777\end{array}$ splicing factor Slu7 $\quad-\begin{array}{llll}-0.4391017 & 6.56060619 & 0.00074676\end{array}$ CLASP family microtubule-associated protein mitochondrial ribosomal protein subunit S17 (predicted) splicing factor Cwf28 $\begin{array}{rrr}-0.43933 & 7.6010329 & 8.36 \mathrm{E}-06\end{array}$ $\begin{array}{lll}-0.4401599 & 6.23983666 & 0.00401601\end{array}$ $\begin{array}{lll}-0.440749 & 6.63414864 & 0.00123585\end{array}$ $\begin{array}{lll}-0.44116 & 4.98916442 & 0.04635528\end{array}$ $\begin{array}{lrrr}\text { GTPase activating protein Gyp2 (predicted) } & -0.4415565 & 7.81470712 & 1.57 \mathrm{E}-06 \\ \text { anaphase-promoting complex, TPR lobe subcomplex subuni } & -0.4415667 & 7.1825889 & 0.00021346\end{array}$ ATP-dependent RNA helicase, spliceosomal (predicted) $\quad \begin{array}{llll}-0.4419374 & 6.67049931 & 0.0004846\end{array}$ $\begin{array}{lllll}\text { ATP-dependent RNA helicase, spliceosomal (predicted) } & -0.4419374 & 6.67049931 & 0.0004846 \\ \text { mitochondrial RNA polymerase specificity factor, predicted } & -0.4419944 & 6.28607554 & 0.00630367\end{array}$ wtf element Wtf21 $\quad-0.4420225 .54783804 \quad 0.02396952$ $\begin{array}{llll}\text { U2 snRNP-associated protein Uap2 } & -0.4422445 & 7.0408384 & 0.00321561\end{array}$ $\begin{array}{lllll}\text { exocyst complex subunit Sec3 } & -0.4426388 & 8.5598875 & 1.84 \mathrm{E}-07\end{array}$ nucleoporin nup211

DNA repair nuclease Rad13

calreticulin/calnexin homolog (predicted) mitochondrial iron-sulfur cluster exporter Atm1

Schizosaccharomyces specific protein

yippee-like protein

cell wall glucan 1,3-beta-glucosidase Exg3

$$
\text { \#N/A }
$$

double-strand siRNA ribonuclease Eri1

$$
\# \mathrm{~N} / \mathrm{A}
$$

ubiquinone biosynthesis protein Coq11

CLRC ubiquitin ligase complex subunit Raf2

$\begin{array}{lll}-0.4426681 & 8.31729117 & 1.62 \mathrm{E}-07\end{array}$

$\begin{array}{lll}-0.4433347 & 7.51290875 & 0.000314\end{array}$

$\begin{array}{lll}-0.4438085 & 5.78579391 & 0.01085082\end{array}$ $\begin{array}{lll}-0.4445153 & 6.78191231 & 0.00158274\end{array}$ $\begin{array}{llll}-0.4447268 & 6.44179087 & 0.00531126\end{array}$ $\begin{array}{lll}-0.4448014 & 6.10014613 & 0.01223498\end{array}$ $\begin{array}{lll}-0.4454296 & 7.32661131 & 0.00597065\end{array}$ $\begin{array}{llll}-0.4458597 & 6.14438931 & 0.00278901\end{array}$ $\begin{array}{llll}-0.4460574 & 6.85036392 & 0.00036957\end{array}$ $\begin{array}{lll}-0.4461667 & 4.67648728 & 0.16391841\end{array}$ $\begin{array}{llll}-0.4464292 & 6.42892758 & 0.00256046\end{array}$ $\begin{array}{llll}-0.4470109 & 6.2232122 & 0.00341747\end{array}$ Nem1-Spo7 phosphatase complex catalytic subunit Nem1（ $\begin{array}{lllll}-0.4471292 & 6.73525541 & 0.00088537\end{array}$ DNA polymerase delta subunit $\mathrm{Cdc} 27$
0.152065368 0.008263492 3.61E-07 0.000103609

0.001971626 0.000338456

1.27E-07

$4.01 \mathrm{E}-05$

0.109083467

0.03996166

0.03314582

$6.27 \mathrm{E}-08$

0.01459376

0.606314507

0.389862733

0.001680274

0.024636733

$1.34 \mathrm{E}-06$

0.451660254

0.083509087

0.052399562

0.002077352

3.36E-05

0.009327282

0.003247097

0.081091956

7.07E-06

0.000666323

0.001408664

0.013963688

0.04585678

0.007672586

9.35E-07

$8.28 \mathrm{E}-07$

0.000948808

0.02268504

0.004055181

0.011967931

0.025245314

0.013338884

0.006749355

0.001099167

0.238245365

0.006249626

0.008103094

0.002407093

0.025915467 
SPNCRNA.624

SPNCRNA.985

SPCC1281.08

SPBC56F2.15

SPNCRNA.1637

SPCC18.04

SPAC688.12C

SPBC21B10.07

SPNCRNA.1638

SPBC8D2.03C

SPAC11E3.14

SPAC25G10.07C

SPBC2D10.18

SPBC29A10.02

SPNCRNA.1197

SPNCRNA.1195

SPBC15D4.07c

SPBC26H8.05C

SPBC1652.01

SPBC1685.12C

SPAC7D4.03C

SPCC663.03

SPCC613.09

SPAC16E8.02

SPNCRNA.1566

SPCC1020.10

SPNCRNA.125

SPAC27E2.07

SPAC17G6.05c

SPNCRNA.554

SPBC1709.09

SPAC750.02C

SPAC16C9.07

SPCC16C4.16C

SPCC4F11.02

SPCC1393.05

SPAC6C3.04

SPBPB2B2.19c

SPBC21C3.05

SPAC24H6.06

SPAC6B12.09

SPCC895.09C

SPBC17D1.07C tam13

$$
\# \text { N/A }
$$

pof6

SPAC688.12C

SPBC21B10.07

\#N/A

SPAC11E3.14

cut7

abc1

spo5

$\# \mathrm{~N} / \mathrm{A}$

$\# \mathrm{~N} / \mathrm{A}$

$\operatorname{atg} 9$

ppe2

stb3

ahk1

pmd1

$\operatorname{sen} 54$

mpo1

oca2

pvg2

bro1

\#N/A

rrf1

SPAC750.02c

pom2

cbc3

ptc1

ers1

cit1

SPBPB2B2.19c

sap62

sld3

trm10

ucp12

npg1
$\#$ N/A

tRNA 2'-O-methyltransferase Trm13 (predicted)

U4/U6 x U5 tri-snRNP complex subunit Prp31

$\# N / A$

$\# N / A$

wtf element Wtf11

Schizosaccharomyces specific protein Tam13

\#N/A

F-box protein Pof6

Schizosaccharomyces specific protein

glycosyl hydrolase family (predicted)

$\# \mathrm{~N} / \mathrm{A}$

histone $\mathrm{H} 4 \mathrm{~h} 4.2$

$\begin{array}{lll}0.4473646 & 7.53341641 & 6.05 \mathrm{E}-05\end{array}$

$\begin{array}{llll}-0.4477633 & 5.24721439 & 0.01518201\end{array}$

$\begin{array}{lll}-0.4481567 & 6.88851675 & 0.00172971\end{array}$

$\begin{array}{lll}-0.4483367 & 6.32501953 & 0.0105643\end{array}$

$\begin{array}{lll}-0.4493876 & 5.55845059 & 0.00935031\end{array}$

$\begin{array}{lll}-0.4495116 & 4.66444411 & 0.15559976\end{array}$

$\begin{array}{lll}-0.4498693 & 7.82641124 & 3.45 \mathrm{E}-06\end{array}$

$\begin{array}{lll}-0.4502925 & 7.02037056 & 0.00184368\end{array}$

$\begin{array}{lll}-0.4503143 & 6.41439194 & 0.00163516\end{array}$

$-0.450411 \quad 7.21446944 \quad 1.71 \mathrm{E}-05$

$\begin{array}{lll}-0.4504449 & 6.46223221 & 0.00033993\end{array}$

$\begin{array}{llll}-0.4510802 & 7.77277865 & 1.58 \mathrm{E}-07\end{array}$

$\begin{array}{lll}-0.4526471 & 6.52108788 & 0.00102995\end{array}$

$\begin{array}{lll}-0.4526548 & 7.53029793 & 1.49 \mathrm{E}-05\end{array}$

$\begin{array}{lllll}\text { protein with a role in clearing protein aggregates (predicter }-0.4529441 & 8.05527484 & 8.32 \mathrm{E}-06\end{array}$

kinesin-5 family plus-end directed microtubule motor, bimc $\quad-0.453024 \quad 7.48995804 \quad 3.21 \mathrm{E}-06$

ABC1 kinase family ubiquinone biosynthesis ATPase Abc1/C $\quad-0.453112 \quad 7.45439724 \quad 7.72 \mathrm{E}-05$

meiotic RNA-binding protein 1

\section{$\# N / A$}

$\begin{array}{lll}-0.4533049 & 4.43820039 & 0.13295497\end{array}$

$-0.4533907 \quad 6.60738541 \quad 0.00051663$

$-0.4539195 \quad 8.21769497 \quad 7.12 \mathrm{E}-07$

autophagy associated protein Atg

$-0.4539424 \quad 6.85132036 \quad 4.65 \mathrm{E}-05$

serine/thro a

transcription coregulator Stb3 (predicted)

\#N/A

$-0.4549057 \quad 7.52232322 \quad 2.47 \mathrm{E}-06$

$\begin{array}{lll}-0.4550809 & 6.57716529 & 0.00815517\end{array}$

$\begin{array}{llll} & 1.82 \mathrm{E}-09\end{array}$

$\begin{array}{lllll}\text { leptomycin transmembrane transporter Pmd1 } & -0.4554942 & 9.43455207 & 1.38 \mathrm{E}-09\end{array}$

tRNA-splicing endonuclease subunit Sen54 (predicted) $\quad \begin{array}{llll}-0.455749 & 6.70667433 & 0.0004509\end{array}$

ER membrane sphingoid metabolism protein Mpo1 (predic1 - 0.45623014 .080449930 .16917788

$$
\text { \#N/A }
$$

serine/threonine protein kinase Oca2

$$
\# \mathrm{~N} / \mathrm{A}
$$

$\begin{array}{lll}-0.4563027 & 5.59781982 & 0.0316572\end{array}$

$-0.4567905 \quad 7.45670607 \quad 5.41 \mathrm{E}-05$

$-0.457253 \quad 3.04345937 \quad 0.32873357$

Golgi 4,6-pyruvylated galactose (PvGal) residue biosynthesi $-0.4573559 \quad 6.96991143 \quad 2.68 \mathrm{E}-05$

BRO1 domain protein Bro1 (predicted)

$$
\text { \#N/A }
$$

mitochondrial translation termination factor Rrf1

transmembrane transporter (predicted)

DYRK family protein kinase Pom2

nuclear cap-binding complex variant subunit $\mathrm{Cbc} 3$

MAP kinase threonine phosphatase Ptc1

RNA-silencing factor Ers1

citrate synthase Cit1

S. pombe specific $5 \mathrm{Tm}$ protein family

U2 snRNP Sap62

DNA replication pre-initiation complex subunit SId3

$\begin{array}{lll}0.4581393 & 7.43154559 & 2.82 \mathrm{E}-05\end{array}$

$\begin{array}{lll}-0.4583489 & 5.0917055 & 0.03318654\end{array}$

$\begin{array}{lll}-0.4585048 & 5.23370446 & 0.021239\end{array}$

$\begin{array}{lll}-0.458613 & 5.57883312 & 0.0103191\end{array}$

$\begin{array}{lll}-0.4588364 & 7.09106172 \quad 4.50 \mathrm{E}-05\end{array}$

$\begin{array}{lll}-0.4593361 & 6.42006021 & 0.00056084\end{array}$

$\begin{array}{lll}-0.4594658 & 7.72510234 & 2.47 \mathrm{E}-05\end{array}$

$\begin{array}{lll}-0.4601646 & 6.04316606 & 0.002756\end{array}$

$\begin{array}{lll}-0.4604761 & 9.1058149 & 3.04 \mathrm{E}-10\end{array}$

$\begin{array}{lll}-0.4626047 & 8.0785646 \quad 8.80 \mathrm{E}-07\end{array}$

$\begin{array}{lll}-0.4632473 & 6.14184488 & 0.00229054\end{array}$

$\begin{array}{lll}-0.4635724 & 6.07817593 & 0.00407203\end{array}$

$\begin{array}{llll}-0.4638911 & 6.43573961 & 0.00058887\end{array}$

ATP-dependent RNA helicase Ucp12, unknown location and $-0.4645188 \quad 7.62723825 \quad 5.25 \mathrm{E}-05$

$\begin{array}{llll}\text { GTPase regulator Rng2-like (predicted) } & -0.4651179 & 6.48871879 & 0.00414669\end{array}$
0.000207184

0.030665955

0.004401892

0.022147281

0.019834422

0.22805435

$1.48 \mathrm{E}-05$

0.004655368

0.004173758

6.51E-05

0.001019942

8.11E-07

0.00275395

5.73E-05

$3.35 \mathrm{E}-05$

1.38E-05

0.000259251

0.200079289

0.001491061

3.35E-06

0.000162677

0.003237779

$1.08 \mathrm{E}-05$

0.017522213

1.23E-08

9.47E-09

0.001316897

0.244362879

0.058381374

0.000186848

0.423439706

9.84E-05

0.000102827

0.060855407

0.041274135

0.021659921

0.000157659

0.001600217

9.13E-05

0.006683303

2.26E-09

4.09E-06

0.005680429

0.009454163

0.001672475

0.000181877

0.009611116 
SPBC30D10.08 SPAC23A1.15C SPBC18H10.09 SPCC24B10.11C SPBC29A10.04 SPBC947.09

SPCC737.09c

SPBC776.10c

SPNCRNA. 557

SPNCRNA.26

SPAC144.15C

SPCC63.11

SPBC691.03C

SPAC19D5.09c

SPBC19F8.03C

SPCC569.02C

SPNCRNA.1025

SPRRNA.52

SPCC1393.11

SPNCRNA.573

SPBC337.11

SPAC688.10

SPAC227.15

SPAC694.06c

SPBC1348.02

SPAC4A8.05C

SPAC1B2.06

SPBC317.01

SPBC3D6.11C

SPNCRNA.972

SPAC23A1.06C

SPAPYUG7.02c

SPAC1952.15C

SPNCRNA.218

SPBC428.20C

SPNCRNA.266

SPAC328.02

SPBC1778.09

SPAC589.12

SPNCRNA.1297

SPAC644.06C

SPAC1002.19

SPBC36B7.05C

SPNCRNA.1384

SPBC409.03

SPBC18E5.09C

SPCC162.10 hsp3103

hmt1

$\operatorname{cog} 6$

\#N/A

$\# N / A$

$\operatorname{cog} 1$

prp28

apl3

Tf2-8

yap18

SPCC569.02C $\#$ N/A

SPRRNA.52

mrpl20 \#N/A

SPBC337.11

rev3

reg1

mrc1

$\mathrm{ftm} 5$

myp2

SPAC1B2.06

$\mathrm{mbx} 2$

slx8

$\# N / A$

cmk2

$\sin 1$

rec24

\#N/A

alp6

\#N/A

$\mathrm{dbl} 4$

SPBC1778.09

cwh43

\#N/A

cdr1

urg1

pib1

wi5

\#N/A

SPBC18E5.09c ppk33 mitochondrial DNA repair protein (predicted)

SNARE Sec20 (predicted)

zf-CHY type zinc finger protein

THO complex subunit Tho7 (predicted)

$\begin{array}{llll}0.4654668 & 6.21604902 & 0.00111322\end{array}$

$\begin{array}{lll}-0.4655559 & 6.52236209 & 0.00072264\end{array}$

$-0.4657215 \quad 6.66565583 \quad 0.00265838$

$\begin{array}{llrr}\text { mitotic cohesin complex ATPase subunit Psm1/Smc1 } & -0.4657471 & 7.98611348 & 3.07 \mathrm{E}-06 \\ \text { ThiJ domain protein, implicated in cellular detoxification } & -0.4660077 & 5.80735359 & 0.0034743\end{array}$

$\begin{array}{llll}\text { vacuolar phytochelatin and glutathione S-conjugate ABC far } & -0.4660688 & 8.67221427 & 9.61 \mathrm{E}-07\end{array}$

Golgi transport complex peripheral subunit $\operatorname{Cog} 6$ (predicted - $0.4662803 \quad 7.66520381$ 1.13E-05

$\begin{array}{lll}-0.4669233 & 5.58264974 \quad 0.01169232\end{array}$

$-0.46743593 .97833558 \quad 0.20703534$

$-0.4675913 \quad 8.16920152 \quad 1.15 \mathrm{E}-07$

Golgi transport complex subunit $\operatorname{Cog} 1$ (predicted)

U5 snRNP-associated ATP-dependent RNA helicase Prp28 ( $\mid-0.46774147 .53386435 \quad 5.85 \mathrm{E}-05$

$\begin{array}{lllll}\text { AP-2 adaptor complex alpha subunit Alp3 } & -0.4680584 & 8.19960983 & 1.12 \mathrm{E}-07\end{array}$

retrotransposable element/transposon Tf2-type $\quad \begin{array}{llll}-0.468113 & 6.96189424 & 0.0002202\end{array}$

ENTH/VHS domain protein (predicted) $\quad-0.4684117 \quad 8.25453236 \quad 1.55 \mathrm{E}-08$

S. pombe specific UPF0321 family protein $2 \quad-0.4685405 \quad 3.9276763 \quad 0.12845001$

\#N/A

5.8S ribosomal RNA

\begin{tabular}{rrr}
-0.4685405 & 3.9276763 & 0.12845001 \\
-0.469501 & 5.45574756 & 0.01531071 \\
\hline
\end{tabular}

$-0.469678 \quad 7.9444663 \quad 6.12 \mathrm{E}-08$

mitochondrial ribosomal protein subunit L20, Mrpl20 (predi $\quad-0.469841 \quad 6.99077309 \quad 8.29 \mathrm{E}-06$$$
\# \mathrm{~N} / \mathrm{A}
$$

$-0.47026 \quad 7.13056455 \quad 0.00355919$

mitochondrial inner membrane $\mathrm{CH}-\mathrm{OH}$ group oxidoreductas $\quad-0.4707167 \quad 5.32359887 \quad 0.01407405$

DNA polymerase zeta catalytic subunit Rev3 (predicted)

protein phosphatase regulatory subunit Reg1 (predicted)

claspin, Mrc1

sub-telomeric 5Tm protein family Ftm5

myosin II heavy chain Myo3

Schizosaccharomyces pombe specific protein

MADS-box transcription factor Pvg4

SUMO-targeted ubiquitin-protein ligase E3 SIx8

$$
\# N / A
$$

MAPK-activated protein kinase $\mathrm{Cmk2}$

stress activated MAP kinase interacting protein $\operatorname{Sin} 1$

meiotic recombination protein $\operatorname{Rec} 24$

$$
\# \mathrm{~N} / \mathrm{A}
$$

gamma tubulin complex Spc98/GCP3 subunit Alp6

$$
\# \mathrm{~N} / \mathrm{A}
$$

ubiquitin-protein ligase E3 involved in sporulation Dbl4

GTPase activating protein (predicted)

glycosylceramide biosynthesis protein Cwh43 (predicted)

$$
\# \text { N/A }
$$

$\begin{array}{lll}0.4707167 & 5.32359887 & 0.01407405\end{array}$

$\begin{array}{lll}-0.4714251 & 7.77584762 \quad 3.62 \mathrm{E}-07\end{array}$

$\begin{array}{lll}-0.4715107 & 7.47577324 & 0.00018112\end{array}$

$\begin{array}{lll}-0.4719281 & 8.13783062 \quad 9.04 \mathrm{E}-07\end{array}$

$\begin{array}{lll}-0.4723624 & 9.33899419 & 1.38 \mathrm{E}-07\end{array}$

$\begin{array}{lll}-0.4730656 & 2.42195393 & 0.42567327\end{array}$

$\begin{array}{llll}-0.4734835 & 8.35068386 & 1.27 \mathrm{E}-06\end{array}$

$\begin{array}{lll}-0.4735435 & 7.01748433 & 0.00010432\end{array}$

$\begin{array}{lll}-0.4735924 & 7.47490585 & 0.00015934\end{array}$

$\begin{array}{lll}-0.473613 & 6.61288748 & 0.00024874\end{array}$

$\begin{array}{lll}-0.4736513 & 7.32609752 & 6.47 \mathrm{E}-06\end{array}$

$\begin{array}{lll}-0.4736948 & 5.43939473 & 0.01031609\end{array}$

$\begin{array}{llll}-0.4740936 & 3.47719175 & 0.21532947\end{array}$

$\begin{array}{lll}-0.4742426 & 6.58849504 & 0.00026446\end{array}$

$\begin{array}{lll}-0.4747695 & 1.16964603 & 0.77294204\end{array}$

$\begin{array}{lll}-0.4748693 & 6.94831804 & 5.22 \mathrm{E}-05\end{array}$

$\begin{array}{lll}-0.4753629 & 6.07503013 & 0.00759939\end{array}$

$\begin{array}{lll}-0.4756123 & 7.53977372 \quad 2.31 \mathrm{E}-05\end{array}$

$-0.4759405 \quad 4.51963872 \quad 0.05311851$

$\begin{array}{lll}-0.4765167 & 7.83253469 & 2.97 \mathrm{E}-05\end{array}$

$\begin{array}{lll}-0.4770814 & 6.44877592 & 0.00078446\end{array}$

GTP cyclohydrolase II Urg1 (predicte

endosomal and vacuolar ubiquitin-protein ligase E3/phosph $\# \mathrm{~N} / \mathrm{A}$

Swi5 protein

$\begin{array}{lll}0.4771772 & 5.6854095 & 0.00543151\end{array}$

$\begin{array}{llll}-0.477265 & 4.03582031 & 0.12067142\end{array}$

$\begin{array}{llll}-0.4775521 & 6.46283476 & 0.00073067\end{array}$

$\begin{array}{lll}-0.477613 & 5.11250913 & 0.03998692\end{array}$

Schizosaccharomyces specific protein

serine/threonine protein kinase Ppk33 (predicted)
0.001596293

0.002955705

0.002017879

0.006460819

1.33E-05

0.008219961

4.45E-06

$4.42 \mathrm{E}-05$

0.024250608

0.289059397

6.03E-07

0.000200991

$5.86 \mathrm{E}-07$

0.000685805

9.21E-08

0.194158661

0.030880081

3.35E-07

3.33E-05

0.008383627

0.028674822

5.96E-05

$1.77 \mathrm{E}-06$

0.000571147

4.19E-06

7.13E-07

0.52070944

5.79E-06

0.000342618

0.000507639

0.000766268

2.66E-05

0.021659921

0.299460686

0.000809924

0.831600087

0.000180694

0.016469387

$8.58 \mathrm{E}-05$

0.091280675

0.00010763

0.002162982

0.012226728

0.184325262

0.002038632

0.071514057

0.002120812 


\begin{tabular}{|c|c|c|c|c|c|c|}
\hline SPAC18G6.10 & lem2 & LEM domain nuclear inner membrane protein Heh1/Lem2 & -0.4794154 & 6.95888195 & 9.39E-06 & 3.73E-05 \\
\hline SPCC970.12 & $\operatorname{mis} 18$ & kinetochore protein Mis18 & -0.4801105 & 5.55766335 & 0.00950009 & 0.020115138 \\
\hline SPAC1834.09 & mug51 & variant protein kinase 19 family protein & -0.4804654 & 4.72293515 & 0.05556989 & 0.094894132 \\
\hline SPBC21C3.01c & vps1301 & chorein homolog Vps13a (predicted) & -0.4805117 & 8.5795419 & 4.38E-08 & $2.46 \mathrm{E}-07$ \\
\hline SPCC188.07 & ccq1 & shelterin complex HEAT repeat subunit Ccq1 & -0.4806456 & 6.60061934 & 0.00017899 & 0.00056467 \\
\hline SPBC31F10.13c & hip1 & histone $\mathrm{H} 3.3 \mathrm{H} 4$ chaperone, hira family Hip1 & -0.4809179 & 8.03042086 & 1.60E-07 & 8.20E-07 \\
\hline SPAC14C4.13 & rad17 & RFC related checkpoint protein Rad17 & -0.4813212 & 6.57571224 & 0.00077105 & 0.002131866 \\
\hline SPNCRNA.901 & $\# \mathrm{~N} / \mathrm{A}$ & \#N/A & -0.4817842 & 5.20944294 & 0.01512875 & 0.030567434 \\
\hline SPCC736.07c & uri1 & unconventional prefoldin chaperone involved protein compli & -0.482049 & 6.92039695 & 0.00021811 & 0.000680212 \\
\hline SPAC3H1.08c & SPAC3H1.08c & mitochondrial calcium uniporter regulator (predicted) & -0.4829834 & 6.06813035 & 0.00089233 & 0.002425042 \\
\hline SPNCRNA.1328 & $\# \mathrm{~N} / \mathrm{A}$ & $\# N / A$ & -0.4831969 & 7.2454219 & 8.64E-06 & 3.45E-05 \\
\hline SPNCRNA.1037 & $\# N / A$ & $\# N / A$ & -0.4837192 & 6.7860994 & $3.34 \mathrm{E}-05$ & 0.000119595 \\
\hline SPCC737.08 & $\mathrm{mdn} 1$ & midasin, Mdn1 & -0.484265 & 9.90160426 & $1.26 \mathrm{E}-11$ & 1.10E-10 \\
\hline SPAC6F6.06c & $\operatorname{rax} 2$ & cell polarity factor Rax2 & -0.4844842 & 8.46030807 & $5.34 \mathrm{E}-08$ & $2.95 \mathrm{E}-07$ \\
\hline SPAC8E11.05c & SPAC8E11.05c & DUF5102 family conserved fungal protein, associated with & -0.484744 & 7.23364894 & $4.52 \mathrm{E}-06$ & $1.90 \mathrm{E}-05$ \\
\hline SPAC12B10.01c & SPAC12B10.01c & HECT-type ubiquitin-protein ligase $E 3$, implicated in negativ & -0.4853547 & 9.2529247 & $1.76 \mathrm{E}-09$ & $1.19 \mathrm{E}-08$ \\
\hline SPBC354.08c & rsn1 & Golgi to plasma membrane transport protein Rsn1 (predict & -0.4857244 & 7.81296154 & $2.25 \mathrm{E}-08$ & 1.30E-07 \\
\hline SPBC660.08 & SPBC660.08 & Schizosaccharomyces specific protein & -0.4859926 & 7.37266096 & $1.90 \mathrm{E}-06$ & $8.44 \mathrm{E}-06$ \\
\hline SPCC23B6.03c & tel1 & ATM checkpoint kinase & -0.4861231 & 7.80737818 & 4.09E-07 & 1.99E-06 \\
\hline SPNCRNA.1650 & $\# N / A$ & \#N/A & -0.4866131 & 4.60460316 & 0.15249912 & 0.224234337 \\
\hline SPBC428.17c & wpl1 & cohesin loading/unloading factor (WAPL) Wpl1 & -0.4874967 & 6.75654858 & 0.00180188 & 0.004563374 \\
\hline SPAC23C4.16c & $\operatorname{atg} 15$ & autophagy associated lysophospholipase Atg15 & -0.4878848 & 7.54671971 & 1.34E-07 & 6.93E-07 \\
\hline SPNCRNA.776 & $\# N / A$ & \#N/A & -0.4883003 & 6.06585878 & 0.00079963 & 0.002200368 \\
\hline SPAC18B11.04 & ncs1 & neuronal calcium sensor related protein Ncs1 & -0.4884223 & 7.35607485 & 0.00024226 & 0.000748676 \\
\hline SPNCRNA.638 & $\# N / A$ & $\# N / A$ & -0.4886385 & 5.81431886 & 0.00360062 & 0.008464167 \\
\hline SPBC32C12.02 & ste11 & transcription factor Ste11 & -0.4891491 & 8.8841278 & 0.00078932 & 0.002173757 \\
\hline SPAC23C11.03 & mpp10 & U3 snoRNP-associated protein Mpp10 (predicted) & -0.4893958 & 7.02530481 & 4.43E-05 & 0.000155078 \\
\hline SPBC336.01 & fbh1 & DNA helicase I, ubiquitin ligase F-box adaptor Fbh1 & -0.4896702 & 7.03348451 & 4.89E-06 & 2.05E-05 \\
\hline SPNCRNA.1428 & $\# N / A$ & \#N/A & -0.4896886 & 6.97554453 & $5.14 \mathrm{E}-05$ & 0.000178523 \\
\hline SPCC63.14 & eis1 & eisosome assembly protein eis 1 & -0.4897181 & 9.14573365 & $8.21 \mathrm{E}-05$ & 0.000273842 \\
\hline SPCC11E10.08 & rik1 & CLRC ubiquitin ligase complex WD repeat protein Rik1 & -0.4902953 & 7.1305286 & $5.03 E-06$ & 2.10E-05 \\
\hline SPAC8F11.02c & dph3 & diphthamide biosynthesis protein Dph3 (predicted) & -0.4906999 & 5.76250581 & 0.00432792 & 0.009976804 \\
\hline SPNCRNA.800 & \#N/A & \#N/A & -0.4907876 & 4.57270716 & 0.04564871 & 0.080020464 \\
\hline SPAC1B3.04c & guf1 & mitochondrial elongation factor GTPase Guf1 (predicted) & -0.4909524 & 6.80278877 & 2.23E-05 & $8.28 \mathrm{E}-05$ \\
\hline SPAC56F8.16 & esc1 & transcription factor Esc1 (predicted) & -0.4917436 & 8.24796907 & $1.82 \mathrm{E}-06$ & 8.11E-06 \\
\hline SPBC30B4.02c & sqs1 & $\mathrm{R} 3 \mathrm{H}$ and G-patch domain protein Sqs1 (predicted) & -0.4918135 & 6.97675254 & 4.99E-05 & 0.000173813 \\
\hline SPNCRNA.1236 & $\# \mathrm{~N} / \mathrm{A}$ & \#N/A & -0.4920552 & 5.16400275 & 0.05965842 & 0.100613517 \\
\hline SPNCRNA.1213 & $\# \mathrm{~N} / \mathrm{A}$ & \#N/A & -0.4921237 & 6.25581649 & 0.00097653 & 0.00262673 \\
\hline SPCC330.20 & $\operatorname{tam} 14$ & stress associated Endoplasmic Reticulum protein Tam14 & -0.4923644 & 4.47841289 & 0.09019599 & 0.143844316 \\
\hline SPBC13E7.02 & cwf24 & ubiquitin-protein ligase E3/GCN5-related $\mathrm{N}$ acetyltransfera: & -0.4925728 & 6.54476537 & 0.00014453 & 0.000464289 \\
\hline SPBC119.07 & ppk19 & serine/threonine protein kinase Ppk19 & -0.4928747 & 7.3469935 & 3.60E-05 & 0.000128315 \\
\hline SPAC1952.03 & otu2 & ubiquitin specific cysteine protease, OTU family, Otu2 & -0.4931171 & 5.8844567 & 0.0020149 & 0.005050246 \\
\hline SPBC12C2.03c & SPBC12C2.03c & methionine synthase reductase (predicted) & -0.4934322 & 6.5319807 & 0.00145509 & 0.003756793 \\
\hline SPCC1884.02 & nic1 & plasma membrane NiCoT heavy metal ion transmembrane & -0.4937302 & 7.16259317 & 6.39E-05 & 0.000217992 \\
\hline SPNCRNA.104 & \#N/A & $\# \mathrm{~N} / \mathrm{A}$ & -0.494014 & 3.06094314 & 0.36601263 & 0.461329127 \\
\hline SPAC13G6.14 & aps1 & diadenosine 5',5'"'-p1,p6-hexaphosphate hydrolase Aps1 & -0.4947524 & 6.75683513 & 0.0005531 & 0.001580111 \\
\hline SPAC6B12.08 & mug185 & DNAJ domain protein Mug185 (predicted) & -0.4949688 & 6.8469256 & 9.31E-06 & $3.71 \mathrm{E}-05$ \\
\hline
\end{tabular}


SPNCRNA.175 SPNCRNA.24 SPBC1711.15C SPBC13E7.01

SPAC4H3.05

SPCC1281.07C

SPAC664.15

SPBC405.05

SPBP35G2.10

SPNCRNA.1206

SPAC12G12.09

SPAC20G8.08c

SPNCRNA.721

SPAC2F7.17

SPNCRNA.05

SPAC56F8.06C

SPAC30D11.11

SPAC977.03

SPNCRNA.1012

SPAC2F3.14C

SPNCRNA.696

SPCC16C4.02C

SPCC1183.11

SPAC5D6.07C

SPAC22E12.18

SPAC22F8.07C

SPBC29A10.05

SPBC29A10.14

SPAC11H11.01

SPAC1420.01C

SPCC10H11.01

SPNCRNA.911

SPAC3C7.05C

SPNCRNA.538

SPBC30D10.09c

SPAC630.11

SPAC57A10.05C

SPNCRNA.1029

SPBC31F10.09C

SPAC23D3.11

SPBC26H8.09c

SPNCRNA.1305

SPNCRNA.1142

SPAC17D4.03C

SPBP8B7.23

SPAC4H3.08

SPBC3E7.05C
$\# N / A$
$\# N / A$

$\# N / A$

SPBC1711.15C

cwf22

srs2

gst4

caf4

$\operatorname{atg} 16$

mit1

\#N/A

fft1

mrf1

\#N/A

$\# N / A$

alg10

izh3

SPAC977.03

\#N/A

saf2

$\#$ N/A

SPCC16C4.02C

msy1

pxa1

SPAC22E12.18

rtf1

exo1

rec8

sst6

SPAC1420.01C

prp11

$\# \mathrm{~N} / \mathrm{A}$

mug191

$\# N / A$

SPBC30D10.09c

vps55

pof1

\#N/A

nut2

ayr1

snf59

$\# N / A$

$\# N / A$

cis4

rnf10

SPAC4H3.08

mic60
\#N/A

Schizosaccharomyces pombe specific protein splicing factor Cwf22

ATP-dependent DNA helicase, UvrD subfamily

glutathione S-transferase (predicted)

CCR4-Not complex subunit Caf4/Mdv1 (predicted)

autophagy associated protein Atg16

SHREC complex ATP-dependent DNA helicase subunit Mit1

$$
\# N / A
$$

$0.4952389 \quad 2.6843569 \quad 0.42248371$ $\begin{array}{llll}-0.4953631 & 3.21914336 & 0.28785931\end{array}$

$\begin{array}{lll}-0.4962085 & 4.63633466 & 0.11685218\end{array}$ $-0.4962105 \quad 7.7181717 \quad 2.22 \mathrm{E}-05$

$-0.4963548 \quad 6.93586919 \quad 1.05 \mathrm{E}-05$

$\begin{array}{lll}-0.4963606 & 7.50925713 \quad 2.06 \mathrm{E}-05 \\ -0.496849 & 7.34566393 & 0.00051667\end{array}$

$-0.49668497 .34566393 \quad 0.00051667$

$\begin{array}{lll}-0.4975174 & 5.09189462 & 0.01485519\end{array}$

$\begin{array}{llll}\text { WD repeat protein associated with stress granule } & -0.4979536 & 7.36866936 & 1.64 \mathrm{E}-07\end{array}$

SMARCAD1 family ATP-dependent DNA helicase Fft1 (predi $\quad-0.498061 \quad 7.1796704 \quad 3.67 \mathrm{E}-05$

$$
\# \mathrm{~N} / \mathrm{A}
$$

mitochondrial translation release factor (predicted)

$\begin{array}{lll}-0.4980953 & 2.73200877 & 0.35394008\end{array}$

$\begin{array}{lll}-0.4989041 & 6.41128361 & 0.0003378\end{array}$

$-0.4989986 \quad 5.95231509 \quad 0.00206686$

dolichyl-phosphate-glucose-glycolipid alpha-glucosyltransfe $\begin{gathered}-0.4990124 \\ 5.03640585\end{gathered} 0.02565269$

ER membrane Haemolysin-III family protein involved in zinc $\begin{array}{rrr}-0.4990826 & 7.88446059 & 9.33 \mathrm{E}-09\end{array}$

methyltransferase (predicted)

$$
\text { \#N/A }
$$

splicing associated factor Saf2

$\begin{array}{lll}0.4992 & 4.18823326 & 0.08602963\end{array}$

$\begin{array}{lll}-0.4992342 & 5.22078019 & 0.03158327\end{array}$

$\begin{array}{lll}-0.4992847 & 5.81207528 & 0.00836098\end{array}$

$\begin{array}{lll}-0.4994989 & 4.69109268 & 0.0405022\end{array}$

$\begin{array}{llll}\text { Armadillo-type fold protein, DUF1941 family protein, huma } & -0.4997851 & 7.448348 & \text { 1.04E-06 }\end{array}$

$\begin{array}{llll}\text { MS calcium ion channel protein Msy1 } & -0.5001987 & 7.97886929 & 1.35 \mathrm{E}-09\end{array}$

PXA domain protein Pxa1

human CCNDBP1 ortholog

replication termination factor Rtf1

exonuclease I Exo1

meiotic cohesin complex subunit Rec 8

ESCRT I complex subunit $\mathrm{Vps} 23$

GATA-like domain protein (predicted)

ATP-dependent RNA helicase Prp11

$$
\text { \#N/A }
$$

alpha-1,6- mannanase (predicted)

\#N/A

$\begin{array}{llll}-0.5004775 & 5.49536924 & 0.01710364\end{array}$

$\begin{array}{llll}-0.5006287 & 6.28281576 & 0.00076849\end{array}$

$\begin{array}{lll}-0.5009635 & 5.80151778 & 0.00277815\end{array}$

$-0.5010388 \quad 8.33009778 \quad 5.51 \mathrm{E}-10$

$\begin{array}{llll}-0.5012449 & 4.87393219 & 0.04846748\end{array}$

$\begin{array}{llll}-0.5017394 & 5.33704148 & 0.02300833\end{array}$

$\begin{array}{lll}-0.5019824 & 8.10807815 & 4.57 \mathrm{E}-09\end{array}$

$\begin{array}{llll}-0.5020489 & 7.43581687 & 0.00037174\end{array}$

$\begin{array}{lll}-0.5021293 & 4.074193 & 0.1093613\end{array}$

$\begin{array}{lll}-0.5025224 & 7.95307055 & 1.22 \mathrm{E}-05\end{array}$

$\begin{array}{lll}-0.5039393 & 2.23742336 & 0.5795262\end{array}$

$\begin{array}{llllll} & 0.5039393 & 2.23742336 & 0.5795262\end{array}$

vacuolar sorting protein $\mathrm{Vps} 55$ (predicted)

F-box/WD repeat protein Pof1

$\# \mathrm{~N} / \mathrm{A}$

$\begin{array}{lll}-0.5055546 & 5.88654157 & 0.00152067\end{array}$

$\begin{array}{lll}-0.5059087 & 6.8217224 & 0.00050915\end{array}$

$\begin{array}{llll}-0.5060075 & 4.8899017 & 0.04955172\end{array}$

$\begin{array}{lll}-0.5063418 & 6.21504937 & 0.00033871\end{array}$

mediator complex subunit Med10

$\begin{array}{llll}-0.5064539 & 6.80291741 & 0.00012807\end{array}$

SWI/SNF complex subunit Snf59

$\# N / A$

$\begin{array}{lll}-0.5065168 & 6.50082455 & 0.00248039\end{array}$

$\begin{array}{lll}-0.506519 & 8.96639992 & 2.59 \mathrm{E}-11\end{array}$

$\begin{array}{lll}-0.5068067 & 5.21215263 & 0.0123677\end{array}$

Golgi cation diffusion family zinc transmembrane transport $-0.5068664 \quad 7.15370734 \quad 3.57 \mathrm{E}-05$

ubiquitin-protein ligase $\mathrm{E3}$ (predicted)

3-hydroxyacyl-CoA dehydrogenase (predicted)

MICOS complex subunit Mic60 (predicted)

$\begin{array}{lll}-0.5074412 \quad 8.18591323 & 2.59 \mathrm{E}-08\end{array}$

$\begin{array}{lll}-0.508218 & 7.48080208 & 0.00184114\end{array}$

$\begin{array}{llll}-0.5087899 & 6.10701644 & 0.00074258\end{array}$

0.517738462

0.380513323

0.179215484

8.26E-05

$4.15 \mathrm{E}-05$

$7.71 \mathrm{E}-05$

0.001491061

0.03008618

0.00011034

$7.74 \mathrm{E}-08$

8.37E-07

0.000130694

0.44944248

0.001014441

0.005163364

0.048652891

5.72E-08

0.137878386

0.058324056

0.017914506

0.072283625

4.80E-06

$9.28 \mathrm{E}-09$

0.034122035

0.002126704

0.00672787

3.96E-09

0.084288578

0.044279324

2.92E-08

0.001105151

0.169175635

4.77E-05

0.66661186

0.04276387

0.003912356

0.00147337

0.085998333

0.001016737

0.000414794

0.006084737

2.19E-10

0.025503703

0.000127747

1.50E-07

0.004650696

0.002066762 
SPAC17G8.09

SPCC320.14

SPAC15A10.12C

SPBC14C8.02

SPAC17C9.05C

SPBP35G2.13C

SPNCRNA.620

SPAC1751.04

SPAC3C7.04

SPAC8E11.06

SPCC970.09

SPAC29E6.10c

SPAC1F5.11C

SPCC548.06c

SPAC23C11.04C

SPAC1952.01

SPAC56E4.06c

SPAC688.08

SPBC2G5.07C

SPNCRNA.454

SPAPB1A10.11C

SPAC16.01

SPCC1919.15

SPNCRNA.1276

SPNCRNA.1425

SPAC343.03

SPBC776.16

SPAC4F8.13C

SPAC13A11.04C

SPNCRNA 1391

SPBC8E4.02C

SPBC25H2.14

SPNCRNA.889

SPAPB15E9.03C

SPCC1442.02

SPBP8B7.22

SPCC16C4.21

SPCC737.07c

SPNCRNA.189
SPBC15D4.13C SPAC27E2.01

mde 7

$\# \mathrm{~N} / \mathrm{A}$

kha1 \#N/A

cwg2

SPAC56F8.15

shg1

sry1

tca17

tim44

pmc3

swc2

\#N/A

loc1

SPAC3C7.04

SPAC8E11.06

sec8

nst1

tra2

ght8

pnk1

gab1

ggt2

srb8

rpc25

\#N/A

mse1

rho2

brl1

\#N/A

$\# N / A$

apc11

mis20

rng2

ubp8

$\# N / A$

mug16

$\# \mathrm{~N} / \mathrm{A}$

Tf2-5

gIm2

erd2

\#N/A

SPCC737.07C

\#N/A activating signal cointegrator 1 complex subunit, human AS $\quad \begin{array}{llll}-0.5092015 & 4.37786664 & 0.0683663\end{array}$ alpha-amylase homolog (predicted)

RNA-binding protein Mde?

$\#$ N/A

$\begin{array}{lll}0.5092136 & 5.62956265 \quad 0.03605062\end{array}$

$\begin{array}{lll}-0.5095899 & 6.3292633 & 0.00263736\end{array}$

$-0.509765 \quad 4.75999874 \quad 0.04918941$

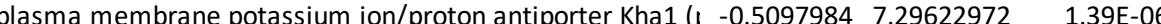

$$
\# \mathrm{~N} / \mathrm{A}
$$

geranylgeranyltransferase I beta subunit Cwg2

Schizosaccharomyces pombe specific protein

$\begin{array}{lll}-0.5098867 & 5.34312198 & 0.00764969\end{array}$

$-0.5100465 \quad 5.91156242 \quad 0.00254955$

$\begin{array}{lll}-0.5107452 & 6.18244174 & 0.00170024\end{array}$

$\begin{array}{lll}-0.5121167 & 5.22658957 & 0.00814011\end{array}$

$\begin{array}{lll}-0.5127003 & 7.44107359 & 2.59 \mathrm{E}-07\end{array}$

$\begin{array}{lll}-0.5127094 & 5.83631968 & 0.00359484\end{array}$

$\begin{array}{lll}0.5128011 & 6.48855247 \quad 8.16 \mathrm{E}-05\end{array}$

$\begin{array}{lll}-0.5129379 & 5.00169503 & 0.08106567\end{array}$

\begin{tabular}{lll}
$-0.513048 \quad 6.85279703 \quad 1.10 \mathrm{E}-05$ \\
\hline
\end{tabular}

$\begin{array}{lll}-0.5133748 & 5.90849985 & 0.00251955\end{array}$

$\begin{array}{lll}-0.5134382 & 5.84187825 & 0.0010562\end{array}$

$\begin{array}{lll}-0.5136709 & 7.9984334 & 5.72 \mathrm{E}-10\end{array}$

$\begin{array}{lll}-0.513699 & 3.09936705 & 0.28596401\end{array}$

$-0.5137465 \quad 7.97478129 \quad 7.18 \mathrm{E}-07$

$-0.5146914 \quad 7.82436391 \quad 8.96 \mathrm{E}-07$

exocyst complex subunit sec8

conserved fungal NST1 family protein

$\begin{array}{lllll}\text { NuA4 complex phosphatidylinositol pseudokinase complex } & -0.515943 & 10.2082488 & 4.55 \mathrm{E}-15 \\ \text { plasma membrane hexose:proton symporter, unknown spe } & -0.5161838 & 9.90082704 & 5.96 \mathrm{E}-12\end{array}$

DNA kinase/phosphatase Pnk1

Pig-U, Gab1 (predicted)

gamma-glutamyltranspeptidase Ggt2

mediator complex subunit Med12

DNA-directed RNA polymerase III complex subunit Rpc25

$$
\text { \#N/A }
$$

mitochondrial glutamyl-tRNA ligase Mse1 (predicted)

Rho family GTPase Rho2

ubiquitin-protein ligase E3 Brl1

$\# N / A$

$\# \mathrm{~N} / \mathrm{A}$

$\begin{array}{lll}0.5164214 & 7.30634212 & 6.61 \mathrm{E}-07\end{array}$

$-0.5164411 \quad 5.91881701 \quad 0.00675902$

$-0.51650757 .78698454 \quad 1.12 \mathrm{E}-07$

$-0.5167218 \quad 7.72677416 \quad 1.98 \mathrm{E}-08$

$\begin{array}{lll}-0.5168943 \quad 6.45356058 & 0.00028293\end{array}$

$\begin{array}{lll}-0.5173654 & 4.79158068 & 0.01985562\end{array}$

$\begin{array}{lll}-0.5182181 & 6.26851464 & 0.00078104\end{array}$

$\begin{array}{lll}-0.5187259 & 6.04882252 & 0.0009782\end{array}$

$\begin{array}{lll}-0.5191051 & 6.8333516 & 2.93 \mathrm{E}-05\end{array}$

$-0.5191051 \quad 6.8333516 \quad 2.93 E-05$

$\begin{array}{lll}-0.5191497 & 1.90514662 & 0.58905608\end{array}$

anaphase-promoting complex ubiquitin -protein ligase E3 s| $-0.5194548 \quad 6.51823423 \quad 6.57 \mathrm{E}-05$

centromere protein Mis20/Eic2

$-0.519798 \quad 4.83431527 \quad 0.06402305$

$\begin{array}{lll}-0.5199381 & 8.81538676 \quad 2.98 \mathrm{E}-11\end{array}$

$\begin{array}{lll}-0.5204691 & 7.27865096 & 8.06 \mathrm{E}-07\end{array}$

$\begin{array}{lll}-0.5213684 & 6.42508467 \quad 8.77 \mathrm{E}-05\end{array}$

$\begin{array}{lll}-0.5217392 & 7.1222645 & 8.73 \mathrm{E}-06\end{array}$

UNC-50 family protein, implicated in vesicle-mediated tran $\begin{array}{cccc}-0.5218809 & 5.51862741 & 0.00549334\end{array}$ \#N/A

$\begin{array}{lll}-0.522677 & 6.37228267 & 4.41 \mathrm{E}-05\end{array}$

$-0.5226988 \quad 7.13715683 \quad 5.56 \mathrm{E}-05$

$-0.5228253 \quad 6.53561124 \quad 3.27 \mathrm{E}-05$

$\begin{array}{lll}-0.5233075 & 6.96065281 & 6.71 \mathrm{E}-06\end{array}$

$\begin{array}{llll}0.5245794 & 3.30113352 & 0.19139972\end{array}$

DNA polymerase alpha-associated DNA helicase A (predicte $-\begin{array}{llll}0.5248551 & 5.62850766 & 0.00261579\end{array}$

\#N/A
0.113030287

0.065384986

0.006414313

0.08544406

$6.29 \mathrm{E}-06$

0.01656784

0.006231925

0.004335011

0.017501868

$1.29 \mathrm{E}-06$

0.008455879

0.000272518

0.130940392

4.31E-05

0.006169672

0.00281859

4.10E-09

0.378964958

3.38E-06

4.16E-06

5.49E-14

$5.41 \mathrm{E}-11$

3.13E-06

0.014832348

5.90E-07

1.16E-07

0.000860672

0.038983688

0.002154418

0.002630049

0.0001061

0.0001061

0.674722541

0.000223714

0.107072222

2.50E-10

3.76E-06

0.000291624

3.49E-05

0.012351382

0.000154502

0.000191558

0.000117167

2.74E-05

0.270728565

0.006368682

0.881178125 
SPNCRNA.1684 SPNCRNA.1547 SPBC1652.02

SPAC637.03

SPCC895.05

SPCC $417.05 \mathrm{C}$

SPBC16E9.20

SPNCRNA.937

SPBP8B7.04

SPBC530.12C

SPAC11G7.03

SPNCRNA.1537

SPAC15E1.09

SPBC21C3.06

SPCC1393.02c

SPBC649.03

SPNCRNA.949

SPNCRNA.577

SPBC27B12.07

SPAC2G11.03C

SPNCRNA.329

SPAC1556.05c

SPAC1093.06C

SPBC1604.17C

SPAC1486.08

SPAC1F12.08

SPAC4G8.12C

SPAC19G12.04

SPBC1709.11C

SPNCRNA.863

SPAC19B12.08

SPAC6F 12.14

SPAC1786.01C

SPNCRNA.1558

SPBC3B8.04C

SPNCRNA.74

SPAC1834.03C

SPNCRNA.1409

SPCC338.08

SPBC409.12C

SPNCRNA.328

SPAC2G11.15C

SPBC119.04

SPNCRNA.1221

SPCC4G3.08

SPAC1B3.07C

SPBP35G2.04C $\begin{array}{llll}0.5253756 & 5.2528987 & 0.01240741\end{array}$ $\begin{array}{llll}-0.526937 & 3.84388547 & 0.15852006\end{array}$ SPBC1652.02 SPAC637.03

cfh2

\#N/A

$\# \mathrm{~N} / \mathrm{A}$

mug 45

pdf1

idh1

\#N/A

grx2

SPBC21C3.06

spt2

rhp14

$\# N / A$

$\# \mathrm{~N} / \mathrm{A}$

SPBC27B12.07

vps 45

$\# N / A$

cgr1

dhc1

tti2

$\operatorname{cox} 16$

cab4

smp3

dal1

png2

atg 4

cut23

$\mathrm{pt} / 2$

$\# N / A$

SPBC3B8.04

$\# \mathrm{~N} / \mathrm{A}$

hhf1

$\# \mathrm{~N} / \mathrm{A}$

ctp1

stn1

tgs1

mei3

psk1

vps 28

SPBP35G2.04c

$\begin{array}{lllll}\text { APC amino acid transmembrane transporter (predicted) } \quad-0.5269643 & 8.21173133 & 1.13 \mathrm{E}-10\end{array}$ DUF1774 family multi-spanning conserved fungal membrar $\quad \begin{array}{llll}-0.527136 & 8.14711136 & 3.62 \mathrm{E}-07\end{array}$ formin For3

$\begin{array}{lll}-0.5279584 & 7.11305626 & 7.36 \mathrm{E}-07\end{array}$ SEL1 repeat protein, chitin synthase regulatory factor-like C $\begin{array}{llll}-0.5281858 & 8.68944255 & 2.59 \mathrm{E}-11\end{array}$

$\# N / A$

$\begin{array}{lll}-0.52833 & 2.94684795 & 0.36256197\end{array}$

Schizosaccharomyces specific protein Mug45

$\begin{array}{llll}-0.5294935 & 6.13802475 & 0.00033665\end{array}$ $\begin{array}{llll}-0.529499 & 5.33582125 & 0.00842401\end{array}$ palmitoyl protein thioesterase/ dolichol pyrophosphate pho $-0.5299725 \quad 8.18576875 \quad 3.07 \mathrm{E}-06$ isocitrate dehydrogenase (NAD+) subunit $1 \mathrm{Idh} 1 \quad-0.5320415 \quad 8.35004839 \quad 2.31 \mathrm{E}-09$

$\# \mathrm{~N} / \mathrm{A}$

glutaredoxin Grx2

Schizosaccharomyces specific protein

non-specific DNA binding protein Spt2 (predicted)

XP-A family homolog Rhp14

$$
\# N / A
$$

\#N/A

mitochondrial calcium uniporter regulator

vacuolar sorting protein $\mathrm{Vps} 45$ \#N/A

ribosome biogenesis CGR1 family (predicted)

$-0.5321218 \quad 4.11599101 \quad 0.13655356$

$\begin{array}{llr}-0.5322072 & 6.29783174 & 5.99 \mathrm{E}-05\end{array}$

$\begin{array}{lll}-0.532417 & 6.40308988 & 0.00014144\end{array}$

$\begin{array}{lll}-0.5333326 & 7.21340163 \quad 1.61 \mathrm{E}-07\end{array}$

$\begin{array}{lll}0.5333548 & 6.1101595 & 0.00024927\end{array}$

$\begin{array}{lll}-0.5342095 & 7.74844358 & 1.32 \mathrm{E}-08\end{array}$

$-0.53520558 .18000055 \quad 5.62 \mathrm{E}-11$

$\begin{array}{lll}-0.5357225 & 6.74630766 & 0.00022643\end{array}$

$\begin{array}{lll}-0.5359238 & 7.60398077 & 2.22 \mathrm{E}-07\end{array}$

$\begin{array}{lll}-0.5364037 & 5.02075169 & 0.02445129\end{array}$

$-0.5365817 \quad 6.53609953 \quad 0.00053591$

$\begin{array}{llll}\text { minus-end directed microtubule motor, dynein heavy chain } & -0.5369308 & 8.23488861 & 1.38 \mathrm{E}-05\end{array}$

ASTRA complex subunit Tti2

$\begin{array}{lll}-0.5369451 & 5.81471863 \quad 0.00139332\end{array}$

mitochondrial copper chaperone for cytochrome c oxidase $\left(\begin{array}{cccc}-0.5370641 & 6.19229135 & 0.00041805\end{array}\right.$

cytidylyltransferase (predicted)

alpha-1,2-mannosyltransferase Smp3 (predicted)

ureidoglycolate hydrolase (predicted)

$\begin{array}{lll}0.5372112 & 6.13762947 & 0.00025931\end{array}$

$\begin{array}{lll}-0.5379807 & 5.35028898 & 0.05616134\end{array}$

$-0.5389135 \quad 5.28002686 \quad 0.00505818$

ING family histone acetyltransferase complex PHD-type zin $\quad \begin{array}{llll}-0.5394775 & 7.13069612 & 2.89 E-05\end{array}$

$$
\# \text { N/A }
$$

$-0.5396155 \quad 6.92317924 \quad 0.01523888$ $-0.5396781 \quad 6.44324097 \quad 7.61 \mathrm{E}-05$

Atg8 deconjugator Atg4 (predicted)

anaphase-promoting complex, TPR lobe subcomplex subuni -0.53970996 .759612850 .00015665$ triacylglycerol lipase pt|2

$\# \mathrm{~N} / \mathrm{A}$

$\begin{array}{lll}-0.5398674 & 8.45375368 & 6.15 \mathrm{E}-08\end{array}$

$\begin{array}{lll}-0.5400848 \quad 5.99634513 & 0.00117391\end{array}$

$-0.5409437 \quad 8.30458872 \quad 2.89 \mathrm{E}-08$

$\begin{array}{lll}-0.541026 & 6.40839251 & 9.96 \mathrm{E}-05\end{array}$

$\begin{array}{lll}-0.5421891 & 7.94545672 & 1.03 E-08\end{array}$

$\begin{array}{llll}-0.5434877 & 3.6837232 & 0.30737145\end{array}$

$\begin{array}{lll}-0.5435091 & 6.93526343 \quad 1.07 E-06\end{array}$

$\begin{array}{llll}-0.5439819 & 6.25252462 & 0.00020972\end{array}$

$\begin{array}{llll}-0.5442116 & 2.39227375 & 0.38199077\end{array}$

$\begin{array}{llll}-0.5446054 & 5.20680101 & 0.00358705\end{array}$

$\begin{array}{lll}-0.5448797 & 1.47697924 & 0.8016669\end{array}$

$\begin{array}{lll}-0.5450077 & 2.5838234 & 0.34514815\end{array}$

$\begin{array}{lll}-0.5454646 & 7.31863285 & 1.49 \mathrm{E}-06\end{array}$

$\begin{array}{lll}-0.5455776 & 6.24827183 & 0.00027719\end{array}$

$\begin{array}{lll}-0.5456612 & 5.21970705 & 0.01047966\end{array}$
0.025570094

0.231636032

8.77E-10

1.77E-06

$3.45 \mathrm{E}-06$

2.19E-10

0.458082842

0.001011434

0.018038193

$1.33 \mathrm{E}-05$

$1.52 \mathrm{E}-08$

0.204499779

0.000205185

0.00045464

$8.24 \mathrm{E}-07$

0.000767211

7.90E-08

$4.52 \mathrm{E}-10$

0.000702618

$1.12 \mathrm{E}-06$

0.046673509

0.001538756

$5.34 \mathrm{E}-05$

0.003612015

0.001228349

0.000795216

0.095682409

0.011462165

0.000105079

0.030753442

0.000255533

0.000500239

3.36E-07

0.003099917

1.66E-07

0.000328768

$6.26 \mathrm{E}-08$

0.400778443

4.93E-06

0.000655542

0.477576182

0.008440487

0.855014347

0.440002469

$6.72 \mathrm{E}-06$

0.000844344

0.021983388 
SPNCRNA.1524

SPCC10H11.02

SPAC26F1.08c

SPNCRNA.1270

SPAC14C4.02C

SPCC777.15

SPCC1840.02C

SPBC211.06

SPBC713.07C

SPBC337.07c

SPCC550.08

SPAC823.04

SPCC622.04

SPNCRNA.111

SPAC30D11.01C

SPAC22H10.07

SPBC26H8.04C

SPAC1834.04

SPBC29A3.05

SPAC1A6.04C

SPBC1711.11

SPNCRNA.861

SPNCRNA.948

SPNCRNA.642

SPAC19G12.09

SPAC22F8.09

SPNCRNA.1356

SPCC417.11C

SPCC4B3.10c

SPCC1020.05

SPAC922.09

SPNCRNA.772

SPCC663.02

SPNCRNA.1279

SPAC589.08C

SPBC16C6.03C

SPBC56F2.14

SPNCRNA.1326

SPAC2F3.10

SPNCRNA.945

SPCC1442.15C

SPBC18E5.13

SPAC977.10

SPNCRNA.970

SPNCRNA.877
SPBC887.08

SPAC19B12.07C

$\# N / A$

CWf23

SPAC26F1.08c $\#$ N/A

smc5

dus4

bgs 4

gfh1

SPBC713.07c

ecm 14

SPCC550.08

rrp36

$\# N / A$

$\# N / A$

gto2

scd2

iml1

hht1

vps71

plb1

atg2402

$+N / A$

$\# N / A$

$\# \mathrm{~N} / \mathrm{A}$

SPAC19G12.09

rrp16

\#N/A

SPCC417.11C

ipk1

dcr2

$\# N / A$

$\# \mathrm{~N} / \mathrm{A}$

wtf14

$\# \mathrm{~N} / \mathrm{A}$

dam1

rsa1

mrpl44

$\# \mathrm{~N} / \mathrm{A}$

vps54

$$
\# \mathrm{~N} / \mathrm{A}
$$

$\operatorname{cox} 18$

mti3

nhe1

\#N/A
Schizosaccharomyces specific protein

lein $\quad-0.5464336 \quad 4.642620890 .03513199$

\#N/A HN/A

malate transmembrane transporter (predicted)

$$
\# \text { N/A }
$$

Smc5-6 complex SMC P-loop ATPase subunit Smc5

tRNA dihydrouridine synthase Dus4 (predicted)

cell wall and secondary septum 1,6 branched 1,3-beta-gluci

gamma tubulin complex subunit Gfh1

vacuolar polyphosphatase (predicted)

carboxypeptidase Ecm14 (predicted)

$\mathrm{N}$-acetyltransferase (predicted)

rRNA processing protein Rrp36 (predicted)

$$
\# N / A
$$

$\# N / A$

alpha-glucosidase (predicted)

Cdc42 GTPase complex scaffold subunit Scd 2

GTPase-activating protein subunit of SEA and Iml1 complex

histone H3 h3.1

Swr1 complex subunit Vps71

phospholipase B homolog Plb1

autophagy associated protein Atg24c

$$
\# N / A
$$

$\# N / A$

$\mathrm{NADH} / \mathrm{NADPH}$-dependent indole-3-acetaldehyde reductase,

rRNA processing protein Rrp16 (predicted)

$$
\text { \#N/A }
$$

glutamate-1-semialdehyde 2,1-aminomutase (predicted)

inositol 1,3,4,5,6-pentakisphosphate (IP5) kinase

phosphoprotein phosphatase involved in unfolded protein $r$

$$
\# N / A
$$$$
\# N / A
$$

wtf element Wtf14

\section{\#N/A}

DASH complex subunit Dam 1

ribosome assembly protein Rsa1 (predicted)

mitochondrial ribosomal protein subunit I44 (predicted) \#N/A

GARP complex subunit Vps54 (predicted)

$$
\text { \#N/A }
$$

mitochondrial inner membrane insertase Cox18

mitochondrial translation initiation factor Mti3 (predicted)

plasma membrane/prospore membrane sodium ion/protor

$$
\# N / A
$$

$\# N / A$ $\begin{array}{lll}0.5468582 & 5.91886956 & 0.00092552\end{array}$ $\begin{array}{llll}-0.5471045 & 6.44282968 & 0.00013276\end{array}$ $\begin{array}{llll}-0.5474748 & 5.76745129 & 0.00336024\end{array}$ $\begin{array}{lll}0.5480872 & 6.97464982 & 7.03 \mathrm{E}-07\end{array}$ $\begin{array}{lll}-0.5482957 & 5.5348948 & 0.00250113\end{array}$ $\begin{array}{lll}-0.5489405 & 7.57644027 & 3.57 \mathrm{E}-07\end{array}$ $\begin{array}{llll}-0.5490111 & 5.82626719 & 0.00738702\end{array}$

$\begin{array}{lll}0.5505609 & 9.77782619 & 2.59 \mathrm{E}-12\end{array}$ $-0.5511387 \quad 7.26117614 \quad 7.11 \mathrm{E}-06$ $-0.5512105 \quad 8.45131342 \quad 1.90 \mathrm{E}-10$ $\begin{array}{lll}-0.5513259 & 8.05685223 & 3.04 \mathrm{E}-11\end{array}$ $\begin{array}{lll}-0.5514357 & 6.02585512 & 0.00027275\end{array}$ $\begin{array}{llll}-0.552378 & 4.92241057 & 0.01161492\end{array}$ $\begin{array}{llll}-0.5525221 & 4.31651665 & 0.04590266\end{array}$ $\begin{array}{lll}-0.5525532 & 7.64534036 & 1.03 \mathrm{E}-07\end{array}$ $\begin{array}{lll}-0.5527156 & 7.43735137 & 1.12 \mathrm{E}-06\end{array}$ $\begin{array}{lll}-0.5532612 & 7.31557521 & 5.10 \mathrm{E}-08\end{array}$ $\begin{array}{lll}0.5535304 & 7.71908209 & 1.73 \mathrm{E}-09\end{array}$ $-0.5541272 \quad 8.74424046 \quad 4.70 \mathrm{E}-11$ $\begin{array}{lll}-0.5547487 & 4.79505221 & 0.10512265\end{array}$ $\begin{array}{lll}-0.5547601 & 10.3911557 \quad 1.89 \mathrm{E}-12\end{array}$ $\begin{array}{lll}-0.5550153 & 5.51714945 & 0.00145373\end{array}$ $\begin{array}{llll}-0.5553622 & 5.20678802 & 0.02081955\end{array}$ $\begin{array}{llll}-0.5556407 & 5.31561943 & 0.00296036\end{array}$ $\begin{array}{llll}-0.5564091 & 3.87618327 & 0.07836716\end{array}$ $\begin{array}{lll}-0.556534 & 8.54996983 \quad 9.04 \mathrm{E}-11\end{array}$ $-0.5566276 \quad 6.74462416 \quad 1.80 \mathrm{E}-05$ $\begin{array}{llll}-0.5567591 & 4.21271478 & 0.11096773\end{array}$ $\begin{array}{lll}-0.5569585 & 6.87090987 & 1.53 \mathrm{E}-06\end{array}$ $\begin{array}{lll}-0.5572692 & 5.20212265 & 0.00432632\end{array}$ $\begin{array}{lll}0.5576667 \quad 8.69392076 & 2.84 \mathrm{E}-14\end{array}$ $\begin{array}{llll}-0.5579572 & 3.2855828 & 0.16055903\end{array}$ $\begin{array}{llll}-0.5584317 & 5.8857292 & 0.00071065\end{array}$ $\begin{array}{llll}-0.5591581 & 6.97949123 & 0.00058174\end{array}$ $\begin{array}{lll}-0.5591817 & 6.8576051 & 5.55 \mathrm{E}-06\end{array}$ $\begin{array}{lll}-0.5592816 & 5.14515908 & 0.00468858\end{array}$ $\begin{array}{llll}-0.5595684 & 5.45587698 & 0.00197269\end{array}$ $\begin{array}{llll}-0.5596873 & 5.20349063 & 0.00726215\end{array}$ $\begin{array}{lll}-0.560514 & 1.82117668 & 0.41881226\end{array}$ $\begin{array}{lll}-0.5606943 & 7.75766505 & 3.12 \mathrm{E}-09\end{array}$ $\begin{array}{lll}-0.5606982 & 4.66867341 & 0.0186957\end{array}$ $\begin{array}{llll}-0.5610758 & 5.31149225 & 0.00369472\end{array}$ $\begin{array}{llll}-0.5614626 & 5.68438223 & 0.00076284\end{array}$ $\begin{array}{lll}0.5617303 & 7.90789972 & 7.13 \mathrm{E}-09\end{array}$ $\begin{array}{lll}-0.5619802 & 5.43521687 & 0.00538343\end{array}$ $\begin{array}{llll}-0.5620353 & 2.33236165 & 0.40308347\end{array}$
0.063889154

0.002505889

0.000428971

0.007970163

3.32E-06

0.006131183

1.75E-06

0.016061226

2.45E-11

2.89E-05

1.44E-09

$2.55 \mathrm{E}-10$

0.000832329

0.024126845

0.080403502

$5.42 \mathrm{E}-07$

5.13E-06

2.83E-07

$1.17 \mathrm{E}-08$

3.83E-10

0.16339872

$1.82 \mathrm{E}-11$

0.003755733

0.04056028

0.007110926

0.127003698

7.07E-10

6.79E-05

0.171349085

$6.89 \mathrm{E}-06$

0.009976491

$3.17 \mathrm{E}-13$

0.2340626

0.001987646

0.001654294

2.31E-05

0.010742693

0.004960875

0.015819113

0.513887028

2.03E-08

0.037037623

0.008648973

0.002113653

4.45E-08

0.012122503

0.498629408 
SPBC16D10.05 SPBC18H10.11C SPBC3D6.10 SPAC12G12.10

SPAC22F8.05

SPNCRNA.743

SPBC24C6.06

SPAC29B12.13

SPAC17A2.09C

SPBP22H7.04

SPNCRNA.1569

SPNCRNA.1600

SPBC1347.11

SPNCRNA.1625

SPNCRNA.980

SPBP22H7.05C

SPBC2D10.15C

SPBP4H $10.06 \mathrm{C}$

SPAC23C4.05C

SPBC577.05C

SPNCRNA.997

SPBC17A3.06

SPCC132.03

SPBC713.06

SPCC16A11.08

SPAC19A8.02

SPNCRNA.1683

SPBC16E9.15

SPAC22F3.09C

SPBC354.11C

SPNCRNA.1046

SPBC32F12.05C

SPBP19A11.04C

SPNCRNA.862

SPAC3A11.05C

SPBC1604.02C

SPNCRNA.1492

SPNCRNA.1546

SPCC24B10.18

SPCC1020.14

SPBC19C2.04C

SPBC30D10.04

SPAC23G3.01

SPCC970.04C

SPAC2G11.08c

SPAC11H11.03C

SPAC2E1P3.03C apn2

wdr21

SPAC22F8.05

$\# \mathrm{~N} / \mathrm{A}$

gpa1

SPAC29B12.13

CSX1

SPBP22H7.04

$\# \mathrm{~N} / \mathrm{A}$

$\# \mathrm{~N} / \mathrm{A}$

sro

$\# N / A$

$\# \mathrm{~N} / \mathrm{A}$

abo2

pth1

cut14

SPAC23C4.05C rec27

$\#$ N/A

SPBC17A3.06

SPCC132.03

adl1

$\operatorname{atg} 20$

sip3

$\# \mathrm{~N} / \mathrm{A}$

SPBC16E9.15

res2

$\# N / A$

$\# \mathrm{~N} / \mathrm{A}$

cwf12

mor2

$\# N / A$

kms1

ppr1

$\# \mathrm{~N} / \mathrm{A}$

$\# N / A$

SPCC24B 10.18

Tf2-12

ubp1

swi3

$\mathrm{rpb} 2$

mob2

smn1

SPAC11H11.03C

Tf2-3 alpha-1,3-glucan synthase Mok13

mitochondrial PPR repeat protein Ppr2

$\begin{array}{lll}0.5629229 & 8.82670978 \quad 4.61 \mathrm{E}-13\end{array}$

$\begin{array}{lll}-0.5644762 \quad 5.62545664 & 0.00546658\end{array}$

$\begin{array}{lll}-0.5645823 & 7.343144 & 3.68 \mathrm{E}-08\end{array}$

WD repeat protein, human DDB1 and CUL4-associated factı $-0.5648403 \quad 7.09718775 \quad 3.69 \mathrm{E}-05$

alpha,alpha-trehalose-phosphate synthase (predicted) $\quad \begin{array}{llll}-0.5657398 & 8.67647483 & 0.00028832\end{array}$

$$
\# \mathrm{~N} / \mathrm{A}
$$

$\begin{array}{lll}-0.565836 & 5.64002803 & 0.00220144\end{array}$

$-0.5668855 \quad 7.7013504 \quad 6.51 \mathrm{E}-10$

S-(hydroxymethyl)glutathione synthase activity (predicted) $\quad \begin{array}{llll}-0.56702 & 5.81274198 & 0.00119971\end{array}$

$\begin{array}{lllll}\text { RNA-binding protein Csx1 } & -0.5675102 & 8.13985788 & 2.16 \mathrm{E}-10\end{array}$

mitochondrial membrane protein, human TMEM186 ortholı $\begin{array}{lll}-0.5683846 & 6.80496019 & 9.22 \mathrm{E}-07\end{array}$ $\# N / A$

$$
\# N / A
$$

$\# N / A$

$\# \mathrm{~N} / \mathrm{A}$

ATPase with bromodomain protein (predicted)

mitochondrial peptidyl-tRNA hydrolase Pth1 (predicted)

condensin complex SMC subunit Smc2

LEA domain protein

meiotic recombination protein $\operatorname{Rec} 27$

$$
\# \mathrm{~N} / \mathrm{A}
$$

phosphoprotein phosphatase (predicted)

Schizosaccharomyces specific protein

DNA ligase (predicted)

autophagy associated PX/BAR domain sorting nexin Atg20

ER-plasma membrane tethering protein Sip3 (predicted)

$$
\text { \#N/A }
$$

$\begin{array}{lll}-0.568735 & 4.64789459 & 0.02260416\end{array}$

$\begin{array}{llll}-0.5690606 & 5.49392116 & 0.01624417\end{array}$

$\begin{array}{lll}-0.5695717 & 6.83929524 & 2.37 \mathrm{E}-05\end{array}$

$\begin{array}{lll}-0.5710436 & 6.12086987 & 0.00013156\end{array}$

$\begin{array}{lll}-0.5717759 & 6.89245745 \quad 7.14 \mathrm{E}-06\end{array}$

$\begin{array}{lll}-0.5729305 & 7.55462659 \quad 3.58 \mathrm{E}-08\end{array}$

$\begin{array}{lll}-0.5732978 & 5.18733229 & 0.01020206\end{array}$

$\begin{array}{lll}-0.57498 & 7.86412175 & 4.05 \mathrm{E}-08\end{array}$

$-0.5756243 \quad 7.47219818 \quad 1.23 \mathrm{E}-06$

$\begin{array}{lll}-0.5763352 & 2.26841394 & 0.37017701\end{array}$

$\begin{array}{lll}-0.5763774 & 5.34567665 & 0.00199799\end{array}$

$\begin{array}{lll}-0.5763916 & 5.27125807 & 0.00497722\end{array}$

$\begin{array}{lll}-0.577773 & 6.56542637 \quad 2.72 \mathrm{E}-06\end{array}$

$\begin{array}{lll}-0.5781472 & 8.65849654 & 2.01 \mathrm{E}-12\end{array}$

$\begin{array}{lll}0.5791859 \quad 5.75647707 \quad 0.00135684 \\ -0.5800301 & 8.7003582 & 2.36 E-10\end{array}$

$\begin{array}{lll}-0.5800301 & 8.7003582 & 2.36 \mathrm{E}-10\end{array}$

$\begin{array}{llll}-0.5800966 & 4.67382716 & 0.02549577\end{array}$

heat shock factor binding protein, implicated in trancription $\quad \begin{array}{llll}-0.58129 & 6.12042103 & 0.00029098\end{array}$

$\begin{array}{llll}\text { MBF transcription factor complex subunit Res2 } & -0.5817213 & 6.91652995 & 4.15 \mathrm{E}-06\end{array}$

$\begin{array}{lll}-0.5829751 & 6.35575633 & 3.69 \mathrm{E}-05\end{array}$

$\begin{array}{lll}-0.5830737 \quad 6.03325461 & 0.00021873\end{array}$

$-0.5833827 \quad 6.54814446 \quad 7.07 \mathrm{E}-06$

$\begin{array}{lll}-0.5834121 & 8.14397985 & 4.62 \mathrm{E}-08\end{array}$

$\begin{array}{lll}-0.5844777 & 7.20047225 & 0.0005094\end{array}$

$-0.5848525 \quad 5.9440895 \quad 0.00162943$

$-0.5853275 \quad 8.27228443 \quad 1.08 \mathrm{E}-09$

$\begin{array}{lll}-0.5854078 & 6.29184649 \quad 8.56 \mathrm{E}-06\end{array}$

$-0.5856181 \quad 6.89636696 \quad 4.20 \mathrm{E}-07$

$\# N / A$

DUF2462 family protein, human Leydig cell tumor $10 \mathrm{kDa}$ p $-0.5858475 \quad 5.87744475 \quad 0.00039075$

retrotransposable element/transposon Tf2-type

ubiquitin C-terminal hydrolase Ubp11

$\begin{array}{lll}-0.5863948 & 5.17846162 & 0.00492139\end{array}$

$\begin{array}{lll}-0.5864179 & 5.98929999 & 0.00216998\end{array}$

$\begin{array}{lll}-0.5885861 & 5.38629767 \quad 0.00126742\end{array}$

$\begin{array}{lll}-0.5887374 & 8.15966009 & 7.87 \mathrm{E}-12\end{array}$

$-0.5888094 \quad 6.85880891 \quad 1.41 \mathrm{E}-06$

$\begin{array}{lll}-0.5890902 & 5.26049881 & 0.00132959\end{array}$

protein kinase activator Mob2

SMN family protein Smn1

ATP-dependent polydeoxyribonucleotide 5'-hydroxyl-kinase $\quad \begin{array}{lllll}-0.5898438 & 4.43537064 & 0.0402045\end{array}$

retrotransposable element/transposon Tf2-type

$\begin{array}{lll}-0.5902515 & 7.6970509 & 6.06 \mathrm{E}-08\end{array}$
4.70E-12

0.012297556

$2.10 \mathrm{E}-07$

0.000131364

0.000875243

0.00547544

4.63E-09

0.003164361

$1.63 \mathrm{E}-09$

$4.28 \mathrm{E}-06$

0.043600141

0.032540931

$8.76 \mathrm{E}-05$

0.000425343

2.91E-05

$2.04 \mathrm{E}-07$

0.021480508

2.29E-07

$5.62 \mathrm{E}-06$

0.465620502

0.00501562

0.011305075

1.19E-05

1.93E-11

0.003522822

$1.78 \mathrm{E}-09$

0.048382233

0.000881608

1.76E-05

0.00013136

0.000681851

$2.88 \mathrm{E}-05$

2.59E-07

0.00147337

0.004160697

7.54E-09

3.43E-05

2.04E-06

0.001156117

0.0112065

0.00540312

0.003323623

$7.08 \mathrm{E}-11$

6.37E-06

0.003465544

0.071789989

3.32E-07 
SPAC6B12.19

SPAC29A4.22

SPAC11D3.08C

SPNCRNA.1014

SPBC1711.14

SPAC20G4.05C

SPCC970.10c

SPAC1F3.02C

SPCC16C4.01

SPAC29B12.11C

SPAC1952.05

SPAC3A11.06

SPBC3B8.11

SPCC736.05

SPCC417.07c

SPNCRNA.671

SPBC1105.14

SPBC216.01C

SPAC2F3.16

SPBC16C6.02C

SPAC23C11.10

SPBC713.11C

SPAC824.05

ScpofMt29

SPAC20G8.02

SPNCRNA.1526

SPBC216.03

SPNCRNA.672

SPNCRNA.1151

SPBC336.07

SPBC11G11.01

SPNCRNA.108

SPNCRNA.66

SPAC513.02

SPBC3B9.21

SPBC1683.03C

SPAC17A5.11

SPBC725.02

SPNCRNA.1026

SPCC188.05

SPAPB2B4.06

SPNCRNA.57

SPBC17G9.05

SPACUNK4.17

SPNCRNA.780

SPNCRNA.438

SPCC24B10.14C rsa3
$\# \mathrm{~N} / \mathrm{A}$

SPAC11D3.08C

\#N/A

rec15

SPAC20G4.05c

brl2

mkh1

SPAC29B12.11C

gen5

mvp1

rrn6

wtf7

mto1

$\# N / A$

rsv2

psy2

SPAC2F3.16

vps 1302

mpn1

pmp3

vps16

\#N/A

SPAC20G8.02

$\# \mathrm{~N} / \mathrm{A}$

SPBC216.03

$\# \mathrm{~N} / \mathrm{A}$

sfc3

$\# N / A$

$\# \mathrm{~N} / \mathrm{A}$

SPAC513.02

SPBC1683.03c

rec12

mpr1

$\# \mathrm{~N} / \mathrm{A}$

$\# \mathrm{~N} / \mathrm{A}$

SPAPB2B4.06

\#N/A

rct1

SPACUNK4.17

$\#$ N/A

$\# \mathrm{~N} / \mathrm{A}$ ribosome assembly protein Rsa3 (predicted)

\#N/A

amino acid transmembrane transporter (predicted)

$\# \mathrm{~N} / \mathrm{A}$

meiotic recombination protein Rec15

$\begin{array}{lll}0.5907985 & 5.45454799 \quad 0.00160888\end{array}$

$\begin{array}{lll}-0.590806 & 7.74233353 \quad 1.60 \mathrm{E}-08\end{array}$

$-0.5908845 \quad 5.79391215 \quad 0.00076211$

$\begin{array}{lll}-0.5918372 & 3.59821521 & 0.14201405\end{array}$

mitochondrial protein, UPF0061 family, human SELENOO ol $\begin{array}{llll}-0.5932661 & 7.04972199 & 3.46 E-07\end{array}$

ubiquitin-protein ligase E3 Brl2 $\quad-0.5933765 \quad 7.42547313 \quad 4.93 \mathrm{E}-08$

mitochondrial protein, involved in mitochondrial gene expre $-0.5945827 \quad 6.48989876 \quad 6.08 \mathrm{E}-06$

human WW domain binding protein-2 ortholog $\quad-0.594762 \quad 6.45549399 \quad 6.13 \mathrm{E}-05$

SAGA complex histone acetyltransferase catalytic subunit $\&-0.5955957 \quad 6.90544401 \quad 3.19 \mathrm{E}-07$

sorting nexin Mvp1 (predicted)

$\begin{array}{llll}\text { RNA polymerase I general transcription initiation factor suk } & -0.5960694 & 7.18892648 & 6.11 \mathrm{E}-07\end{array}$

wtf element Wtf7

gamma tubulin complex linker Mto1

$\begin{array}{lll}-0.5966988 & 5.285419 & 0.00964613\end{array}$

$\begin{array}{lll}0.5969106 & 7.79711305 \quad 1.70 \mathrm{E}-08\end{array}$

$\begin{array}{lll}-0.5972497 \quad 6.72058031 & 0.00010312\end{array}$

$\begin{array}{lll}-0.5976899 & 7.09862012 & 6.79 \mathrm{E}-07\end{array}$

transcription factor Rsv2

$$
\# \mathrm{~N} / \mathrm{A}
$$

protein phosphatase PP4 complex regulatory subunit 3 Psy2 -0.59769

Nepair (predir -0.59871567 .2974866$

$1.62 \mathrm{E}-10$

$6.49 \mathrm{E}-09$

$-0.5988514 \quad 9.88066816 \quad 1.82 \mathrm{E}-14$

poly(U)-specific exoribonuclease, producing 3' uridine cyclic $\begin{array}{cllll}-0.5991935 & 4.4783731 & 0.02608651\end{array}$

HOPS/CORVET complex subunit Vps16 (predicted) $\# \mathrm{~N} / \mathrm{A}$

mitochondrial DDHD family phospholipase (predicted)

$$
\text { \#N/A }
$$

NADP binding superfamily conserved fungal protein

$$
\# N / A
$$

$\# N / A$

$\begin{array}{rrr}0.5993683 & 7.26414635 & 4.72 \mathrm{E}-08 \\ -0.5994926 & 7.44448384 & 8.17 \mathrm{E}-09\end{array}$

$\begin{array}{lll}-0.5995669 & 2.77624901 & 0.25298749\end{array}$

$-0.60149047 .66144203 \quad 1.93 \mathrm{E}-09$

$-0.60212376 .85886836 \quad 6.60 \mathrm{E}-07$

$-0.6025898 \quad 7.17082465 \quad 9.48 \mathrm{E}-09$

$-0.6026498 \quad 6.60228123 \quad 9.26 \mathrm{E}-05$

$\begin{array}{lll}-0.6026938 & 5.45024956 & 0.00225773\end{array}$

transcription factor TFIIIC complex B box binding subunit Sf $\quad \begin{array}{llll}-0.6032595 & 7.04056992 & 8.56 \mathrm{E}-08\end{array}$

$\begin{array}{llll}\text { mitochondrial fission protein Fis1 (predicted) } & -0.6035593 & 7.43028133 & 2.02 \mathrm{E}-10\end{array}$

$\# N / A$

$\begin{array}{lll}-0.6047857 & 3.60958411 & 0.1278613\end{array}$

$\begin{array}{lll}-0.6048302 & 3.23525313 & 0.24714201\end{array}$

phosphoglycerate mutase/6-phosphofructo-2-kinase family $\begin{array}{lllll}-0.6055863 & 4.88800485 & 0.0604589\end{array}$

mRNA decapping complex regulatory subunit Dcp1

$\begin{array}{lll}0.6056465 & 4.98385325 & 0.0080477\end{array}$

$-0.6079072 \quad 7.6968621 \quad 9.29 \mathrm{E}-12$

$-0.6081934 \quad 4.83359944 \quad 0.01615829$

meiotic recombination endonuclease Rec12

2.53E-12

\#N/A $\quad-0.6086672 \quad 6.77613186 \quad 2.52 \mathrm{E}-06$

\#N/A $\quad-0.609657 \quad 1.731037090 .40315342$

acyl-coenzyme $\mathrm{A}$ thioesterase

$\begin{array}{lll}-0.6099243 & 6.44752461 & 5.13 \mathrm{E}-06\end{array}$

\#N/A

$\begin{array}{lll}-0.6107365 & 1.36231072 & 0.60260688\end{array}$

cyclophilin family peptidyl-prolyl cis-trans isomerase, RRM- $-0.6111952 \quad 6.3478878 \quad 4.16 \mathrm{E}-06$

$\begin{array}{llll}\text { NAD binding dehydrogenase family protein, human DHDH o } & -0.6115016 & 7.1254357 & 6.41 \mathrm{E}-06\end{array}$

$\# N / A$

$\begin{array}{llll}-0.611552 & 2.8854436 & 0.17559373\end{array}$ $\begin{array}{lll}0.6119315 & 4.02501815 & 0.09387765\end{array}$ $\begin{array}{lllllll}\text { XRCC4-like nonhomologous end joining factor, Cernunnon X } & -0.6128644 & 4.27166883 & 0.02362787\end{array}$
0.106305816

0.004112856

$9.45 \mathrm{E}-08$

0.00211248

0.211374798

1.70E-06

2.75E-07

$1.54 \mathrm{E}-08$

2.51E-05

0.000209425

$1.58 \mathrm{E}-06$

0.000205349

$2.91 \mathrm{E}-06$

0.020404689

$1.00 \mathrm{E}-07$

0.000339013

3.21E-06

$1.23 \mathrm{E}-09$

4.08E-08

2.06E-13

0.049338164

2.64E-07

5.06E-08

0.34308088

$1.29 \mathrm{E}-08$

3.13E-06

5.81E-08

0.000306629

0.005607248

$4.58 \mathrm{E}-07$

$1.52 \mathrm{E}-09$

0.193483687

0.336831844

0.101786933

0.017314131

8.26E-11

0.032397512

2.41E-11

1.10E-05

0.498629408

2.14E-05

0.685971861

1.76E-05

2.64E-05

0.252251494

0.148843762

0.045317818 
SPBC27B12.14

SPNCRNA.765

SPBP35G2.06C

SPBC2D10.16

SPCC330.08

SPAC25B8.08

SPAC14C4.08

SPAC17C9.16C

SPCC1919.10c

SPCC594.04c

SPCPB1C11.02

SPNCRNA.384

SPCC18B5.09C

SPAC1D4.14

SPAP27G11.05

SPCC965.08C

SPBC12C2.01C

SPNCRNA.903

SPCC594.05C

SPCP31B10.03C

SPAC23H3.03C

SPBC9B6.02C

SPBC16A3.02C

SPAC26A3.13C

SPNCRNA.1387

SPAC22F $3.08 \mathrm{C}$

SPBC6B1.05C

SPBC1778.02

SPACUNK4.20

SPAC16E8.12C

SPAC23H4.14

SPBC18H10.10c

SPBC543.08

SPBC15D4.12C

SPNCRNA.1013

SPBC365.13C

SPAC16A10.01

SPCC1442.04c

SPNCRNA.1167

SPAC1F3.01

SPAC750.05c

SPNCRNA.1362

SPNCRNA.857

SPAC27D7.11C

SPBP18G5.02

SPAC823.05C

SPNCRNA.1581 cbp4

$\mathrm{N} / \mathrm{A}$

nup131

mhf1

SPAC25B8.08

mug5

mfs1

myo52

SPCC594.04c

SPCPB1C11.02

\#N/A

SPCC18B5.09C

tho2

vps41

SPBC12C2.01C

$\# \mathrm{~N} / \mathrm{A}$

spf1

$\operatorname{med} 31$

npr2

Tf2-9

SPBC16A3.02C

Tf2-4

$\# \mathrm{~N} / \mathrm{A}$

rok1

$\operatorname{atg} 7$

rap1

$\# \mathrm{~N} / \mathrm{A}$

png3

vam6

saf4

SPBC543.08

mug98

$\#$ N/A

hba1

SPAC16A10.01

SPCC1442.04C

$\#$ N/A

rrp6

ftm4

$\# N / A$

$\# \mathrm{~N} / \mathrm{A}$

SPAC27D7.11C

pgs1

tlg2

\#N/A mitochondrial respiratory complex assembly protein Cbp4 $(-0.61416895 .518840510 .00215387$ $\begin{array}{lll}-0.6149876 & 3.533649 & 0.19278169\end{array}$ $\begin{array}{lll}-0.6157603 & 7.53096954 & 6.52 \mathrm{E}-09\end{array}$ $-0.6161944 \quad 4.81134554 \quad 0.01084845$ nucleoporin, WD repeat Nup131 N/A

$\begin{array}{lllll}\text { GDP-Man:Man3GIcNAc2-PP-Dol alpha-1,2-mannosyltransfe } & -0.6166666 & 7.64345333 & 8.90 \mathrm{E}-12 \\ \text { Golgi Ras-interacting protein with DIL domain, human RAD } & -0.6170599 & 6.94182642 & 2.84 \mathrm{E}-07\end{array}$

$\begin{array}{llrlr}\text { Golgi Ras-interacting protein with DIL domain, human RAD } & -0.6170599 & 6.94182642 & 2.84 \mathrm{E}-07 \\ \text { dynactin complex subunit Mug5 } & -0.617086 & 4.45089418 & 0.01759214\end{array}$

plasma membrane spermidine transmembrane transporter -0.61716518 .78672909$

myosin type $\mathrm{V}$

steroid oxidoreductase superfamily protein (predicted)

amino acid transmembrane transporter (predicted)

$$
\text { \#N/A }
$$

Schizosaccharomyces specific protein

THO complex subunit Tho2 (predicted)

HOPS complex subunit Vps41 (predicted)

alanine racemase Alr1

Schizosaccharomyces specific protein

$$
\text { \#N/A }
$$

Set1C PHD Finger protein Spf1

mediator complex subunit Med31

$\mathrm{SEA} / \mathrm{Iml} 1 / \mathrm{Npr} 2 / 3$ complex subunit Npr2

$\begin{array}{lll}-0.6178318 & 8.29928444 & 2.92 \mathrm{E}-12\end{array}$

$-0.6180591 \quad 6.3866299 \quad 1.85 \mathrm{E}-05$

$-0.6184034 \quad 7.73777702 \quad 1.15 \mathrm{E}-10$

$\begin{array}{llll}-0.6186343 & 3.31659885 & 0.11538949\end{array}$

$\begin{array}{lll}-0.6196319 & 5.68993446 & 0.00903157\end{array}$

$\begin{array}{lll}-0.6196692 & 8.97226385 & 8.34 \mathrm{E}-17\end{array}$

$\begin{array}{lll}-0.6201626 & 7.37738436 \quad 4.68 \mathrm{E}-10\end{array}$

$\begin{array}{lll}-0.6213789 & 6.11504774 & 0.00017253\end{array}$

$\begin{array}{lll}-0.621589 & 6.32817999 & 0.00095871\end{array}$

$\begin{array}{llll}-0.6220122 & 2.9474472 & 0.25011074\end{array}$

$\begin{array}{llll}-0.622171 & 6.10294021 & 0.00025166\end{array}$

$-0.6229335 \quad 5.97415258 \quad 0.00088461$

$\begin{array}{lll}-0.6229459 & 6.524568 & 7.57 \mathrm{E}-05\end{array}$

$\begin{array}{lrrr}\text { retrotransposable element/transposon Tf2-type } & -0.622948 & 7.9508481 & 9.51 \mathrm{E}-09 \\ \text { mitochondrial CH-OH group oxidoreductase, human RTN4IP } & -0.6234835 & 8.76290714 & 7.27 \mathrm{E}-13\end{array}$

retrotransposable element/transposon Tf2-type $\quad \begin{array}{lllll}-0.6236237 & 8.13250429 & 1.86 \mathrm{E}-09\end{array}$

$-0.6247738 \quad 5.70697177 \quad 0.0049796$

$\begin{array}{lll}-0.625117 & 6.0948312 & 8.78 \mathrm{E}-06\end{array}$

$-0.62650546 .75210716 \quad 1.08 \mathrm{E}-06$

$-0.6272425 \quad 5.94257033-5.10 \mathrm{E}-05$

$-0.6281827 .48638281 \quad 2.61 \mathrm{E}-11$

$-0.6297285 \quad 6.37256028 \quad 2.86 \mathrm{E}-06$$$
\text { \#N/A }
$$

ING family homolog Png3 (predicted)

$\begin{array}{lll}-0.6309879 & 6.28207674 \quad 1.69 \mathrm{E}-05 \\ -0.6316155 & 6.33003754 & 2.89 \mathrm{E}-05\end{array}$

$-0.6316155 \quad 6.33003754 \quad 2.89 \mathrm{E}-05$

$-0.6329112 \quad 3.93905643 \quad 0.06616709$

$-0.6336748 \quad 5.838209840 .00028386$

$-0.63369597 .40370694 \quad 7.17 \mathrm{E}-10$

Ran GTPase binding protein Hba

$\begin{array}{lll}-0.6338951 & 6.26197857 \quad 8.23 \mathrm{E}-06\end{array}$

$-0.63394957 .74504392 \quad 8.27 \mathrm{E}-13$

$\begin{array}{lll}-0.6352972 & 5.3970898 & 0.0005498\end{array}$

$\begin{array}{lll}-0.6360921 & 7.83285186 \quad 7.85 \mathrm{E}-09\end{array}$

$\begin{array}{llll}-0.6366078 & 7.26797792 & 4.02 \mathrm{E}-07\end{array}$

$\begin{array}{llll}-0.6367742 & 6.28747667 & 0.00038292\end{array}$

$-0.6368637 \quad 7.42061916 \quad 1.31 \mathrm{E}-07$ $\begin{array}{lll}-0.6369723 & 7.73755486 & 1.37 \mathrm{E}-05\end{array}$

$\begin{array}{lllll} & 0.6370147 & 6.52085876 & 2.71 \mathrm{E}-05\end{array}$ SNARE TIg2 (predicted)

$\begin{array}{lll}-0.637267 & 6.69714019 \quad 2.87 \mathrm{E}-07\end{array}$

$\begin{array}{llll}-0.6377023 & 3.46307741 & 0.17593538\end{array}$
0.00536496

0.272400206

4.09E-08

0.02268504

7.94E-11

$1.41 \mathrm{E}-06$

0.035014489

$3.52 \mathrm{E}-15$

2.74E-11

6.97E-05

$8.92 \mathrm{E}-10$

0.177171808

0.019206196

1.17E-15

3.40E-09

0.000545317

0.002584781

0.339992724

0.000774204

0.002405977

0.000254441

$5.81 \mathrm{E}-08$

7.30E-12

$1.25 \mathrm{E}-08$

0.011306688

$3.51 \mathrm{E}-05$

4.97E-06

0.000177324

2.21E-10

1.24E-05

$1.18 \mathrm{E}-05$

$6.42 \mathrm{E}-05$

0.000105079

0.110009052

0.000863132

5.08E-09

3.32E-05

$8.27 \mathrm{E}-12$

0.001572015

$4.88 \mathrm{E}-08$

1.96E-06

0.00113542

6.80E-07

5.31E-05

$9.92 \mathrm{E}-05$

$1.42 \mathrm{E}-06$

0.252630021 
SPBC14C8.11C

SPNCRNA.1394

SPAC11D3.09

SPAC22G7.08

SPBC2D10.17

SPAC13C5.07

SPAC1834.07

SPAC27D7.12C

SPAC227.19c

SPNCRNA.1679

SPNCRNA.996

SPAC9E9.14

SPAC1D4.10

SPCC777.03C

SPBP35G2.17

SPAC458.03

SPAC7D4.04

SPNCRNA.1044

SPAC5D6.04

SPAC13C5.03

SPAC144.12

SPAC1002.06c

SPBC25H2.08c

SPCC1919.12C

SPAC57A10.08C

SPAC1952.17c

SPNCRNA.519

SPAC27E2.09

SPAC7D4.13C

SPBC1685.05

SPBC19F5.01C

SPAC10F6.12C

SPCC16A11.04

SPNCRNA.1516

SPCC18B5.11C

SPCC830.09c

SPNCRNA.427

SPNCRNA.190

SPNCRNA.1461 wtf12

SPBC14C8.11C

$\# \mathrm{~N} / \mathrm{A}$

SPAC11D3.09

ppk8

clr1

mre11

but1

SPAC227.19c

$\# \mathrm{~N} / \mathrm{A}$

$\# N / A$

vps 24

trz1

SPCC777.03C

\#N/A

tel2

$\operatorname{atg} 11$

$\# \mathrm{~N} / \mathrm{A}$

SPAC5D6.04

tht1

rki1

bqt2

mrs2

erm2

SPAC57A10.08 tbc13

$\# N / A$

mak2

SPAC7D4.13C

htr11

puc1

mam4

snx12

$$
\text { \#N/A }
$$

cds1

pop5

$\#$ N/A
$\#$ N/A
\#N/A

$\begin{array}{llll}-0.6377023 & 3.46307741 & 0.17593538\end{array}$

$\begin{array}{lllll}\text { \#N/A } & -0.638771 & 4.20540389 & 0.03646518\end{array}$

$\# N / A$

$\# N / A$

F-box protein Pof2

bouquet formation protein Bqt1

Schizosaccharomyces pombe specific protein

$$
\# \mathrm{~N} / \mathrm{A}
$$

agmatinase (predicted)

serine/threonine protein kinase Ppk8 (predicted)

SHREC complex intermodule linker subunit CIr1

Mre11 nuclease

kinesin-like protein Klp3

thioredoxin family protein But1

$\begin{array}{lll}-0.6438768 & 4.36493366 & 0.01447081\end{array}$

$\begin{array}{lll}0.638553 & 4.71033989 & 0.00555684\end{array}$

$\begin{array}{lll}-0.6393841 & 6.74654214 & 4.83 \mathrm{E}-08\end{array}$

$\begin{array}{lll}-0.6395206 & 4.71446216 & 0.00767679\end{array}$

$\begin{array}{llll}-0.640082 & 4.63188635 & 0.01893446\end{array}$

$\begin{array}{lll}-0.6412504 & 5.13052929 & 0.00397417\end{array}$

$\begin{array}{lll}-0.6417308 & 5.08426597 & 0.00154008\end{array}$

$\begin{array}{lll}-0.6419175 & 6.82523893 & 3.49 \mathrm{E}-06\end{array}$

$-0.6419987 \quad 6.46605567 \quad 2.76 \mathrm{E}-07$

$-0.6422644 \quad 7.72866814 \quad 1.42 \mathrm{E}-10$

$\begin{array}{lll}-0.6423871 & 8.00181904 & 8.33 \mathrm{E}-15\end{array}$

$\begin{array}{llll}-0.6455078 & 4.25106184 & 0.16860209\end{array}$

$\# N / A$

$\# \mathrm{~N} / \mathrm{A}$

$\begin{array}{llll}0.6460352 & 4.04389621 & 0.07733715\end{array}$

$\begin{array}{lll}-0.6464329 & 6.61768042 & 1.60 \mathrm{E}-07\end{array}$

$-0.6467805 \quad 6.21876493 \quad 4.12 \mathrm{E}-05$

ESCRT III complex subunit Vps24

$-0.6470053 \quad 7.15554633 \quad 1.12 \mathrm{E}-08$

$\begin{array}{lll}-0.6471509 & 6.4756176 & 5.80 \mathrm{E}-06\end{array}$

$-0.6474018 \quad 5.91174693 \quad 2.05 \mathrm{E}-05$

$-0.6476276 \quad 6.95810419 \quad 4.13 \mathrm{E}-08$

$-0.6484115 \quad 8.52924463 \quad 3.42 \mathrm{E}-17$

$\begin{array}{lll}-0.6484753 & 5.28068781 & 0.00116184\end{array}$

$\begin{array}{lll}-0.6488127 & 8.19504344 \quad 4.56 \mathrm{E}-15\end{array}$

$\begin{array}{lll}-0.6494783 & 5.7618359 & 0.00031589\end{array}$

$-0.6494886 \quad 7.82160117 \quad 1.04 \mathrm{E}-10$

$\begin{array}{lll}-0.6518711 & 2.3003563 & 0.31522166\end{array}$

nuclear membrane protein involved in karyogamy Tht1

ribose 5-phosphate isomerase Rki1 (predicted)

bouquet formation protein Bqt2

$-0.6529578 \quad 5.66814984 \quad 0.00044614$

$\begin{array}{lllll}\text { multi-spanning } & 0.6530689 & 8.33964704 & 1.19 \mathrm{E}-11\end{array}$

$\begin{array}{lllll}\text { lipid particle hydrolase (predicted) } & -0.6538386 & 5.83013823 & 0.00015755\end{array}$

GTPase activating protein, involved in vesicle-mediated tral $-0.6541751 \quad 6.58466291 \quad$ 4.90E-06

$$
\# \mathrm{~N} / \mathrm{A}
$$

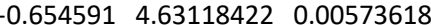

$\begin{array}{lll}-0.655202 & 8.8870319 & 2.26 \mathrm{E}-15\end{array}$

$\begin{array}{lll}-0.6554032 & 5.79588675 & 0.0002367\end{array}$

$-0.6555003 \quad 7.44341976 \quad 8.41 \mathrm{E}-10$

$-0.6559093 \quad 6.43395655 \quad 5.88 \mathrm{E}-05$

$\begin{array}{lll}-0.6570124 & 6.71307908 & 0.0007756\end{array}$

$\begin{array}{lll}-0.6570253 & 7.65389939 & 6.89 E-08\end{array}$

$\begin{array}{llll}-0.6589701 & 4.20387837 & 0.03577229\end{array}$

$\begin{array}{lll}-0.6598177 & 7.47965886 & 2.16 \mathrm{E}-07\end{array}$

$\begin{array}{lll}-0.660016 & 5.99883379 & 0.00063344\end{array}$

$\begin{array}{llll}-0.6611994 & 2.13402 & 0.35384029\end{array}$

$\begin{array}{llll}-0.6612488 & 2.77875722 & 0.28385898\end{array}$

$\begin{array}{lll}-0.6614692 & 6.45622999 & 2.59 \mathrm{E}-07\end{array}$
0.252630021

0.000799804

0.06599615

0.012475844

0.448382828

2.69E-07

0.016621249

0.037445221

0.009239571

0.003959282

$1.50 \mathrm{E}-05$

1.37E-06

$1.09 \mathrm{E}-09$

$9.81 \mathrm{E}-14$

7.73E-06

0.029381179

0.243958586

0.125648744

$8.22 \mathrm{E}-07$

0.000145313

$6.81 \mathrm{E}-08$

2.40E-05

7.66E-05

2.33E-07

$5.02 \mathrm{E}-16$

0.003070411

$5.49 \mathrm{E}-14$

0.000953698

$8.12 \mathrm{E}-10$

0.409210868

0.001306346

$1.05 \mathrm{E}-10$

0.000502871

2.05E-05

0.01286152

2.80E-14

0.000732479

$5.92 \mathrm{E}-09$

0.000201886

0.002141158

3.73E-07

0.064914785

$1.08 \mathrm{E}-06$

0.001788608

0.449419173

0.376762309

1.29E-06 
SPBC1105.12

SPNCRNA.1248

SPNCRNA.995

SPNCRNA.561

SPNCRNA.1481

SPCC63.04

SPBC660.05

SPNCRNA.647

SPAC8E 11.12

SPAC29B12.03

SPNCRNA.1274

SPNCRNA.584

SPNCRNA.1285

SPBC18E5.03C

SPAC24C9.11

SPAC17A2.06C

SPCC297.04c

SPNCRNA.253

SPNCRNA.833

SPNCRNA.1283

SPCPJ732.03

SPNCRNA.931

SPBC577.13

SPBC18H10.05

SPCC757.02C

SPNCRNA.773

SPBC1683.01

SPAC4D7.14

SPBP22H7.02C

SPBC1718.06

SPNCRNA.506

SPAC23D3.13C

SPCC965.05C

SPAC15E1.02C

SPAC27E2.08

SPAC10F6.09c

SPNCRNA.725

SPBC11C11.11C

SPAC4A8.04

SPNCRNA.987

SPAC1687.14C

SPAC6F 12.02

SPAC27D7.03C jmj1

mug126

Tf2-2

hhf3

$\# \mathrm{~N} / \mathrm{A}$

$\# N / A$

$\# N / A$

$\# N / A$

$\#$ N/A

mok14

SPBC660.05

$\# \mathrm{~N} / \mathrm{A}$

SPAC8E11.12

spd1

$\# N / A$

$\# N / A$

$\# N / A$

$\operatorname{sim} 4$

sgd1

vps8

set7

$\# \mathrm{~N} / \mathrm{A}$

$\# \mathrm{~N} / \mathrm{A}$

$\# \mathrm{~N} / \mathrm{A}$

meu15

\#N/A

syj2

SPBC18H10.05

SPCC757.02C

$\# \mathrm{~N} / \mathrm{A}$

SPBC1683.01

new13

mrd1

msp1

\#N/A

SPAC23D3.13C

thp1

SPAC15E1.02C

Tf2-6

psm3

$\# \mathrm{~N} / \mathrm{A}$

irc3

isp6

\#N/A

SPAC1687.14c

rst2

mei2 histone demethylase Jmj1 (predicted)

Schizosaccharomyces pombe specific protein

retrotransposable element/transposon Tf2-type

$\#$ N/A

histone $\mathrm{H} 4 \mathrm{~h} 4.3$

$\# \mathrm{~N} / \mathrm{A}$

$\# N / A$

$\# N / A$

$\# \mathrm{~N} / \mathrm{A}$

alpha-1,4-glucan synthase Mok14

WW domain containing conserved fungal protein

$$
\text { \#N/A }
$$

Schizosaccharomyces pombe specific protein

ribonucleotide reductase (RNR) inhibitor

$$
\# N / A
$$$$
\# \text { \#N/A }
$$

CENP-K ortholog Sim 4

\#N/A

ribosome small subunit biogenesis protein Sgd1 (predicted)

CORVET complex WD repeat/ ubiquitin-protein ligase E3 su

histone lysine methyltransferase Set7 (predicted)

$$
\# N / A
$$$$
\# N / A
$$

Schizosaccharomyces pombe specific protein Meu15

$$
\text { \#N/A }
$$

$\begin{array}{lll}0.6619137 & 5.04617126 & 0.00534463\end{array}$

$\begin{array}{lll}-0.6622897 & 5.09315457 & 0.01403693\end{array}$

$\begin{array}{lll}-0.6627366 & 9.23315758 \quad 2.55 \mathrm{E}-12\end{array}$

$\begin{array}{lll}-0.6636267 & 2.63512196 & 0.23845952\end{array}$

$\begin{array}{lll}-0.6643656 & 7.8668804 & 3.01 \mathrm{E}-13\end{array}$

$\begin{array}{lll}-0.6646687 & 3.00521569 & 0.20275003\end{array}$

$\begin{array}{lll}-0.6654188 & 6.62120635 \quad 8.67 \mathrm{E}-08\end{array}$

$\begin{array}{lll}-0.6655302 & 5.74392969 & 7.54 \mathrm{E}-05\end{array}$

$\begin{array}{lll}-0.665686 & 4.44379088 & 0.01163774\end{array}$

$\begin{array}{lll}-0.66592 & 7.44675111 \quad 8.58 \mathrm{E}-09\end{array}$

$\begin{array}{lll}-0.6678054 & 5.24897054 & 0.00068228\end{array}$

$\begin{array}{lll}-0.6707495 & 6.96375839 & 6.13 \mathrm{E}-09\end{array}$

$\begin{array}{lll}0.6710906 & 2.58396417 & 0.27298764\end{array}$

$\begin{array}{lll}-0.671112 & 6.86935289 & 4.63 \mathrm{E}-09\end{array}$

$\begin{array}{lll}-0.6716189 & 7.38889057 \quad 4.45 E-11\end{array}$

$-0.67281067 .38291431 \quad 4.12 \mathrm{E}-11$

$-0.67381415 .90713856 \quad 5.96 \mathrm{E}-06$

$\begin{array}{lll}-0.6744187 & 5.47847709 & 0.00013533\end{array}$

$\begin{array}{lll}-0.6755234 & 7.2354539 & 8.62 \mathrm{E}-09\end{array}$

$-0.6756375 \quad 8.10249427 \quad 6.62 \mathrm{E}-11$

$\begin{array}{lll}-0.6767454 & 6.4266526 & 6.13 \mathrm{E}-06\end{array}$

$\begin{array}{llll}-0.6773266 & 2.23624907 & 0.29432647\end{array}$

$-0.6776873 \quad 6.84963132 \quad 8.90 \mathrm{E}-08$

$\begin{array}{llll}-0.6780578 & 5.02785194 & 0.00060046\end{array}$

$\begin{array}{llll}-0.6785087 & 1.86477774 & 0.33312797\end{array}$

$-0.6785773 \quad 5.7559139 \quad 3.63 \mathrm{E}-05$

inositol-polyphosphate 5-phosphatase, synaptojanin homolı $-0.6787885 \quad 7.53570063 \quad 1.15 \mathrm{E}-12$

WD repeat protein, human WDR44 family $\quad-0.6788887 \quad 6.70808544 \quad 8.07 E-08$

dehydrogenase (predicted)

$$
\# \mathrm{~N} / \mathrm{A}
$$

$2.13 \mathrm{E}-05$

$\begin{array}{lll}-0.6797738 & 6.30031247 & 2.13 \mathrm{E}-05 \\ -0.6797771 & 6.60508743 & 6.84 \mathrm{E}-07\end{array}$

$\begin{array}{lllll}\text { inorganic phosphate transmembrane transporter (predictec } & -0.680832 & 7.66172256 & 1.41 \mathrm{E}-10\end{array}$

conserved fungal protein of unknown function

$0.6812431 \quad 5.30990527 \quad 0.00107951$

ribosome biogenesis RNA-binding protein Mrd1 (predicted) $\quad-0.6819439 \quad 6.33588758 \quad 8.80 \mathrm{E}-06$

mitochondrial dynamin family fusion GTPase Msp1

$$
\text { \#N/A }
$$

$-0.6826095 \quad 8.89839497 \quad 8.59 \mathrm{E}-21$

$\begin{array}{lll}0.6843972 \quad 1.23599102 & 0.76938276\end{array}$

$-0.6849427 \quad 8.57717436 \quad 1.95 \mathrm{E}-18$

$\begin{array}{lll}-0.6849591 & 6.71104308 \quad 3.00 \mathrm{E}-09\end{array}$

$-0.68546547 .34574445$

$-0.6855052 \quad 8.77867297 \quad 8.66 \mathrm{E}-13$

$\begin{array}{lll}-0.6856218 & 8.42149622 \quad 7.66 \mathrm{E}-11\end{array}$

$\begin{array}{lll}-0.6856448 & 8.42373868 \quad 8.10 \mathrm{E}-11\end{array}$

retrotransposable element/transposon Tf2-type

mitotic cohesin complex ATPase subunit Psm3/Smc3

\#N/A

mitochondrial DNA branch migration helicase Irc3 (predicte $\quad-0.686403 \quad 7.01895775 \quad 3.20 \mathrm{E}-08$

vacuolar serine protease Isp6

$\# \mathrm{~N} / \mathrm{A}$

EF hand family protein, centrin-like $\begin{array}{lll}-0.6869763 & 9.28395729 & 1.08 \mathrm{E}-12\end{array}$ $-0.68726563 .317093540 .08818333$

$\begin{array}{lll}-0.6876215 & 5.47315029 & 0.00207538\end{array}$

$\begin{array}{lll}-0.6887258 & 7.86106749 \quad 4.83 \mathrm{E}-15\end{array}$

$-0.68922678 .67137072 \quad 2.22 \mathrm{E}-07$
0.012039122

0.028607765

$2.41 \mathrm{E}-11$

0.326633865

3.14E-12

0.283774982

4.64E-07

0.000253653

0.024166866

5.30E-08

0.001917756

3.86E-08

0.364670456

2.96E-08

3.64E-10

3. $40 \mathrm{E}-10$

2.46E-05

0.000436879

5.32E-08

$5.26 \mathrm{E}-10$

2.53E-05

0.387032196

4.74E-07

0.001701123

0.427481775

0.000129199

1.13E-11

4.33E-07

7.93E-05

3.23E-06

1.09E-09

0.002876301

3.51E-05

$1.80 \mathrm{E}-19$

0.828687822

3.19E-17

$1.95 \mathrm{E}-08$

$1.30 \mathrm{E}-10$

$8.62 \mathrm{E}-12$

6.03E-10

6.35E-10

1.84E-07

1.07E-11

0.141130657

0.00518084

5.80E-14

$1.12 \mathrm{E}-06$ 
SPAC30.02C

SPAPB24D3.04c

SPNCRNA.379

SPAC607.08C

SPNCRNA.68

SPAC1006.04C

SPAC110.01

SPAC16E8.08

SPNCRNA.1427

SPAC3A11.14C

SPAC16A10.02

SPNCRNA.1368

SPCC338.03C

SPNCRNA.1008

SPAC22A12.01c

SPNCRNA.1689

SPNCRNA.70

SPAC607.07C

SPBC3B9.06C

SPBC582.06C

SPBC16C6.14

SPBC29A10.10c

SPBC16C6.01C

SPNCRNA.1458

SPAC22G7.11C

SPNCRNA.1284

SPAC3H1.14

SPAC9.04

SPNCRNA.1572

SPAC823.16C

SPAC17H9.01

SPCC569.04

SPBC27.03

SPAC22H10.10

SPBC1718.01

SPNCRNA.1611

SPAC1002.10c

SPNCRNA.738

SPBC4C3.08

SPAC1002.20

SPCC18B5.03

SPBC1703.04

SPCC417.04

SPNCRNA.1579

SPAC22G7.07c

ScpofMr11

SPNCRNA.1443 ub1

$\# N / A$

$\# N / A$

$\#$ N/A

$\# N / A$

$\# \mathrm{~N} / \mathrm{A}$

SPAC607.07C

atg3

mcp6

spo2

SPBC16C6.01c

\#N/A

cum1

$\# N / A$

\#N/A

$\operatorname{atg} 1802$

cid16

SPCC569.04

meu25

alp21

pop1

\#N/A

sgt1

\#N/A

otg2

SPAC1002.20

wee1

mlh1

\#N/A

$\# N / A$

ime4

$\# N / A$

$\# N / A$ elongator complex associated protein Kti12 (predicted)

DNA-3-methyladenine glycosylase Mag1

$$
\# N / A
$$

$0.69071315 .86130298 \quad 0.00017228$

$-0.69120015 .60850083 \quad 6.05 \mathrm{E}-05$

$-0.6914805 \quad 1.23596255 \quad 0.76509305$

lipase/AP-1 adaptor complex binding protein Mil1 (predicte $-0.6929824 \quad 6.70754047 \quad 6.15 \mathrm{E}-08$

$$
\# \mathrm{~N} / \mathrm{A}
$$

Schizosaccharomyces specific protein Mcp 3

serine/threonine protein kinase Ppk1 (predicted)

Spa2 interacting protein Pos1

$$
\text { \#N/A }
$$

$\begin{array}{lll}-0.6931304 & 1.23627586 & 0.76264901\end{array}$

$\begin{array}{lll}-0.6943372 & 5.3994089 & 0.00045024\end{array}$

$-0.694794 \quad 7.11413621 \quad 1.16 \mathrm{E}-07$

$\begin{array}{llll}-0.6954059 & 3.97835671 & 0.02033354\end{array}$

$\begin{array}{llll}-0.6958033 & 5.96541383 & 0.00010132\end{array}$

kinesin-14 family minus-end directed microtubule motor Pk $\quad-0.6961085 \quad 7.14488873 \quad 1.14 \mathrm{E}-10$

transcription coactivator PC4

\section{$\# N / A$}

$\# N / A$

DNA 5' exonuclease (predicted)

\#N/A

$\# N / A$

Schizosaccharomyces specific protein

autophagy associated protein Atg3

horsetail movement protein Hrs1/Mcp6

sporulation specific protein $\mathrm{Spo} 2$

ATP-dependent RNA helicase Dbl8

$\begin{array}{lll}0.6963432 & 7.67333288 & 7.71 \mathrm{E}-12\end{array}$

$\begin{array}{lll}-0.6966477 & 6.4647392 & 1.16 \mathrm{E}-07\end{array}$

$\begin{array}{lll}-0.6968925 & 4.94522357 & 0.00465387\end{array}$

$\begin{array}{llll}-0.6971133 & 5.6589456 & 0.00015372\end{array}$

$\begin{array}{llll}-0.6989935 & 6.79796432 \quad 237 \mathrm{E}-09\end{array}$

$\begin{array}{lll}-0.7002347 & 4.17901561 & 0.01485141\end{array}$

$\begin{array}{lll}-0.7004943 & 1.23658302 & 0.76608323\end{array}$

$-0.70246976 .64463076 \quad 7.97 \mathrm{E}-09$

$-0.7036645 \quad 6.16916228 \quad 1.74 \mathrm{E}-06$

$-0.70376714 .04513168 \quad 0.03702945$

$\begin{array}{lll}-0.7045751 & 6.64919299 \quad 6.69 \mathrm{E}-08\end{array}$

$-0.7047544 \quad 7.82774571 \quad 3.23 \mathrm{E}-13$

lysine methyltransferase, human SETD6 ortholog (predictec $\begin{array}{llll}-0.7052682 & 5.82822704 & 1.65 \mathrm{E}-05\end{array}$ \#N/A

$\begin{array}{lll}-0.7055172 & 5.34365252 & 0.01545942\end{array}$

$\begin{array}{llll}-0.7064823 & 7.04672835 & 8.50 \mathrm{E}-09\end{array}$

$\begin{array}{lll}-0.7064894 & 3.34824734 & 0.17836654\end{array}$

$\begin{array}{llll}-0.707181 & 5.32070143 & 0.00354167\end{array}$

$-0.70851358 .80954668 \quad 4.54 \mathrm{E}-14$

$-0.7092634 \quad 5.08935404 \quad 0.00080746$

$-0.7095731 \quad 6.46311283 \quad 3.48 \mathrm{E}-08$

$\begin{array}{lll}-0.7114567 & 7.75647024 & 1.02 \mathrm{E}-12\end{array}$

$\begin{array}{llll}-0.7118646 & 3.36274531 & 0.07068237\end{array}$

$-0.71208845 .72845725 \quad 1.80 \mathrm{E}-05$

$\begin{array}{llll}-0.7122183 & 5.24209176 & 0.00060008\end{array}$

$-0.71538767 .11084974 \quad 1.22 \mathrm{E}-08$

$\begin{array}{lll}-0.718359 & 7.90791711 & 9.95 \mathrm{E}-16\end{array}$

$\begin{array}{lll}-0.7184166 & 6.34600709 \quad 7.11 \mathrm{E}-08\end{array}$

$\begin{array}{lll}-0.7186468 & 4.4950225 & 0.01186371\end{array}$

$\begin{array}{lll}-0.721064 & 6.45458362 & 6.35 \mathrm{E}-06\end{array}$

$-0.72263513 .636260260 .03660649$

$\begin{array}{lll}-0.7233258 & 7.66617484 & 4.36 \mathrm{E}-13\end{array}$

$\begin{array}{llll}-0.7236796 & 6.25084055 \quad 4.69 \mathrm{E}-07\end{array}$

$\begin{array}{lll}-0.7237123 & 4.77471419 & 0.0019996\end{array}$

$\begin{array}{lll}-0.7243162 & 5.52447368 & 4.95 \mathrm{E}-05\end{array}$$$
\# \mathrm{~N} / \mathrm{A}
$$

$4.95 \mathrm{E}-05$

mRNA (N6-adenosine)-methyltransferase Ime4 (predicted) $-0.7258771 \quad 6.62935592 \quad 7.94 \mathrm{E}-05$

$\begin{array}{lrrr}\text { \#N/A } & -0.726162 & 11.1240026 & 1.13 \mathrm{E}-15 \\ \text { \#N/A } & -0.7262687 & 7.11052639 & 1.68 \mathrm{E}-09\end{array}$

0.000544782

0.000207065

0.825243017

3.36E-07

0.822955581

0.001315532

$6.04 \mathrm{E}-07$

0.039727171

0.000334199

$8.83 \mathrm{E}-10$

$6.94 \mathrm{E}-11$

$6.04 \mathrm{E}-07$

0.010673946

0.00049158

$1.56 \mathrm{E}-08$

0.03008618

0.826049184

4.94E-08

7.79E-06

0.066821901

3.64E-07

3.35E-12

$6.28 \mathrm{E}-05$

0.031152307

$5.25 \mathrm{E}-08$

0.255533539

0.008351022

5.00E-13

0.002217417

1.99E-07

1.01E-11

0.116210074

6.79E-05

0.001701123

7.33E-08

$1.28 \mathrm{E}-14$

3.84E-07

0.024568629

2.61E-05

0.066216686

4.46E-12

2.27E-06

0.00501742

0.000172355

0.000265617

1.44E-14

1.14E-08 
SPBC776.05

SPBC16D10.04c

SPNCRNA.1509

SPAC2G11.09

SPAC9G1.02

SPBC1289.17

SPNCRNA.944

SPBC1105.19

SPNCRNA.124

SPAC4G8.09

SPAC22H10.13

SPNCRNA.257

SPBC1198.06C

SPNCRNA.1315

SPNCRNA.683

SPAC29A4.11

SPNCRNA.1580

ScpofMr12

SPNCRNA.1493

SPAC24B11.14

SPBC27.05

SPBPB2B2.02

SPCC1739.09C

SPAC3A12.09c

SPAC1782.03

SPAC1F3.06C

SPNCRNA.1571

SPNCRNA.813

SPBC543.10

SPAC1565.07c

SPBC1D7.01

SPAC11E3.05

SPAC20G4.02C

SPAP27G11.14c

SPAC13F5.05

SPNCRNA.1153

SPBC947.15C

SPNCRNA.529

SPNCRNA.1155

SPCC191.01

SPBC29A3.03C

SPNCRNA.1164

SPAC977.01

SPAC6B12.02C

SPNCRNA.35

SPBC9B6.03

SPBC530.07C
Tf2-11

$\# \mathrm{~N} / \mathrm{A}$

tam12

$\#$ N/A

SPAC4G8.09

zym1

\#N/A

SPBC1198.06C

$\# \mathrm{~N} / \mathrm{A}$

$\# \mathrm{~N} / \mathrm{A}$

rgas

$\# N / A$

$\# N / A$

$\# \mathrm{~N} / \mathrm{A}$

$\# \mathrm{~N} / \mathrm{A}$

say1

$\operatorname{cox} 13$

ure4

saf3

spo15

$\# N / A$

$\# \mathrm{~N} / \mathrm{A}$

get1

knd1

gim6

sea3

fus1

SPAP27G11.14c

mpd1

$\# N / A$

nde1

$\# N / A$

$\# N / A$

SPCC191.01

gid2

\#N/A

ftm1

mus7

$\# \mathrm{~N} / \mathrm{A}$

SPBC9B6.03

SPBC530.07C glycerophosphocholine acyltransferase (GPCAT) Gpc1 (pred $-0.72639946 .15507028 \quad 7.58 \mathrm{E}-06$ DNA replication endonuclease-helicase Dna2

$$
\text { \#N/A }
$$

$0.7267177 \quad 7.77980451$

$1.50 \mathrm{E}-13$

$\begin{array}{llll}-0.7276951 & 4.56614339 & 0.00918947\end{array}$

calcium ion transmembrane transporter (predicted)

$-0.7280777 \quad 7.44158421$

$1.41 \mathrm{E}-11$

$-0.728417 \quad 8.07213243-1.34 \mathrm{E}-16$

\begin{tabular}{lll}
-0.7291129 & 8.69123876 & $6.45 \mathrm{E}-15$ \\
\hline
\end{tabular}

$\begin{array}{lll}-0.7294086 & 3.5841637 & 0.05074533\end{array}$

$\begin{array}{lll}-0.7322147 & 4.75151223 & 0.00094561\end{array}$

$\begin{array}{lll}-0.7323669 & 1.53206359 & 0.50511003\end{array}$

$-0.7323845 \quad 7.44762085 \quad 1.25 \mathrm{E}-14$

$\begin{array}{lll}-0.7325504 & 5.68104898 \quad 3.19 \mathrm{E}-05\end{array}$

$\begin{array}{lll}-0.7327187 & 3.08235137 & 0.0944101\end{array}$

$\begin{array}{lll}-0.7327429 & 7.87373992 & 3.98 \mathrm{E}-17\end{array}$

$\begin{array}{lll}-0.7346376 & 2.1695998 & 0.29035565\end{array}$

$-0.7347724 \quad 7.36115944 \quad 1.43 \mathrm{E}-14$

$-0.7352559 \quad 9.11044293 \quad 2.19 \mathrm{E}-17$

$-0.736815 \quad 6.43725839 \quad 4.19 \mathrm{E}-06$

$\begin{array}{lll}-0.7382067 & 9.9562044 & 6.64 \mathrm{E}-16\end{array}$

$\begin{array}{lll}-0.7388416 & 2.16869456 & 0.29747945\end{array}$

$\begin{array}{llll}-0.7390599 & 3.82159942 & 0.02580302\end{array}$

$\begin{array}{llll}-0.7391673 & 3.96739395 & 0.05591137\end{array}$

$\begin{array}{llll}-0.7401031 & 4.98781527 & 0.00628125\end{array}$

$-0.74042796 .33914901 \quad 1.65 \mathrm{E}-05$

$-0.74062845 .55054237 \quad 1.53 \mathrm{E}-05$

$-0.7411102 \quad 5.98731698 \quad 1.07 \mathrm{E}-05$

$-0.74212359 .27309938 \quad 1.78 \mathrm{E}-15$

$\begin{array}{llll}-0.7422376 & 5.02852948 & 0.00070278\end{array}$

$\begin{array}{llll}-0.7427787 & 3.11855707 & 0.16789452\end{array}$

$\# \mathrm{~N} / \mathrm{A}$

$\begin{array}{llll}\text { GET complex (ER membrane insertion) subunit Get1 (predi } & -0.7436309 & 6.65955936 & 1.66 \mathrm{E}-09 \\ \text { Cullin-associated NEDD8-dissociated protein Knd1 (predicte } & -0.7441182 & 7.16658283 & 1.39 \mathrm{E}-10\end{array}$

$\begin{array}{llll}\text { Cullin-associated NEDD8-dissociated protein Knd1 (predicte } & -0.7441182 & 7.16658283 & 1.39 \mathrm{E}-10\end{array}$

SEA complex ubiquitin-protein ligase E3 subunit Sea3 (pred $-0.744898 \quad 7.42080315 \quad 1.38 \mathrm{E}-13$

formin Fus1

$-0.745932 \quad 7.339124 \quad 7.34 \mathrm{E}-13$

$\begin{array}{lllll}\text { Schizosaccharomyces pombe specific protein } & -0.7459466 & 6.45831681 & 8.89 \mathrm{E}-06\end{array}$

thioredoxin family protein Mpd1 (predicted) $\quad-0.74641145 .52733906 \quad 0.00025928$

$$
\text { \#N/A }
$$

$-0.7464895 \quad 6.48126829 \quad 2.70 \mathrm{E}-06$

mitochondrial NADH dehydrogenase (ubiquinone) Nde1 (prr $\begin{array}{llll}\text {-0.748537 } & 8.85276606 & 3.22 \mathrm{E}-13\end{array}$

\#N/A $\quad-0.74856513 .137021940 .12155526$

$\# \mathrm{~N} / \mathrm{A}$

$\begin{array}{lll}-0.7492713 & 6.69264154 \quad 6.47 \mathrm{E}-07\end{array}$

$-0.74982718 .04949723 \quad 5.35 \mathrm{E}-15$

Schizosaccharomyces specific protein

$\begin{array}{crrrr}\text { GID complex ubiquitin-protein ligase E3 subunit Gid2/RmdE } & -0.7500306 & 6.80973163 & 1.19 \mathrm{E}-10 \\ \text { \#N/A } & -0.7502677 & 4.45880153 & 0.00486461\end{array}$

sub-telomeric 5Tm protein family Ftm1

$\begin{array}{lll}-0.75152 & 7.02986928 \quad 1.87 \mathrm{E}-05\end{array}$

$-0.75198528 .00334821 \quad 6.18 \mathrm{E}-19$

$\begin{array}{lrrr}\text { \#N/A } & -0.7550869 & 3.68558533 & 0.0224343 \\ \text { protein, localizations and processe: } & -0.7556124 & 6.9675624 & 4.59 \mathrm{E}-11\end{array}$

$\begin{array}{lrrr}\text { zf-FYVE type zinc finger protein, localizations and processe؛ } & -0.7556124 & 6.9675624 & 4.59 E-11 \\ \text { TENA/THI family protein, domain found in context with a bI } & -0.7558726 & 5.27889941 & 0.00013766\end{array}$
3.07E-05

$1.59 \mathrm{E}-12$

0.019511503

1.23E-10

$1.85 \mathrm{E}-15$

7.66E-14

0.087801159

0.00255657

0.598515372

$1.43 \mathrm{E}-13$

0.000114782

0.149548589

$5.76 \mathrm{E}-16$

0.382920587

1.63E-13

3.24E-16

1.77E-05

$8.66 \mathrm{E}-15$

0.390423843

0.048891616

0.095290187

0.013927636

6.30E-05

5.85E-05

4.21E-05

2.23E-14

0.001969682

0.243089831

$1.12 \mathrm{E}-08$

1.07E-09

2.87E-08

1.46E-12

7.35E-12

3.55E-05

0.000795216

$1.18 \mathrm{E}-05$

3.35E-12

0.185374252

3.07E-06

6.39E-14

9.21E-10

0.011101166

7.05E-05

1.05E-17

0.043333919

3.76E-10

0.000443563 
SPNCRNA.1662 SPNCRNA.229 SPCC645.05C

SPCC970.11C

SPNCRNA.1336

SPBC14F5.01

SPAC1399.06

SPNCRNA.992

SPNCRNA.1441

SPNCRNA.1446

SPNCRNA.1191

SPCC1322.08

SPAC29B12.14C

SPAP8A3.14C

SPNCRNA.186

SPNCRNA.887

SPBC1604.01

SPNCRNA.93

SPBC1683.07

ScpofMp08

SPACUNK4.08

SPCC1827.08C

SPCC1322.07

SPAC688.06c

SPCC320.06

SPNCRNA.1677

SPNCRNA.581

SPBC609.04

SPNCRNA.1396

SPNCRNA.700

SPAC4D7.11

SPNCRNA.873

SPCPB16A4.07

SPAC3F10.19

SPNCRNA.1623

SPNCRNA.133

SPNCRNA.960

SPAC227.13C

SPCC1281.04

SPCC24B10.03

SPNCRNA.1355

SPAC27D7.13C

SPAC16A10.03C

SPCC550.12

SPAC23H3.15C

SPBC20F10.10

SPBC27B12.02
SPBC14F5.01

SPAC1399.06

$\# \mathrm{~N} / \mathrm{A}$

$\# N / A$

$\# N / A$

$\# N / A$

srk1

SPAC29B12.14 sls1

$\# N / A$

\#N/A

egt1

\#N/A

mal1

$\# N / A$

dpp2

pof7

mug150

slx4

SPCC320.06

$\# \mathrm{~N} / \mathrm{A}$

$\# N / A$

caf5

$\# N / A$

$\# N / A$

dsc4

$\# \mathrm{~N} / \mathrm{A}$

SPCPB16A4.07 spd2

$\# N / A$

$\# N / A$

isu1

akr7

SPCC24B10.03

\#N/A

ssm4

SPAC16A10.03C

arp6

ddr48

psl1

mis19

myosin II heavy chain

wtf element Wtf9

$\# \mathrm{~N} / \mathrm{A}$

$$
\begin{aligned}
& \# N / A \\
& \# N / A \\
& \# N / A \\
& \# N / A
\end{aligned}
$$

$$
\# N / A
$$

$$
\# \mathrm{~N} / \mathrm{A}
$$

$$
\text { \#N/A }
$$

maltase alpha-glucosidase Mal1

$\# \mathrm{~N} / \mathrm{A}$

dipeptidyl peptidase (predicted)

F-box protein Pof7

conserved fungal protein

$$
\# N / A
$$

$$
\text { \#N/A }
$$

$\# N / A$

$\# N / A$ actin-like protein Arp6

centromere protein Mis19/Eic1
DUF4504 family protein, human C1orf74 ortholog

Schizosaccharomyces pombe specific protein

MAPK-activated protein kinase Srk1

plasma membrane purine transmembrane transporter (pre $\begin{array}{cccc}-0.7654407 & 7.49519659 & 3.65 \mathrm{E}-13\end{array}$ mitochondrial inner membrane protein Sls1 (predicted) $\quad \begin{array}{llll}-0.7679029 & 6.42338602 & 4.38 \mathrm{E}-08\end{array}$

ergothioneine biosynthesis protein Egt1

Schizosaccharomyces pombe specific protein Mug150

structure-specific endonuclease subunit SIX4

$\begin{array}{lll}0.7564215 & 6.12350978 \quad 6.24 \mathrm{E}-07\end{array}$

$\begin{array}{llll}-0.7567493 & 3.18613821 & 0.08717837\end{array}$

$\begin{array}{lll}-0.7570197 & 8.35054459 & 2.39 \mathrm{E}-15\end{array}$

$\begin{array}{lll}0.7583691 & 5.70998197 \quad 2.69 \mathrm{E}-06\end{array}$

$\begin{array}{lll}-0.7602044 & 4.59823748 \quad 0.00950038\end{array}$

$\begin{array}{llr}-0.7603926 & 5.6502644 & 1.09 \mathrm{E}-05\end{array}$

$\begin{array}{lll}-0.7617556 & 4.1773784 & 0.01788003\end{array}$

$\begin{array}{lll}-0.7623696 & 8.12390158 \quad 3.14 \mathrm{E}-14\end{array}$

$\begin{array}{llll}-0.7626313 & 2.68498898 & 0.13463992\end{array}$

$-0.7628874 \quad 5.74326972 \quad 4.02 \mathrm{E}-06$

$-0.7645437 \quad 6.08226839 \quad 1.22 \mathrm{E}-07$

$\begin{array}{rrr}-0.7654407 & 7.49519659 & 3.65 \mathrm{E}-13 \\ -0.7679029 & 6.42338602 & 4.38 \mathrm{E}-08\end{array}$

$\begin{array}{lll}-0.7685874 & 4.48488356 & 0.01145404\end{array}$

$\begin{array}{lll}-0.7693407 & 5.98519634 & 1.29 \mathrm{E}-05\end{array}$

$-0.7702328 .22803319 \quad 6.06 \mathrm{E}-14$

$\begin{array}{lll}-0.7702454 & 2.86380529 & 0.1375561\end{array}$

$\begin{array}{lll}-0.7706727 & 9.17484701 & 1.01 \mathrm{E}-25\end{array}$

$\begin{array}{lll}-0.7722232 & 2.09883468 & 0.25931681\end{array}$

$\begin{array}{lll}-0.7744703 & 8.22454586 & 1.23 \mathrm{E}-18\end{array}$

$-0.77615436 .30100336 \quad 2.51 \mathrm{E}-07$

$\begin{array}{lll}-0.7771851 & 6.15498266 & 3.09 \mathrm{E}-06\end{array}$

$-0.7772996 \quad 6.00226838 \quad 1.08 \mathrm{E}-05$

$\begin{array}{lll}-0.7787783 & 7.89184412 \quad 3.57 \mathrm{E}-17\end{array}$

$\begin{array}{lll}-0.7800363 & 5.07970463 & 0.00324364\end{array}$

$-0.7809515 \quad 5.96658161 \quad 2.63 \mathrm{E}-06$

plasma membrane spermine family transmembrane transk - $-0.7846396 \quad 6.70177537$

$-0.7866469 \quad 6.18531901-5.16 \mathrm{E}-08$

$-0.7867547 \quad 6.88568833 \quad 4.21 \mathrm{E}-11$

$-0.79028376 .96264003-2.31 \mathrm{E}-11$

$-0.79117796 .32099893 \quad 3.16 \mathrm{E}-06$

$-0.7914242 \quad 6.36936156 \quad 5.15 \mathrm{E}-08$

$-0.7918476 \quad 5.53583321 \quad 5.52 \mathrm{E}-05$

$\begin{array}{lll}-0.7923916 & 4.42331217 & 0.00707722\end{array}$

$-0.79304397 .85361068 \quad 3.44 \mathrm{E}-10$

$-0.79653146 .58244312 \quad 1.06 \mathrm{E}-09$

$-0.7968114 \quad 6.8870378 \quad 1.43 \mathrm{E}-06$

$-0.7979266 \quad 4.92612992 \quad 0.00129264$

$\begin{array}{lll}-0.7986297 & 4.9392046 & 0.0001962\end{array}$

$-0.7994435 \quad 2.58358749 \quad 0.15596003$

$\begin{array}{llrrr}\text { dynactin microtubule-binding subunit, p150-Glued Ssm4 } & -0.7996113 & 5.41690224 & 0.0002867 \\ \text { ubiquitin-protein ligase E3 involved in vesicle docking Pep5/ } & -0.7996577 & 7.94774125 & 1.24 \mathrm{E}-16\end{array}$

$0.8005556 \quad 5.66907426 \quad 0.00016326$

$\begin{array}{lll}0.8022267 & 8.99292302 & 1.11 \mathrm{E}-08\end{array}$

$-0.80232075 .08846499 \quad 6.53 \mathrm{E}-05$

$\begin{array}{lll}-0.8028087 & 5.15599731 & 0.00014469\end{array}$

cyclin pho85 family Psl1 (predicted)
2.97E-06

0.139587952

$2.96 \mathrm{E}-14$

$1.18 \mathrm{E}-05$

0.020115138

4.28E-05

0.035525143

$3.50 \mathrm{E}-13$

0.201989558

1.71E-05

6.37E-07

1.87E-07

$3.76 \mathrm{E}-12$

$2.46 \mathrm{E}-07$

0.023814472

5.01E-05

$6.59 \mathrm{E}-13$

0.205684307

2.70E- 24

0.349782135

2.06E-17

1.26E-06

$1.34 \mathrm{E}-05$

4.22E-05

$5.18 \mathrm{E}-16$

0.007731342

$1.15 \mathrm{E}-05$

$1.45 \mathrm{E}-09$

2.86E-07

$3.46 \mathrm{E}-10$

$1.98 \mathrm{E}-10$

1.37E-05

2.85E-07

0.000190294

0.015460825

2.55E-09

7.41E-09

$6.45 \mathrm{E}-06$

0.003384548

0.000615827

0.228385621

0.000870981

$1.72 \mathrm{E}-15$

0.000519401

6.73E-08

0.000222412

0.000464443 


\begin{tabular}{|c|c|c|c|c|c|c|}
\hline SPNCRNA.832 & $\# N / A$ & $\# \mathrm{~N} / \mathrm{A}$ & -0.803443 & 8.99293836 & 1.12E-08 & $6.81 \mathrm{E}-08$ \\
\hline SPBC3D6.16 & $\# N / A$ & $\# N / A$ & -0.8054323 & 4.79162644 & 0.00056797 & 0.001619188 \\
\hline SPNCRNA.1585 & $\# \mathrm{~N} / \mathrm{A}$ & \#N/A & -0.8065957 & 5.8434315 & 1.16E-06 & $5.32 \mathrm{E}-06$ \\
\hline SPCC4B3.02c & got1 & Golgi transport protein Got1 (predicted) & -0.80698 & 6.31448162 & $3.76 \mathrm{E}-06$ & $1.60 \mathrm{E}-05$ \\
\hline SPNCRNA.521 & $\# N / A$ & \#N/A & -0.8077459 & 3.4501927 & 0.02778889 & 0.052111993 \\
\hline SPBC887.18c & hfi1 & SAGA complex subunit Hfi1/Ada1 & -0.8083631 & 5.85112048 & 2.40E-06 & $1.06 \mathrm{E}-05$ \\
\hline SPNCRNA.01 & $\# N / A$ & \#N/A & -0.8089067 & 6.54900057 & $1.86 \mathrm{E}-09$ & $1.25 \mathrm{E}-08$ \\
\hline SPNCRNA.517 & $\# N / A$ & $\# N / A$ & -0.8089237 & 4.10081524 & 0.02640391 & 0.049910719 \\
\hline SPNCRNA.448 & $\# N / A$ & $\# N / A$ & -0.8097048 & 5.09309445 & 0.00013881 & 0.000446911 \\
\hline SPNCRNA.651 & $\# \mathrm{~N} / \mathrm{A}$ & $\# \mathrm{~N} / \mathrm{A}$ & -0.8101227 & 3.54770267 & 0.07380282 & 0.120727926 \\
\hline SPNCRNA.1071 & $\# N / A$ & $\# \mathrm{~N} / \mathrm{A}$ & -0.8119228 & 4.63103157 & 0.00069941 & 0.001961857 \\
\hline SPBC119.14 & rti1 & Rad22 homolog Rti1 & -0.8122775 & 3.08232246 & 0.05573163 & 0.09513464 \\
\hline SPNCRNA.1168 & \#N/A & $\# \mathrm{~N} / \mathrm{A}$ & -0.8129464 & 4.45864776 & 0.00361749 & 0.00849449 \\
\hline SPNCRNA.1317 & \#N/A & \#N/A & -0.8133691 & 6.94899433 & $1.35 \mathrm{E}-12$ & 1.33E-11 \\
\hline SPNCRNA.1282 & $\# N / A$ & $\# N / A$ & -0.8135279 & 5.26144813 & 1.59E-05 & 6.07E-05 \\
\hline SPAC6G9.16c & $\operatorname{xrc4}$ & XRCC4 nonhomologous end joining factor Xrc4 & -0.8137987 & 5.987214 & $2.45 \mathrm{E}-06$ & $1.07 \mathrm{E}-05$ \\
\hline SPAC22F3.11C & snu23 & U4/U6 x U5 tri-snRNP complex subunit Snu23 & -0.8147404 & 5.18912805 & 0.0001233 & 0.000401091 \\
\hline SPNCRNA.487 & $\# N / A$ & $\# N / A$ & -0.8159927 & 3.62494567 & 0.02332221 & 0.044832671 \\
\hline SPAC22H12.05c & fsc1 & fasciclin domain protein Fsc1 & -0.8160868 & 7.49136971 & 6.39E-13 & $6.44 \mathrm{E}-12$ \\
\hline SPAC17A2.07c & SPAC17A2.07c & Schizosaccharomyces specific protein & -0.8172121 & 5.34636935 & 0.00036065 & 0.001075478 \\
\hline SPAC2OH4.11C & rho5 & Rho family GTPase Rho5 & -0.8173323 & 5.63327484 & $2.08 \mathrm{E}-05$ & 7.74E-05 \\
\hline SPCC1682.06 & SPCC1682.06 & Schizosaccharomyces specific protein & -0.8175653 & 6.11210004 & $1.31 \mathrm{E}-07$ & $6.80 \mathrm{E}-07$ \\
\hline SPBC685.04c & aps2 & AP-2 adaptor complex sigma subunit Aps2 (predicted) & -0.8184466 & 5.94106865 & $1.43 \mathrm{E}-06$ & 6.47E-06 \\
\hline SPAC631.02 & bdf2 & BET family double bromodomain protein Bdf2 & -0.8189392 & 8.65703769 & $1.30 E-24$ & $3.30 \mathrm{E}-23$ \\
\hline SPAC56F8.13 & $\# N / A$ & $\# N / A$ & -0.8194998 & 7.06515257 & $1.66 \mathrm{E}-13$ & $1.75 \mathrm{E}-12$ \\
\hline SPCC794.02 & wtf5 & wtf element Wtf5 & -0.8204972 & 5.81693297 & $7.08 \mathrm{E}-07$ & $3.34 \mathrm{E}-06$ \\
\hline SPNCRNA.646 & $\# N / A$ & $\# \mathrm{~N} / \mathrm{A}$ & -0.8224865 & 5.66329731 & $6.65 \mathrm{E}-06$ & $2.72 \mathrm{E}-05$ \\
\hline SPCC1919.06c & wtf25 & wtf element & -0.8228851 & 6.241025 & $9.48 \mathrm{E}-08$ & $5.03 \mathrm{E}-07$ \\
\hline SPNCRNA.966 & $\# \mathrm{~N} / \mathrm{A}$ & $\# N / A$ & -0.8235481 & 3.81118894 & 0.02060193 & 0.040159309 \\
\hline SPCC1906.04 & wtf20 & wtf element Wtf20 & -0.8246711 & 4.53093041 & 0.00356206 & 0.008387479 \\
\hline SPAC19A8.08 & upf2 & nonsense-mediated decay protein Upf2 & -0.8257329 & 7.38722549 & 8.95E-17 & $1.25 \mathrm{E}-15$ \\
\hline SPCC645.12c & SPCC645.12c & Schizosaccharomyces specific protein & -0.8262508 & 4.90116274 & 0.00025267 & 0.000776621 \\
\hline SPCP25A2.03 & tho1 & THO complex subunit Tho1 (predicted) & -0.8270638 & 5.77691479 & 2.33E-06 & $1.03 \mathrm{E}-05$ \\
\hline SPNCRNA.1294 & $\# N / A$ & \#N/A & -0.8273968 & 6.16032264 & $1.06 \mathrm{E}-07$ & $5.60 \mathrm{E}-07$ \\
\hline SPNCRNA.1696 & $\# \mathrm{~N} / \mathrm{A}$ & $\# N / A$ & -0.8304431 & 6.0097517 & $1.95 \mathrm{E}-06$ & 8.67E-06 \\
\hline SPBC13A2.04c & ptr2 & plasma membrane PTR family peptide transmembrane tra! & -0.8304883 & 9.2235289 & $6.01 \mathrm{E}-19$ & 1.03E-17 \\
\hline SPAC18G6.01c & SPAC18G6.01c & chalcone related protein family & -0.8314026 & 6.74492817 & $2.21 \mathrm{E}-08$ & $1.28 \mathrm{E}-07$ \\
\hline SPBC31E1.04 & pep12 & SNARE Pep12 & -0.8317834 & 7.83483349 & $4.48 \mathrm{E}-20$ & $8.71 \mathrm{E}-19$ \\
\hline SPCC737.03c & ima1 & inner nuclear membrane protein Ima1 & -0.8318269 & 8.57190464 & $1.96 \mathrm{E}-24$ & $4.93 \mathrm{E}-23$ \\
\hline SPCC1620.01c & Ism2 & Lsm2-8 complex subunit Lsm2 & -0.8322579 & 5.2043742 & 0.00749797 & 0.016275503 \\
\hline SPCC825.04c & naa40 & histone N-acetyltransferase Naa40 (predicted) & -0.8329259 & 4.80814976 & 0.00111051 & 0.002949664 \\
\hline SPAC2C4.17c & msy2 & MS ion channel protein 2 (predicted) & -0.8333036 & 8.61945675 & 5.65E-26 & $1.54 \mathrm{E}-24$ \\
\hline SPNCRNA.214 & ter1 & telomerase RNA & -0.8336677 & 7.89272794 & $6.47 \mathrm{E}-17$ & $9.19 \mathrm{E}-16$ \\
\hline SPNCRNA.882 & $\# N / A$ & $\# N / A$ & -0.8340591 & 4.23123334 & 0.00516961 & 0.011691318 \\
\hline SPNCRNA.467 & $\# N / A$ & $\# \mathrm{~N} / \mathrm{A}$ & -0.8344467 & 1.58394586 & 0.54903511 & 0.639100912 \\
\hline SPNCRNA.884 & $\# N / A$ & $\# N / A$ & -0.8347308 & 7.89339955 & 5.75E-17 & $8.21 \mathrm{E}-16$ \\
\hline SPBC19F8.07 & $\operatorname{mcs} 6$ & TFIIH associated cyclin-dependent protein kinase Mcs6 & -0.8353683 & 7.67420098 & $3.22 \mathrm{E}-19$ & $5.68 \mathrm{E}-18$ \\
\hline
\end{tabular}


ScpofMp10

SPNCRNA.658

SPBC365.11

SPCC285.10C

SPAC6F12.03c

SPNCRNA.155

SPBC1604.09c

SPNCRNA.417

SPNCRNA.1092

SPNCRNA.119

SPBC21C3.02C

SPBC2A9.05C

SPBC1677.02

SPAC222.05C

SPAC18B11.11

SPNCRNA.1647

SPNCRNA.1304

SPAC25H1.04

SPBC6B1.09C

SPBC25B2.02C

SPAC3C7.03C

SPAC57A7.05

SPNCRNA.717

SPCP1E11.07C

SPAC3H8.04

SPNCRNA.848

SPAC18B11.09c

SPNCRNA.760

SPNCRNA.1257

SPNCRNA.681

SPBC4F6.09

SPNCRNA.58

SPCC1672.14

SPCC1827.07c

SPAPB17E12.09

SPNCRNA.900

SPBC15C4.06C

SPBC1A4.06C

SPAC1687.08

SPAC19G12.06C

SPCPJ732.02C

SPNCRNA.1622

SPCC645.02

SPCC1529.01

SPNCRNA.1074

SPNCRNA.443

SPAC1002.05C dep1

tvp23

dpm 3

$\mathrm{mss} 1$

SPAC18B11.11

$\# N / A$

$\# N / A$

mug105

nbs 1

mam1

rad55

SPAC57A7.05 $\#$ N/A

CWf18

SPAC3H8.04 \#N/A

SPAC18B11.09C

$\#$ N/A

$\# N / A$

\#N/A

str1

$\# N / A$

\#N/A

SPCC1827.07C

SPAPB17E12.09 $\# \mathrm{~N} / \mathrm{A}$

SPBC15C4.06c

tam41

SPAC1687.08

hta2

xks1

\#N/A

gep4

SPCC1529.01

\#N/A

$\# \mathrm{~N} / \mathrm{A}$

jmj2
\#N/A

$\# \mathrm{~N} / \mathrm{A}$

Golgi GRIP domain protein Grp2 (predicted)

SPRY domain membrane protein, specificity factor required

SNARE Fsv1

\#N/A

exoribonuclease Rex4 (predicted)

$\# N / A$

$\# N / A$

$\# \mathrm{~N} / \mathrm{A}$

Sds3-like family protein Dep1

Golgi transport protein Tvp23 (predicted)

dolichol-phosphate mannosyltransferase subunit 3

$\begin{array}{lll}0.8355017 & 9.20807824 & 1.88 \mathrm{E}-08\end{array}$

$\begin{array}{lll}-0.8360951 & 3.17320906 & 0.11623777\end{array}$

$\begin{array}{lll}-0.8400447 & 7.41212437 & 3.66 \mathrm{E}-13\end{array}$

$\begin{array}{lll}-0.842524 & 6.73463098 & 9.94 \mathrm{E}-11\end{array}$

$\begin{array}{lll}-0.8426549 & 6.67500662 & 1.50 \mathrm{E}-12\end{array}$

$\begin{array}{lll}-0.8438819 & 1.5836122 & 0.4956262\end{array}$

$\begin{array}{lll}-0.8452495 & 5.6944475 & 2.00 \mathrm{E}-06\end{array}$

$\begin{array}{lll}-0.8454164 & 4.13025086 & 0.00898701\end{array}$

$\begin{array}{llll}-0.8458214 & 3.43639268 & 0.02046063\end{array}$

$\begin{array}{llll}-0.8461589 & 3.27055077 & 0.03965289\end{array}$

$\begin{array}{lll}-0.8466417 & 4.19904492 & 0.00874745\end{array}$

$\begin{array}{lll}-0.8493453 & 6.64198846 & 2.72 \mathrm{E}-11\end{array}$

$-0.85026647 .60252689 \quad 1.08 \mathrm{E}-19$

mitochondrial tRNA wobble uridine modification GTPase M $-0.8513024 \quad 6.9457288 \quad 8.83 \mathrm{E}-14$

$-0.8543156 \quad 7.42611388 \quad 1.42 \mathrm{E}-15$

$-0.8550776 \quad 5.70050971 \quad 1.28 \mathrm{E}-06$

$\begin{array}{lll}-0.8570091 & 6.66489626 & 4.42 \mathrm{E}-10\end{array}$

$-0.8576902 \quad 5.65336256 \quad 6.00 \mathrm{E}-07$

$-0.85769956 .49207485 \quad 6.72 \mathrm{E}-09$

$-0.8613113 \quad 9.05153053 \quad 4.86 \mathrm{E}-26$

$-0.8613247 \quad 6.2296618 \quad 2.81 \mathrm{E}-08$

$\begin{array}{lll}-0.8648189 & 8.73992751 \quad 2.35 \mathrm{E}-20\end{array}$

$\begin{array}{lll}-0.8658291 & 2.60963414 & 0.10955982\end{array}$

$\begin{array}{lll}-0.8667852 & 5.54361951 & 2.84 \mathrm{E}-05 \\ -0.867036 & 6.85553434 & 3.01 \mathrm{E}-13\end{array}$$$
\text { \#N/A }
$$

3434

DUF4210 domain protein, human FAM214A ortholog impli $\quad \begin{array}{lllll}-0.867036 & 6.85553434 & 3.01 \mathrm{E}-13\end{array}$ $\# \mathrm{~N} / \mathrm{A}$

serine O-acetyltransferase activity (predicted)

$\# N / A$

$\# N / A$

$\begin{array}{lll}-0.8704309 & 6.64163103 & 6.61 \mathrm{E}-11\end{array}$

$\begin{array}{lll}-0.8709968 & 5.01340022 & 0.00077129\end{array}$

$\begin{array}{llll}-0.8711986 & 4.19622898 & 0.00388011\end{array}$

$-0.87428094 .95875786 \quad 9.26 \mathrm{E}-05$

plasma membrane siderophore-iron transmembrane transk $-0.8773734 \quad 7.38571471 \quad 3.10 \mathrm{E}-14$

$-0.87824125 .80224207 \quad 1.99 \mathrm{E}-05$

$\# N / A$

$\begin{array}{lll}0.8783593 & 4.05648559 & 0.01081288\end{array}$

$-0.8795834 \quad 6.95941556 \quad 2.19 \mathrm{E}-11$

SPX/EXS domain protein (predicted)

$\begin{array}{lll}-0.8804089 & 3.5207785 & 0.01394165\end{array}$

\#N/A $\quad-0.88180913 .080322790 .05336019$

ubiquitin-protein ligase E3 Meu34, human RNF13 family ho $-0.8818424 \quad 7.44703919 \quad 7.13 \mathrm{E}-17$

mitochondrial phosphatidate cytidylyltransferase Tam41 ( $p-0.8818493 \quad 6.29477286 \quad 1$ - $19 \mathrm{E}-09$

Schizosaccharomyces pombe specific protein

$\begin{array}{lll}-0.8825484 & 3.95769968 & 0.13095033\end{array}$

histone $\mathrm{H} 2 \mathrm{~A}$ beta

xylulose kinase Xks1 (predicted)

$\begin{array}{ll}-0.8827782 & 7.62957956 \quad 3.81 \mathrm{E}-15\end{array}$

$\begin{array}{lll}-0.8862644 & 8.58625399 \quad 9.63 \mathrm{E}-25\end{array}$

$\begin{array}{lll}0.8866582 & 4.28708821 & 0.00606167\end{array}$

phosphatidylglycerol phosphate phosphatase Gep4 (predictı $-0.8880132 \quad 5.23036001 \quad 7.20 \mathrm{E}-05$

transmembrane transporter (predicted)

$-0.8917554 \quad 6.34593611 \quad 7.54 \mathrm{E}-11$

\#N/A $\quad-0.891845 \quad 7.2613029 \quad 1.07 E-18$

$\# \mathrm{~N} / \mathrm{A}$

histone demethylase Jmj2 $\begin{array}{llll}-0.8928174 & 2.55760512 & 0.13968621\end{array}$

$-0.8935762 \quad 6.488065 \quad 4.30 \mathrm{E}-12$
1.10E-07

0.178353553

3.77E-12

7.75E-10

$1.47 \mathrm{E}-11$

0.589742313

8.84E-06

0.019129375

0.039929618

0.07098655

0.018677873

2.29E-10

2.03E-18

9.47E-13

$1.81 \mathrm{E}-14$

5.81E-06

3.22E-09

2.86E-06

4.21E-08

$1.34 \mathrm{E}-24$

$1.62 \mathrm{E}-07$

$4.68 \mathrm{E}-19$

0.169444224

0.00010329

$3.14 \mathrm{E}-12$

2.72E-06

5.26E-10

0.002131866

0.00903942

0.000306629

3.46E-13

7.46E-05

0.022626588

$1.88 \mathrm{E}-10$

0.028430597

0.091576586

$1.01 \mathrm{E}-15$

$8.25 \mathrm{E}-09$

0.197498995

4.61E-14

$2.45 \mathrm{E}-23$

0.013506769

0.000243213

5.95E-10

1.79E-17

0.208320114

3.97E-11 
SPCC1235.13

SPNCRNA.1118

SPNCRNA.632

SPAC12G12.16C

SPCC63.07

SPNCRNA.390

SPNCRNA.1100

SPBC1105.13C

SPNCRNA.1668

ScpofMt17

SPNCRNA.532

SPNCRNA.786

SPNCRNA.1052

SPBC1604.18c

SPBC1347.03

SPBC1348.15

SPBCPT2R1.03

SPNCRNA.43

SPBC337.12

SPBC17D11.05

SPCC757.03C

SPAC20G4.03C

SPBC1198.01

SPAC13F5.04c

SPCC737.04

SPAC3C7.02C

SPBC18H10.18c

SPBC660.09

SPBC1348.04

SPBP8B7.27

SPNCRNA.1333

SPNCRNA.1320

SPNCRNA.301

SPAC11D3.01C

SPBC19C7.09c

SPAC23H3.13C

SPBC29A3.14C

SPNCRNA.1433

SPNCRNA.1093

SPBC36.10

SPNCRNA.1108

ScpofMp01

SPAC9E9.01

SPBC1347.01C

SPNCRNA.740

SPCC1235.12C

SPBC211.07C ght6

$\# N / A$

$\# \mathrm{~N} / \mathrm{A}$

SPAC12G12.16

thy 1

$\# \mathrm{~N} / \mathrm{A}$

$\#$ N/A

SPBC1105.13C

$\# N / A$

$\# \mathrm{~N} / \mathrm{A}$

$\# N / A$

$\# N / A$

$\# \mathrm{~N} / \mathrm{A}$

cmp7

meu14

SPBC1348.15

SPBCPT2R1.03

\#N/A

red5

tif32

hsp3101

hri1

fmd2

vta1

SPCC737.04

pil2

SPBC18H10.18C

mug 168

SPBC1348.04

mug30

$\# N / A$

$\# \mathrm{~N} / \mathrm{A}$

$\# \mathrm{~N} / \mathrm{A}$

SPAC11D3.01C

uve1

gpa2

trt1

$\# N / A$

$\#$ N/A

ups2

$\# N / A$

$\# N / A$

$\# \mathrm{~N} / \mathrm{A}$

rev1

$\# \mathrm{~N} / \mathrm{A}$

mug146

ubc8 plasma membrane glucose/fructose:proton symporter Ght $\quad-0.8947738 \quad 6.44506492$

$\# N / A$

$-0.8949352 \quad 7.21776863$

$-0.89529926 .02060806$

$-0.89578815 .59449588$

XP-G family (predir

$\# N / A$

$\# N / A$

Schizosaccharomyces pombe specific protein

$\# \mathrm{~N} / \mathrm{A}$

$\# N / A$

$\# N / A$

$\# N / A$

$\# N / A$

ESCRT III complex subunit Cmp7 (predicted)

sporulation specific PIL domain protein Meu14

Schizosaccharomyces pombe specific protein

hypothetical protein

$\# \mathrm{~N} / \mathrm{A}$

$\begin{array}{lll}-0.8977853 & 2.92641181 & 0.04551021\end{array}$

$-0.8981297 \quad 6.77496684 \quad 1.18 \mathrm{E}-06$

$-0.90109465 .41914863 \quad 5.68 \mathrm{E}-06$

$\begin{array}{lll}-0.901122 & 7.64292779 \quad 8.15 \mathrm{E}-08\end{array}$

$-0.9026515 \quad 2.06287196 \quad 0.14798606$

$\begin{array}{lll}-0.9029507 & 5.63579819 & 1.31 \mathrm{E}-06\end{array}$

$\begin{array}{lll}-0.9034109 & 4.37857793 & 0.009485\end{array}$

$-0.903415 \quad 5.63364049 \quad 7.84 \mathrm{E}-08$

$-0.9040691 \quad 5.13967622 \quad 7.75 \mathrm{E}-06$

$\begin{array}{lll}-0.9041752 & 5.52429 & 2.88 \mathrm{E}-05\end{array}$

$\begin{array}{lll}-0.9042493 & 1.30013156 & 0.56753043\end{array}$

$\begin{array}{lll}-0.9042493 & 1.30013156 & 0.56753043\end{array}$

$\begin{array}{llll}-0.9047038 & 2.23763185 & 0.15673439\end{array}$

zf-CCCH type zinc finger protein, NURS complex subunit Rer $\begin{array}{lll}-0.9052832 & 6.68369585 & 2.97 \mathrm{E}-10\end{array}$

$\begin{array}{llll}\text { translation initiation factor elF3a } & -0.9075785 & 7.21610502 & 9.97 \mathrm{E}-17\end{array}$

$\begin{array}{lllll}\text { glyoxylase III Hsp3101 } & -0.9092324 & 6.77858402 & 3.38 \mathrm{E}-07\end{array}$

elF2 alpha kinase Hri1 $\quad-0.9112388 \quad 8.37178388 \quad 5.95 \mathrm{E}-18$

glutathione-dependent formaldehyde dehydrogenase Fmd2 $-0.9118237 \quad 7.05905861 \quad 9.87 \mathrm{E}-12$

$\begin{array}{llll}\text { Vps20 associated protein Vta1 (predicted) } & -0.913142 & 6.2982755 & 6.35 \mathrm{E}-09\end{array}$

$-0.913531-9.28611304-1.04 E-38$

meiotic eisosome BAR domain protein Pil2 $\quad \begin{array}{llll}-0.9142686 & 6.16308266 & 3.62 \mathrm{E}-10\end{array}$

hecific protein

(2)

$\begin{array}{lll}-0.9142686 & 6.16308266 & 3.62 \mathrm{E}-10 \\ -0.9144636 & 6.10722016 & 2.29 \mathrm{E}-09\end{array}$

$-0.9144636 \quad 6.10722016 \quad 2.29 \mathrm{E}-09$

0.91453595 .83690249

$-0.9146828 \quad 4.31909393 \quad 0.00493984$

HECT-type ubiquitin-protein ligase E3, found in association ' $-0.9152662 \quad 7.21282673 \quad 1.15 \mathrm{E}-14$

$-0.91692083 .18643158 \quad 0.05749149$

$\begin{array}{lll}-0.918689 & 7.0413771 \quad 1.34 \mathrm{E}-11\end{array}$

$\# N / A$

Con-6 family conserved fungal protein

endonuclease Uve1

heterotrimeric $\mathrm{G}$ protein alpha-2 subunit $\mathrm{Gpa2}$

telomerase reverse transcriptase 1 protein Trt1

$\# N / A$

$\# N / A$

phosphatidic acid transfer protein Ups2 (predicted)

$\# N / A$

$\# N / A$

$\# N / A$

deoxycytidyl transferase Rev1 (predicted)

\#N/A

Schizosaccharomyces specific protein Mug46

ubiquitin conjugating enzyme E2 Ubc8 (predicted)
$-0.9212244 \quad 3.68490375 \quad 0.01567359$

$\begin{array}{lll}-0.92254 & 5.91035139 & 2.13 \mathrm{E}-08\end{array}$

$-0.9239916 \quad 6.84037254 \quad 4.99 \mathrm{E}-14$

$-0.9241484 \quad 5.8649302 \quad 6.66 \mathrm{E}-08$

$\begin{array}{lll}-0.9251827 & 6.7109457 & 3.95 \mathrm{E}-12\end{array}$

$\begin{array}{llll}-0.9254977 & 3.81151387 & 0.00595557\end{array}$

$\begin{array}{llll}-0.9257783 & 4.08325143 & 0.00084884\end{array}$

$\begin{array}{lll}-0.9262521 & 6.44661444 & 3.67 \mathrm{E}-12\end{array}$

$\begin{array}{llll}-0.9286297 & 3.52082088 & 0.01834534\end{array}$

$\begin{array}{lll}-0.9327833 & 10.5733703 \quad 1.18 \mathrm{E}-12\end{array}$

$\begin{array}{lll}0.9332428 & 1.86480698 & 0.25396083\end{array}$

$\begin{array}{lll}-0.9347238 & 6.07916863 & 4.23 \mathrm{E}-09\end{array}$

$\begin{array}{llll}-0.9348279 & 28225713 & 0.09561045\end{array}$

$\begin{array}{lll}-0.9348614 & 2.70789005 & 0.06266193\end{array}$

$\begin{array}{lll}-0.9367158 \quad 7.04358577 \quad 1.16 \mathrm{E}-13 & \end{array}$
4.04E-11

4.04E-11

2.06E-13

3.33E-08

3.80E-06

0.079839382

5.39E-06

2.36E-05

4.37E-07

0.218637682

5.96E-06

0.020101337

4.22E-07

3.14E-05

0.000104589

0.656586977

0.656586977

0.229420844

2.22E-09

$1.39 \mathrm{E}-15$

1.66E-06

9.32E-17

$8.76 \mathrm{E}-11$

4.00E-08

$6.20 \mathrm{E}-37$

2.66E-09

$1.51 \mathrm{E}-08$

3.77E-07

0.011231454

$1.33 \mathrm{E}-13$

0.097612167

1.17E-10

0.031518552

1.24E-07

$5.49 \mathrm{E}-13$

3.63E-07

3.67E-11

0.013309563

0.002317972

$3.42 \mathrm{E}-11$

0.036396514

1.16E-11

0.344195074

2.72E-08

0.1510411

0.105105851

1.24E- 12 
SPAC26H5.04 SPAC11E3.02C SPAP8A3.13C SPCC1919.02

SPNCRNA.1330 SPAP11E10.02C SPNCRNA.1630 SPAC3C7.13C

SPNCRNA.1339 SPAC11H11.05C SPNCRNA.516

SPAC20G8.10C SPBC215.14C SPBC215.10

SPAC1002.04c SPNCRNA.650

SPNCRNA.134

SPNCRNA.319

SPNCRNA.389

SPAC6C3.03C

SPBC20F10.05

SPACUNK4.10

SPAC732.02C

SPAC11H11.02C

SPAC14C4.16

SPNCRNA.1193

SPAC2F 3.08

SPAC11E3.08C

SPCC16A11.15C

SPAC16A10.08C

SPNCRNA.1663

SPNCRNA.921

SPCC5E4.10C

SPAC1F12.03C

SPBC725.03

ScpofMp05

SPNCRNA.1576

SPAC1071.13

SPAC11D3.19

SPNCRNA.982

SPNCRNA.1497

SPBC3B9.09

SPNCRNA.919

SPNCRNA.1205

SPAC11E3.03

SPNCRNA 399

SPBC83.09C gid5

ync13

SPAP8A3.13C

pbn1

\#N/A

mam3

$\# \mathrm{~N} / \mathrm{A}$

SPAC3C7.13C

\#N/A

fta6

$\# N / A$

atg6

vps20

odr1

taf11

$\# \mathrm{~N} / \mathrm{A}$

$\# N / A$

$\# N / A$

$\# \mathrm{~N} / \mathrm{A}$

SPAC6C3.03C

nrl1

gor1

SPAC732.02C

mug162

dad3

$\# \mathrm{~N} / \mathrm{A}$

sut1

SPCC16A11.15C

mug74

$\# \mathrm{~N} / \mathrm{A}$

$\# N / A$

SPCC5E4.10C

\#N/A

SPBC725.03

\#N/A

$\# N / A$

$\# \mathrm{~N} / \mathrm{A}$

SPAC11D3.19

$\# N / A$

$\# \mathrm{~N} / \mathrm{A}$

vps36

$\# N / A$

$\# \mathrm{~N} / \mathrm{A}$

$\# N / A$
GID complex armadillo repeat subunit Gid5 (predicted)

Munc family exocytic/endocytic regulator Ync13

$-0.9367287 \quad 7.445198$

$-0.9368128 .44168184$

$-0.93783457 .21566865$

Vid24 family protein (predicted)

$-0.94075346 .39580254$

$$
\text { \#N/A }
$$

$-0.9421275 \quad 4.74094458$

$-0.94280897 .17249982$

$\begin{array}{lll}-0.9431825 & 6.61184159\end{array}$

$-0.94411528 .61251652$

$\# N / A$

glucose-6-phosphate 1-dehydrogenase (predicted)

$$
\text { \#N/A }
$$

Mis6-Sim4 complex Fta6

$\#$ N/A

$0.9451247 \quad 2.26920974 \quad 0.16749596$

$\begin{array}{llll}-0.9459176 & 3.25240247 & 0.0374399\end{array}$

$\begin{array}{lll}-0.9467216 & 2.2685595 & 0.12443491\end{array}$

$\begin{array}{lll}-0.9470301 & 5.92856077 \quad 2.14 \mathrm{E}-09\end{array}$

$-0.9474022 \quad 5.78170421 \quad 6.47 \mathrm{E}-08$

$-0.94759147 .91071318 \quad 2.68 \mathrm{E}-26$

ESCRT III complex subunit Vps20

HAD superfamily hydrolase, unknown role

$\begin{array}{lllll}\text { transcription factor TFIID complex subunit Taf11 (predicted } & -0.9487582 & 5.71616566 & 8.61 \mathrm{E}-07\end{array}$

\#N/A $\quad-0.94934453 .76786126 \quad 0.00409001$

\#N/A $\quad-0.95130236 .79437632 \quad 2.43 E-13$

$\# \mathrm{~N} / \mathrm{A}$

$\begin{array}{lll}-0.9514084 & 2.58439386 & 0.09015273\end{array}$

$\begin{array}{lll}-0.9541991 & 2.84306892 & 0.04379285\end{array}$

$\begin{array}{lrrr}\text { Schizosaccharomyces pombe specific protein } & -0.9556894 & 5.0560903 & 2.71 \mathrm{E}-05 \\ \text { RNAi-mediated silencing protein, human NRDE2 ortholog N } & -0.9559175 & 7.10760349 & 1.71 \mathrm{E}-14\end{array}$

slyoxylate reductase (predicted)

fructose-2,6-bisphosphate 2-phosphatase activity (predicter $-0.9612259 \quad 7.34344659 \quad 3.50 \mathrm{E}-17$

$\begin{array}{llll}\text { Schizosaccharomyces specific protein Mug162 } & -0.9623316 & 5.92070864 & 1.76 \mathrm{E}-07\end{array}$

DASH complex subunit Dad3

\#N/A

$\begin{array}{lll}-0.9624467 & 5.11715963 & 0.0001111\end{array}$

$-0.9637867 \quad 3.80142295 \quad 0.00736481$

plasma membrane sucrose/maltose:proton symporter Sut1 $-0.9640329 \quad 8.0434168 \quad 6.00 \mathrm{E}-20$

Smc5-6 complex non-SMC subunit Nse6 $\quad-0.96504845 .35741142$

Schizosacch

Schizosaccharomyces specific protein Mug74

$\# N / A$

$\# \mathrm{~N} / \mathrm{A}$

human leukocyte receptor 1 ortholo

$\# \mathrm{~N} / \mathrm{A}$

pyridoxamine 5 '-phosphate oxidase (predicted)

$\# N / A$

$\# N / A$

$\# \mathrm{~N} / \mathrm{A}$

Schizosaccharomyces pombe specific protein

$\# N / A$

$\# N / A$

ESCRT II complex subunit Vps36

\#N/A

$\begin{array}{lll}-0.9654803 & 6.08057912 & 1.19 \mathrm{E}-08\end{array}$

$-0.9661655 \quad 5.22469547 \quad 1.43 \mathrm{E}-06$

$-0.9663922 \quad 5.66874905 \quad 1.66 \mathrm{E}-06$

$\begin{array}{lll}-0.967522 & 4.77206084 & 0.00080497\end{array}$

$\begin{array}{lll}-0.9682257 & 5.41883878 & 7.27 \mathrm{E}-07\end{array}$

$\begin{array}{lll}-0.9685458 & 2.65931919 & 0.12414491\end{array}$

$-0.9714269 \quad 6.15246688 \quad 1.28 \mathrm{E}-09$

$\begin{array}{lll}0.9715226 & 10.9589732 & 9.19 \mathrm{E}-14\end{array}$

$\begin{array}{lll}-0.9718441 \quad 5.15336342 \quad 4.55 E-06 \\ -0.9726816 & 5.97811073 & 5.95 E-10\end{array}$

$-0.9726816 \quad 5.97811073 \quad 5.95 \mathrm{E}-10$

$-0.974865 .57072916 \quad 1.36 \mathrm{E}-07$

$\begin{array}{lll}-0.9750721 & 5.31741091 \quad 8.37 \mathrm{E}-08\end{array}$

$\begin{array}{lll}-0.9761672 & 2.0984728 & 0.12622697\end{array}$

$\begin{array}{lll}-0.9771508 & 5.77986278 \quad 7.99 \mathrm{E}-06\end{array}$

$-0.9781222 \quad 4.77614335 \quad 1.60 \mathrm{E}-05$

$\begin{array}{lll}-0.9791541 & 5.26929256 & 1.25 \mathrm{E}-05\end{array}$

microtubule-site clamp monopolin complex subunit Csm1/F $-0.9814452 \quad 5.2536888 \quad 3.03 \mathrm{E}-05$ \#N/A

$\begin{array}{lll}-0.9846782 & 2.60939701 & 0.072281\end{array}$

$\begin{array}{lll}-0.9848015 & 6.08970533 \quad 1.56 \mathrm{E}-08\end{array}$

U5 snRNP subunit Snu40 (predicted)
$5.02 \mathrm{E}-16$

$2.58 \mathrm{E}-29$

2.30E-18

$1.83 \mathrm{E}-11$

0.000128002

2.65E-06

$7.26 \mathrm{E}-13$

$9.58 \mathrm{E}-27$

0.242577383

0.067490996

0.189055497

$1.42 \mathrm{E}-08$

3.53E-07

$7.52 \mathrm{E}-25$

$4.01 \mathrm{E}-06$

0.009489442

2.54E-12

0.143809031

0.077405278

9.92E-05

$1.95 \mathrm{E}-13$

2.20E-32

5.10E-16

8.97E-07

0.000363652

0.016027358

$1.14 \mathrm{E}-18$

5.29E-05

7.17E-08

6.47E-06

7.43E-06

0.00221146

3.41E-06

0.188673552

8.83E-09

9.83E-13

1.91E-05

4.26E-09

7.04E-07

4.49E-07

0.191265755

3.23E-05

6.10E-05

4.87E-05

0.000109449

0.118666392

9.26E-08 


\begin{tabular}{|c|c|c|c|c|c|c|}
\hline SPNCRNA.451 & $\# N / A$ & $\# N / A$ & -0.985368 & 2.44980736 & 0.09004441 & 0.143700716 \\
\hline SPNCRNA.1605 & $\# N / A$ & $\# N / A$ & -0.9854788 & 4.11917848 & 0.00037957 & 0.001126461 \\
\hline SPNCRNA.881 & $\# N / A$ & $\# N / A$ & -0.9858872 & 4.25645193 & 0.00070486 & 0.00197308 \\
\hline SPNCRNA.885 & \#N/A & \#N/A & -0.9866737 & 5.41081238 & $3.90 \mathrm{E}-07$ & $1.91 \mathrm{E}-06$ \\
\hline SPAC27D7.02c & grp1 & Golgi GRIP domain protein Grp1 (predicted) & -0.9868725 & 7.52936979 & $4.88 \mathrm{E}-17$ & 7.03E-16 \\
\hline SPNCRNA.500 & \#N/A & \#N/A & -0.9877636 & 3.47920369 & 0.00824506 & 0.017688406 \\
\hline SPAC4A8.09c & cwf21 & complexed with Cdc5 protein Cwf21 & -0.9888362 & 6.93628185 & 4.94E-19 & 8.55E-18 \\
\hline SPBC1826.01c & mot1 & TATA-binding protein-associated transcription initiation rep & -0.9890518 & 9.04756331 & $5.25 \mathrm{E}-42$ & 3.97E-40 \\
\hline SPAC869.09 & SPAC869.09 & Con- 6 family conserved fungal protein & -0.990909 & 4.04644958 & 0.00050946 & 0.00147337 \\
\hline SPNCRNA.227 & $\# N / A$ & \#N/A & -0.9910399 & 4.67264603 & 0.00063046 & 0.001780924 \\
\hline SPAC1486.04c & alm1 & nucleoporin Alm1 & -0.9913204 & 8.09320774 & $3.26 \mathrm{E}-22$ & 7.43E-21 \\
\hline SPBC21B10.15 & SPBC21B10.15 & Schizosaccharomyces specific protein & -0.9918835 & 5.00592511 & 0.00047256 & 0.001376603 \\
\hline SPBC887.17 & SPBC887.17 & nucleobase transmembrane transporter (predicted) & -0.9919573 & 8.62348964 & $6.41 \mathrm{E}-28$ & $2.01 \mathrm{E}-26$ \\
\hline SPAC23C11.06c & SPAC23C11.06c & vacuolar membrane hydrolase, implicted in protein cataboli & -0.9924436 & 9.11131819 & $7.90 \mathrm{E}-22$ & $1.78 \mathrm{E}-20$ \\
\hline SPAC1F7.08 & fio1 & plasma membrane iron transport multicopper oxidase Fio1 & -0.9934008 & 8.37177509 & $2.58 \mathrm{E}-22$ & $5.92 \mathrm{E}-21$ \\
\hline SPCC1494.02c & taf13 & transcription factor TFIID complex subunit Taf13 (predicted & -0.994012 & 5.87386996 & 7.73E-08 & 4.16E-07 \\
\hline SPBC543.05c & bor1 & plasma membrane borate efflux transmembrane transport & -0.9945018 & 5.93189565 & 1.19E-09 & $8.25 E-09$ \\
\hline SPNCRNA.798 & $\# N / A$ & \#N/A & -0.9946472 & 9.10966833 & $7.91 \mathrm{E}-22$ & $1.78 \mathrm{E}-20$ \\
\hline SPNCRNA.1053 & $\# N / A$ & $\# N / A$ & -0.9950002 & 5.45446681 & $7.55 \mathrm{E}-08$ & 4.07E-07 \\
\hline SPCC1919.04 & SPCC1919.04 & Schizosaccharomyces specific protein & -0.9956859 & 5.49248197 & $1.39 \mathrm{E}-07$ & 7.19E-07 \\
\hline SPCC63.03 & SPCC63.03 & mitochondrial MIB complex subunit, human DNAJC11 ortho & -0.9960242 & 6.53976043 & $6.41 \mathrm{E}-14$ & $6.96 \mathrm{E}-13$ \\
\hline SPNCRNA.570 & \#N/A & \#N/A & -0.9987078 & 5.69692808 & 1.37E-07 & 7.11E-07 \\
\hline SPNCRNA.1303 & $\# N / A$ & $\# N / A$ & -1.0035223 & 2.92711365 & 0.0189542 & 0.037473367 \\
\hline SPAC1A6.11 & $\# N / A$ & $\# N / A$ & -1.0062312 & 4.52146494 & 3.70E-05 & 0.000131605 \\
\hline SPNCRNA.1604 & $\# N / A$ & $\# N / A$ & -1.0062594 & 4.18026422 & 0.00023087 & 0.000715413 \\
\hline SPNCRNA.1302 & $\# N / A$ & $\# N / A$ & -1.0072318 & 8.64608148 & 8.37E-23 & $1.94 \mathrm{E}-21$ \\
\hline SPNCRNA.306 & $\# N / A$ & $\# N / A$ & -1.0076718 & 2.68433795 & 0.04795008 & 0.083495462 \\
\hline SPBC215.04 & git11 & heterotrimeric $\mathrm{G}$ protein gamma subunit Git11 & -1.0084936 & 4.57362424 & 0.00080286 & 0.002207452 \\
\hline SPNCRNA.1660 & $\# N / A$ & $\# N / A$ & -1.0085124 & 6.82727855 & 2.03E-18 & 3.30E-17 \\
\hline SPNCRNA.263 & $\# N / A$ & $\# N / A$ & -1.0095422 & 1.63556724 & 0.29339481 & 0.386105529 \\
\hline SPAC1556.01c & $\operatorname{rad} 50$ & DNA repair protein Rad50 & -1.0099885 & 7.89230365 & 4.66E-20 & 8.96E-19 \\
\hline SPBC16C6.10 & chp2 & heterochromatin (HP1) family chromodomain protein Chp2 & -1.0100622 & 5.4625318 & $2.28 \mathrm{E}-06$ & $1.00 \mathrm{E}-05$ \\
\hline SPNCRNA.1054 & $\# N / A$ & \#N/A & -1.0103518 & 2.39266697 & 0.1327732 & 0.199849957 \\
\hline SPBC30B4.09 & SРВС30B4.09 & Schizosaccharomyces specific protein & -1.0106373 & 5.73813445 & 3.40E-08 & 1.95E-07 \\
\hline SPAC869.06c & hry1 & HHE domain cation binding protein (predicted) & -1.0115666 & 4.30315597 & 0.00101113 & 0.002708949 \\
\hline ScpofMp04 & $\# N / A$ & \#N/A & -1.0136931 & 11.3147181 & $2.58 \mathrm{E}-12$ & 2.44E-11 \\
\hline SPNCRNA.173 & $\# N / A$ & $\# N / A$ & -1.0150382 & 0.94659076 & 0.69611748 & 0.768570505 \\
\hline SPBPB21E7.01c & eno102 & enolase (predicted) & -1.0172275 & 8.74166426 & $1.56 \mathrm{E}-23$ & 3.77E-22 \\
\hline SPNCRNA.13 & \#N/A & \#N/A & -1.0198413 & 3.04359894 & 0.04577402 & 0.080198812 \\
\hline SPAC23D3.12 & SPAC23D3.12 & plasma membrane inorganic phosphate transmembrane tri & -1.0206069 & 8.11441987 & 1.37E-28 & 4.51E-27 \\
\hline SPAC6C3.07 & mug68 & Schizosaccharomyces specific protein Mug68 & -1.0247615 & 5.24941359 & $3.40 \mathrm{E}-07$ & 1.67E-06 \\
\hline SPNCRNA.205 & $\# N / A$ & \#N/A & -1.0258352 & 5.59096267 & 4.69E-07 & $2.27 \mathrm{E}-06$ \\
\hline SPAC57A10.04 & mug10 & meiotic Rho guanine nucleotide exchange factor (predicted & -1.0262663 & 4.46922061 & 0.00022595 & 0.000701787 \\
\hline SPNCRNA.626 & $\# N / A$ & \#N/A & -1.0265401 & 5.70206106 & 1.71E-09 & $1.16 \mathrm{E}-08$ \\
\hline SPNCRNA.101 & $\# \mathrm{~N} / \mathrm{A}$ & $\# N / A$ & -1.0273652 & 4.873521 & 8.69E-06 & 3.47E-05 \\
\hline SPNCRNA.91 & $\# N / A$ & $\# N / A$ & -1.0274212 & 1.90552565 & 0.18371282 & 0.261869655 \\
\hline SPNCRNA.1439 & \#N/A & $\# N / A$ & -1.0279096 & 5.88997617 & 8.77E-09 & $5.40 \mathrm{E}-08$ \\
\hline
\end{tabular}


SPNCRNA.62

SPAC12B10.10

SPAC9E9.09C

SPAP27G11.11C

SPBC685.02

SPBPB21E7.02C

SPAC1952.09c

SPNCRNA.936

SPAC4F10.16C

SPNCRNA.495

SPAC22H10.09

SPBC1306.01C

SPNCRNA. 275

SPNCRNA.188

SPNCRNA.791

SPNCRNA.1499

SPBC56F2.06

SPAC23E2.03C

SPNCRNA.1281

SPBC1A4.03C

SPAC328.08C

SPNCRNA.916

SPNCRNA.825

SPAC27F1.04C

SPNCRNA.920

SPAC31G5.06

SPBC1685.04

SPNCRNA.756

SPAC110.05

SPNCRNA. 274

SPAC56F8.14C

SPNCRNA.79

SPCC70.04C

SPAC3A12.19

SPNCRNA.1200

SPNCRNA.815

SPBC36.13

SPAC6G10.06

SPAC25G10.04C

SPBC14C8.09c

SPAC607.09c

SPAC1006.09

SPAC12G12.11C

SPAC2G11.02

SPNCRNA.1366

SPNCRNA.898

SPNCRNA.256
\#N/A

$-1.0289999 \quad 4.88950777 \quad 6.74 \mathrm{E}-06$

$-1.0296437 \quad 4.82228645 \quad 0.00010137$

$-1.0301467 \quad 9.12042596 \quad 2.01 \mathrm{E}-30$

$\begin{array}{lll}-1.0308672 & 0.94684644 & 0.69620422\end{array}$

aldehyde dehydrogenase (predicted)

$$
\# N / A
$$

$\begin{array}{llll}\text { mitochondrial single stranded DNA specific 5'-3' exodeoxyri } & -1.0337351 & 5.48780551 & 2.71 \mathrm{E}-09 \\ \text { phosphoglycerate mutase/6-phosphofructo-2-kinase family } & -1.0339566 & 4.57413638 & 6.81 \mathrm{E}-05\end{array}$

$\begin{array}{lrrr}\text { phosphoglycerate mutase/6-phosphofructo-2-kinase family } & -1.0339566 & 4.57413638 & 6.81 \mathrm{E}-05 \\ \text { acetyl-CoA hydrolase (predicted) } & -1.0366114 & 7.79842 & 1.07 \mathrm{E}-20\end{array}$

$$
\begin{array}{lll}
-1.0375227 & 7.01244574 \quad 1.03 E-16
\end{array}
$$

plasma membrane phospholipid-translocating ATPase com $-1.0380647 \quad 8.41994035 \quad 2.47 \mathrm{E}-32$

$\#$ N/A

1.03822385 .02729828

7.36E-06

$-1.03921016 .39471473 \quad 2.11 \mathrm{E}-05$

$\begin{array}{llllll} & \\ \text { mito } & -1.0397367 & 6.70081426 & 3.85 \mathrm{E}-14\end{array}$

mef1

$\# N / A$

$\# \mathrm{~N} / \mathrm{A}$

$\# N / A$

$\# \mathrm{~N} / \mathrm{A}$

mug147

ste7

\#N/A

top2

tbc1

$\# N / A$

$\# \mathrm{~N} / \mathrm{A}$

nuf2

\#N/A

rrg8

SPBC1685.04

$\# \mathrm{~N} / \mathrm{A}$

$\# \mathrm{~N} / \mathrm{A}$

$\# \mathrm{~N} / \mathrm{A}$

mug115

\#N/A

SPCC70.04C

SPAC3A12.19

$\# \mathrm{~N} / \mathrm{A}$

$\# N / A$

tda3

$\# N / A$

rec10

$\mathrm{db} / 3$

btn1

win1

miy1

urb2

$\# N / A$

$\# \mathrm{~N} / \mathrm{A}$

$\# N / A$
$-1.03978745 .68301639 \quad 1.16 \mathrm{E}-08$

$\begin{array}{lrrr}\text { \#N/A } & -1.0398917 & 2.13372661 & 0.13360614 \\ \text { \#N/A } & -1.0407619 & 5.95912761 & 4.83 \mathrm{E}-10 \\ \text { \#N/A } & -1.0417764 & 2.30110148 & 0.13871086\end{array}$

$\begin{array}{lrrr}\text { \#N/A } & -1.0398917 & 2.13372661 & 0.13360614 \\ \text { \#N/A } & -1.0407619 & 5.95912761 & 4.83 \mathrm{E}-10 \\ \text { \#N/A } & -1.0417764 & 2.30110148 & 0.13871086\end{array}$

$\# N / A$

$-1.04225457 .75896186 \quad 6.68 \mathrm{E}-11$

$\begin{array}{lll}-1.0440354 & 7.8222085 & 1.70 \mathrm{E}-17\end{array}$

$\begin{array}{lll}-1.0454289 & 4.56035738 & 0.00033623\end{array}$

$\begin{array}{lll}-1.0455098 & 6.60290173 \quad 4.80 \mathrm{E}-13\end{array}$

DNA topoisomerase II

$\# \mathrm{~N} / \mathrm{A}$

$\begin{array}{lll}-1.0455347 & 5.29668285 & 6.68 \mathrm{E}-07\end{array}$

tubulin specific chaperone cofactor C, GTPase activating prr $\begin{array}{rrrr}-1.0472804 & 3.84615707 & 0.00221031\end{array}$

\#N/A $\quad-1.04944553 .063452230 .00946093$

$-1.04954545 .95809518 \quad 7.87 \mathrm{E}-09$

$\begin{array}{lll}-1.0500088 & 4.8375126 & 6.53 \mathrm{E}-06\end{array}$

NMS complex subunit Nuf2

$\begin{array}{lllll}\text { mitochondrial conserved fungal protein Rrg8 (predicted) } & -1.0507184 & 4.03449115 & 0.00226276\end{array}$

$\begin{array}{lllll}\text { Schizosaccharomyces specific protein } & -1.0579695 & 5.59753891 & 8.10 \mathrm{E}-09\end{array}$

\#N/A $\quad-1.06055263 .519843020 .00276252$

\#N/A $\quad-1.061276 \quad 4.112537380 .00070095$

$\# N / A$

Schizosaccharomyces pombe specific protein Mug115

\#N/A

Schizosaccharomyces pombe specific protein

mitochondrial ribosomal protein subunit L27 (predicted)

$$
\# N / A
$$

$\#$ \#N/A

$\begin{array}{rrr}-1.061276 & 4.11253738 & 0.00070095 \\ -1.0614037 & 3.51998816 & 0.00256922\end{array}$

$\begin{array}{rrr}-1.0614037 & 3.51998816 & 0.00256922 \\ -1.0615315 & 7.90569283 & 3.18 \mathrm{E}-32\end{array}$

$\begin{array}{lll}-1.0630836 & 3.92027005 & 0.00084109\end{array}$

$\begin{array}{lll}-1.0641148 & 6.61219112 & 7.77 \mathrm{E}-15\end{array}$

$\begin{array}{lll}-1.0657071 & 5.27478008 \quad 5.24 \mathrm{E}-07\end{array}$

$\begin{array}{lll}-1.0659152 & 4.26271042 \quad 0.00015401\end{array}$

$-1.0724164 \quad 5.33642037 \quad 6.89 \mathrm{E}-08$

$\begin{array}{lll}-1.0738093 & 4.48276788 \quad 3.14 \mathrm{E}-05\end{array}$

FAD-dependent amino acid oxidase involved in late endosol $-1.07454913 .90716058 \quad 0.00152831$

meiotic recombination protein Rec10

$1.08096 \quad 6.94214625 \quad 2.57 \mathrm{E}-19$

$\begin{array}{lllll}\text { IMPACT domain protein, possible chaperone (predicted) } & -1.0809842 & 4.65837633 & 4.76 \mathrm{E}-05\end{array}$

battenin CLN3 family protein

MAP kinase kinase kinase Win1

Lys48-specific deubiquitinase Mindy family, Miy1

ribosome biogenesis protein Urb2 (predicted)

$\# N / A$

$\#$ \#N/A

$-1.08395025 .91777734$

$1.0848438 \quad 8.03669521 \quad 9.38 \mathrm{E}-34$

$\begin{array}{lll}-1.0862275 & 5.66498926 & 7.36 \mathrm{E}-09\end{array}$

$-1.08806996 .74334527 \quad 1.21 \mathrm{E}-17$

$1.09048394 .83505763 \quad 6.10 \mathrm{E}-06$

$\begin{array}{lll}-1.0928621 & 6.63409524 & 3.44 \mathrm{E}-13\end{array}$

$\begin{array}{lll}-1.0959532 & 1.36201371 & 0.3054826\end{array}$
2.76E-05

0.000334219

$7.38 \mathrm{E}-29$

0.768570505

1.77E-08

0.000231519

2.21E-19

$1.43 \mathrm{E}-15$

1.04E-30

2.99E-05

7.87E-05

4.27E-13

7.03E-08

0.200925915

3.50E-09

0.207047029

5.30E-10

$2.54 \mathrm{E}-16$

0.00101062

$4.88 \mathrm{E}-12$

3.16E-06

0.005495478

0.020056581

$4.88 \mathrm{E}-08$

$2.68 \mathrm{E}-05$

0.005617687

5.01E-08

0.006694771

0.001965377

0.006266505

$1.32 \mathrm{E}-30$

0.002301422

$9.16 \mathrm{E}-14$

2.52E-06

0.000492261

3.73E-07

0.000113033

0.003930525

4.60E-18

0.000166172

3.12E-09

4.31E-32

$4.58 \mathrm{E}-08$

$1.83 \mathrm{E}-16$

2.52E-05

3.56E-12

0.399003662 
SPAC4G9.13C

SPAC186.02C

SPNCRNA.621

SPNCRNA.100

SPBC16E9.17C

SPNCRNA.1175

SPNCRNA.1057

SPCC1753.02C

SPAC23H3.04

SPAC14C4.03

SPNCRNA.71

SPBC1D7.02C

SPNCRNA.644

SPBC19G7.08C

SPNCRNA.502

SPNCRNA.1227

SPNCRNA.42

SPNCRNA.1463

SPCC4G3.03

SPNCRNA.1649

SPNCRNA 212

SPNCRNA.268

SPBC8E4.05C

SPBC31F10.08

SPNCRNA.1010

SPNCRNA.1218

SPAC6G9.06C

SPNCRNA.178

SPNCRNA.20

SPAC1399.01C

SPAC167.06c

SPNCRNA.289

SPNCRNA.854

SPNCRNA.830

SPCC663.17

SPNCRNA.260

SPNCRNA.1385

SPNCRNA.1272

SPBC23G7.04C
SPBC13G1.16

$\# N / A$

$\# N / A$

$\# N / A$

SPAC13G6.13

SPAC22F8.03C

$\#$ N/A

cwf16

vps26

SPAC186.02C

$\# \mathrm{~N} / \mathrm{A}$

$\# N / A$

rem 1

$\# \mathrm{~N} / \mathrm{A}$

$\# \mathrm{~N} / \mathrm{A}$

git3

SPAC23H3.04

mek1

$\# \mathrm{~N} / \mathrm{A}$

scr1

\#N/A

art1

$\# N / A$

$\# \mathrm{~N} / \mathrm{A}$

\#N/A

SPCC4G3.03

$\# \mathrm{~N} / \mathrm{A}$

$\# N / A$

\#N/A

SPBC8E4.05C

mde2

$\# N / A$

\#N/A

pcp1

$\# \mathrm{~N} / \mathrm{A}$

\#N/A

SPAC1399.01C

mug143

$\# N / A$

$\# N / A$

$\# \mathrm{~N} / \mathrm{A}$

wtf15

$\# N / A$

$\# N / A$

$\# \mathrm{~N} / \mathrm{A}$

nif1
Schizosaccharomyces specific protein

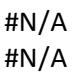

\#N/A

Schizosaccharomyces pombe specific protein

Schizosaccharomyces pombe specific protein \#N/A

splicing factor Cwf16

retromer complex subunit Vps 26

hydroxyacid dehydrogenase, implicated in cellular detoxifici $\# N / A$

$\# N / A$

meiosis-specific cyclin Rem 1

$$
\# \mathrm{~N} / \mathrm{A}
$$

$\# N / A$

G-protein coupled receptor Git3

conserved fungal multispanning membrane protein

Cds1/Rad53/Chk2 family protein kinase Mek1

$$
\# \text { N/A }
$$

transcription factor Scr1

$$
\# \mathrm{~N} / \mathrm{A}
$$

arrestin family protein Art1

$\# \mathrm{~N} / \mathrm{A}$

$\# N / A$

$\# N / A$

$\# \mathrm{~N} / \mathrm{A}$

WD40/YVTN repeat-like protein

$$
\# N / A
$$$$
\# \text { \#/A }
$$

A

fumarate lyase superfamily, unknown specificity, bacterial :

Mde2 protein

\section{\#N/A}

$\# N / A$

gamma tubulin complex linker, pericentrin/kendrin Pcp1

$\# \mathrm{~N} / \mathrm{A}$

$\# N / A$

nucleobase transmembrane transporter (predicted)

Schizosaccharomyces specific protein Mug143

\#N/A

$\# N / A$

wtf element Wtf15

$$
\# N / A
$$

$\# N / A$

$\#$ N/A

protein kinase inhibitor, SEL1 repeat protein Nif1 $\begin{array}{lll}-1.0986673 & 3.46430948 & 0.01336966\end{array}$ $\begin{array}{lll}1.0990584 & 1.53131778 & 0.33464709\end{array}$

$\begin{array}{lll}-1.102172 & 4.6892931 & 2.80 \mathrm{E}-06\end{array}$

- 1.10693541 .683632580 .26495356

$\begin{array}{llll}-1.1094703 & 1.68383019 & 0.19923981\end{array}$

$\begin{array}{lll}-1.1099761 & 1.68422919 & 0.19471744\end{array}$

$\begin{array}{lll}-1.1104284 & 3.95794023 & 0.00085546\end{array}$

$-1.1113036 \quad 6.48610109 \quad 8.60 \mathrm{E}-13$

$-1.1114712 \quad 8.59337765 \quad 4.71 \mathrm{E}-38$

$\begin{array}{llll}-1.114746 & 3.73402774 & 0.00202349\end{array}$

$\begin{array}{llll}-1.1163948 & 4.0930113 & 0.00124012\end{array}$

$\begin{array}{llll}-1.120804 & 3.34781058 & 0.00667568\end{array}$

$\begin{array}{llll}-1.1221265 & 3.43581479 & 0.00362448\end{array}$

$\begin{array}{lll}-1.1225737 & 4.76605219 & 1.90 \mathrm{E}-05\end{array}$

$\begin{array}{lll}-1.1303614 & 2.2695032 & 0.05197193\end{array}$

$\begin{array}{lll}-1.1349751 & 8.40284311 & 5.18 \mathrm{E}-42\end{array}$

$\begin{array}{lll}-1.135991 & 5.33303152 & 3.18 \mathrm{E}-09\end{array}$

$\begin{array}{llll}-1.1363017 & 4.04000931 & 0.00254172\end{array}$

$\begin{array}{lll}-1.1372954 & 2.53198765 & 0.03295919\end{array}$

$\begin{array}{lll}-1.1397024 & 7.19536422 & 1.62 \mathrm{E}-09\end{array}$

$-1.1401744 \quad 4.294317530 .00052578$

$\begin{array}{llll}-1.1414307 & 6.34707771 \quad 5.92 \mathrm{E}-14\end{array}$

$\begin{array}{lll}-1.1461911 & 3.0063648 & 0.00695651\end{array}$

$\begin{array}{lll}-1.1495211 & 7.6200281 & 9.62 \mathrm{E}-32\end{array}$

$\begin{array}{lll}-1.1496335 & 3.87778641 & 0.0088431\end{array}$

$\begin{array}{llll}-1.1498037 & 3.11858776 & 0.00606533\end{array}$

$\begin{array}{lll}-1.1503053 & 6.33127374 & 2.92 \mathrm{E}-12\end{array}$

$-1.15127535 .93288624 \quad 6.15 \mathrm{E}-12$

$\begin{array}{llll}-1.151835 & 4.16485988 & 0.00012527\end{array}$

$\begin{array}{llll}-1.1526499 & 2.53269195 & 0.03633464\end{array}$

$\begin{array}{lll}-1.1571085 & 5.76218648 \quad 1.19 \mathrm{E}-12\end{array}$

$\begin{array}{lll}-1.1658575 & 4.69314316 \quad 2.57 \mathrm{E}-06\end{array}$

$\begin{array}{lll}-1.1698522 & 3.13599844 & 0.007488\end{array}$

$\begin{array}{lll}-1.1727093 & 4.25584029 & 0.00013654\end{array}$

$1.1755532 \quad 7.50012319$

$\begin{array}{lll}-1.1764438 & 4.71246829 & 7.95 \mathrm{E}-06\end{array}$

$\begin{array}{lll}-1.1806773 & 5.57121143 \quad 3.20 \mathrm{E}-10\end{array}$

$-1.1810493 \quad 5.36032437$

$\begin{array}{lll}-1.1820978 & 6.44658384 & 4.96 \mathrm{E}-17\end{array}$

$\begin{array}{lll}-1.1844994 & 2.7082352 & 0.0193259\end{array}$

-1.1848899 $4.13681268 \quad 7.95 \mathrm{E}-05$

$\begin{array}{lll}-1.1919492 & 3.20295056 & 0.0073865\end{array}$

$\begin{array}{lll}-1.1940053 & 5.00971154 & 5.21 \mathrm{E}-07\end{array}$

$\begin{array}{lll}-1.1950053 & 2.30142252 & 0.03848733\end{array}$

$\begin{array}{lll}-1.1956185 & 4.27272088 \quad 5.86 \mathrm{E}-06\end{array}$

$\begin{array}{lll}-1.1957318 & 4.81910807 & 5.96 \mathrm{E}-07\end{array}$

$\begin{array}{lll}-1.19838 & 6.95906258 \quad 3.09 \mathrm{E}-20\end{array}$
0.027363264

0.428945838

$1.22 \mathrm{E}-05$

0.355974134

0.279956004

0.274679138

0.00233417

$8.57 \mathrm{E}-12$

2.69E-36

0.005068028

0.003257051

0.01468262

0.008505028

7.14E-05

0.089559131

$3.96 \mathrm{E}-40$

2.07E-08

0.006215019

0.060471092

$1.10 \mathrm{E}-08$

0.001513508

$6.45 \mathrm{E}-13$

0.015241151

$3.83 \mathrm{E}-30$

0.01885845

0.013510495

$2.74 \mathrm{E}-11$

5.57E-11

0.00040632

0.065794894

1.17E-11

1.12E-05

0.016259033

0.000440358

$1.64 \mathrm{E}-25$

3.21E-05

2.37E-09

9.60E-09

7.12E-16

0.038097546

0.000266119

0.016061226

2.51E-06

0.069170174

2.43E-05

2.84E-06

6.07E-19 


\begin{tabular}{|c|c|c|c|c|c|c|}
\hline SPNCRNA.247 & \#N/A & $\# \mathrm{~N} / \mathrm{A}$ & -1.2013053 & 2.47798403 & 0.03907477 & 0.070066879 \\
\hline SPNCRNA.1606 & $\# \mathrm{~N} / \mathrm{A}$ & $\# \mathrm{~N} / \mathrm{A}$ & -1.2039073 & 8.23393679 & $3.11 \mathrm{E}-39$ & $1.92 \mathrm{E}-37$ \\
\hline SPBC106.02c & srx1 & sulfiredoxin & -1.2053981 & 4.09369683 & $5.32 \mathrm{E}-05$ & 0.000184015 \\
\hline SPNCRNA.141 & $\# \mathrm{~N} / \mathrm{A}$ & $\# \mathrm{~N} / \mathrm{A}$ & -1.2054324 & 2.84450081 & 0.03721631 & 0.067123512 \\
\hline SPNCRNA.723 & $\# N / A$ & $\# N / A$ & -1.2090348 & 4.25637801 & 2.43E-05 & $9.00 \mathrm{E}-05$ \\
\hline SPNCRNA.954 & $\# \mathrm{~N} / \mathrm{A}$ & \#N/A & -1.2119538 & 7.45011708 & $6.29 E-31$ & $2.38 \mathrm{E}-29$ \\
\hline SPBC18E5.08 & SPBC18E5.08 & $\mathrm{N}$-acetyltransferase (predicted) & -1.2146673 & 5.35012353 & $1.28 \mathrm{E}-08$ & 7.68E-08 \\
\hline SPBC365.12c & ish1 & nuclear envelope LEA domain protein Ish1 & -1.2164724 & 8.9011738 & $5.78 \mathrm{E}-13$ & $5.85 \mathrm{E}-12$ \\
\hline SPNCRNA.40 & $\# N / A$ & \#N/A & -1.2183711 & 1.86532895 & 0.12235276 & 0.186349709 \\
\hline SPNCRNA.358 & $\# \mathrm{~N} / \mathrm{A}$ & \#N/A & -1.2214969 & 2.73164728 & 0.03044878 & 0.056443453 \\
\hline SPAC513.04 & SPAC513.04 & Schizosaccharomyces pombe specific protein & -1.2219864 & 1.73109507 & 0.14065028 & 0.209620053 \\
\hline SPAC1039.01 & SPAC1039.01 & amino acid transmembrane transporter (predicted) & -1.2221833 & 6.9223323 & 2.04E-28 & 6.57E-27 \\
\hline SPNCRNA.664 & \#N/A & $\# N / A$ & -1.2223779 & 1.58349576 & 0.25813546 & 0.348739608 \\
\hline SPAP27G11.08c & meu32 & Schizosaccharomyces specific protein Meu32 & -1.2229957 & 4.110703 & 2.99E-05 & 0.000108244 \\
\hline SPCC285.06c & wtf17 & wtf element Wtf17 & -1.2262872 & 5.26772304 & 2.09E-09 & $1.39 \mathrm{E}-08$ \\
\hline SPNCRNA.1350 & $\# N / A$ & \#N/A & -1.2282372 & 3.57427545 & 0.00082358 & 0.002258036 \\
\hline SPNCRNA.437 & $\# \mathrm{~N} / \mathrm{A}$ & $\# N / A$ & -1.2295223 & 1.73130975 & 0.08317332 & 0.134026621 \\
\hline SPNCRNA.376 & \#N/A & \#N/A & -1.2311919 & 1.58403623 & 0.23583268 & 0.323491515 \\
\hline SPNCRNA.962 & $\# N / A$ & \#N/A & -1.2341119 & 2.58492386 & 0.01775254 & 0.035313082 \\
\hline SPBC4.01 & dni2 & tetraspan protein, claudin Dni2 & -1.2373497 & 4.4893511 & 1.17E-05 & 4.58E-05 \\
\hline SPNCRNA.159 & $\# \mathrm{~N} / \mathrm{A}$ & \#N/A & -1.2381875 & 4.63092837 & $5.84 \mathrm{E}-06$ & $2.42 \mathrm{E}-05$ \\
\hline SPAC644.18c & bet3 & TRAPP complex subunit Bet3 (predicted) & -1.2387637 & 3.93960849 & $6.98 \mathrm{E}-05$ & 0.000236566 \\
\hline SPNCRNA.33 & \#N/A & \#N/A & -1.2441653 & 1.42060843 & 0.29694548 & 0.38999401 \\
\hline SPNCRNA.177 & $\# N / A$ & \#N/A & -1.2460193 & 1.42022806 & 0.31626179 & 0.410326313 \\
\hline SPBC1E8.05 & SPBC1E8.05 & conserved fungal cell surface protein, Kre9/Knh1 family & -1.2515865 & 8.00203619 & $1.05 \mathrm{E}-25$ & $2.79 \mathrm{E}-24$ \\
\hline SPCC1442.01 & ste6 & guanyl-nucleotide exchange factor Ste6 & -1.2519351 & 7.40126714 & $9.84 \mathrm{E}-32$ & $3.89 \mathrm{E}-30$ \\
\hline SPNCRNA.571 & $\# \mathrm{~N} / \mathrm{A}$ & \#N/A & -1.252582 & 3.42284373 & 0.0009731 & 0.002621486 \\
\hline SPNCRNA.305 & $\# \mathrm{~N} / \mathrm{A}$ & $\# N / A$ & -1.2573722 & 2.42192183 & 0.01997175 & 0.039177782 \\
\hline SPNCRNA.801 & $\# \mathrm{~N} / \mathrm{A}$ & $\# \mathrm{~N} / \mathrm{A}$ & -1.260801 & 6.47778736 & 5.17E-18 & 8.15E-17 \\
\hline SPNCRNA.1267 & $\# N / A$ & \#N/A & -1.2624002 & 3.91846167 & 0.00480221 & 0.010977163 \\
\hline SPAC1039.11c & gto1 & alpha-glucosidase (predicted) & -1.2632606 & 8.26887816 & 4.05E-54 & 5.10E-52 \\
\hline SPNCRNA.405 & $\# N / A$ & \#N/A & -1.2658434 & 2.75459439 & 0.01325969 & 0.02717836 \\
\hline SPNCRNA.131 & $\# N / A$ & $\# N / A$ & -1.2664746 & 4.31931649 & 2.11E-06 & $9.34 \mathrm{E}-06$ \\
\hline SPNCRNA.1031 & $\# N / A$ & \#N/A & -1.2665789 & 7.24856745 & $3.83 E-26$ & 1.07E-24 \\
\hline SPBC359.05 & abc3 & $A B C$ transmembrane transporter $A b c 3$ & -1.2669718 & 8.3350568 & $1.36 \mathrm{E}-51$ & $1.50 \mathrm{E}-49$ \\
\hline SPBC13G1.15c & SPBC13G1.15c & Schizosaccharomyces pombe specific protein & -1.2687479 & 2.23643034 & 0.04706849 & 0.082212799 \\
\hline SPNCRNA.1691 & $\# N / A$ & \#N/A & -1.2697492 & 4.08325863 & 8.69E-05 & 0.000289214 \\
\hline SPCC1183.10 & wtf10 & wtf element Wtf10 & -1.2757568 & 4.9652279 & 3.95E-08 & $2.24 \mathrm{E}-07$ \\
\hline SPNCRNA.1255 & $\# N / A$ & $\# \mathrm{~N} / \mathrm{A}$ & -1.277545 & 2.23742559 & 0.05759251 & 0.097759297 \\
\hline SPNCRNA.47 & $\# \mathrm{~N} / \mathrm{A}$ & $\# \mathrm{~N} / \mathrm{A}$ & -1.277938 & 3.73203568 & 0.00036919 & 0.001098531 \\
\hline SPNCRNA.1312 & $\# N / A$ & $\# \mathrm{~N} / \mathrm{A}$ & -1.2789117 & 7.56597631 & 2.19E-35 & 1.07E-33 \\
\hline SPCC553.05c & wtf6 & wtf element Wtf6 & -1.2792736 & 5.39239276 & 7.19E-08 & $3.88 \mathrm{E}-07$ \\
\hline SPAC4F $10.15 c$ & wsp1 & WASp homolog & -1.2801388 & 7.57494902 & $6.18 \mathrm{E}-31$ & $2.35 \mathrm{E}-29$ \\
\hline SPCP31B10.06 & tcb2 & tricalbin, C2 domain protein (phospholipid binding) ER-plasI & -1.2810156 & 7.94743262 & $4.28 \mathrm{E}-18$ & $6.81 \mathrm{E}-17$ \\
\hline SPAC1556.06.1 & $\# N / A$ & \#N/A & -1.2821568 & 5.32269297 & 3.79E-09 & $2.45 \mathrm{E}-08$ \\
\hline SPAC1805.15c & pub2 & HECT-type ubiquitin-protein ligase E3 Pub2 & -1.2823704 & 7.05037982 & $8.25 E-29$ & $2.74 \mathrm{E}-27$ \\
\hline SPNCRNA.1224 & \#N/A & \#N/A & -1.2825146 & 4.09297276 & $1.96 \mathrm{E}-05$ & 7.35E-05 \\
\hline
\end{tabular}


SPAC2F7.06C

SPNCRNA.774

SPCC1235.14

SPNCRNA.1437

SPNCRNA.1148

SPNCRNA.821

SPNCRNA.1352

SPAC750.03C

SPNCRNA.828

SPNCRNA.1075

SPCC1393.12

SPNCRNA.1133

SPCC569.05c

SPNCRNA.267

SPAC1002.16C

SPAC16E8.18

SPNCRNA.243

SPNCRNA.239

SPNCRNA.951

SPNCRNA.1318

SPNCRNA.283

SPBC25B2.08

SPBC8D2.05C

SPNCRNA.95

SPNCRNA.1062

SPNCRNA.04

SPAC1F8.03C

SPBC839.06

SPAC458.04c

SPNCRNA.1327

SPBC1683.09c

SPNCRNA.1598

SPNCRNA.1374

SPNCRNA.1163

SPNCRNA.478

SPBC1685.14C

SPAC26F1.11

SPAPB15E9.02C

SPNCRNA.520

$\operatorname{rim} 20$

$\# N / A$

$\# \mathrm{~N} / \mathrm{A}$

SPAC4H3.03C

$\mathrm{mfm} 2$

ksh1

$\#$ N/A

$\# N / A$

pols

\#N/A

ght5

$\# \mathrm{~N} / \mathrm{A}$

$\# N / A$

$\# N / A$

$\# \mathrm{~N} / \mathrm{A}$

SPAC750.03C

$\# \mathrm{~N} / \mathrm{A}$

SPCC1393.12

$\# \mathrm{~N} / \mathrm{A}$

SPCC569.05C

$\# \mathrm{~N} / \mathrm{A}$

SPAC1002.16C

SPAC16E8.18

$\# \mathrm{~N} / \mathrm{A}$

$\# N / A$

$\# N / A$

$\# N / A$

$\# N / A$

SPBC25B2.08

sfi1

$\# N / A$

$\# N / A$

$\# \mathrm{~N} / \mathrm{A}$

str3

cta3

dli1

\#N/A

frp1

$\# N / A$

$\# N / A$

$\# N / A$

$\# \mathrm{~N} / \mathrm{A}$

vid27

$\# \mathrm{~N} / \mathrm{A}$

$\# N / A$

$\# \mathrm{~N} / \mathrm{A}$
BRO1 domain protein Rim20

$\# N / A$

lucan 1,4-alpha-glucosidase (predicted)

M-factor precursor Mfm2

FGolgi kish family protein Ksh1 (predicted)

$\# N / A$

DNA polymerase $X$ family

$\# N / A$

$\# N / A$

$\begin{array}{lll}-1.3003862 & 6.15751181 \quad 3.95 \mathrm{E}-14\end{array}$

$\begin{array}{llll}-1.3042201 & 3.6619991 & 0.00023894\end{array}$

$\begin{array}{lll}-1.3079686 & 3.50754394 & 0.00091653\end{array}$

$-1.3102904 \quad 8.10929549 \quad 2.83 \mathrm{E}-29$

$\begin{array}{lll}-1.3104058 & 6.51045587 \quad 4.61 \mathrm{E}-08\end{array}$

$\begin{array}{lll}-1.3121342 & 2.63495059 & 0.01728967\end{array}$

$\begin{array}{lll}-1.3121954 & 6.5315507 & 1.04 \mathrm{E}-17\end{array}$

$-1.31420016 .49440869 \quad 1.68 \mathrm{E}-18$

$\begin{array}{lll}-1.3143983 & 6.52980338 & 2.74 \mathrm{E}-21\end{array}$

$\begin{array}{lll}-1.3188294 & 2.23847346 & 0.07411668\end{array}$

plasma membrane high-affinity glucose/fructose:proton sy $\quad \begin{array}{llll}-1.321228 & 9.92101954 & 1.50 \mathrm{E}-60\end{array}$

\#N/A $\quad-1.33268093 .59914573 \quad 0.00053876$

$\begin{array}{llll}\# N / A & -1.3346537 & 1.77751576 & 0.10232888\end{array}$

\#N/A $\quad-1.33632512 .26962857 \quad 0.02454172$

\#N/A $\quad-1.3440047 \quad 3.23677210 .00124661$

$\begin{array}{lll}-1.3462961 & 3.28683111 & 0.00040874\end{array}$

$-1.34643376 .87471714 \quad 2.01 \mathrm{E}-28$

$\# \mathrm{~N} / \mathrm{A}$

Schizosaccharomyces specific protein

$\# \mathrm{~N} / \mathrm{A}$

$\begin{array}{lll}1.3511528 & 4.35178397 \quad 1.05 \mathrm{E}-06\end{array}$

$\begin{array}{lll}-1.3548892 & 8.22593941 & 4.44 \mathrm{E}-34\end{array}$

$-1.3554757 \quad 5.48802129 \quad 2.08 \mathrm{E}-12$

plasma membrane spermidine family transmembrane tran $\begin{array}{llll}-1.3593425 & 7.1989359 & 8.03 \mathrm{E}-37\end{array}$

$$
\text { \#N/A }
$$

$\begin{array}{lll}-1.3596979 & 1.63524073 & 0.1283419\end{array}$

carboxylic acid transmembrane transporter (predicted) $\quad \begin{array}{lllll}-1.3607225 & 3.87936184 & 0.00039393\end{array}$

$\begin{array}{lllll}\text { Schizosaccharomyces pombe specific protein } & -1.3630177 & 4.92715973 & 4.92 \mathrm{E}-09\end{array}$

\#N/A $\quad-1.3641426 \quad 2.94767 \quad 0.00302615$

$\begin{array}{lll}-1.3726036 & 2.47793221 & 0.02006689\end{array}$

$\begin{array}{lll}-1.3726036 & 2.47793221 & 0.02006689\end{array}$

$\begin{array}{lll}-1.3797999 & 8.31843869 \quad 9.49 E-57\end{array}$

$\# N / A$

$\#$ N/A

Schizosaccharomyces pombe specific protein

spindle pole body half bridge protein Sfi1

$\#$ \#N/A

$\begin{array}{lll}-1.3813065 & 1.63582942 \quad 0.14199707\end{array}$

$-1.3821899 \quad 4.02862291 \quad 7.38 \mathrm{E}-06$

$-1.3846977 \quad 4.94185951 \quad 9.92 \mathrm{E}-10$

$\begin{array}{lll}-1.3862455 & 2.88645831 & 0.00670665\end{array}$

$\begin{array}{lll}-1.387556 & 3.89946964 & 0.00011104\end{array}$

$\begin{array}{lll}-1.3880329 & 1.47715398 & 0.21172994\end{array}$

$\#$ N/A

$\begin{array}{lllll}\text { plasma membrane siderophore-iron transmembrane transF } & -1.3902608 & 6.87662942 & 3.14 \mathrm{E}-25 \\ \text { P-type ATPase, potassium exporting Cta3 } & -1.3920674 & 8.06291459 & 1.31 \mathrm{E}-39\end{array}$

P-type ATPase, potassium exporting Cta3

$-1.39462865 .02052506 \quad 3.47 \mathrm{E}-10$

$-1.39484095 .51652335$

$\# \mathrm{~N} / \mathrm{A}$

plasma membrane ferric-chelate reductase Frp1

$\# \mathrm{~N} / \mathrm{A}$

$\# N / A$

$\# N / A$

$-1395000$

$1.55 \mathrm{E}-14$

$\begin{array}{lll}-1.3952461 & 3.68610755 & 6.50 \mathrm{E}-05\end{array}$

$-1.39651997 .95882826 \quad 2.49 \mathrm{E}-41$

$\begin{array}{lll}-1.4045881 & 4.77869708 & 4.54 \mathrm{E}-09\end{array}$

$-1.4062162 \quad 4.70257811 \quad 5.35 \mathrm{E}-08$

WD repeat protein, Vid27 family, conserved in fungi and plè $-1.4130474 \quad 7.0916868 \quad 1.10 \mathrm{E}-38$

\#N/A $\quad-1.41313575 .17625741 \quad 1.13 E-08$

\#N/A $\quad-1.41454157 .42618965 \quad 2.25 \mathrm{E}-45$

\#N/A $\quad-1.4174783 .101729410 .00229487$
4.37E-13

0.00073875

0.002486831

9.59E-28

$2.59 \mathrm{E}-07$

0.034442688

$1.58 \mathrm{E}-16$

$2.76 \mathrm{E}-17$

6.01E-20

0.121153978

2.32E-58

0.001544989

0.159896222

0.046806722

0.003271562

0.001203583

$6.51 \mathrm{E}-27$

4.85E-06

2.07E-32

2.00E-11

4.17E-35

0.194038353

0.001165007

$3.14 \mathrm{E}-08$

0.007256153

0.039307772

0.039307772

1.34E-54

0.211374798

2.99E-05

6.92E-09

0.014741209

0.000363625

0.295069068

8.26E-24

8.49E-38

2.56E-09

1.77E-13

5.79E-39

0.000221428

$1.79 \mathrm{E}-39$

2.91E-08

2.95E-07

$6.50 \mathrm{E}-37$

$6.82 \mathrm{E}-08$

$1.99 \mathrm{E}-43$

0.005689106 


\begin{tabular}{|c|c|c|c|c|c|c|}
\hline SPAPB15E9.06 & $\# \mathrm{~N} / \mathrm{A}$ & $\# \mathrm{~N} / \mathrm{A}$ & -1.4222354 & 5.50007974 & $1.70 \mathrm{E}-15$ & $2.14 \mathrm{E}-14$ \\
\hline SPNCRNA.69 & $\# \mathrm{~N} / \mathrm{A}$ & \#N/A & -1.4234932 & 5.48955432 & $4.89 \mathrm{E}-15$ & $5.86 \mathrm{E}-14$ \\
\hline SPCC330.04c & mug135 & mug2/mug $135 /$ meu2 family, with repeat expansion & -1.4238217 & 5.02362847 & 5.80E-09 & $3.66 \mathrm{E}-08$ \\
\hline SPNCRNA.917 & \#N/A & \#N/A & -1.4244196 & 1.82173372 & 0.06725725 & 0.111494661 \\
\hline SPNCRNA.552 & $\# N / A$ & $\# N / A$ & -1.4250077 & 6.1470968 & 2.93E-19 & $5.20 \mathrm{E}-18$ \\
\hline SPNCRNA.660 & $\# N / A$ & $\# N / A$ & -1.4277607 & 6.26390682 & $1.27 \mathrm{E}-20$ & 2.57E-19 \\
\hline SPNCRNA.1426 & $\# N / A$ & $\# N / A$ & -1.4492634 & 3.25409306 & 0.00372289 & 0.008708928 \\
\hline SPNCRNA.1697 & $\# N / A$ & $\# N / A$ & -1.4499075 & 7.17003473 & $5.21 \mathrm{E}-25$ & $1.35 \mathrm{E}-23$ \\
\hline SPNCRNA.1249 & $\# \mathrm{~N} / \mathrm{A}$ & $\# N / A$ & -1.453365 & 1.30023338 & 0.25968949 & 0.350215412 \\
\hline SPNCRNA.89 & $\# N / A$ & $\# N / A$ & -1.4575934 & 1.30034148 & 0.24533326 & 0.334634767 \\
\hline SPAC4G9.12 & idn1 & gluconokinase & -1.4619663 & 8.07088528 & $2.12 \mathrm{E}-63$ & 3.61E-61 \\
\hline SPBC18E5.14c & SPBC18E5.14c & Schizosaccharomyces specific protein & -1.4662551 & 4.63369111 & $2.10 \mathrm{E}-08$ & $1.22 \mathrm{E}-07$ \\
\hline SPAC32A11.02c & SPAC32A11.02c & DUF4449 family conserved fungal protein & -1.4678538 & 9.37640764 & 1.77E-27 & $5.41 \mathrm{E}-26$ \\
\hline SPNCRNA.84 & $\# N / A$ & $\# N / A$ & -1.4708216 & 2.70885052 & 0.00504042 & 0.011433342 \\
\hline SPAC186.04c & SPAC186.04c & $\mathrm{N}$-terminal of transmembrane channel, truncated & -1.4765909 & 2.63587517 & 0.00422306 & 0.009764834 \\
\hline SPNCRNA.130 & \#N/A & \#N/A & -1.4793029 & 7.6180485 & 4.66E-20 & 8.96E-19 \\
\hline SPNCRNA.994 & $\# \mathrm{~N} / \mathrm{A}$ & $\# N / A$ & -1.4793029 & 7.6180485 & 4.66E-20 & $8.96 \mathrm{E}-19$ \\
\hline SPNCRNA.16 & $\# N / A$ & $\# N / A$ & -1.4794415 & 1.68417413 & 0.1035156 & 0.161344946 \\
\hline SPAC869.08 & $\mathrm{pcm} 2$ & protein-L-isoaspartate O-methyltransferase Pcm 2 (predicte & -1.4794546 & 4.8851858 & 2.95E-10 & 2.20E-09 \\
\hline SPAC869.05c & SPAC869.05c & plasma membrane sulfate transmembrane transporter (pr€ & -1.4803845 & 6.75857348 & $1.21 \mathrm{E}-26$ & $3.44 \mathrm{E}-25$ \\
\hline ScpofMp03 & $\# N / A$ & $\# N / A$ & -1.4811106 & 7.33458541 & 3.74E-18 & 5.99E-17 \\
\hline SPNCRNA.45 & $\# \mathrm{~N} / \mathrm{A}$ & $\# N / A$ & -1.4839118 & 2.4511474 & 0.00610623 & 0.013588269 \\
\hline SPAPB1A10.02 & $\mathrm{scm} 3$ & CENP-A histone chaperone $\mathrm{Scm} 3$ & -1.4864382 & 5.22761205 & 4.23E-11 & $3.48 \mathrm{E}-10$ \\
\hline SPNCRNA.1616 & $\# \mathrm{~N} / \mathrm{A}$ & $\# N / A$ & -1.4873809 & 5.6889849 & $1.20 \mathrm{E}-13$ & $1.28 \mathrm{E}-12$ \\
\hline SPNCRNA.940 & $\# N / A$ & $\# N / A$ & -1.4916759 & 2.75603955 & 0.05331611 & 0.091550879 \\
\hline SPNCRNA.106 & $\# \mathrm{~N} / \mathrm{A}$ & $\# N / A$ & -1.4938897 & 3.15316806 & 0.00036176 & 0.001077364 \\
\hline SPNCRNA.1210 & $\# N / A$ & $\# N / A$ & -1.4983475 & 5.80679003 & 3.44E-16 & 4.59E-15 \\
\hline SPBC215.13 & $\mathrm{mtl} 3$ & plasma membrane-associated serine-rich cell wall sensor $\mathrm{N}$ & -1.5019321 & 5.75388947 & $9.60 \mathrm{E}-13$ & $9.52 \mathrm{E}-12$ \\
\hline SPNCRNA.10 & $\# \mathrm{~N} / \mathrm{A}$ & \#N/A & -1.5039014 & 4.32011244 & $1.60 \mathrm{E}-07$ & $8.22 \mathrm{E}-07$ \\
\hline SPBPB2B2.11 & $\operatorname{tgd} 1$ & nucleotide-sugar 4,6-dehydratase (predicted) & -1.5056934 & 9.16102418 & 8.84E-65 & $1.54 \mathrm{E}-62$ \\
\hline SPNCRNA.139 & $\# N / A$ & \#N/A & -1.5064594 & 2.96686824 & 0.00232737 & 0.00575917 \\
\hline SPBC15D4.11c & SPBC15D4.11c & mitochondrial Mam33 family protein (predicted) & -1.5098514 & 4.03876458 & 5.15E-05 & 0.000178571 \\
\hline SPAC9E9.17c & $\# N / A$ & \#N/A & -1.5098899 & 1.86457817 & 0.05668674 & 0.096461422 \\
\hline SPNCRNA.1505 & $\# N / A$ & \#N/A & -1.5152413 & 6.16651206 & 3.95E-21 & $8.61 \mathrm{E}-20$ \\
\hline SPBC646.17c & \multicolumn{2}{|c|}{35764 meiotic dynein intermediate chain Dic1 } & -1.5167956 & 4.9638101 & $4.93 \mathrm{E}-12$ & 4.50E-11 \\
\hline SPBC24C6.09c & SPBC24C6.09c & phosphoketolase family protein (predicted) & -1.5170157 & 6.15988058 & $2.28 \mathrm{E}-21$ & $5.01 \mathrm{E}-20$ \\
\hline SPNCRNA.1123 & $\# N / A$ & \#N/A & -1.5192369 & 2.13599294 & 0.01648698 & 0.033007917 \\
\hline SPNCRNA.194 & $\# \mathrm{~N} / \mathrm{A}$ & $\# \mathrm{~N} / \mathrm{A}$ & -1.5287977 & 2.26974833 & 0.02737597 & 0.051504977 \\
\hline SPNCRNA.469 & $\# N / A$ & $\# N / A$ & -1.5338741 & 2.58487592 & 0.00781945 & 0.016876411 \\
\hline SPBPB2B2.12C & gal10 & UDP-glucose 4-epimerase/aldose 1-epimerase Gal10 & -1.533993 & 9.20849537 & 3.45E-65 & $6.18 \mathrm{E}-63$ \\
\hline SPNCRNA.604 & $\# \mathrm{~N} / \mathrm{A}$ & $\# N / A$ & -1.5373911 & 2.98646013 & 0.00201815 & 0.00505652 \\
\hline SPNCRNA.1162 & $\# N / A$ & $\# N / A$ & -1.5467683 & 5.0047699 & 1.23E-11 & $1.08 \mathrm{E}-10$ \\
\hline SPCC1906.03 & wtf19 & wtf element Wtf19 & -1.549513 & 6.61501806 & $1.06 \mathrm{E}-26$ & $3.08 \mathrm{E}-25$ \\
\hline SPNCRNA.605 & $\# \mathrm{~N} / \mathrm{A}$ & $\# \mathrm{~N} / \mathrm{A}$ & -1.5527996 & 6.95174296 & $3.22 \mathrm{E}-42$ & $2.52 \mathrm{E}-40$ \\
\hline SPNCRNA.1141 & \#N/A & $\# N / A$ & -1.5575361 & 1.09840313 & 0.30892589 & 0.402342708 \\
\hline SPNCRNA.168 & $\# \mathrm{~N} / \mathrm{A}$ & $\# \mathrm{~N} / \mathrm{A}$ & -1.5616586 & 3.06503347 & 0.00043354 & 0.001271097 \\
\hline SPNCRNA.720 & $\# N / A$ & $\# N / A$ & -1.5616586 & 3.06503347 & 0.00043354 & 0.001271097 \\
\hline
\end{tabular}




\begin{tabular}{|c|c|c|c|c|c|c|}
\hline SPCC1739.07 & cti1 & exosome C1D family subunit Cti1 & -1.5652155 & 7.25691545 & $1.92 \mathrm{E}-29$ & $6.64 \mathrm{E}-28$ \\
\hline SPCC663.06c & osr1 & short chain dehydrogenase, unknown specificity (predicted) & -1.5675085 & 5.69711438 & $1.44 \mathrm{E}-19$ & $2.66 \mathrm{E}-18$ \\
\hline SPNCRNA.1431 & \#N/A & $\# N / A$ & -1.5706938 & 4.38868808 & $5.18 \mathrm{E}-06$ & 2.16E-05 \\
\hline SPNCRNA.1381 & \#N/A & \#N/A & -1.5711456 & 5.97803536 & $8.41 \mathrm{E}-25$ & 2.17E-23 \\
\hline SPNCRNA.1486 & \#N/A & \#N/A & -1.5751442 & 3.53573274 & $2.20 \mathrm{E}-05$ & 8.17E-05 \\
\hline SPAC1F8.05 & isp3 & spore wall structural constituent Isp3 & -1.5774926 & 5.7019766 & $6.55 \mathrm{E}-18$ & $1.02 \mathrm{E}-16$ \\
\hline SPNCRNA.834 & \#N/A & \#N/A & -1.5802014 & 5.32427304 & $3.06 \mathrm{E}-12$ & 2.87E-11 \\
\hline SPNCRNA.974 & \#N/A & \#N/A & -1.5814186 & 4.47089189 & $1.02 \mathrm{E}-07$ & $5.38 \mathrm{E}-07$ \\
\hline SPBPB2B2.13 & gal1 & galactokinase Gal1 & -1.5895889 & 7.6150636 & $2.80 \mathrm{E}-33$ & $1.26 \mathrm{E}-31$ \\
\hline SPNCRNA.388 & \#N/A & $\# N / A$ & -1.5947889 & 3.54899043 & $1.57 \mathrm{E}-05$ & $6.01 \mathrm{E}-05$ \\
\hline SPNCRNA.398 & $\# \mathrm{~N} / \mathrm{A}$ & $\# \mathrm{~N} / \mathrm{A}$ & -1.6015032 & 2.86646625 & 0.00129454 & 0.003388206 \\
\hline SPCC162.04c & wtf13 & wtf element Wtf13 & -1.6039875 & 6.45119425 & $8.69 \mathrm{E}-30$ & $3.11 \mathrm{E}-28$ \\
\hline SPNCRNA.993 & \#N/A & $\# N / A$ & -1.6129148 & 3.10044352 & 0.00016855 & 0.000533713 \\
\hline SPNCRNA.28 & \#N/A & \#N/A & -1.6139157 & 5.68523876 & $5.16 \mathrm{E}-21$ & $1.12 \mathrm{E}-19$ \\
\hline SPNCRNA.1034 & $\# N / A$ & $\# \mathrm{~N} / \mathrm{A}$ & -1.6171634 & 2.88715427 & 0.00063683 & 0.001796696 \\
\hline SPNCRNA.627 & \#N/A & \#N/A & -1.6278569 & 4.58866101 & 1.97E-09 & $1.31 \mathrm{E}-08$ \\
\hline SPAC11H11.04 & mam2 & pheromone $\mathrm{p}$-factor receptor & -1.6278618 & 7.87070468 & $2.15 \mathrm{E}-48$ & $2.15 \mathrm{E}-46$ \\
\hline SPNCRNA.1203 & $\# N / A$ & \#N/A & -1.6351576 & 7.39829839 & $3.81 \mathrm{E}-58$ & $5.63 \mathrm{E}-56$ \\
\hline SPNCRNA.1348 & $\# \mathrm{~N} / \mathrm{A}$ & \#N/A & -1.6385087 & 3.88823342 & 8.77E-07 & 4.08E-06 \\
\hline SPNCRNA.1364 & \#N/A & \#N/A & -1.6468066 & 5.57717415 & $1.31 \mathrm{E}-18$ & $2.19 \mathrm{E}-17$ \\
\hline SPAC1F8.04c & SPAC1F8.04c & hydrolase, implicated in cellular detoxification (predicted) & -1.6491118 & 6.78821591 & $1.78 \mathrm{E}-36$ & $9.03 E-35$ \\
\hline SPNCRNA.943 & $\# N / A$ & $\# N / A$ & -1.6560152 & 2.9067703 & 0.00093716 & 0.002535725 \\
\hline SPCC1442.11c & SPCC1442.11c & Schizosaccharomyces pombe specific protein & -1.6603462 & 2.3327587 & 0.00528224 & 0.011918316 \\
\hline SPAC16E8.18c & $\operatorname{tam} 5$ & Schizosaccharomyces specific protein Tam5 & -1.6669923 & 4.48702594 & 1.59E-07 & 8.18E-07 \\
\hline SPAC4D7.15 & new12 & Schizosaccharomyces specific protein New12 & -1.6822031 & 4.06724943 & 1.17E-08 & $7.08 \mathrm{E}-08$ \\
\hline SPAC4F10.17 & SPAC4F10.17 & conserved fungal protein & -1.6830531 & 5.81038028 & $1.47 \mathrm{E}-19$ & $2.70 \mathrm{E}-18$ \\
\hline SPNCRNA.237 & $\# N / A$ & \#N/A & -1.6872274 & 2.84370745 & 0.00087618 & 0.002384963 \\
\hline ScpofMp02 & $\# N / A$ & $\# N / A$ & -1.708158 & 7.52410939 & $1.52 \mathrm{E}-17$ & $2.28 \mathrm{E}-16$ \\
\hline SPNCRNA.879 & $\# N / A$ & \#N/A & -1.7113228 & 2.36324944 & 0.00255484 & 0.006240661 \\
\hline SPAC29A4.12c & mug108 & Schizosaccharomyces specific protein Mug108 & -1.7154383 & 7.65714274 & $5.12 \mathrm{E}-61$ & 8.30E-59 \\
\hline SPNCRNA.956 & $\# N / A$ & $\# N / A$ & -1.7192209 & 3.30261142 & $2.92 \mathrm{E}-05$ & 0.000106085 \\
\hline SPCC548.03c & wtf4 & wtf element Wtf4 & -1.7211331 & 6.2266616 & $8.47 E-33$ & $3.70 \mathrm{E}-31$ \\
\hline SPNCRNA.184 & \#N/A & $\# N / A$ & -1.7212457 & 2.23805236 & 0.0059174 & 0.013237314 \\
\hline SPBC947.05c & frp2 & ferric-chelate reductase Frp2 (predicted) & -1.7346418 & 5.36898342 & $1.76 \mathrm{E}-16$ & $2.40 \mathrm{E}-15$ \\
\hline SPNCRNA.103 & sme2 & meiRNA sme2 & -1.7429084 & 4.96889406 & $2.13 E-08$ & $1.24 \mathrm{E}-07$ \\
\hline SPNCRNA.869 & $\# N / A$ & $\# N / A$ & -1.7457156 & 1.4204924 & 0.12130812 & 0.185090079 \\
\hline ScpofMt37 & $\# N / A$ & \#N/A & -1.7503063 & 2.80078926 & 0.00020182 & 0.000632605 \\
\hline SPNCRNA.1602 & $\# N / A$ & $\# \mathrm{~N} / \mathrm{A}$ & -1.7507136 & 4.46749534 & 1.90E-09 & $1.27 \mathrm{E}-08$ \\
\hline SPNCRNA.156 & \#N/A & \#N/A & -1.7594412 & 1.63474265 & 0.06308699 & 0.105714595 \\
\hline SPNCRNA.628 & $\# N / A$ & $\# N / A$ & -1.7603578 & 3.92957328 & 4.26E-08 & 2.40E-07 \\
\hline SPBPB8B6.02c & SPBPB8B6.02c & plasma membrane urea transmembrane transporter (predi & -1.761606 & 4.22577919 & $3.35 \mathrm{E}-08$ & $1.92 \mathrm{E}-07$ \\
\hline SPNCRNA.1472 & $\# N / A$ & \#N/A & -1.762728 & 4.04705936 & $9.98 \mathrm{E}-06$ & 3.95E-05 \\
\hline SPNCRNA.197 & \#N/A & $\# N / A$ & -1.7676649 & 5.26704686 & $2.13 \mathrm{E}-18$ & $3.45 \mathrm{E}-17$ \\
\hline SPNCRNA.327 & $\# \mathrm{~N} / \mathrm{A}$ & \#N/A & -1.770444 & 1.16895367 & 0.19988982 & 0.2805218 \\
\hline SPNCRNA.296 & $\# N / A$ & $\# \mathrm{~N} / \mathrm{A}$ & -1.7756945 & 1.16892511 & 0.20204549 & 0.282963486 \\
\hline SPNCRNA.466 & \#N/A & \#N/A & -1.7763263 & 3.39532964 & 0.000349 & 0.00104438 \\
\hline SPNCRNA.606 & \#N/A & \#N/A & -1.7773344 & 5.10257483 & $7.53 \mathrm{E}-15$ & 8.89E-14 \\
\hline
\end{tabular}




\begin{tabular}{|c|c|}
\hline SPNCRNA.512 & $\# \mathrm{~N} / \mathrm{A}$ \\
\hline SPNCRNA.276 & $\# \mathrm{~N} / \mathrm{A}$ \\
\hline SPNCRNA.338 & $\# N / A$ \\
\hline SPBC83.19c & SPBC83.19c \\
\hline SPNCRNA.1219 & $\# N / A$ \\
\hline SPCC1753.03c & rec7 \\
\hline SPAC17A2.11 & SPAC17A2.11 \\
\hline SPBC1718.02 & hop1 \\
\hline SPNCRNA.831 & $\# N / A$ \\
\hline SPNCRNA.244 & $\# \mathrm{~N} / \mathrm{A}$ \\
\hline SPNCRNA.587 & $\# N / A$ \\
\hline SPAC19A8.16 & prl65 \\
\hline SPAC186.05c & gdt1 \\
\hline SPCC1620.02 & wtf23 \\
\hline SPNCRNA.1055 & $\# N / A$ \\
\hline SPNCRNA.144 & $\# N / A$ \\
\hline SPNCRNA.534 & $\# N / A$ \\
\hline SPNCRNA.1128 & $\# N / A$ \\
\hline SPNCRNA.1172 & $\# N / A$ \\
\hline SPBC16E9.16c & Isd90 \\
\hline SPNCRNA.145 & $\# N / A$ \\
\hline SPNCRNA.1216 & $\# N / A$ \\
\hline SPAC5H10.02c & hsp3102 \\
\hline SPCC1235.18 & $\# N / A$ \\
\hline SPAC1D4.07c & $\# N / A$ \\
\hline SPNCRNA.1109 & $\# N / A$ \\
\hline SPAC869.07c & mel1 \\
\hline SPAC1687.23c & SPAC1687.23c \\
\hline SPNCRNA.505 & \#N/A \\
\hline SPNCRNA.1617 & $\# N / A$ \\
\hline SPNCRNA.613 & $\# N / A$ \\
\hline SPCC338.18 & SPCC338.18 \\
\hline SPNCRNA.1048 & $\# N / A$ \\
\hline SPNCRNA.802 & $\# \mathrm{~N} / \mathrm{A}$ \\
\hline SPAC4F8.08 & mug114 \\
\hline SPNCRNA.187 & $\# \mathrm{~N} / \mathrm{A}$ \\
\hline SPBPB2B2.10c & gal7 \\
\hline SPNCRNA.1087 & $\# N / A$ \\
\hline SPAC13F5.07c & hpz2 \\
\hline SPNCRNA.311 & $\# N / A$ \\
\hline SPBC359.04c & $\mathrm{pfl} 7$ \\
\hline SPNCRNA.876 & $\# \mathrm{~N} / \mathrm{A}$ \\
\hline SPAC3G6.07 & SPAC3G6.07 \\
\hline SPNCRNA.1186 & $\# N / A$ \\
\hline SPAC1F7.06 & hsp3105 \\
\hline SPNCRNA.1420 & $\# N / A$ \\
\hline SPRRNA.13 & SPRRNA.13 \\
\hline
\end{tabular}

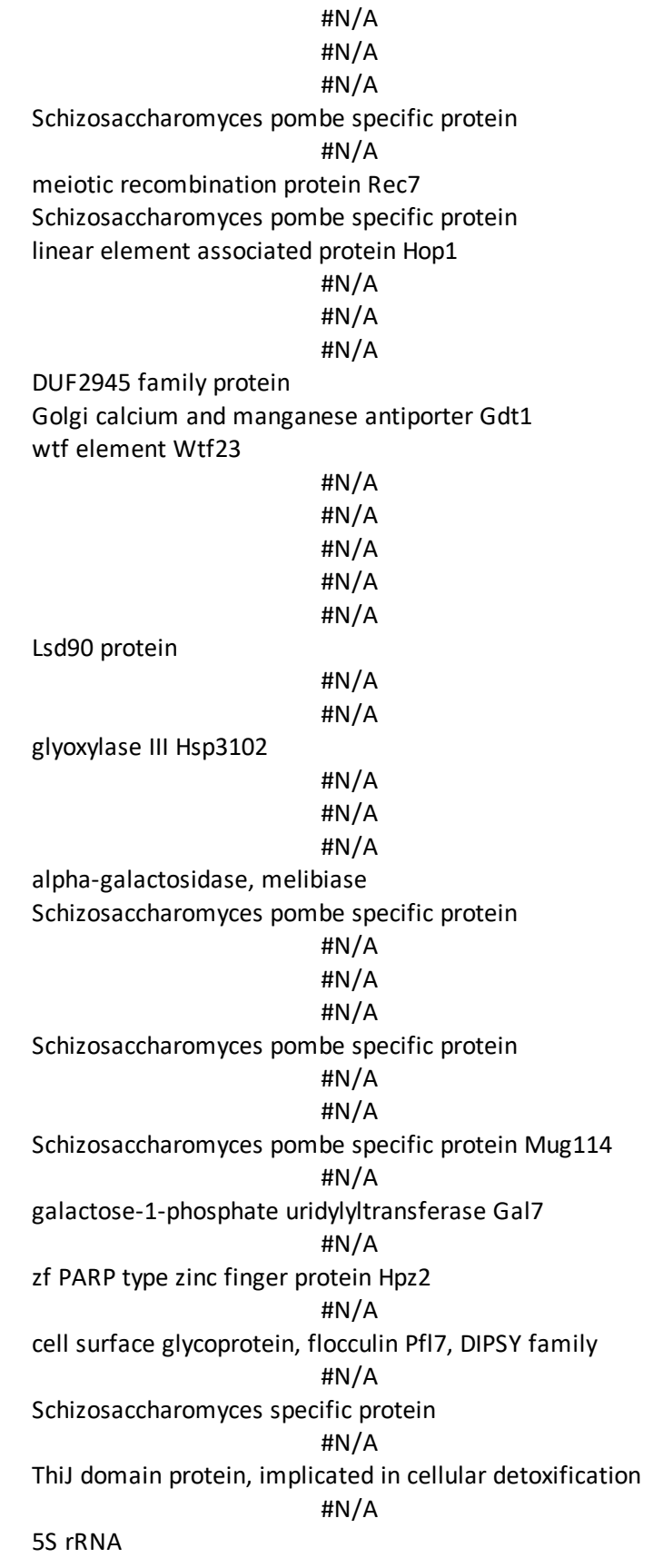

$\begin{array}{lll}-1.7805609 & 2.30048012 & 0.02269204\end{array}$ $\begin{array}{lll}-1.7858917 & 4.13944002 & 2.54 \mathrm{E}-09\end{array}$

$\begin{array}{lll}-1.7865237 & 1.16947709 & 0.19642247\end{array}$ $\begin{array}{llll}-1.7902823 & 3.40910839 & 0.00033353\end{array}$ $1.7956223 \quad 4.82890896 \quad 130 \mathrm{E}-05$

$\begin{array}{lll}-1.7986041 & 6.27706701 & 2.18 \mathrm{E}-34\end{array}$

$\begin{array}{lll}-1.8012203 & 3.22259742 & 1.10 \mathrm{E}-05\end{array}$

$-1.8014573 \quad 4.50279756 \quad 5.32 \mathrm{E}-10$

$\begin{array}{lll}-1.8041881 & 5.29366975 & 2.50 \mathrm{E}-19\end{array}$

$\begin{array}{lll}-1.8065315 & 4.99721793 & 1.38 \mathrm{E}-12\end{array}$

$\begin{array}{lll}-1.8148741 & 3.79086012 & 2.08 \mathrm{E}-07\end{array}$

$\begin{array}{lll}-1.8218593 & 5.36784443 & 5.38 \mathrm{E}-17\end{array}$

$\begin{array}{lll}-1.8229126 & 2.02557386 & 0.01246004\end{array}$

$\begin{array}{lll}-1.82567 & 6.50620158 & 2.73 \mathrm{E}-36\end{array}$

$\begin{array}{lll}-1.8339078 & 3.30452094 & 3.61 \mathrm{E}-05\end{array}$

$\begin{array}{lll}-1.8381489 & 4.18163462 & 1.49 \mathrm{E}-08\end{array}$

$\begin{array}{lll}-1.8462718 & 2.02631924 & 0.04372945\end{array}$

$\begin{array}{lll}-1.8468048 & 2.94873981 & 4.41 \mathrm{E}-05\end{array}$

$\begin{array}{lll}-1.8479004 & 2.02647935 & 0.0153664\end{array}$

$\begin{array}{lll}-1.8493591 & 9.24472421 & 5.47 \mathrm{E}-38\end{array}$

$\begin{array}{lll}-1.8532234 & 1.6834764 & 0.04683408\end{array}$

$-1.85468194 .70330068 \quad 1.60 \mathrm{E}-12$

$\begin{array}{lll}-1.8581564 & 5.22587692 & 2.24 \mathrm{E}-14\end{array}$

$\begin{array}{lll}-1.8655773 & 5.16876651 & 2.98 \mathrm{E}-16\end{array}$

$\begin{array}{lll}-1.8659479 & 1.68441151 & 0.03016218\end{array}$

$\begin{array}{lll}-1.8670272 & 4.36823727 & 7.46 \mathrm{E}-10\end{array}$

$\begin{array}{lll}-1.871309 & 6.17547081 & 6.05 \mathrm{E}-32\end{array}$

$\begin{array}{lll}-1.8791095 & 1.68438625 & 0.02972643\end{array}$

$\begin{array}{llll}-1.8839831 & 1.68527452 & 0.03344893\end{array}$

$\begin{array}{lll}-1.8938701 & 3.89950302 & 9.50 \mathrm{E}-08\end{array}$

$-1.9032898 \quad 4.85074344 \quad 3.23 \mathrm{E}-10$

$-1.92045245 .30963844 \quad 1.38 \mathrm{E}-18$

$\begin{array}{lll}-1.9328587 & 4.38238437 & 5.62 \mathrm{E}-09\end{array}$

$\begin{array}{lll}-1.9563664 & 5.83643154 & 1.50 \mathrm{E}-27\end{array}$

$\begin{array}{lll}-1.959771 & 7.15979342 & 8.23 \mathrm{E}-68\end{array}$

$\begin{array}{lll}-1.9653505 & 1.23639932 & 0.12346449\end{array}$

$\begin{array}{lll}-1.967032 & 6.54168563 & 7.98 \mathrm{E}-28\end{array}$

$-1.9780214 \quad 7.34017832 \quad 5.96 \mathrm{E}-61$

$\begin{array}{lll}-1.9827018 & 5.85620014 & 8.14 \mathrm{E}-30\end{array}$

$-1.99805823 .73403222 \quad 1.43 \mathrm{E}-05$

$-2.00219315 .76685396 \quad 1.70 \mathrm{E}-22$

$\begin{array}{llll}-2.0037432 & 2.53394138 & 0.00034484\end{array}$

$\begin{array}{llll}-2.0100264 & 2.42159406 & 0.00038782\end{array}$

$\begin{array}{llll}-2.012092 & 2.42217083 & 0.00037632\end{array}$

$\begin{array}{lll}-2.0186567 & 4.98986364 & 2.20 \mathrm{E}-18\end{array}$

\begin{tabular}{lll|l}
-2.0190656 & 3.9990701 & $7.64 \mathrm{E}-08$
\end{tabular}

$\begin{array}{llll}-2.0429616 & 1.77707117 & 0.02469888\end{array}$
0.043732464

$1.67 \mathrm{E}-08$

0.276912142

0.00100295

5.05E-05

$1.03 \mathrm{E}-32$

4.31E-05

3.83E-09

4.47E-18

$1.35 \mathrm{E}-11$

1.05E-06

7.70E-16

0.025670765

$1.38 \mathrm{E}-34$

0.00012876

$8.88 \mathrm{E}-08$

0.077313298

0.000154502

0.030983214

$3.08 \mathrm{E}-36$

0.08184538

$1.55 \mathrm{E}-11$

$2.53 \mathrm{E}-13$

$4.01 \mathrm{E}-15$

0.05598845

5.26E-09

$2.44 \mathrm{E}-30$

0.055285154

0.061320036

5.04E-07

2.39E-09

2.29E-17

3.56E-08

4.65E-26

1.60E-65

0.187832744

$2.48 \mathrm{E}-26$

$9.44 \mathrm{E}-59$

2.93E-28

5.49E-05

3.92E-21

0.001032948

0.001148447

0.001117309

3.55E-17

4.12E-07

0.047053711 


\begin{tabular}{|c|c|c|c|c|c|c|}
\hline SPCC285.07c & wtf18 & wtf element Wtf18 & -2.0510156 & 5.22461476 & $3.89 E-19$ & $6.81 \mathrm{E}-18$ \\
\hline SPBC1348.12 & SPBC1348.12 & transcription factor (predicted) & -2.072421 & 5.42915269 & $1.82 \mathrm{E}-28$ & $5.95 \mathrm{E}-27$ \\
\hline SPBC32H8.02c & nep2 & NEDD8 protease Nep2 & -2.0787179 & 8.07466661 & $1.68 \mathrm{E}-102$ & $6.34 \mathrm{E}-100$ \\
\hline SPNCRNA.1361 & $\# N / A$ & $\# \mathrm{~N} / \mathrm{A}$ & -2.1000673 & 3.11907034 & 0.0001068 & 0.000350413 \\
\hline SPNCRNA.282 & $\# N / A$ & $\# N / A$ & -2.1031801 & 3.49511407 & 2.12E-08 & $1.24 \mathrm{E}-07$ \\
\hline SPCPB16A4.06c & SPCPB16A4.06c & Schizosaccharomyces specific protein & -2.1147671 & 7.02322347 & $2.02 \mathrm{E}-68$ & 4.29E-66 \\
\hline SPNCRNA.1370 & $\# N / A$ & $\# N / A$ & -2.1358984 & 2.02475293 & 0.00190792 & 0.004808666 \\
\hline SPNCRNA.54 & \#N/A & \#N/A & -2.1462355 & 2.96845876 & $9.38 \mathrm{E}-06$ & 3.73E-05 \\
\hline SPBC1711.01c & mat3-Mi & mating type M-specific polypeptide Mi at silenced MAT3 lo & -2.1493492 & 1.8222487 & 0.00769883 & 0.016647768 \\
\hline SPBC23G7.17c & mat1-Mi & M-specific trancription factor $\mathrm{Mi}$ & -2.1493492 & 1.8222487 & 0.00769883 & 0.016647768 \\
\hline SPNCRNA.12 & $\# N / A$ & \#N/A & -2.1642238 & 2.20488765 & 0.0006634 & 0.001867773 \\
\hline SPCC417.16 & SPCC417.16 & mitochondrial protein (predicted) & -2.1654488 & 5.81478008 & $5.13 \mathrm{E}-26$ & $1.41 \mathrm{E}-24$ \\
\hline SPNCRNA.486 & $\# \mathrm{~N} / \mathrm{A}$ & $\# N / A$ & -2.1694585 & 2.9868634 & 4.75E-05 & 0.000166013 \\
\hline SPBC1348.07 & SPBC1348.07 & S. pombe specific DUF999 protein family 6 & -2.1804778 & 3.005602 & 0.00014717 & 0.000472185 \\
\hline SPNCRNA.973 & $\# \mathrm{~N} / \mathrm{A}$ & \#N/A & -2.1834524 & 4.78537338 & $1.56 \mathrm{E}-18$ & $2.57 \mathrm{E}-17$ \\
\hline SPNCRNA.649 & $\# \mathrm{~N} / \mathrm{A}$ & $\# N / A$ & -2.1837228 & 2.66020679 & $5.14 \mathrm{E}-05$ & 0.000178523 \\
\hline SPNCRNA.1319 & $\# \mathrm{~N} / \mathrm{A}$ & $\# \mathrm{~N} / \mathrm{A}$ & -2.1847152 & 6.87892009 & $1.01 \mathrm{E}-54$ & $1.32 \mathrm{E}-52$ \\
\hline SPBC336.16 & $\# N / A$ & $\# N / A$ & -2.1969464 & 2.77932036 & $2.53 \mathrm{E}-05$ & $9.32 \mathrm{E}-05$ \\
\hline SPBPB21E7.10 & SPBPB21E7.10 & Schizosaccharomyces specific protein & -2.2054879 & 6.40834579 & $1.82 \mathrm{E}-53$ & 2.21E-51 \\
\hline SPAC17A2.10c & SPAC17A2.10c & Schizosaccharomyces pombe specific protein & -2.212797 & 4.13049717 & $3.51 \mathrm{E}-11$ & $2.91 \mathrm{E}-10$ \\
\hline SPNCRNA.132 & $\# N / A$ & \#N/A & -2.217454 & 5.79856361 & $2.95 \mathrm{E}-35$ & $1.43 \mathrm{E}-33$ \\
\hline SPNCRNA.1619 & $\# \mathrm{~N} / \mathrm{A}$ & $\# \mathrm{~N} / \mathrm{A}$ & -2.2377605 & 3.0266174 & 8.25E-06 & 3.32E-05 \\
\hline SPNCRNA.02 & $\# N / A$ & $\# N / A$ & -2.2407526 & 2.4214671 & 0.00016307 & 0.00051902 \\
\hline SPNCRNA.147 & $\# \mathrm{~N} / \mathrm{A}$ & $\# \mathrm{~N} / \mathrm{A}$ & -2.248285 & 1.63590483 & 0.01253125 & 0.02580967 \\
\hline SPNCRNA.1154 & $\# N / A$ & $\# N / A$ & -2.2554662 & 1.36148488 & 0.05321437 & 0.091399238 \\
\hline SPNCRNA.323 & $\# N / A$ & $\# \mathrm{~N} / \mathrm{A}$ & -2.2567185 & 2.27018162 & 0.00060571 & 0.001715293 \\
\hline SPNCRNA.1262 & $\# N / A$ & $\# N / A$ & -2.2587971 & 2.58521924 & 0.0004043 & 0.001191547 \\
\hline SPNCRNA.137 & $\# \mathrm{~N} / \mathrm{A}$ & $\# \mathrm{~N} / \mathrm{A}$ & -2.2698174 & 1.36175451 & 0.04744076 & 0.082693238 \\
\hline SPNCRNA.1324 & \#N/A & \#N/A & -2.2703235 & 9.15478903 & $1.01 \mathrm{E}-131$ & $5.70 \mathrm{E}-129$ \\
\hline SPNCRNA.208 & $\# N / A$ & $\# \mathrm{~N} / \mathrm{A}$ & -2.2707109 & 1.36208952 & 0.04779061 & 0.08323909 \\
\hline SPNCRNA.126 & $\# \mathrm{~N} / \mathrm{A}$ & $\# N / A$ & -2.273448 & 2.94894411 & 1.14E-06 & 5.22E-06 \\
\hline SPNCRNA.1621 & $\# \mathrm{~N} / \mathrm{A}$ & \#N/A & -2.291966 & 3.94275139 & 4.33E-10 & $3.16 \mathrm{E}-09$ \\
\hline SPCC569.09 & SPCC569.09 & Schizosaccharomyces specific protein & -2.293865 & 7.33340391 & $1.28 \mathrm{E}-80$ & $3.48 \mathrm{E}-78$ \\
\hline SPNCRNA.1631 & $\# N / A$ & $\# N / A$ & -2.2958153 & 3.57550825 & $8.80 \mathrm{E}-08$ & 4.69E-07 \\
\hline SPNCRNA.279 & \#N/A & \#N/A & -2.3022703 & 4.32322578 & $4.11 \mathrm{E}-13$ & $4.22 \mathrm{E}-12$ \\
\hline SPAPB8E5.05 & $\mathrm{mfm} 1$ & $\mathrm{M}$-factor precursor $\mathrm{Mfm} 1$ & -2.3035009 & 4.95817352 & $2.03 E-17$ & $3.02 \mathrm{E}-16$ \\
\hline SPNCRNA.1165 & $\# N / A$ & \#N/A & -2.3112448 & 3.17193764 & $1.31 \mathrm{E}-07$ & $6.79 \mathrm{E}-07$ \\
\hline SPNCRNA.428 & $\# \mathrm{~N} / \mathrm{A}$ & $\# \mathrm{~N} / \mathrm{A}$ & -2.3128361 & 3.58840792 & $5.54 \mathrm{E}-08$ & $3.05 \mathrm{E}-07$ \\
\hline SPNCRNA.1159 & $\# N / A$ & $\# N / A$ & -2.3206707 & 4.20633517 & $4.90 \mathrm{E}-08$ & 2.73E-07 \\
\hline SPNCRNA.127 & $\# N / A$ & $\# N / A$ & -2.3228308 & 3.27201299 & $2.33 \mathrm{E}-08$ & $1.35 \mathrm{E}-07$ \\
\hline SPNCRNA.181 & $\# N / A$ & $\# N / A$ & -2.3265774 & 2.75529437 & $9.05 \mathrm{E}-06$ & 3.61E-05 \\
\hline SPNCRNA.424 & $\# N / A$ & $\# N / A$ & -2.3484888 & 2.33175054 & 0.00018291 & 0.000576507 \\
\hline SPBPJ4664.03 & $\mathrm{mfm} 3$ & $\mathrm{M}$-factor precursor $\mathrm{Mfm} 3$ & -2.3931637 & 5.43879018 & $3.18 \mathrm{E}-34$ & $1.49 \mathrm{E}-32$ \\
\hline SPAC5D6.10c & mug116 & Schizosaccharomyces pombe specific protein Mug116 & -2.4318083 & 2.68565601 & 3.89E-05 & 0.000137765 \\
\hline SPNCRNA.1039 & $\# \mathrm{~N} / \mathrm{A}$ & \#N/A & -2.4364456 & 1.98689571 & 0.00544626 & 0.012255876 \\
\hline SPNCRNA.989 & $\# N / A$ & $\# \mathrm{~N} / \mathrm{A}$ & -2.4393197 & 5.04579181 & $1.31 \mathrm{E}-25$ & $3.46 \mathrm{E}-24$ \\
\hline SPAPB1A10.14 & pof15 & F-box protein (predicted) & -2.4519513 & 6.66036733 & $3.06 \mathrm{E}-67$ & $5.79 E-65$ \\
\hline
\end{tabular}


SPCC1183.12

SPNCRNA.210

SPBC1198.14C

SPNCRNA.1024

SPBC1683.08

SPAC977.06

SPNCRNA.431

SPAPB1A11.01

SPNCRNA.36

SPNCRNA.480

SPNCRNA.326

SPBPB21E7.11

SPNCRNA.1560

SPNCRNA.868

SPNCRNA.439

SPNCRNA.423

ScpofMp06

SPBPB21E7.05

SPNCRNA.1372

SPNCRNA.316

SPBC887.16

SPNCRNA.1311

SPNCRNA.293

SPBP4H10.09

SPNCRNA.1295

SPNCRNA.29

SPNCRNA.182

SPAC13F5.03C

SPNCRNA. 312

SPNCRNA.23

SPCC1235.17

SPCC548.07c

SPNCRNA.1549

SPNCRNA.394

SPCC1739.08c

ScpofMp07

SPCC794.04c

SPNCRNA.21

SPNCRNA.154

SPAC6B12.03C

SPNCRNA.298

SPNCRNA.102

SPAC2F3.07C
$\# \mathrm{~N} / \mathrm{A}$

SPBPB2B2.06C

SPAC23C11.07

rbd3

spo13

\#N/A

fbp1

$\# \mathrm{~N} / \mathrm{A}$

ght4

SPAC977.06

\#N/A

mfc1

$\# N / A$

$\# \mathrm{~N} / \mathrm{A}$

$\# \mathrm{~N} / \mathrm{A}$

SPBPB21E7.11

$\#$ N/A

$\# N / A$

$\# N / A$

$\# N / A$

$\# N / A$

SPBPB21E7.05

$\# N / A$

$\# N / A$

$\# N / A$

$\# N / A$

rsv1

$\# N / A$

$\# N / A$

$\# N / A$

gld1

\#N/A

$\# N / A$

$\# \mathrm{~N} / \mathrm{A}$

ght1

$\# N / A$

$\# \mathrm{~N} / \mathrm{A}$

SPCC1739.08C

$\#$ N/A

SPCC794.04c

$\# \mathrm{~N} / \mathrm{A}$

$\# N / A$

bit2

\#N/A

$\# \mathrm{~N} / \mathrm{A}$

SPAC2F3.07C
\#N/A

$-2.4583805 \quad 5.62911179 \quad 5.75 \mathrm{E}-32$

$\begin{array}{lrrr}\text { extracellular 5'-nucleotidase, human NT5E family (predicte } & -2.4870635 & 5.6751241 & 1.28 \mathrm{E}-29 \\ \text { Schizosaccharomyces pombe specific protein } & -2.5035856 & 2.02506387 & 0.00102401\end{array}$

$\begin{array}{lllll}\text { mitochondrial rhomboid family protease } & -2.5202919 & 6.13193081 & 9.87 \mathrm{E}-48\end{array}$

sporulation specific guanyl-nucleotide exchange factor Spo $1-2.5267888 \quad 2.75614885 \quad 2.25 \mathrm{E}-06$

\#N/A $\quad-2.56988572 .063345640 .0004491$

$-2.5794797 \quad 9.03693401 \quad 8.99 \mathrm{E}-144$

$-2.5842002 \quad 3.35162574$

$\# \mathrm{~N} / \mathrm{A}$

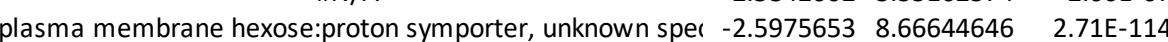

$\begin{array}{llll}\text { S. pombe specific DUF999 family protein } 3 & -2.6148015 & 2.50530948 & 1.26 \mathrm{E}-05\end{array}$

\#N/A $\quad-2.62512142 .82334675 \quad 1.73 E-07$

prospore membrane copper transmembrane transporter $M \cdot-2.6383565 \quad 5.51133989 \quad 6.34 \mathrm{E}-39$

\#N/A $\quad-2.6760743 \quad 1.86514290 .00120474$

\#N/A $\quad-2.68889462 .135953820 .00020256$

\#N/A $\quad-2.69733152 .36345298 \quad 3.18 \mathrm{E}-05$

$\begin{array}{lll}-2.6983944 & 7.86392712 & 4.90 \mathrm{E}-150\end{array}$

$-2.7115696 \quad 6.78345468 \quad 2.43 \mathrm{E}-63$

$-2.72717165 .13704534 \quad 7.72 \mathrm{E}-33$

$-2.7392884 \quad 1.16928044 \quad 0.04561358$

$\begin{array}{lll}-2.7450088 & 1.16963333 & 0.04553144\end{array}$

$\begin{array}{lll}-2.7469819 & 7.03153368 & 2.07 \mathrm{E}-22\end{array}$

$\begin{array}{lll}-2.7486827 & 1.58494902 & 0.00382112\end{array}$

$-2.7781192 \quad 3.30483405 \quad 9.73 \mathrm{E}-10$

$-2.7977056 \quad 3.32039114 \quad 5.84 \mathrm{E}-10$

$\begin{array}{lll}-2.8021426 & 2.20534182 & 0.0001117\end{array}$

$\begin{array}{lll}2.8043766 & 5.37583361 & 1.71 \mathrm{E}-29\end{array}$

$-2.8210442 \quad 5.33336568 \quad 2.88 \mathrm{E}-29$

$-2.826222 \quad 4.65826065 \quad 6.80 \mathrm{E}-22$

$-2.84830215 .13520902 \quad 2.12 \mathrm{E}-29$

$-2.84830215 .13520902 \quad 2.12 \mathrm{E}-29$

$\begin{array}{llll}-2.8852406 & 1.98778554 & 0.00024502\end{array}$

$\begin{array}{llll}-2.900158 & 8.83329575 & 3.93 \mathrm{E}-151\end{array}$

$-2.96789591 .68530655 \quad 0.00449506$

$-2.9724822 \quad 3.34922149 \quad 6.47 \mathrm{E}-10$

$-2.9903882 \quad 6.29990799 \quad 9.06 \mathrm{E}-52$

plasma membrane high-affinity glucose:proton symporter $\begin{array}{rrrr}r & -3.013788 & 7.67616272 & 2.11 \mathrm{E}-154\end{array}$

$\# N / A$

$\begin{array}{llr}-3.0554881 & 2.80253053 & 4.25 \mathrm{E}-08\end{array}$

$\begin{array}{lll}-3.0939947 & 1.77747682 & 0.00053826\end{array}$

$\begin{array}{lll}-3.1405241 & 8.241741 & 3.20 \mathrm{E}-212\end{array}$

$\begin{array}{llr}-3.1671149 & 5.60715445 & 1.16 \mathrm{E}-32\end{array}$

-3.1715326 $8.73056817 \quad 3.46 \mathrm{E}-250$

$\begin{array}{llll}-3.1887532 & 1.36267154 & 0.00511639\end{array}$

$\begin{array}{lll}-3.1904392 & 2.70904326 & 4.65 \mathrm{E}-08\end{array}$

$\# N / A$

$\# \mathrm{~N} / \mathrm{A}$

$-3.19924064 .72446184$

$7.26 \mathrm{E}-31$

3.21740696 .44555519

$\begin{array}{lll}-3.2316023 & 2.20554211 & 1.96 \mathrm{E}-05\end{array}$

$\# \mathrm{~N} / \mathrm{A}$

$-3.2525797 \quad 4.31861135$

5.73E-21

$2.33 \mathrm{E}-30$

$4.50 \mathrm{E}-28$

0.002741196

9.46E-46

9.92E-06

0.00131332

$5.56 \mathrm{E}-141$

$1.01 \mathrm{E}-06$

1.23E-111

4.89E-05

8.83E-07

3.85E-37

0.003172707

0.000634351

0.000114299

3.71E-147

4.03E-61

3.39E-31

0.0799795

0.079856038

4.75E-21

0.008911136

6.80E-09

4.18E-09

0.000365249

5.94E- 28

9.70E-28

$1.54 \mathrm{E}-20$

7.24E-28

$7.24 \mathrm{E}-28$

0.0007565

$3.82 \mathrm{E}-148$

0.010334077

4.61E-09

$1.01 \mathrm{E}-49$

2.39E-151

2.39E-07

0.001544214

7.26E-209

$5.00 \mathrm{E}-31$

$1.18 \mathrm{E}-246$

0.011578661

2.60E-07

2.72E-29

$3.86 \mathrm{E}-84$

7.35E-05

Schizosaccharomyces pombe specific protein

1.22E-19 
SPAC5D6.09C

SPAC3G9.11C

SPBPB2B2.14C

SPNCRNA.1121

SPNCRNA.07

SPNCRNA.1314

SPCC191.11

SPBPB2B2.07C

SPNCRNA.473

SPNCRNA.1098

SPNCRNA.1120

SPCC417.15

SPAPB24D3.10

SPNCRNA.1313

SPNCRNA.1256

SPNCRNA.1223

SPNCRNA.549

SPCC191.10

SPNCRNA.32

SPNCRNA.217

SPBC359.06

SPNCRNA.393

SPNCRNA.513

SPAC589.11

SPNCRNA.1460

SPAC1F8.01

SPAC1F8.02C

SPNCRNA.1559

SPNCRNA.352

SPNCRNA.37

SPCC794.16

SPNCRNA.209

SPBC1348.14

SPNCRNA.22

SPNCRNA.603

SPNCRNA.410

SPNCRNA.30

SPBC32H 8.15 mug86

SPBPB2B2.14C

$\# N / A$

N/A

inv1

PBBPB2B2.07C

$\# \mathrm{~N} / \mathrm{A}$

$\# N / A$

$\# N / A$

\#N/A

agl1

$\# \mathrm{~N} / \mathrm{A}$

$\# \mathrm{~N} / \mathrm{A}$

$\# N / A$

$\# \mathrm{~N} / \mathrm{A}$

SPCC191.10

$\# \mathrm{~N} / \mathrm{A}$

$\# N / A$

mug14

$\# N / A$

$\# \mathrm{~N} / \mathrm{A}$

pth4

\#N/A

ght3

shu1

$\#$ N/A

$\# N / A$

$\# N / A$

$\# N / A$

$\# \mathrm{~N} / \mathrm{A}$

ght7

\#N/A

$\# N / A$

$\# N / A$

$\# N / A$

$\# N / A$ plasma membrane acetate transmembrane transporter ( $\mathrm{pr}$

pyruvate decarboxylase (predicted)

S. pombe specific DUF999 protein family 8

$\# N / A$

$\# N / A$

$$
\# N / A
$$

external invertase, beta-fructofuranosidase Inv1

S. pombe specific DUF999 protein family 7

$$
\text { \#N/A }
$$

$\# N / A$

$\# N / A$

$\# N / A$

maltose alpha-glucosidase $\mathrm{Agl1}$

$\# N / A$

$\# N / A$

$\# N / A$

$\# N / A$

Schizosaccharomyces pombe specific protein

$\# \mathrm{~N} / \mathrm{A}$

$\# N / A$

adducin, involved in actin cytoskeleton organization

$\# N / A$

$\# N / A$

mitochondrial translation release factor

\#N/A

plasma membrane gluconate:proton symporter Ght3

cell-surface heme aquisition protein Shu1

$\# N / A$

$\# N / A$

$\# N / A$

$\# N / A$

\#N/A

$\begin{array}{lll}-3.31633 & 8.10346345 \quad 4.38 \mathrm{E}-147\end{array}$

$\begin{array}{lll}-3.3205653 & 8.70514074 & 1.02 \mathrm{E}-71\end{array}$

$\begin{array}{lll}-3.3485412 & 2.82289839 & 2.18 \mathrm{E}-09\end{array}$

$-3.3650973 \quad 5.99736839 \quad 5.18 \mathrm{E}-68$

$\begin{array}{lll}-3.3715427 & 5.25004444 \quad 1.71 E-37\end{array}$

$\begin{array}{lll}-3.3849829 & 2.8461931 & 1.16 \mathrm{E}-09\end{array}$

$\begin{array}{lll}-3.394427 & 7.91200419 & 2.66 \mathrm{E}-126\end{array}$

$\begin{array}{lll}-3.5124376 \quad 1.53181995 \quad 0.00180544 & 0\end{array}$

$\begin{array}{llll}-3.6710462 & 2.13567407 \quad 4.04 \mathrm{E}-06\end{array}$

$-3.7306254 \quad 5.07030263 \quad 2.85 E-38$

$-3.7440056 \quad 4.41607548 \quad 1.21 \mathrm{E}-29$

$-3.74923542 .20357143 \quad 2.36 \mathrm{E}-05$

$\begin{array}{lll}-3.7559431 & 10.2519919 & 3.35 \mathrm{E}-40\end{array}$

$\begin{array}{lll}-3.8920071 & 2.98974174 & 1.53 \mathrm{E}-12\end{array}$

$-3.9671783 \quad 3.52404357 \quad 5.84 \mathrm{E}-18$

$-3.98576094 .04403194 \quad 1.01 \mathrm{E}-20$

$\begin{array}{lll}-4.0457667 & 3.59024479 \quad 9.79 E-19\end{array}$

$\begin{array}{lll}-4.1943415 & 2.91059612 & 3.48 \mathrm{E}-11\end{array}$

$\begin{array}{lll}-4.2446057 & 2.55929871 \quad 6.58 \mathrm{E}-07\end{array}$

$-4.36466373 .06442292 \quad 3.94 \mathrm{E}-12$

$\begin{array}{lll}-4.41263 & 8.69486144 \quad 2.59 \mathrm{E}-17\end{array}$

$\begin{array}{lll}-4.5615878 & 1.09920238 & 0.00953619\end{array}$

$-4.6347392 \quad 2.84551955 \quad 2.26 \mathrm{E}-13$

$\begin{array}{lll}-4.6623818 & 3.29045077 \quad 1.49 \mathrm{E}-16\end{array}$

$\begin{array}{lll}-4.6950636 & 2.88905799 & 5.20 \mathrm{E}-14\end{array}$

$\begin{array}{lll}-4.9772568 & 8.91307991 \quad 2.59 \mathrm{E}-28\end{array}$

$-5.1496523 \quad 4.95191346 \quad 3.37 \mathrm{E}-51$

$\begin{array}{lll}-5.2672471 & 2.70955697 & 7.46 \mathrm{E}-12\end{array}$

$\begin{array}{llll}-5.3380081 & 1.47783518 & 0.00022477\end{array}$

$\begin{array}{lll}-5.3402188 & 1.47798839 & 0.00013577\end{array}$

$-5.4359375 .88486551 \quad 1.37 \mathrm{E}-91$

$-5.60688161 .63535113 \quad 4.77 \mathrm{E}-05$

plasma membrane hexose transmembrane transporter Ght -5.63456457 .94113774$

\#N/A

$-5.8435967 \quad 1.77795487$

$\begin{array}{ll}-6.16101853 .44260486 \\ -6.5511707 & 2.27015158\end{array}$

$\begin{array}{ll}-6.5511707 & 2.27015158\end{array}$

$-7.53051583 .04822762$

$\# N / A$

$\# N / A$

$\# N / A$

$-7.55375383 .06698216$
$2.98 \mathrm{E}-144$

$2.24 \mathrm{E}-69$

$1.45 \mathrm{E}-08$

1.04E-65

$9.17 \mathrm{E}-36$

8.09E-09

$1.39 \mathrm{E}-123$

0.00456788

$1.72 \mathrm{E}-05$

$1.66 \mathrm{E}-36$

4.30E-28

$8.74 \mathrm{E}-05$

2.22E-38

1.49E-11

9.16E-17

2.10E-19

$1.64 \mathrm{E}-17$

2.89E-10

$3.12 \mathrm{E}-06$

3.66E-11

3. $80 \mathrm{E}-16$

0.020184689

2.37E-12

2.05E-15

$5.68 \mathrm{E}-13$

$8.31 \mathrm{E}-27$

3.64E-49

$6.72 \mathrm{E}-11$

0.000698421

0.000438083

4.23E-89

0.000166463

2.13E-05

6.16E-19

$1.78 \mathrm{E}-09 \quad 1.20 \mathrm{E}-08$

5.02E-18 7.92E-17

$1.00 \mathrm{E}-15$ 